\title{
REPÈRES POUR \\ L'ORIENTATION
}

Sous la direction de Jonas Masdonati, Koorosh Massoudi et Jérôme Rossier

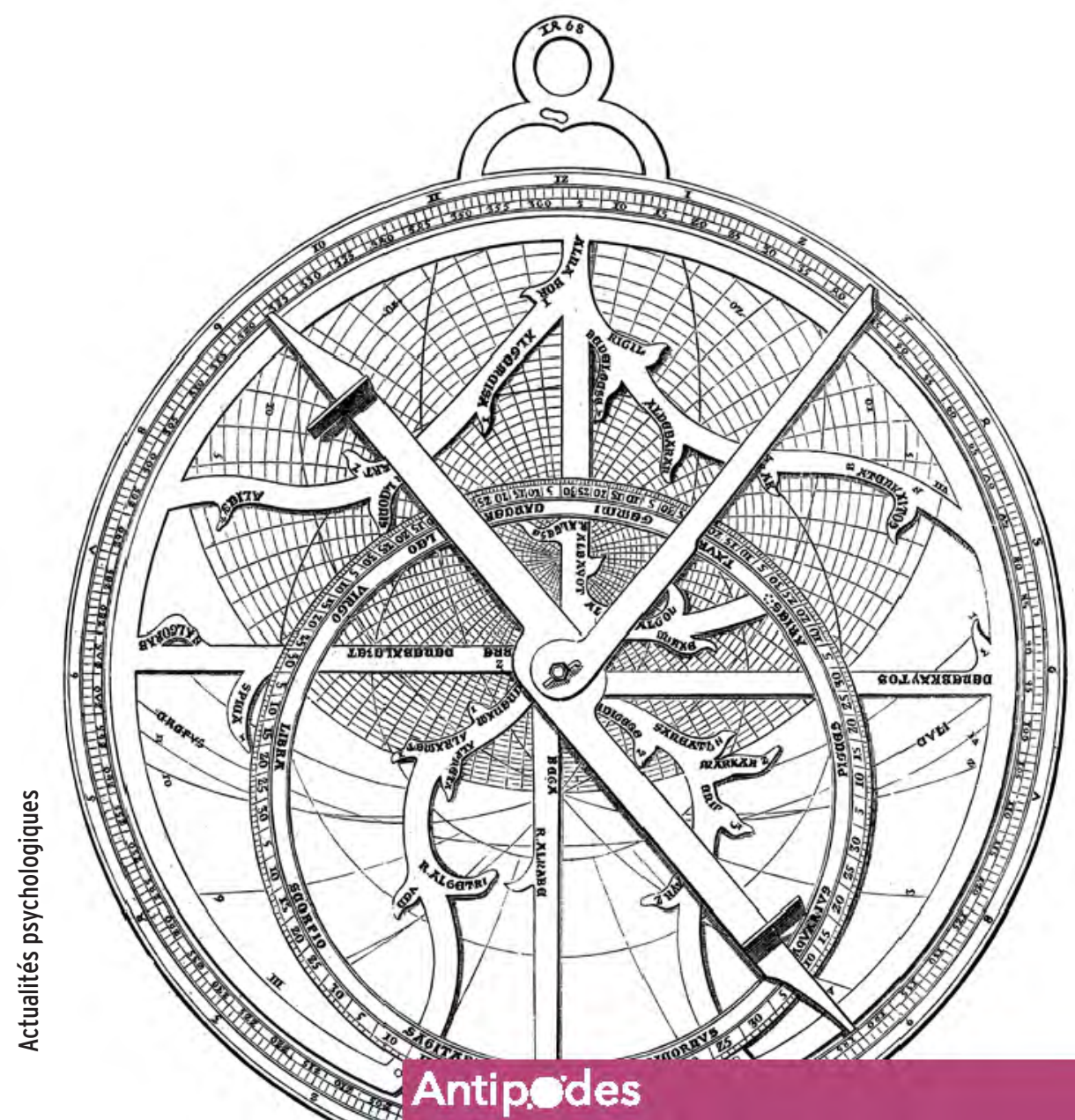





\section{REPÈRES POUR L'ORIENTATION}




\section{REMERCIEMENTS}

L'édition de ce livre a reçu le soutien de la Fondation Chuard Schmid, de l'Université de Lausanne et de la Société académique vaudoise.

L'étape de la prépresse de cette publication a été soutenue par le Fonds national suisse de la recherche scientifique.

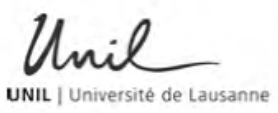

\section{Société \\ Académique Vaudoise}

Les Éditions Antipodes bénéficient d'une prime d'encouragement de l'Office fédéral de la culture pour les années 2019-2020.

\section{MISE EN PAGE}

Fanny Tinner | chezfanny.ch

\section{CORRECTION}

Adeline Vanoverbeke

\section{ILLUSTRATION DE COUVERTURE}

(C) Nastasic/iStockphoto.com

\section{(c) $(i)(8) \Theta$}

Ce texte est sous licence Creative Commons: elle vous oblige, si vous utilisez cet écrit, à en citer l'auteur.e, la source et l'éditeur original, sans modification du texte ou de l'extrait et sans utilisation commerciale.

(C) 2019, Éditions Antipodes

École-de-Commerce 3, 1004 Lausanne, Suisse

www.antipodes.ch - editions@antipodes.ch

DOI : 10.32551/ANTIPODES.11551

Papier, ISBN : 978-2-88901-155-1

PDF, ISBN : 978-2-88901-977-9

EPUB, ISBN : 978-2-88901-978-6 
Sous la direction de Jonas Masdonati,

Koorosh Massoudi et Jérôme Rossier

\section{REPÈRES POUR L'ORIENTATION}

Antipodes Collection Actualités psychologiques 



\section{PRÉFACE}

[ n proposant comme titre d'un nouvel ouvrage « repères pour - l'orientation", Jonas Masdonati, Koorosh Massoudi et Jérôme L Rossier amènent d'abord à nous interroger sur un détail cocasse. L'orientation, dont la vocation est d'aider des individus à trouver des repères pour prendre des directions de vie, aurait-elle besoin elle-même de s'y repérer? À y regarder de près, c'est pourtant une question capitale qui est posée. En effet, les actes d'orientation s'appuient sur des pratiques et des cadres conceptuels diversifiés et complexes, qui impliquent de mobiliser un grand nombre de compétences de nature technique, humaine, procédurale, gestionnaire, informationnelle, etc. Il importe donc, dans ce domaine, de tenir compte de l'exposé des idées récentes tout en s'appuyant sur de nouvelles approches. L'orientation comme discipline scientifique et professionnelle apparaît ancrée dans les évolutions du paysage économique, social et politique, sans être à l'écart des changements générés par les débats scientifiques. Force est de constater que depuis les premières réflexions de Parsons (1909), à l'origine de ses fondements, l'orientation a beaucoup évolué dans ses repères et ce phénomène s'amplifie depuis l'avènement de la mondialisation et de la libéralisation de l'économie. Rien ne serait plus dommageable que de laisser s'imposer des théories obsolètes, des pratiques d'un autre âge ou, pire encore, des cadres de référence implicites. L'orientation se doit d'être pensée comme une science, qui s'appuie sur des cadres empiriques, des théories bien construites, des méthodes innovantes et actualisées, et ce à tous les âges de la vie.

À ce titre, l'ouvrage Repères pour l'orientation apporte tout un ensemble de contributions qui vont dans le sens de l'histoire, en exposant des pratiques et théories nouvelles, étayées par des exposés de cas concrets qui permettent de saisir ce qui se joue dans 
les pratiques et de définir comment articuler le savoir et l'intervention. Il prend appui sur des sujets particulièrement légitimes aujourd'hui: comprendre et analyser les parcours et leur impact sur les personnes, prendre en compte les publics les plus fragiles et penser leur prise en charge, définir comment s'appuyer sur des atouts et ressources pour permettre aux personnes de s'adapter ou de transformer le monde auquel ils doivent faire face.

Les contributions des auteur.e.s s'appuient sur l'expérience accumulée dans de nombreux pays, par le biais de recherches et synthèses de travaux qui, au XXI ${ }^{\mathrm{e}}$ siècle, ont permis de considérer que l'orientation se situe comme une discipline dynamique, en progrès. Ainsi une place singulière est-elle accordée aux méthodes qualitatives qui, il y a une vingtaine d'années, étaient encore marginales dans le champ de l'orientation.

Les lecteurs et lectrices pourront avantageusement utiliser cet ouvrage comme un manuel d'autoformation théorique et pratique, ouvrant vers l'actualisation des connaissances, permettant d'enrichir des interventions et de questionner une posture réflexive. Il peut être considéré comme une mine pour les chercheurs et chercheuses grâce à l'apport et à la mise à jour de notions très récentes dans le giron de la recherche, mais aussi pour les praticien.ne.s, qui y puiseront de quoi renouveler leurs cadres d'intervention et leurs méthodes. De nombreux schémas et des ressources bibliographiques contemporaines complètent très utilement les exposés. C'est enfin un ouvrage qui répond à une fonction noble, qui est celle d'amener les lecteurs et lectrices à "penser» l'orientation, non pas comme une succession d'outils ou de solutions à des problèmes, mais bien comme une question scientifique et de société, fertile et fondamentale pour le développement de l'humanité.

Jean-Luc Bernaud, professeur des universités en psychologie au Conservatoire national des arts et métiers, Paris 


\section{INTRODUCTION}

KOOROSH MASSOUDI ET JONAS MASDONATI

(UNIVERSITÉ DE LAUSANNE)

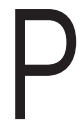

endant longtemps, la psychologie du conseil et de l'orientation s'est focalisée principalement sur l'accompagnement de la transition de l'école vers l'emploi. Fortement inspirée du paradigme de l'appariement individu-travail, l'idée centrale était ici que le parcours professionnel, fondé sur un premier choix mûrement réfléchi et bien documenté, se déroulerait de manière harmonieuse et prévisible dans un contexte socioéconomique relativement stable (Guichard, 2015). Or, depuis au moins deux décennies, praticien-ne-s et chercheurs-ses observent, si ce n'est une complexification, au moins un changement des problématiques qu'elles et ils rencontrent. D'abord, le contexte dans lequel se projettent les individus devient plus mouvant et instable, et par conséquent les choix qui s'y opèrent deviennent éphémères, les transitions multiples et les parcours imprévisibles (Olry-Louis, Vonthron, Vayre, \& Soidet, 2017; Vuori, Blonk, \& Price, 2015). De plus, le public cible de l'orientation se diversifie, présentant des caractéristiques variées, des besoins spécifiques et des demandes inédites, auxquels on ne saurait plus répondre par des méthodes d'intervention préétablies et standardisées. Ainsi, la discipline traverse aujourd'hui une période de réformes et de remises en question, tant du point de vue de ses modèles théoriques de compréhension du comportement vocationnel (Kenny, Blustein, \& Meerkins, 2018; Swanson, 2014) que de ses logiques et méthodes d'intervention (Maree, 2010; McMahon, 2017). Plus précisément, les nouveaux défis de la psychologie du conseil et de l'orientation se conjuguent selon trois niveaux, et les trois parties qui composent cet ouvrage sont conçues comme autant de suggestions de réponses, certes partielles, et parfois encore en gestation, à ces trois types de défis. 
Le premier niveau de défi résulte de la complexification croissante des "parcours d'orientation». Aux cheminements traditionnels, linéaires, ponctués par des étapes prévisibles et structurés par des moments charnières institutionnalisés (la fin de l'école obligatoire, l'insertion professionnelle, la sortie du marché du travail) s'ajoutent différentes configurations de parcours plus hétérogènes et relativement inédits (Olry-Louis et al., 2017; Vuori et al., 2015). Ces nouvelles formes de trajectoires sont marquées par des alternances ou des intermittences professionnelles (travail-chômage, travail-formation, etc.), et des transitions de carrière (école-travail, travail-formation, travail-retraite, etc.) dont la fréquence, la durée et l'imprévisibilité vont grandissantes (Fournier, Zimmermann, Gauthier, Masdonati, \& Lachance, 2016). Dans les textes réunis dans la première partie de l'ouvrage, il est donc question de s'atteler à des problématiques qui relèvent des étapes caractéristiques des parcours professionnels et des défis qu'elles réservent aux individus, depuis leurs efforts pour une première insertion dans le monde du travail jusqu'aux préparations nécessaires pour en sortir, en passant par les adaptations aux transitions de carrière et les tentatives d'articulation plus ou moins réussies entre les sphères de vie. Ainsi, Valérie Cohen-Scali, Jacques Pouyaud et Jean Guichard ouvrent les feux avec les adultes émergent.e.s et les défis auxquels elles et ils font face. Ils rappellent que cette période de la vie - qui s'étend de 18 à 29 ans - est centrale pour la construction de soi, mais également critique pour le parcours professionnel, car souvent marquée par les premiers pas dans le monde du travail. Grégoire Bollmann, Éva Clot-Siegrist et Levke Henningsen poursuivent avec une réflexion sur les défis qui s'imposent aux hommes et aux femmes déjà engagé.e.s dans leur carrière professionnelle, et qui doivent à ce titre concilier les différentes sphères de leur vie et leurs exigences en termes de rôles et de responsabilités. Le chapitre 3, proposé par Jonas Masdonati, Sylvie Franz et Marc Abessolo, rappelle la multiplication des transitions tout au long des parcours professionnels. À travers une réflexion sur les reconversions professionnelles, les auteur.e.s proposent aussi bien une conceptualisation des enjeux et des conjugaisons de cette transition particulière que des pistes d'intervention et d'accompagnement de celles et ceux qui s'y essaient. Enfin, Ariane Froidevaux et Christian Maggiori permettent de «boucler la boucle» de ce survol des parcours professionnels en s'intéressant à la dernière transition qui les caractérise: 
celle vers la retraite, avec ses défis, son lot d'ajustements et ses nouvelles configurations dans un contexte marqué par la perspective des carrières durables et des "seniors" actifs-ves.

Un deuxième type de défi découle de la diversification des populations qui transitent par les mondes de l'éducation et du travail et qui pourraient, à un moment ou un autre de leur parcours, bénéficier des prestations d'orientation (Duffy, Blustein, Diemer, \& Autin, 2016). Au public «typique» et «traditionnel» de l'orientation, composé d'adolescent.e.s qui élaborent un projet de carrière à l'approche de la fin du secondaire, s'ajoutent ainsi d'autres groupes, plus hétéroclites, qui courent le risque d'une exclusion à plus ou moins long terme face aux exigences et à l'intransigeance du marché du travail. Afin de renforcer une approche inclusive et socialement engagée de l'orientation, il nous semble essentiel d'étudier ces nouveaux publics, de mieux comprendre leurs besoins spécifiques et d'identifier les obstacles qu'ils rencontrent, pour favoriser leur intégration professionnelle et leur inclusion sociale (Guichard, 2016; Nota \& Rossier, 2015). C'est ainsi que, dans la deuxième partie, le regard est plutôt porté sur des groupes, encore peu ou mal connus dans notre champ, qui se trouvent en situation de vulnérabilité sur le marché du travail et dont l'insertion socioprofessionnelle est à risque. Au chapitre 5, rédigé par Philippe Handschin et Marco Schiavetta, il est donc question des écueils rencontrés par les «NEETs», ces jeunes adultes qui ne sont ni en emploi, ni en formation, ni en stage et qui, dans des marchés du travail survalorisant la qualification et les diplômes, rencontrent d'importantes difficultés d'insertion professionnelle déjà en début de parcours. Au chapitre 6, Kokou Amenyona Atitsogbe, Shagini Udayar et Federico Durante s'attellent pour leur part aux enjeux particuliers de l'accompagnement des migrant.e.s, que ce soit pour les aider à faire un choix d'orientation ou pour faciliter leur entrée dans le marché du travail, en Suisse en l'occurrence. Une autre population peu connue dans notre domaine et pourtant particulièrement exposée au risque de l'exclusion socioprofessionnelle est celle des personnes en situation de handicap, qui sont au cœur des réflexions proposées au chapitre 7 par Ilaria Di Maggio, Sara Santilli, Maria Cristina Ginevra, Giuseppe Martella et Laura Nota. Dans le Chapitre 8, Saliha Kozan, Jonas Masdonati, Lily Konowitz et David Blustein se focalisent enfin sur les stratégies qu'il est nécessaire et souhaitable de déployer pour permettre à tout individu, et 
en particulier aux personnes en situation de marginalisation ou aux faibles ressources socio-économiques, d'accéder à un travail considéré comme « décent».

Enfin, la complexification des parcours et la diversification des publics appellent naturellement à une réadaptation, voire un renouvellement des pratiques afin de répondre à des demandes et des problématiques inédites. Ainsi, un troisième niveau de défi s'adresse aux praticien.e.s, les poussant à dépasser une pratique «orthodoxe» de l'orientation, fortement teintée de l'évaluation psychométrique et du choix professionnel par appariement, ou du moins à la compléter par d'autres formes d'intervention. Outre les dispositions individuelles stables - traits de personnalité, intérêts ou valeurs -, ces nouvelles pratiques tiennent compte des enjeux identitaires, des processus de construction de soi et des influences systémiques et contextuelles impliqués dans les parcours et les transitions (McMahon, 2017). Enfin, tout en reconnaissant les obstacles et les difficultés que rencontrent les personnes sollicitant un accompagnement, ces approches émergentes du counseling d'orientation insistent également sur l'identification et le renforcement de leurs ressources - personnelles ou sociales - afin de les aider à combler leurs vulnérabilités (Hartung \& Taber, 2008; Massoudi et al., 2018). Dans la troisième partie, le regard passe donc des vulnérabilités aux ressources et les logiques d'interventions proposées visent le renforcement des forces et des atouts plutôt que le traitement et la réduction des troubles et des déficits. Dans le chapitre 9, Koorsoh Massoudi, Ieva Urbanaviciute, Jenny Hoffman et Fabian Gander proposent d'aborder la santé et le bien-être au travail, non pas comme résultant automatiquement de l'absence de symptômes de détresse et d'épuisement, mais également au regard des manifestations positives de l'engagement, du sens et du plaisir au travail. Le chapitre 10 donne l'occasion à Yann Le Bossé, Bruno Bourassa, Manon Chamberland, Geneviève Fournier, Simon-Pierre Tremblay et Bernard Vallerie de proposer leur conception d'une approche centrée sur les ressources à travers le développement du pouvoir d'agir et son application dans le domaine de l'accompagnement en orientation. Shékina Rochat et Éloïse Banet abordent la problématique typique de l'orientation, celle du choix professionnel, mais avec un éclairage original qui implique l'identification et l'intégration des émotions. Les auteures proposent ainsi le travail sur et avec les émotions comme une 
activité utile pour dépasser l'indécision vocationnelle. Le chapitre final, écrit par Peter McIlveen et Allison Creed, vise quant à lui à mettre en évidence les avantages d'une approche narrative de l'accompagnement en orientation, avec le processus dialogique et l'utilisation de la métaphore de la conversation au service de la construction de sens.

En somme, cet ouvrage constitue un tour d'horizon, nous semble-t-il, des principaux enjeux qui marquent la psychologie du conseil et de l'orientation contemporaine, sans prétendre en offrir une vision exhaustive ou y apporter des solutions définitives. Comme son titre l'indique, son objectif est plutôt de proposer des points de repère pour saisir les problématiques nouvelles dans toute leur complexité et pour discerner des pistes d'accompagnement et d'intervention auprès des personnes qui y font face. Ainsi, pour renforcer le dialogue entre la recherche et la pratique, chaque chapitre est le fruit de la collaboration d'auteur.e.s issu.e.s aussi bien du domaine académique que du monde de l'intervention en orientation. Les textes proposés allient donc une approche conceptuelle et distanciée des phénomènes abordés - à travers un état des lieux des concepts théoriques et des résultats empiriques sur le sujet abordé - à une vision pragmatique et concrète - via des vignettes cliniques, des propositions d'outils ou des pistes d'intervention. Signalons enfin que, même si les problématiques sont parfois adaptées à un contexte précis, avec ses normes socioculturelles, son marché du travail et son système d'éducation spécifiques, le livre réunit plus de 40 auteur.e.s venant de six pays différents, et que chaque chapitre a été expertisé à l'aveugle par deux évaluateurs-trices. De ce fait, des textes centrés sur la réalité suisse, voire suisse romande, s'alternent avec des textes plus généraux et couvrant une réalité plus internationale. Nous invitons les lectrices et lecteurs à naviguer à leur guise à travers cette constellation de textes, et espérons qu'elles et ils y trouveront, qui quelques repères pour sa pratique future, qui de nouveaux horizons pour ses projets de recherche. 


\section{RÉFÉRENCES}

Duffy, R. D., Blustein, D. L., Diemer, M. A., \& Autin, K. L. (2016). The psychology of working theory. Journal of Counseling Psychology, 63, 127-148. Doi: 10.1037/cou0000140.

Fournier, G., Zimmermann, H., Gauthier, C., Masdonati, J., \& Lachance, L. (2016). Vers l'élaboration d'un cadre d'analyse des parcours professionnels: l'éclairage de l'approche du Parcours de vie et des théories récentes en développement de carrière. In G. Fournier, E. Poirel, \& L. LaChance (éds), Education et vie au travail: Perspectives contemporaines sur les parcours de vie professionnelle (pp. 93-143). Québec: Presses de l'Université Laval.

Guichard, J. (2016). Life- and working-design interventions for constructing a sustainable human(e) world. Studia Poradoznawczel Journal of Counsellogy, 5, 179-190.

Guichard, J. (2015). From vocational guidance and career counseling to Life Design dialogues. In L. Nota \& J. Rossier (éds.), Handbook of Life Design: From practice to theory and from theory to practice (pp. 11-25). Göttingen: Hogrefe.

Hartung, P. J., \& Taber, B. J. (2008). Career Construction and Subjective Well-Being. Journal of Career Assessment, 16, 75-85. Doi : $10.1177 / 1069072707305772$.

Kenny, M. E., Blustein, D. L., \& Meerkins, T. M. (2018). Integrationg relational perspectives in career counseling practice. The Career Development Quarterly, 66, 135-148. Doi: 10.1002/ cdq. 12128.

Maree, K. (éd.) (2010). Career counseling: Methods that work. Le Cape: Juta.

Massoudi, K., Abessolo, M., Atitsogbe, K. A., Banet, E., Bollmann, G., Dauwalder, J.-P., Handschin, P., Maggiori, C., MasDOnati, J., Rochat, S., \& Rossier, J. (2018). A value-centered approach to decent work. In V. Cohen-Scali, J. Pouyaud, V. M. Podgorny, V. Drabik-Podgorna, G. Aisenson, J.-L. Bernaud, I. A. Moumoula \& J. Guichard (éds), Interventions in Career Design and Education: Transformation for Sustainable Development and Decent Work. UNESCO Chair on Life Long Guidance and Counseling: Springer. 
McMahon, M. (éd.) (2017). Career counseling: Constructivist approaches ( $2^{\mathrm{e}}$ édition). Londres/New York: Routledge.

Nota, L., \& Rossier, J. (éds.) (2015). Handbook of the Life Design paradigm: From practice to theory and from theory to practice. Göttingen: Hogrefe.

Olry-Louis, I., Vonthron, A.-M., Vayre, E., \& Soidet, I. (éds) (2017), Les transitions professionnelles: Nouvelles problématiques psychosociales. Paris: Dunod.

Swanson, J. L. (2014). Traditional and emerging career development theory and the psychology of working. In D. L. BLusteIN (éd.), The Oxford handbook of the psychology of working (pp. 49-67). New York: Oxford University Press.

Vuori, J., Blonk, R., \& Price, R. H. (éds) (2015). Sustainable working lives: Managing work transitions and health throughout the life course. Dordrecht: Springer. Doi: 10.1007/978-94-017-9798-6. 

PREMIÈRE PARTIE

ORIENTATION ET PARCOURS 



\section{LES MÉTHODES DE LIFE DESIGN POUR LA CONSTRUCTION DE SOI ET L'ORIENTATION PROFESSIONNELLE DES ADULTES ÉMERGENT·E.S}

VALÉRIE COHEN-SCALI, JACQUES POUYAUD ET JEAN GUICHARD (CNAM/INETOP, PARIS)

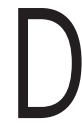

ans la plupart des pays industrialisés, les jeunes gens traversent une période de transition de l'école au travail qui, sur la base notamment des travaux d'Arnett (2006) concernant l'adulte émergent.e, peut être décrite comme plus longue, chaotique et aléatoire dans un monde où les repères d'étayage se réduisent. Cette liquéfaction de la société (Bauman, 2007) ramène la question de la construction de soi et de l'identité au centre des parcours personnels et professionnels. Pour les adultes émergent.e.s, la transition de l'école au travail peut être définie comme un moment d'autonomisation associant des temps de formation, d'activité et de recherche d'emploi, mais aussi un moment de bouleversements psychologiques notamment dus aux changements dans la représentation du monde tels qu'ils sont mobilisés lors des transitions psychosociales (Parkes, 1971).

L'approche du Life Design (Savickas et al., 2009) développe depuis une dizaine d'années une réflexion sur les modalités d'interventions d'accompagnement à l'orientation permettant de faire face à ces défis de la construction d'un sujet dans les sociétés de la modernité tardive (Rosa, 2012). L'objectif de cette approche centrée sur l'accompagnement à la construction de soi est d'aider les individus à construire des perspectives d'avenir et à formuler leurs propres normes de vie leur permettant de donner sens et direction à leur existence (Savickas et al., 2009). Qu'en est-il de cette construction dans le cas de la transition de l'école à l'emploi des adultes émergent.e.s d'aujourd'hui? Dans quelle mesure ces approches peuvent permettre de comprendre et d'accompagner la construction identitaire à cette période de la vie? L'objectif de ce chapitre est de présenter deux modalités d'accompagnement s'inscrivant dans le paradigme du Life Design, dans la perspective 
d'un accompagnement à la construction de soi des adultes émergent.e.s. Une première partie détaillera les concepts principaux des modèles constructivistes sur lesquels ces approches reposent (Guichard, 2009; Collin Guichard, 2011), ainsi que les principaux enjeux liés à la construction identitaire des adultes émergent.e.s. Une deuxième partie présentera les Dialogues de conseil en Life Design (DCLD) (Guichard, Bangali, Cohen-Scali, Pouyaud, \& Robinet, 2017), ainsi que l'entretien de construction de carrière (Career Construction Interview - CCI - Savickas, 2011) comme deux modalités d'intervention utilisant ce cadre théorique. Dans une troisième partie, le cas de Marc, adulte émergent changeant d'emploi après une première expérience professionnelle, sera détaillé comme l'illustration de l'utilisation de ces méthodes dans les situations de transition psychosociale. Enfin, la discussion mettra en évidence certains points communs et différences entre ces méthodes et la conclusion reprendra les apports de ces approches dans la perspective d'un enjeu plus large pour le développement d'un rapport décent au travail (BIT, 2013).

\section{LIFE DESIGN ET CONSTRUCTION DE SOI}

\section{EN CONTEXTE CHEZ LES ADULTES ÉMERGENTS}

Les approches d'accompagnement présentées s'inscrivent dans une perspective constructiviste qui permet de rendre compte et d'accompagner les transformations identitaires des individus.

\subsection{LE LIFE DESIGN: UNE PERSPECTIVE CONSTRUCTIVISTE}

Les interventions de conseil en Life Design ont pour objet la construction de soi. Elles se réferent à une épistémologie de type "constructiviste-constructionniste» (Collin Guichard, 2011), dans laquelle la connaissance que les individus ont sur le monde n'est pas considérée comme donnée ou à enregistrer, mais plutôt à construire activement dans les interactions sociales, le langage, les actions, afin d'y donner du sens. Le soi (self) est également une de ces connaissances à construire, non pas comme une entité ou un ensemble de traits de personnalité, mais comme "un système complexe de processus actifs et interactifs s'auto-organisant» (Mahoney, 2002, p. 748), «un édifice mental complexe que chacun construit en mettant en œuvre une variété de processus mentaux" (Bruner, 1994, p. 41). Les modèles constructivistes soulignent l'importance des 
contextes dans lesquels les individus sont en interactions et interrelations (Pepper, 1942). L'individu est «socialement constitué dans les limites de sa culture, de ses contextes d'interaction et de son langage» (Raskin, 2002, p. 18). En ce sens, ces approches accordent une place centrale au langage et au récit dans la construction du sujet. "La manière dont les gens parlent d'eux-mêmes et de leur monde détermine la nature de leurs expériences» (Raskin, 2002, p. 18). Le soi est vu comme une "fiction utile» qui «désigne le lieu d'où l'homme perçoit le monde et peut agir plutôt que d'être la somme singulière de tout ce qu'il ressent» (Harré, 1998, pp. 3-4). Le soi en tant qu' "histoire perpétuellement réécrite" (Bruner, 1994, p. 53) s'apparente ainsi à un outil psychologique de développement et d'adaptation. La construction de soi est alors un processus continu et dynamique, à partir duquel émerge un soi jamais achevé. C'est un processus actif de négociation, d'organisation, de synthèse, d'intégration et de réflexion.

Si ce processus entre particulièrement en jeu dans les moments de transitions psychosociales, il est singulièrement important au moment du passage à l'âge adulte. L'adolescence comme lieu de construction d'une "thèse de soi" (Dumora \& Boy, 2008) est suivie d'une phase plus ou moins longue et diffuse de mise à l'épreuve de cette thèse, notamment dans le rapport au travail, constitutif de la vie adulte. Le sens, alors construit activement dans le rapport au contexte de vie et de travail mouvant, est central. Il s'agit d'un moment où se jouent la consolidation et l'ajustement de ses propres normes, et corrélativement de celles d'une société qui pourrait être pensée comme plus durable et humaine.

\subsection{LES ADULTES ÉMERGENT·E·S: UNE PÉRIODE CENTRALE}

\section{POUR LA CONSTRUCTION DE SOI}

Les travaux d'Arnett (2000) sur les adultes émergent.e.s ont mis au jour le fait que cette période de la vie (entre 18 à 25 ans et parfois jusqu'à 29 ans; Arnett, 2012) dans les pays développés se caractérise par une certaine anomie et une absence de comportement prédéfini. Les adultes émergent.e.s développent des activités plus variées que les individus d'autres âges de la vie, car ils seraient moins contraints par les demandes associées aux rôles sociaux. Cette exploration se manifeste dans leurs modes de vie.

Ainsi, elles et ils ont une plus grande liberté pour élaborer des rôles singuliers et peuvent inventer des manières d'être et de vivre 
inédites. Elles et ils connaissent des changements de lieux de vie, car la plupart de ces personnes quittent le domicile familial, mais aussi d'activités au quotidien, puisqu'une partie d'entre elles passent de l'enseignement secondaire à l'enseignement supérieur ou au monde du travail. Selon Arnett (2015), l'influence de la globalisation est particulièrement importante au cours de cette période de la vie, car les migrations sont principalement opérées par des jeunes de moins de 30 ans. Ces jeunes sont les plus mobiles. Elles et ils partent de chez eux pour étudier ou pour travailler. Elles et ils sont, davantage que les autres tranches d'âge, confronté.e.s à des cultures variées, à des nouveaux modes de vie, ce qui les conduit à devoir construire une «identité globale» leur permettant de s'adapter à une variété de contextes et d'échanges culturels. Il s'agit alors de négocier leur appartenance à une variété de cultures.

Cette période de la vie et les explorations identitaires rendues alors possibles leur permettent de se préparer aux rôles et de développer des réflexions en lien avec certaines questions essentielles pour leur vie professionnelle future telles que: à quel type de travail suis-je bon-ne? Dans quel type de travail puis-je m'engager sur le long terme? Quelles sont mes chances d'obtenir un travail dans un secteur qui m'intéresse? (Arnett, 2000). Ainsi, au cours de cette période de la vie, les individus présentent des réactions psychologiques caractérisées par l'exploration identitaire, l'instabilité, la centration sur soi, où domine le sentiment "d'être entre deux".

À ces expérimentations identitaires s'associe une recherche d'un sens à donner à sa vie future. Ces jeunes déclarent davantage que les individus d'autres âges de la vie vouloir assumer la responsabilité de leurs actions et décider par elles et eux-mêmes de leurs croyances et de leurs valeurs pour conduire leur vie. C'est en cela que l'adulte émerge chez l'adolescent.e, car au cours de cette période faite de multiples expériences se développe une réflexion sur le type d'adulte qu'elles ou ils peuvent ou souhaitent devenir.

Selon Arnett (2001), les adultes émergent.e.s adopteraient progressivement les attitudes propres à la culture individualiste des pays industrialisés. Les individus de cette catégorie d'âge s'interrogent sur les choix à faire pour leur vie future. Ils s'intéressent aux idéologies existantes et disponibles dans la société et cherchent à les évaluer et les classer de façon à les relier à leurs préoccupations et à ce qu'elles constituent, pour certaines, des possibilités ou des perspectives pouvant donner sens à leur vie. Ce processus est perçu 
comme central dans le fait de devenir adulte (Arnett, Ramos, \& Arnett, 2001).

Ces jeunes ne se perçoivent plus comme des adolescent.e.s et pas encore comme des adultes. Elles et ils ont des sentiments partagés concernant le fait de devenir adultes, car elles et ils en perçoivent les bénéfices, mais également les difficultés et les coûts. Pour McAdams \& Olson (2010), les adultes émergent.e.s développent un modèle de vie qui fournit à leur existence un semblant d'unité, de finalité et de sens. Il s'agit d'un processus «d'orchestration des différentes voix des sois à l'intérieur d'une conversation narrative continue» (McAdams \& Olson, 2010, p. 533). Ainsi, la tâche de cette période est de devenir auteur.e de son identité narrative. À la fin de cette période, les individus doivent pouvoir articuler et internaliser une histoire plus ou moins cohérente à propos de qui elles et ils sont et souhaitent devenir.

Comment donc permettre au mieux une construction active et libre de ses propres normes de développement dans la consolidation de l'âge adulte? Dans ce monde, les jeunes adultes doivent être capables de gérer par elles-eux-mêmes leur vie professionnelle. Cela implique de produire une activité d'anticipation: se préparer à rencontrer des difficultés, envisager des solutions possibles, développer par avance certaines compétences utiles en cas de problème. Il s'agit aussi de s'informer sur les formations, les emplois et l'évolution des secteurs professionnels; de produire des compétences pour résoudre des problèmes nouveaux; de développer des projets innovants, et de réaménager continuellement ses priorités. Les premières expériences de travail constituent ainsi une phase de socialisation à cette nouvelle forme de gestion de sa carrière, qui apparaît centrale en tant que moment de développement de soi. Dans quelle mesure ces expériences peuvent-elles permettre les apprentissages indispensables pour se percevoir comme un.e professionnel.e en devenir?

\subsection{EXPÉRIENCE DE TRAVAIL ET SENS DE SOI}

De manière générale, l'expérience est directement associée à la notion de vécu et de conscience (Jodelet, 2006), c'est-à-dire à un état où le sujet éprouve et ressent son travail et sa vie de manière émotionnelle. Mais l'expérience comporte également une dimension cognitive favorisant l'expérimentation du monde et concourant à la construction de la réalité selon des catégories socialement données. Ainsi, l'expérience est mise en forme par les représentations 
sociales elles-mêmes utilisées pour donner sens à l'expérience. L'expérience intervient donc comme médiation dans la construction des connaissances tout en mobilisant les émotions des sujets et en contribuant à la création et à la transformation des représentations sociales. Elle permet de faire émerger des connaissances nouvelles pour le sujet.

Les expériences de travail tendent tout particulièrement à interroger plusieurs objets sociaux : le soi (à cause des enjeux identitaires, de reconnaissance et de valorisation dans le milieu professionnel), le futur (car les jeunes adultes n'en sont qu'aux premières étapes de leur carrière), le travail (car elles et ils abordent le travail avec certaines attitudes et opinons acquises tout au long de leur vie). Pour Eraut (2004), l'engagement dans des activités de travail induit des apprentissages informels concernant le développement personnel (auto-évaluation, automanagement, gestion des émotions, des relations, apprentissage par l'expérience).

Toutefois, pour que l'expérience puisse aboutir à des connaissances et des compétences nouvelles, il est souvent nécessaire qu'elle soit l'objet d'une élaboration cognitive. Comme le souligne Vygotski (1994, p. 226), "l'action passée au crible de la pensée se transforme en une autre action qui est réfléchie». L'activité consistant à élaborer l'expérience, c'est-à-dire à la transformer en un récit de soi, présente plusieurs caractéristiques (Barbier, 2013). Elle présuppose l'engagement d'un sujet dans des actions intentionnelles de transformation du monde. Elle est une action du sujet sur luimême, dont le produit est une ressource pour l'action. Elle contribue à la construction du soi comme représentation par le sujet de ses ressources, de son pouvoir d'agir, de sa compétence et de son agentivité. Pour Barbier (2013), l'élaboration de l'expérience (qu’il est possible de définir comme transformation de l'expérience en expérience pour soi) survient dans le cadre d'actions singulières de transformation du monde physique, mental ou social, associées à des émotions. Plus particulièrement, elle apparaît dans des situations considérées par le sujet comme nouvelles, inconnues, non contrôlées. Les premières expériences de travail sont des situations nouvelles qui favorisent le processus de réflexivité pour donner un sens pour soi à ce qui nous arrive. C'est aussi ce que Pastré (2013) nomme «le travail de l'expérience». Pour Pastré, cette ascription (ou le fait de reconnaittre comme sien ce qu'on a vécu) ne porte pas sur la totalité du vécu, mais sur certains événements considérés comme 
fondateurs, exemplaires. Cette ascription n'est pas immédiate, mais se prolonge par d'autres événements qui viennent corroborer ces premiers éléments. Les premières expériences de travail sont donc importantes, car il est possible qu'elles se composent d'événements fondateurs et permettent ainsi de constituer la source de l'identité narrative relativement au champ du travail. Les expériences de travail, aussi disparates soient-elles, réélaborées de manière réflexive dans une situation d'entretiens développés dans le cadre du Life Design peuvent ainsi aider les jeunes adultes à donner du sens à leur vie et à poursuivre leur parcours de manière plus favorable. Le rôle de la conseillère ${ }^{1}$ peut être également interpellé pour faciliter la réflexion et l'intégration de ces premières expériences de travail pour la construction de soi de cette population.

\section{LES MODÈLES «SE FAIRE SOI ॥ \\ ET "LA CONSTRUCTION DE CARRIÈRE »}

Les modèles de Guichard et de Savickas sont successivement présentés, dans leurs principes théoriques d'abord, puis du point de vue des méthodes d'intervention qui ont été mises au point pour aider les individus à réfléchir sur leurs expériences et construire leur vie.

\subsection{LE MODÈLE «SE FAIRE SOI » POUR ACCOMPAGNER \\ LE PASSAGE À L'ÂGE ADULTE À TRAVERS LA RÉFLEXIVITÉ SUR L'EXPÉRIENCE PROFESSIONNELLE.}

Le modèle "se faire soi» de Guichard (2004, 2005, 2009) apparaît comme particulièrement intéressant pour analyser la place de ces expériences professionnelles dans la construction de soi et pour aider les jeunes individus à s'engager dans un processus réflexif et dans un ensemble d'activités utiles pour construire des compétences à s'orienter.

Ainsi, pour Guichard (2004, 2009), le processus de construction de soi s'appuie sur les représentations sociales. Cette conceptualisation des représentations de soi et d'autrui est fondée sur l'hypothèse de l'existence de deux structures: les cadres cognitifs identitaires et les formes identitaires subjectives. L'individu disposerait en mémoire à long terme d'un système de cadres cognitifs identitaires (ou système de CCId), c'est-à-dire d'une structure (dont il n'a pas conscience)

1. Nous adoptons une écriture inclusive et, pour cela, nous parlerons de la conseillère et du demandeur afin de ne pas trop alourdir la lecture. 
organisant sa mémorisation personnelle de l'offre identitaire de la société où il vit. Autrement dit, sa mémorisation comprend l'ensemble des informations (représentations, croyances, savoirs, attitudes, affects, etc.) relatives aux catégories sociales, aux modes de catégorisation, aux formes biographiques, etc. relatives aux personnes, aux collectifs et à leurs interactions, ayant cours dans cette société.

Au sein de cette structure, une substructure organise les informations en mémoire de l'individu relatives à lui-même: le système des cadres cognitifs identitaires subjectifs (système de CCIdS). Chaque CCIdS est une structure de mémorisation de l'information relative à soi dans un certain contexte ou domaine de la vie de l'individu (passé, présent ou anticipé). L'information mémorisée dans un CCIdS est celle de schémas de soi, de scripts d'actions, d'attitudes, de sentiments de compétences, d'affects, de schémas d'interactions et d'interlocutions, de représentations d'autrui et d'objets, etc., pertinents dans le contexte correspondant de la vie de l'individu. L'entrée au travail d'un.e adulte émergent.e peut correspondre, pour lui ou elle, à un enrichissement de l'offre identitaire. Cette expérience le ou la conduit à construire en mémoire à long terme au moins un nouveau CCIdS : la mémorisation des informations relatives à ellemême ou lui-même en tant que ce.tte professionnel.le. D'autres nouveaux CCIdS peuvent aussi se construire à cette occasion, par exemple si ce.te jeune professionnel-le commence à pratiquer un sport d'équipe avec des collègues de l'entreprise. Certains CCIdS antérieurement établis ont toutes les chances d'être affectés par la construction de ce (ou ces) nouveau CCIdS. Par exemple, le CCIdS de mémorisation de l'information relative à "soi-étudiant» sera probablement réorganisé.

Le système de CCidS se compose d'un ensemble de formes identitaires subjectives (FIS) dont il ou elle a, à chaque instant, une représentation plus ou moins explicite, claire et précise d'au moins quelques aspects. En effet, une FIS correspond à l'activationactualisation d'un CCIdS dans une certaine circonstance (interaction effective, réflexions sur soi, imagination d'une certaine situation, etc.). Les FIS d'un individu correspondent à l'ensemble de ses représentations potentielles de soi dans ses différents contextes d'interaction. Certaines FIS concernent le présent, mais d'autres peuvent être passées. D'autres peuvent émerger: les FIS anticipées. Chaque FIS se caractérise par un certain rapport à soi (une certaine façon de se voir dans ce contexte-là), aux objets (un intérêt 
particulier à utiliser tel objet), aux actions (un certain intérêt à effectuer telle action dans ce contexte), à autrui (certains types ou qualités de relations). Chez tout individu, cet ensemble est généralement organisé d'une manière telle qu'une (parfois quelques) FIS y tient un rôle majeur, car elle correspond à des attentes de réalisation de soi dans ce domaine: une certaine perfection que l'individu veut atteindre. Les périodes de transition peuvent manifester une réorganisation d'un ensemble de FIS de l'individu.

Certaines expériences directes de travail peuvent enrichir les CCIdS en mémoire et amener à de nouvelles possibilités d'activation ou d'actualisation dans des FIS qui peuvent alors constituer des objets de mobilisation de soi dans des futurs ou des situations qui deviennent souhaitables pour soi. Certaines nouvelles FIS se construisent à partir de processus de personnalisation et l'identification de rôles professionnels originaux pour soi-même. C'est cette mobilisation des FIS qui est en jeu dans les dialogues de conseil, car ceux-ci questionnent de manière réflexive l'expérience singulière de travail des jeunes adultes. C'est donc dans la réflexivité sur son expérience vécue que se fondent les processus de construction de soi en jeu dans ces dialogues. L'objectif des Dialogues de conseil en Life Design est d'aider l'individu à avoir la perception la plus complète et précise de ses FIS afin d'y repérer ou d'y définir celles qui sont l'objet d'attentes de réalisation de soi. Autrement dit: celle(s) correspondant à certaines perspectives d'avenir donnant sens à son existence actuelle.

Dans ce modèle, les processus réflexifs sont centraux, car ils permettent de construire et de renouveler le système des formes identitaires subjectives des individus. Plus précisément, deux formes de réflexivité peuvent être distinguées qui permettent de décrire ces phénomènes d'interprétation par les individus des éléments marquant le cours de leur vie. La première - la réflexivité duelle - les conduit à s'y rapporter du point de vue d'un état de perfection qu'ils veulent atteindre (Erikson, 1980; Lacan, 1966; Foucault, 1984). La seconde forme de réflexivité peut être qualifiée de ternaire ou trinitaire, car elle prend la forme d'un dialogue intra ou interindividuel au cours duquel "je dis à tu», "tu réponds à je», «je et tu font référence à il ou elle» (Colapietro, 1989; Jacques, 1982): cette réflexivité trinitaire permet aux personnes de se rapporter à ces mêmes phénomènes et de les combiner, en vue d'en produire des perspectives d'avenir potentielles, susceptibles de donner un sens et 
une orientation renouvelés à leur vie. La réflexivité trinitaire permet ainsi de faire émerger des positions nouvelles de l'individu, de nouveaux points de vue grâce à un phénomène de décentration de soi et de recentration; tandis que la réflexivité duelle est plus statique en permettant de se constituer comme objet pour soi-même. Ces formes de réflexivité participent d'un premier stade d'élaboration de l'expérience. Le cas de Marc, présenté dans la suite de ce chapitre, illustrera l'importance de ces premières expériences de travail.

\subsection{LA PERSPECTIVE NARRATIVE}

\section{DE LA CONSTRUCTION DE CARRIËRE (SAVICKAS, 2011)}

La théorie de la construction de carrière constitue une autre manière d'investiguer l'identité narrative comme ressource de construction de soi. La théorie distingue le soi de l'identité. Le soi est ici considéré comme le produit d'une histoire racontée sur soi-même. Culturellement et socialement située, cette histoire constitue une "thèse» langagière sur soi. L'identité correspond à une interprétation de ce soi mis en ouvre dans les contextes de vie à travers les rôles sociaux. L'identité, comme «synthèse» langagière du soi, sert à construire des réponses pour s'engager et faire face aux contraintes et réalités sociales. C'est dans le récit que s'articulent les notions de soi et d'identité. Par le récit, l'individu interprète le soi comme s'il était un.e autre (Ricœur, 1990) et ouvre ainsi les possibles de la (re) création de soi.

Pour faire face aux transitions et événements de la vie, l'individu a besoin de construire une histoire de vie cohérente qui le protège d'une remise en question trop radicale et d'une déconstruction de soi. Il doit pouvoir consolider les éléments centraux, les fondamentaux d'un soi servant de pilier. Mais il doit aussi pouvoir lire les aléas de l'existence et les ruptures professionnelles comme des modalités temporaires invitant à la possibilité de variantes narratives. L'appartenance à des groupes sociaux divers amène l'individu à un ajustement identitaire permanent visant l'intégration des expériences significatives dans un récit cohérent. En partageant les valeurs du groupe, en poursuivant des objectifs communs, les individus vont pouvoir mettre à l'épreuve des actes leurs récits identitaires et les peaufiner en les incorporant dans des histoires collectives. Ces histoires collectives sont des scénarios qui supportent la construction identitaire, car ils fonctionnent à la fois comme source d'ajustement et de consolidation du récit identitaire. 
Dans le récit, le thème de vie structure la logique globale de l'identité. Il s'agit d'un motif, une quête pour l'assouvissement de désirs profonds, à travers lequel l'ensemble des événements peut être lu et prendre du sens. Le thème, en ajoutant de l'intentionnalité au récit identitaire, joue le rôle d'une unité fondamentale de sens. Mais l'intrigue de ce récit est soutenue également par des personnages qui se définissent par les besoins et valeurs qui les poussent à agir. Ces besoins et valeurs, en définissant des buts à l'action, participent de la manière dont les personnes surmontent leurs failles et deviennent plus qu'elles ne sont et se construisent. L'intrigue du récit peut alors être vue comme ce qui permet de transformer les besoins en buts. Le parcours de vie est ainsi comme la tentative des individus pour transformer des préoccupations (visibles dans les thèmes de vie évoqués au cours du récit) en métiers (visible dans les choix et activités professionnelles), le tout structuré par des intrigues.

Dans le cas des adultes émergent·e.s, les premières expériences professionnelles peuvent être reprises avec ce modèle dans une perspective de consolidation du récit identitaire. La démarche narrative apparaît comme bien complémentaire du travail dialogique de réflexivité sur l'expérience dans le sens où elle permet la reprise de l'ascription de l'expérience, dans un scénario permettant de consolider les choix utiles pour le développement vers l'âge adulte, servant d'étayage là où le contexte peut être vécu comme fuyant ou impalpable.

\section{LE CONSEIL EN LIFE DESIGN MIS EN CEUVRE DANS LES DEUX MODÈLES}

La méthodologie commune aux interventions en Life Design consiste à offrir au demandeur une situation telle que celle-ci ou celui-ci puisse "tenir conseil» avec elle-même ou lui-même (Lhotellier, 2001), grâce au soutien d'un entretien avec une conseillère. Les interventions de conseil en Life Design visent à aider les demandeurs à trouver leur réponse à la question générique d'orientation comme permettant de donner sens et direction à leur existence (Savickas et al., 2009). Les deux principales méthodes de conseil en Life Design - le Dialogue de conseil en Life Design (DCLD) (Guichard, 2004, 2005, Guichard et al., 2017) et l'entretien de construction de carrière (ECC) (Savickas, 2011, 2013) - partagent ce fondement épistémologique en abordant l'interaction de conseil de manière à la fois différente et complémentaire. Après avoir présenté les caractéristiques des deux démarches, nous 
illustrerons à partir du cas de Marc une mise en ouvre de cette complémentarité.

\subsection{LES DIALOGUES DE CONSEIL EN LIFE DESIGN}

Les dialogues de conseil en Life Design (DCLD) visent à aider les personnes à définir des perspectives d'avenir - pas nécessairement d'ordre professionnel - donnant sens à leur vie, et à s'engager dans la voie de leur réalisation. Ce sont des entretiens de conseil en orientation entre un demandeur et une conseillère qui se rencontrent trois ou quatre fois au cours d'une période allant de deux à six semaines. Ils sont conduits sur le mode de l'entretien semi-directif par la conseillère, qui utilise des interventions telles que l'écho, le reflet, les reformulations, etc. propres à ce type d'entretien. Ces dialogues suivent la logique globale suivante. D'abord l'établissement d'une alliance de travail comme dans tout type d'entretien. Il s'agit pour la conseillère de bien préciser que la démarche est un dialogue au cours duquel le demandeur réfléchit, avec l'aide de la conseillère, sur sa situation présente dans ses différents aspects (le travail, la famille, les relations qui comptent, les loisirs, etc.), sur certaines expériences ou événements passés qui ont compté ou l'ont marqué et sur certaines de ses anticipations pour l'avenir. Il convient également de préciser que cette réflexion prend du temps (trois ou quatre rencontres étalées sur une période d'environ un mois).

Dans un deuxième temps, le dialogue se focalise sur l'exploration de l'expérience. La conseillère et le demandeur démarrent par une exploration des différentes sphères de vie signifiantes (passées, présentes ou futures). Après cette "cartographie" des sphères de vie, conseillère et demandeur choisissent celles qu'il convient d'explorer de manière plus systématique en termes d'actions, activités, rapport à soi, à autrui, aux objets. Il s'agit en effet d'explorer chaque sphère pour se former une vue aussi claire et précise que possible des différents aspects des FIS qui y sont associées. Tout en faisant ce travail sur une sphère, puis deux, puis trois, etc., le demandeur découvre et esquisse pour lui-même un système signifiant de soi dans son contexte. Le dialogue engagé stimule les deux formes de réflexivité, la production d'associations (cognitives et émotives) et de mises en relation des discours. C'est par ce travail dialogique à deux niveaux que le demandeur peut alors découvrir dans la mise en récit (en acte, en affectivité, en cognition) de son expérience passée, présente et future ce qui constitue ses perspectives d'avenir et attentes fondamentales dans l'existence. 
Dans la dernière partie des dialogues, la conseillère devra s'assurer que ce qui est élaboré constitue bien des souhaits fondamentaux qui donnent sens à la vie actuelle du demandeur. La conseillère sera attentive aux expressions qui montrent que le demandeur "se voit» en imagination dans cette forme souhaitée, quelles sont les personnes de l'entourage qui peuvent la renforcer, et quelles actions mettre en place pour avancer dans cette direction.

\subsection{L'ENTRETIEN DE CONSTRUCTION DE CARRIËRE (ECC)}

L'entretien de construction de carrière a aussi pour objet la construction de soi, mais en utilisant des modalités d'interaction différentes. Savickas, en référence aux travaux d'Alfred Adler (1931, 1956), pose que certains thèmes de vie - ancrés dans des expériences précoces de la personne - structurent l'existence de celle-ci. Les entretiens de construction de carrière ont, de ce fait, pour objectif d'aider le demandeur à s'appuyer sur certaines de ses caractéristiques personnelles (son caractère, sa personnalité, ses intérêts) pour faire face au problème d'orientation auquel il est confronté. Ces caractéristiques personnelles fondamentales sont considérées comme étant les solutions trouvées par la personne au cours de son développement pour "transformer les tensions en intentions, les problèmes en opportunités et les préoccupations en occupations" (Savickas, 2011, p. 125). L'ECC est donc une intervention visant à révéler les tensions, problèmes et préoccupations ainsi que le caractère, la personnalité et les intérêts que la personne a construits sur cette base afin de lui permettre de s'appuyer sur ses caractéristiques fondamentales pour faire face à ce nouveau problème.

L'entretien en lui-même se structure en deux sessions organisées autour de questions suscitant des "microrécits» que la conseillère et le demandeur vont réinvestir dans un "macrorécit» identitaire à la fin de l'accompagnement. Cinq domaines sont explorés: les concepts de soi du demandeur (microrécits sur les héros de l'enfance); les intérêts (microrécits sur les magazines préférés); l'appariement soi-environnement (microrécits sur le roman/film préféré); les conseils que le demandeur s'adresse à lui-même (microrécits sur la devise); les thèmes de vie (microrécits sur les plus anciens souvenirs). À l'issue de ces narrations, la conseillère doit construire alors un macrorécit qui agit comme un scénario possible pour faire face à la problématique énoncée initialement par le demandeur. Selon Savickas, se met alors en place « un processus d'apprentissage transformatif qui [lui] permet 
de faire face à son dilemme d'orientation ou au bouleversement de sa vie professionnelle» (2006, p. 68). Ce scénario est présenté comme une nouvelle mise en acte de l'histoire du demandeur qui était mise en suspens. Le scénario (macrorécit) est alors évalué, discuté, et mis à l'épreuve de l'entourage proche du demandeur.

\section{ILLUSTRATION CLINIQUE: LE CAS DE MARC}

Pour illustrer ces démarches et leur complémentarité dans le cadre de l'accompagnement des adultes émergent.e.s par l'analyse réflexive de leur expérience, le cas de Marc est maintenant présenté, puisqu'il lui a été proposé, dans le cadre de ses activités de recherche d'emploi, un entretien DCLD, puis un ECC.

\subsection{CONTEXTE DE L'ENTRETIEN}

Marc est un jeune homme de 25 ans et rentre de ce fait dans la catégorie qui nous intéresse ici des adultes émergents. Il arrive au premier entretien en ayant déposé quelques semaines plus tôt sa démission pour tenter de trouver un nouveau travail lui correspondant mieux, et doit quitter ce tout premier emploi dans les semaines qui viennent. L'accompagnement qui lui est proposé va s'appuyer sur l'analyse de cette expérience pour l'aider à avancer et trouver ses propres perspectives et attentes dans la vie. Marc a été rencontré dans le cadre d'un travail de recherche à l'université visant à mettre en ouvre et à étudier les dialogues de conseil en Life Design. Marc était volontaire et demandeur pour participer à ces entretiens. Il a contacté l'université à la suite d'une annonce proposant un service de conseil en orientation. Marc a donc été reçu à la fois pour répondre à sa demande de conseil en orientation et pour participer à la recherche.

Le premier contact a été téléphonique pour préciser les conditions spécifiques de la recherche (lieux, possibilité d'enregistrement audio et vidéo) et convenir d'une date de premier entretien. Durant celui-ci, après la signature d'un consentement éclairé, les séances de dialogue de conseil se déroulent comme pour un accompagnement traditionnel (outre le fait qu'ils soient filmés). Une procédure d'autoconfrontation est aussi proposée à la fin de l'accompagnement pour pouvoir le prolonger si la personne le souhaite et analyser les dynamiques en jeu dans les dialogues dans le cadre de la recherche (voir Pouyaud et al., 2016). L'autoconfrontation consiste à choisir en amont des moments signifiants pour la conseillère et 
le demandeur, d'en faire un montage vidéo et d'utiliser ce montage comme support de discussion entre la conseillère, le demandeur et la ou le chercheur-se lors d'un entretien collectif.

La démarche d'entretien s'est poursuivie sur un mois à raison de quatre entretiens. Les trois premiers entretiens ont concerné la mise en œuvre des dialogues de conseil tels que décrits plus haut, le quatrième entretien a complété l'accompagnement avec la méthode des entretiens de construction de carrière (Savickas, 2011). Il s'agissait de tester avec un sujet volontaire les deux techniques, qui correspondent généralement à deux démarches différentes. Nous avons commencé par les DCLD, car cette démarche est encore peu étudiée, contrairement au ECC. Cela permettait d'investiguer a postrriori les éléments travaillés lors des dialogues (en comparaison au contenu du ECC). L'objectif de cette double méthode est d'analyser les liens entre ces deux modalités d'interaction qui partagent une même logique dialogique et constructiviste pouvant s'inscrire dans le paradigme du Life Design (Savickas et al., 2009). Durant les DCLD, un support (une grande feuille format A1) a été utilisé pour noter avec Marc les éléments discutés sous forme de schémas.

\subsection{LA SITUATION DE MARC ET SON PROBLÈME D'ORIENTATION}

Lors du premier entretien, à la question de départ «En quoi puis-je vous être utile? », Marc répond: "Personnellement, [ces entretiens] ça peut permettre de m'entraîner, de me jauger, de faire une sorte de bilan par rapport à mon parcours, autant professionnel que personnel, et ce qui peut être bénéfique pour la suite, en prévision de futurs entretiens et de choix d'orientation." Marc travaille actuellement dans une banque comme gestionnaire à la direction des affaires immobilières. Son travail consiste à gérer les dossiers de prêts des sociétés de constructions individuelles ou collectives. Il est en poste depuis un an et demi. C'est le premier travail qu'il a trouvé après ses études de droit (niveau master), principalement parce qu'il y avait des débouchés. Il dit avoir accepté parce que cela lui permettait d'avoir une première expérience, de "se mettre le pied à l'étrier" pour travailler dans le domaine de l'urbanisme. Aujourd'hui, il est dans le domaine du montage immobilier du côté "financier» et "banque» et c'est cet aspect qui lui déplaît: "C'est trop froid et ça ne correspond pas parce que c'est ni ma formation ni ma vocation." Il rêve plutôt d'architecture, d'urbanisme: "Je préfere être plus sur un coté urbaniste, sociologique, penser la ville, 
qu'est-ce qu'on peut faire de bien pour que demain un maximum d'habitants puissent vivre, c'est utopique hein, mais puissent vivre du mieux possible.»

À présent, Marc souhaite être accompagné pour ne pas se tromper dans ses choix à la suite de sa démission et préparer ses futurs entretiens d'embauche en faisant le point sur ce qui lui plaît "vraiment" et sa situation. Il dit: "Oui c'est ça, le sens qu'on peut donner à son travail, et derrière il en découle [...] une sorte d'épanouissement au final. [...] Être le plus large et le plus ouvert et le plus curieux au début, pour ensuite arriver sur un entonnoir avec vraiment des points plus précis et plus concrets vers la fin. C'est avancer dans le noir avec plein de questions au début pour ensuite essayer de trouver des réponses.» Une autre problématique émerge de sa demande derrière le terme "ce qui me plaît vraiment" réside dans son questionnement (voire son inquiétude) à choisir de lui-même, non pas par conformisme vis-à-vis de son histoire, de son entourage familial, mais par un désir plus intrinsèquement construit.

\subsection{LE SYSTÈME DES FORMES IDENTITAIRES SUBJECTIVES}

\section{DE MARC ET SES ÉVOLUTIONS}

La première phase de l'entretien a consisté à explorer la sphère professionnelle dans laquelle il travaille encore du point de vue des rapports à soi, aux autres, aux actions et aux objets. Plusieurs caractéristiques relevant de la FIS «moi comme travailleur» ont ainsi pu être détaillées. En même temps que ces éléments sont abordés dans les dialogues, la conseillère et le demandeur les notent à tour de rôle sur la feuille A1 sous forme d'un graphique qui se coconstruit progressivement. Dans les rapports à soi, on trouve par exemple: «je crois que je vais plutôt marcher à l'affect et à la confiance»; "assidu, qui ne rechigne pas à travailler»; "débonnaire et jovial»; "j'aime bien rigoler ou essayer de faire rire». Dans les rapports aux actions: "pas de déplacements, c'est vraiment un travail qui est sédentaire»; "assez répétitif»; "c'est un travail qui est assez exécutant en fait et pas vraiment dans la création au niveau cérébral». Pour les rapports aux autres: "on prend plaisir à se voir tous les matins et à travailler ensemble"; "on travaille tous dans la même pièce, y a beaucoup d'interactions». Enfin, pour le rapport à l'objet travail: «je trouve que c'est trop austère»; "c'est vraiment de la gestion pure et dure»; "construire des logements tout carré, tout blanc, tout classique qui sont pas très beaux au final et dont la qualité laisse à désirer, et qui 
ont un prix qui peut parfois affoler, ça n'a aucun intérêt en soi; moi, ce qui me plairait, c'est vraiment de donner un sens à tout ça, pourquoi on fait ça, pour que des gens puissent se loger».

Une première forme identitaire anticipée «immobilière» apparait. L'ensemble des éléments discutés durant ce dialogue sur la sphère travail aboutit à l'émergence d'une première forme identitaire subjective anticipée et valorisée qui apparaît comme un compromis biographique, comme une anticipation "plausible» dans laquelle il se verrait bien. C'est une anticipation professionnelle dans laquelle il pourrait arriver à combiner ce qui lui convient de son travail actuel et ce qui lui manque comme signification pour s'épanouir. Nous pouvons décrire cette forme identitaire subjective anticipée (FISA) sous l'angle d'un "concepteur-réalisateur de programmes d'aménagement urbain de logements (notamment sociaux) permettant aux habitants de mener ensemble une vie bonne». Marc se projette à la fois de manière cognitive et affective dans cette forme identitaire. Il "s'y voit» parce que cela correspond à ses compétences et expériences, mais aussi parce que cela est cohérent avec la logique narrative de son parcours. Il y voit une manière d'être la personne rationnelle, ouverte sur la société, l'actualité, la politique, l'histoire et la sociologie, tout en étant dans une logique rassurante, réaliste et stratégique du point de vue de l'emploi, conforme aux conventions et aux attentes de son entourage, dans la lignée logique de son parcours tout en l'enrichissant. Cette forme, même si elle s'élabore dans la logique de la réflexivité duelle comme une projection possible, se heurte au questionnement plus profond de Marc sur sa motivation intrinsèque. Est-ce vraiment mon propre choix? Ne suis-je pas en train de reproduire ce qu'ont vécu mes parents et dans quoi je ne veux certainement pas me retrouver? La routine, le train-train, la vie réglée, conforme, sans aventures, ni questionnements, ni voyages.

Une seconde forme identitaire anticipée alternative "théâtrale» se manifeste également. Durant les dialogues qui vont suivre avec la conseillère et dans l'exploration des autres sphères de vie va alors se construire une autre forme identitaire subjective anticipée concurrente, fondée sur les décalages produits dans le dialogue. C'est alors la mise en réflexivité trinitaire, sous-tendue par ce questionnement de remise en question de la première FISA, qui va être le support de cette élaboration. Cette seconde FISA est une forme identitaire subjective rêvée qui «s'oppose» et "s'articule» de 
manière systémique avec la première. Si Marc n’avait pas de compromis économiques à faire, sa vie rêvée serait ainsi d'écrire des pièces de théâtre qu'il jouerait dans une troupe qu'il dirigerait. Cette forme identitaire subjective s'élabore avec la conseillère à partir de l'exploration des activités de loisirs et notamment la participation de Marc à une troupe de théâtre amateur. Cette activité a commencé réellement pour lui en même temps que son emploi dans la banque. Quand on explore avec Marc cette sphère, on y voit de multiples proximités (chevauchement-emboîtement) avec la sphère professionnelle.

Les rapports à soi sont inscrits dans une logique de création et de plaisir, de faire rire: «j'aime bien faire rire la galerie»; "c'est l'improvisation qui me fait un peu plus peur». Les rapports aux autres sont ceux de l'amusement et la camaraderie: "je pense qu'on est une bande de gamins"; "ce que je ressens - une solidarité - une ambiance de groupe ». Le contenu de l'activité (rapport aux actions) demande tout de même de la rigueur et un sérieux dans l'investissement, comme le travail, notamment dans l'écriture; on doit «écrire des sketchs sur des sujets d'actualité, les préparer en dehors». Enfin, le rapport à l'objet "théâtre» renvoie à un fort investissement subjectif et émotionnel: "le but est de toujours faire de l'humour»; "c'est quelque chose qui me tient à cœur que ça soit écrire ou sur scène, je ne saurais pas dire pourquoi, ça met en valeur quelque chose... je crois que ça me correspond».

L'intégration de ces deux formes identitaires se réalise difficilement. Les deux formes élaborées dans les dialogues sont enchâssées dans un système de tensions reliées à la problématique de Marc. Ici, les deux FIS élaborées ne s'opposent pas complètement. Pour Marc, le travail est un lieu où "on porte un costume au sens propre... où il faut faire semblant...». Mais le choix qui se pose à lui aujourd'hui pour retrouver du travail et «ne pas se tromper» s'inscrit dans cette tension entre un engagement rassurant, mais potentiellement ennuyeux, versus un engagement stimulant, plus personnel, mais risqué.

La résolution de la tension entre ces deux formes identitaires s'organise finalement. Après le premier entretien, Marc a donné sa démission. Entre le deuxième et le troisième entretien, il a effectué un entretien d'embauche. Au quatrième entretien, il annoncera à la conseillère qu'il a été recruté sur le poste. Ce poste est finalement un poste de "responsable de développement et chargé d'affaires » pour 
la prospection foncière et l'établissement de gros projets immobiliers. Il correspond en tout point à la première FISA valorisée pour son caractère rassurant et cohérent dans l'expérience de Marc, qui se dit très heureux de ce développement. Comment s'est résolue la tension identitaire? Pour Marc, l'entretien permet finalement de reconstruire une signification aux différents engagements et formes entre elles. Ainsi, la forme "théâtrale» est re-signifiée pour s'articuler avec la forme "immobilière": "Le fait d'être au théâtre... c'est faire un travail sur soi pour se sentir plus à l'aise pour parler aux autres et d'apprendre à travailler à plusieurs...» "Mon objectif c'est d'être original et de ne pas rester dans l'humour basique où on sait très bien ce que la personne sur scène va dire [...] et du coup au travail c'est dans les moments moins intentionnels où j'aime bien faire rire la galerie et où je me sens plus libre de le faire... ça se fait au fur et à mesure... c'est dans l'imbrication des deux, l'apprentissage des usages au travail et de continuer au théâtre."

\subsection{EXPLORATION ET LIENS AVEC L'ENTRETIEN}

\section{DE CONSTRUCTION DE CARRIÈRE}

Le contenu de l'entretien de construction de carrière avec Marc n'est pas développé afin que nous nous centrions sur les principaux points communs identifiés dans l'histoire. Les deux méthodes ont en effet permis d'observer les complémentarités qui existent entre les microrécits du ECC et les éléments travaillés dans le cadre des DCLD.

Certaines activités de loisirs de l'enfance (ECC) apparaissent déterminantes pour les FIS actuelles (DLCD). Lors des dialogues de conseil en Life Design, Marc a établi un lien entre son intérêt actuel pour le théâtre et une expérience de dix années au moment de l'adolescence (entre l'âge de 10 et 20 ans) au sein des associations de scouts. Dans ces associations religieuses, rigueur et discipline sont nécessaires. Un sens aigu de l'entraide et de la camaraderie, un rapport direct à la nature, un apprentissage de la débrouillardise, du commandement, du respect et de la hiérarchie sont aussi très importants. Durant cette expérience, Marc relève deux dimensions fondatrices de cette seconde identité rêvée. La première est liée à l'activité d'écriture. À l'occasion des veilles de nuit solitaires chez les scouts où l'ennui domine, il écrivait des notes humoristiques sur les cahiers de veille à l'attention des quarts de garde suivants, pour les faire rire et tromper leur ennui. Marc construit alors un lien direct avec son activité actuelle d'écriture de sketchs. 
La deuxième est liée à une figure marquante qui lui sert de modèle. Ce chef scout était d'origine juive et, pourtant, il officie dans les groupes de scouts catholiques. De ce fait, il représente pour Marc un exemple de diversité et de non-conformisme, de quelqu'un qui ne se limite pas aux clichés habituels, aux idées toutes faites. C'est quelqu'un qu'il décrit comme ayant une grande élégance intellectuelle, une prise de distance critique et un regard neuf sur les choses. S’il séduit autant Marc, c'est qu'il répond de manière très prégnante à sa volonté d'inscrire son parcours dans un choix personnel, qui répond à ses propres envies et non aux attentes conformistes de son contexte de vie.

Des personnages de fiction (ECC) permettent de structurer le récit de Marc et de concevoir certaines FIS (DLCD). Durant l'entretien de construction de carrière, les héros choisis reprennent très précisément cette problématique et le profil rêvé de Marc. Bob Morane d'un côté et Arsène Lupin de l'autre. Deux personnages de roman dont la caractéristique principale relevée par Marc est leur caractère double à l'image du chef scout: Bob Morane est un aventurier mi-justicier, mi-espion, et Arsène Lupin est un gentleman cambrioleur au grand cœur qui fait le bien. Cette dualité se retrouve aussi chez Marc durant l'élaboration des dialogues de conseil, entre la forme identitaire subjective "conformiste rassurante» de conception immobilière et celle "non conformiste risquée» d'écrivain-acteur de théâtre. Il aimerait certainement comme ses héros pouvoir retenir ses deux facettes de la personne qu'il pense être. Ce décalage est aussi le fruit d'une réflexivité trinitaire qui met en tension le système de formes identitaires subjectives coconstruit durant les dialogues, et qui pousse la personne dans une problématique de choix qui nécessite d'évaluer ces formes sur l'engagement affectif et effectif qu'elles sous-tendent. La proximité dans le choix des héros (Arsène Lupin, Bob Morane) rejoint donc la problématique identitaire de Marc: concilier deux choses en même temps, essayer de faire coexister deux formes identitaires subjectives plus ou moins compatibles entre elles.

Les romans préférés (ECC) permettent une totalisation de son expérience dans une FIS complexe (DLCD). Cette dualité se retrouve également exprimée dans le choix du livre de chevet: Le Seigneur des anneaux et un épisode d'Arsène Lupin. Dans ces histoires, «le personnage s'enrichit, il se complexifie... on découvre d'autres facettes... on arrive dans une ouvre qui se complexifie, 
ça permet de mieux le découvrir et le comprendre». Ce sont des histoires qui racontent un développement, un moyen de devenir adulte en se complexifiant. Comment, dans l'histoire, Arsène Lupin ou le héros du Seigneur des anneaux devient adulte? En faisant des choix personnels dans des situations critiques - Arsène Lupin "se refuse à tuer alors qu'il a lui-même été meurtrier par le passé». Ainsi, la nécessité pour Marc aujourd'hui de faire ce choix personnel, pour grandir, passer d'une première expérience professionnelle à une autre plus en phase avec lui-même, s'exprime.

Les microrécits sur d'anciens souvenirs d'enfance (ECC) éclairent Marc sur son intérêt pour les personnages doubles (DCLC). Cette problématique qui consiste à vouloir devenir adulte par soi-même, différemment de ses parents, est également présente dans les premiers souvenirs, exprimée comme un thème de vie. "Je rentre dans la chambre de mon grand frère, y a deux fenêtres - un flash, mais y s'est rien passé... je suis allé dans le garage avec des outils, je voulais fouiller, mais ma mère m'a dit de revenir... J'étais content, le jardin, c'était hyper grand... la découverte de la nouvelle maison... tout de suite le jardin, le garage, une caisse bleue, je voulais toucher ce qu'il y avait dedans et ma mère m'a dit de revenir.» Peut-être peut-on voir ici l'expression de la problématique du choix qui s'exprime, entre ce qui me plaît et ce qui est bon, valorisé socialement, ou familialement. L'important est que cette interdiction n'est pas vécue comme une contrainte, une punition ou une soumission, mais comme un plaisir. Nous pouvons y voir l'ambivalence de la problématique de Marc: le choix «rationnel» n'est pas complètement une contrainte, il ne s'oppose pas complètement au plaisir.

Le microrécit sur la devise préférée (ECC) montre la manière dont Marc surmonte des contradictions ou le conflit entre certaines formes identitaires (DCLD). L'effort et le plaisir vont de pair, comme cela transparaît également dans le choix de sa devise: "qui veut aller loin ménage sa monture», autrement dit «si je veux aller loin, faut que je m'accroche». Comme meilleur conseil que l'on se donne à soi-même, cette devise traduit l'issue vers laquelle l'ont mené les dialogues de conseil: non pas un choix, mais un compromis, une conciliation des formes identitaires subjectives qui lui paraissaient au départ en opposition, et qui se traduisent par une formule d'action telle qu' «il me faut faire des compromis «raisonnables» si je veux atteindre mes ambitions personnelles». 


\section{DISCUSSION}

Dans le contexte de globalisation tel que décrit plus haut, un des nouveaux enjeux des adultes émergent.e.s est d'être capables de transformation, de construction, d'adaptation de qui elles ou ils sont, en lien avec des expériences toujours de plus en plus variées, chaotiques et multiples. Ainsi, le travail réflexif qui consiste à modeler (designer) son système de formes identitaires subjectives selon les circonstances et événements de vie, tout en déployant un dialogue continué référant à soi, est devenu une tâche développementale centrale de ce moment du parcours de vie.

L'exemple de Marc va dans ce sens. Nous voyons comment il arrive, dans le cadre de cet accompagnement, à modeler un compromis identitaire fondé à la fois sur l'analyse de son expérience et les besoins d'ajustement qu'il ressent dans la phase de transition dans laquelle il se trouve. Il réélabore ainsi son système de formes identitaires subjectives autour de la prise de conscience de la tension qui construit sa problématique de développement. La forme identitaire subjective professionnelle qu'il construit est bien un compromis issu de la réflexivité autour de cette tension. Le dialogue de Life Design met particulièrement au jour les spécificités identitaires de Marc comme adulte émergent: il est ouvert aux expériences et essaie de trouver une finalité à son travail. Ces observations vont dans le sens de celles d'Arnett, Ramos et Arnett (2001) : cet âge de la vie se caractérise par une réflexion relative à la recherche de croyances, d'idéologies à adopter, ce qui manifeste l'importance de la construction d'une identité éthique ou politique. Identifiant clairement sa principale FIS, Marc peut alors interroger son importance et la revisiter: l'immobilier, est-ce vraiment son propre choix? La méthode des dialogues de conseil permet des explorations identitaires par la mise au jour de FIS, puis la réflexion sur ces FIS. Cette activité est centrale pour la construction de soi des adultes émergent.e.s qui se questionnent sur leurs rôles sociaux futurs (Arnett, 2000). Marc, au cours des dialogues, a pu mettre au jour deux formes identitaires subjectives majeures. Il a ensuite pu les articuler. La FIS «théâtrale» est resignifiée et articulée à la FIS «immobilière». Les dialogues permettent d'enrichir les sois, en construisant un faisceau de liens entre eux, et offrent ainsi une plus grande souplesse et adaptabilité à l'ensemble des FIS. Il permet surtout aux adultes émergent.e.s de produire des activités de personnalisation et d'imaginer des formes identitaires subjectives 
singulières utiles dans une société où les expériences culturelles et professionnelles se multiplient (Arnett, 2015).

Chez Marc, l'entretien de construction de carrière permet précisément de faire le lien avec le passé. Les questions portant sur les personnages marquants de son histoire ont été particulièrement utiles, car ceux-ci apparaissent comme des piliers de son identité narrative. C'est le cas, par exemple, du chef scout rencontré des années auparavant, capable de son point de vue de développer une identité hybride complexe "juif-catholique-intellectuel-sportif», ce qu'il aimerait lui aussi parvenir à réaliser. De même, les aventures vécues par les héros auxquels il s'identifie lui permettent de passer progressivement vers l'âge adulte, en surmontant par lui-même les épreuves rencontrées. Le choix de sa devise, qui utilise la métaphore du voyage à cheval, exprime tout particulièrement le processus de construction et l'évolution à l'œuvre au cours de ces réflexions. L'entretien de construction de carrière, grâce à ces ensembles de questions, offre une opportunité de reconsidérer les expériences passées. Il permet de mettre au point une identité narrative et de guider son évolution, ce qui est une tâche essentielle des adultes émergent.e.s (McAdams \& Olson, 2010).

Dans cette tâche développementale, nous voyons ainsi que deux activités sont mises en œuvre dans le cadre de l'accompagnement. L'une consiste à mettre à jour les dimensions cognitives, représentationnelles de soi de manière systémique, l'autre consiste à produire une narration structurante. Ainsi, les deux méthodes d'accompagnement présentées semblent fournir des supports complémentaires dans le sens où elles soutiennent plus singulièrement chacune un type d'activité, les DCLD pour le premier, l'ECC pour le second. Les deux méthodes comportent cependant aussi des différences importantes.

Ces méthodes ne reposent pas complètement sur les mêmes ressorts théoriques et modalités concrètes d'interaction. Les entretiens de construction de carrière proposent des thèmes pour orienter la narration, alors que les dialogues de conseil en Life Design laissent la personne déterminer les sphères de vie qu'elle souhaite évoquer (Guichard, 2016). La conseillère qui conduit un entretien de construction de carrière pose en effet une série de questions précise amenant le demandeur à produire des microrécits sur lesquels une synthèse des grands thèmes autour desquels il organise sa vie et la construit. La conseillère qui dialogue avec un demandeur dans 
le cadre des DCLD en vue de l'aider à s'orienter dans l'existence laisse ce dernier choisir des situations significatives qu'il narre à sa manière. Cette narration lui permet d'effectuer des rapprochements entre certains de ces événements, en relation notamment avec les affects et émotions qu'il éprouve à l'occasion de ses récits. Il élabore ainsi progressivement une certaine signification les reliant, constituant un fil rouge qui s'ouvre sur une certaine perspective d'avenir, une aspiration à se réaliser dans une certaine forme de vie active.

Les dialogues de conseil en Life Design accordent plus de poids au présent et à la capacité de résilience de la personne que les entretiens de construction de carrière, qui mettent l'accent sur le socle des expériences précoces dans la construction d'un sens de la vie relativement stable. Ainsi, les DCLD ont peut-être l'avantage d'être plus ouverts au développement spontané du discours de la personne, mais la contrepartie en est le nécessaire besoin de temps. Si les ECC sont plus directifs, ils permettent aussi d'être plus rapides. En termes de perspectives de recherches et d'interventions, cette question du temps disponible et/ou nécessaire de l'intervention au regard du travail sur soi à réaliser est un enjeu important.

Ces constats laissent penser qu'il serait peut-être intéressant de concevoir une démarche globale intégrant ces deux approches malgré leurs différences théoriques et techniques. Toutefois, avant de s'engager dans un tel projet, il serait sans doute nécessaire de formaliser davantage les compétences requises par les conseillers.ères souhaitant s'engager dans ces méthodes, et d'identifier quels savoirs et savoir-faire, quelle formation théorique et expérientielle permettent de les mettre en œuvre. Par exemple, la manière dont les conseillers ères élaborent la narration à partir des microrécits des demandeurs-ses mériterait d'être plus explicitée pour être réalisée à la manière de l'auteur de la méthode lui-même. Du côté des demandeurs.ses, les motifs à entrer dans cette démarche, les capacités langagières, les aptitudes cognitives nécessaires à cette activité réflexive devraient donner lieu à des analyses plus nombreuses afin de cerner les conditions qui permettent de réussir dans cette réorganisation ou construction de soi. La poursuite de recherches visant l'évaluation, l'enrichissement et la mise en perspective de ces méthodes reste donc aujourd'hui un enjeu important pour l'utilité, l'efficacité et la faisabilité de la mise en œuvre de ce paradigme du Life Design. 


\section{CONCLUSION}

L'objectif de ce chapitre était de présenter comment les approches constructivistes actuelles du Life Design peuvent permettre de prendre en compte les spécificités des problématiques d'orientation des adultes émergent.e.s dans le contexte actuel des sociétés liquides. Pour ces individus, il a été noté que les premières expériences professionnelles pouvaient jouer un rôle fondamental dans la construction de telles ressources de développement, ressources identitaires et compétences à s'orienter. Les approches du Life Design suggèrent qu'il est possible d'étayer la construction de ces ressources identitaires sur la base d'un travail d'analyse réflexive sur soi et de son expérience, menant à une construction de soi. Le cas présenté de Marc permet de se rendre compte de la manière dont deux méthodes d'intervention en conseil de Life Design peuvent permettre de faire ce travail réflexif. Dans les deux cas, la focalisation sur le sens que l'on peut construire à partir de l'analyse de l'expérience est centrale. C'est en ce sens que ces modalités d'accompagnement se distinguent des interventions "traditionnelles» de guidance en employabilité. Leur finalité est d'aider les personnes à mener une vie qu'elles jugent bonne, avec comme objectif d'aider les demandeurs.ses à construire des perspectives d'avenir et à formuler des normes de vie leur permettant de donner sens et direction à leur existence. Pour les jeunes adultes en situation de transition psychosociale, cette question du développement de l'agentivité ou du "pouvoir d'agir» dépasse ainsi la question du bien-être individuel. Au cours des activités d'exploration caractéristiques des adultes émergents, les normes de conduites qui seront mises en œuvre dans la société de demain sont élaborées ou négociées. Ainsi, cette période de la vie est centrale puisqu'y sont définies des attitudes et des conduites qui permettront l'existence de travail décent dans le cadre d'une société durable. La question de l'agentivité des jeunes concerne ainsi la possibilité de transformation de la société à travers les parcours individuels. Elle implique le développement d'une socialisation active (Malrieu, 2003), d'une démarche de personnalisation permettant à chacun.e de participer par ses choix à la construction d'une société plus juste, plus durable, et élaborant des environnements décents de travail. 


\section{RÉFÉRENCES BIBLIOGRAPHIQUES}

Adler, A. (1931). What Life Could Mean to You. Oxford: Oneworld.

Adler, A. (1956). The individual psychology of Alfred Adler: A systematic presentation in selections from his writing. H. L. Ansbacher \& R. R. Ansbacher (éds). New York: Basic Books.

Arnett, J. J. (2000). Emerging adulthood. A theory of development from the late teens through the twenties. American Psychologist, 5. 469-480. Doi: 10.1037//0003-066X.55.5.469.

Arnett, J. J., Ramos, K. D., \& Arnett, J. L. (2001). Ideological views in emerging adulthood: Balancing autonomy and community. Journal of Adult Development. 2, 69-79. Doi: 10.1023/A:1026460917338.

ARnetT, J. J. (2001). Conceptions of the transition to adulthood: perspectives from adolescence through midlife. Journal of adult development, 2, 133-142.

ARnetT, J. J. (2015). Identity Development from Adolescence to Emerging Adulthood: What We Know and (Especially) Don't Know. In K.C. McLean \& M. Syed (éds). The Oxford Handbook of Identity Development (pp. 53-65). New York: Oxford University Press.

ARnett, L.J, \& ARnetT, J.J. (2012). Going global: new pathways for adolescents and emerging adults in a changing world. Journal of Social Issues, 3, 473-492. Doi :10.1111/j.1540-4560.2012.01759.x.

Barbier, J. M. (2013). Expérience, Apprentissage, Éducation. In L. Albarello, J. M. Barbier, E. Bourgeois \& M. Durand (éds), Expérience, activité, apprentissage (pp.65-85). Paris: PUF.

Bauman, Z. (2007). Liquid Times: Living in an Age of Uncertainty. Cambridge: Polity Press.

Bruner, J. S. (1994). The «remembered" self. In U. NeISSER \& R. FIVUSH (éds), The remembering self: Construction and accuracy in self-narrative (pp. 41-54). Cambridge: Cambridge University Press.

Bureau international du travail (BIT) (2013). Decent Work indicators. Guidelines for producers and users of statistical and legal framework indicators. Genève: BIT.

Colapietro, V. M. (1989). Peirce's approach to the self. A semiotic 
perspective on human subjectivity. Albany (NY) : State University of New York Press.

Collin, A., \& Guichard, J. (2011). Constructing self in career theory and counseling interventions. In P. J. Hartung \& L. M. Subich (éds), Constructing Self in Work and Career. Concepts, cases and contexts (pp. 89-106). Washington: American Psychological Association.

Dumora, B., \& Boy, T., (2008). Les perspectives constructivistes et constructionnistes de l'identité ( $2^{\mathrm{e}}$ partie) : Modèles constructivistes et constructionnistes et psychologie du conseil. L'Orientation scolaire et professionnelle, 37 (3), 365-386. Doi : 10.4000/osp.1729.

ERIKSON, E. (1980). Identity and the life cycle. New York: Norton.

Eraut, M. (2004). Informal learning at the workplace. Studies in Continuing Education. 26, 247-273. Doi: 10.1080/ 158037042000225245.

Foucault, M. (1984). Histoire de la sexualité 3 - Le souci de soi. Paris: Gallimard.

GUICHARD, J. (2004). Se faire soi. L'Orientation scolaire et professionnelle, 33, 499-534. Doi : 10.4000/osp.226.

GUICHARD, J. (2005). Life-long self-construction. International Journal for Educational and Vocational Guidance, 5, 111-124. Doi: 10.1007/s10775-005-8789-y.

Guichard, J. (2009). Self-constructing. Journal of Vocational Behavior, 75, 251-258. https://doi.org/10.1016/j.jvb.2009.03.004

Guichard, J. (2016). Reflexivity in Life Design interventions: Comments on life and career design dialogues. Journal of Vocational Behavior, 97, 78-83. doi : 10.1016/j.jvb.2016.08.001

Guichard, J., Bangali, M., Cohen-Scali, V., Pouyaud, J., \& Robinet, M.-L. (2017). Concevoir et orienter sa vie: les dialogues de conseil en Life Design. Paris: Qui Plus Est.

Harré, R. (1998). The singular self: An introduction to the psychology of personhood. Londres: Sage.

Jacques, F. (1982). Différence et Subjectivité. Paris: Aubier.

Jodelet, D. (2006). Place de l'expérience vécue dans les processus de formation des représentations sociales. In V. HAAS (éd.). 
Les savoirs au quotidien (pp. 235-256). Rennes: Presses universitaires de Rennes.

LACAN, J. (1966). Le stade du miroir comme formateur de la fonction du je telle qu'elle nous est révélée dans l'expérience psychanalytique. In J. LACAN, Écrits (pp. 93-100). Paris: Seuil.

Lhotellier, A. (2001). Tenir conseil: délibérer pour agir. Paris: Seli Arslan.

Mahoney, M. J. (2002) Constructivism and positive psychology. In C. R. SNyder \& S. J. Lopez (éds), Handbook of Positive Psycho$\log y$ (pp. 745-751). New York: Oxford University Press.

Malrieu, P. (2003). La construction du sens dans les dires autobiographiques. Toulouse: Érès.

McAdams, D. P., \& Olson, B. D. (2010). Personality Development: Continuity and change over the life course. Annual Review of Psychology, 61, 517-542.

PARKes, C. M. (1971). Psycho-social transitions: A field for study. Social Science \& Medicine, 5, 101-115.

Pouyaud, J., Bangali, M., Cohen-Scali, V., Robinet, M.-L., \& Guichard, J. (2016). Exploring changes during Dialogues for Lifeand Career- Designing. Journal of Vocational Behavior, 97, 3-12. Doi : 10.1016/j.jvb.2016.07.008.

Pepper, S. C. (1942). World hypotheses: A study in evidence. Berkeley: University of California Press.

Pastré, P. (2013). Le travail de l'expérience. In L. Albarello, J. M. Barbier, E. Bourgeois, \& M. Durand (éds), Expérience, activité, apprentissage (pp. 93-110). PUF.

Raskin, J. D. (2002). Constructivism in psychology: Personal construct psychology, radical constructivism, and social constructionism. In J. D. Raskin \& S. K. Bridges (éds), Studies in meaning: Exploring constructivist psychology (pp. 1-25). New York: Pace University Press.

Ricceur, P. (1990). Soi-même comme un autre. Paris: Seuil.

Rosa, H. (2012). Aliénation et accélération. Vers une théorie critique de la modernité tardive. Paris: La Découverte.

SAVICKAS, M. L. (2011). Career Counseling. Washington: APA. 
SAVICKAS, M. L. (2013). Career construction theory and practice. In R. W. Lent \& S. D. BRown (éds), Career development and counseling: Putting theory and research to work ( $2^{\mathrm{e}}$ édition, pp. 147-183). Hoboken (NJ) : Wiley.

Savickas, M. L., Nota, L., Rossier, J., Dauwalder, J. P., Duarte, M. E., Guichard, J., Soresi, S., Van Esbroeck, R., \& Van Vianen, A. E. M. (2009). Life designing: A paradigm for career construction in the 21st century. Journal of Vocational Behavior, 75, 239250. Doi : $10.1016 /$ j.jvb.2009.04.004.

Vygotski, L. (1994). Défectologie et déficience mentale. Lausanne: Delachaux et Niestlé. 


\section{APPENDIX}

\begin{tabular}{|c|c|c|}
\hline & | DCLD & ECC \\
\hline OBJECTIF & $\begin{array}{l}\text { Permettre à l'individu } \\
\text { d'être en accord avec } \\
\text { ses attentes profondes. }\end{array}$ & $\begin{array}{l}\text { Aider une personne } \\
\text { à construire sa carrière. }\end{array}$ \\
\hline DÉROULEMENT & $\begin{array}{l}\text { De trois à cinq temps } \\
\text { de dialogue visant la mise } \\
\text { au jour des principales } \\
\text { formes identitaires dans } \\
\text { ses différentes sphères } \\
\text { de vie par le développement } \\
\text { de la réflexivité. }\end{array}$ & $\begin{array}{l}\text { Deux sessions devant } \\
\text { permettre d'identifier } \\
\text { des microrécits } \\
\text { qui seront réorganisés } \\
\text { en une histoire de vie } \\
\text { par la conseillère. }\end{array}$ \\
\hline $\begin{array}{l}\text { LES ÉTAPES } \\
\text { DES } \\
\text { ENTRETIENS }\end{array}$ & $\begin{array}{l}\text { Quatre étapes } \\
\text { 1. Alliance de travail } \\
\text { 2. Exploration des sphères } \\
\text { de vie et identification } \\
\text { des principales FIS } \\
\text { 3. Analyse de chaque FIS } \\
\text { 4. Planification des actions } \\
\text { à mettre en œuvre }\end{array}$ & $\begin{array}{l}\text { Cinq questions stimuli } \\
\text { sont proposées au cours } \\
\text { du premier entretien. } \\
\text { Le second entretien } \\
\text { est consacré } \\
\text { à la construction } \\
\text { d'un nouveau récit. }\end{array}$ \\
\hline QUESTIONS & $\begin{array}{l}\text { Quelles sont vos principales } \\
\text { activités aujourd'hui? } \\
\text { Comment vous voyez } \\
\text { vous dans ce contexte-là? } \\
\text { Qu'est-ce qui vous satisfait } \\
\text { tout particulièrement } \\
\text { dans cette activité-là? } \\
\text { Comment vous voyez } \\
\text { vous plus tard en tant que...? }\end{array}$ & $\begin{array}{l}\text { Cinq questions stimuli } \\
\text { concernant: } \\
\text { 1. Les personnages } \\
\text { importants de l'enfance } \\
\text { 2. Les magazines } \\
\text { préférés } \\
\text { 3. L'histoire préférée } \\
\text { 4. Le proverbe utile } \\
\text { dans la vie } \\
\text { 5. Les souvenirs } \\
\text { les plus anciens }\end{array}$ \\
\hline $\begin{array}{l}\text { RÔLE } \\
\text { DE LA } \\
\text { CONSEILLĖRE }\end{array}$ & $\begin{array}{l}\text { Aide à la mise au jour } \\
\text { des FIS passées, présentes } \\
\text { et anticipées par quelques } \\
\text { questions ouvertes et } \\
\text { interventions semi-directives. }\end{array}$ & $\begin{array}{l}\text { Note les réponses } \\
\text { aux questions stimuli } \\
\text { et reconstitue } \\
\text { la narration à partir } \\
\text { des microrécits. }\end{array}$ \\
\hline
\end{tabular}




\section{CONCILIATION DES DOMAINES DE VIE: LE RÔLE DU CONTEXTE SOCIAL ET DES RESSOURCES D'ADAPTABILITÉ 1}

GRÉGOIRE BOLLMANN (UNIVERSITÉ DE ZURICH), EVA CLOT-SIEGRIST (UNIVERSITÉ DE LAUSANNE)

ET LEVKE HENNINGSEN (UNIVERSITÉ DE ZURICH)

$\mathrm{L}$

e début du $\mathrm{XXI}^{\mathrm{e}}$ siècle voit une série de changements sociétaux importants autant dans les rapports de travail que dans les ingovations technologiques, dans la diversification des structures familiales ou dans la démographie de nombreux pays, dont la Suisse. Ces changements modifient les attitudes et normes sociales liées aux rôles que femmes et hommes occupent dans différents domaines de vie. Ils donnent un nouveau visage à ce que la population conçoit comme une carrière professionnelle et plus généralement une vie épanouie et équilibrée (Greenhaus \& Kossek, 2014) Ainsi, les interdépendances entre le domaine professionnel et celui du foyer - qu'il s'agisse du couple, des enfants, des parents ou des amis - occupent aujourd'hui une place de choix dans les préoccupations de nombreux adultes, et d'autres domaines, tels que les loisirs et les activités de développement personnel, gagnent continuellement en importance. Or ces interdépendances, les difficultés à les concilier comme les bénéfices qu'il est possible d'en retirer jouent un rôle sur la santé et le bien-être subjectif (McNall, Nicklin, \& Masuda, 2010; Nohe, Meier, Sonntag, \& Michel, 2015).

Malgré leur évolution, les normes sociales concernant la répartition des rôles dans le domaine professionnel et familial sont encore distinctes entre femmes et hommes, ce qui appelle d'autres transformations (Williams, Berdahl, \& Vandello, 2016). Des différences entre les sexes peuvent être observées aujourd'hui encore sur de nombreux indicateurs descriptifs des carrières professionnelles (Csonka

1. Cette publication a bénéficié du soutien du Pôle de recherche national LIVES-Surmonter la vulnérabilité: perspective du parcours de vie, financé par le Fonds national suisse (numéro de subside: 51NF40-160590). Les auteur.e.s remercient le Fonds national suisse de la recherche scientifique de son aide financière. 
\& Mosimann, 2017). De fait, comprendre les facteurs influençant la manière dont la population gère les domaines de vie et leurs effets sur les parcours professionnels et le bien-être revêt un enjeu crucial pour la pratique et la recherche en psychologie des organisations et du conseil en orientation.

En mobilisant la théorie des rôles sociaux (Eagly \& Wood, 2012) et celle de la construction de carrière (Savickas et al., 2009), ce chapitre met en avant, d'une part, le rôle du contexte social et des normes de genre y régnant et, d'autre part, le potentiel des ressources d'adaptabilité de carrière dans les dynamiques de conciliation des domaines de vie. Dans un premier temps, la question de la conciliation des différents domaines de vie sera abordée du point de vue du paradigme "construire sa vie» (en anglais, Life Design), dérivé de la théorie de la construction de carrière, avant de présenter les différents types d'interdépendances existantes entre domaines de vie, puis le rôle du contexte social et le potentiel des ressources d'adaptabilité que les individus peuvent déployer pour les concilier. Dans un deuxième temps, deux outils d'intervention seront présentés, permettant ainsi d'explorer ces questions dans le cadre d'un entretien de conseil en orientation et d'offrir des perspectives de réflexion sur les forces et les limites de ces outils, ainsi que sur le rôle que le contexte social et les ressources d'adaptabilité peuvent jouer dans la conciliation des domaines de vie.

\section{CONSTRUIRE UNE VIE ÉPANOUIE}

\section{EN CONCILIANT DIFFÉRENTS DOMAINES DE VIE}

Le paradigme d'orientation "construire sa vie» a pour objectif de comprendre comment les individus construisent activement leur vie au sein de leur contexte social (Savickas et al., 2009). Il part de cinq présupposés, à savoir que (1) la réussite du conseil en orientation ne se mesure pas à l'aide d'un indicateur unique, mais nécessite d'offrir une place à la subjectivité de la personne et à sa propre définition d'une vie épanouie; (2) les parcours professionnels ne sont plus seulement linéaires, mais ils se diversifient; (3) cette diversité reflète l'équilibre auquel aspirent les personnes entre la multitude des rôles sociaux qu' elles occupent; (4) les femmes et les hommes ne peuvent être considérés sans leur contexte social, avec qui ils forment une entité dynamique; 
(5) les transitions nécessitent de réfléchir aux ressources à leur disposition et aux stratégies d'adaptation possibles. Le paradigme adopte une perspective dynamique qui met en exergue l'évolution des individus tout au long de leur vie; il se veut holistique en ce qu'il concerne non seulement le domaine professionnel, mais aussi les autres domaines qui leur sont chers, contextuel, à savoir qu'il prend en compte différents environnements sociaux communs à toute une frange de la population (contexte institutionnel national ou régional) et propre à l'individu (son organisation, sa famille), et préventif en promulguant une aptitude à gérer la situation présente tout en envisageant l'avenir de manière à maintenir un éventail de possibilités et à pouvoir faire face à des situations à risque. Ce paradigme est ici appliqué à la conciliation des différents domaines de vie. La carrière professionnelle et la satisfaction que les individus en retirent peut ainsi être vue comme résultant des interdépendances entre domaines et des stratégies mises en place grâce à leurs dispositions et leurs ressources adaptatives pour concilier les rôles sociaux inhérents aux domaines de vie, comme l'illustre la Figure 1.

Figure 1. Modèle de conciliation des domaines de vie

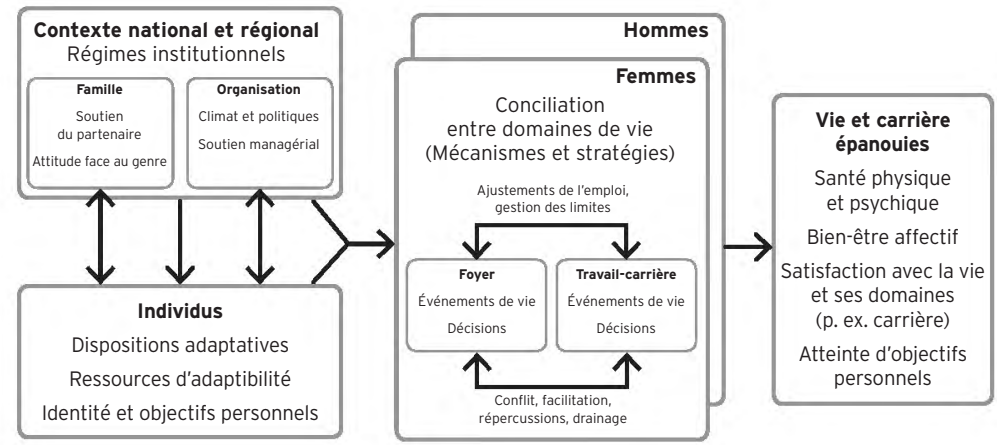

\subsection{UNE VIE ET UN PARCOURS PROFESSIONNEL ÉPANOUIS ET ÉQUILIBRÉS}

Dans le cadre du paradigme "construire sa vie», une grande part de subjectivité est laissée aux individus pour définir une vie épanouie. Celle-ci peut se caractériser par un épanouissement dans les domaines de vie qui ont du sens pour eux et qui satisfont leurs 
besoins de base, ainsi que par un bien-être subjectif durable (Rossier, Ginevra, Bollmann, \& Nota, 2017). Une vie épanouie comprend donc aussi bien la poursuite et l'atteinte d'objectifs chers à l'individu (bien-être eudémonique) qu'un sentiment entremêlant affects positifs et négatifs ainsi que satisfaction de vie au sens large (Bollmann, Johnston, Maggiori, \& Rossier, en prép.) ou à l'égard de domaines spécifiques tels que le travail, la carrière, la famille, les études ou les loisirs (bien-être hédonique; Diener, Scollon, \& Lucas, 2003). Cette conception d'une vie épanouie est aussi cohérente avec la notion d'équilibre travail-famille défini comme «l'évaluation globale de la cohérence entre l'efficacité et la satisfaction d'une personne dans ses rôles professionnels et familiaux et ses valeurs personnelles à un moment donné» (Greenhaus \& Allen, 2011, p. 174).

Dans cette approche, le domaine professionnel - l'emploi et plus généralement la carrière - s'intègre dans un parcours de vie plus large, défini comme un enchevêtrement de phases où les individus concilient les différents rôles sociaux qu'ils occupent. Le domaine professionnel est alors en interdépendance constante avec d'autres domaines, comme celui du couple, de la famille, des amis, ou de l'engagement communautaire ${ }^{2}$. A la différence du schéma normatif de la carrière traditionnelle au cours de laquelle se succèdent les emplois à temps complet suivant une ascension progressive au sein de la hiérarchie d'une même entreprise ou d'un nombre limité d'entre elles (encore très répandu), les parcours professionnels se définissent aussi par une succession d'emplois et/ou de périodes de non-emploi, caractérisées par des rapports de travail de diverses natures (Arthur, Khapova, \& Wilderom, 2005; Williams et al., 2016). Dans ces nouveaux parcours professionnels, les personnes occupent des emplois dans plusieurs branches ou professions, à différents niveaux hiérarchiques et pour plusieurs organisations se distinguant elles-mêmes par des cultures et pratiques de mobilité interne plus ou moins élaborées; on parle ainsi de carrière sans frontière ou de carrière personnalisée (Arthur et al., 2005; Greenhaus \& Kossek, 2014). Témoin de cette diversification des parcours professionnels, la proportion de personnes occupant un emploi à durée déterminée, travaillant à temps partiel ou occupant plus d'un emploi est en progression constante depuis près de vingt-cinq ans

2. En accord avec les perspectives récentes (Greenhaus \& Kossek, 2014), l'accent est mis ici sur les interdépendances entre le domaine du travail et celui du foyer, qui regroupe autant le couple et/ou la famille que les amis, ou le développement personnel. 
dans de nombreux pays de l'OCDE, dont la Suisse (Murier, 2010; OCDE, 2018).

À l'instar de la notion de vie épanouie, la conception du parcours professionnel épanoui laisse également une place importante au récit professionnel relaté par les individus, à leur satisfaction avec leur carrière ou leur emploi, et à l'équilibre entre domaines de vie (Savickas, 2005). Ainsi, bien que les parcours professionnels puissent être décrits par des indicateurs objectifs traditionnels, tels que le salaire, le nombre de promotions, l'ancienneté ou les changements d'emploi (Wang, Olson, \& Shultz, 2013), ces indicateurs objectifs se distinguent du sentiment de succès - avec lequel ils ne présentent, par ailleurs, que des associations modérées (Ng, Eby, Sorensen, \& Feldman, 2005). En effet, si certaines personnes voient une promotion comme un signe de réussite, toutes n'en sont pas toujours avides, même aux niveaux les plus élevés (Packard, 2015). De même, si les formes de travail flexibles telles que le télétravail, les horaires souple ou les postes à temps partiel peuvent répondre aux besoins de certaines personnes, elles peuvent également être vécues comme une source de précarité par d'autres (Duffy, Blustein, Diemer, \& Autin, 2016).

\subsection{INTERDÉPENDANCES ENTRE DOMAINES DE VIE}

\section{ET EFFETS SUR LA SANTÉ ET LE BIEN-ÊTRE}

Les interdépendances entre domaines de vie peuvent s'observer sous une multitude de phénomènes (Edwards \& Rothbard, 2000; Wiese, Seiger, Schmid, \& Freund, 2010). Par exemple, l'interdépendance entre les domaines familial et professionnel peut générer des conflits caractérisés par le fait que les pressions ou exigences vécues dans un domaine entravent le fonctionnement de l'autre domaine (Greenhaus \& Beutell, 1985). À l'inverse, ces interdépendances peuvent conduire à un enrichissement entre domaines lorsque les situations ou les expériences vécues dans l'un des domaines bénéficient à l'autre (Greenhaus \& Powell, 2006). Les effets de répercussion (spillover effects; Edwards \& Rothbard, 2000) s'observent eux dans le cadre de conflits ou d'enrichissements lorsque des humeurs, des valeurs, des compétences ou des comportements d'un domaine se transferrent à un autre domaine. Enfin, un phénomène de drainage des ressources se manifeste lors d'un transfert de ressources limitées (telles que le temps, l'argent ou l'énergie) d'un domaine vers un autre (Edwards \& Rothbard, 2000). 
Plusieurs méta-analyses suggèrent l'existence d'effets de répercussion et de drainage entre les domaines professionnel et familial sur divers indicateurs de santé et de satisfaction (Amstad, Meier, Fasel, Elfering, \& Semmer, 2011; McNall et al., 2010; Shockley \& Singla, 2011; pour une étude sur les interdépendances entre loisirs, travail et famille, Knecht, Wiese, \& Freund, 2016). Ainsi, de manière générale, les personnes qui rapportent que leur travail enrichit leur vie familiale ou que leur vie de famille enrichit leur vie professionnelle témoignent d'une meilleure santé physique et mentale, ainsi que d'une satisfaction de vie plus élevée (McNall et al., 2010). À l'inverse, les personnes rapportant vivre des conflits entre ces différents domaines sont davantage susceptibles que les autres de se sentir en moins bonne santé, de vivre un burnout, de présenter des symptômes physiques et des états dépressifs, ou d'abuser de substances (Amstad et al., 2011). En retour, ces manifestations tendent à renforcer les conflits au cours du temps, au risque de conduire à un cercle vicieux (Nohe et al., 2015). Néanmoins, de nombreux.ses employé.es traversant de tels conflits semblent capables de s'adapter efficacement et de retrouver leur bien-être après une période de souffrance (Matthews, Wayne, \& Ford, 2014). À noter qu'au-delà des effets sur la satisfaction avec le domaine d'où les interdépendances tirent leur origine, ces dernières affectent également la satisfaction des individus envers les domaines qu'elles touchent, bien que dans une plus faible mesure. Par exemple, les interférences du travail sur la vie de famille sont plus étroitement associées à du stress au travail et à une moindre satisfaction envers son emploi qu'envers sa situation maritale et familiale (Amstad et al., 2011; Shockley \& Singla, 2011). De même, l'enrichissement de la vie professionnelle par la vie familiale est systématiquement lié à la satisfaction envers sa vie de famille, et dans une moindre mesure à la satisfaction envers son emploi (McNall et al., 2010; Shockley \& Singla, 2011).

Si le bien-être des femmes et des hommes semble être impacté de manière relativement similaire par les conflits interdomaines (Nohe et al., 2015; Shockley \& Singla, 2011), il apparaît tout de même que la satisfaction des femmes envers leur emploi et envers leur famille profite davantage que celle des hommes d'un enrichissement de leur vie familiale par leur emploi (McNall et al., 2010; Shockley \& Singla, 2011). Les conflits famille-travail semblent en outre plus affecter le parcours professionnel des femmes que celui des hommes. Shockley, Shen, DeNunzio, Arvan et Knudsen 
(2017) relèvent que les femmes témoignent vivre le plus d'interférences entre les domaines familial et professionnel, bien que les hommes passent davantage de temps à leur travail, vraisemblablement parce que les femmes sont plus nombreuses à exercer des professions ou des emplois à des taux d'activité réduits et jouissant de moins d'autonomie tout en assumant un rôle familial plus important. Ainsi, tandis que les hommes ont tendance à maintenir une séparation entre le domaine professionnel et les autres domaines de leur vie, les femmes semblent plus tenir compte des besoins de leurs proches. Plus de femmes que d'hommes annoncent ainsi avoir accommodé leur carrière aux exigences familiales et un plus grand nombre d'entre elles ont déjà déménagé pour leur conjoint (Mainiero \& Sullivan, 2005). Comme le mentionnent Shockley et ses collègues (2017), ces comparaisons interindividuelles restent cependant peu parlantes sans plus d'information quant à la nature des conflits, aux dynamiques intra-individuelles et aux décisions prises pour les régler. Or, lorsqu'on s'intéresse à des événements en particulier, par exemple la naissance du premier enfant, il apparaît que les femmes sont plus affectées que les hommes dans leurs trajectoires de satisfaction avec la vie, le travail et les loisirs dans les années précédant et suivant la maternité (Bernardi, Bollmann, Potarca, \& Rossier, 2017). De même, douze mois après leur retour au travail, les mères qui expérimentent plus de conflits entre le travail et la famille affectant leur santé mentale et physique que les autres semblent aussi plus susceptibles de quitter leur emploi (Carlson et al., 2011). Ce constat pose donc la question des antécédents à ses différences entre femmes et hommes dans les dynamiques d'interdépendance entre domaines de vie. Dans la section qui suit, nous abordons cette question d'abord à la lumière du contexte social et plus précisément des normes de genre qui y règnent, puis à celle des ressources d'adaptabilité de carrière.

\section{RÔLE DU CONTEXTE SOCIAL ET DES RESSOURCES D'ADAPTABILITÉ DANS LES INTERDÉPENDANCES ENTRE DOMAINES DE VIE}

\subsection{CONTEXTE SOCIAL ET NORMES DE GENRE}

Au cours des dernières décennies, la répartition traditionnelle des rôles sociaux entre femmes au foyer et hommes pourvoyeurs de revenus a remarquablement changé. Aux États-Unis par exemple, 
la proportion de ménages à double revenu parmi les couples mariés ayant des enfants de moins de 18 ans est passée de $25 \%$ en 1960 à $60 \%$ en 2012 (Pew Research Center, 2015). En Suisse, la proportion de mères d'enfants de moins de 15 ans occupant un emploi est passée de 57,5\% en 1991 à 74,3\% en 2013 (Lässig \& Vuille, 2014). Malgré des changements dans les structures familiales et la présence accrue des femmes sur le marché du travail, certaines différences entre les sexes sont néanmoins encore marquées. En 2014, en Suisse, parmi les mères d'enfants de moins de 25 ans actives et occupées professionnellement, $81 \%$ travaillaient à temps partiel, alors que les hommes n'étaient que $8,3 \%$ à le faire (Csonka \& Mosimann, 2017). En outre, les femmes continuent d'exécuter la majorité des tâches liées au ménage et à la famille, un constat qui se retrouve dans d'autres pays de l'OCDE tels que l'Autriche, l'Allemagne et les États-Unis (Csonka \& Mosimann, 2017; OECD Family Database, 2016).

La théorie des rôles sociaux (Eagly, 1987; Eagly \& Wood, 2012) avance que la distribution inégale des femmes et des hommes dans diverses professions ainsi que dans le travail non rémunéré a des implications sur les croyances que les individus ont à l'égard des rôles que les deux sexes devraient occuper dans la société (rôles ou normes de genre) et plus généralement à l'égard des traits qui les caractérisent. Cette théorie propose que les caractéristiques physiques des individus - la taille et la force des hommes et les caractéristiques reproductives des femmes en matière de gestation et d'allaitement - en interaction avec les caractéristiques sociostructurelles en place rendent certaines activités plus efficaces pour l'un ou l'autre sexe (Eagly \& Wood, 2012), desquelles découlent les normes liées au genre. Ces normes de genre orientent en outre les comportements et décisions des individus et plus généralement leur parcours de vie par l'intermédiaire de mécanismes biologiques et psychosociaux (Eagly \& Wood, 2012).

La théorie avance que, par des mécanismes d'autorégulation, les femmes et les hommes conçoivent leurs propres comportements et décisions en fonction des normes de genre qu'ils intègrent dans leur identité et leur concept de soi. Et, en effet, les hommes se projetant dans leur trentaine imaginent par exemple plus que les femmes poursuivre des objectifs liés au statut social, tandis que ces dernières s'imaginent plus développer des objectifs en matière de soins, ce qui mène les deux sexes à s'intéresser à des carrières facilitant la 
poursuite de ces objectifs (Evans \& Diekman, 2009). D'autre part, la théorie propose également que, par des mécanismes de régulation sociale, c'est-à-dire au travers des attentes sociales et des réactions de leur entourage, les femmes et les hommes sont poussés à concilier leurs domaines de vie selon les normes de genre prévalant dans les différents contextes sociaux qu'ils côtoient (Eagly \& Wood, 2012). Les attitudes envers les rôles de genre régnant au sein du couple jouent ainsi un rôle dans les conflits travail-famille vécus et les décisions prises par les mères en emploi. Celles dont les conjoints ont des attitudes plus traditionnelles vivent plus de conflits travailfamille (Steiner, Krings, \& Wiese, 2018) et ajustent le temps d'interruption de travail et leur taux d'activité à la suite d'une maternité (Stertz, Grether, \& Wiese, 2017). Or ces décisions concernent des aspects importants des parcours professionnels, sachant que les parents qui se retirent temporairement du marché du travail vont à l'encontre de notions d'engagement et de mérite prévalant dans le monde professionnel. Les parents sont en effet plus durement jugés que les chômeurs ou que les parents ne faisant pas le choix de se retirer du marché du travail (Weisshaar, 2018). De même, à l'embauche, les mères sont perçues comme moins compétentes et moins agentiques que les femmes sans enfant ou les pères (Heilman \& Okimoto, 2008), un phénomène que Correll et ses collègues (2007) nomment "pénalité de maternité» (motherhood penalty).

Ces résultats, en accord avec la théorie des rôles sociaux, suggèrent d'intervenir au niveau des régimes institutionnels et des politiques organisationnelles pour réduire les différences entre femmes et hommes dans la conciliation de leurs domaines de vie (voir aussi, Kossek, Su, \& Wu, 2016; Tomlinson, Baird, Berg, \& Cooper, 2017). Ainsi, certains pays de l'OCDE ont par exemple mis en place des régimes de congés parentaux permettant aussi bien aux hommes qu'aux femmes de prendre des congés payés et protégés lorsqu'ils deviennent parents, des politiques favorisant les structures d'accueil journalier pour enfants, ainsi que plus généralement des lois permettant des modes d'organisation flexible du travail (Hegewisch \& Gornick, 2011). La population soutient largement ce type de régimes dont les bénéfices sont documentés (Lalive \& Zweimüller, 2009; Valarino, 2018). Dans le contexte de la parentalité, les régimes, de congés parentaux sont aussi plus utilisés si les organisations s'y associent par des politiques et des climats organisationnels cohérents (O’Neill et al., 2009). Dans la mesure où 
ces initiatives peuvent réduire le nombre de jours d'absence ou les intentions de changer de poste (Ropponen, Känsälä, Rantanen, \& Toppinen-Tanner, 2016), il apparaît également bénéfique pour ces dernières de soutenir et encourager leur utilisation, et ce, non seulement par les femmes, mais aussi par les hommes (Haas, Allard, \& Hwang, 2002; Lévy, 2018), ainsi que de continuer à développer l'offre d'outils pour les couples (Girardin, Bühlmann, Hanappi, Le Goff, \& Valarino, 2016) comme pour les familles monoparentales (Struffolino, Bernardi, \& Larenza, 2018).

\subsection{RESSOURCES D'ADAPTABILITÉ}

En complément de l'influence du contexte social et des normes de genre, la théorie de la construction de carrière (Savickas, 2005, 2013) considère le développement de la carrière et, par extension, la conciliation de différents domaines de vie comme un processus d'adaptation continu entre les individus et leur environnement. Dans cette théorie, l'environnement est peu formalisé, mais est décrit en cohérence avec la théorie des rôles sociaux comme le contexte social d'une personne (par exemple, famille, entreprise, lieu de résidence, culture locale). Femmes et hommes y concilient leurs domaines de vie et leur identité de manière proactive et adaptée au moyen de dispositions adaptatives et de ressources d'adaptabilité (Hirschi, Herrmann, \& Keller, 2015). Les dispositions adaptatives comprennent la personnalité, l'intelligence, l'estime de soi et les traits de personnalité proactive et résiliente, toutes associées à l'adaptabilité de carrière (pour une méta-analyse, Rudolph, Lavigne, \& Zacher, 2017). Les ressources d'adaptabilité se distinguent des dispositions essentiellement par leur moindre stabilité dans le temps et leurs propriétés autorégulatrices (Rossier, 2015; voir également, Bollmann, Rouzinov, Berchtold, \& Rossier, 2019). Parmi ces ressources, l'adaptabilité de carrière - la capacité à se préparer aux transitions professionnelles, à assumer la responsabilité de leur parcours, à explorer les opportunités et à croire en leur capacité à surmonter les défis de leur carrière (Savickas, 2005) - est considérée comme centrale dans la théorie de la construction de carrière (Hirschi, 2012). Elle se compose de l'intérêt, la volonté de faire des choix pour sa carrière professionnelle, le contrôle, ou le sentiment de responsabilité pour ses choix et décisions de carrière, la curiosité, l'envie d'explorer différentes perspectives et opportunités proposées par le monde du travail, ainsi que la confiance, la 
conviction de pouvoir surmonter les obstacles se dressant sur son parcours professionnel.

De nombreux travaux adoptant une perspective interindividuelle attestent du facteur protecteur que constitue l'adaptabilité de carrière, ainsi que d'autres ressources adaptatives pour le bien-être et la santé au travers des stratégies qu'elles favorisent ( $\mathrm{Ng}$ et al., 2005; Rudolph et al., 2017). Les personnes bénéficiant de plus de ressources d'adaptabilité de carrière témoignent d'une meilleure santé, d'une satisfaction plus élevée envers différents domaines de vie, ainsi qu'envers la vie en général (Maggiori, Johnston, Krings, Massoudi, \& Rossier, 2013; Rudolph et al., 2017) et d'une moindre augmentation de stress au travail au cours du temps que les autres (Fiori, Bollmann, \& Rossier, 2015). Relativement peu d'études se sont jusque-là intéressées aux liens entre l'adaptabilité de carrière et la conciliation des domaines de vie. Néanmoins, une étude qualitative fondée sur 13 interviews de femmes de plus de 50 ans suggère que ces dernières rapportent souvent des ressources élevées en termes d'adaptabilité de carrière et perçoivent une certaine flexibilité et autonomie dans leur travail, leur permettant de concilier les sphères professionnelle et familiale de manière personnelle (Whiston, Feldwisch, Evans, Blackman, \& Gilman, 2015).

Pour illustrer ce potentiel et pallier le manque d'information existant à ce sujet, la section suivante présente deux outils d'intervention et leur illustration clinique, qui mettent en lumière des moyens d'aborder les problématiques de conciliation de domaines dans l'orientation. Ces illustrations servent en outre à relever l'influence du contexte social dans le vécu des conflits interdomaines et offrent des perspectives de réflexion sur le rôle que les ressources d'adaptabilité peuvent jouer dans la conciliation des domaines de vie.

\section{OUTILS D'INTERVENTION ET ILLUSTRATIONS CLINIQUES}

L'inventaire du système des activités et l'analyse des rôles de vie invitent les personnes à se positionner par rapport aux différents domaines de vie dans lesquels elles évoluent et à explorer les relations y existantes. Pour chacun de ces outils, après une brève présentation suit une illustration clinique de leur application dans le cadre d'un accompagnement en orientation. Ces illustrations ainsi que les outils sont ensuite discutés à la lumière des notions théoriques abordées en première partie de chapitre. 


\subsection{INVENTAIRE DU SYSTÈME DES ACTIVITÉS (ISA)}

L'inventaire du système des activités se compose d'un jeu de cartes sur lesquelles sont inscrites des activités-buts liés à quatre domaines de vie représentés chacun par une couleur de carte différente: professionnel, familial, social et personnel (Curie, Hajjar, Marquie, \& Roques, 1990; Pouyaud, 2008). Le domaine familial propose par exemple des items comme "préserver un bon climat familial» ou "me rendre disponible pour m'occuper de mes enfants", tandis que le domaine social est composé d'activités-buts tels que "recevoir des amis chez moi» ou "être, devenir membre d'un club, d'une association ». Pour chacun des quatre domaines, deux cartes vierges sont également proposées afin de permettre à la personne d'indiquer des objectifs personnels.

Dans un premier temps, les consultant.e.s sont invité.e.s à classer les activités-buts propres à chaque domaine par ordre d'importance (de 1 = très important à 3 = pas très important), puis, pour chaque domaine séparément, à choisir une à deux activités prioritaires parmi celles jugées moyennement ou très importantes. Dans un second temps, les consultant.e.s établissent une nouvelle hiérarchie parmi ces activités-buts, mais cette fois avec les quatre domaines confondus. À cette étape, la couleur des cartes leur permet de visualiser le ou les domaines de vie prédominant parmi les activités-buts jugés les plus importants. Dans un troisième temps enfin, les consultant.e.s sélectionnent pour chaque domaine deux activités-buts considérés comme prioritaires et indique si les autres activités-buts soutiennent l'objectif prioritaire (enrichissement), interferent avec celui-ci (conflit) ou n'ont aucun rapport. À chacune de ces étapes, les consultant.e.s sont invité.e.s à commenter leur classement et à partager leurs réflexions et raisonnement.

Initialement développé pour la recherche auprès de différentes populations d'adultes comme de jeunes (Curie et al., 1990; Louche, 2007; Pouyaud, 2008), l'ISA semble aussi très pertinent dans le cadre de démarches d'orientation avec des consultant.e.s qui vivent un mal-être dans leur situation professionnelle actuelle et/ou qui souhaitent identifier des pistes d'évolution professionnelle. L'outil peut ainsi être proposé en complément des investigations et explorations plus classiques (par exemple, intérêts, personnalité, valeurs, compétences), dans le but d'élargir la nature des échanges et d'inclure les autres domaines de vie dans la réflexion sur la situation actuelle de la personne et sur ses désirs d'évolution. 
L'outil permet alors d'initier une réflexion sur son investissement et sur les interdépendances vécues. Les étapes de classement permettent aux consultant.e.s d'identifier les activités-buts qui leur sont prioritaires, de prendre conscience de l'importance relative qu'occupe chaque domaine dans leur vie et de réfléchir aux interdépendances entre les activités-buts qu'elles et ils poursuivent dans ces derniers.

\section{IIllustration clinique}

Claire $^{3}, 30$ ans, jeune pasteure exerçant depuis deux ans dans une paroisse de campagne, exprime un mal-être au niveau de son rôle et de son environnement professionnel. Elle «étouffe dans la grande cure à moitié vide", où elle vit avec son mari, et réfléchit à changer d'orientation. Elle souhaite réaliser un bilan de compétences dans l'espoir de se positionner dans sa situation actuelle et de dégager des pistes d'évolution professionnelle. La consultante se livre facilement en entretien et s'investit fortement en effectuant les tâches proposées avec engagement et sérieux, ce qui atteste, avec son optimisme et sa confiance en ses capacités professionnelles, de ses importantes ressources. Claire élabore en premier lieu une ligne de vie qui frappe par le contraste que cette dernière révèle entre ses trajectoires professionnelle (formation comprise) et personnelle. Alors que la trajectoire professionnelle relate un parcours sans fautes mettant en lumière une jeune fille, puis une femme, avide d'apprentissages, brillante et curieuse sur le plan intellectuel, la trajectoire personnelle dresse un tableau très sombre, évoquant des relations affectives difficiles ayant conduit à des événements traumatiques durant son enfance et son adolescence. La discussion révèle qu'en parallèle de la remise en question professionnelle, Claire vit une crise avec son mari, dont elle s'est temporairement séparée. Cette situation contrastée nous incite à proposer l'ISA.

Claire accueille l'outil avec curiosité et intérêt, elle apprécie les cartes colorées, son aspect ludique et remarque que la réflexion est favorisée par la manipulation du matériel. Le premier exercice nous apprend qu'au niveau professionnel, Claire met l'accent sur son besoin d'apprendre et son désir de développement (l'objectif prioritaire étant de «reprendre des études»). Ses choix dans le domaine social mettent en exergue sa personnalité sociable et extravertie

3. Cette vignette s'inspire d'une situation réelle, anonymisée, rencontrée au Service de consultations de l'Université de Lausanne. Son récit accentue certains éléments dans le but de faciliter l'analyse. 
(l'objectif prioritaire est d' «aller régulièrement chez des amis/les recevoir chez moi»). Le domaine personnel, investi avec enthousiasme, révèle son amour pour la lecture et son besoin d'avoir des moments pour elle, seule (objectifs prioritaires: "prendre du temps pour lire", "acheter des livres"). Le domaine familial est enfin le plus difficile à aborder; le classement s'accompagne de beaucoup d'hésitations et de soupirs, en particulier pour la carte "avoir des enfants/ou un enfant de plus». Après une longue hésitation, Claire choisit comme projet prioritaire "se marier - vivre en couple». Le deuxième exercice révèle la priorité des domaines personnel et professionnel sur ceux social et familial. Claire l'explique par son ambition professionnelle et sa compréhension de son oasis personnelle comme d'une ressource importante. Le dernier exercice, mettant en lumière les relations entre les différentes activités intraet interdomaines, permet de faire deux constats. En premier lieu, Claire observe beaucoup d'interactions positives entre les activités qu'elle identifie comme des "ressources", ce qui l'amène à déclarer que ses objectifs ne devraient pas être difficiles à atteindre. Le deuxième constat concerne le projet d'avoir et d'éduquer des enfants, qui entre le plus souvent en conflit avec les autres projets. Encouragée à réfléchir sur ce point, Claire exprime sa double envie de s'investir entièrement dans une carrière professionnelle et de fonder une famille. Elle n'imagine pas encore comment ces deux projets pourraient cohabiter harmonieusement, les percevant comme incompatibles. Claire est en outre ambivalente quant à son réel désir d'avoir des enfants, tout en reconnaissant les attentes de son entourage proche. Elle en conclut qu'il lui faut entreprendre une thérapie de couple afin de tenter de sauver cette relation.

\subsection{ANALYSE DES RÔLES DE VIE (ARV)}

L'analyse des rôles de vie est un exercice développé par Brott (2004, 2011) sur la base d'un outil créé par Brown et Brooks (1991). Elle vise à aider les consultant.e.s à adopter une position de recul et de guider leur réflexion au-delà du domaine professionnel, en ouvrant la discussion et l'exploration sur d'autres domaines de vie. Une place importante est donnée à l'analyse des interactions entre les différents rôles de vie que chacun·e occupe au quotidien, ainsi qu’à leur évolution, passée ou souhaitée (Gysbers, 2006).

Concrètement, l'outil requiert des consultant.e.s qu'ils.elles représentent les différents rôles (par exemple parent, partenaire, 
employé.e, enfant, ami, choriste) qu'elles et ils occupent à l'aide de cercles. La taille des cercles représente l'importance actuelle des rôles dans la vie de la personne, et leurs chevauchements leur degré d'interdépendance. Les consultant.e.s sont invité.e.s par le ou la psychologue conseiller ère en orientation à rendre compte de leur schéma en indiquant l'importance relative des rôles, la nature de leurs interdépendances, la satisfaction par rapport à l'ensemble, ou encore les changements souhaités selon la problématique en question. À la suite de cette discussion, le ou la psychologue conseiller.ère en orientation peut proposer de réaliser le même exercice en se projetant dans le futur et/ou à une époque passée (par exemple, plus ou moins cinq ans). Alors que la rétrospective permet de souligner le chemin parcouru en général et de réfléchir en termes de changements vécus, de satisfactions retirées ou de ressources activées, la projection dans le futur vise à favoriser la mise en exergue des changements souhaités et la réflexion sur les ressources à mobiliser pour les réaliser.

\section{Illustration clinique}

Paul $^{4}, 44$ ans, exprime une insatisfaction importante quant à la place qu'occupe, dans sa vie, le domaine professionnel. Père de trois enfants en âge scolaire, il est depuis une dizaine d'années père au foyer; sa femme exerce une activité à plein temps. Paul est également engagé à hauteur de $40 \%$ dans une activité d'achat-revente d'objets, qu'il gère avec une associée. Diplômé universitaire, professionnel et post-gradué, il explique ne pas avoir réussi jusqu'à présent à s'engager pleinement et durablement dans une activité professionnelle. Il souhaite comprendre et débloquer «les freins» qui l'empêchent de se construire un parcours professionnel satisfaisant. Paul explique en outre subir une certaine pression de l'entourage étant donné cette situation assez atypique, les pères au foyer étant plutôt rares en Suisse. Son entourage lui suggère régulièrement de "régulariser» sa situation en cherchant un «vrai» travail. Sa femme semble se montrer ambivalente à ce sujet, même si sa carrière professionnelle bénéficie de la répartition actuelle des rôles. Paul réagit aux attentes de son entourage avec une certaine résistance, en revendiquant ce modèle de vie où le travail n'occupe pas le devant

4. Cette vignette s'inspire d'une situation réelle, anonymisée, rencontrée au Service de consultations de l'Université de Lausanne. Son récit accentue certains éléments dans le but de faciliter l'analyse. 
de la scène, mais aussi avec une insatisfaction relative au déséquilibre engendré au sein du couple. Sa femme étant la "pourvoyeuse des besoins" de la famille, il a parfois le sentiment qu'il n'est pas légitime pour lui d'exprimer ses besoins et désirs. Paul réalise l'ARV à domicile entre deux séances, en représentant sa vie actuelle ainsi que son idéal dans cinq ans de la manière suivante (Figure 2).

\section{Figure 2. Rôles de vie - Paul - situation actuelle et dans cinq ans (trait discontinu)}

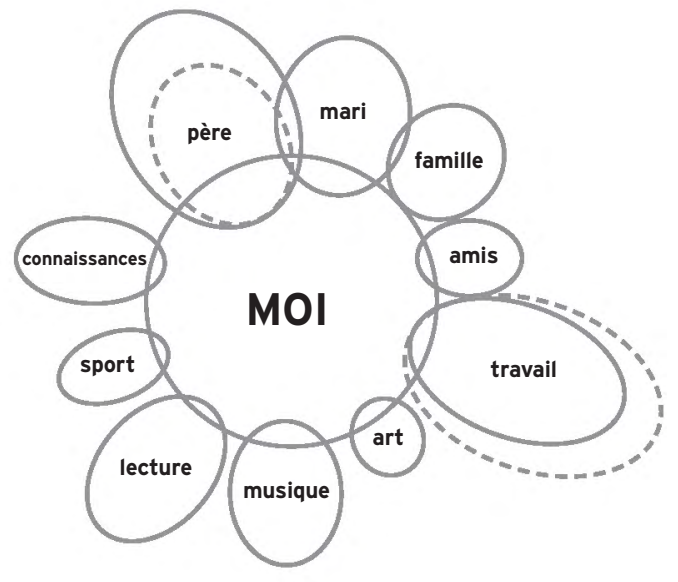

Paul précise que la taille des cercles représente le temps requis pour les activités en question et non leur importance. À ses yeux, tous les rôles ont la même importance, car ils sont nécessaires au maintien de son équilibre et de son bien-être: «Ben, c'est clair, le père, les enfants, ils vont passer avant tout le reste, mais je sais que je dois cultiver le reste pour être un bon père pour mes enfants! C'est pourquoi je me suis mis, moi, au centre. Je sais que j'ai besoin de cet équilibre pour pouvoir affronter toutes ces activités." Il remarque que seul son rôle professionnel l'empêche d'atteindre un équilibre satisfaisant. Selon sa projection dans cinq ans, le travail devrait occuper plus de temps sans pour autant supplanter le rôle du père, qui devrait en occuper moins. Il ne changerait rien aux autres rôles, ne voudrait pas que son activité professionnelle occupe plus que 50-60\% de son temps et ne "souffre» pas d'être homme et père au foyer, au contraire. Son activité indépendante lui convient grâce à la liberté et à la flexibilité qu'elle lui offre pour 
concilier ses différents rôles. Ses constats affermissent sa position visà-vis de son entourage, qui voudrait, selon lui, le voir «rentrer dans la norme». Ils permettent de cerner davantage la source personnelle d'insatisfaction liée à son activité professionnelle: il voudrait que son activité ait plus de sens pour s'y investir et s'y engager pleinement. Forts de ces réflexions, nous décidons avec Paul d'orienter la suite du processus d'accompagnement, d'une part, autour des freins qui l'empêchent de s'engager davantage dans le rôle professionnel et, d'autre part, sur ses désirs et besoins en termes de réalisation professionnelle.

\subsection{DISCUSSION DES ILLUSTRATIONS CLINIQUES}

\section{ET APPORTS DES OUTILS}

L'insatisfaction qui amène Claire et Paul à consulter concerne certes le domaine professionnel, mais il apparaît, conformément au paradigme "construire sa vie», qu'il faille placer cette insatisfaction en relation avec les autres domaines de vie pour la comprendre. En parallèle de sa remise en question professionnelle, Claire vit une crise conjugale, qui engendre probablement des répercussions négatives bidirectionnelles entre les deux domaines. Paul relate également des tensions dans son couple dues à sa situation professionnelle insatisfaisante. Son rôle de père au foyer "draine» une partie du temps et de l'énergie qui lui font défaut pour s'investir pleinement dans son rôle professionnel. L'impact du contexte social suggéré par la théorie des rôles sociaux est également visible dans les deux situations. Les choix et positions de vie de Claire et de Paul vont à l'encontre des normes de leur entourage proche et, plus généralement, du contexte traditionnel suisse. Ces contextes font obstacle à leurs efforts de définir un équilibre de vie satisfaisant. Le profil de Claire se distancie du rôle traditionnellement associé aux femmes par son ambition et son désir fort d'accorder une place importante à sa carrière professionnelle, mais aussi par le temps restreint qu'elle semble avoir prévu pour une famille. Dans sa position de pasteure, qu'elle voit comme une conséquence «logique» de ses études de théologie, elle se rend compte que la teneur très altruiste de ce rôle ne lui convient pas, car elle souffre d'un manque de stimulation intellectuelle et de défis à relever. Se distanciant ainsi du stéréotype de genre, la jeune femme doit non seulement composer avec les attentes sociales générées par son rôle de pasteure, mais aussi avec un conflit interne entre son ambition et son éventuel projet familial. Le rôle de père et d'homme au foyer de Paul est 
en contraste avec le rôle traditionnel de l'homme pourvoyeur de revenus. Les tensions résultantes de cette position anticonformiste s'expriment ici aussi sur le plan individuel et social. Paul montre une ambivalence, fier de vivre en cohérence avec ses valeurs, mais aussi frustré de ne pas s'investir pleinement dans une activité professionnelle. Au niveau social, la distribution «inversée» des rôles sociaux semble perturber l'harmonie de son couple et son entourage proche exerce une pression concrète sur lui, lui enjoignant de suivre un modèle plus traditionnel.

Claire et Paul disposent de ressources adaptatives qu'ils devront mobiliser pour développer des stratégies et solutions leur permettant de concilier les différents domaines de vie qui leur tiennent à cœur. En référence aux ressources d'adaptabilité, de carrière, le récit de Claire permet de supposer qu'elle a construit son parcours de formation de façon pro-active et engagée, caractéristique des ressources de préoccupation et contrôle, et que ses choix ont été guidés par une grande curiosité et une ouverture à la nouveauté. Sa facilité à nommer ses compétences professionnelles témoigne d'une confiance en ses capacités. Claire semble donc jouir d'importantes ressources d'adaptabilité qu'elle active en partie en décidant de suivre une thérapie de couple et qui lui permettront peut-être de trouver une issue à sa situation de crise professionnelle-familiale, par exemple en trouvant une activité qui lui permette de se réaliser pleinement. La curiosité et l'ouverture au changement de Paul sont également visibles dans son parcours professionnel et son attitude anticonformiste. Cependant, son discours révèle une "cassure» au moment de l'insertion et dans son parcours professionnel. De même, son rôle de père au foyer résulte peut-être davantage d'un concours de circonstances. Il relate ainsi des difficultés à se donner une direction et à trouver une satisfaction durable dans ses activités. Paul semble faire preuve de confiance en ses capacités intellectuelles et professionnelles, mais il doute tout de même de sa capacité à se dégager des "freins» qu'il ressent et qui l'empêchent de trouver un équilibre harmonieux. Ces deux points suggèrent un travail nécessaire sur son sentiment de contrôle (faire des choix réfléchis et les assumer) et, dans une moindre mesure, sur sa confiance, qui devraient lui permettre d'ajuster son activité professionnelle à ses désirs et, éventuellement, aux attentes de son entourage.

Ces outils d'intervention ont permis à Claire et à Paul de mener la discussion et la réflexion en prenant en compte l'ensemble de leurs 
domaines de vie et leurs interdépendances. L'ISA, par l'intermédiaire de la visualisation du nombre important d'enrichissements interdomaines, semble susceptible d'avoir renforcé la confiance et l'optimisme de Claire quant à sa capacité à résoudre les défis futurs. La difficulté que ressent la consultante dans le classement lorsqu'il est question de fonder une famille lui a peut-être permis de prendre davantage conscience de ce conflit. Pour finir, la priorisation des projets au sein du domaine familial, où Claire choisit de vouloir vivre en couple pour partager sa vie avec une personne "à côté de qui on se voit vieillir", a peut-être contribué à prendre conscience de l'importance de son couple, encourageant son choix d'entamer une thérapie de couple. Ce choix peut être assimilé à une stratégie adaptative visant à retrouver un bien-être dans les domaines de vie chers à la consultante.

L'ARV offre une grande liberté aux consultant.e.s pour déterminer les domaines de vie pertinents, les rôles qu'elles et ils y occupent et leurs interdépendances. Sa dimension diachronique permet de réfléchir avec les consultant-e.s sur la co-évolution des différentes trajectoires dans le temps, valorisée dans le cadre de l'approche dynamique du paradigme "construire sa vie», un aspect qui peut faire défaut à l'ISA. Dans le cas de Paul, cette mise en perspective temporelle semble l'avoir particulièrement aidé à prendre conscience de ses valeurs et de ses besoins personnels, ainsi qu'à clarifier la provenance des tensions qu'il ressent. L'affirmation de la position et des choix de Paul qui en résultent peut être assimilée à un début de renforcement de son sentiment de contrôle. Ce nouveau positionnement a ainsi permis d'orienter la suite de la démarche d'accompagnement.

\section{IMPLICATIONS PRATIQUES ET PERSPECTIVES DE RECHERCHE}

Les deux outils d'intervention accompagnés de leur illustration ainsi que les perspectives théoriques abordées en début de chapitre mettent en évidence le rôle du contexte social sur les trajectoires professionnelles des consultant.e.s, ainsi que celui de l'agentivité propre de ces derniers.ères. Ces éléments présentent des implications importantes pour les interventions de conciliation de domaines de vie et les perspectives de recherche future.

Premièrement, il apparaît important de mieux rendre compte du contexte social dans lequel évoluent les personnes afin de les 
accompagner de manière optimale dans la conciliation de leurs domaines de vie. Dans le contexte des outils d'intervention, cela pourrait signifier, par exemple, de chercher à identifier les mesures institutionnelles et organisationnelles à disposition des consultant.e.s, ainsi que le soutien reçu de l'éventuel.le partenaire de vie, de la ou du supérieur.e hiérarchique. En accord avec le paradigme «construire sa vie", les psychologues conseillers.ères en orientation devront veiller à ne pas porter de jugement sur les attitudes envers les normes de genre des acteurs impliqués. Au contraire, dans la perspective de ce qu'implique la définition subjective d'une vie épanouie, ils auront pour objectif de chercher à saisir l'agencement de ces normes véhiculées par différents acteurs. Diverses théories (Bronfenbrenner, 1986) et recherches empiriques (Weiss, Freund, \& Wiese, 2012) soulignent, en effet, que des contingences bénéfiques ou, au contraire, des conflits peuvent exister entre les normes sociales véhiculées par différents contextes. L'épanouissement des personnes deviendrait alors fonction de la pertinence de cet agencement pour les objectifs qu'elles cherchent à atteindre dans leurs différents domaines de vie, plutôt que la promotion d'un rôle de genre en particulier.

Du point de vue des mesures institutionnelles et organisationnelles, les transformations sociétales en cours promettent une plus grande flexibilité dans la conciliation des domaines de vie et la gestion des transitions qui y sont liées. Ces transformations semblent engendrer une lente évolution des normes et des pratiques associées aux rôles de genre, faisant tendre les sociétés vers une plus grande subjectivité dans les parcours de vie et une plus grande égalité entre les sexes. L'évolution des régimes institutionnels observée ces dernières décennies dans certains pays de l'OCDE au sujet des congés parentalité ou de la flexibilisation des lois sur l'organisation du travail semble en attester (Hegewisch \& Gornick, 2011). Au niveau organisationnel, cependant, les possibilités de flexibilité accrue en termes de congés ou de lieux et d'horaire de travail ne sont souvent pas accessibles à toutes et à tous de manière identique, ni avec les mêmes bénéfices (Kossek \& Lautsch, 2017). Par ailleurs, l'existence de politiques organisationnelles favorables à la conciliation des domaines de vie n'est bénéfique que lorsqu'elles sont associées au sentiment d'être soutenu par l'organisation et la ou le supérieur.e hiérarchique (Kossek, Pichler, Bodner, \& Hammer, 2011; Leslie, Manchester, Park, \& Mehng, 2012). La mise en place d'outils organisationnels favorables à la conciliation des domaines 
de vie doit donc passer par le développement d'un véritable climat organisationnel, caractérisé également par le soutien managérial et des normes favorables à leur utilisation (O’Neill et al., 2009).

Finalement, malgré les nombreux travaux documentant les effets de l'adaptabilité de carrière sur les stratégies adaptatives (Johnston, 2018; Rudolph et al., 2017), les travaux empiriques sont encore limités en ce qui concerne le rôle de l'adaptabilité de carrière dans le contexte de la conciliation des domaines de vie. Il reste ainsi à découvrir, par exemple, quelles stratégies sont plus facilement mobilisées par les individus ayant des niveaux élevés d'adaptabilité de carrière pour pallier des conflits interdomaines, et comment l'adaptabilité de carrière, ses dimensions, ou encore d'autres ressources d'adaptabilité peuvent permettre de gérer les conflits ou de favoriser les enrichissements interdomaines. Certains travaux suggèrent, par exemple, que ces ressources favoriseraient l'adoption de stratégies adaptatives en termes de planification et d'exploration de carrière (Gunkel, Schlaegel, Peluchette, \& Langella, 2010; Rudolph et al., 2017), des stratégies potentiellement pertinentes dans le conciliation de domaines de vie (Greenhaus \& Kossek, 2014). La décision de participer, ou non, au marché du travail, et si oui, dans quelle mesure, représente une autre stratégie cruciale pour les personnes faisant face à des besoins accrus en termes de conciliation, comme les parents (Valcour \& Ladge, 2008), notamment quant au choix du moment du retour en emploi à la suite de l'arrivée d'un enfant (Wiese $\&$ Ritter, 2012). Dans un contexte plus général, les stratégies de conciliation peuvent également impliquer l'optimisation d'objectifs personnels et/ou professionnels, des ajustements personnels initiés par les employée.es dans les ressources et les exigences de leur emploi (Ilies, Pater, Lim, \& Binnewies, 2012; Tims, Bakker, \& Derks, 2012), ou encore la mise en place de limites plus ou moins perméables entre différents domaines de vie (Ashforth, Kreiner, \& Fugate, 2000). Dans ce cadre d'intérêt, l'influence du contexte social sur le lien entre ressources d'adaptabilité d'un côté et stratégies et résultats adaptatifs de l'autre est un aspect supplémentaire qu'il faudra étudier. Dans un contexte social moins conventionnel, certaines dimensions de l'adaptabilité de carrière, comme la curiosité ou la confiance, pourraient bien influencer des stratégies axées sur l'innovation et les changements, alors que d'autres dimensions, comme le contrôle ou l'intérêt, pourraient favoriser l'adoption de stratégies protectrices des acquis. 


\section{CONCLUSION}

Les évolutions sociétales récentes ont favorisé l'investissement des individus dans une multitude de domaines de vie. Ces multiples investissements génèrent néanmoins des interdépendances qui doivent être gérées de manière efficace et harmonieuse pour prétendre à l'atteinte d'une vie et d'un parcours professionnel épanouis. Ce chapitre a cherché à mettre au jour le rôle du contexte social et le potentiel des ressources d'adaptabilité pour expliquer comment femmes et hommes parviennent à développer et faire face à différents types d'interdépendances entre domaines de vie. Il a aussi tenté d'illustrer la pertinence à tenir compte de ces facteurs dans le cadre des interventions de conseil en orientation à l'aide de deux outils d'intervention accompagnés de leur vignette clinique, ce qui a permis d'identifier une série d'implications pratiques pour les psychologues conseillers.ères en orientation et de pistes de recherche future.

\section{RÉFÉRENCES BIBLIOGRAPHIQUES}

Amstad, F. T., Meier, L. L., Fasel, U., Elfering, A., \& Semmer, N. K. (2011). A meta-analysis of work - family conflict and various outcomes with a special emphasis on cross-domain versus matching-domain relations. Journal of Occupational Health Psychology, 16, 151-169. Doi: 10.1037/a0022170.

Arthur, M. B., Khapova, S. N., \& Wilderom, C. P. M. (2005). Career success in a boundaryless career world. Journal of Organizational Behavior, 26, 177-202. Doi: 10.1002/job.290.

Ashforth, B. E., Kreiner, G. E., \& Fugate, M. (2000). All in a day's work: Boundaries and micro role transitions. Academy of Management Review, 25, 472-491. Doi : 10.5465/amr.2000.3363315.

Bernardi, L., Bollmann, G., Potarca, G., \& Rossier, J. (2017). Multidimensionality of well-being and spillover effects across life domains: How do parenthood and personality affect changes in domain-specific satisfaction? Research in Human Development, 14, 26-51. Doi : 10.1080/15427609.2016.1268893.

Bollmann, G., Johnston, C. S., Maggiori, C., \& Rossier, J. (2018). Reciprocal changes in life satisfaction and affect are moderated by self-rated health. Manuscript in preparation. 
Bollmann, G., Rouzinov, S., Berchtold, A., \& Rossier, J. (2019). Illustrating instrumental variable regressions using the career adaptability - job satisfaction relationship. Frontiers in Psychology, 10, 1481. Doi: 10.3389/fpsyg.2019.01481.

Bronfenbrenner, U. (1986). Ecology of the family as a context for human development: Research perspectives. Developmental Psycho$\log y$, 22, 723-742. Doi : 10.1037/0012-1649.22.6.723.

Brott, P. E. (2004). Constructivist assessment in career counseling. Journal of Career Development, 30, 189-200. Doi: 10.1177/089484530403000302.

BRotT, P. E. (2011). The storied approach : A postmodern perspective for career counseling. The Career Development Quarterly, 49, 304-313. Doi : 10.1002/j.2161-0045.2001.tb00958.x.

Brown, D., \& Brooks, L. (1991). Career counseling techniques. Needham Heights (MA) : Allyn \& Bacon.

Carlson, D. S., Grzywacz, J. G., Ferguson, M., Hunter, E. M., Cuinch, C. R., \& Arcury, T. A. (2011). Health and turnover of working mothers after childbirth via the work - family interface: An analysis across time. Journal of Applied Psychology, 96, 10451054. Doi : $10.1037 / \mathrm{a} 0023964$.

Correll, S. J., Benard, S., \& Paik, I. (2007). Getting a job: Is there a motherhood penalty? American Journal of Sociology, 112, $1297-$ 1339. Doi : $10.1086 / 511799$.

Csonka, Y., \& Mosimann, A. (2017). Les familles en Suisse. Rapport statistique 2017. Neuchâtel: Office fédéral de la statistique.

Curie, J., Hajjar, V., Marquie, H., \& Roques, M. (1990). Proposition méthodologique pour la description du système des activités. Le Travail Humain, 53, 103-118.

Diener, E., Scollon, C. N., \& Lucas, R. E. (2003). The evolving concept of subjective well-being: the multifaceted nature of happiness. In Advances in Cell Aging and Gerontology (vol. 15, pp. 187219). Doi : 10.1016/S1566-3124 (03) 15007-9.

Duffy, R. D., Blustein, D. L., Diemer, M. A., \& Autin, K. L. (2016). The psychology of working theory. Journal of Counseling Psychology, 63, 127-148. Doi: 10.1037/cou0000140. 
Eagly, A. H. (1987). Sex differences in social behavior: A social-role interpretation. Hillsdale (NJ): Erlbaum.

Eagly, A. H., \& Wood, W. (2012). Social Role Theory. In P. A. M. Van Lange, A. W. Kruglanski, \& E. T. Higgins (éds), Handbook of theories of social psychology: Volume two (pp. 458-476). Londres: Sage Publications. Doi: 10.1007/0-387-36921-X_12.

Edwards, J. R., \& Rothbard, N. P. (2000). Mechanisms linking work and family: Clarifying the relationship between work and family constructs. Academy of Management Review, 25, 178-199. Doi : 10.5465/amr.2000.2791609.

Evans, C. D., \& Diekman, A. B. (2009). On motivated role selection: Gender beliefs, distant goals, and career interest. Psychology of Women Quarterly, 33, 235-249. Doi: 10.1111/j.14716402.2009.01493.x.

Fiori, M., Bollmann, G., \& Rossier, J. (2015). Exploring the path through which career adaptability increases job satisfaction and lowers job stress: The role of affect. Journal of Vocational Behavior, 91, 113-121. Doi: 10.1016/j.jvb.2015.08.010.

Girardin, N., Bühlmann, F., Hanappi, D., Le Goff, J.-M., \& VALARINO, I. (2016). The transition to parenthood in Switzerland: Between institutional constraints and gender ideologies. In D. GrünOw \& M. EverTsSOn (éds), Couples'Transitions to Parenthood. Glos (UK) : Edward Elgar Publishing.

Greenhaus, J. H., \& Allen, T. D. (2011). Work - family balance: A review and extension of the literature. In Handbook of occupational health psychology (2 édition, pp. 165-183). Washington: American Psychological Association.

Greenhaus, J. H., \& Beutell, N. J. (1985). Sources of conflict between work and family roles. Academy of Management Review, 10, 76-88. Doi : 10.5465/amr.1985.4277352.

Greenhaus, J. H., \& Kossek, E. E. (2014). The contemporary career: A work - home perspective. Annual Review of Organizational Psychology and Organizational Behavior, 1, 361-388. Doi: 10.1146/annurev-orgpsych-031413-091324.

Greenhaus, J. H., \& Powell, G. N. (2006). When work and family are allies: A theory of work-family enrichment. Academy of Management Review, 31, 72-92. Doi: 10.5465/amr.2006.19379625. 
Gunkel, M., Schlaegel, C., Peluchette, J. V, \& Langella, I. M. (2010). Personality and career decisiveness: An international empirical comparison of business students'career planning. Personnel Review, 39, 503-524. Doi: 10.1108/00483481011045443.

Gysbers, N. C. (2006). Using qualitative career assessments in career counselling with adults. International Journal for Educational and Vocational Guidance, 6, 95-108. Doi: 10.1007/s10775-0069102-4.

Haas, L., Allard, K., \& Hwang, P. (2002). The impact of organizational culture on men's use of parental leave in Sweden. Community, Work \& Family, 5, 319-342. Doi : 10.1080/1366880022000041801.

Hegewisch, A., \& Gornick, J. C. (2011). The impact of workfamily policies on women's employment: A review of research from OECD countries. Community, Work \& Family, 14, 119-138. Doi : 10.1080/13668803.2011.571395.

Heilman, M. E., \& Окimoto, T. G. (2008). Motherhood: A potential source of bias in employment decisions. Journal of Applied Psychology, 93, 189-198. Doi : 10.1037/0021-9010.93.1.189.

Hirschi, A. (2012). The career resources model: an integrative framework for career counsellors. British Journal of Guidance \& Counselling, 40, 369-383. Doi : 10.1080/03069885.2012.700506.

Hirschi, A., Herrmann, A., \& Keller, A. C. (2015). Career adaptivity, adaptability, and adapting: A conceptual and empirical investigation. Journal of Vocational Behavior, 87, 1-10. Doi: 10.1016/j.jvb.2014.11.008.

Ilies, R., Pater, I. E. De Lim, S., \& Binnewies, C. (2012). Attributed causes for work - family conflict: Emotional and behavioral outcomes. Organizational Psychology Review, 2, 293-310. Doi: $10.1177 / 2041386612452288$.

Johnston, C. (2018). A systematic review of the career adaptability literature and future outlook. Journal of Career Assessment, 26, 3-30. Doi : 10.1177/1069072716679921.

Knecht, M., Wiese, B. S., \& Freund, A. M. (2016). Going beyond work and family: A longitudinal study on the role of leisure in the work - life interplay. Journal of Organizational Behavior, 37, 1061-1077. Doi: 10.1002/job.2098. 
Kossek, E. E., \& Lautsch, B. A. (2017). Work - life flexibility for whom? Occupational status and work - life inequality in upper, middle, and lower level jobs. Academy of Management Annals, 12, 5-36. Doi : 10.5465/annals.2016.0059.

Kossek, E. E., Pichler, S., Bodner, T., \& Hammer, B. H. (2011). Workplace social support and work-family conflict: A meta-analysis clarifying the influence of general and work-family-specific supervisor and organizational support. Personnel Psychology, 64, 289-313. Doi : 10.1111/j.1744-6570.2011.01211.x.

Kossek, E. E., Su, R., \& Wu, L. (2016). «Opting out» or "pushed out »? Integrating perspectives on women's career equality for gender inclusion and interventions. Journal of Management, 43, 228254. Doi: $10.1177 / 0149206316671582$.

Lalive, R., \& Zweimüller, J. (2009). How does parental leave affect fertility and return to work? Evidence from two natural experiments. The Quarterly Journal of Economics, 124, 1363-1402. Doi : 10.1162/qjec.2009.124.3.1363.

LÄssig, V., \& Vuille, A. (2014). ESPA en bref 2013 - L'enquête suisse sur la population active (Report $N^{\circ} 361-1300$ ). Neuchâtel: Office fédéral de la statistique.

Leslie, L. M., Manchester, C. F., Park, T.-Y., \& Mehng, S. I. A. (2012). Flexible work practices: A source of career premiums or penalties? Academy of Management Journal, 55, 1407-1428. Doi: 10.5465/amj.2010.0651.

LÉVY, R. (2018). Devenir parents ré-active les inégalités de genre: une analyse des parcours de vie des hommes et des femmes en Suisse. Social Change in Switzerland (vol. 18). En ligne: [https://www.socialchangeswitzerland.ch].

Louche, C. (2007). Psychologie sociale des organisations (2e édition). Paris : Armand Colin.

Maggiori, C., Johnston, C. S., Krings, F., Massoudi, K., \& RosSIER, J. (2013). The role of career adaptability and work conditions on general and professional well-being. Journal of Vocational Behavior, 83, 437-449. Doi : 10.1016/j.jvb.2013.07.001.

Mainiero, L. A., \& Sullivan, S. E. (2005). Kaleidoscope careers: An alternate explanation for the "Opt-out» revolution. Academy of Management Executive, 19, 106-123. Doi : 10.5465/AME.2005.15841962. 
Matthews, R. A., Wayne, J. H., \& Ford, M. T. (2014). A work family conflict/subjective well-being process model: A test of competing theories of longitudinal effects. Journal of Applied Psychology, 99, 1173-1187. Doi : 10.1037/a0036674.

McNall, L. A., Nicklin, J. M., \& Masuda, A. D. (2010). A metaanalytic review of the consequences associated with work-family enrichment. Journal of Business and Psychology, 25, 381-396. Doi : 10.1007/s10869-009-9141-1.

Murier, T. (2010). La multiactivité en Suisse. Une étude basée sur les résultats de l'enquête suisse sur la population active (ESPA) de 1991 à 2009. Neuchâtel: Office fédéral de la statistique.

NG, T. W. H., Eby, L. T., Sorensen, K. L., \& Feldman, D. C. (2005). Predictors of objective and subjective career success: A meta-analysis. Personnel Psychology, 58, 367-408. Doi: 10.1111/j.17446570.2005.00515.x.

Nohe, C., Meier, L. L., Sonntag, K., \& Michel, A. (2015). The chicken or the egg? A meta-analysis of panel studies of the relationship between work - family conflict and strain. Journal of Applied Psychology, 100, 522-536. Doi : 10.1037/a0038012.

O’Neill, J. W., Harrison, M. M., Cleveland, J., Almeida, D., Stawski, R., \& Crouter, A. C. (2009). Work - family climate, organizational commitment, and turnover: Multilevel contagion effects of leaders. Journal of Vocational Behavior, 74, 18-29. Doi: 10.1016/j.jvb.2008.10.004.

OCDE (2018). Taux d'emploi (indicateur). Doi: 10.1787/ f58ea354-fr.

OECD Family Database. (2016). LMF2.5: Time use for work, care and other day-to-day activities.

PACKARD, S. (2015). Why I turned down the chance to be CEO [Web log post]. En ligne: [https://www.refinery29.com/susan-packardwork-life-balance].

Pew Research Center (2015). The rise of dual income households. En ligne: [http://www.pewresearch.org/ft_dual-income -households-1960-2012-2/].

Pouyaud, J. (2008). Transitions, construction de soi et développement vocationnel: L'exemple des collégiens sorientant en lycée professionnel. 
Thèse de doctorat, du Conservatoire national des arts et métiers, Paris.

Ropponen, A., Känsälä, M., Rantanen, J., \& ToppinenTAnner, S. (2016). Organizational initiatives for promoting employee work-life reconciliation over the life course. A systematic review of intervention studies. Nordic Journal of Working Life Studies, 6, 79-100. Doi: 10.19154/njwls.v6i3.5529.

Rossier, J. (2015). Career adaptability and life designing. In L. Nota \& J. Rossier (éds), Handbook of life design: From practice to theory and from theory to practice (pp. 153-168). Berne: Hogrefe.

Rossier, J., Ginevra, M. C., Bollmann, G., \& Nota, L. (2017). The importance of career adaptability, career resilience, and employability in designing a successful life. In K. MareE (éd.), Psychology of Career Adaptability, Employability and Resilience (pp. 65-82). Springer. Doi : 10.1007/978-3-319-66954-0_5.

Rudolph, C. W., Lavigne, K. N., \& Zacher, H. (2017). Career adaptability: A meta-analysis of relationships with measures of adaptivity, adapting responses, and adaptation results. Journal of Vocational Behavior, 98, 17-34. Doi: 10.1016/j.jvb.2016.09. 002 .

SAVICKAS, M. L. (2005). The theory and practice of career construction. In Career development and counseling: Putting theory and research to work. (pp. 42-70). Hoboken (NJ): John Wiley \& Sons.

SAVICKAS, M. L. (2013). Career construction theory and practice. In S. D. Brown \& R. W. Lent (éds), Career Development and Counseling: Putting Theory and Research to Work ( $2^{\mathrm{e}}$ édition, pp. 147-183). Hoboken (NJ): John Wiley \& Sons.

Savickas, M. L., Nota, L., Rossier, J., Dauwalder, J.-P., Duarte, M. E., Guichard, J., van Vianen, A. E. M. (2009). Life designing: A paradigm for career construction in the 21st century. Journal of Vocational Behavior, 75, 239-250. Doi : 10.1016/j.jvb.2009.04.004.

Shockley, K. M., Shen, W., DeNunzio, M. M., Arvan, M. L., \& Knudsen, E. A. (2017). Disentangling the relationship between gender and work - family conflict: An integration of theoretical perspectives using meta-analytic methods. Journal of Applied Psychology, 102, 1601-1635. Doi : 10.1037/ap10000246. 
Shockley, K. M., \& Singla, N. (2011). Reconsidering work - family interactions and satisfaction: A meta-analysis. Journal of Management, 37, 861-886. Doi : 10.1177/0149206310394864

Steiner, R. S., Krings, F., \& Wiese, B. S. (2018). Remember the children, honey! Spouses'gender-role attitudes and working mothers'work-to-family conflict. Applied Psychology. Advance online publication. Doi: 10.1111/apps.12160.

Stertz, A. M., Grether, T., \& Wiese, B. S. (2017). Gender-role attitudes and parental work decisions after childbirth: A longitudinal dyadic perspective with dual-earner couples. Journal of Vocational Behavior, 101, 104-118. Doi: 10.1016/j.jvb.2017.05.005.

Struffolino, E., Bernardi, L., \& Larenza, O. (2018). Lone parenthood and employment trajectories: A longitudinal mixed-method study. LIVES Working Paper, 67, 1-32. Doi: 10.12682/lives.22961658.2018.67.

Tims, M., Bakker, A. B., \& Derks, D. (2012). Development and validation of the job crafting scale. Journal of Vocational Behavior, 80, 173-186. Doi : 10.1016/j.jvb.2011.05.009.

Tomlinson, J., Baird, M., Berg, P., \& Cooper, R. (2017). Flexible careers across the life course: Advancing theory, research and practice. Human Relations, 71, 4-22. Doi : 10.1177/0018726717733313

VALARINO, I. (2018). Is there public support for a longer and more gender equal leave scheme in Switzerland? LIVES Working Papers, 65, 1-29. Doi : 10.12682/lives.2296-1658.2018.65.

VAlcour, M., \& Ladge, J. J. (2008). Family and career path characteristics as predictors of women's objective and subjective career success: Integrating traditional and protean career explanations. Journal of Vocational Behavior, 73, 300-309. Doi: 10.1016/j. jvb.2008.06.002

Wang, M., Olson, D. A., \& Shultz, K. S. (2013). Mid and late career issues: An integrative perspective. New York: Routledge/Taylor \& Francis Group..

Weiss, D., Freund, A. M., \& Wiese, B. S. (2012). Mastering developmental transitions in young and middle adulthood: The interplay of openness to experience and traditional gender ideology on women's self-efficacy and subjective well-being. Developmental Psychology, 48, 1774-1784. Doi : 10.1037/a0028893. 
WeisshaAr, K. (2018). From opt out to blocked out: The challenges for labor market re-entry after family related employment lapses. American Sociological Review, 83, 34-60. Doi: 10.1177/0003122417752355.

Whiston, S. C., Feldwisch, R. P., Evans, K. M., Blackman, C. S., \& Gilman, L. (2015). Older professional women's views on work: A qualitative analysis. The Career Development Quarterly, 63, 98-112. Doi : 10.1002/cdq.12007.

Wiese, B. S., \& RitTer, J. O. (2012). Timing matters: Length of leave and working mothers'daily reentry regrets. Developmental Psychology, 48, 1797-1807. Doi : 10.1037/a0026211.

Wiese, B. S., Seiger, C. P., Schmid, C. M., \& Freund, A. M. (2010). Beyond conflict: Functional facets of the work - family interplay. Journal of Vocational Behavior, 77, 104-117. Doi: 10.1016/j.jvb.2010.02.011.

Williams, J. C., Berdahl, J. L., \& Vandello, J. A. (2016). Beyond work-life "integration". Annual Review of Psychology, 67, 515-539. Doi: 10.1146/annurev-psych-122414-033710. 


\title{
3. COMPRENDRE ET ACCOMPAGNER LES RECONVERSIONS PROFESSIONNELLES
}

JONAS MASDONATI, SYLVIE FRANZ ET MARC ABESSOLO

(UNIVERSITÉ DE LAUSANNE)

\begin{abstract}
e chapitre propose des clés de compréhension et d'intervention permettant de mieux saisir le phénomène de la reconversion professionnelle. Dans un premier temps, il sera question de faire état de la complexité de cette transition professionnelle, en recourant aux réflexions théoriques et aux recherches sur le sujet. Il s'agira ensuite de décrire l'outil de la ligne de vie qui, dans le cadre d'une démarche de bilan de compétences, peut aider à accompagner une personne qui vit une reconversion. Une étude de cas permettra d'incarner les concepts théoriques de la première partie et d'illustrer concrètement l'utilité du recours à l'outil de la ligne de vie. Enfin, des réflexions seront partagées quant aux perspectives d'intervention et de recherche, ainsi qu'aux portées et limites de l'outil proposé.
\end{abstract}

\section{COMPRENDRE LES RECONVERSIONS PROFESSIONNELLES}

Afin de saisir la complexité du phénomène de la reconversion professionnelle, nous proposons de la situer en tant qu'événement marquant du parcours professionnel d'un individu. S'agissant par ailleurs d'une transition de carrière complexe et multiforme, il est également important d'en comprendre les différentes configurations, ainsi que les causes et les étapes qui rythment les reconversions, tout comme ses impacts et les facteurs qui peuvent en affecter le déroulement. Partant de là, nous proposons en fin de section quatre axes de compréhension des reconversions professionnelles, visant à en saisir les enjeux.

\subsection{LA RECONVERSION EN TANT QU'ÉLÉMENT CLÉ DU PARCOURS PROFESSIONNEL}

Depuis environ deux décennies, le marché du travail connaît des transformations structurelles importantes qui ont des répercussions sur la 
configuration des parcours professionnels des individus (Boutinet, 2014; Guichard, 2015). Ceux-ci sont en effet aujourd'hui davantage diversifiés et moins normés, encadrés et rythmés socialement, si bien qu'on assiste "à une forme de dérèglement de «l'horloge sociale» et à une multiplication de transitions souvent plus désordonnées et imprévisibles» (Fournier, Zimmermann, Gauthier, Masdonati, \& Lachance, 2016, p. 98). Partant de ce constat, Fournier et collègues mettent en évidence cinq dimensions clés qui permettent de saisir les enjeux d'un parcours professionnel : sa stabilité, le sentiment de maittrise de l'individu, ainsi que sa subjectivité au travail, la temporalité et l'articulation de la sphère professionnelle avec les autres sphères de vie.

Premièrement, un parcours peut donc être appréhendé du point de vue de sa stabilité (Fournier et al., 2016). Cette dernière est tributaire de ce qui cimente les événements clés traversés par l'individu et de la manière dont les différentes étapes professionnelles s'enchaînent. Un parcours est alors d'autant plus stabilisé, par exemple, que les alternances entre emploi, non-emploi et formation sont réduites - ou fréquentes, mais prévisibles -, que la personne peut transférer ses compétences d'un emploi à l'autre, et qu'il présente une pente globale ascendante ou, en tout cas, horizontale.

Deuxièmement, un parcours professionnel peut être appréhendé en fonction du sentiment de maîtrise que la personne a sur lui, soit de son « regard en perspective [...] sur sa capacité à orienter l'ensemble de son parcours en dépit des impondérables et des contingences» (Fournier et al., 2016, p. 114). Ce sentiment de maîtrise couvre l'ensemble de la carrière et dépend, notamment, du caractère plus ou moins volontaire et prévisible des transitions qui le jalonnent, du sentiment de pouvoir tenir compte de ses priorités de vie dans son engagement au travail, mais aussi des stratégies mises en place pour composer avec les aléas et les imprévisibilités de la vie au travail.

En troisième lieu, la question de la subjectivité au travail met la focale sur le sens que l'individu attribue à son inscription dans la sphère professionnelle et sur la manière dont les représentations qu'il a de lui-même et du monde du travail contribuent à orienter son parcours (Fournier et al., 2016). Cette subjectivité au travail dépend de trois processus. D'abord, elle est influencée par le rapport à l'emploi de la personne, celle-ci pouvant se sentir solidement ancrée au marché du travail ou, à l'opposé, vivre de l'insécurité professionnelle. 
Elle est ensuite tributaire de son rapport au travail, ce dernier pouvant répondre, selon les cas, à des attentes et des finalités intrinsèques - lorsqu'il est une source centrale d'épanouissement - ou extrinsèques - lorsque la personne trouve ses sources d'épanouissement et d'identification en dehors du travail. La subjectivité au travail dépend enfin de l'identité professionnelle, et plus particulièrement de l'estime de soi au travail. Il est question ici du sentiment d'appartenance au monde du travail et de l'identification à un métier ou à une organisation, mais aussi de ce qu'implique et signifie pour un individu d'endosser le statut de travailleur et d'exercer un métier donné, dans une entreprise donnée.

Quatrièmement, un parcours doit être situé dans une temporalité, donc en fonction de l'articulation passé-présent-futur (Fournier $e t$ al., 2016). En ce sens, les expériences passées de la personne et la perception de sa situation présente affectent sa confiance en l'avenir et délimitent le champ des possibles. Porter attention à la temporalité signifie aussi comprendre l'individu en fonction de son âge subjectif, soit de la perception qu'il a de son âge en tant que facteur handicapant ou, à l'opposé, en tant qu'atout. Cette perception est subjective, puisqu'un même âge biologique peut être considéré comme un atout ou comme une entrave dans le parcours professionnel.

En cinquième lieu, un parcours professionnel doit être situé en fonction de son articulation avec les autres sphères de vie, c'est-à-dire des interinfluences entre la vie professionnelle et la vie extraprofessionnelle (Fournier et al., 2016). Cette articulation peut par exemple donner lieu à des effets de cumul ou de «transfert» de ressources ou de handicaps du travail vers le hors-travail, et inversement. Elle peut aussi révéler des situations où le hors-travail assume une fonction de palliatif identitaire, notamment lorsqu'une personne cherche dans la famille ou les loisirs des sources de valorisation qu'elle n'arrive pas à trouver dans le travail. Enfin, un regard systémique sur le parcours permet parfois de comprendre et de situer des décisions de carrière en fonction d'événements, non pas professionnels, mais biographiques. C'est le cas, par exemple, pour les transitions professionnelles s'expliquant par un changement de domicile, un bouleversement familial ou la survenue d'une maladie.

En tant qu'événements qui marquent souvent les parcours professionnels (Boutinet, 2007, 2014), les reconversions peuvent donc être appréhendées en fonction de ces cinq dimensions. Elles favoriseront alors une consolidation ou, au contraire, elles incarneront 
une instabilité générale du parcours professionnel (dimension stabilité). Leur caractère plus ou moins choisi affectera, quant à lui, le sentiment d'emprise de l'individu sur son parcours (dimension sentiment de maîtrise). Une reconversion professionnelle prendra ensuite des significations variées selon son importance aux yeux de l'individu qui la traverse (dimension subjectivité). En tant que processus de mise en projet, elle sollicitera également un vrai travail d'articulation entre passé, présent et futur (dimension temporalité). Enfin, la faisabilité et l'issue d'une reconversion dépendront des enjeux - en termes de possibilités et de contraintes, par exemple - que la personne vit dans ses autres rôles et activités (dimension articulation entre les sphères de vie).

\subsection{LA RECONVERSION EN TANT QUE TRANSITION PROFESSIONNELLE}

Les parcours professionnels contemporains sont également caractérisés par la multiplication et la complexification des transitions qu'une personne doit traverser tout au long de son cheminement professionnel (Boutinet, 2014; Olry-Louis, Vonthron, Vayre, \& Soidet, 2017; Rudisill, Edwards, Hershberger, Jadwin, \& McKee, 2010). Ces dernières prennent aujourd'hui de multiples formes, suivant qu'elles réferent à des transitions internes à une organisation (maintenance transitions), à des mobilités ascendantes (advancement transitions), à l'insertion ou réinsertion professionnelle (entry/reentry transitions), à des réorientations ou des reconversions (leave-or-seek transitions), voire à la sortie du marché du travail (Heppner \& Scott, 2006; Masdonati \& Zittoun, 2012). Au-delà des enjeux qu'elle soulève dans le cadre plus général d'un parcours professionnel, la reconversion peut donc être appréhendée et définie sous un angle transitionnel, soit comme une forme particulière de transition professionnelle (Berton, 2013; Fouad \& Bynner, 2008; Masdonati, sous presse). Elle fait alors référence à un "sous-ensemble des transitions de rôles professionnels comportant un changement d'employeur, la modification concrète de certaines activités et rôles au travail et la perception subjective que de tels changements constituent un "changement de carrière" " (Ibarra, 2006, p. 77, traduction personnelle). Une reconversion renvoie donc à une transition vers un nouvel emploi qui éloigne l'individu d'une progression de carrière typique et attendue. De ce fait, les compétences déployées dans l'ancien emploi deviennent en partie caduques, ce qui oblige la personne à entreprendre de nouveaux apprentissages, formels ou informels (Carless \& Arnup, 2011). 
Au-delà de ces définitions générales, les reconversions professionnelles peuvent elles aussi prendre plusieurs formes et couvrir des situations très variées. Une lecture typologique de cet événement permet ainsi de saisir la variété de situations que l'on peut qualifier de reconversions. En ce sens, en s'appuyant sur les témoignages de 33 salarié.e.s qui avaient démissionné ou qui avaient vécu un licenciement en France deux ans auparavant, Berton (2013) met en évidence cinq types de transition de carrière. Un premier groupe de personnes poursuivent leur carrière dans le même secteur d'emploi, auprès d'un nouvel employeur, et échappent à ce que recouvre une reconversion professionnelle, telle que définie plus haut. Dans un deuxième cas, cette transition permet aux salarié.e.s de trouver leur voie: leur ancien emploi n'était qu'une occupation provisoire et leur nouveau projet professionnel est l'occasion de mettre à profit des compétences, jusque-là inexploitées, dans une voie davantage valorisée. La véritable reconversion ne concerne qu'un troisième groupe de travailleurs et travailleuses qui, à la suite de la démission ou du licenciement, décide de leur initiative d'intégrer un nouveau métier. En quatrième lieu, la démission ou le licenciement oblige d'autres personnes à se réorienter, c'est-à-dire à rompre avec la situation passée en changeant de statut d'emploi ou de secteur. Ce qui différencie les reconversions des réorientations est alors le caractère davantage imposé, contraint et moins abouti de ces dernières. Le cinquième et dernier cas de figure mis en évidence par Berton concerne les personnes qui subissent la rupture, soit des salarié.e.s qui sont licencié.e.s sans qu'elles et ils n'aient mis en place un projet de réinsertion et qui, de ce fait, sont exposés à un risque d'exclusion du marché du travail.

Une autre typologie des reconversions professionnelles est proposée par Fournier et collègues (2017) qui, au Québec, ont analysé le récit de 34 travailleurs et travailleuses ayant eu des parcours professionnels marqués par de la mobilité. Ces auteur.e-s mettent en évidence quatre situations distinctes. Il y a d'abord les situations de repositionnement biographique, qui concernent les personnes qui, à la suite d'un événement de vie personnel (par exemple un déménagement), décident de changer de style de vie et de réinvestir autrement leur travail et, plus généralement, leur vie. Une reconversion vocationnelle est ensuite vécue par les travailleurs et travailleuses ayant perdu l'intérêt pour leur métier et ayant développé de nouvelles aspirations qui les mènent vers un métier plus attrayant, voire idéal. D'autres personnes sont en posture de requalification sociale: face au constat d'un faux départ 
dans le marché du travail, elles sont en quête d'un emploi socialement plus valorisé et personnellement plus valorisant, leur permettant de (se) prouver leur valeur. Enfin, les situations de repositionnement stratégique couvrent des départs forcés qui mènent les personnes à faire le deuil de leur métier et à composer avec les renoncements que cela implique. Leurs objectifs professionnels se limitent alors à l'obtention d'un emploi stable et décemment rémunéré.

Ces typologies insistent sur les déclencheurs d'une reconversion - notamment en opposant reconversions choisies et imposées - ainsi que sur les différents niveaux de changements que celles-ci comportent (par exemple de statut, de métier ou de style de vie). Elles se focalisent donc essentiellement sur les causes et les effets d'une reconversion, sans s'attarder sur les étapes ou processus ayant cours entre les inputs et outputs de cette transition (Ibarra, 2006).

\subsection{LA RECONVERSION EN TANT QUE PROCESSUS}

De manière complémentaire aux approches typologiques, les reconversions professionnelles ont ainsi également été étudiées en tant que processus. Les auteur.e.s ayant adopté cette perspective se sont alors attaqué.e.s aux causes et déclencheurs de cette transition professionnelle, aux étapes à travers lesquelles passent les individus concernés, ainsi qu'aux effets de cet événement sur la personne ou sur sa carrière. Les causes d'une reconversion professionnelle sont généralement divisées entre des push et pull movements (Wise \& Millward, 2005), suivant que celle-ci a été initiée en réponse à une situation professionnelle insatisfaisante ou plutôt en raison de l'attrait pour un nouveau projet professionnel. Une recherche portant sur 30 adultes qui, au Québec, avaient décidé d'intégrer une formation professionnelle afin de réorienter leur carrière montre, par exemple, que les raisons de cette décision sont soit réactives, soit proactives (Masdonati, Fournier, \& Lahrizi, 2017). Dans le premier cas, les travailleurs et travailleuses devaient composer avec des soucis de santé physique ou psychologique rencontrés sur leur ancien lieu de travail ou faisaient état d'une insatisfaction envers leur emploi (envers ses contenus ou les conditions dans lesquelles celui-ci s'exerçait). À l'inverse, les participant.e.s vivant une reconversion proactive étaient plutôt attiré.e.s par un métier proposant des conditions intéressantes (comme de bonnes perspectives d'insertion, ou la qualité de l'emploi ou du travail), leur permettant de se développer personnellement (par du changement et de nouveaux 
apprentissages) ou encore de poursuivre leur vocation (en étant en phase avec leurs valeurs ou en rencontrant leurs véritables intérêts).

Concernant les étapes d'une reconversion professionnelle, elles ont essentiellement été étudiées chez des personnes ayant décidé et choisi de changer de carrière (par exemple, Chevallier, 2017; Denave, 2015; Négroni, 2007). Dans son analyse des récits de 67 cas de reconversion professionnelle volontaire, Négroni met par exemple en exergue cinq phases de ce processus: la vocation contrée, soit le constat de l'impossibilité de réaliser ses intérêts; le désengagement vis-à-vis d'une situation professionnelle insatisfaisante; la latence, période d'entredeux initiant progressivement une prise de décision de reconversion; la bifurcation, concrétisant et marquant la véritable prise de décision; le réengagement dans le nouveau projet professionnel.

Les résultats d'une reconversion professionnelle dépendent, quant à eux, de sa rapidité, de son degré de difficulté, de sa vraisemblance et du caractère plus ou moins radical du changement (Ibarra, 2006). En général, lorsqu'elle est réussie, une reconversion conduit à une amélioration de la satisfaction professionnelle, ainsi qu'à l'augmentation du sentiment de confiance et de maîtrise de sa carrière (Carless \& Arnup, 2012; Hostetler, Sweet, \& Moen, 2007). Toujours est-il qu'un projet de reconversion peut ne pas aboutir ou donner lieu à des résultats ne répondant que partiellement aux attentes de la personne, cette dernière étant alors portée à questionner son projet (Boutinet, 2014; Masdonati, sous presse).

Enfin, tant les effets que l'ensemble du processus de reconversion professionnelle peuvent être modérés par des facteurs individuels, situationnels ou contextuels. Ainsi, la qualité des soutiens sociaux, le degré de réversibilité du changement, la transférabilité des compétences de l'ancienne à la nouvelle situation professionnelle, l'accès à des formations, des enjeux concomitants dans d'autres sphères de vie, le degré d'autonomie de la personne, tout comme son âge et son sexe peuvent faciliter ou compromettre la reconversion (Ibarra, 2006). Dans une recherche longitudinale menée en Australie auprès de plusieurs milliers de salarié.e.s, Carless et Arnup (2012) montrent, par exemple, qu'une reconversion professionnelle est d'autant plus probable pour les personnes faisant état d'une personnalité ouverte et extravertie, pour les plus jeunes, pour les hommes, pour les personnes jouissant d'un bon niveau d'éducation et étant depuis peu dans leur emploi, ainsi que pour les travailleurs et travailleuses vivant de l'insécurité professionnelle. 


\subsection{AXES DE COMPRÉHENSION DES RECONVERSIONS}

Les reconversions professionnelles constituent donc une transition complexe, qui est à situer dans un parcours professionnel qui se décline en des formes plurielles, qui est initié par de multiples raisons, qui s'étale sur un processus plus ou moins long et délicat et qui donne lieu à des résultats variés, eux-mêmes dépendants de plusieurs facteurs modérateurs. Tant pour l'intervenant.e que pour le chercheur ou la chercheuse, il est donc capital de saisir les enjeux spécifiques d'une reconversion, ceux-ci pouvant varier sensiblement suivant les particularités de la situation à l'étude (Masdonati, 2017). En partant d'une lecture transversale de nos propres travaux, ainsi que de la revue de la littérature sur le sujet que nous venons d'esquisser ci-dessus, il est possible de situer ces enjeux sur quatre axes distincts: l'intentionnalité, le degré de mâ̂trise, les enjeux identitaires et le degré de continuité (Tableau 1). Si ces axes peuvent partiellement recouper des facteurs proches aux dimensions clés des parcours professionnels - telles que mises en évidence par Fournier et collègues (2016) et exposées en début de chapitre -, ils se focalisent uniquement sur l'événement spécifique de la reconversion et sur ce qui la colore. Bien que considérant une certaine temporalité, notamment dans l'articulation entre l'avant et l'après-changement, ils ne visent donc pas à saisir les tenants et aboutissants de l'ensemble d'un parcours professionnel et sa dimension longitudinale.

\section{Tableau 1. Les quatre axes de compréhension des reconversions}

\begin{tabular}{l|l|l} 
AXES & PÔLES \\
\hline $\begin{array}{l}\text { 1. Intentionnalité } \\
\begin{array}{l}\text { 2. Degré } \\
\text { de maîtrise }\end{array}\end{array}$ & Reconversion choisie & Reconversion imposée \\
\hline $\begin{array}{l}\text { 3. Enjeux } \\
\text { identitaires }\end{array}$ & $\begin{array}{l}\text { Reconversion anticipée } \\
\text { des remaniements } \\
\text { identitaires }\end{array}$ & $\begin{array}{l}\text { Reconversion comportant } \\
\text { une prise de risque }\end{array}$ \\
\hline $\begin{array}{l}\text { 4. Degré } \\
\text { de continuité }\end{array}$ & $\begin{array}{l}\text { Continuité entre l'avant } \\
\text { et l'après-reconversion }\end{array}$ & $\begin{array}{l}\text { Reconversion à faibles } \\
\text { enjeux identitaires } \\
\text { et l'après-reconversion }\end{array}$
\end{tabular}

La reconversion peut alors se comprendre en fonction d'un premier axe d'intentionnalité, opposant les transitions choisies par la personne - typiquement, une démission - à celles qui lui ont été imposées, malgré elle ou à son insu - comme un licenciement 
(Boutinet, 2014; Fouad \& Bynner, 2008). Entre ces deux extrêmes, on retrouve de nombreuses situations de reconversion qui sont en partie imposées et en partie choisies (Fournier et al., 2017; Masdonati et al., 2017). C'est le cas par exemple lorsqu'une personne fait d'une dégradation de ses conditions d'emploi le déclencheur pour investir un rêve latent.

Le deuxième axe est celui de la maîtrise; il oppose les reconversions que la personne a pu anticiper et préparer à celles impliquant une prise de risque, parce qu'intervenues sans qu'elle ait pu mettre en place un nouveau projet (Berton, 2013; Boutinet, 2014). Ces deux premiers axes de compréhension d'une reconversion permettent de saisir l'agentivité du sujet qui en fait l'expérience, c'està-dire sa marge de manœuvre, l'emprise que celui-ci a sur le processus (Picard, Olympio, Masdonati, \& Bangali, 2015).

Le troisième axe de compréhension d'une reconversion renvoie à ses enjeux identitaires, qui peuvent être plus ou moins importants suivant, justement, l'ampleur de son impact sur l'image que la personne donne et a d'elle même dans la nouvelle situation professionnelle (Masdonati, sous presse; Masdonati \& Zittoun, 2012). Par ailleurs, lorsqu'une reconversion soulève des enjeux identitaires, ceux-ci peuvent tendre soit vers une revalorisation de l'identité personnelle ou sociale (lors de reconversions vers des emplois permettant une meilleure identification ou plus prestigieux), soit vers sa "dégradation" (lorsque la nouvelle situation est dévalorisée et dévalorisante, comparativement à l'ancienne).

Le quatrième axe oppose les reconversions comportant une rupture entre l'avant et l'après à celles s'inscrivant dans la continuité. Ce qui est central ici, ce n'est pas l'écart objectif entre l'ancien emploi et le nouveau projet, mais plutôt la perception qu'en a l'individu (Boutinet, 2014). Ces deux derniers axes touchent à la "signifiance» d'une reconversion professionnelle, c'est-à-dire au sens personnel et social que la personne lui attribue dans son parcours.

Les enjeux soulevés par une reconversion professionnelle résultent ainsi de l'articulation du positionnement de celle-ci sur chacun de ces quatre axes. Par exemple, une femme cadre qui, à 45 ans, décide de devenir agricultrice pour exercer un métier davantage en lien avec ses valeurs a généralement choisi ce changement (intentionnalité forte) et aura vraisemblablement préparé ce projet, notamment en termes financiers ou de formation (maitrise élevée). On présume que, pour elle, cette reconversion comporte une redéfinition de son 
identité (enjeux identitaires forts) et qu'elle marque une rupture radicale avec son métier antérieur (continuité faible). La situation est différente, en revanche, pour un infirmier de 22 ans licencié à la suite d'une restructuration opérée par l'hôpital qui l'engageait et qui, faute de postes d'infirmier proches de chez lui, postule pour un emploi de secrétaire dans un cabinet médical. Dans ce cas, la reconversion est plutôt imposée (intentionnalité faible) et comporte une prise de risque (maîtrise faible). Si, de plus, ce changement ne met pas en branle de gros bouleversements identitaires (enjeux identitaires faibles) et que la personne considère qu'elle reste dans le même champ professionnel - le secteur de la santé - (continuité forte), on comprend bien que ces deux exemples couvrent des situations tout à fait distinctes.

\section{ACCOMPAGNER LA RECONVERSION PROFESSIONNELLE}

Afin de rendre compte des applications possibles des théories sur la reconversion professionnelle dans le champ de la psychologie du conseil et de l'orientation, nous définissons tout d'abord une démarche d'accompagnement des adultes, le bilan de compétences. Un outil qualitatif, la ligne de vie, permettant de travailler sur la reconversion au regard du parcours professionnel, est alors présenté. Nous terminons par une illustration de cas de reconversion où l'outil a été appliqué et par les apports de cet outil dans une démarche de reconversion professionnelle.

Il existe plusieurs interventions possibles, en psychologie du conseil et de l'orientation, permettant d'accompagner la personne dans une transition professionnelle. En revanche, il n'y a pas, à notre connaissance, de suivi spécifique à la reconversion professionnelle qui s'étayerait sur les modèles compréhensifs présentés ci-dessus. Le caractère de celle-ci étant multiforme et complexe, comme nous venons de le voir dans le chapitre précédent, cela dépendra des difficultés soulevées, des buts poursuivis et de l'approche dans laquelle s'inscrivent les interventions. L'approche de la transition de Schlossberg, par exemple, se focalisera sur le moment de la reconversion et mettra en évidence différentes composantes en jeu, comme la situation ayant conduit à une reconversion, le soi, les soutiens et les stratégies pour faire face à la transition (Anderson, Goodman, \& Schlossberg, 2012; Schlossberg, 2011). L'accompagnement visera à aider l'individu à prendre conscience de ces quatre 
éléments, lui permettant ainsi d'amorcer le changement. Pour sa part, Heppner (1998; Fernandez, Fouquereau, \& Heppner, 2008) invite à évaluer et mobiliser les ressources d'une personne face à une transition, telles que sa préparation (readiness), sa confiance en sa capacité à l'affronter, son sentiment de contrôle de la situation, sa perception de soutiens sociaux et son indépendance dans la prise de décision. Dans la même veine, il est possible de comprendre et accompagner une reconversion en s'appuyant sur l'adaptabilité de la personne qui la traverse, soit sur sa préoccupation (concern), sa maîtrise (control), sa curiosité, sa confiance (Savickas \& Porfeli, 2012) et sa capacité à coopérer et à travailler en relation avec autrui (McMahon, Watson, \& Bimrose, 2012).

\subsection{LE BILAN DE COMPÉTENCES}

La démarche d'accompagnement de la personne en reconversion que nous retenons ici est celle du bilan de compétences (Joras, 2007; Le Boterf, 1997). Cette démarche a été introduite en Suisse dans les années 1990, dans un contexte socio-économique instable et incertain, au sein duquel les salariés doivent organiser, anticiper et planifier leur transition professionnelle (Évéquoz, 2012). Elle répond bien aux enjeux soulevés par les parcours professionnels contemporains non linéaires. Le bilan de compétences regroupe plusieurs outils, méthodes, processus différents, et donc de multiples pratiques. Nous décrivons ici une démarche développée au sein du service de consultations de l'Université de Lausanne et s'inscrivant dans une approche intégrative du counseling, qui prône une combinaison cohérente d'outils qualitatifs et quantitatifs (Rottinghaus \& Eshelman, 2015). Dans ce cadre, le bilan de compétences se déroule en trois phases. La première phase est rétrospective et porte sur le déroulement de la vie de la personne et les différents événements survenus, autant professionnels que personnels. La deuxième phase porte sur le présent et se centre sur l'exploration de soi (valeurs, intérêts, image de soi, compétences, motivation, freins à la reconversion, etc.). La troisième phase porte sur la projection dans l'avenir, la formulation d'un projet s'appuyant sur les découvertes dans les phases précédentes de l'accompagnement.

Différents outils peuvent être proposés à chaque étape d'un bilan de compétences (Tableau 2): par exemple, la ligne de vie lors de la première phase, des questionnaires d'intérêts, de personnalité, de valeurs, mais aussi des outils qualitatifs comme l'analyse 
des expériences lors de la deuxième phase, un plan d'action lors de la dernière phase. Parmi ceux-ci, l'outil de la ligne de vie (Brott, 2004, 2017) se révèle particulièrement intéressant et mérite une attention singulière. Il permet en effet de situer et de saisir finement les enjeux spécifiques d'une reconversion donnée et de respecter ainsi l'unicité et les particularités de chaque situation. De ce fait, il est idéal pour initier et asseoir un processus de bilan de compétences.

Tableau 2. Les phases et exemples d'outils du bilan de compétences

\begin{tabular}{l|l} 
PHASES DU BILAN DE COMPÉTENCES & EXEMPLES D'OUTILS \\
\hline 1) Rétrospective & Ligne de vie \\
\hline 2) Présente & $\begin{array}{l}\text { Évaluation des ressources } \\
\text { et compétences }\end{array}$ \\
\hline 3) Prospective & Plan d'action
\end{tabular}

\subsection{LA LIGNE DE VIE}

La ligne de vie (Brott, 2004, 2017) est un outil qualitatif et interactif qui s'inscrit dans les approches constructivistes et narratives de l'orientation (Guichard, 2016; McMahon, Watson, \& Lee, 2018; Savickas, 2013; Young \& Colin, 2004). Elle vise à retracer l'histoire, le passé des personnes et à y ancrer leurs projets d'avenir, en les amenant à « revisiter et réfléchir aux différentes périodes de leur vie et aux événements ou non-événements ayant marqué, positivement ou négativement, leur expérience» (Chope, 2015, p. 80, traduction personnelle). Le sens de la reconversion apparaît ainsi, notamment, au regard de l'histoire de vie, des thèmes de vie et des choix faits par la personne dans son passé.

La ligne de vie est souvent proposée au début de la démarche, après un premier entretien, comme "devoir" à domicile. Conformément à la démarche proposée par Chan (2008), il s'agit de dessiner sur une feuille blanche une ligne du temps horizontale, en inscrivant à l'extrémité gauche sa date de naissance et à l'extrémité droite son âge actuel. La ou le consultant.e va alors noter sur cette ligne les événements marquants, professionnels et personnels, aux différentes dates clés qui ont jalonné son parcours (par exemple, fin de l'école enfantine à 5 ans, première orientation scolaire à 12 ans, certificat du secondaire à 15 ans, etc.). On peut demander de situer les événements marquants dans le temps quelque part entre le bas et 
le haut de la page pour indiquer quel impact ils ont eu: un impact plutôt positif vers le haut de la page, un impact plutôt négatif vers le bas de la page. Les événements professionnels et personnels sont ensuite reliés entre eux par deux courbes distinctes, ce qui permet d'avoir une vision globale de l'évolution de ces deux pans de vie.

Le deuxième entretien va être consacré à la reprise de la ligne de vie. La ou le psychologue conseillère ou conseiller peut laisser la personne se raconter librement, mais généralement, elle ou il la guide dans son récit en explorant différentes dimensions: par exemple, la signification des événements de vie, les croyances, les attitudes, les valeurs et préférences, la manière de faire des choix, les thèmes de vie, l'articulation entre son histoire professionnelle et son histoire personnelle, etc. Ces dimensions sont mises en lien avec la reconversion professionnelle présente, pour laquelle la personne est venue consulter. À titre d'illustration, nous détaillons ici trois de ces dimensions - la manière de faire des choix, les thèmes de vie et l'articulation entre son histoire professionnelle et personnelle - en les incarnant dans de courtes vignettes. De plus, le Tableau 3 propose des exemples de questions qui permettent d'investiguer chacune de ces dimensions.

\section{Tableau 3. Exemples de questions permettant d'investiguer trois dimensions de la ligne de vie}

\begin{tabular}{l|l} 
DIMENSION & EXEMPLES DE QUESTIONS \\
\hline Manière faire des choix & $\begin{array}{l}\text { Pourquoi avez-vous fait ce choix? } \\
\text { Comment avez-vous fait ce choix? } \\
\text { Quelles similitudes et différences voyez-vous } \\
\text { avec d'autres choix de votre parcours? } \\
\text { Qu'est-ce que cette manière de faire des choix } \\
\text { dit sur vous? } \\
\text { Aujourd'hui, qu'est-ce que vous souhaiteriez garder } \\
\text { de la manière dont vous faites vos choix ? Et qu'est- } \\
\text { ce que vous aimeriez changer? }\end{array}$ \\
\hline Thèmes de vie & $\begin{array}{l}\text { Qu'est-ce qui vous a suivi tout au long de votre vie? } \\
\text { Ou qu'est-ce qui a traversé votre parcours de vie? } \\
\text { Quelles répétitions percevez-vous } \\
\text { dans votre parcours de vie? } \\
\text { Aujourd'hui, dans quelle mesure ce thème de vie } \\
\text { est-il toujours actuel? }\end{array}$ \\
\hline
\end{tabular}


Articulation entre histoire professionnelle et histoire personnelle
Quel(s) lien(s) voyez-vous entre votre parcours

de vie personnelle et professionnelle?

Quel sens donnez-vous à votre parcours professionnel au regard de votre parcours personnel? Et vice versa?

Aujourd'hui, quelle place souhaiteriez-vous accorder à votre travail dans votre vie?

Et quelle place souhaiteriez-vous accorder à votre vie privée?

Au sujet de la première dimension, la manière de faire des choix (Gati, Landman, Davidovitch, Asulin-Peretz, \& Gadassi, 2010), le focus de l'entretien est mis sur les différentes transitions jalonnant le parcours et sur la reconversion professionnelle actuelle. Juliette a fait le choix, dans son passé, de quitter un emploi de bibliothécaire scolaire, car elle ne trouvait pas claires les attentes de la hiérarchie. Aujourd'hui, elle regrette d'avoir laissé un travail qu'elle affectionnait beaucoup et pense qu'elle aurait pu rester en affirmant davantage ses besoins. À propos de la manière de faire ce choix, Juliette dira qu'elle a fonctionné au feeling et qu'elle a tranché à un moment donné. Elle voit des similitudes avec d'autres décisions prises dans sa vie, et en retient qu'elle est assez impulsive dans sa façon de décider. Aujourd'hui, elle souhaiterait être toujours proche de son ressenti, mais désirerait être plus réfléchie afin d'être plus confiante dans le choix qu'elle va effectuer.

À propos de la deuxième dimension, les thèmes de vie (McIlveen, 2011), l'attention porte ici sur les aspects transversaux de l'histoire de vie de la personne, à savoir les répétitions qu'elle perçoit, ce qui l'a suivi tout au long de sa vie. Loïc, infirmier de profession, a passé sa vie à soigner les autres. Il raconte que, depuis son plus jeune âge, il s'occupait de sa mère malade. À l'adolescence, il est parti effectuer une mission humanitaire en Afrique, où il travaillait comme éducateur de la petite enfance dans un orphelinat. Au moment du choix professionnel, il s'est décidé pour un métier de soins, car «aider les autres» était tout ce qu'il connaissait. À l'âge adulte, il a soutenu sa femme, qui vivait des dépressions chroniques. À la suite d'un épuisement professionnel, il remet en question sa carrière d'infirmier, en disant qu'il souhaiterait, aujourd'hui, s'octroyer le droit de penser à lui avant les autres.

En ce qui concerne la troisième dimension, l'articulation entre son histoire professionnelle et son histoire personnelle, l'attention porte 
sur les liens que la personne fait, à différents moments de son parcours, entre sa vie professionnelle et sa vie personnelle, et la manière dont elle explique l'enchaînement des différents événements. Hugo, journaliste de profession, a toujours placé le travail au centre de sa vie. Il a fait une ascension continuelle depuis son master en journalisme, en occupant d'abord des postes de journaliste de presse écrite, puis de rédacteur en chef et, finalement, de directeur d'une maison d'édition. Sa ligne de vie personnelle est pauvre en événements, plutôt en dents de scie. Il ne s'est jamais marié. Aujourd'hui, Hugo, âgé de 43 ans, remet en question la place que prend le travail dans sa vie et ne se voit pas continuer dans son emploi actuel. Ayant rencontré une femme, il a le projet de fonder une famille et aimerait accorder plus de temps à sa passion, la peinture. Il souhaite donc équilibrer autrement les différentes sphères de sa vie.

À la suite de ces investigations, la ou le psychologue conseiller ou conseillère peut demander à faire une copie de la ligne de vie afin de prendre le temps de l'analyser après coup. En effet, celle-ci est souvent riche en éléments divers et ne peut être appréhendée en une seule fois sans un temps de recul et après un premier approfondissement. On peut également inviter la personne à identifier des chapitres de sa vie et à donner un titre à chacun d'eux (Brott, 2004; McIlveen, 2015).

Lors d'un troisième entretien, la ou le psychologue pourra soumettre à la personne son analyse de la ligne de vie et reprendre certains éléments qu'elle ou il n'a pas pu approfondir lors de l'entretien précédent. En fin de démarche, c'est-à-dire lors de la troisième phase du bilan de compétences, où il s'agit de définir un projet de reconversion, la ligne de vie peut être reprise pour favoriser une projection dans le futur, en demandant à la personne de nommer le prochain chapitre de sa vie et de faire état tant des éléments nécessitant un changement que de ceux qu'elle désire maintenir dans le futur.

\subsection{ILLUSTRATION CLINIQUE: LE CAS DE MÉLODIE'}

Mélodie, 47 ans, travaille depuis deux ans comme documentaliste en contrat à durée déterminée (CDD) pour les archives d'un festival de musique. Nous la rencontrons au mois de mai et son contrat

1. Ce cas s'inspire d'une situation réelle rencontrée au Service de consultations de l'Université de Lausanne et reprise avec l'autorisation de la personne concernée. Le cas a été anonymisé et certains éléments le caractérisant ont été adaptés pour en favoriser la compréhension et l'analyse. 
se termine vraisemblablement à la fin de l'année. Passionnée de chant, elle se dit contente de son travail, car proche de la musique, mais frustrée, car elle souhaiterait être sur le devant de la scène, alors qu'elle est devant un ordinateur toute la journée. Elle a toujours espéré devenir chanteuse et vivre de son art, sans avoir jamais réalisé son rêve. D'ailleurs, selon l'avis de son père, cela n'était pas "un vrai métier». Au terme de sa scolarité obligatoire, elle a donc fait un choix par défaut, dicté par la raison et la voix paternelle, et a entrepris un apprentissage d'électronicienne en radio et télévision. Elle a exercé dans plusieurs domaines variés : secrétaire à deux reprises, dont une pour sa propre entreprise, technicienne dans les systèmes de surveillance, assistante technique et assistante de production, présentatrice dans une télévision locale, représentante de vente. Elle a alterné des périodes de chômage et de travail.

À propos de son emploi actuel, Mélodie apprécie la diversité des activités, l'indépendance de son poste et l'appartenance à une équipe. Elle se sent toutefois globalement frustrée, car elle ne sait pas ce que l'on attend d'elle. Son responsable lui a reproché de ne pas prendre assez d'initiatives. Or elle dit qu'elle ne peut pas le faire, les missions des membres de l'équipe n'étant ni explicites, ni coordonnées.

En parallèle de ses activités professionnelles, Mélodie développe ses intérêts pour l'art, en particulier le chant, le spectacle et la peinture. Elle a monté une association avec une troupe de comédiens et créé une comédie musicale. Elle en parle de manière enjouée, en transmettant tout le plaisir qu' elle a eu à créer, à participer à un grand projet et à mener les choses comme elle l'entendait. Elle a dû développer de nouvelles compétences, ce qu'elle apprécie tout particulièrement. En outre, elle s'est produite de manière souvent bénévole sur plusieurs scènes et festivals. À une période de sa vie, elle a également fait de la peinture et, depuis peu, elle donne un cours de chant à une adulte. Mélodie explique avoir recherché un équilibre dans sa vie en menant des activités extraprofessionnelles qui comblaient ce qu'elle ne trouvait pas dans le travail.

Au niveau personnel, elle a connu son mari à l'adolescence et est mariée depuis vingt ans. Bien qu'à un moment donné de sa vie elle ait souhaité avoir des enfants, celui-ci n'en voulait pas. Ce fut une période pénible et un choix qu'elle a dû accepter malgré elle. Son mari la soutient dans sa démarche de réorientation, la sentant peu épanouie dans son travail actuel. 
Durant son parcours, Mélodie a déjà accompli des démarches de bilan de compétences et se sent familière avec celles-ci. Cela fait longtemps qu'elle se pose la question de son orientation. Elle a le sentiment de ne pas avoir trouvé sa place dans ses différents emplois et d'avoir toujours été habitée par son rêve de devenir chanteuse. À chaque fois qu'elle y a réfléchi, elle a laissé tomber la possibilité de réorienter sa carrière, percevant un nombre important d'obstacles: elle a notamment plusieurs craintes, comme de se lasser après quelque temps, de ne pas réussir à se faire une place dans un "milieu de requins", de ne pas y arriver faute de diplômes et d'apprentissages de base. Elle redoute également la précarité du travail dans ce domaine. De plus, elle pense que si elle avait vraiment voulu le faire, elle l'aurait déjà fait.

Ainsi, Mélodie se trouve bloquée dans sa situation et a le sentiment de tourner en rond. À 47 ans, elle pense que c'est sa dernière chance de réorienter sa carrière. Elle souhaiterait savoir où se diriger en fonction de ses compétences et questionner la possibilité d'exercer la musique comme activité principale. Pour réaliser ses projets, elle se dit prête à refaire une formation si cela s'avère nécessaire, mais n'exclut pas l'abandon du projet de devenir chanteuse. Elle a besoin de retrouver du plaisir dans son travail et un environnement avec un management qui la stimule, mais aussi une stabilité, car elle a beaucoup changé de travail dans sa vie.

\subsubsection{Conceptualisation du cas}

Le cas de Mélodie peut être compris selon les dimensions caractérisant son parcours, tout comme du point de vue du type, du processus et des caractéristiques - en fonction des quatre axes - de la reconversion qu'elle semble envisager.

Concernant son parcours, partant des travaux de Fournier et collègues (2016), on peut supposer que Mélodie fait état d'un parcours professionnel qui peut être qualifié d'instable, alternant périodes d'emploi et de non-emploi de manière souvent imprévisible, occupant des travaux exigeant des compétences différentes (administratives, techniques, vente). En ce qui concerne le sentiment de maîtrise, elle ne paraît pas avoir eu beaucoup d'emprise sur ses choix: son premier choix s'est fait par défaut et elle a ensuite occupé des emplois plus par besoin de sécurité que par réalisation personnelle, en compensant son insatisfaction par l'exercice de la musique à titre de loisir. Au sujet de la subjectivité au travail, le 
rapport à l'emploi de Mélodie semble ambigu. Elle recherche en effet de la sécurité financière et de la stabilité, mais n'a pas eu d'emplois stables, a souvent quitté ses emplois et est actuellement en CDD. On peut supposer des besoins contradictoires, de stabilité, de sécurité financière et d'un cadre clair et bien défini d'une part, et de liberté, de créativité, de prise de risque et d'apprentissages nouveaux d'autre part. Elle souhaite aujourd'hui que son travail soit sa source centrale d'épanouissement et recherche plus d'accomplissement dans ce qu'elle fait. Au sujet de la temporalité, la manière dont les événements passés s'articulent entre eux et avec le présent n'est pas claire. Nous savons que son âge, 47 ans, a joué un rôle dans son questionnement actuel, parce qu'il est perçu comme une dernière chance pour changer d'emploi et réaliser son rêve. Finalement, en ce qui concerne l'articulation entre la sphère privée et professionnelle, comme mentionné plus haut, on peut penser que Mélodie a compensé une insatisfaction dans ses différents emplois par ses activités extraprofessionnelles créatives, celles-ci jouant le rôle de palliatifs identitaires.

En référence à la typologie des reconversions de Fournier et collègues (2017), Mélodie semble vivre une reconversion vocationnelle: bien que satisfaisant, son travail de documentaliste ne répond en effet pas à son désir d'être sur le devant de la scène; son élan vers un éventuel changement est alimenté par cette envie de vivre de son art et de s'investir enfin dans le métier de chanteuse, qu'elle a toujours rêvé exercer.

D'un point de vue processuel, il peut être intéressant de comprendre les raisons de cet élan vers une éventuelle reconversion, ainsi que la phase où elle en est au moment où elle rencontre un·e psychologue et les facteurs qui peuvent modérer le processus et son aboutissement. Comme cela est souvent le cas lors d'une reconversion vocationnelle, les raisons d'une éventuelle reconversion sont à la fois réactives (push) et proactives (pull) (Masdonati et al., 2017; Wise \& Millward, 2005). Mélodie fait en effet état d'une relative insatisfaction envers sa situation actuelle, tant dans le contenu de son travail (par exemple être devant l'ordinateur toute la journée) que du point de vue des conditions d'emploi (le contrat qui arrive à échéance). En même temps, elle est attirée par l'espoir de réaliser sa vocation de chanteuse et de réaliser enfin un rêve professionnel.

Par ailleurs, on peut situer Mélodie dans la phase de latence du processus de reconversion professionnelle volontaire décrit par 
Négroni (2007): consciente de l'existence d'une vocation contrée (ne pas avoir réussi à vivre de son art) et de l'urgence qu'elle ressent pour la "réactiver", elle semble se désengager progressivement de son emploi de documentaliste, qu'elle qualifie de globalement frustrant, et réfléchir à un changement de carrière. Le fait qu'elle ait sollicité un accompagnement d'orientation symbolise cette latence, car il s'agit des premiers pas vers la prise de décision d'une éventuelle reconversion. Cela dit, Mélodie n'en est pas encore à l'étape de bifurcation, car la décision n'est pas encore prise et il se peut que le bilan d'orientation aboutisse au renoncement du projet de devenir chanteuse professionnelle.

Les facteurs pouvant modérer ce processus et son aboutissement sont multiples (Carless \& Arnup, 2012; Ibarra, 2006). D’une part, le soutien du mari, les compétences déjà développées dans le chant, les connaissances du domaine artistique, l'ouverture à entreprendre une nouvelle formation et l'apparente absence d'enjeux concomitants semblent favoriser une reconversion. D'autre part, des facteurs contraignants pourraient l'empêcher ou la fragiliser dans ce projet, tels que son âge, perçu comme étant avancé par rapport à une carrière artistique, ainsi que la conscience du risque de précarité. Ses craintes de ne pas se sentir compétente, de ne pas être suffisamment affirmée pour se défendre et de se lasser après un certain temps peuvent également constituer des freins pour sa reconversion.

Enfin, il est possible de situer le cas de Mélodie sur chacun des quatre axes de compréhension d'une reconversion. Concernant le premier axe, on peut parler d'une intentionnalité relative: la reconversion est en partie imposée par l'échéance de son CDD et par une frustration professionnelle de moins en moins supportable, mais également choisie, du fait du désir de devenir chanteuse professionnelle. Quant au deuxième axe, il semble s'agir d'une situation de maîtrise, car Mélodie entame un processus accompagné de réflexion sur la faisabilité de son projet et vise une entrée progressive dans le monde du chant - notamment en baissant son taux d'activité de salariée seulement en cas de réussite de sa carrière de chanteuse. Ainsi, le croisement de ces deux premiers axes fait état d'une agentivité plutôt élevée de Mélodie, qui œuvre pour garder une emprise sur sa vie professionnelle.

Une analyse sous l'angle identitaire (troisième axe de compréhension) indique de forts enjeux identitaires sous-jacents au projet de reconversion de Mélodie. On peut en effet émettre l'hypothèse qu'une bifurcation vers le chant impliquerait la construction d'une 
nouvelle identité professionnelle assumée et valorisée, alors que l'éventuel abandon de ce projet pourrait affecter négativement son regard sur elle-même. Pour ce qui a trait au quatrième axe, Mélodie perçoit une certaine continuité entre sa situation présente et l'éventuelle entrée dans une carrière artistique, puisque son travail actuel est déjà en lien avec la musique et qu'elle a toujours côtoyé le monde artistique. Le croisement de ces deux derniers axes suggère une forte "signifiance" d'une reconversion vers le chant: ce projet aurait pour Mélodie un sens particulier, car il la rapprocherait de ce qu'elle a toujours rêvé, mais sans jamais l'investir pleinement. Cette forte signifiance porte d'ailleurs également à considérer attentivement les possibles répercussions négatives que pourrait avoir le fait de ne pas s'engager dans un tel projet.

\subsubsection{Application de la ligne de vie}

Nous présentons ici les éléments qui ont émergé des discussions sur la ligne de vie de Mélodie (Figure 1) en nous centrant sur les trois dimensions parcourues au chapitre 2.3 : la manière de faire des choix, les thèmes de vie, l'articulation entre la vie professionnelle et la vie personnelle.

\section{Figure 1. Ligne de vie de Mélodie}

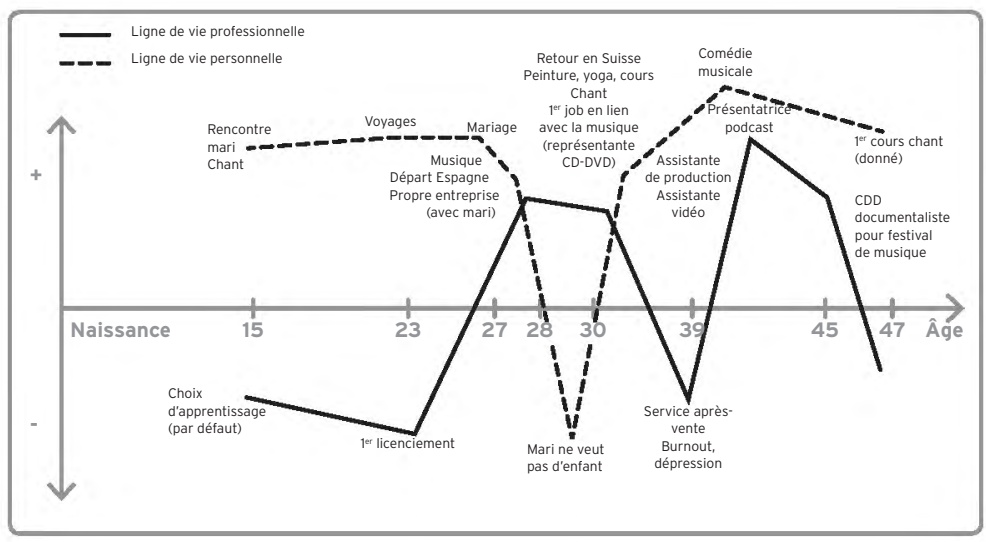

Au sujet de la manière de faire des choix, Mélodie explique que les nombreux changements d'emploi, durant son parcours, étaient en partie dus à des licenciements ou à des fins d'emplois abrupts et inattendus. Parfois, l'entreprise a fait faillite; d'autres fois, Mélodie 
est partie de son propre chef parce qu'elle ne se plaisait pas ou que la situation était tellement mauvaise qu'elle ne la supportait plus. Lorsqu'elle a eu le choix de partir, elle rapporte qu'elle s'ennuyait et avait le sentiment de ne plus rien apprendre. Elle constate, en regardant sa ligne de vie, que le plus souvent elle est restée deux ans en poste. Mélodie raconte qu'elle a accepté par défaut la plupart de ses emplois, alors qu'elle souhaitait faire autre chose en rapport avec le domaine de la musique. Cette manière de faire des choix parle de qui elle est: une personne curieuse, qui s'adapte facilement, avide de nouvelles connaissances, ouverte aux opportunités et qui ne se sent pas avoir réellement effectué de choix en fonction de ses valeurs et intérêts. Aujourd'hui, elle dit souhaiter changer sa manière de faire des choix, en prenant une décision réfléchie, qui lui corresponde, et en étant proactive. Comme nous l'avons vu, c'est une des raisons qui l'ont motivée à venir consulter.

En ce qui concerne ses thèmes de vie, Mélodie rapporte que l'art, en particulier la musique, l'a suivie tout au long de sa vie. Elle constate qu'elle s'en rapproche au niveau professionnel depuis l'âge de ses 40 ans. Cela fait partie de son identité et elle ne pourrait pas s'en passer. Elle a tout particulièrement aimé l'expérience d'avoir monté une association avec la troupe d'une comédie musicale: elle était dans son élément et a apprécié être libre de faire les choses comme elle l'entendait. Mélodie pense également qu'elle a manqué de confiance tout au long de son parcours et que cela explique en partie le fait qu'elle ne se soit pas lancée dans la musique et qu'elle ait choisi la voie de la sécurité: elle ne croyait pas en ses compétences, se sentait incapable de "sortir du lot» et avait toutes sortes de peurs. Ainsi, deux thèmes de vie ressortent du récit de Mélodie: la créativité via la musique et le manque de confiance en soi. Aujourd'hui, elle pense que ces deux thèmes sont toujours d'actualité dans son désir de reconversion. Elle souhaiterait renforcer le premier, en osant «franchir le pas» et, peut-être, vivre de son art, et réduire le deuxième, en gagnant en confiance en elle et en prenant plus de risques.

Au sujet de l'articulation entre sa vie professionnelle et personnelle, Mélodie raconte avec beaucoup d'émotions avoir pris conscience, en effectuant sa ligne de vie, qu'elle a mis en place plusieurs activités artistiques pour combler l'impossibilité d'enfanter. Elle perçoit que la musique, exercée à titre personnel, a pallié des hauts et des bas sur le plan professionnel. Aujourd'hui, Mélodie a pu surmonter 
l'épreuve de ne pas avoir été mère et l'exercice de la ligne de vie lui a permis de mettre en évidence son besoin, accentué par son âge, de laisser une trace à travers sa création. Ainsi, elle aimerait que la musique ait un rôle central dans sa vie et pense que cela serait pleinement le cas si elle pouvait en vivre.

\subsubsection{Apports de la ligne de vie}

L'utilisation de la ligne de vie pour accompagner Mélodie a permis de consolider la compréhension à la fois de son parcours et des enjeux de son éventuelle reconversion. En lien avec les dimensions mises en évidence par Fournier et collègues (2016), la Figure 1 exacerbe en effet le caractère instable de son parcours et la discussion autour de l'exercice a amené Mélodie à réaliser qu'elle a toujours eu peu d'influence sur ses choix. De plus, l'exercice a pu enrichir la compréhension de sa subjectivité au travail, par une meilleure perception de ses besoins et valeurs, tels que la liberté et le besoin de créativité et de nouveauté. Au sujet de la temporalité, on comprend mieux comment s'articulent les événements de sa vie; par exemple, on peut supposer que son fort besoin de créer découle en partie du fait de ne pas avoir été maman. En ce qui concerne l'articulation entre les sphères de vie, l'on perçoit mieux que sa vie privée a probablement joué un rôle de palliatif identitaire par rapport à sa vie au travail, la ligne personnelle étant haute et positive, alors que celle du travail est en "dents de scie».

Les apports de la ligne de vie pour mieux comprendre la situation de reconversion de Mélodie sont également multiples. L'exercice confirme par exemple qu'il s'agit bel et bien d'un projet de reconversion vocationnelle (Fournier et al., 2017), puisque la musique a toujours été présente dans son histoire, sans pour autant éclore en tant que projet professionnel à part entière. En lien avec ce constat, la ligne de vie a mis en évidence une certaine prédominance des raisons proactives (Masdonati et al., 2017) d'une éventuelle reconversion, le chant étant un thème de vie qui a traversé son histoire, indirectement (dans ses emplois) ou directement (dans ses loisirs). Du point de vue de ce qui motive le changement, l'élan vers la vocation contrée semble ainsi avoir le dessus sur la fuite d'une situation présente insatisfaisante.

L'exercice permet aussi de situer avec plus de finesse le cas de Mélodie sur les axes de compréhension d'une reconversion. La prévalence de raisons proactives sur des raisons réactives mise en 
évidence par la ligne de vie porte à situer cette reconversion plutôt dans le pôle du choix sur l'axe d'intentionnalité. Bien que l'arrivée à échéance de son contrat suggère une situation en partie imposée, son désir de se prendre en main et de faire enfin un véritable choix assumé conforte cette analyse. Concernant le deuxième axe, l'outil de la ligne de vie met en exergue un dilemme important qui semble habiter Mélodie, tiraillée entre son réflexe d'opter pour des options de carrière sécuritaires, comme elle l'a toujours fait jusque-là, et l'envie de rompre avec ce fonctionnement et de prendre des risques, en investissant une carrière artistique, vraisemblablement plus instable. Ce dilemme semble d'ailleurs exacerbé, d'une part, par une personnalité prudente et un certain manque de confiance en soi (induisant à la mâ̂trise) et, d'autre part, par le sentiment d'urgence et la sensation d'en être arrivée, au mitan de la vie, à une dernière chance de réaliser son rêve (favorisant la prise de risque) (Barclay, Stoltz, \& Chung, 2011). Du point de vue des enjeux identitaires, la ligne de vie montre que l'art et la créativité constituent un thème de vie marquant le parcours de Mélodie et que celle-ci sidentifie fortement dans ce domaine, devenu aujourd'hui saillant et central. Une reconversion vers un métier artistique comporterait ainsi une forte implication identitaire et serait d'autant plus importante qu'elle pourrait constituer un moment de reprise de confiance en soi. La ligne de vie confirme enfin le fait que devenir chanteuse s'inscrirait à ses yeux dans une sorte de continuité et constituerait même un aboutissement à la fois idéal et logique de son parcours.

Comme nous l'avons vu, la ligne de vie a permis à Mélodie de prendre conscience d'éléments nouveaux sur elle et sa manière de fonctionner, ce qui a contribué à augmenter la conscience d'ellemême. Par conséquent, elle pourra plus facilement s'engager dans les prochaines étapes de la reconversion, en ayant une vision plus claire de ses besoins, de sa personnalité et de ses préférences (Brott, 2004). En partant de cette compréhension consolidée du parcours et du projet de reconversion de Mélodie, il est donc possible d'identifier des pistes d'intervention visant à vérifier la pertinence et la faisabilité de ce projet. À ce stade du processus d'orientation, la prise de décision peut en effet découler d'une mise en perspective éclairée des éléments parlant en faveur ou contre une reconversion. Par exemple, les avantages seraient la réalisation d'un choix différent des autres, avec plus de prise de risque, un sentiment d'aboutissement de son parcours, la concrétisation d'un rêve et un regain de 
confiance en elle; a contrario, les inconvénients consisteraient surtout en une perte de stabilité et de sécurité d'emploi. Une discussion autour de ces éléments, par exemple sous la forme de balance décisionnelle (Miller \& Rose, 2013), constituerait une suite logique du processus d'accompagnement d'orientation que l'on pourrait proposer à Mélodie. Nous pourrions alors travailler sur les obstacles ou freins à sa reconversion, et sur ses ressources, notamment ses compétences, pour l'aider à avancer dans ce processus.

\section{BILAN ET PERSPECTIVES}

La proposition d'un cadre conceptuel visant une compréhension fine des processus de reconversion professionnelle répond à une nécessité d'innover dans les théories en psychologie de l'orientation afin qu'elles soient au pas avec les enjeux qui caractérisent les parcours professionnels contemporains (Blustein, 2014; Guichard, 2015; Lent, 2014). Or les reconversions professionnelles sont des transitions qui n'ont étonnamment que peu été étudiées dans notre champ. En même temps, elles sont en plein essor et se révèlent d'une complexité extrême pour les individus qui les traversent : tantôt moments clés d'une prise en main de sa biographie, tantôt véhicules d'une dégradation du rapport au travail, elles sous-tendent un travail d'accompagnement en orientation qui reste à asseoir (Fournier et al., 2017). Il est donc essentiel, pour les psychologues de l'orientation accompagnant des personnes en reconversion, d'être en mesure de situer ce moment en fonction de l'ensemble de leur parcours professionnel et de vie, d'identifier le type de reconversion dont il est question et l'étape où se trouve l'individu dans ce processus, de comprendre ce qui en a été la cause, mais aussi les facteurs spécifiques à chaque situation qui peuvent influencer, positivement ou négativement, son déroulement et son issue. Cet accompagnement doit également être ajusté selon que la reconversion a été choisie ou imposée, d'après le degré de maîtrise de la personne sur cet événement, en fonction des enjeux identitaires plus ou moins forts et plus ou moins positifs qu'elle véhicule et suivant qu'elle s'inscrit dans une continuité ou une rupture. Une attention particulière devrait être portée aux modalités d'accompagnement de personnes vivant une reconversion imposée, qui devrait intégrer un travail de deuil et de séparation de l'ancienne situation professionnelle. 
L'outil de la ligne de vie, ainsi que le dialogue qui l'accompagne et en explicite la compréhension, peut se révéler précieux pour saisir les caractéristiques et enjeux d'une reconversion ou d'un projet de reconversion. En tant qu'outil d'évaluation qualitative, il permet "de se focaliser sur les expériences, les situations et les conditions dans lesquelles ces enjeux sont imbriqués» (Gysbers, 2006, p. 96), ce qui aide à comprendre les racines et les contextes d'une reconversion. Ainsi, la personne peut prendre conscience des valeurs, besoins et motivations qui ont guidé ses choix, mettre en contexte ses phases de vie, événements et décisions en créant des liens entre différentes sphères de son existence, retracer une historicité des événements et comprendre ce qui l'a conduite à la situation actuelle, identifier des répétitions et trouver un fil rouge à son parcours. Le bilan de ce qu'elle a fait dans le passé et d'où elle en est dans le présent la conduit à clarifier et renforcer son identité et, par conséquent, à percevoir des directions plus claires pour le futur (Brott, 2004; McIlveen, 2017).

Toujours est-il que la compréhension de ce phénomène est loin d'être satisfaisante et que des recherches sont encore nécessaires afin d'en dresser un portrait exhaustif. Premièrement, peu de travaux se sont penchés sur les enjeux spécifiques des reconversions professionnelles non choisies, intervenant, par exemple, à la suite d'un accident, d'un licenciement ou d'une pénurie d'emplois dans un secteur donné (Berton, 2013; Blustein, Kozan, \& Connors-Kellgren, 2013; Fournier et al., 2017). Logiquement, on peut s'attendre à ce que ces enjeux et les impacts (notamment identitaires) de ce type de reconversion soient distincts, voire opposés, de ceux liés à des changements initiés par la personne. Dans ce même ordre d'idées, des travaux sont également nécessaires afin de saisir les caractéristiques spécifiques des reconversions vécues par des groupes sociaux vivant des situations plus vulnérables, comme les migrant.e.s dont les diplômes ne sont pas reconnus dans le pays d'accueil (Abkhezr \& McMahon, 2017) ou les travailleuses et travailleurs "seniors", qui ont plus de probabilités de rencontrer des écueils dans leur réinsertion professionnelle (Fournier, Zimmermann, Gauthier, \& Masdonati, 2014).

Un deuxième champ de recherche qui reste à mieux investir est celui menant à une compréhension plus approfondie des facteurs qui viennent modérer ou moduler une reconversion (Carless \& Arnup, 2012; Ibarra, 2006). De toute évidence, la qualité des 
soutiens sociaux, les ressources personnelles et matérielles dont bénéficie la personne, tout comme la phase de vie qu'elle traverse colorent le processus de reconversion et peuvent en influencer significativement l'issue (Vogelsang, Schutz, \& Olson, 2018).

Une troisième piste de recherche consisterait à complexifier le cadre de compréhension des reconversions, en croisant les dimensions qui caractérisent un parcours de vie avec les axes qui marquent cette transition. Cela permettrait d'adopter un regard longitudinal et de s'interroger sur la variété des impacts d'une reconversion sur le parcours de vie, mais aussi d'ajouter aux axes de compréhension des reconversions une dimension temporelle qui peut être centrale afin de comprendre l'articulation entre soi passés et soi possibles (Obodaru, 2017). Dans cette même veine, à notre connaissance, aucune recherche n'a porté sur les reconversions professionnelles avortées, inabouties ou donnant lieu à de résultats inattendus, à des déceptions ou à des retours à la case de départ. Or, comme le souligne Boutinet (2014), "c'est là le coût attaché à toute transition, ce risque de conduire vers l'anecdotique ou la désillusion" (p. 8).

En termes d'intervention aussi, des perspectives s'ouvrent et peuvent venir consolider les apports de l'outil de la ligne de vie, qui, en soi, n'est pas suffisant pour accompagner une reconversion. Partant de l'analyse de la ligne de vie, il nous paraît ainsi essentiel de compléter l'accompagnement avec d'autres outils encore à tester et permettant de cerner les ressources et obstacles à une reconversion, tant externes (formes de soutien, ressources financières, disponibilité de dispositifs de formation, etc.) qu'internes (personnalité, caractéristiques sociobiographiques, etc.). Par ailleurs, bien que cet outil soit un support précieux pour une réflexion sur l'avenir - notamment du point de vue de ce que la personne veut préserver de son histoire et de sa manière de fonctionner ou, au contraire, de ce qu'elle veut changer à travers une éventuelle reconversion -, il est insuffisant pour satisfaire la partie prospective d'un bilan de compétences. Celle-ci mériterait d'être complétée par un travail de projection et d'anticipation de soi plus approfondi, intégrant notamment une préparation aux aléas du marché du travail contemporain et aux implications identitaires d'un changement de rôle professionnel (Boutinet, 2007; Lent, 2013). 


\section{RÉFÉRENCES BIBLIOGRAPHIQUES}

ABKhezR, P., \& McMAHON, M. (2017). Narrative career counselling for people with refugee backgrounds. International Journal for the Advancement of Counselling, 39, 99-111. Doi: 10.1007/ s10447-017-9285-z.

Anderson, M. L., Goodman, J., \& SchlossberG, N. K. (2012). Counseling adults in transition: Linking Schlossberg's theory with practice in a diverse world (4 édition). New York: Springer.

Barclay, S. R., Stoltz, K. B., \& Chung, Y. B. (2011). Voluntary midlife career change: Integrating the transtheoretical model and the Life-Span, Life-Space approach. The Career Development Quarterly, 59, 386-399. Doi : 10.1002/j.2161-0045.2011.tb00966.x.

BERTON, F. (2013) Démissions et licenciements face aux changements dans les entreprises: la diversité des transitions professionnelles. Travail et Emploi, 136, 49-68. Doi : 10.4000/travailemploi.6142.

Blustein, D. L. (éd.) (2014). The Oxford handbook of the psychology of working. New York, : Oxford University Press. Doi: 10.1093/ oxfordhb/9780199758791.001.0001.

Blustein, D. L., Kozan, S., \& Connors-Kellgren, A. (2013). Unemployment and underemployment: A narrative analysis about loss. Journal of Vocational Behavior, 82, 256-265. Doi : 10.1016/j. jvb.2013.02.005.

Boutinet, J.-P. (2007). L'espace contradictoire des conduites à projet: Entre le projet d'orientation du jeune et le parcours atypique de l'adulte. L'Orientation scolaire et professionnelle, 36, 19-32. Doi : 10.4000/osp.1259.

Boutinet, J.-P. (2014). Actualité des transitions dans les parcours de vie adulte. L'Orientation scolaire et professionnelle, 43 (4). En ligne: [http://journals.openedition.org/osp/4463]. Doi: 10.4000/ osp.4463 (consulté le 7 février 2018).

Brott, P. E. (2004). Constructivist assessment in career counseling. Journal of Career Development, 30, 189-200. Doi : 10.1023/B : JOCD.0000015539.21158.53.

BRotT, P. E. (2017). The storied approach. In M. McMahon (éd.), Career counseling: Constructivist approaches (2 édition) (pp. 93-103). Londres/New York: Routledge. 
Carless, S. A., \& Arnup, J. L. (2011). A longitudinal study of the determinants and outcomes of career change. Journal of Vocational Behavior, 78, 80-91. Doi: 10.1016/j.jvb.2010.09.002.

Chan, A. (2008). Lifeline exercise. En ligne: [https://www.gsb. stanford.edu/sites/gsb/files/alumni-migration/files/alumni_migration/career/careerlifevision/pdf/Lifeline_Exercise.pdf].

Chevallier, E. (2017). Les ruptures intentionnelles de carrière chez les cadres français souhaitant redonner du sens à leur vie professionnelle: description du concept et du processus. L'Orientation scolaire et professionnelle, 46, 427-453. Doi : 10.4000/osp.5484.

Chope, R. C. (2015). Card sorts, sentence completions, and other qualitative assessments. In P. J. Hartung, M. L. Savickas, \& W. B. Walsh (éds), APA Handbook of Career Intervention: vol. 2 (pp. 71-84). Washington, DC: APA. Doi : 10.1037/14439-006.

Denave, S. (2015). Reconstruire sa vie professionnelle. Sociologie des bifurcations biographiques. Paris: PUF.

ÉvÉquOZ, G. (2012). La validation des acquis de l'expérience en Suisse: Quel rôle pour l'orientation? L'Orientation scolaire et professionnelle, 41. Doi: 10.4000/osp.3812.

Fernandez, A., Fouquereau, E., \& Heppner, M. J. (2008). The Career Transition Inventory: A psychometric evaluation of a French version (CTI-F). Journal of Career Assessment, 16, 384-398. Doi: 10.1177/1069072708317384.

Fouad, N. A., \& Bynner, J. (2008). Work transitions. American Psychologist, 63, 241-251. Doi: 10.1037/0003-066X.63.4.241.

Fournier, G., Gauthier, C., Perron, F., Masdonati, J., Zimmermann, H., \& Lachance, L. (2017). Processus de reconversion professionnelle de travailleur.euse.s inscrit.e.s dans des parcours professionnels marqués par la mobilité: entre le deuil du métier et le désir de réinvestir sa vie autrement. L'Orientation scolaire et professionnelle, 46, 363-399. Doi: 10.4000/osp.5465.

Fournier, G., Zimmermann, H., Gauthier, C., \& Masdonati, J. (2014). L'expérience du chômage chez les travailleurs seniors: de la crise identitaire à la réflexion sur l'avenir. L'Orientation scolaire et professionnelle, 43, 337-366. Doi: 10.4000/osp.4431.

Fournier, G., Zimmermann, H., Gauthier, C., Masdonati, J., 
\& Lachance, L. (2016). Vers l'élaboration d'un cadre d'analyse des parcours professionnels: l'éclairage de l'approche du Parcours de vie et des théories récentes en développement de carrière. In G. Fournier, E. Poirel, \& L. Lachance (éds), Éducation et vie au travail: Perspectives contemporaines sur les parcours de vie professionnelle (pp. 93-143). Québec: PUL.

Gati, I., Landman, S., Davidovitch, S., Asulin-Peretz, L., \& GADASSI, R. (2010). From career decision-making styles to career decision-making profiles: A multidimensional approach. Journal of Vocational Behavior, 76, 277-291. Doi: 10.1016/j.jvb.2009.11.001. GUICHARD, J. (2004). Se faire soi. L'Orientation scolaire et professionnelle, 33, 499-533. Doi : 10.4000/osp.226.

Guichard, J. (2015). From vocational guidance and career counseling to Life Design dialogues. In L. Nota \& J. Rossier (éds), Handbook of the Life Design paradigm: From practice to theory and from theory to practice (pp. 11-25). Göttingen: Hogrefe.

Guichard, J. (2016). Une comparaison des apports des modèles de la construction de la carrière et de la construction de soi au life designing counseling. Psychologie française, 61, 15-29. Doi: 10.1016/j. psfr.2013.03.002.

Gysbers, N. C. (2006). Using qualitative career assessments in career counselling with adults. International Journal for Educational and Vocational Guidance, 6, 95-108. Doi : 10.1007/s10775-006-9102-4.

Heppner, M. J., \& Scott, A. B. (2006). Career transitions. In J. H. Greenhaus \& G. A. Callanan (éds), Encyclopedia of Career Development (pp. 157-159). Thousand Oaks (CA): Sage. Doi: 10.4135/9781412952675.n57.

Hostetler, A. J., Sweet, S., \& Moen, P. (2007). Gendered career paths: A life course perspective on returning to school. Sex Roles, 56, 85-103. Doi : 10.1007/s11199-006-9150-8.

Ibarra, H. (2006). Career change. In J. H. Greenhaus \& G. A. Callanan (éds.), Encyclopedia of Career Development (pp. 77-83). Thousand Oaks (CA) : Sage. Doi: 10.4135/9781412952675.n57.

Jorat, M. (2007). Le bilan de compétences. Paris: PUF.

Le Boterf, G. (1997). De la compétence à la navigation professionnelle. Paris: Éditions d'Organisation. 
LENT, R.W. (2013). Career-life preparedness: Revisiting career planning and adjustment in the new workplace. Career Development Quarterly, 61, 2-14. Doi : 10.1002/j.2161-0045.2013.00031.x.

Masdonati, J. (2017). Introduction: les reconversions professionnelles. L'Orientation scolaire et professionnelle, 46 (3), 335-338.

Masdonati, J. (sous presse). Le travail identitaire lors d'un changement de carrière. In T. Perez-Roux, M. Deltand, C. Duchesne, \& J. MASDONATI (éds). Parcours professionnels, transitions et transformations identitaires: le sujet au cour des évolutions dans le champ de l'éducation et de la formation. Montpellier: Presses universitaires de la Méditerranée.

Masdonati, J., Fournier, G., \& Lahrizi, I. Z. (2017). The reasons behind a career change through vocational education and training. International Journal for Research in Vocational Education and Training, 4, 249-269. Doi: 10.13152/IJRVET.4.3.4.

Masdonati, J., \& ZitToun, T. (2012). Les transitions professionnelles: processus psychosociaux et implications pour le conseil en orientation. L'Orientation scolaire et professionnelle, 41, 229-253. Doi : $10.4000 /$ osp.3764.

McIlveen, P. (2011). Life themes in career counselling. In M. McMaHON \& M. B. WATSON (éds), Career counseling and constructivism: Elaboration of constructs (pp. 73-85). New York: Nova Science Publishers.

McIlveen, P. (2015). My Career Chapter and the Career Systems Interview. In M. MCMAHON \& M. WATSON (éds), Career assessment: Qualitative approaches (pp. 123-128). Rotterdam: Sense.

McIlveen, P. (2017). Dialogical self: Co-investigator in career self-research. In M. McMaHON (éd.), Career counselling: Constructivist approaches ( $2^{\mathrm{e}}$ édition) (pp. 153-163). Londres: Routledge.

McMahon, M., Watson, M., \& Bimrose, J. (2012). Career adaptability: A qualitative understanding from the stories of older women. Journal of Vocational Behavior, 80, 762-768. Doi: 10.1016/j. jvb.2012.01.016.

McMahon, M., Watson, M., \& Lee, M. C. Y. (2018). Qualitative career assessment: A review and reconsideration. Journal of Vocational Behavior. Advance online publication. Doi: 10.1016/j. jvb.2018.03.009. 
Miller, W. R., \& Rose, G.S. (2015). Motivational interviewing and decisional balance: Contrasting responses to client ambivalence. Behavioural and Cognitive Psychotherapy, 43, 129-141. Doi: $10.1017 /$ S1352465813000878.

NÉGRONI, C. (2007). Reconversion professionnelle volontaire. Changer d'emploi, changer de vie: un regard sociologique sur les bifurcations. Paris: Armand Colin.

ObODARU, A. (2017). Forgone, but not forgotten : Toward a theory of forgone professional identities. Academy of Management Journal, 60 (2), 523-553. Doi: 10.5465/amj.2013.0432.

Olry-Louis, I., Vonthron, A.-M., Vayre, E., \& Soidet, I. (éds) (2017). Les transitions professionnelles: Nouvelles problématiques psychosociales (pp. 9-16). Paris: Dunod.

Picard, F., Olympio, N., Masdonati, J., \& Bangali, M. (2015). Justice sociale et orientation scolaire: l'éclairage de l'approche par les «capabilités» d'Amartya Sen. L'Orientation scolaire et professionnelle, 44 (1). Doi : 10.4000/osp.4515.

Rottinghaus, P. J., \& Eshelman, A. J (2015). Integrative approaches to career intervention. In P. J. Hartung, M. L. SAVICKAS, \& W. B. WALSH (éds), APA Handbook of Career Intervention: Vol. 2. Applications (pp. 25-39). Washington: American Psychological Association. Doi : 10.1037/14439-003.

Rudisill, J. R., Edwards, J. M., Hershberger, P. J., Jadwin, J. E., \& McKee, J. M. (2010) Coping with job transitions over the work life. In T. W. MiLler (éd.), Handbook of stressful transitions across the lifespan (pp. 111-131). New York: Springer. Doi: 10.1007/978-1-4419-0748-6_6.

SAVICKAS, M.L. (2013). Career construction theory and practice. In R.W. Lent \& S. D. BRown (éds), Career development and counseling: Putting theory and research to work ( $2^{\mathrm{e}}$ édition, pp. 147-183). Hoboken (NJ) : Wiley.

Savickas, M. L., \& Porfeli, E. J. (2012). Career Adapt-Abilities Scale: Construction, reliability, and measurement equivalence across 13 countries. Journal of Vocational Behavior, 80, 661-673. Doi : $10.1016 /$ j.jvb.2012.01.011.

Schlossberg, N. K. (2011). The challenge of change: The transition model and its applications. Journal of Employment Counseling, 48, 159-162. Doi: 10.1002/j.2161-1920.2011.tb01102.x. 
Vogelsang, E. M., Schultz, K. S., \& Olson, D. A. (2018). Emotional wellbeing following a later life career change: The roles of agency and resources. The International Journal of Aging and Human Development. Advance online publication. Doi: 10.1177/0091415017745972.

Wise, A. J., \& Millward, L. J. (2005). The experiences of voluntary career change in 30-somethings and implications for guidance. Career Development International, 10, 400-417. Doi: $10.1108 / 13620430510615328$.

Young, R. A., \& Collin, A. (2004). Introduction : Constructivism and social constructionism in the career field. Journal of Vocational Behavior, 64, 373-388. Doi : 10.1016/j.jvb.2003.12.005. 


\title{
4. TRANSITION À LA RETRAITE
}

\author{
ARIANE FROIDEVAUX (UNIVERSITÉ DE FLORIDE) ${ }^{1}$ \\ ET CHRISTIAN MAGGIORI \\ (HAUTE ÉCOLE DE TRAVAIL SOCIAL DE FRIBOURG)
}

\begin{abstract}
[ n considérant le contexte professionnel et démographique actuel, le présent chapitre s'intéresse aux différentes étapes - allant de la préparation jusqu’à l'adaptation - qui caractérisent le processus de la transition à la retraite dans les sociétés occidentales au $\mathrm{XXI}^{\mathrm{e}}$ siècle. Notre analyse portera sur les enjeux non seulement au niveau micro- (éléments théoriques, partie 1 ; et étude de cas, partie 2), mais aussi aux niveaux meso- et macro-sociaux (mise en perspective, partie 3). Une attention particulière sera portée à la réalité des travailleuses et travailleurs âgé.e.s en Suisse.
\end{abstract}

\section{LES APPROCHES THÉORIQUES DE LA TRANSITION À LA RETRAITE}

Tandis que l'espérance de vie à l'âge à 65 ans indique un nombre significatif d'années encore à vivre une fois atteint l'âge officiel de la retraite, de plus en plus de travailleuses ou travailleurs seniors sont et seront concerné.e.s par la transition à la retraite, définie comme "la sortie d'un individu du monde du travail, accompagnée par une diminution de l'engagement psychologique envers le travail et par un retrait comportemental» (Wang \& Shi, 2014, p. 211, traduction personnelle). De nos jours, les chemins qui mènent à la retraite - définie en tant que retrait du marché du travail - sont donc multiples, puisque de plus en plus de travailleuses et

1. La contribution de la $\mathrm{D}^{\mathrm{re}}$ Ariane Froidevaux à ce chapitre est soutenue par une bourse de mobilité postdoctorale attribuée par le Fonds national suisse de la recherche scientifique $\left(\mathrm{n}^{\circ} 168394\right)$. En accord avec les magazines sous-mentionnés, ce chapitre reprend et étend le contenu paru une première fois dans deux brefs articles écrits par Ariane Froidevaux pour la revue Panorama - Magazine du Centre suisse de services de formation professionnelle, Orientation professionnelle, universitaire et de carrière (CSFO), $\mathrm{n}^{\circ} 3$, année 2014, pages 20-21 et n 3, année 2012, pages 22-23, respectivement. Le texte de la partie «Étude de cas" de ce chapitre a également paru une première fois dans Psychoscope - Magazine de la Fédération suisse des Psychologues (FSP), n 2, année 2017, pages 18-21. 
travailleurs seniors décident de rester actives et actifs sur le marché du travail bien qu'elles ou ils perçoivent déjà leur pension de retraite. Le terme de "carrières tardives" (late careers en anglais) a été défini par la littérature scientifique comme étant des nouvelles formes de carrière concernant les individus dès 55 ans et jusqu'audelà de 65 ans (Greenhaus, Callanan, \& Godshalk, 2009). Les late careers incluent donc la retraite au sens traditionnel, mais ne se limitent pas à celle-ci. Ainsi, le passage à la retraite n'est plus seulement synonyme d'un passage unique dans le temps d'une activité professionnelle à un retrait complet du marché du travail. Cela est spécialement le cas dans des pays comme le Canada ou les ÉtatsUnis, qui ont abrogé l'âge officiel de la retraite pour offrir davantage de flexibilité.

\subsection{LA PERSPECTIVE DES CARRIÈRES DURABLES}

Au même titre que le XXI siècle se concentre de plus en plus sur le développement durable (par exemple en termes énergétiques), la perspective des carrières durables (De Vos \& van der Heijden, 2015) conçoit les carrières comme pouvant durer tout au long de la vie. Selon Newman (2011), au cours de la vie, non seulement la formation ne se limite plus au début de la vie professionnelle, mais encore le temps consacré aux sphères de vie non professionnelles ne se limite plus à la retraite. En effet, les carrières dites durables sont avant tout caractérisées par le renouvellement de soi tout au long du développement de carrière, à travers une meilleure intégration des sphères de vie non professionnelles à la sphère professionnelle (par exemple, augmentation des temps sabbatiques). Concrètement, la négociation de cette phase de vie des late careers peut permettre un certain renouveau professionnel (career renewal en anglais). Une caractéristique des carrières durables est la nécessité d'acquérir une certaine flexibilité: de par l'engagement continu dans des formations (Newman, 2011), de par la mobilité physique et psychologique et de par l'importance des valeurs personnelles et des capacités d'autodirection de l'individu comparés à celles de l'organisation. Appliquée aux late careers, la perspective des carrières durables souligne la continuité entre les différents épisodes de carrière, tandis que des connaissances régulièrement mises à jour contribuent positivement à la possibilité de continuer à travailler au-delà de 65 ans (Froidevaux \& Hirschi, 2015). 


\subsection{LE PROCESSUS DE LA TRANSITION}

\section{À LA RETRAITE (OU LATE CAREERS)}

Au niveau psychologique, le fait de quitter son travail lors de la transition à la retraite (que ce soit complètement ou en partie) implique pour les individus la perte de plusieurs fonctions du travail qui allaient auparavant de soi, et qui doivent maintenant être réinventées: non seulement la gestion du temps et les relations interpersonnelles, mais aussi le statut, les réalisations, le sens et l'utilité (Hall \& Mirvis, 2013). Dans la suite de ce chapitre, nous illustrons comment cet enjeu se traduit lors des différentes étapes de la transition à la retraite reconnues par la littérature scientifique, en adaptant le modèle temporel de la retraite tel que décrit par Wang et Shi (2014) en un modèle dynamique qui permette l'intégration des différentes étapes et chemins jusqu’à la retraite au sens propre (Cahill, Giandrea, \& Quinn, 2015). En effet, l'ensemble de ces chemins représente des possibilités uniques pour les individus de s'engager dans leur étape de late career.

\section{FIGURE 1. RÉSUMÉ DU PROCESSUS DE LA TRANSITION À LA RETRAITE OU LATE CAREERS AU XXIE SIÈCLE}

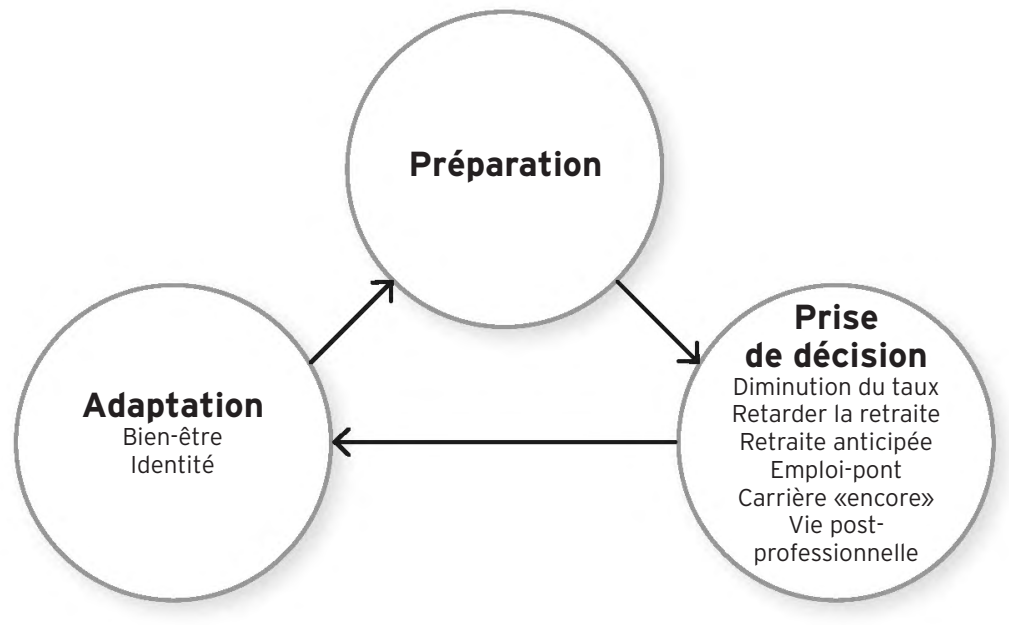

\subsubsection{La préparation}

Comme illustré dans la Figure 1, l'étape de préparation permet tout d'abord aux travailleuses et travailleurs seniors d'explorer les différents enjeux liés au désengagement de la vie professionnelle, notamment en termes de lieu où vivre ou de manière de gérer son 
temps. Dans le cadre de la préparation à la retraite, la recherche a identifié différents facteurs individuels qui sont associés à une probabilité réduite de s'engager dans des comportements de planification, comme avoir un niveau d'études moins élevé, une situation financière plus difficile, une préférence pour des gratifications à court terme quitte à ce qu'elles soient aussi plus faibles, et des attitudes négatives envers la retraite (par exemple "les retraité.e.s s'ennuient»). D'un autre côté, d'autres facteurs individuels ont été identifiés comme étant associés à une probabilité plus élevée de s'engager dans des comportements de planification, comme une personnalité proactive et la tendance à définir ses objectifs sur le plan financier, de loisirs ou de travail. Planifier sa retraite est important, car la recherche montre qu'il s'agit d'un exercice bénéfique non seulement pour la sécurité financière des individus, mais aussi pour leur santé physique et leur bien-être personnel et interpersonnel (pour une revue détaillée, voir Froidevaux, Baumann, Maggiori, Wieber, \& Rossier, 2017). Ces effets positifs s'expliquent par le fait que la planification augmente la confiance et le sentiment d'efficacité que les travailleuses et travailleurs seniors ont de leurs compétences à gérer leur étape de late careers. Concrètement, planifier cette étape de carrière permet aux individus de développer des attitudes plus positives envers la retraite (par exemple, «c'est un temps pour profiter de la vie») et des attentes plus réalistes, grâce à la définition d'objectifs concrets pour cette étape de carrière et de vie.

Bien que la recherche se soit principalement concentrée sur la préparation à la retraite, il nous semble raisonnable d'étendre cette préparation à l'entier de la phase de late careers dans une perspective de carrières durables. Ainsi, différentes phases de préparation peuvent survenir à différents moments du late career development, et seront associées, chaque fois, à des objectifs différents. Par exemple, une personne à qui son entreprise n’offre pas de possibilité de travailler au-delà de l'âge officiel de la retraite pourra planifier la prochaine étape de son développement de carrière en incluant ou non sa participation au marché du travail.

\subsubsection{La prise de décision}

La préparation permet d'atteindre l'étape de la prise de décision (voir Figure 1) entre les différentes options possibles dans l'étape de vie des late careers. Alors que traditionnellement cette prise de décision concernait le moment de la retraite (c’est-à-dire anticipée, 
à l'âge légal ou retardée), de nos jours, la prise de décision concerne davantage la forme de la retraite (c'est-à-dire la gestion de son temps, entre les différentes sphères de vie, incluant la possibilité d'une continuation dans le marché du travail, ou le choix entre l'investissement dans plusieurs sphères non professionnelles) (Wang \& Shi, 2014). Globalement, la recherche a montré que des facteurs individuels comme l'âge (être plus âgé), la situation financière (être plus aisé financièrement), l'éligibilité à recevoir une pension de retraite, la (mauvaise) santé des individus, mais aussi de leur partenaire, la (non-) satisfaction au travail et les (non-) possibilités de travail flexible augmentent la probabilité de prendre une décision de se retirer complètement du marché du travail (Jex \& Grosch, 2013).

Comme illustré dans la Figure 1, les formes de retraite ou de late careers incluent: la réduction graduelle du taux de travail dans le temps, l'emploi-pont, la carrière "encore» et l'engagement au sein des différentes sphères de vie non professionnelles. Le départ à la retraite dans sa conception traditionnelle implique un retrait complet de la sphère professionnelle pour se diriger vers les sphères non professionnelles comme le bénévolat, les activités de loisirs et le soin apporté aux autres (care en anglais; notamment auprès des parents âgés et des petits-enfants). Au cours des dernières décennies, de plus en plus d'entreprises ont commencé à offrir la possibilité aux travailleuses et travailleurs seniors de réduire petit à petit leur taux de travail, passant par exemple d'un $100 \%$ à un $80 \%$, voire à un $50 \%$, avant un désengagement complet. Cette retraite "graduelle» permet aux individus d'apporter des modifications à leurs style de vie et emploi du temps; toutefois l'élément clef pour le bien-être social et psychologique identifié par une recherche australienne est le contrôle sur le moment et la manière de se désengager du travail rémunéré (De Vaus, Wells, Kendig, \& Quine, 2007).

L'emploi-pont se définit par un nouvel emploi se situant entre le départ de l'emploi exercé dans la "carrière principale» d'un individu et son désengagement complet du monde du travail (Shultz, 2003). Ainsi, cet emploi peut commencer à tout moment au sein de l'étape de late career, soit de 50 ans jusqu'à plus de 65 ans. Concrètement, cet emploi peut s'exercer dans le même domaine professionnel (par exemple, impliquant le même métier ou fonction) ou dans un autre domaine. Par exemple, dans sa recherche, Froidevaux (2016) décrit le cas d'un représentant de commerce qui, 
après avoir été licencié à 60 ans, est devenu chauffeur de taxi afin de maintenir ses finances jusqu'à l'obtention complète de sa pension de retraite. L'emploi-pont peut aussi s'exercer en tant qu'indépendant.e, et il peut être temporaire (par exemple, travail saisonnier) ou à durée indéterminée. Quant à la carrière "encore» (encore career en anglais), elle représente un type spécifique d'emploi-pont associé à une réorientation professionnelle nécessitant un retour en formation, dans le but d'exercer un emploi avec un plus grand sens et au bénéfice de la communauté (Freedman, 2007). Par exemple, lors de son travail sur le terrain, Froidevaux (2016) a rencontré plusieurs personnes qui, ayant travaillé comme responsables dans le domaine de la finance durant une trentaine d'années, ont décidé de se former pour devenir coachs en entreprise afin d'aider les cadres dans leurs transitions de carrière au sein de et à l'extérieur de l'entreprise. Dans cet exemple, la carrière "encore» est caractérisée par un travail à visée altruiste afin d'accompagner le cheminement personnel d'individus en transition.

La recherche a mis en avant différents facteurs individuels qui influencent la probabilité de s'engager dans une forme de late career plutôt qu'une autre. Ainsi, comme résumé par Jex et Grosch (2013), être au bénéfice d'un revenu confortable est associé positivement au choix d'une retraite traditionnelle, tandis que subir des difficultés financières et/ou avoir son partenaire qui travaille et/ou des enfants encore à charge augmentent la probabilité de poursuivre avec un emploi-pont. Toutefois, le rôle de l'attachement au travail modère cette relation: des individus au bénéfice de revenus confortables peuvent choisir de continuer à travailler à la retraite en raison de cet attachement. Bénéficier de relations sociales quotidiennes au travail constitue également un motif pour s'engager dans un emploi-pont, indépendamment de l'état de ses finances. De plus, le choix d'un emploi-pont au sein de la même profession a été associé à la satisfaction au travail. Finalement, la santé et le bien-être en général, un meilleur statut de travailleuse ou travailleur senior (par exemple, moins de discrimination liée à l'âge) et une autonomie et sécurité financière accrues ont été identifiés comme d'autres facteurs propices à l'engagement dans un emploi-pont (Alcover, 2017).

\subsubsection{L'adaptation}

L'étape de l'adaptation à la retraite en tant que départ du marché du travail - ou adaptation à toute autre forme de late career - 
a été définie comme le processus au travers duquel les individus s'habituent aux changements intervenus dans leur vie quotidienne pour atteindre un certain niveau de bien-être psychologique (Wang \& Shi, 2014). Deux principaux enjeux ont été identifiés dans la littérature scientifique pour cette étape: développer un style de vie satisfaisant et se détacher de son travail à la fois psychologiquement et socialement (van Solinge \& Henkens, 2008). Tandis que nous développons ci-dessous les aspects de l'adaptation liés au bien-être, ceux relatifs à l'identité seront examinés en lien avec l'outil décrit dans l'étude de cas présentée plus loin dans ce chapitre.

Différents patterns d'adaptation à la retraite ont été identifiés dans la littérature scientifique (pour une analyse détaillée, voir Froidevaux et al., 2017). En particulier, Schlossberg (2003) a proposé une typologie consistant en cinq types d'adaptation à la retraite, issus de sa pratique en orientation aux États-Unis. Cette typologie peut être utilisée pour expliquer le déroulement des différents chemins que peut prendre l'étape des late careers, et la manière dont il est possible de s'adapter aux changements qui y surviennent. Il est toutefois important de préciser qu'il est possible d'appartenir à plusieurs types à la fois, et que cela peut évoluer au cours du temps passé à la retraite (surtout au début). Premièrement, «ceux et celles qui continuent" (continuers en anglais) adaptent, en les modifiant quand nécessaire, leurs compétences utilisées par le passé, ainsi que leurs valeurs et leurs intérêts, au moment de toucher leur pension de retraite. Ainsi, même si certains aspects importants de leur vie ont changé, ces personnes continuent de maintenir un certain niveau d'identification à leur travail en s'engageant dans un emploi-pont, une retraite graduelle ou des activités de volontariat souvent en lien avec leur précédent métier (par exemple, un.e enseignant.e s'engageant dans une association ayant pour but d'aider des enfants de milieux défavorisés à faire leurs devoirs).

Deuxièmement, les "aventuriers et aventurières" (adventurers en anglais) perçoivent la retraite comme une opportunité d'organiser leur temps et leur espace d'une manière différente. Le critère d'inclusion à cette catégorie repose sur le fait de vouloir développer des nouvelles compétences dans cette nouvelle étape de late career. Par exemple, la retraite peut amener l'opportunité de se lancer dans le projet d'une "carrière encore", ou de s'inscrire à de nouvelles formations (aussi bien de courte durée que certificatives, telles que cours de langues ou formation pour devenir coach agréé.e). 
Troisièmement, les personnes dites «au jour le jour» (easy gliders en anglais) tendent à voir leur retraite comme une période de liberté où elles ne veulent pas générer explicitement de nouveaux projets de vie. L'expression "se laisser porter par le vent" serait particulièrement caractéristique de ces individus qui apprécient le fait de passer du temps avec les amis et la famille un jour, et de s'engager le lendemain dans des activités sportives ou même de retourner travailler.

Quatrièmement, pour les personnes "en recherche» (searchers en anglais), la retraite marque la nécessité d'explorer de nouvelles options pour structurer son quotidien avec différentes activités. Les individus de cette catégorie n'ont pas encore trouvé leur "niche» et peuvent avoir le sentiment de bouger d'une activité à une autre sans trouver de satisfaction. Ces personnes se questionnent sur les opportunités qui peuvent être amenées par la retraite. Elles peuvent avoir de nombreuses idées et rencontrer de la difficulté à choisir entre celles-ci. Généralement, cette étape fait partie du processus d'adaptation et est souvent limitée dans le temps.

Enfin, un dernier profil concerne les individus dits "en retrait" (retreaters en anglais). Ces personnes ont une vision négative de la retraite, comme celle de la fin d'une carrière riche et intéressante. De ce fait, leur adaptation est bien souvent accompagnée d'émotions négatives (comme la tristesse) liées au deuil de leur travail, et d'un certain retrait comportemental (par exemple, rester chez soi toute la journée). Bien que ce chemin d'adaptation de retraite soit le plus difficile, il s'agit heureusement le plus souvent d'un état passager. Toutefois, un accompagnement psychologique peut s'avérer nécessaire lorsque la phase de deuil du travail dure un certain temps et/ou est ressentie de façon particulièrement aiguë.

Tandis que la typologie développée par Schlossberg (2003) permet de qualifier les différents chemins menant à l'adaptation à la retraite, des études longitudinales allemande (Pinquart \& Schindler, 2007) et américaine (Wang, 2007) ont permis de les quantifier. Le premier élément à retenir de ces études est que, pour la plupart des individus (environ 75\%), la transition à la retraite n'apporte pas de modification notable dans leur bien-être, mais bel et bien un maintien de leur état antérieur. Ce type de résultat a également été mis en évidence concernant la satisfaction de couple à l'orée de la retraite: alors qu'elle tend à augmenter chez les couples qui faisaient déjà l'expérience de la félicité conjugale, elle tend à diminuer chez ceux qui rencontraient déjà certaines difficultés (Rauer \& Jensen, 2016). 
Ensuite, tandis qu'environ $10 \%$ des retraités rencontrent une baisse de satisfaction dans leur vie en raison de la transition, 15\% d'entre eux font l'expérience d'une amélioration (Pinquart \& Schindler, 2007; Wang, 2007). Ainsi, pour certains, la retraite représente une étape difficile, notamment à cause de la perte de nombreuses fonctions importantes du travail qui n'existaient peut-être pas (encore) dans d'autres sphères de vie (par exemple, les relations au quotidien). En revanche, pour d'autres personnes, la retraite représente un soulagement, par exemple si elles ont eu un travail pénible ou exercé dans des conditions (de plus en plus) insatisfaisantes.

La recherche a mis en évidence cinq catégories de facteurs influençant la qualité de l'adaptation à la retraite et donc le type de trajectoire dont les individus peuvent faire l'expérience (Wang, Henkens, $\&$ van Solinge, 2011). D'abord, ces chercheurs et chercheuses ont identifié des facteurs (1) individuels et (2) familiaux ${ }^{2}$ : l'adaptation est meilleure si la personne possède une bonne santé physique et mentale et une situation financière aisée, est mariée, heureuse en ménage, avec un partenaire qui ne travaille pas, et n'ayant pas/plus d'enfants à charge. Ensuite, des facteurs liés à (3) la situation professionnelle antérieure peuvent influencer l'adaptation à la retraite: la personne connaît moins de difficultés d'adaptation si elle a subi du stress au cours de sa dernière activité, y a ressenti de l'insatisfaction ou était sans emploi. Une identification forte à l'entreprise diminue en revanche la qualité de son adaptation, tandis qu'avoir été habituée à occuper plusieurs rôles sociaux avant la retraite permet de se séparer plus facilement de son rôle de travailleur ou de travailleuse, puisque plusieurs des fonctions du travail sont alors également assumées par d'autres rôles. Un autre type de facteur concerne (4) la transition à la retraite en elle-même: l'adaptation sera plus facile si cette dernière a été décidée et planifiée. Si, pour des raisons de santé par exemple, des personnes ont été contraintes de prendre leur retraite plus tôt que prévu, la qualité de l'adaptation baissera. Il peut également s'agir de problèmes de santé du partenaire ou d'un.e autre membre de la famille qui demandent des soins de la part du ou de la retraité.e. Enfin, (5) des pressions organisationnelles, comme l'impossibilité institutionnelle d'établir des contrats de travail pour des personnes au-delà de l'âge légal de la retraite (comme c'est le cas dans de nombreux emplois du secteur public en Romandie) peuvent

2. À l'heure actuelle, la recherche ne permet pas de conclure à des différences hommesfemmes dans ces facteurs. 
aussi rendre le départ à la retraite involontaire. En revanche, un désir personnel de réaliser d'autres activités ou de toucher sa rente est favorable pour l'adaptation à la retraite.

\section{APPLICATION}

\subsection{OFFRES EXISTANTES POUR RÉPONDRE AUX ENJEUX PSYCHOLOGIQUES}

\section{DE LA TRANSITION À LA RETRAITE EN SUISSE ROMANDE}

Aujourd'hui en Suisse, rares sont les structures qui proposent un soutien ciblé sur le thème de l'accompagnement à la retraite. Ce dernier se limite bien souvent à celui du conseil financier. De ce fait, la population senior est encore relativement peu intégrée dans les dispositifs classiques d'orientation du secteur public. Comme expliqué par Froidevaux (2014), différentes croyances bien répandues concernant la retraite peuvent expliquer cet état de fait, la principale d'entre elles assumant que la retraite constitue un état de repos, voire de vacances permanentes, pour des personnes ayant travaillé toute leur vie et ayant de ce fait mérité ce statut privilégié de rentier ou de rentière. De l'autre côté, les principaux désavantages généralement associés à la retraite sont la détérioration de l'état de santé - de par l'association généralement effectuée entre retraite et vieillissement - et les potentielles difficultés financières: deux types de problèmes qui ne sont pas directement du ressort des psychologues du conseil et de l'orientation.

Cette étape de l'existence soulève pourtant beaucoup de questions pratiques ([Froidevaux-] Rosselet \& Hirschi, 2012): comment structurer son temps, continuer à se sentir utile, se trouver un hobby ou encore vivre en couple? Enfin, et surtout, naît le souci de trouver un nouveau sens à sa vie au terme de sa carrière. Les psychologues du conseil et de l'orientation sont à même d'aider les consultant.e.s seniors et futurs retraité.e.s et d'apporter une réponse à leurs préoccupations. À la connaissance des auteur.e. $s^{3}$, les principaux organismes en Suisse romande qui organisent des cours de préparation à la retraite et/ou entretiens individuels sont

3. Il est important de préciser que les auteur.e.s n'ont pas reçu de demande de la part des organismes mentionnés pour apparaître dans ce chapitre. Ces mentions sont fondées sur les expériences personnelles des auteur.e.s en matière de collaborations existantes et passées avec les organismes en question et se limitent à des informations factuelles. Plus d'informations sont disponibles sur les sites internet de ces organismes: http://www.fr.avantage.ch/; http:// www.force-nouvelle.ch et http://cercledelegs.org/. 
Avant'Âge, Force Nouvelle et les Cercles de Legs. Avec le credo "l'expérience en plus», le programme Avant'Âge, basé à Lausanne, a été créé par Pro Senectute, avec pour objectif un soutien à la préparation à la retraite et des conseils aux entreprises pour favoriser l'employabilité des travailleuses et travailleurs seniors. Avec le credo "centre d'information retraite", la fondation Force Nouvelle, basée à Genève, à but social et reconnue d'utilité publique, a pour objectif un soutien à la préparation à la retraite par différents cours offerts aux travailleuses et travailleurs seniors par le biais de leur entreprise. Finalement, avec le credo "pour un troisième tiers de carrière harmonieux et pour transmettre ses savoirs et ses bonnes pratiques", les différents intervenant.e.s de la méthode du Cercle de Legs (Limoges, 2008), basé.e.s à Lausanne et à Genève, offrent aux individus de plus de 50 ans, et aux employé.e.s via leur entreprise, un espace de groupe avec pour objectif de favoriser la réflexion sur les dernières années au travail et la transition à la retraite.

\subsection{L'APPORT DE LA PSYCHOLOGIE DU CONSEIL ET DE L'ORIENTATION}

La psychologie du conseil et de l'orientation permet de porter un regard particulier sur l'entrée dans la retraite en tant que transition de carrière. En ce début de XXI siècle, un nouveau paradigme est né dans cette sous-discipline de la psychologie, sous l'impulsion de praticien-ne.s chercheur-se-s de plusieurs universités dans le monde, dont celle de Lausanne. Intitulé Life Design (construire sa vie, en français), ce paradigme permet de prendre en compte le passage d'une société standardisée et institutionnalisée (où l'on cherchait à associer un individu avec un métier stable) à des biographies individuelles multiples (Savickas et al., 2009). En raison de transitions de carrière et d'emploi de plus en plus fréquentes, les psychologues ont pour rôle d'accompagner les personnes à construire leur vie de manière continuelle, en fonction de leurs valeurs de vie fondamentales, dans le but qu'elles puissent s'adapter lorsque la prochaine étape surviendra.

Jean Guichard, professeur émérite au Conservatoire national des arts et métiers de Paris, a proposé un modèle pour construire sa vie. Dans le cadre de son approche de la construction de soi, il propose la notion de forme identitaire subjective, définie comme la manière dont une personne se voit, se pense et parle d'elle-même dans un rôle social donné, en relation avec son passé et son futur (Guichard, 2004). Selon cet auteur, l'identité individuelle se comprend comme un système dynamique de formes identitaires subjectives, 
impliquant des représentations de soi dans différents contextes. Cet outil vise à élaborer avec la cliente ou le client ses formes identitaires subjectives à trois périodes du temps: le présent, représentant les contextes actuels d'activité; le passé, à savoir les interactions passées jouant un rôle dans la vie présente; et le futur, ces anticipations de soi qui donnent au système présent son unité, en l'ancrant dans une perspective future.

\section{3 ÉTUDE DE CAS}

En prenant appui sur une étude de cas réalisée dans le cadre d'un entretien de recherche (Froidevaux, 2018), nous suivrons le parcours de Pierre (pseudonyme, cas anonymisé) et compléterons cette étude de cas avec des éléments pratiques d'accompagnement de groupes et d'individus en transition vers la vie post-professionnelle ou en situation de retraite. Pierre, 69 ans, habite en Suisse romande et est à la retraite depuis quatre ans et demi. Il vit avec son épouse, avec laquelle il est marié depuis quarante ans. Il a deux fils adultes et trois petits-enfants. Un de ses fils vit dans une certaine proximité géographique, alors que le second vit sur un autre continent. Pierre a également un chien. Une grande partie de son activité professionnelle s'est réalisée comme boulanger indépendant. Il possédait une affaire familiale avec un de ses cousins. Il a pris sa retraite de manière volontaire à l'âge de 65 ans, âge légal de la retraite en Suisse.

\subsubsection{Rôles et domaines de vie centraux avant la prise de retraite (passé)}

Pierre peut clairement identifier les sphères de vie principales lors du temps de vie professionnelle. Le travail est le domaine principal, avec un fort investissement personnel, tant d'un point de vue psychologique que financier. Le second domaine central est la vie de famille, en tant qu'époux et père. Il évoque d'ailleurs des regrets concernant le fait de ne pas avoir pu voir grandir ses enfants autant qu'il l'aurait souhaité. Dans la pratique de l'accompagnement, des hommes évoquent souvent cet élément lors du travail en individuel ou en groupe. La mise en balance identitaire des différentes sphères de vie met également en évidence ce que le sujet peut avoir investi en termes de temporalité (nombre d'heures dans la vie professionnelle), mais également en termes de rôles sociaux (l'époux ou l'épouse de..., le père de..., la mère de..., etc.) et psychologique (investissement identitaire et psychoaffectif). Enfin, lorsqu'il travaillait, Pierre manquait de temps pour ses loisirs. 


\subsubsection{Rôles et domaines de vie centraux}

\section{quatre ans et demi après la retraite (présent)}

Si l'on regarde le processus de transition et d'ajustement entre la fin de la vie professionnelle et sa situation de retraité actuelle, Pierre a fait l'expérience d'étapes qui ont du sens pour lui. Il a identifié la durée subjective d'une année pour se "sentir» complètement dans son nouveau statut de retraité, durée qui lui a permis de répondre à son principal défi au départ de la transition: savoir comment se détacher de son rôle professionnel. Dans cet "entre-deux", il dit avoir beaucoup pensé à son travail, avoir continué de suivre le rythme des réveils nocturnes comme pour se rendre au travail. Il ajoute qu'il se rendait régulièrement dans «sa» boulangerie. Avec l'aide de son épouse, Pierre a pu prendre conscience qu'il ne se rendrait plus au travail, qu'il n'y travaillerait plus. Depuis cette prise de conscience, Pierre ne retournera qu'occasionnellement sur son ancien lieu de travail, pour faire des pâtisseries en lien avec ses engagements personnels et bénévoles. Il ajoute qu' «il est important de réaliser que vous faites quelque chose de nouveau à la retraite et de vieillir d'une manière différente».

Tandis que l'entretien avec Pierre se déroule quatre ans après la prise de retraite, dans l'accompagnement de groupe dans les entreprises pour préparer cette transition de carrière, les personnes sont encore en activité professionnelle. Un des éléments importants à considérer dans l'accompagnement de la transition à la retraite est le cycle de vie au travail. En effet, les questions d'attachement et de détachement du monde du travail ne sont pas vécues de manière identique entre les individus, dont la singularité resurgit dans ce bilan identitaire qui s'invite lors de cette transition. Qu'il s'agisse de sessions de groupe (offertes ou non par l'entreprise) ou d'une consultation individuelle, les personnes sont invitées à se décentrer progressivement du monde du travail, à le regarder, à le mettre en perspective dans un passé, présent, futur; d'en faire une histoire, leur propre histoire. Le passage à la retraite constitue une rupture d'avec le monde du travail qui questionne les «équilibres» de vie, les «rôles» et le «sens» de cette étape de vie, tout en se projetant certaines fois dans des perspectives temporelles où la question du vieillissement, de la maladie ou de la mort s'invite.

Pour Pierre, une nouvelle subjectivité identitaire se dessine, entre continuité et changement avec le passé avant la retraite. Il dit "continuer la même route, mais faire les choses différemment»; 
et il précise: "Mon travail a été une part de moi durant les quarante ans dernières années et il sera encore une part de moi jusqu’à la fin.» Dans la pratique d'accompagnement individuel, la question de l'identification au rôle professionnel reste effectivement un élément significatif. Il est en effet important que le psychologue du conseil et de l'orientation explore avec la personne la part d'identité professionnelle qu'elle ou il souhaite garder en tant que retraité.e, afin de l'inscrire, comme le dit Pierre, comme une partie de soi qui peut perdurer au-delà du temps professionnel. Pierre dit maintenant pouvoir passer plus de temps avec son épouse, dans son rôle de père et de grand-père; plus de temps pour le domaine des loisirs; et deux nouveaux domaines de vie ont émergé, le bénévolat et le fait d'avoir un chien, son cadeau de retraite.

\subsubsection{Rôles et domaines de vie centraux: projection dans le futur}

Pierre, dans sa projection future, voit son rôle dans la famille et en tant que bénévole comme domaines de vie principaux. Dans un second temps, il peut imaginer développer davantage un de ses loisirs, tandis qu'un troisième et nouveau domaine est identifié autour des voyages, en fonction des relations familiales: son épouse et lui auront plus de temps pour voyager et rendre visite à leur fils à l'étranger, ce qui est difficile actuellement en raison de leur chien.

\subsection{TRAVAILLER LE REMANIEMENT IDENTITAIRE AU CENTRE}

\section{DE LA TRANSITION TRAVAIL-RETRAITE}

Selon le paradigme du Life Design, étant donné que les individus peuvent de moins en moins compter sur leur entreprise pour se définir, de par le fait que les emplois fixes à long terme se raréfient, il devient nécessaire qu'ils utilisent leur identité comme "compas" pour les guider dans la construction de leur vie, tant professionnelle que personnelle. Dans ce chapitre, cela est décrit sous la forme des formes identitaires subjectives (Guichard, 2004). Dans le cas de Pierre, ses rôles d'époux, de grand-père ou encore de boulanger indépendant constituent des formes identitaires subjectives importantes, dans son passé, tout comme dans son présent et/ou futur.

Ainsi, le passage à la retraite implique une redéfinition de soi, c'est-à-dire du système formé par les différentes formes identitaires subjectives. Se détacher psychologiquement et socialement de son travail a d'ailleurs été identifié par la recherche comme l'un des principaux défis pour se sentir bien dans sa retraite. La valeur du 
travail est questionnée, mais également ses fonctions (revenu, intégration, plan relationnel, etc.), qui nécessitent d'être réinventées. Concrètement, il s'agit pour les psychologues d'aider les personnes à devenir pleinement conscientes de la manière dont elles articulent leurs rôles et domaines de vie centraux (ou formes identitaires subjectives), de même que cette articulation par le passé, afin de les relier à leurs projets et envies au sein de l'un ou l'autre de ces rôles dans le futur. Cela permettra à une certaine compréhension de l'identité d'émerger: identités au travail, en transition, et en tant que «nouvelle et nouveau» retraité.e. Les psychologues aideront également à cerner les stratégies développées par les sujets face au départ annoncé (différentes formes d'annonce) et le vécu singulier de chacun s'y rapportant. La manière de faire face au changement (coping) et de vivre les transitions sont en arrière-fond des thématiques abordées. Les consultations en individuel et en groupe ne sont finalement pas dénuées d'un questionnement existentiel et d'un rapport aux échéances: vieillissement "young olds" - 65-79 ans; versus les "old olds", 80-94 ans -, pertes, finitude, mort.

\section{PERSPECTIVES}

Nous poursuivons à présent notre réflexion en adoptant une perspective davantage méso- et macro-social, à savoir focalisée sur les enjeux pour les organisations et la société. Il est en effet important de rappeler que le travail individuel nécessite aussi la prise en compte des ressources et des contraintes apportées par le contexte global dans lequel elle ou il évolue, à savoir les environnements familial, organisationnel (par exemple, les pratiques de ressources humaines sont mises en place pour favoriser l'intégration des seniors dans l'entreprise) et sociétal (comme les lois sur la retraite et la culture/norme d'une retraite plutôt anticipée ou tardive). Nous présenterons donc brièvement le possible impact du vieillissement démographique actuel sur le fonctionnement social et le monde du travail, ainsi que des situations spécifiques à la réalité actuelle des travailleuses et travailleurs âgé.e.s, telles que l'âgisme au travail.

\subsection{UN MARCHÉ DU TRAVAIL VIEILLISSANT?}

Sur le plan démographique, la Suisse connaît depuis la fin du $\mathrm{XIX}^{\mathrm{e}}$ siècle un vieillissement important de sa population. Plus spécifiquement, le vieillissement démographique d'un groupe indique 
une modification de la structure par âge liée à l'augmentation de la proportion des personnes âgées (65 ans et plus) et la diminution de celle des plus jeunes (0 à 19 ans) (Kohli, Bläuer Herrmann, \& Babel, 2010). En Suisse, les personnes âgées de plus de 65 ans représentent à présent $18 \%$ de la population, et le nombre de centenaires est en constante augmentation (en 2016, ils étaient 1809 vivant en Suisse, pour seulement 106 centenaires en 1970; OFS, 2017a). Un tel changement démographique a des répercussions importantes pour notre société à plusieurs niveaux. Au niveau macro-social, les enjeux sont de taille, notamment sur les plans économique, politique, sociétaire et sanitaire, et plus précisément en termes de marché du travail, de comportements de consommation ou de votes, de cohésion sociale et de rapports intergénérationnels et de santé de la population. Sur un plan méso-social se posent des questions relatives aux dispositifs, aux institutions et aux services de soutien ou de prise en charge qui doivent continuellement être adaptés aux besoins changeants des différents groupes de la population (comme les crèches pour les enfants en bas âge ou les foyers de jour pour les seniors).

Comme indiqué ci-dessus, le vieillissement démographique a aussi des répercussions sur le marché du travail et sur différentes transitions professionnelles, comme le passage à la retraite. En effet, le vieillissement démographique comporte également un vieillissement de la population active qui risque de provoquer une certaine pénurie de main-d'œuvre dans plusieurs domaines professionnels. Ceci est essentiellement dû aux passages à la retraite massifs dans un laps de temps relativement bref des baby-boomers et au manque de travailleuses et travailleurs pour les remplacer. Cette tendance devrait également se maintenir dans les prochaines décennies. Bien évidemment, cette situation nécessite un ajustement important des politiques d'emploi (et d'embauche) des entreprises, et de soutien aux travailleurs plus âgés. Enfin, elle pose aussi un défi de taille pour les assurances sociales de notre pays.

En Suisse, entre 1991 et 2016, l'âge moyen de la population active est passé de 39 à 41,5 ans (OFS, 2018). Cet âge varie bien évidemment selon les branches économiques, allant de 39,6 dans le domaine de la construction à 46,9 pour l'agriculture et sylviculture. Pour leur part, les indépendant.e.s avaient un âge moyen de 50,5 ans en 2016 (OFS, 2018). Quant à la participation au marché du travail des 55-64 ans en Suisse, on a observé une augmentation au cours des deux dernières décennies. En effet, parmi les 55-64 ans, le taux 
d'emploi a progressé de $63 \%$ à 74\% entre 1996 et 2016 (SECO, 2017). En comparaison avec les autres pays de l'Organisation de Coopération et de développement économiques (OCDE), en 2017, seuls l'Islande, la Nouvelle-Zélande, la Suède et le Japon ont enregistré un taux supérieur. Ce résultat peut, au moins en partie, s'expliquer par les efforts produits dans divers secteurs professionnels pour améliorer les conditions favorisant le maintien en emploi des travailleuses et travailleurs âgé.e.s ainsi que par une participation accrue au marché du travail de la part des femmes. Par rapport aux 65 ans et plus, si après l'âge de la retraite ordinaire le taux d'emploi diminue fortement, il reste tout de même important et a même progressé entre 1996 et 2016. En effet, le taux des personnes au-delà de 65 ans ayant choisi de continuer à être professionnellement actives est passé de $15 \%$ à $19 \%$ (SECO, 2017). Cela représente, pour l'ensemble de la population suisse, un taux d'activité professionnelle de $11,7 \% \mathrm{chez}$ les plus de 65 ans (OFS, 2016). Parmi les personnes actives après l'âge de la retraite, on retrouve surtout des hommes, des personnes avec une formation de degré tertiaire, des indépendant.e.s ou encore des gens travaillant pour l'entreprise ou l'exploitation familiale. Le temps partiel est la forme de travail la plus répandue auprès des 65 ans et plus (OFS, 2008). Enfin, l'âge moyen à la sortie du marché du travail a également augmenté au cours de la dernière décennie. En 2016, il se situait à 65,2 ans (à 65,6 ans pour les hommes et à 64,9 ans pour les femmes), alors qu'en 2006 il était de 64,3 ans (OFS, 2017b). Cela place la Suisse parmi les pays de l'OCDE ayant les âges de retraite effectifs les plus élevés (OCDE, 2014). Spécifiquement, $45 \%$ des femmes prennent leur retraite entre 62 et 64 ans, et $35 \%$ des hommes à l'âge de 65 ans (Madero-Cabib, Gauthier, \& Le Goff, 2015).

\subsection{FAVORISER LA PARTICIPATION DES TRAVAILLEUSES}

\section{ET TRAVAILLEURS ÂGÉ•E·S: LES FACTEURS INDIVIDUELS}

Comme l'indiquent plusieurs auteur.e.s (par exemple, Faurie et al., 2008; Swift et al., 2017; OFAS 2016; Trageser, Hammer, \& Fliedner, 2012), la participation des travailleuses et travailleurs âgé.e.s au marché du travail est un élément essentiel pour faire face à nombre de défis actuels liés au vieillissement de la population active (tels que la pénurie de main-d'œuvre actuelle et à venir dans plusieurs secteurs). Tant en Suisse que dans les autres pays occidentaux, l'intégration des seniors dans le marché du travail a d'ailleurs été reconnue comme 
l'un des principaux défis du XXI siècle (Shultz \& Adams, 2007). Selon le Conseil fédéral (OFAS, 2016), l'encouragement à l'emploi des travailleuses et travailleurs âgé.e.s de 58 ans et plus signifie motiver les personnes à rester plus longtemps professionnellement actives. Ceci impliquerait la réduction du nombre de retraites anticipées ainsi que l'incitation à rester professionnellement actives-actifs même au-delà de l'âge légal de la retraite (Trageser et al., 2012). En effet, malgré le fait que toujours plus de personnes continuent à travailler au-delà de l'âge légal de la retraite, et que l'on observe une perte de vitesse de la tendance à prendre une retraite anticipée, le nombre de travailleuses et travailleurs quittant de manière anticipée le marché du travail reste élevé en Suisse (OFAS, 2016). De manière plus spécifique, entre 2002 et 2012 le taux de personnes actives prenant une retraite anticipée dans les cinq ans précédant l'âge de la retraite ordinaire est passé d'environ $27 \%$ à $22 \%$. Donc, un.e travailleur ou travailleuse âgé.e sur cinq quittait prématurément le marché du travail (SECO, 2017). De récentes études réalisées en Suisse ont montré que les facteurs favorisant un départ à la retraite anticipée sont des finances élevées, le fait d'être un homme et celui d'avoir des problèmes de santé; tandis que les facteurs associés à une prise de retraite tardive sont un parcours de vie incluant la parentalité et le fait d'être une femme séparée ou divorcée (Madero-Cabib et al., 2015; Madero-Cabib \& Kaeser, 2016).

D'autre part, d'après une étude mandatée par l'OFAS et réalisée par Trageser et al. (2012), en Suisse, parmi les 58 ans et plus, les principales motivations pour rester en activité jusqu’à l'âge légal de la retraite sont liées à la santé ainsi qu'aux conditions et au type de travail. En effet, un bon état de santé ainsi que des conditions de travail favorables - caractérisées notamment par une bonne ambiance, des horaires flexibles et qui permettent de concilier vie professionnelle et vie privée - sont associés à l'intention de continuer à travailler. Il est intéressant de remarquer que les aspects économiques (tels qu'une continuité dans le salaire ou des rentes vieillesse plus élevées) ont un rôle moins important dans cette décision par rapport aux facteurs cités avant. Parmi les 58-63/64 ans, environ 20\% prévoyaient de rester actives-actifs au-delà de l'âge de la retraite. Des conditions de travail favorables, le plaisir à travailler ainsi qu'une mise en valeur de la part de l'entreprise des compétences apportées sont les principales raisons expliquant cette intention (OFAS, 2016; Trageser et al., 2012). En revanche, le désir de bénéficier de plus de liberté, 
des problèmes de santé, des conditions de travail négatives ou encore un licenciement sont les principales motivations pour sortir de la vie active en Suisse (Trageser et al., 2012).

\subsection{FAVORISER LA PARTICIPATION DES TRAVAILLEUSES ET TRAVAILLEURS}

\section{ÂGÉ•E-S: LES FACTEURS ORGANISATIONNELS ET SOCIÉTAUX}

Au niveau des organisations, l'étude menée par Trageser et al. (2012) met en évidence qu'environ $60 \%$ des entreprises interrogées considère utile et important de garder les travailleuses et travailleurs jusqu'à l'âge de retraite (voire même au-delà). Les principales motivations sont liées aux compétences cumulées dans le temps par ces travailleuses et travailleurs et à la nécessité de transmettre ce savoir-faire afin de garantir une certaine continuité avec les collaboratrices et collaborateurs plus jeunes (Trageser et al., 2012). Cependant, selon ces mêmes entreprises, en cas de pénurie de main-d'œuvre, la possibilité d'engager des travailleuses et travailleurs âgé.e.s (58 ans et plus) est une stratégie rarement envisagée et à laquelle on tend à préférer le recrutement de personnes depuis l'étranger et/ou de travailleuses et travailleurs plus jeunes (Trageser et al., 2012). Par ailleurs, même si des actions ponctuelles pour encourager le maintien en activité des 58 ans et plus ont été mises en place, la plupart des entreprises interrogées n'ont pas encore de politique d'encouragement systématique de l'emploi des travailleuses et travailleurs âgé.e.s de 58 ans et plus.

Concrètement, comment faire pour favoriser la participation au marché du travail des travailleuses et travailleurs âgé.e.s? La littérature issue de la psychologie du travail et des ressources humaines (RH) offre également plusieurs pistes. Un exemple de pratique RH serait de former les managers et responsables en ressources humaines à la reconnaissance de leurs propres stéréotypes liés à l'âge. Une étude allemande a démontré l'utilité d'une telle formation pour les cadres dirigeant des équipes avec une forte diversité d'âge, en diminuant les stéréotypes liés à l'âge plus avancé et les conflits, et en augmentant l'innovation dans ces équipes de travail (Wegge et al., 2012). Toutefois, la recherche a également montré que l'implémentation de pratiques $\mathrm{RH}$ centralisées sur les seniors peut avoir un effet contre-productif, ces derniers se sentant discriminés. En conséquence, Oliveira (2018) recommande des pratiques qui insistent sur les apports positifs des différents groupes d'âge pour le développement d'une identité positive dans l'entreprise, et un accès aux formations et autres opportunités (par exemple pour les promotions) pour tous les groupes d'âge. 
Au niveau sociétal finalement, le Conseil fédéral a souligné l'importance de travailler en priorité sur trois axes: (1) la réinsertion des travailleuses et travailleurs âgé.e.s; (2) le maintien et le développement de l'aptitude au travail ainsi que de la motivation à poursuivre une carrière professionnelle; (3) l'adaptation de la législation relative à la prévoyance sociale (par exemple, la possibilité de percevoir une rente AVS partielle) (SECO, 2016; Trageser et al., 2012). Les entreprises ont donc un rôle fondamental à jouer concernant les deux premiers éléments, notamment en proposant de bonnes conditions de travail (par exemple en termes de flexibilité des horaires et de possibilité d'adapter ou de développer des nouvelles compétences, et en valorisant les compétences des employé.e.s) (Trageser et al., 2012). Enfin, le système législatif suisse a décidé de plusieurs mesures institutionnelles pour renforcer l'activité professionnelle chez les seniors. Ces mesures incluent, d'abord, la possibilité de continuer à contribuer au premier pilier (AVS) au-delà de l'âge officiel de la retraite (64 ans pour les femmes, 65 ans pour les hommes), à savoir jusqu'à 69 ans pour les femmes, et 70 ans pour les hommes. Ensuite, des avantages financiers supplémentaires concernant le deuxième pilier en cas de retardement de l'âge de la retraite et des pénalités en cas de retraite anticipée (Madero-Cabib et al., 2015).

\subsection{RÉFLEXIONS SUR L'AVENIR DU MONDE DU TRAVAIL}

Parallèlement, à cause notamment des changements au niveau économique et social, le monde du travail a connu de profondes transformations au cours des dernières décennies. C'est dans ce contexte en pleine évolution que les travailleuses et travailleurs âgé.e.s doivent gérer la suite de leur carrière et penser à réaliser le passage à la retraite.

\subsubsection{Le chômage après 50 ans}

Depuis le début des années 2000, le taux de chômage au sens du Bureau international du travail (BIT) chez les 50-64 ans est inférieur à celui des autres groupes d'âge (SECO, 2016; SECO, 2017). À titre d'exemple, en 2016, le taux de chômage des 50-64 ans était d'environ 3,6\%, alors que celui des 15-24 ans et des 25-49 ans était respectivement de 7,8\% et de 4,7\% (OFS, 2017a). Il faut cependant relativiser en partie ce constat, car chez les 50 ans et plus, de nombreuses personnes doivent quitter leur emploi pour 
des raisons de santé, ou doivent prendre une retraite anticipée à la suite de la perte d'un emploi (souvent involontaire) et ne vont donc pas compter parmi les chômeuses et chômeurs (OFS, 2008). En même temps, parmi les 25-49 ans existe une partie non négligeable de chômage frictionnel (donc, dû au temps nécessaire pour [re] trouver un emploi après une période d'inactivité volontaire ou involontaire) liée aux personnes - notamment des femmes - qui désirent réintégrer le marché du travail à la suite d'une période dédiée aux responsabilités familiales (OFS, 2008).

Bien que les travailleuses et travailleurs âgé.e.s semblent moins souvent touché.e.s par le chômage que les jeunes, ils et elles sont davantage concerné.e.s par le chômage de longue durée (c'est-àdire pendant une année ou plus; Marmora \& Ritter, 2015; SECO, 2017). En effet, en comparaison aux autres groupes d'âge, les demandeuses et demandeurs d'emploi de 50 ans et plus ont davantage de difficulté à (re) trouver un emploi et passent plus de temps à la recherche d'une nouvelle occupation (Moser, Egger, \& Thom, 2008; SECO, 2017). En 2016, en Suisse, chez les 55-64 ans, la part des chômeuses et chômeurs de longue durée était de $56 \%$, alors que chez les 25-39 ans et les 40-54 ans, elle était respectivement de $37 \%$ et $47 \%$. D'une part, comme indiqué plus haut dans ce chapitre, les employeurs et employeuses sont parfois réticent.e.s à engager des personnes âgées de plus de 50 ans et leur préferent des travailleuses et travailleurs plus jeunes. Parmi les principales raisons, on retrouve, par exemple, les charges sociales en général plus élevées (c'est-à-dire la contribution au deuxième pilier) ou encore l'idée que les qualifications (et par conséquent les compétences) ne sont plus suffisamment actuelles (SECO, 2017). D'autre part, parmi les 50-64 ans sans emploi, seulement $21,5 \%$ seraient d'accord d'aller vivre ailleurs (donc de déménager) pour un nouvel emploi, alors que c'est le cas d'environ 30\% des 25-49 ans. Enfin, on remarque également que parmi les personnes âgées de 50 ans et plus, ce sont celles et ceux qui exercent une fonction non qualifiée et les femmes qui rencontrent le plus de difficultés à retrouver une place de travail (Moser, Egger, \& Thom, 2008).

Une des principales conséquences du chômage à long terme est représentée par le nombre de personnes qui se voient obligées (ou poussées) par la situation à prendre une retraite anticipée, ce qui n'était pas nécessairement leur intention à ce moment de leur vie. Tout comme pour les autres groupes d'âge, la littérature actuelle 
montre que le chômage - et notamment le chômage de longue durée - a un effet négatif sur le bien-être et la santé des seniors, en augmentant la fréquence des symptômes dépressifs (Brugiavini, Croda, \& Dewey, 2008) et de la consommation d'alcool (Gallo, Bradley, Siegel, \& Kasl, 2000). Cette dégradation de l'état de santé et du bien-être est à son tour associée à une difficulté accrue à retrouver un emploi, augmentant ainsi la probabilité de prendre une retraite anticipée (Brugiavini et al., 2008; Marmora \& Ritter, 2015).

\subsection{2 Âgisme au travail}

L'âgisme - à savoir les stéréotypes et les discriminations systématiques à l'encontre de la personne âgée (Butler, 1989) - est un phénomène malheureusement très répandu dans la société actuelle et encore trop souvent ignoré ou nié. Le domaine professionnel n'est malencontreusement pas épargné par ce phénomène. En effet, malgré une attention grandissante et l'existence dans plusieurs pays d'outils législatifs spécifiques (par exemple, l'Employment Act de 1967 aux États-Unis, qui punit toute discrimination envers les employé.e.s âgé.e.s de 40 ans et plus), les travailleuses et travailleurs âgé.e.s restent malgré tout victimes d'âgisme sur le lieu de travail, tant au niveau interpersonnel qu'organisationnel (Swift et al., 2017). En effet, une enquête menée en 2006 dans 28 pays dont la Suisse montre que l'âgisme - avec le sexisme - est la forme de discrimination la plus fréquente sur le lieu du travail (Kelly Services, 2006). On observe, par exemple, des situations âgistes au niveau des critères d'embauche ou de promotion, des opportunités de formation et des conditions favorables de travail, ou encore dans les attitudes au quotidien des supérieur.e.s et des collègues (Swift et al., 2017). Une série d'expériences menées en Suisse auprès de professionnelles et professionnels des ressources humaines et d'étudiant.e.s universitaires par Krings, Sczesny et Kluge (2011) indique que, de manière stéréotypée, d'une part, les candidat.e.s plus âgé.e.s sont considéréee.s comme étant plus chaleureux et chaleureuses mais moins compétent.e.s par les employeurs et, d'autre part, ces mêmes candidat.e.s sont moins souvent choisi.e.s même lorsque l'emploi requiert de la personne qu'elle soit chaleureuse.

Ces stéréotypes (par exemple, celui qui dépeint le-la travailleur ou travailleuse âgé.e comme étant moins engagé·e et motivé·e, avec une formation dépassée, plus limité.e physiquement, plus lent·e à la tâche ou encore moins performant.e et moins capable 
de s'adapter aux nouvelles exigences du monde du travail) sont particulièrement injustes, puisqu'ils ne sont pas soutenus par la littérature scientifique, qui montre au contraire une situation beaucoup plus nuancée et positive ( $\mathrm{Ng} \&$ Feldman, 2008). Plus spécifiquement, d'une part, les études montrent que la productivité générale ne décline pas avec l'âge, tandis que les collaboratrices et collaborateurs âgé.e.s aident davantage leurs collègues, sont moins souvent absent.e.s et en retard, et qu'ils et elles font preuve de moins de comportements agressifs, de comportements risqués (par exemple dans la manipulation de matériaux dangereux) et d'abus de substances sur le lieu de travail (par exemple Greller \& Simpson, 1999; McEvoy \& Cascio, 1989; Moser, Egger, \& Thom, 2008; Ng \& Feldman, 2008). D'autre part, les études centrées sur les attitudes professionnelles indiquent que, dans l'ensemble, les travailleuses et travailleurs âgé.e.s font davantage preuve d'attitudes professionnelles positives (et/ou de moins d'attitudes négatives) que les plus jeunes. Par exemple, la méta-analyse réalisée par $\mathrm{Ng}$ et Feldman (2010) met en évidence - entre autres - que les travailleuses et travailleurs âgé.e.s sont davantage satisfait.e.s avec leur emploi, rapportent un plus grand sentiment de contrôle au travail ainsi que plus de loyauté envers l'entreprise et d'identification avec l'organisation. De plus, par rapport aux personnes plus jeunes, elles et ils font également preuve de moins d'épuisement affectif et de conflits avec les collègues ( $\mathrm{Ng} \&$ Feldman, 2010). De manière générale, les études montrent que des différences peuvent apparaître avec l'âge, mais que celles-ci ne sont pas simplement la conséquence de l'avancement en âge, mais plutôt celle de l'interaction entre les caractéristiques personnelles de l'individu, de son parcours personnel et de l'environnement professionnel. Il est donc important de ne pas perdre de vue l'individualité de la personne et de ne pas considérer toutes et tous les travailleuses et travailleurs âgé.e.s de 50 ans et plus, dans les divers domaines professionnels, comme un seul groupe homogène ayant les mêmes ressources, les mêmes compétences et les mêmes difficultés.

\section{CONCLUSION}

En résumé, le mot "retraite», au sens littéral de "se retirer» de la société, ne semble donc plus correspondre à cette redéfinition. À cet égard, il est intéressant de s'interroger sur les différents termes 
utilisés dans d'autres langues (Froidevaux, 2014): le retirement anglais comporte la même idée de retrait qu'en français; le Ruhestand allemand renforce l'idée de calme; le pensionamento italien souligne le droit social à une rente. Le terme contrastant le plus avec le mot français est espagnol: la retraite devient jubilación, une forme de jouissance de la vie, davantage en accord avec le vécu des jeunes retraités issus de la génération du baby-boom. C'est pourquoi de nouveaux termes ont été proposés (Dennis \& Fike, 2012): "re-traiter" ou "re-création de soi", autrement dit considérer la retraite comme une invitation à une réflexion sur soi-même et sur comment vivre cette nouvelle étape de vie, en se renouvelant. Les psychologues du conseil et de l'orientation disposent de toutes les compétences pour accompagner cette réflexion, non seulement dans l'anticipation, mais aussi dans le vécu de cette transition.

\section{LIMITATIONS}

Il est également important de clarifier certaines limitations à la perspective adoptée dans ce chapitre. D'abord, nous nous sommes parfois limités au cas de la Suisse, qui fait partie des pays à culture de départ à la retraite tardive (late-exit cultures en anglais; Gould \& Saurama, 2004). À l'inverse, la France et la Belgique font partie des pays à culture de départ à la retraite de manière anticipée (early-exit cultures en anglais). En effet, le taux de participation au marché du travail chez les 55-64 ans est de 72,5\% en Suisse, de 52,2\% en France et de $49,7 \%$ en Belgique - la moyenne des pays de l'OCDE se situant à 60,8\% (OCDE, 2017).

Ensuite, nous nous sommes concentrés sur l'application et l'analyse de l'outil des formes identitaires subjectives développé par Jean Guichard, or il ne s'agit que d'un exemple parmi de multiples autres possibles. Par exemple, Bernard et Treille (2008) proposent un exercice créatif autour du projet de retraite où il s'agit pour la personne de dessiner, sculpter ou faire des collages de sa retraite idéale, des lieux de résidence et d'activités et des personnes les plus importantes. Une alternative serait le génogramme de carrière (Okiishi, 1987) pour une réflexion autour des modèles familiaux concernant la retraite. 


\section{RÉFÉRENCES BIBLIOGRAPHIQUES}

Alcover, C.-M. (2017). Bridge employment: Transitions from career employment to retirement and beyond. In E. Parry \& J. McCarthy (éds), The Palgrave Handbook of Age Diversity and Work (pp. 225262). Londres: Springer.

Bernard, S., \& Treille, L. (2008). Enfin la retraite! Genève: Jouvence.

Blanché, A. (2014). La retraite, une nouvelle vie: Une odyssée personnelle et collective. Paris: Odile Jacob.

Brugiavini, A., Croda, E., \& Dewey, M. (2008). Retirement and mental health. In A. Börsch-Supan, A. Brugiavini, H. JÜrges, A. Kapteyn, J. Mackenbach, J. Siegrist, \& G. Weber (éds), First Results from the Survey of Health, Ageing, and Retirement in Europe (2004-2007): Starting the longitudinal dimension (pp. 247-254). Mannheim: Mannheim Research Institute for the Economics of Aging. En ligne: [http://www.share-project.org/fileadmin/pdf_ documentation/FRB2/FRB2_all_chapters.pdf].

Butler, R. N. (1989). Dispelling ageism: The cross-cutting intervention. The Annals of the American Academy of Political and Social Science, 503, 138-147. Doi : 10.1177/0002716289503001011.

Cahill, K. E., Giandrea, M. D., \& Quinn, J. F. (2015). Retirement patterns and the macroeconomy, 1992-2010: The prevalence and determinants of bridge jobs, phased retirement, and reentry among three recent cohorts of older Americans. The Gerontologist, 55, 384-403. Doi: 10.1093/geront/gnt146.

De Vaus, D., Wells, Y., Kendig, H., \& Quine, S. (2007). Does gradual retirement have better outcomes than abrupt retirement? Results from an Australian panel study. Ageing and Society, 27, 667-682. Doi: $10.1017 /$ S0144686X07006228.

De Vos, A., \& van der Heijden, B. (2015). Handbook of research on sustainable careers. Cheltenham (UK)/Northampton (MA): Edward Elgar Publishing.

Dennis, H., \& Fike, K. T. (2012). Retirement planning: New context, process, language, and players. In J. W. HedGe \& W. C. Borman (éds), The Oxford Handbook of Work and Aging (pp. 538548). New York: Oxford University Press. 
Faurie, I., Fraccaroli, F., \& Le Blanc, A. (2008). Âge et travail: Des études sur le vieillissement au travail à une approche psychosociale de la fin de la carrière professionnelle. Le Travail Humain, 2, 137-172. Doi: 10.3917/th.712.0137.

Freedman, M. (2007). Encore: Finding work that matters in the second half of life. New York: Public Affairs.

[Froidevaux-] Rosselet, A., \& Hirschi, A. (2012). La fin de la vie professionnelle: Une nouvelle étape à accompagner. Panorama, 3, 22-23.

Froidevaux, A. (2014). Accompagner la construction d'une nouvelle identité. Panorama, 3, 20-21.

Froidevaux, A. (2016). Adjusting successfully to retirement: Qualitative and quantitative investigations on identity, meaning and mattering. Thèse de doctorat en psychologie, non publiée, Université de Lausanne.

Froidevaux, A. (2018). A life design perspective on the work to retirement transition. In V. Cohen-Scali, J. Rossier, \& L. Nota (éds), New Perspectives on Career Counseling and Guidance in Europe (pp. 89-104). Cham (Suisse) : Springer.

Froidevaux, A., Baumann, I., Maggiori, C., Wieber, F., \& Rossier, J. (2017). Retirement planning: How to deal with different adjustment trajectories? In B. J. ERTELT \& M. ScharpF (éds), Berufliche Beratung für Ältere [Career Guidance for Older People] (pp. 25-53). Francfort: Peter Lang.

Froidevaux, A., \& Hirschi, A. (2015). Managing the transition to retirement: From meaningful work to meaning in life at retirement. In A. De Vos \& B. VAN DER Heijden (éds), Handbook of Research on Sustainable Careers (pp. 350-363). Cheltenham (UK)/ Northampton (MA) : Edward Elgar Publishing.

Gallo, W. T., Bradley, E. H., Siegel, M., \& Kasl, S. V. (2000). Health effects of involuntary job loss among older workers. Findings from the health and retirement survey. The Journals of Gerontology: Series B, 55, 131-140. Doi : 10.1093/geronb/55.3.S131.

Gould, R., \& Saurama, L. (2004). From early exit culture to the policy of active ageing - the case of Finland. In T. Maltby, B. De Vroom, M. L. Mirabile, \& E. Øverbye (éds), Ageing and the Transition to Retirement. A comparative Analysis of European Welfare States. Aldershot / New York: Routledge. 
Greenhaus, J. H., Callanan, G. A., \& Godshalk, V. M. (2009). The middle and late career stages. In J. H. Greenhaus, G. A. CaLLanan, \& V. M. GodshalK (éds), Career Management (pp. 230 261). Los Angeles: Sage Publications.

Greller, M. M., \& Simpson, P. (1999). In search of late career: A review of contemporary social science research applicable to the understanding of late career. Human Resource Management Review, 9, 309-347. Doi : 10.1016/S1053-4822 (99) 00023-6.

Guichard, J. (2004). Se faire soi [Making oneself self]. L'Orientation scolaire et professionnelle, 33, 499-533. Doi : 10.4000/osp.226.

Hall, D. T., \& Mirvis, P. H. (2013). Redefining work, work identity, and career sucess. In D. L. Buustein (éd.), The Oxford Handbook of the Psychology of Working (pp. 203-217). New York: Oxford University Press.

Jex, S. M., \& Grosch, J. (2013). Retirement decision making. In M. Wang (éd.), The Oxford Handbook of Retirement (pp. 267279). New York: Oxford University Press.

Kelly Services (2006). Discrimination emerging in new forms in the global jobs market. En ligne (site du Business and Human Rights Resource Center): [http://www.reports-and-materials.org/Kellydiscrimination-survey-2006.pdf].

Kohli, R., Bläuer Herrmann, A., \& Babel, J. (2006). Les scénarios de l'évolution de la population de la Suisse - 2005-2050. Neuchâtel: Office fédéral de la statistique.

Krings, F., Sczesny, S., \& Kluge, A. (2013). Stereotypical inferences as mediators of age discrimination: The role of competence and warmth. British Journal of Management, 22, 187-201. Doi: 10.1111/j.1467-8551.2010.00721.x.

Limoges, J. (2008). Pour un troisième tiers de carrière porteur de vie: Vademecum (2 ${ }^{\mathrm{e}}$ édition). Sherbrooke: G.G.C. Itée.

Madero-Cabib, I., Gauthier, J.-A., \& Le Goff, J.-M. (2015). The influence of linterlocked employment-family trajectories on retirement timing. Work, Aging and Retirement, 2, 38-53. Doi : 10.1093/ workar/wav023.

Madero-Cabib, I., \& Kaeser, L. (2016). How voluntary is the active ageing life? A life-course study on the determinants of extending 
careers. European Journal of Ageing, 13, 25-37. Doi: 10.1007/ s10433-015-0355-y.

Marmora, P., \& Ritter, M. (2015). Unemployment and the retirement decisions of older workers. Journal of Labor Research, 36, 274-290. Doi : 10.1007/s12122-015-9207-y.

McEvoy, G. M., \& CAScio, W. F. (1989). Cumulative evidence of the relationship between employee age and job performance. Journal of Applied Psychology, 74, 11-17. Doi :10.1037/0021-9010.74.1.11.

NeWMan, K. L. (2011). Sustainable careers: Lifecycle engagement in work. Organizational Dynamics, 40, 136-143. Doi: 10.1016/j. orgdyn.2011.01.008.

NG, T. W. H, \& Feldman, D. C. (2008). The relationship of age to ten dimensions of job performance. Journal of Applied Psychology, 93, 392-423. Doi : 10.1037/0021-9010.93.2.392.

NG, T. W. H, \& Feldman, D. C. (2010). The relationship of age with job attitudes: A meta-analysis. Personnel Psychology, 63, 677718. Doi : 10.1111/j.1744-6570.2010.01184.x.

OCDE (2014). Vieillissement et politiques de l'emploi: Suisse 2014: Mieux travailler avec l'âge. En ligne: http://www.oecd-ilibrary.org/ social-issues-migration-health/vieillissement-et-politique-de-l-emploi-suisse-2014_9789264222823-fr.

OCDE (2017). Employment rate by age group: 55-64 years-old, Q3 2017. En ligne: [https://data.oecd.org/emp/employment-rate-byage-group.htm].

Office fédéral de la statistique (OFS) (2008). L'activité professionnelle des personnes de 50 ans et plus. Une étude basée sur les résultats de l'enquête suisse sur la population active et de l'enquête sur la structure des salaires. En ligne: [https://www.bfs.admin.ch/bfs/fr/home/ statistiques/catalogues-banques-donnees/publications.assetdetail.344116.html].

Office fédéral de la statistique (OFS) (2016). Activité professionnelle et temps de travail: Données détaillées. En ligne: [http://www.bfs.admin. $\mathrm{ch} / \mathrm{bfs} /$ portal/fr/index/themen/03/02/blank/data/03.html].

Office fédéral de la statistique (OFS) (2017a). La population de la Suisse 2016. En ligne: [https://www.bfs.admin.ch/bfs/fr/home/ actualites/quoi-de-neuf.gnpdetail.2017-0586.html]. 
Office fédéral de la statistique (OFS) (2017b). L'ESPA en bref 2016 - L'enquête suisse sur la population active. En ligne: [https://www.bfs.admin.ch/bfs/fr/home/statistiques/catalogues-banques-donnees/publications.assetdetail.3079804.html].

Office fédéral de la statistique (OFS) (2017c). Rapport de dépendance des personnes âgées. En ligne: [https://www.bfs.admin.ch/bfs/ fr/home/statistiques/themes-transversaux/ mesure-bien-etre/indicateurs/rapport-personnes-agees.html].

Office fédéral de statistique (OFS) (2018). Prévoyance vieillesse 2020. En ligne: [https://www.bsv.admin.ch/bsv/fr/home/assurances-sociales/ahv/reformes-et-revisions/altersvorsorge2020.html].

Office fédéral des assurances sociales (OFAS) (2016). Départ à la retraite et démographie: Une étude révèle une tendance et une disposition à travailler plus longtemps. En ligne: [https://www.admin.ch/ gov/fr/accueil/documentation/communiques.msg-id-46218.html].

OkishI, R. W. (1987). The genogram as a tool in career counseling. Journal of Counseling \& Development, 66, 139-143. Doi: 10.1002/j.1556-6676.1987.tb00820.x.

Oliveira, E. (2018). Buffers or boosters? Human resource management practices shape the way older workers see themselves in the workplace. En ligne: [http://agingandwork.bc.edu/blog/buffersor-boosters-human-resource-management-practices-shape-theway-older-workers-see-themselves-in-the-workplace/].

PAUL, K. I., \& Moser, K. (2009). Unemployment impairs mental health: Meta-analyses. Journal of Vocational Behavior, 74, 264-282. Doi: $10.1016 /$ j.jvb.2009.01.001

PinquarT, M., \& SCHIndLer, I. (2007). Changes of life satisfaction in the transition to retirement: A latent-class approach. Psychology and Aging, 22, 442-455. Doi: 10.1037/08827974.22.3.442.

Rauer, A., \& Jensen, J. F. (2016). These happy golden years? The role of retirement in marital quality. In J. BoOKWALA (éd.), Couple Relationships in the Middle and Later Years: Their Nature, Complexity, and Role in Health and Illness (pp. 157-176). Washington: American Psychological Association.

Savickas, M. L., Nota, L., Rossier, J., Dauwalder, J.-P., Duarte, M. E., Guichard, J., van Vianen, A. E. (2009). Life designing: 
A paradigm for career construction in the 21st century. Journal of Vocational Behavior, 75, 239-250. Doi : 10.1016/j.jvb.2009.04.004.

SCHLOSSBERG, N. K. (2003). Retire smart, retire happy: Finding your true path in life. Washington: American Psychological Association.

Secrétariat d'État à l'économie (SECO) (2016). L'évolution démographique et ses conséquences - Observations concernant l'état de la population active jusqu'en 2004 et ses évolutions possibles d'ici 2050. En ligne: [https://www.seco.admin.ch/seco/fr/home/ Arbeit/Arbeitsbedingungen/Arbeitnehmerschutz/Aeltere-Arbeitnehmende/Betriebliches-Altersmanagement/DemografischerWandel-und-seine-Folgen.html].

Secrétariat d'État à l'économie (SECO) (2017). Indicateurs de la situation des travailleuses et travailleurs âgés sur le marché suisse du travail - Documents de base pour la conférence nationale du 25 avril 2017. En ligne: [https://www.newsd.admin.ch/newsd/ message/attachments/48023.pdf].

ShulTz, K. S. (2003). Bridge employment: Work after retirement. In G. A. Adams \& T. A. Beehr (éds), Retirement: Reasons, processes, and results (pp. 214-241). New York: Springer.

Shultz, K. S., \& Adams, G. A. (2007). Aging and work in the 21st century. Mahwah: Lawrence Erlbaum Associates.

Swift, H. J., Abrams, D., Lamont, R. A., \& Drury, L. (2017). The risks of ageism model: How ageism and negative attitudes toward age can be a barrier to active aging. Social Issues and Policy Review, 11, 195-231. Doi : 10.1111/sipr.12031.

Trageser, J., Hammer, S., \& Fliedner, J. (2012). Le départà la retraite dans le contexte de l'évolution démographique. En ligne: [https://www. news.admin.ch/message/index.html?lang=fr\&msg-id=46218].

van Solinge, H., \& Henkens, K. (2008). Adjustment to and satisfaction with retirement: Two of a kind? Psychology and Aging, 23, 422-434. Doi : 10.1037/0882-7974.23.2.422.

WAnG, M. (2007). Profiling retirees in the retirement transition and adjustment process: Examining the longitudinal change patterns of retirees' psychological well-being. Journal of Applied Psychology, 92, 455-474. Doi: 10.1037/0021-9010.92.2.455.

Wang, M., Henkens, K., \& van Solinge, H. (2011). Retirement 
adjustment: A review of theoretical and empirical advancements. American Psychologist, 66, 204-213. Doi: 10.1037/a0022414.

WANG, M., \& SHI, J. (2014). Psychological research on retirement. Annual Review of Psychology, 65, 209-233. Doi : 10.1146/annurevpsych-010213-115131.

Wegge, J., Jungmann, F., Liebermann, S., Shemla, M., Ries, B., Diestel, S., \& Schmidt, K.-H. (2012). What makes age diverse teams effective? Results from a six-year research program. Work: A Journal of Prevention, Assessment and Rehabilitation, 41, 5145-5151. Doi: 10.3233/WOR-2012-0084-5145. 

DEUXIÈME PARTIE

ORIENTATION ET VULNÉRABILITÉS 



\title{
5. L'INSERTION PROFESSIONNELLE DES JEUNES ADULTES NI EN EMPLOI, NI EN FORMATION, NI EN STAGE (NEET) ${ }^{1}$
}

\author{
PHILIPPE HANDSCHIN (UNIVERSITÉ DE LAUSANNE) \\ ET MARCO SCHIAVETTA (UNIVERSITÉ DE GÊNES)
}

a crise économique de 2008 a induit une augmentation très importante du chômage des jeunes adultes dans les pays occidentaux. Alors que la situation avait évolué de manière favorable depuis le milieu des années 1990, le taux de chômage des jeunes entre 15 et 29 ans a augmenté de $6 \%$ entre 2008 et 2010 dans les pays membres de l'Organisation de coopération et de développement économiques (OCDE), contre 2,5\% pour les adultes (OCDE, 2010). Certains pays comme l'Espagne, la Suède ou la République slovaque affichaient des taux supérieurs à $25 \%$, alors que pour d'autres, tels que la Corée, l'Allemagne ou le Mexique, les chiffres étaient inférieurs à $10 \%$. Cette évolution est allée de pair avec une augmentation importante du nombre de jeunes adultes non actifs (Mawn et al., 2017; Zuccotti \& O'Reilly, 2018). Cette catégorie de jeunes adultes entre 15 et 29 ans a été désignée au Royaume-Uni dès les années 1990 par le terme de NEET, pour "Not in Employment, Education or Training" ("ni en emploi, ni en formation, ni en stage») (Eurofound, 2012; Furlong, 2006). En 2011, 12,9\% des jeunes entre 15 et 24 ans appartenaient à la catégorie NEET (Eurofound, 2012); elles·ils étaient 12\% en 2015 (Eurofound, 2016). L'OCDE (2018) estime qu'en 2016, $13,9 \%$ des 15-29 ans de ses pays membres entraient dans la catégorie NEET. Les disparités entre pays sont cependant considérables. Ainsi, en Europe, le Luxembourg affichait un taux de 7,6\%, alors que l'Italie comptait la proportion de NEET la plus élevée, avec 26,0\%. Parmi les pays européens francophones, la France comptait $17,2 \%$ de NEET, la Belgique $13,0 \%$ et la Suisse $8,7 \%$.

1. Les auteurs remercient Cecilia Toscanelli pour son aide pour la traduction de l'italien au français de la contribution de Marco Schiavetta. La contribution de Philippe Handschin a bénéficié du soutien du Fonds national suisse de la recherche scientifique (n 26041365). 
Si les pays qui connaissent un taux de chômage élevé ont souvent une proportion de NEET également élevée (Tableau 1), les différences internationales ne peuvent être uniquement expliquées par l'état du marché de l'emploi, mais dépendent également de facteurs socio-économiques plus larges. Par exemple, au sein des États européens qui présentent les taux de NEET les plus élevés, les pays du sud-ouest, l'Irlande et les pays baltes sont caractérisés par des proportions d'hommes et de diplômé.e.s du supérieur plus élevées que les pays de l'Est, où les femmes et les personnes peu qualifiées sont davantage touchées par le phénomène (Eurofound, 2012).

\section{Tableau 1. Taux de NEET (15-29 ans) et taux de chômage dans la population générale pour différents pays de I'OCDE. Données issues d'OCDE (2018a, 2018b).}

\begin{tabular}{l|c|c} 
PAYS & TAUX DE NEET & TAUX DE CHÔMAGE GLOBAL \\
\hline Islande & 5,3 & 3,0 \\
\hline Luxembourg & 7,6 & 6,3 \\
\hline Pays-Bas & 7,8 & 5,7 \\
\hline Suède & 8,2 & 7,0 \\
\hline Danemark & 8,2 & 6,2 \\
\hline Suisse & 8,7 & 4,9 \\
\hline Norvège & 9,4 & 4,7 \\
\hline Allemagne & 9,6 & 4,1 \\
\hline Autriche & 10,9 & 6,0 \\
\hline Nouvelle-Zélande & 11,3 & 5,1 \\
\hline Lituanie & 11,4 & 7,9 \\
\hline Australie & 11,4 & 5,7 \\
\hline Slovénie & 11,6 & 8,0 \\
\hline République tchèque & 11,6 & 4,0 \\
\hline Belgique & 13,0 & 7,8 \\
\hline Finlande & 13,2 & 8,8 \\
\hline Canada & 13,2 & 7,0 \\
\hline Royaume-Uni & 13,2 & 4,8 \\
\hline Israël & 13,8 & 4,8 \\
\hline Russie & 14,1 & 5,5 \\
\hline États-Unis & 14,1 & 4,9 \\
\hline Lettonie & 14,4 & 9,6 \\
\hline Estonie & 15,1 & 6,8 \\
\hline Pologne & & \\
\hline & & \\
\hline
\end{tabular}




\begin{tabular}{l|c|c}
\hline Hongrie & 15,2 & 5,1 \\
\hline Portugal & 15,6 & 11,1 \\
\hline Slovaquie & 15,9 & 9,7 \\
\hline France & 17,2 & 10,1 \\
\hline Colombie & 21,2 & 8,8 \\
\hline Espagne & 21,7 & 19,7 \\
\hline Mexique & 21,8 & 3,9 \\
\hline Grèce & 23,5 & 23,5 \\
\hline Italie & 26,0 & 11,7 \\
\hline Turquie & 28,2 & 10,8
\end{tabular}

Note. On observe que, bien que plus le taux de chômage est élevé, plus la proportion de NEET l'est également, les différences entre pays ne peuvent être résumées à ce paramètre.

La catégorie NEET est utilisée comme indicateur statistique, notamment par l'UE, l'Australie ou le Japon, ainsi que par des organisations internationales comme l'OCDE ou l'Organisation internationale $\mathrm{du}$ travail. Elle est également employée comme catégorie de bénéficiaires cibles par certaines politiques sociales et programmes de lutte contre le chômage et de (ré) insertion sociale et professionnelle. Dans ce chapitre, nous proposons de décrire cette population de jeunes adultes et de présenter certaines interventions socioprofessionnelles qui leur sont destinées. Après avoir retracé les origines de l'indicateur NEET et décrit son usage dans les politiques européennes, nous examinerons deux exemples de programmes destinés aux NEET, celui de la Garantie Jeunes dans l'UE, notamment en Italie et en France, et celui du programme Forjad en Suisse. Ces exemples nous permettront de discuter du rôle de la psychologie du conseil et de l'orientation dans les dispositifs développés pour cette population caractérisée par une précarité et une vulnérabilité sociale plus importante.

\section{NI EN EMPLOI, NI EN FORMATION, NI EN STAGE: UNE NOUVELLE RÉALITÉ?}

Dans cette première partie, nous retracerons brièvement les origines du terme NEET et proposerons une brève analyse de ses fondements et de sa pertinence. Nous examinerons ensuite son importance grandissante dans les politiques publiques des pays occidentaux. 


\section{1 ÉMERGENCE DU CONCEPT DE NEET}

Le terme de NEET est apparu au Royaume-Uni avec la mise en œuvre du Social Security Act en 1986, dont l'une des conséquences a été de restreindre fortement l'accès aux prestations d'assurance chômage des jeunes adultes. À ce moment, le terme "Status ZerO" a d'abord été employé pour désigner des jeunes de 16 à 18 ans qui n'étaient ni en formation ni en emploi. Considéré comme porteur d'une connotation trop négative, l'acronyme NEET lui a été progressivement substitué. Il est à présent utilisé aussi bien comme un indicateur statistique que comme un concept de recherche et un outil intégré à différentes politiques sociales, non seulement dans l'UE, mais également dans d'autres pays comme la Suisse (Baggio et al., 2015), la Norvège (Holte, 2018) ou l'Australie (O'Dea et al., 2014; Rodwell et al., 2017). L'Enquête européenne sur les forces de travail calcule par exemple le taux de NEET dans un pays donné en divisant le nombre de jeunes ni en emploi, ni en stage et ni en formation par le nombre total de jeunes du même âge. Cet indicateur est décrit comme offrant une alternative à l'opposition usuelle entre personnes actives et inactives en rendant compte de manière générale des «jeunes qui n'accumulent pas de capital humain à travers des canaux formels comme la participation au marché du travail» (Eurofound, 2016, p. 28; traduction personnelle).

Le terme de NEET englobe cependant des situations et des parcours très divers (Eurofound, 2016) et, assurément, le fait qu'un·e jeune adulte ne soit pas en formation ou en emploi ne peut que caractériser un état, et non nous renseigner sur ses causes. En ce sens, un rapport de la Fondation européenne pour l'amélioration des conditions de vie et de travail (Eurofound, 2012) met en avant l'hétérogénéité des situations vécues par les NEET et distingue cinq catégories de personnes: 1 . les chômeuses et les chômeurs de courte ou longue durée; 2 . celles et ceux qui ne peuvent travailler ou étudier en raison de charges familiales ou de problèmes de santé; 3. celles et ceux qui sont découragés et ont arrêté de chercher un emploi (le terme anglais est disengaged); 4. celles et ceux qui sont en recherche d'emploi mais attendent de trouver un poste qui réponde à certaines attentes et aspirations spécifiques; et 5 . les volontaires qui sont engagés dans des circuits alternatifs (arts, apprentissage en autodidacte, musique, etc.). En 2016, un nouveau rapport propose une classification plus détaillée des situations de NEET sur la base de cinq variables de l'Enquête européenne 
sur les forces de travail (Eurofound, 2016) : recherche d'un emploi durant les quatre dernières semaines; raisons pour lesquelles la personne ne cherche pas un emploi; disponibilité à débuter un nouvel emploi dans les deux prochaines semaines; raisons pour lesquelles la personne n'est pas disponible pour débuter un nouvel emploi (e.g., maladies, responsabilités familiales); et durée du chômage. Sur la base des réponses à ces cinq variables, la nouvelle classification aboutit à sept catégories: les chômeuses et chômeurs de courte et longue durée, qui représentent deux catégories distinctes; les personnes indisponibles pour des raisons de santé ou à cause de responsabilités familiales (deux catégories); les travailleuses et travailleurs découragés et celles et ceux qui sont sur le point de débuter un emploi ou une formation. Le septième groupe est composé des personnes ne rentrant dans aucune de ces catégories et est donc, par conséquent, très hétérogène. Pour comparaison, cette dernière catégorie représentait, en 2013, 12,5\% des NEET, alors que les deux groupes les plus importants sont ceux des chômeuses et chômeurs de courte et de longue durée (respectivement 29,8 et 22\%). Pour la Fondation européenne pour l'amélioration des conditions de vie et de travail, les objectifs de ces distinctions entre catégories de situations sont notamment de permettre des comparaisons plus précises entre pays européens et de décrire le "phénomène» avec plus de détails (Eurofound, 2016).

\subsection{CARACTÉRISTIQUES ASSOCIÉES À LA SITUATION DE NEET}

Ainsi que nous venons de le voir, il est possible de faire apparaître, sur la base d'un petit nombre de variables, une hétérogénéité importante des situations chez les personnes répondant aux critères de NEET. Malgré cette diversité, certaines caractéristiques sont associées de façon générale à la situation de NEET. Notamment, un parcours scolaire marqué par des difficultés ou de mauvais résultats semble le prédicteur le plus important et serait donc un facteur de risque d'appartenir à la catégorie NEET (Bynner \& Parsons, 2002; Furlong, 2006). D'autres variables telles que le genre, le pays de résidence ou l'origine (Eurofound, 2012, 2016), la précarité sociale ou encore le lieu d'habitation (Bynner \& Parsons, 2002) sont associées à la situation de NEET. Guégnard, Giret, Joseph et Murdoch (2017) ont montré, dans le cadre de "l'Enquête génération» (Céreq), qu'un cursus scolaire difficile, des parents sans activité professionnelle, la lassitude de l'école, des problèmes de santé ou 
encore le défaut d'une formation à proximité prédisent significativement le fait de devenir NEET. Il faut noter que ces facteurs de risques socio-économiques tendent à se cumuler (Schoon, 2014) et à interagir (par exemple, l'effet des difficultés scolaires s'exprime différemment selon l'origine culturelle; Zuccotti \& O'Reilly, 2018).

Par ailleurs, la santé physique et mentale est également associée au statut de NEET. Nous savons qu'activité professionnelle et santé mentale sont étroitement liées (Blustein, 2008; Creed \& Reynolds, 2001 ; OCDE, 2015). On observe notamment une détérioration de celle-ci dès le début d'une période de chômage et une amélioration postérieurement à la reprise d'une activité (Paul \& Moser, 2009). De même, les adolescent.e.s qui terminent leur scolarité obligatoire et se retrouvent sans activité professionnelle montrent une dégradation de leur santé mentale, alors que celles et ceux qui réussissent à s'engager dans une activité professionnelle montrent une réduction de symptômes indiquant une détresse psychologique. Différentes recherches ont montré une association entre le statut de NEET et la santé mentale. Des études réalisées en Australie (O’Dea et al., 2014; Rodwell et al., 2017), au Canada (Henderson, Hawke, \& Chaim, 2017), en Suisse (Baggio et al., 2015) ou encore en France (Guégnard et al., 2017; Robert et al., 2017) ont montré que, comparés à des pairs employés ou engagés dans une formation, les NEET montrent davantage de symptômes dépressifs et, de façon plus générale, un risque plus élevé de souffrir d'un trouble mental. Néanmoins, la question de la causalité demeure. Bien que peu d'études se soient penchées sur le sens de cette relation, il semble que si un état de santé mentale fragile peut en partie prédire le statut de NEET, ce dernier peut également favoriser l'apparition ou l'aggravation de symptômes (Baggio et al., 2015; Goldman-Mellor et al., 2016); on serait donc en présence d'une causalité dynamique réciproque ou circulaire.

\subsection{UN PHÉNOMÈNE COMPLEXE}

Différentes remarques s'imposent à la lecture de ces constats. En premier lieu, il apparaît qu'un vaste ensemble de variables contextuelles et individuelles sont associées au statut de NEET, et ce dans des relations souvent complexes et réciproques. Afin de comprendre le phénomène NEET, tant au niveau collectif qu'individuel, il importe d'être critique et particulièrement vigilant quant aux explications simplistes et stigmatisantes qui peuvent résulter de la dimension politique du phénomène. Il nous faut ainsi 
souligner, à l'instar de Furlong (2006), que «la vulnérabilité n'est pas la simple conséquence d'un ensemble de déficits personnels» (p. 567, traduction personnelle). Il nous faut également préciser que l'indicateur NEET et les catégorisations qui en découlent, bien qu'utiles pour mieux appréhender la situation des NEET, se limitent à une vision catégorielle et statique du phénomène. Ainsi que Zuccotti et O'Reily (2018) le mentionnent, "à mesure que le concept [de NEET] est devenu de plus en plus employé, la population qu'il décrit est devenue plus diverse, incluant celles et ceux qui sont là par choix ou par contrainte» (p. 3, traduction personnelle). Ainsi, le terme de NEET, avant de désigner une catégorie de personnes, se réfere en réalité davantage à un statut, un état, ou plus simplement à un moment donné de la vie d'un individu entre 15 et 29 ans durant lequel il n'est pas en formation ou inséré dans le marché du travail.

Les catégories des NEET ont l'avantage de mettre l'accent sur la pluralité et l'individualisation des situations, mais ne permettent pas d'avoir une vision réellement holistique des parcours de ces jeunes. Comme le souligne Thompson (2011), "il est malheureux que l'hétérogénéité des NEET soit parfois présentée comme une découverte "empirique" plutôt que comme une conséquence logique de la définition» (p. 791, traduction personnelle). De plus, la situation d'un grand nombre de NEET semble fortement liée aux évolutions récentes de l'économie et du marché de l'emploi, caractérisée notamment par une tertiarisation de l'économie, où la primo-insertion apparaît de plus en plus précaire en ce qu'elle est souvent marquée par l'alternance entre périodes de stage, contrats à durée déterminée, chômage ou retour en formation. Par exemple, la Suisse, un pays où le modèle de la formation duale (apprentissage) est choisi par environ deux tiers des jeunes, connaît différents problèmes liés aux mutations du marché du travail (Perriard, 2005). Les formations de type apprentissage concernent en effet principalement le secteur secondaire, alors que l'on assiste parallèlement à une automatisation de celui-ci et à une tertiairisation du monde professionnel. Les attentes des jeunes ne sont pas toujours en lien avec l'offre de formation professionnelle, certains secteurs ayant de la difficulté à trouver des jeunes intéressés. D'autre part, l'offre en places d'apprentissage étant tributaire de l'économie, elle a été naturellement réduite à la suite de la crise financière. Le déséquilibre entre offre et demande qui en résulte défavorise ainsi les postulant.e.s à un apprentissage qui disposent d'un bagage scolaire 
plus faible, les patron-ne.s se montrant de plus en plus exigeant.e.s au moment d'embaucher un.e apprenti.e.

Plus globalement, on observe une dé-standardisation des parcours individuels dans nos structures sociales contemporaines, que l'on peut qualifier de liquides et moins structurantes (Nico, 2014). La situation des NEET illustre ces nouveaux parcours marqués par des phases d'alternance. Il devient ainsi probablement de plus en plus commun pour les jeunes d'intégrer la catégorie NEET à un ou plusieurs moments de leur primo-insertion et il est donc essentiel, notamment pour les psychologues conseillères et conseillers en orientation, de dépasser une approche statique et catégorielle s'ils visent à comprendre la singularité des parcours individuels, et d'éviter l'illusion d'uniformité d'une catégorie de la population qui, de par sa définition même, est extrêmement hétérogène. Si certaines caractéristiques telles qu'une scolarité difficile ou des problèmes de santé sont effectivement plus fréquentes chez les NEET, un grand nombre d'entre elles et eux ne présentent pas de difficultés particulières (Maguire, 2015). Il est ainsi indispensable pour les politiques d'aller au-delà de ces catégories pour s'interroger et intervenir sur les déterminants sociaux qui influencent l'insertion de ces jeunes, le risque de tomber dans une image prototypique et stéréotypée de ces derniers et dernières, sans remettre en question les structures politiques et éducatives dans lesquels leurs parcours évoluent, étant un risque bien réel (Holte, 2018). Enfin, il est tout aussi nécessaire pour les chercheuses et chercheurs en sciences sociales d'aborder les NEET non comme un concept théorique, mais comme un objet d'étude (Holte, 2018).

Que le terme NEET caractérise davantage une situation qu'une catégorie d'individus n'implique bien évidemment pas qu'on s'en désintéresse: même transitoire, cette situation comporte des risques réels de conséquences futures négatives (scarring effect) et de pérennisation, sans compter les coûts financiers et sociaux associés. Différents programmes ont ainsi été mis en œuvre dans l'UE et dans différents pays (Blustein, Connors-Kellgren, Olle, \& Diamonti, 2017; Maguire, 2015). Dans la section suivante, après avoir brièvement décrit quelques-uns de ces programmes, nous nous pencherons plus en détail sur deux exemples: celui de la Garantie Jeunes, proposée dans le cadre d'une politique européenne globale de lutte contre le chômage des jeunes, et celui du programme Forjad, en Suisse. 


\section{PRATIQUES ET INTERVENTIONS DE SOUTIEN}

\section{À L'INSERTION SOCIALE ET PROFESSIONNELLE}

Dans l'UE, le Fonds social européen (FSE) alloue des ressources importantes à des initiatives dans différents pays membres pour aider les adolescent.e.s et jeunes adultes à s'insérer dans la vie professionnelle. De nombreux projets soutenus par le FSE visent ainsi à doter les jeunes chômeuses et chômeurs des compétences et qualifications demandées sur le marché du travail actuel. Le manque de formation étant un facteur de risque de premier ordre quant aux difficultés d'insertion que connaissent les NEET (Bynner \& Parsons, 2002), les projets qui leur sont dédiés visent avant tout à leur fournir des compétences professionnelles pour un emploi stable, en commençant parfois par l'apprentissage même des démarches à effectuer pour trouver un emploi. Les NEET présentant un niveau scolaire faible et un manque de formation ont notamment des difficultés accrues à s'insérer professionnellement et nécessitent donc un accompagnement spécifique leur permettant de se fixer des objectifs réalistes. Parallèlement, le FSE fournit un financement substantiel pour la réforme structurelle et pour l'investissement à long terme et nécessaire dans l'avenir des jeunes et de l'économie (Commission européenne, 2016). De fait, l'ensemble des pays concernés par une augmentation du taux de NEET ont tenté de développer des politiques publiques pour combattre cette évolution et éviter que ces personnes ne finissent par dépendre durablement de l'aide sociale. Parmi les projets soutenus par le FSE, la Garantie Jeunes vise à permettre aux jeunes adultes de moins de 25 ans de bénéficier d'offres d'emploi de qualité, d'une formation continue, d'un apprentissage ou d'un stage dans les quatre mois suivant la fin de l'école ou le début du chômage. Nous discuterons plus en détail de ce programme dans la section suivante.

D'autres pays hors de l'UE ont également pris des mesures pour lutter contre le chômage des jeunes, dont certaines ont débuté avant la crise économique de 2008. Le Japon a ainsi mis en place des Jobs Cafés, dans lesquels les jeunes peuvent se présenter sans rendez-vous afin d'utiliser différentes ressources pour chercher un emploi et, si elles et ils le souhaitent, obtenir des services personnalisés sur rendez-vous (OCDE, 2017). De plus, les NEET jusqu’à 39 ans qui connaissent des obstacles importants à leur insertion professionnelle peuvent se rendre à l'un des 160 services régionaux qui proposent des prestations d'orientation, la possibilité de réaliser des expériences professionnelles ou encore d'améliorer leurs 
compétences interpersonnelles. Il est à noter que tant les Jobs Cafés que les services régionaux sont en interaction avec les services d'emploi. Enfin, les services sociaux offrent également des services spécialement dédiés aux NEET. En Australie, le Conseil des gouvernements australiens (Council of Australian Governements) a voté en 2009 le plan National Partnership on Youth Attainment and Transition. Celui-ci contenait, entre autres, le programme Youth Connections, destiné aux jeunes en risque de décrochage, qui n’ont pas achevé leur éducation secondaire ( $12^{\mathrm{e}}$ année) ou qui n’ont pas réussi à s'insérer dans le monde professionnel, ainsi qu'une série de mesures visant par exemple à obliger les moins de 21 ans bénéficiant d'aides sociales à compléter leur scolarité secondaire jusqu'à la $12^{\mathrm{e}}$ année (OCDE, 2016). Le National Partnership on Youth Attainment and Transition a été suivi en 2015 par la Youth Employment Strategy. En Suisse, le principal outil pour aider les adolescents.e.s et jeunes adultes à faire face à des difficultés dans leur insertion professionnelle est le système de "case management formation professionnelle». L'implémentation de ce système a été décidée en novembre 2006, soit avant la crise financière de 2008 (Office fédéral de la formation professionnelle et de la technologie [OFFT], 2008). L'objectif principal était alors d'augmenter la proportion des moins de 25 ans titulaires d'un diplôme du secondaire II de 90 à $95 \%{ }^{2}$. Ce projet a été initialement soutenu par la Confédération, mais son implémentation effective dépendait de chaque canton. Dans le canton de Vaud, le case management formation professionnelle intervient notamment dans les difficultés de transition entre la fin de l'école obligatoire et l'entrée en formation professionnelle (Canton de Vaud, s. d.). Les jeunes peuvent être adressés par les psychologues en orientation de leur école ou s'adresser eux-mêmes à un guichet régional de la transition. Ce dernier pourra les orienter vers trois types de solution:

- une mesure de transition d'une durée de six mois à un an durant laquelle elle ou il sera accompagné.e dans l'élaboration d'un projet professionnel, la découverte du monde du travail et un soutien scolaire;

- un suivi individuel de type coaching par un.e psychologue en orientation;

- un bilan d'orientation.

2. Les dernières analyses effectuées par l'Office fédéral de la statistique (2018) montrent qu'en $2015,90,9 \%$ des jeunes ont obtenu un diplôme du secondaire II avant 25 ans. 
Dans la suite de ce chapitre, après avoir discuté de la Garantie Jeunes, nous présenterons un exemple de programme développé par le canton de Vaud, le programme Forjad, destiné aux jeunes adultes bénéficiaires de l'aide sociale (revenu d'insertion) qui ne disposent pas d'un diplôme du secondaire II.

\subsection{L'EXEMPLE DE LA GARANTIE JEUNES}

La création et la mise en œuvre de la Garantie Jeunes découle de la recommandation de la Commission européenne d'avril 2013 (Recommandation du Conseil de l'Europe 2013/C, 2013), qui vise à lutter contre l'inactivité des jeunes et à faciliter leur entrée sur le marché du travail. L'objectif principal de ce programme d'insertion socioprofessionnelle est de permettre à ces jeunes de bénéficier d'une offre d'emploi valable, de poursuivre leurs études ou d'entamer une formation professionnelle, un stage ou un service civil, dans un délai de quatre mois après le début du chômage ou de la sortie du système de formation. La Garantie Jeunes bénéficie d'un appui logistique et d'un soutien financier de l'UE, principalement à travers l'Initiative pour l'emploi des jeunes. Créée en 2013 par le Conseil et le Parlement européen, elle assure un soutien financier à destination des NEET et des régions dont le taux de chômage des 15-24 ans était supérieur à $25 \%$ en 2012 (20\% dans certains cas). Sa dotation initiale était de 6,4 milliards d'euros et elle sera majorée de 2 milliards d'ici à 2020.

Les modalités précises de l'implémentation de la Garantie Jeunes dépendent de chaque pays. En Italie, par exemple, les jeunes NEET de 15 à 29 ans sont contactés dans un délai de soixante jours après l'inscription au programme par le guichet régional et conviés à un premier rendez-vous d'orientation. Un programme personnalisé d'insertion ou de réinsertion est alors défini, en tenant compte des caractéristiques personnelles de chaque bénéficiaire, sous la forme principale d'un accompagnement personnalisé. Les jeunes peuvent bénéficier de formations brèves, d'un accompagnement au travail, de stages, d'un service civil, d'un soutien à l'auto-entrepreneuriat, d'un soutien à la mobilité professionnelle ou d'un apprentissage à distance. En France, l'expérimentation de la Garantie Jeunes a été confiée aux missions locales (à savoir des structures établies sur l'ensemble du territoire et spécialisées dans la prise en charge des difficultés d'insertion professionnelle des jeunes), tout en s'appuyant sur des commissions à l'échelle départementale. L'accompagnement est réalisé par un 
binôme de conseillères et conseillers et débute, pour chaque cohorte de 10 à 20 jeunes, par une première période de six semaines d'ateliers collectifs, poursuivie par un accompagnement individuel.

Dans sa communication du 4 octobre 2016, la Commission européenne mettait en avant une diminution du taux de chômage de $3,4 \%$ trois ans après la création du programme, ainsi qu'une diminution du taux de NEET au sein de l'UE de 13 à $12 \%$. Depuis janvier 2014, 14 millions de jeunes ont participé à la Garantie Jeunes et participant.e.s et 9 millions de participant.e.s ont accepté une offre d'emploi, de stage ou de formation. Pour la Commission, la Garantie a en outre permis de mettre les préoccupations des NEET au centre des débats et a facilité des réformes structurelles dans les pays membres. Elle souligne néanmoins des disparités parfois importantes entre ceux-ci et la nécessité d'une meilleure coordination entre les différents acteurs, notamment entre éducateurs (ou sens large) et employeurs (la Commission encourage également le développement du système par apprentissage). Ce bilan intermédiaire, globalement positif, a été nuancé par une évaluation de la Cour des comptes européenne publiée en 2017: pour elle, les résultats et progrès encourageants apportés par la Garantie Jeunes ne sont "pas à la hauteur des attentes suscitées par le lancement de ce dispositif»(p. 7). Elle souligne par exemple que la baisse du nombre de NEET est due à une hausse du nombre de jeunes en formation et non en emploi, une baisse du taux de jeunes NEET au chomage, mais une stagnation du taux de NEET inactifs ainsi qu'une baisse du nombre de sorties du dispositif à l'issue positive entre 2014 et 2015. Elle fait en outre état d'importantes lacunes dans le repérage et le suivi des NEET, et signale la qualité insuffisante des données recueillies par les États pour le suivi des participant.e.s et l'évaluation des effets du programme.

Les points de divergence entre les appréciations respectives de la Commission et de la Cour des comptes semblent indiquer que l'évaluation de la Garantie Jeunes souffre non seulement d'un manque de recul, mais également de rigueur scientifique. En effet, distinguer à l'heure actuelle l'effet de la Garantie Jeunes des influences contextuelles socio-économiques plus larges paraît un exercice difficile. Ceci apparaît d'autant plus regrettable que, de par les options laissées aux pays membres dans l'implémentation du programme, les études et procédures d'évaluation rigoureuses pourraient bénéficier des comparaisons de méthodes et instruments à travers des contextes locaux, nationaux et transnationaux. De fait, 
nous rejoignons les recommandations de la Cour des comptes en soulignant l'opportunité qu'offre la mise en place de collaboration entre pays et régions (que celles-ci appartiennent à des pays différents ou non). D’une part, car les comparaisons évoquées précédemment représentent un outil précieux pour identifier l'efficacité de certaines pratiques en rapport à des contextes et publics donnés. D'autre part, car les réflexions critiques qui émergent des études comparatives permettent de mettre en relief des problématiques et besoins communs, et de fonder, voire organiser l'action politique sur des bases dépassant le cadre local ou national.

\subsection{L'EXEMPLE DU PROGRAMME FORJAD}

Le programme Forjad a été créé en 2006 sur mandat du Département de la santé et de l'action sociale du canton de Vaud (Suisse) face à une augmentation progressive du nombre de jeunes adultes (18-25 ans) bénéficiaires de l'aide sociale. Le but du programme Forjad est de permettre à ces jeunes adultes d'intégrer ou de réintégrer le marché du travail par le biais d'une formation. Parmi les trois étapes que comporte le programme, la première vise la préparation à l'entrée en formation. Lors de celle-ci, les jeunes adultes intègrent une mesure d'insertion sociale (MIS), c'est-à-dire une structure d'accompagnement à la définition et à l'élaboration d'un projet professionnel. Celui-ci est mis à l'épreuve de la réalité à travers des stages en entreprise. Parallèlement, les participant.e.s à la MIS sont accompagnés dans leurs démarches, telles que la rédaction d'un curriculum vitae ou la recherche d'une place d'apprentissage ou en école. La durée normale de participation à une MIS est de trois mois et une même personne peut participer successivement à plusieurs MIS. La deuxième étape débute dès l'entrée en formation, et durant toute la durée de celle-ci. La personne bénéficie alors d'un accompagnement fondé sur quatre piliers (Nunez, 2018) :

- professionnel (consistant principalement à créer un lien avec l'entreprise formatrice et/ou l'école afin d'assurer de bonnes conditions de formation et d'intervenir en cas de conflits);

- scolaire (suivi des résultats et difficultés, accompagnement individualisé);

- personnel (identification et résolution des problèmes entravant la réussite de la formation, tels que comportements inadéquats sur le lieu de travail, problèmes de santé physique ou mentale, etc.);

- social (soutien aux démarches administratives, à la demande de 
revenus complémentaires tels qu'indemnités ou allocations, et aux situations du quotidien telles que logement ou garde des enfants).

La personne se voit également allouer une bourse d'apprentissage, dont le but est de lui permettre (avant tout symboliquement) de ne plus dépendre de l'aide sociale. En cas d'échec ou d'arrêt de la formation, la personne a l'obligation de rembourser l'intégralité du montant de la bourse qui lui a été alloué. Enfin, la dernière étape débute à la fin de la formation et consiste au placement en emploi.

Depuis le début du programme, 1945 jeunes sont entrés en formation, avec un taux global de réussite de $65 \%$ (Centre d'orientation et de formation professionnelles, 2017). Si les succès du programme sont donc réels, il convient de souligner que ces chiffres indiquent qu'un tiers des participant.e.s ne terminent pas leur formation, ce qui implique alors pour elles et eux le remboursement du montant de la bourse. De nombreux jeunes adultes, dont la situation est en équilibre fragile et marquée par des difficultés financières, peuvent donc se retrouver non seulement au point de départ - c'est-à-dire sans formation -, mais avec une dette qui parfois s'ajoute à d'autres. D'autre part, l'égalité des chances, notamment quant au droit à essayer et à se tromper, n'est pas garantie: les jeunes bénéficiaires du programme Forjad doivent faire face à des contraintes financières qui rendent un éventuel changement d'orientation plus complexe que pour le reste des pairs de même âge, qui peuvent davantage se permettre d'abandonner une formation qui ne leur convient pas (Bonvin, Dif-Pradalier, \& Rosenstein, 2012). Il faut également préciser que près de $80 \%$ de l'ensemble des jeunes adultes de 18-25 à l'aide sociale ne disposent pas d'une formation du niveau secondaire II (lycée, apprentissage, etc.; Département de la santé et de l'action sociale du Canton de Vaud, 2017). Parallèlement, seul un quart d'entre eux sont "en mesure d'entrer dans un processus de formation professionnelle et motivés par cette perspective» (Bonvin et al., 2012, p.13). Les jeunes qui ne peuvent entrer dans le programme sont fréquemment entravés par des difficultés et obstacles importants (endettement, addictions, retards scolaires, précarité sociale, etc.). Des mesures dites «bas seuil» peuvent alors être intégrées, mais ne ciblent qu'indirectement l'insertion professionnelle. Enfin, si le programme Forjad est utile pour les jeunes qui n'ont pas réussi à réaliser la transition école-travail, on ne peut perdre de vue que différentes contraintes 
structurelles influencent aujourd'hui cette transition. Ainsi, Perriard (2005), dans une analyse des facteurs influençant la transition école-travail en Suisse, relève un déséquilibre entre l'offre et la demande de places d'apprentissage (une modalité de formation choisie par environ deux tiers des jeunes en 2003), la majorité de celles-ci concernant le secteur secondaire, largement influencé par l'automatisation et la mondialisation. Les employeuses et employeurs reçoivent donc de nombreuses candidatures pour des places d'apprentissage et peuvent dès lors se montrer plus exigeants quant au bagage scolaire nécessaire pour être embauché. Les jeunes les mieux formés ont donc davantage de chances d'être embauchés et tendent à occuper les "niches» qui étaient autrefois celles d'élèves moins favorisé.e.s. L'aptitude de l'école à préparer les jeunes à l'entrée dans le monde du travail est également pointée du doigt par les employeur.e.s. Cette préparation est rendue d'autant plus délicate que les évolutions rapides du monde du travail empêchent une définition claire des attentes et besoins en matière de savoirs et compétences nécessaires à l'insertion dans l'univers professionnel.

Les programmes d'insertion professionnelle sont donc essentiellement des solutions qui interviennent lorsque les problèmes ont déjà été posés. Dans un monde marqué par une instabilité structurelle et par les difficultés des politiques et institutions à s'y adapter et à se renouveler, la psychologie de l'orientation peut jouer un rôle important, à la fois en aidant les jeunes à s'orienter en leur fournissant des outils critiques pour le comprendre et y construire leur parcours (Blustein, Kenna, Gill, \& DeVoy, 2008), notamment grâce à des approches constructivistes et holistiques (Guichard, 2016; Patton \& McMahon, 2006), mais également en participant aux débats sociétaux et politiques autour des évolutions des mondes du travail et de la formation. Dans la troisième et dernière partie de ce chapitre, nous proposons des pistes de réflexion et d'intervention au sujet des difficultés d'insertion professionnelle, et notamment de la transition école-travail qui la conditionne.

\section{PERSPECTIVES}

En 2012, la Commission européenne estimait le coût associé aux NEET à 153 milliards d'euros pour les pays de l'UE, soit 1,2\% du PIB (Eurofound, 2012). Cette estimation exclut les coûts relatifs aux allocations de chômage, aux revenus non perçus, aux cotisations non 
versées ainsi qu'aux impôts non perçus. En outre, elle ne tient pas compte des effets sur la santé physique et mentale, sur le taux de criminalité ainsi que sur la cohésion sociale (Mawn et al., 2017). Enfin, il ne faut pas oublier que les jeunes les plus touchés sont ceux ayant un statut socio-économique et un niveau d'instruction et de compétences peu élevées. Aujourd'hui, l'édition 2017 du rapport annuel sur l'évolution de l'emploi et de la situation sociale en Europe (ESDE) confirme que le nombre des jeunes au chômage a diminué de 1,8 million depuis le pic de la crise en 2013, et le nombre de NEET d'un million (Commission européenne, 17 juillet 2017).

Cependant, les difficultés que rencontrent les NEET ne sont pas spécifiques aux jeunes générations: face aux mutations du monde du travail, l'orientation est devenue un processus qui n'est plus limité aux adolescents, mais qui se fait tout au long de la vie (Cedefop, 2011; Masdonati Zitoum, 2013). De plus, l'automatisation des processus de production, la levée des frontières et la compétition accrue qui en résulte ont généré la crise de la grande entreprise, la délocalisation des postes de travail et la nécessité accrue de structures de production plus flexibles. La nécessité de s'orienter tout au long de l'existence pose aussi la question de l'orientation permanente, de l'apprentissage permanent et de la reconnaissance des compétences formelles et informelles face à des scénarios sociaux encore incertains dus à la rapide transformation du monde du travail (Schiavetta, 2015).

La situation des NEET ne révèle pas uniquement l'existence d'obstacles dans le processus de transition de l'école au travail, elle met en lumière la pluralité et la diversité des parcours du monde actuel: la carrière est aujourd'hui non plus envisagée comme déterminée par un choix réalisé tôt dans la vie de l'individu, mais comme une série de transitions, d'adaptations et de décisions tout au long de l'existence, et ce dans une pluralité de contextes (Fouad \& Bynner, 2008). Pour la majorité des individus, il s'agit donc d'un état transitoire inséré dans une séquence faite d'autres moments tels que travail à temps partiel, contrats à durée déterminée, retour en formation ou réorientations (Bynner \& Parsons, 2002; Furlong, 2006; Holte, 2018). Néanmoins, des attentes croissantes d'auto-actualisation et de mobilisation de ressources individuelles aboutissent au fait que les inégalités sont redéfinies en termes de risques individuels et les problèmes sociaux en termes d'attributs et de dispositions psychologiques (Thompson, 2011). Le risque d'individualisation ou de psychologisation des NEET, perçus comme 
une catégorie discrète, peut avoir comme conséquence, ainsi que nous l'avons vu, de reléguer au second plan l'influence du contexte social, éducatif et professionnel sur les parcours individuels.

En outre, les situations des jeunes NEET peuvent être accompagnées par un ensemble de caractéristiques et facteurs de risque au niveau de la santé physique et mentale (par exemple, Baggio et al., 2015; Guégnard et al., 2017), de logement (Henderson et al., 2017) ou encore des difficultés familiales (Eurofound, 2012). Ces résultats rejoignent les recommandations de l'OCDE (2010) en plaidant en faveur de «mesures globales [pour les jeunes défavorisés] intégrant intervention précoce, aide à la recherche d'emploi, tutorat par des adultes, rattrapage scolaire, soutien professionnel et garantie de ressources» (p. 136). Plus spécialement, les interventions en orientation auprès de publics présentant des handicaps sociaux gagnent à ne pas séparer difficultés d'ordre professionnel et difficultés d'ordre socio-affectif, ces deux dimensions n'étant, dans la réalité, pas indépendantes (Blustein et al., 2008). Sans prise en compte des problèmes et facteurs de risque associés au statut NEET, il semble peu raisonnable de penser que les difficultés d'insertion professionnelle puissent être résolues de manière indépendante. En ce qui concerne le soutien psychologique en orientation, une démarche holistique, dans laquelle l'individu est vu au centre de différents systèmes s'influençant réciproquement, paraît particulièrement apte à répondre à ces difficultés (Schiavetta, 2015). L'approche systémique en orientation permet de tenir compte de trois systèmes - la personne, les établissements d'enseignement et le monde professionnel -, tout en tenant compte de manière plus générale du contexte social et culturel. L'intervention se fait au niveau du système plutôt qu'à celui de l'individu et se réalise à travers trois dimensions: celles de l'information, de l'éducation et du conseil (Guichard, 2016).

En effet, si les psychologues en orientation sont amenés, de par la spécificité de leur domaine d'intervention, à se centrer sur les problèmes de leurs client.e.s liés aux mondes du travail et de la formation, le cas des NEET plaide en faveur d'une exploration et d'une compréhension nécessaire des facteurs entourant les difficultés de transition école-travail de la personne. Pour Masdonati et Fournier (2015), quatre processus centraux influencent la transition de l'école au monde professionnel: l'école et le contexte du marché du travail, l'environnement relationnel de l'individu, sa construction 
identitaire et sa relation à l'école et au monde du travail. Ces quatre processus peuvent être utilement intégrés dans un cadre d'analyse lors d'interventions auprès de client.e.s NEET. Nous insisterons brièvement sur l'importance de la construction identitaire, et notamment de l'identité vocationnelle, tant leur rôle dans la primo-insertion semble fondamental. En effet, l'impossibilité pour les jeunes de construire une identité liée au travail positive, notamment quand ils font l'expérience du chômage directement ou à travers leurs proches et amis, peut les amener à séparer leur identité de leur futur professionnel (Koen, Van Vianen, Klehe, \& Zikic, 2016). Ainsi, Seddon, Hazenberg et Denny (2013), dans leurs analyses des effets d'un programme d'insertion destiné à des NEET, relèvent que nombre d'entre eux évoquent un manque de confiance en leurs capacités à cause de mauvaises performances à l'école, un manque qui débouche parfois sur une anxiété dans des situations sociales nouvelles. Leurs perspectives professionnelles sont parfois vagues, rigides ou irréalistes, et ont peu de rapport avec les capacités de la personne ou les opportunités disponibles dans l'environnement. À l'issue du programme, ils sont davantage à décrire des projets plus réalistes et à montrer une plus grande confiance en soi. De fait, l'émergence d'une identité liée au travail n'est pas nécessairement motivée par des intentions claires ou des soi possibles, mais peut au contraire émerger lors de la transition, parfois en rendant moins saillante leur ancienne identité (Koen et al., 2016). Les motivations (ou l'absence de motivations) au début du programme ne semble pas avoir eu d'influence sur leur succès futur de transition professionnelle. Pour les auteurs, il est ainsi important de ne pas évaluer la réussite d'une intervention uniquement par le placement en emploi ou en formation, mais également en termes de changements identitaires. L'identité, et notamment l'identité vocationnelle, est au cœur des parcours de transition et l'évaluation des soft outcomes est donc primordiale dans les programmes d'insertion et dans le travail des psychologues conseillers.ères en orientation. Elles et ils pourront ainsi favoriser la mobilisation et la création de ressources individuelles face à la transition en intégrant dans leurs évaluations les dimensions d'identité, de capital social et de capital identitaire (Fouad \& Bynner, 2008).

Dans cette optique d'accompagnement à la construction d'une identité vocationnelle positive, les psychologues conseillers pourront se fonder sur différentes approches constructivistes telles que le para- 
digme "concevoir et construire sa vie» (life designing; Nota \& Rossier, 2015; Savickas \& Pouyaud, 2016), le modèle de la construction de carrière (Savickas, 2005) ou encore le modèle "se faire soi» (Guichard, 2016). Ce dernier propose par exemple d'aider les client.e.s à prendre conscience des différentes dimensions de leur identité (définie plus précisément par Guichard comme un système de "formes identitaires subjectives»), à repérer comment celles-ci s'articulent entre elles et à définir les ressources à mobiliser afin de réaliser ce qu'ils souhaitent être. Ces interventions, en ce qu'elles «ne visent $[\ldots]$ pas à révéler quelque chose qui serait déjà là, mais à construire, à créer une certaine intention» (Guichard, 2016, p. 26), peuvent être pertinentes pour les NEET, dont les projets sont parfois mal définis ou irréalistes (Seddon et al., 2013) et dont l'identité professionnelle est peu construite (Koen et al., 2016). Elles gagnent enfin sans doute à être intégrées dans le cadre de la "psychologie de l'activité de travail " (psychology of working framework, développé par Blustein et al., 2008), centrée sur la situation des personnes marginalisées par rapport au marché du travail et connaissant différents obstacles dans leur accès à celui-ci.

En outre, comme le mentionne Del Core, les références éthiques et éducatives qui soutiennent les processus de prise de décision sont devenues plus vagues: "Dans une société définie comme «éthiquement neutre", qui ne fait désormais plus de choix éthiques ni ne les indique, qui insiste néanmoins sur le fait que le choix est personnel [...] puisqu'il n'y a pas de règle sociale commune, les jeunes ne sont pas aidés dans leurs choix et décisions. Au niveau de l'expérience psychologique individuelle, la peur $d u$ choix est donc largement amplifiée, ce qui peut se traduire soit par un ajournement continu des choix, soit par l'indifférence, jusqu'au refus d'effectuer des choix décisifs» (2007, p. 58; traduction personnelle). L'orientation permanente est un processus de connaissance de soi et d'autonomie qui favorise chez la personne la prise de décision et la prise de responsabilité face aux choix futurs. Il s'agit d'aider, quel que soit l'âge de la personne, à "apprendre » à être autonome dans son jugement, à développer une capacité critique et autocritique et à acquérir une capacité de décision proactive.

Le conseil en orientation n'est cependant qu'une des composantes de programmes tels que la Garantie Jeunes ou Forjad. L'évaluation rigoureuse de ces derniers demeure essentielle pour garantir et améliorer leur efficacité. Mawn et al. (2017) ont par exemple 
réalisé une méta-analyse de recherches évaluant les effets des programmes destinés aux personnes en situation de NEET. Elles et ils y soulignent différents problèmes statistiques et méthodologiques (notamment l'absence, dans de nombreuses études, de données nécessaires aux méta-analyses) et proposent cinq objectifs prioritaires dans l'évaluation de tels programmes:

1. déterminer les pratiques efficaces pour aider les jeunes à se réinsérer et, plus précisément,

2. comparer l'efficacité de ces pratiques en fonction du type de bénéficiaires du programme;

3. déterminer l'efficacité des composantes des programmes (par exemple, conseil en orientation, stages) et non pas seulement l'efficacité globale des programmes;

4. conduire davantage d'évaluations portant sur des interventions construites sur des fondements théoriques clairs et, finalement,

5. conduire davantage de recherches portant sur la santé physique et mentale des NEET.

Les auteurs notent également que les études portant sur l'évaluation de programmes de réinsertion devraient utiliser des méthodes plus rigoureuses, notamment en adhérant à des protocoles standardisés (par exemple Prisma). En effet, sur 18 études retenues, seules trois répondent aux critères nécessaires à l'inclusion dans une méta-analyse. Bien qu'elles aient montré une augmentation significative de quatre points sur le taux de chômage des participant.e.s, il est bien entendu difficile de se prononcer sur un nombre d'études si faible. Récemment, Kluve et al. (2016) ont conduit une méta-analyse de 87 programmes destinés aux jeunes (c'est-à-dire non restreints aux NEET) dans 31 pays et trouvent qu'environ un tiers des interventions montrent des résultats positifs. Il semble néanmoins qu'il n'existe pas un type de programme meilleur que les autres en termes de résultats, mais que le succès d'une intervention semble davantage lié à sa construction, son implémentation et son adéquation avec les objectifs recherchés. Cependant, les programmes qui incorporent différents types d'outils et d'interventions sont plus susceptibles de montrer des résultats positifs, possiblement car l'une des composantes au moins est susceptible de toucher un individu dans la diversité du groupe. Si ces résultats semblent encourageants, il existe un réel besoin de recherches qui tentent de saisir 
plus finement les processus et changements à l'œuvre en fonction du type d'intervention et de ses composantes.

\section{CONCLUSION}

De plus en plus employé, le terme NEET recouvre une pluralité de parcours et de vécus. Indicateur d'une situation généralement transitoire dans les parcours des individus, il est révélateur des difficultés actuelles d'accès aux marchés du travail et de la formation et de la complexité croissante des parcours de transition école-travail. Ainsi que nous l'avons évoqué, différents programmes d'insertion ont été proposés et différents outils peuvent être appliqués par les psychologues conseillers.ères pour aider les personnes en situation de NEET. Ainsi que nous venons de le souligner, il est nécessaire que ces programmes soient construits et évalués de façon plus rigoureuse.

Néanmoins, comme le soulignent Blustein et al. (2017), les politiques ciblant l'insertion ou la réinsertion professionnelle visent davantage à accroître l'employabilité des personnes qu’à augmenter le nombre d'emplois ou que, reformulé de façon plus générale, à agir sur les structures sociales et économiques dans lesquelles nous évoluons. Bien que les programmes d'insertion professionnelle soient des outils d'intervention importants, nous rejoignons les positions de ces auteur.e.s en soulignant qu'il est urgent de repenser et d'agir sur les structures qui rendent l'accès au travail et à la formation complexes pour un grand nombre de personnes, et que les interventions de réinsertion doivent mettre à la disposition des individus des outils d'auto-affirmation (empowerment) et de pensée critique. Les individus capables de comprendre les structures dans lesquels ils évoluent peuvent non seulement y naviguer plus facilement, mais surtout les questionner et agir sur elles, notamment par le biais des associations et syndicats, et ainsi contribuer à un monde professionnel davantage façonné par ses principaux protagonistes. 


\section{RÉFÉRENCES BIBLIOGRAPHIQUES}

Baggio, S., Iglesias, K., Deline, S., Studer, J., Henchoz, Y., Mohler-Kuo, M., \& Gmel, G. (2015). Not in education, employment, or training status among young Swiss men. Longitudinal associations with mental health and substance use. Journal of Adolescent Health, 2, 238-243. Doi : 10.1016/j.jadohealth.2014.09.006.

BlusteIn, D. L. (2008). The role of work in psychological health and well-being: A conceptual, historical, and public policy perspective. American Psychologist, 4, 228-240. Doi: 10.1037/0003066X.63.4.228.

Blustein, D. L., Connors-Kellgren, A., Olle, C. D., \& DiaMONTI, A. J. (2017). Promising career and workforce development programs and services in supporting the needs of unemployed populations. In V. S. H. SolberG \& S. R. Ali (éds), The handbook of career and workforce development: Research, practice and policy (pp. 97-123). New York: Routledge.

Blustein, D. L., Kenna, A. C., Gill, N., \& DeVoy, J. E. (2008). The psychology of working: A new framework for counseling practice and public policy. The Career Development Quarterly, 4, 294-308. Doi: 10.1002/j.2161-0045.2008.tb00095.x.

Bonvin, J.-M., Dif-Pradalier, M., \& Rosenstein, E. (2012). L'activation des "Jeunes Adultes en Difficulté»: Le cas du programme Forjad dans le canton de Vaud. Chroniques du Travail, 2, 3-21.

Bynner, J., \& Parsons, S. (2002). Social exclusion and the transition from school to work: The case of young people not in education, employment, or training (NEET). Journal of Vocational Behavior, 2, 289-309. Doi : 10.1006/jvbe.2001.1868.

Canton de Vaud (s. d.). Transition 1 - Portail Migration. En ligne: [https://www.vd.ch/themes/formation/orientation/formations/transition-1-portail-migration/].

Cedefop (2011). Lifelong guidance across Europe: Reviewing policy progress and future prospects. Luxembourg: Publications Office of the European Union.

Centre d'orientation et de formation professionnelles. (2017, octobre 4). Forjad-VD.CH. En ligne: [https:/www.vd.ch/etablissements-de-formation/centre-dorientation-et-de-formation-professionnelles/forjad/]. 
Commission européenne (2016). ESF Ex-post Evaluation Synthesis 2007-2013: EU synthesis report - final version. Luxembourg: Publications Office of the European Union.

Commission européenne (17 juillet 2017). Évolution de l'emploi et de la situation sociale en Europe: Le rapport 2017 montre des tendances positives, mais souligne la charge élevée qui pèse sur les jeunes. En ligne: [http://europa.eu/rapid/press-release_IP-17-1988_fr.htm].

Commission européenne (4 octobre 2016). Communication de la Commission au Parlement européen, au Conseil européen, au Conseil, au Comité économique et social européen et au comité des régions: La garantie pour la jeunesse et l'intiative pour l'emploi des jeunes, trois ans après. En ligne: [https://eur-lex.europa.eu/legal-content/ EN/TXT/?qid=1477901398883\&uri=CELEX:52016DC0646] (consulté le 24 octobre 2018).

Cour des comptes européenne (2017). Chômage des jeunes: les politiques de l'UE ont-elles changé le cours des choses? Évaluation de la garantie pour la jeunesse et de l'initiative pour l'emploi des jeunes accompagnée des réponses de la Commission (rapport spécial $n^{\circ}$ 5/2017). Luxembourg. En ligne: [https://www.eca.europa.eu/ fr/Pages/DocItem.aspx?did=41096] (consulté le 24 octobre 2018).

Creed, P. A., \& Reynolds, J. (2001). Economic deprivation, experiential deprivation and social loneliness in unemployed and employed youth. Journal of Community \& Applied Social Psycho$\log y$, 3, 167-178. Doi : 10.1002/casp.612.

Del Core, P. (2007). Atteggiamenti e stili decisionali degli adolescenti e dei giovani. Rivista di Scienze dell'Educazione, 1, 55-77. En ligne: [http://rivista.pfse-auxilium.org/it/riv_fascicolo2.cfm? fascicolorivista $=1$ \&annorivista $=2007 \& \mathrm{tab}=8]$.

Département de la santé et de l'action sociale du Canton de Vaud (2017). Rapport social vaudois 2017 (n d.). Lausanne. En ligne: [https://www.google.ch/search?source=hp\&ei=n2N oWvb9KMTDwAK3uJKYDQ\&q=rapport+social +vad ois $+2017 \&$ oq $=$ rapp \&gs_l=psy-ab.3.0.35i $39 \mathrm{k} 1 \mathrm{j} 0 \mathrm{i} 67 \mathrm{k} 1$ j018.8963.9530.0.10639.5.4.0.0.0.0.84.292.4.4.0...0...1.1.64. psy-ab..1.4.291.0..0i131k1j0i131i67k1.0.ky19k0M_NdU].

Escudero, V., \& López Mourelo, E. (2017). The European Youth Guarantee: A systematic review (Working paper $n^{\circ} 21$ ). Interna- 
tional Labour Office. En ligne: [https://www.google.ch/search? source $=$ hp\&ei $=x M-e$ WovNLo6XkwXdwKnYBA\&q=the+european +youth+guarante+a+systematic+review\&oq =the+european+youth + guarantee+a+systematic+re\&gs_l=psy-ab.3.0.33i2 $1 \mathrm{k} 1 \mathrm{j} 33 \mathrm{i} 160 \mathrm{k} 1$ $.1485 .13833 .0 .14961 .46 .34 .3 .8 .8 .0 .192 .2732 .26 j 8.34 .0 \ldots . . .$. 1c.1.64.psy-ab..1.43.2515.0..0j35i39k1j0i131k1j0i203k1j0i13k1j0i 22i30k1.0.qpBGhpRjI5w].

Eurofound (2012). NEET- Young people not in employment, education or training: Characteristics, costs and policy responses in Europe. Luxembourg: Publications Office of the European Union. Doi: 10.2806/41578.]

Eurofound (2016). Exploring the diversity of NEET. Luxembourg: Publications Office of the European Union. En ligne: [https://www.eurofound.europa.eu/fr/publications/report/2016/ labour-market-social-policies/exploring-the-diversity-of-neets].

European Comission (2017). Youth Guarantee country by country: France, March 2017. En ligne: [http://ec.europa.eu/social/main. jsp?catId=1161

European Commission (2017). Youth Guarantee country by country: Italy, March 2017. En ligne: [http://ec.europa.eu/social/main.jsp ?catId $=1161]$.

European Commission. (2017). Youth Guarantee country by country: United Kingdom, March 2017. En ligne: [http://ec. europa.eu/social/main.jsp?catId=1161].

Fouad, N. A., \& Bynner, J. (2008). Work transitions. American Psychologist, 4, 241-251. Doi : 10.1037/0003-066X.63.4.241.

Furlong, A. (2006). Not a very NEET solution: Representing problematic labour market transitions among early schoolleavers. Work, Employment and Society, 3, 553-569. Doi: $10.1177 / 0950017006067001$.

Goldman-Mellor, S., Caspi, A., Arseneault, L., Ajala, N., Ambler, A., Danese, A., Moffitt, T. E. (2016). Committed to work but vulnerable: Self-perceptions and mental health in NEET 18-year-olds from a contemporary British cohort. Journal of Child Psychology and Psychiatry, 2, 196-203. Doi : 10.1111/jcpp.12459.

Guégnard, C., Giret, J.-F., Joseph, O., \& Murdoch, J. (2017). Les situations de NEET dans les parcours d'insertion des jeunes 
en France. In J. Calmand, T. Couppié, \& V. Henrard (éds), Rendement éducatif, parcours et inégalités dans l'insertion des jeunes: Recueil d'études sur la génération 2010 (Céreq Échanges $N^{\circ}$ 5) (pp. 225-246). Marseille: Centre d'études et de recherches sur les qualifications. En ligne: [http://www.cereq.fr/publications/CereqEchanges/Rendement-educatif-parcours-et-inegalites-dans-l-insertion-des-jeunes.-Recueil-d-etudes-sur-la-Generation-2010].

Guichard, J. (2016). Une comparaison des apports des modèles de la construction de la carrière et de la construction de soi au life designing counseling. Psychologie française, 1, 15-29. Doi : 10.1016/j. psfr.2013.03.002.

Henderson, J. L., Hawke, L. D., \& Chaim, G. (2017). Not in employment, education or training: Mental health, substance use, and disengagement in a multi-sectoral sample of service-seeking Canadian youth. Children and Youth Services Review, 75 (Supplement C), 138-145. Doi : 10.1016/j.childyouth.2017.02.024.

HolTe, B. H. (2018). Counting and meeting NEET young people: Methodology, perspective and meaning in research on marginalized youth. Young, 1, 1-16. Doi: 10.1177/1103308816677 618.

Kluve, J., Puerto, S., Robalino, D., Romero, J. M., Rother, F., STÖTERAU, J.,..., WitTE, M. (2016). Do youth employment programs improve labor market outcomes? A systematic review (Ruhr Economic Papers $n^{\circ}$ 648). Essen: RWI / Leibniz-Institut für Wirtschaftsforschung. Doi: 10.44119/86788754.

Koen, J., Van Vianen, A., Klehe, U.-C., \& Zikic, J. (2016). "A whole new future" - identity construction among disadvantaged young adults. Career Development International, 7, 658-681. https://doi.org/10.1108/CDI-02-2016-0019.

Maguire, S. (2015). NEET, unemployed, inactive or unknown - why does it matter? Educational research, 2, 121132. Doi : 10.1080/00131881.2015.1030850.

Masdonati, J., \& Fournier, G. (2015). Life design, young adults and the school-to-work transition. In L. Nota \& J. Rossier (éds), Handbook of life design: From practice to theory and from theory to practice (pp. 117-133). Göttingen: Hogrefe. Doi: 10.11588/nice.2015.1. 20479. 
Masdonati, J., \& ZitToun, T. (2012). Les transitions professionnelles: processus psychosociaux et implications pour le conseil en orientation. L'Orientation scolaire et professionnelle, 41, 229-253.

Mawn, L., Oliver, E. J., Akhter, N., Bambra, C. L., Torgerson, C., Bridle, C., \& Stain, H. J. (2017). Are we failing young people not in employment, education or training (NEET)? A systematic review and meta-analysis of re-engagement interventions. Systematic Reviews, 1, 16. Doi : 10.1186\% 2Fs13643-016-0394-2.

Mucchielli, R. (1987). Apprendere il counseling, Trente: Erickson. Nico, M. (2014). Variability in the transitions to adulthood in Europe: A crticial approach to de-standardization of the life course. Journal of Youth Studies, 2, 166-182. Doi: 10.1080/13676261.2013.805877.

Nota, L., \& Rossier, J. (éds). (2015). Handbook of life design: From practice to theory and from theory to practice. Göttingen: Hogrefe. Doi : 10.11588/nice.2015.1.20479.

Nunez, A. (2018, septembre). Séance d'information AccentForjad. Présenté à séance d'information Accent-Forjad, Lausanne.

OCDE (2010). Des débuts qui comptent! Des emplois pour les jeunes. En ligne: [http://www.oecd-ilibrary.org/fr/employment/prendreun-bon-depart-des-emplois-pour-les-jeunes_9789264096110-fr].

OCDE (2015). Fit mind, fit job: From evidence to practice in mental health and work. Paris: Auteur. En ligne: [http://www.oecd.org/ els/fit-mind-fit-job-9789264228283-en.htm].

OCDE (2017). Investing in Youth: Japan. En ligne: [https:// www.oecd-ilibrary.org/social-issues-migration-health/investingin-youth-japan/executive-summary_9789264275898-3-en]. Doi : 10.1787/9789264275898-en (consulté le 29 juillet 2018).

OCDE (2018a). Unemployment rate (indicator). En ligne: [https:// data.oecd.org/unemp/unemployment-rate.htm]. Doi : 10.1787/997 c8750-en (consulté le 29 juillet 2018).

OCDE (2018b). Youth not in employment, education or training (NEET) (indicator). En ligne: [https://data.oecd.org/youthinac/ youth-not-in-employment-education-or-training-neet.htm]. Doi : 10.1787/72d1033a-en (consulté le 29 juillet 2018). 
OFFT (2008). Case management "Formation professionnelle»: Soutien et accompagnement de la mise en ouvre dans les cantons: Mandat de projet OFFT - CSFP (Mise à jour du 22 juillet 2008). En ligne: [http://www.sbbk.ch/dyn/20280.php].

O’Dea, B., Glozier, N., Purcell, R., McGorry, P. D., Scott, J., FeILDS, K.-L., ... et al. (2014). A cross-sectional exploration of the clinical characteristics of disengaged (NEET) young people in primary mental healthcare. BMJ open, 4 (12), e006378. Doi: 10.1136/bmjopen-2014-006378.

Patton, W., \& McMahon, M. (2006). The Systems Theory Framework of Career Development and Counseling: Connecting theory and practice. International Journal for the Advancement of Counselling, 2, 153-166. Doi : 10.1007/s10447-005-9010-1.

Paul, K. I., \& Moser, K. (2009). Unemployment impairs mental health: Meta-analyses. Journal of Vocational Behavior, 3, 264-282. Doi: $10.1016 /$ j.jvb.2009.01.001.

Perriard, V. (2005). Transition de l'école obligatoire vers la formation professionnelle: Les facteurs explicatifs des difficultés actuelles ( $\left.n^{\circ} 120\right)$. Lausanne: URSP.

Pombeni, M. L., \& Angelo, M. G. D. (1994). L'orientamento di gruppo. Rome: NIS.

Premier ministre de la République française (20 décembre 2013). Plan national de mise en ouvre de la Garantie européenne pour la jeunesse: Réponse des autorités françaises. En ligne: [http://travail-emploi. gouv.fr/emploi/mesures-jeunes/article/garantie-europeenne-pour-lajeunesse] (consulté le 29 juillet 2018).

Recommandation du Conseil de l'Europe 2013/C 120/01 du 22 avril 2013 sur l'établissement d'une garantie pour la jeunesse (2013). Journal officiel de l'Union européenne, C120, 1-7 case management formation professionnelle

Robert, S., Lesieur, S., Chastang, J., Kergoat, V., Dutertre, J., \& Chauvin, P. (2017). Santé et recours aux soins des jeunes en insertion âgés de 18 à 25 ans suivis en mission locale. Revue d'épidémiologie et de santé publique, 4, 265-276. Doi: 10.1016/j.respe.2017.01.120.

Rodwell, L., Romaniuk, H., Nilsen, W., Carlin, J. B., Lee, K. J., \& Patton, G. C. (2017). Adolescent mental health and behavioural predictors of being NEET: A prospective study of young adults 
not in employment, education, or training. Psychological Medicine, 5, 1-11. Doi: 10.1017/S0033291717002434.

Savickas, M. L., \& Pouyaud, J. (2016). Concevoir et construire sa vie: Un modèle général pour l'accompagnement en orientation au XXI ${ }^{\mathrm{e}}$ siècle. Psychologie française, 1, 5-14. Doi: 10.1016/j. psfr.2013.11.003.

SAVICKAS, M. L. (2005). The theory and practice of career construction. In S. D. Brown, \& R. W. Lent (éds), Career development and counseling: Putting theory and research to work (pp. 42-70). Hoboken (NJ): Wiley.

SCHIAVETTA, M. (21 mai 2015). Auto-orientamento: una competenza necessaria. TELLUSfolio (supplemento telematico quotidiano). En ligne: [http://www.tellusfolio.it/index.php?prec=/index.php?lev=61 $\& \mathrm{cmd}=\mathrm{v} \& \mathrm{lev}=61 \& \mathrm{id}=18715$ ].

SCHOON, I. (2014). Parental worklessness and the experience of NEET among their offspring: Evidence from the longitudinal study of young people in England (LSYPE). Longitudinal and Life Course Studies, 5, 129-150. Doi: 10.14301/llcs.v5i2.279.

Seddon, F., Hazenberg, R., \& Denny, S. (2013). Effects of an employment enhancement programme on participant NEET. Journal of Youth Studies, 4, 503-520. Doi: 10.1080/ 13676261.2012.733808.

Thompson, R. (2011). Individualisation and social exclusion: The case of young people not in education, employment or training. Oxford Review of Education, 6, 785-802. Doi : 10.1080/03054985.2011.636507.

Zuccotti, C. V., \& O’Reilly, J. (2018). Ethnicity, gender and household effects on becoming NEET: An intersectional analysis. Work, Employment and Society, n.d., 1-23. Doi: 10.1177\% 2F0950017017738945. 


\section{ORIENTATION ET INSERTION EN CONTEXTE MIGRATOIRE ${ }^{1}$}

KOKOU A. ATITSOGBE, SHAGINI UDAYAR

ET FEDERICO DURANTE (UNIVERSITÉ DE LAUSANNE)

a migration est un terme polysémique qui désigne les mouvements de personnes allant de petits déplacements au niveau local jusqu'à ceux atteignant les destinations les plus lointaines. Le terme "migrant.e» s'emploie pour désigner les personnes effectuant de tels déplacements. Selon l'Organisation internationale pour les migrations (OIM, 2018), il n'existe pas une définition universellement acceptée de ce terme. Parmi les différentes formes que peuvent prendre les migrations, les migrations internationales ${ }^{2}$ ont toujours mobilisé l'attention des pouvoirs publics, des associations et même celle des citoyens, étant donné les enjeux économiques, sociaux et culturels dont elles sont porteuses. L'Organisation des Nations unies a estimé à 258 millions le nombre de migrant.e.s internationaux.les à travers le monde en 2017 (United Nations, 2017) : un individu sur 25 serait donc un migrant. S'établir dans une nouvelle société peut nécessiter beaucoup d'efforts et d'ajustements, surtout chez les migrant.e.s dont les normes, les valeurs et les codes culturels different notablement de ceux du milieu d'accueil. Comprendre les processus psychosociaux en jeu dans ces migrations aiderait à développer pour cette population des dispositifs d'accompagnement en orientation scolaire et professionnelle (OSP) plus efficaces. Dans ce chapitre, le terme migration sera employé en référence à la migration de type international et celui de migrant.e pour désigner plus largement les personnes ayant quitté leur pays d'origine ou de naissance pour un autre, en vue d'améliorer leurs conditions.

1. Cette publication a bénéficié du soutien du Pôle de recherche national LIVES - Surmonter la vulnérabilité: perspective du parcours de vie, financé par le Fonds national suisse (Fonds $\mathrm{n}^{\circ}$ 51NF40-160590).

2. Déplacement des personnes d'un pays vers un autre. 


\section{MIGRATION, ACCULTURATION, ADAPTATION ET ORIENTATION}

\subsection{UN PHÉNOMÈNE AUX ENJEUX MULTIPLES QUI PREND DE L'AMPLEUR}

\subsubsection{Migration: chiffres et faits}

Des flux migratoires de grande ampleur vers l'Europe ont été enregistrés ces dix dernières années, avec une amplification du phénomène en particulier dans certains pays de l'Organisation de coopération et de développement économiques (OCDE). Les chiffres récents concernant les migrations internationales indiquent une entrée d'environ 5 millions de personnes dans la zone en 2016, une augmentation de $7 \%$ par rapport à l'année 2015 (OCDE, 2017). La littérature distingue plusieurs catégories de migrants, suivant qu'ils sont issus d'une migration volontaire ou forcée (Berry, 1997), légale ou illégale (Reitz, 2007), pour raisons économiques ou d'instabilité politique (Fargues, 2016). La migration fait désormais partie des enjeux globaux, avec une accentuation du phénomène due aux déplacements massifs de migrant.e.s forcé.e.s, notamment des réfugié.e.s et des demandeurs.ses d'asile (UN High Commissioner for Refugees, 2017). La Suisse a dénombré jusqu’à fin 2017 un total de 65775 personnes inscrites dans le processus d'asile, dont 41544 admises provisoirement (Secrétariat d'État aux migrations, 2018). L'Association américaine de psychologie (American Psychological Association [APA], 2012) a identifié trois principales causes de la forte migration récente à travers le monde: la recherche de travail, le regroupement familial et les raisons humanitaires. Indépendamment des raisons qui sous-tendent les migrations, la gestion des migrant.e.s en provenance des pays extra-européens semble représenter un défi majeur (OCDE, 2017). Des dispositions ont été prises par des institutions internationales telles que l'OCDE et le $\mathrm{HCR}$, encourageant les États à développer et à mettre en œuvre des politiques et pratiques d'intégration des migrant.e.s. Si la plupart des pays faisant face à une forte migration essaient de se conformer aux dispositions internationales en la matière, les politiques et pratiques qui ont pour objectif l'intégration de ces migrant.e.s varient beaucoup (OCDE, 2017). Les migrant.e.s représentant une population très hétérogène, les enjeux de leur intégration pour les institutions internationales, les pouvoirs publics, les associations et même les citoyen-ne.s se situent à différents niveaux, notamment politique, économique ou encore social (OCDE, 2017). 


\subsubsection{Implications culturelles et psychosociales de la migration}

La migration implique différentes transitions que sous-entendent des processus complexes sur les plans culturel, psychosocial et professionnel (Cohen, Arnold, \& O'Neill, 2011). Comme le relèvent Masdonati et Zittoun (2012), ces transitions qui représentent des situations mettant les personnes "au défi de quitter des lieux ou des situations connues et d'agir de manière compétente dans une situation insolite demandent des actions spécifiques et, avec elles, l'acquisition de nouvelles compétences sociales, professionnelles, cognitives, etc.» (p. 4). En effet, d'un point de vue culturel, les migrant.e.s, au contact de codes différents de ceux de leur société d'origine, s'ajustent à leur milieu d'accueil en développant différents comportements ou attitudes: garder l'héritage culturel d'origine, s'approprier celui du milieu d'accueil, concilier les deux ou encore les renier (Berry, 1997). D'un point de vue psychosocial, la migration génère des transformations identitaires, mettant les migrant·e.s dans une position où l'acquisition de nouvelles connaissances est inévitable (Masdonati et Zittoun, 2012). Ces dernières peuvent comprendre l'apprentissage de langues, l'adoption de normes et codes culturels nouveaux ou le développement des compétences scolaires, professionnelles et sociales. D'un point de vue professionnel, la migration représente également un enjeu pour les migrant.e.s qui espèrent poursuivre et réaliser leur projet professionnel, individuellement ou collectivement, dans la société d'accueil. Ainsi, s'épanouir dans la société d'accueil implique d'y être intégré.e.

L'intégration socioprofessionnelle exige de ces personnes une adaptation au milieu d'accueil. Selon Berry (1997), l'adaptation se réfere aux changements mis en place, que ce soit au niveau individuel ou groupal, pour faire face aux exigences de l'environnement. En ce sens, elle peut revêtir une dimension psychologique ou une dimension socioculturelle (Berry \& Sam, 1997). L'adaptation sur le plan psychologique peut se mesurer non seulement en termes de satisfaction avec sa nouvelle vie ou ses nouvelles conditions, mais également en termes de bien-être psychologique et émotionnel (Jong, Chamratrithirong, \& Tran, 2002). Ladaptation sur le plan socioculturel suppose que la personne migrante acquière les connaissances culturelles et linguistiques telles que véhiculées par la société d'accueil (Berry, Poortinga, Breugelmans, Chasiotis, \& Sam, 2011). Ensemble, l'adaptation psychologique et l'adaptation socioculturelle peuvent faciliter l'intégration des migrant.e.s dans 
la société d'accueil et sont donc nécessaires dans l'élaboration et la mise en œuvre de leurs projets professionnels, ces derniers dépendant fortement des caractéristiques du marché du travail et des politiques sur l'emploi dans la société d'accueil (OCDE, 2017).

\subsection{ACCULTURATION ET STRATÉGIES D'ACCULTURATION}

Se reconstruire dans une nouvelle culture nécessite de s'y adapter et les efforts d'adaptation sont fonction des caractéristiques de la culture d'accueil, des ressources personnelles et de la distance culturelle entre la culture d'accueil et la culture d'origine (Demes \& Geeraert, 2014; Hofstede, 1991). Les migrant.e.s au contact d'une nouvelle culture y découvrent des modes de vie, des normes sociales et des systèmes de valeurs différents des leurs. Ces différences, parfois sources de conflits et de déséquilibre et de stress, suscitent chez les migrant.e.s des ajustements au niveau psychologique et comportemental (Berry, 1997; Camilleri, 1990). La façon dont les individus opèrent cet ajustement, en prenant en considération aussi bien les normes de la culture d'origine que celles du milieu d'accueil, a été conceptualisée comme processus d'acculturation et les attitudes et comportements y afférents comme stratégies d'acculturation (Berry, 2006). Une des définitions les plus anciennes, mais toujours partagée du concept d'acculturation est celle de Redfield, Linton et Herskowits (1936), qui la conçoivent comme "l'ensemble des phénomènes résultant du contact direct et continu entre des groupes d'individus de cultures différentes, avec des changements subséquents dans les types de cultures originales de l'un ou des deux groupes» (p. 149). Selon Berry (1997), les populations migrantes, tout comme les populations autochtones peuvent être confrontées à deux problèmes fondamentaux: d'une part, une préférence relative pour la conservation de l'héritage culturel et l'identité forgée dans le milieu d'origine et, d'autre part, une préférence relative du contact aussi bien avec l'ensemble de la société qu'avec les différents groupes ethnoculturels composant la société d'accueil. De ces deux problèmes fondamentaux découlent quatre stratégies d'acculturation: l'assimilation, la séparation, l'intégration et la marginalisation (Berry, 1997). On parle d'assimilation lorsque les personnes migrantes se distancient de leur culture d'origine ou la renient au profit d'une socialisation active dans le milieu d'accueil. La séparation procède par conservation de l'héritage culturel du milieu d'origine tout en se soustrayant des échanges avec les 
membres de la société d'accueil. La stratégie d'intégration procède par conciliation des deux cultures en maintenant des éléments de la culture d'origine et en se socialisant activement dans la société hôte. Enfin, on parle de marginalisation lorsque les personnes migrantes prennent des distances vis-à-vis de leur culture d'origine sans toutefois adopter celle de la société d'accueil. Bien que distinctes, ces stratégies peuvent être utilisées conjointement (Berry et al., 2011). Par exemple, certaines populations de migrant.e.s peuvent adopter la stratégie d'intégration dans l'espace public et la séparation dans leur vie privée (Arends-Tóth \& van de Vijver, 2003).

Les stratégies d'acculturation influencent notablement les parcours professionnels des migrant.e.s. Par exemple, Sarot, Girard, Chomentowski, Revah-Lévy, Falissard et Moro (2017) rapportent que la réussite professionnelle des mères de famille issues de cultures collectivistes où la communauté prime sur l'accomplissement individuel semble parfois être obtenue "au prix d'une renonciation à la culture d'origine, source de fragilité identitaire» dans les sociétés individualistes (p. 186). Le mode d'acculturation peut donc avoir une influence sur l'élaboration et la mise en ouvre des projets professionnels. Parmi les quatre stratégies d'acculturation, l'intégration semble la stratégie qui présente le plus de bénéfices, car elle génère moins de stress et facilite l'adaptation au milieu d'accueil tout en préservant une certaine continuité chez les migrant.e.s (Meca et al., 2018). Elle est également la stratégie la plus fréquemment mise en ouvre (Kanouté, 2002). De plus, certains contextes culturels se prêteraient mieux à cette stratégie d'acculturation (Kamanzi, Bastien, Doray, \& Magnan, 2016).

\subsection{ADAPTATION ET PROJETS SCOLAIRES ET PROFESSIONNELS}

Berry et ses collègues (2011) distinguent deux niveaux d'adaptation selon l'âge: l'adaptation des enfants et jeunes au milieu scolaire, qui dépend fortement des dispositifs d'accueil proposés dans chaque pays, et l'adaptation professionnelle pour les adultes, en particulier dans des sociétés où le travail est un facteur d'accomplissement et d'intégration. À celles-ci peut s'ajouter également l'adaptation en formation professionnelle, qui concerne les jeunes qui décident de poursuivre une voie professionnalisante après l'école obligatoire dans certains pays comme la Suisse ou l'Allemagne.

L'adaptation au système scolaire contribue à structurer les projets d'avenir (Guichard \& Huteau, 2006). Pour Vedder et Motti-Stefanidi (2016), la réussite scolaire/académique, avec une performance 
supérieure ou égale à la moyenne de leurs pairs de la société d'accueil, est un indicateur d'une bonne adaptation au système scolaire des enfants/jeunes ayant émigré. Selon ces auteurs, l'absence de décrochage scolaire est un second indicateur. Pour les allophones, l'acquisition de la langue du pays d'accueil est un élément crucial pour l'adaptation à un nouveau système scolaire et l'insertion sociale ou professionnelle (Sarot et al., 2017). L'adaptation en formation professionnelle pour les jeunes adultes migrant.e.s peut se traduire par l'obtention d'un diplôme de fin d'apprentissage et une progression dans le domaine professionnel au fil des années. Pour Berry et ses collègues (2011), l'adaptation des migrant.e.s en emploi pourrait être appréhendée à travers l'atteinte des objectifs professionnels, mais également par le fait que le travail obtenu dans la société d'accueil soit en lien avec leur formation initiale. Il convient de souligner que ce dernier critère n'est pas toujours pertinent. C'est en effet le cas lorsque la personne migrante décide par elle-même de changer complètement de métier ou de domaine ou qu'en raison de certaines conditions, elle ne pourra exercer un métier en lien avec sa formation initiale.

\subsection{L'ORIENTATION SCOLAIRE ET PROFESSIONNELLE AVEC UNE POPULATION MIGRANTE}

\subsubsection{L'empathie culturelle}

Lors d'une démarche d'orientation avec les migrant.e.s, les psychologues conseillers ères en orientation (P-CO) risquent d'interpréter les interactions avec les consultant.e.s sur fond de leurs propres références culturelles (Cardu \& Sanschagrin, 2004). Selon Deen (2002), lorsqu'on travaille avec les migrant.e.s, il faut dans un premier temps avoir une idée exacte sur ses propres préjugés. Dans un second temps, il est important d'utiliser l'empathie culturelle, qui est un processus permettant "d'identifier les valeurs et les attentes qui peuvent déterminer le comportement du consultant et la signification que prend pour ce dernier la recherche de l'aide d'un conseiller qui n'est ni de sa famille ni de son groupe culturel » (p. 11). Il s'agit donc pour le P-CO de prendre en considération les influences culturelles qui peuvent agir sur la démarche d'orientation, et ainsi proposer une intervention adaptée à la situation de la personne migrante. Toutefois, il ne faut pas réduire complètement la personne et son fonctionnement psychologique à sa 
culture d'origine. Il faut également garder à l'esprit que certain·e.s migrant.e.s peuvent être dans le rejet de certaines valeurs de leur culture initiale ou présenter déjà certaines formes d'acculturation et, du coup, identifier dans la société d'accueil les valeurs culturelles qui leur sont signifiantes (Hofstede, 1991).

Lors d'une démarche d'orientation avec les migrant.e.s, les cadres théoriques développés dans un contexte occidental sont certes applicables, mais ont leurs limites. En effet, la plupart des modèles théoriques considèrent le choix avant tout comme un processus individuel (Schultheiss \& Davis, 2015; Stebleton, 2007). Cependant, il s'avère que lorsqu'on travaille avec des migrant.e.s issu.e.s des cultures collectivistes, le choix est également un processus collectif impliquant souvent les différents membres de la famille ou la communauté (Kennedy \& Chen, 2012). Avec les migrant.e.s pour qui la religion occupe une place importante dans le choix de carrière, l'utilisation de certains modèles de choix professionnel issus de la psychologie dominante n'est pas non plus pertinente (Schultheiss \& Davis, 2015). Ainsi, être sensible à ces différences culturelles permettrait une meilleure prise en charge de la personne. Certain.e.s auteur.e.s ont essayé d'inclure ces éléments culturels dans le développement des théories sensibles à la culture (pour une revue de la question, voir Leong \& Flores, 2015).

\subsubsection{Les compétences multiculturelles}

Prendre en charge des migrant.e.s nécessite donc pour les $\mathrm{P}-\mathrm{CO}$ de développer des compétences multiculturelles dans les trois domaines suivants (APA, 2003; Flores, Hsieh, \& Chiao, 2011; Schultheiss \& Davis, 2015) :

- au niveau du savoir-être, par la prise de conscience des croyances personnelles et des attitudes envers les individus issus de cultures très diversifiées. Ainsi, les conseillers-ères reconnaissent que la culture est un élément important à la compréhension des processus psychologiques en jeu lors de la prise en charge;

- au niveau du savoir, par les connaissances développées sur les différentes cultures. Ceci implique, pour la ou le conseiller·ère, non seulement, à travers des formations continues, de se familiariser avec les différents concepts culturels, sociaux, psychologiques, politiques, économiques et historiques liés à un groupe spécifique de migrants, mais également de reconnaître ses propres limites et, si nécessaire, de faire appel à des expert.e.s; 
- au niveau du savoir-faire, en recourant à des outils d'intervention appropriés pour ces types de population. Certains outils sont développés dans une culture spécifique et sont donc parfois démunis de sens pour les personnes qui ont grandi dans une tout autre culture. Par exemple, les outils classiques d'orientation tels que les questionnaires d'intérêt, de personnalité et de valeur, requérant une bonne maîtrise de la langue, sont souvent inutilisables avec une population migrante (Perdrix, Charvoz, \& Rossier, 2009; Rossier $\&$ Duarte, sous presse). Les P-CO doivent donc tenir compte de ces limites lors de la prise en charge des migrant.e.s.

Acquérir ces compétences multiculturelles permet ainsi aux P-CO de développer une sensibilité à la culture et d'empêcher ainsi les stéréotypes de nuire à la bonne prise en charge de cette population (Stuart, 2004). Si les aspects culturels sont très importants, ils ne "déterminent" pas complètement les migrant.e.s. En effet, les parcours individuels (vécu, expériences, connaissances) sont à considérer autant que les influences contextuelles. Par exemple, les migrant.e.s humanitaires viennent souvent avec des histoires de vie douloureuses et traumatiques qu'il ne faut pas négliger lors de la prise en charge. Les $\mathrm{P}-\mathrm{CO}$ doivent donc avoir des compétences leur permettant de reconnaître de pareils cas et de diriger les personnes vers des spécialistes pour les situations sensibles.

Un dernier point à prendre en considération lors d'une démarche d'orientation avec les migrant.e.s est la triade que forment la ou le conseiller-ère, la ou le consultant·e et l'interprète. Au-delà d'une relation classique conseiller.ère-consultant.e, la prise en charge d'un.e migrant.e fait souvent intervenir un nouvel acteur dans cette relation, à savoir l'interprète. L'alliance de travail, élément central pour une intervention efficace (Whiston, Rossier, \& Hernandez Barón, 2016), peut être mise à rude épreuve avec la présence d'un·e interprète. L'interprète peut être aussi bien une personne externe à l'intervention psychologique (par exemple, recours à des interprètes professionnelles via divers associations et organisations) qu'une personne interne (par exemple, membres du personnel éducatif du milieu scolaire qui improvisent une interprétation). Dans ce second cas, l'interprète peut facilement être tenté.e de reformuler les propos de la ou du consultant.e afin de donner une réponse plus claire, ce qui peut impacter l'efficacité de l'intervention. De plus, l'interprète, étant souvent un·e référent.e culturel.le 
qui rappelle explicitement ou implicitement les normes culturelles, la ou le migrant.e peut ne pas se sentir pleinement libre de s'exprimer (Hatoss, Neill, \& Eacersall, 2012). Par exemple, un garçon voulant aller vers des métiers perçus comme "féminins» dans sa culture d'origine pourrait se sentir gêné de parler de son projet professionnel devant l'interprète. Il s'agit alors pour les P-CO d'être attentifs.ves à la relation entre les consultant.e.s et les interprètes et d'identifier les éventuels non-dits des migrant-e.s liés à la présence de ce ou cette référent.e culturel.le au cours des entretiens. La ou le P-CO doit donc développer des compétences additionnelles afin de gérer des entretiens à trois (Yakushko, Backhaus, Watson, Ngaruiya, \& Gonzalez, 2008).

\section{5 ÉTAT DE LA RECHERCHE}

La méconnaissance de la démarche d'orientation (Sultana \& Watt, 2007) et l'incertitude liée à leur statut de séjour (Jackson \& Bauder, 2014) ont été identifiées comme des facteurs influençant le processus d'orientation et l'insertion professionnelle des migrant.e.s, mais ce ne sont pas les seuls. Des chercheurs.ses, notamment anglosaxon-ne.s (principalement États-Unis, Australie et Canada) se sont penché.e.s sur la question et ont ainsi relevé non seulement des barrières, mais également des ressources que les migrant.e.s utiliseraient pour les surmonter. Parmi ces facteurs, certains dépendent du contexte économique et sociopolitique du pays d'accueil. D'autres sont généralisables à l'ensemble de la population migrante.

Les recherches ont montré que les facteurs influençant les trajectoires professionnelles des migrant.e.s se situent à trois niveaux: individuel, collectif et contextuel (Yakushko et al., 2008). Le niveau individuel regroupe tous les facteurs propres à la personne ayant un impact sur le choix et l'insertion professionnelle. Ainsi, des études ont identifié les facteurs sociodémographiques tels que le pays d'origine (Mamgain \& Collins, 2003), le genre (Hatoss et al., 2012; Mamgain \& Collins, 2003), le niveau de maîtrise de la langue nationale du pays d'accueil (Chen \& Hong, 2016), ainsi que le niveau d'études (Codell, Hill, Woltz, \& Gore, 2011). D'autres facteurs, notamment le manque de connaissances et d'informations concernant le marché du travail (Amundson, Yeung, Sun, Chan, \& Cheng, 2011; Koert, Borgen, \& Amundson, 2011), le manque de connaissances concernant les codes et normes culturelles du pays d'accueil (Chen \& Hong, 2016), le manque ou l'insuffisance 
d'expériences et compétences professionnelles (Koert et al., 2011), le manque de buts professionnels (Amundson et al., 2011), ainsi que le sens et les représentations que se font les jeunes migrant·e.s du travail (Abkhezr, McMahon, \& Rossouw, 2015), ont également été identifiés comme des variables individuelles affectant leurs choix et leur insertion professionnelle. En effet, le travail peut représenter pour certaines migrant.e.s un élément central à la survie, et pas dans l'immédiat un facteur de développement personnel comme cela peut l'être pour la plupart des autochtones (Blustein, McWirther, \& Perry, 2005). Le stress d'immigration et les expériences pré- ou post- migratoires peuvent influencer le processus de choix de profession (Chen \& Hong, 2016; Yakushko et al., 2008b). Par exemple, les traumatismes vécus dans le pays d'origine peuvent limiter leurs choix et leur insertion professionnelle (Hatoss et al., 2012). Certaines études ont mis en évidence la résilience et le sentiment d'efficacité comme des ressources que les migrant.e.s mobilisent lors d'une démarche d'orientation professionnelle (Obschonka, Hahn, \& Bajwa, 2018; Yakushko et al., 2008). Ces études mentionnent que ces ressources aident les migrant.e.s à se motiver, à s'adapter à la situation et à s'impliquer dans cette démarche.

Le niveau collectif considère l'individu dans son rapport à l'autre. Ainsi, il englobe non seulement les influences de différents groupes sociaux sur l'individu, mais également l'influence de tous les facteurs liés à la quantité et à la qualité des relations interpersonnelles entretenues avec ces différents groupes sociaux lors d'une démarche d'orientation professionnelle. Ainsi, le manque de réseau social et de soutien social est un facteur agissant comme une barrière à l'insertion professionnelle des jeunes migrant.e.s (Colic-Peisker \& Tilbury, 2006). La famille/la communauté est un facteur influençant le choix professionnel. En effet, il est fort probable que les décisions prises par les migrant.e.s provenant de cultures collectivistes soient le fruit d'une réflexion collective. Ainsi, la famille, les amis, la communauté peuvent influencer de manière considérable le choix professionnel des migrant.e.s (Arulmani, 2014) et doivent être considérés comme des éléments centraux dans le processus d'orientation (Cohen et al., 2011). Les attentes liées au genre peuvent influencer leur choix de profession (Hatoss et al., 2012). En effet, souvent, la famille et la communauté attendent des femmes qu'elles s'occupent des tâches ménagères et des enfants; elles pourraient donc s'attendre à maintenir ce rôle dans la société d'accueil (Cardu \& Sanschagrin, 
2004). Ainsi, lors d'un processus d'orientation, de telles responsabilités peuvent limiter leurs attentes et leurs choix. Les obligations familiales peuvent être un autre facteur collectif influençant le choix d'orientation des migrant.e.s (Stebleton, 2012). Par exemple, le devoir de soutien du ou de la migrant.e envers la famille qui est à ses côtés, mais également envers celle qui est restée dans le pays d'origine, peut influencer le choix professionnel de ce ou cette dernier-ère (Hatoss et al., 2012). En effet, il est souvent attendu des migrant.e.s qu'elles et ils apportent une aide financière à leur famille. Ainsi, cette pression supplémentaire peut agir comme un frein en limitant leurs aspirations professionnelles, ou au contraire agir comme une motivation les poussant à atteindre leurs buts professionnels. La discrimination est une barrière au choix et à l'insertion professionnelle relevée par de nombreuses études (Chen \& Hong, 2016; Constantine, Miville, Warren, Gainor, \& Lewis-Coles, 2006). En revanche, la présence d'autres migrant.e.s de la même communauté peut agir comme une ressource dans le cadre d'une démarche d'orientation et d'insertion professionnelle (Yakushko et al., 2008b). La religion et la spiritualité sont d'autres ressources mobilisées par les jeunes migrant.e.s lorsqu'elles et ils doivent faire un choix d'orientation professionnelle. Par exemple, Constantine et collègues (2006) ont montré que la religion et la spiritualité, en donnant du sens au travail, motivent certain.e.s migrant.e.s à choisir certaines carrières et à saisir les opportunités afin d'y réussir. De plus, ces éléments joueraient un rôle de soutien émotionnel dans le processus de choix de carrière.

Le niveau contextuel regroupe tous les événements ou circonstances qui peuvent affecter la vie professionnelle d'un individu (Stebleton, 2007). En contexte migratoire, il s'agit principalement de tenir compte des circonstances socioculturelles et politiques qui influencent la carrière des migrant.e.s. Ainsi, la transition vers une société occidentale post-industrielle est un facteur influençant le processus d'insertion professionnelle des jeunes migrant.e.s (Abkhezr et al., 2015). En effet, beaucoup de ces jeunes proviennent de pays où l'économie est toujours à l'ère industrielle ou parfois pré-industrielle. Lors de la transition, un écart important peut apparaître entre les représentations de l'éducation et du travail construites de leur pays d'origine et du pays d'accueil. Les écarts peuvent être également grands au niveau des compétences et expériences professionnelles exigées dans les deux contextes. Un autre facteur contextuel est celui de la non-reconnaissance des diplômes 
(Chen \& Hong, 2016; Muller Mirza \& Alber, sous presse). Comme évoqué précédemment, le statut de réfugié.e et sa durée constitueraient des entraves à une démarche d'orientation et d'insertion (Codell et al., 2011 ; Yakushko et al., 2008). Les événements sociopolitiques du pays d'origine influenceraient également le choix d'orientation des jeunes migrant.e.s (Stebleton, 2012). En effet, ces événements peuvent influencer le sens que donnent ces derniers.ères au choix de projet professionnel.

En somme, l'orientation et l'insertion professionnelle des migrant.e.s sont marquées par des facteurs à la fois contextuels et individuels (Lent, Brown, \& Hackett, 1994). Il s'agit alors pour les P-CO de tenir compte de ces potentiels déterminants et de mettre en pratique des compétences multiculturelles afin de proposer des interventions adéquates (Durante \& Favre, 2012).

\section{BILAN D'ORIENTATION POUR UN PUBLIC EN CONTEXTE MIGRATOIRE}

Deux types d'intervention peuvent être appliqués en parallèle lors de la démarche d'orientation avec les migrant.e.s, à savoir une intervention collective ou individuelle. Une intervention en orientation professionnelle collective avec des migrant.e.s a généralement pour objectif de transmettre aux participant.e.s les clés de lecture du contexte de formation ou de travail du pays d'accueil: expliciter le système de formation, les différentes options proposées, de manière transparente par rapport aux logiques et objectifs politiques qui les sous-tendent et aux éventuelles différences d'avec celles des pays d'origine. Une approche du monde du travail par des stages ou des séances d'information, ainsi qu'une préparation aux techniques de recherche d'emploi, est également essentielle (Deen, 2002). Les codes et les attentes des nouveaux contextes professionnels pourront ainsi être progressivement intégrés, en fonction de l'importance des distances culturelles. Les thèmes traités sont les mêmes que pour une population tout-venant. Il s'agit toutefois d'appliquer la perspective d'empathie culturelle, telle que décrite plus haut, prenant en compte les différences culturelles qu'il peut y avoir entre pays d'origine et pays d'accueil, en cherchant à contrôler et minimiser leur influence dans le processus d'orientation, tout en apportant les outils nécessaires à l'autonomisation de la ou du consultant.e dans son futur contexte de travail ou de formation (APA, 2003; Schultheiss \& Davis, 2015). 
Concernant les interventions individuelles, nous pouvons relever deux éléments concrets qu'il s'agira de mettre en ouvre, à savoir l'utilisation des outils non verbaux et l'application de l'approche narrative. Nous avons mentionné plus haut l'importance de travailler avec des interprètes communautaires, donnant la possibilité aux consultant.e.s de s'exprimer dans leur langue d'origine. L'interprétation ne se limite pas au discours, mais s'étend aux coutumes, pratiques et significations, qui peuvent ainsi être regardées sous le prisme des cultures des pays d'origine et d'accueil (APA, 2003). Comme nous l'avons relevé, la population migrante est souvent en cours d'acquisition de la langue du pays d'accueil. Il s'agit donc de privilégier des instruments non verbaux, se fondant sur l'image plutôt que le langage, pour illustrer des intérêts professionnels ou évaluer des compétences cognitives (Steger, Shim, Rush, Brueske, Shin, \& Merriman, 2013). Nous pouvons citer notamment l'Echelle non verbale d'Intelligence de Wechsler et Naglieri (2006), pour les aspects cognitifs, et le Test d'intérêts en photos (Stoll, Jungo, \& Toggwiler, 2012). Les tests non-verbaux peuvent parfois être également sensibles aux biais culturels. Dans le cas d'utilisation de tests traduits, il est important de vérifier la validité d'une telle traduction sur un plan culturel (Leong \& Hartung, 1997; APA, 2003).

Une approche narrative dans les entretiens d'orientation (Morgan, 2010; White, 2009) permettra d'intégrer à la démarche l'influence des contextes culturels. En effet, une telle approche permet d'aider la ou le consultant.e à se constituer auteur de sa vie (reauthoring). Dans cette démarche non normative, les éléments culturels sont alors intégrés au récit et au processus d'individuation. Avec l'aide de la ou du praticien-ne narratif-ve, les croyances, les théories et les pratiques de l'environnement culturel de la personne peuvent ainsi être mises en lumière et déconstruites (Morgan, 2010). Adoptant ainsi une perspective systémique et postmoderne (Busacca \& Rehfuss, 2016), la ou le P-CO considérera sa ou son consultant.e à la fois comme détenteur-trice d'une histoire plurielle, influencée par différents contextes de vie, et auteur.e d'un futur s'écrivant autour de possibles, également pluriels. L'impact d'un double contexte (pays d'origine et pays d'accueil) et de leur culture sera ainsi pris en compte.

Deux cas cliniques seront présentés ci-dessous afin d'illustrer ce qu'implique une démarche d'orientation en contexte migratoire. Les cas d'Alberto et de Jaganmay, le premier tiré d'un service d'orientation scolaire publique du canton de Vaud et le second d'un service 
privé d'orientation du même canton, permettront de mieux comprendre comment les différents concepts évoqués dans la première partie du chapitre sont mobilisés lors d'une démarche d'orientation.

\subsection{LE CAS D'ALBERTO}

Alberto est un jeune homme âgé de 17 ans, d'origine albanaise. Arrivé en Suisse avec sa famille pour des raisons économiques, il a effectué l'entier de sa scolarité en Italie, pays vers lequel ont migré ses parents avant sa naissance. En Suisse, il entame sa formation en classe d'accueil ${ }^{3}$, au sein de laquelle il apprend le français et consolide ses connaissances scolaires. Il acquiert ainsi une troisième langue après l'albanais, sa langue maternelle, et l'italien, langue de scolarisation dans son pays de provenance. Il est reçu une première fois par le $\mathrm{P}-\mathrm{CO}$ de l'école, afin de l'aider à définir un projet professionnel. Auparavant, ce dernier a expliqué, lors d'une séance en classe, le système de formation suisse, et notamment la formation professionnelle duale ${ }^{4}$, peu pratiquée de manière aussi généralisée à l'étranger (Sefri, 2018).

Plusieurs projets sont mentionnés: juge, psychologue, physiothérapeute ou gérant d'une entreprise liée au tourisme. Afin de mieux définir un choix, il est proposé à Alberto de passer un test d'intérêts (dans sa langue), évaluant les domaines d'activité (j'aime faire) et les métiers (j'aimerais être) qu'il préfere (Gendre, Capel, \& Rossé, 2006). Ce test correspond tout à fait aux attentes d'Alberto. Les domaines de la santé et du travail social sont identifiés de manière marquée. La question se pose alors de savoir s'il vise une voie académique afin de se former dans les professions évoquées, ou s'il privilégie la formation professionnelle, permettant de rapprocher l'entrée dans le monde du travail. Selon les enseignants consultés, Alberto a de bonnes compétences scolaires. Il apprend effectivement le français rapidement. Il se dit toutefois paresseux et n'est pas sûr de vouloir entreprendre des études supérieures.

Plusieurs mois s'écoulent à la suite de la passation du test. Les élèves de la classe d'Alberto ayant alors acquis une certaine autonomie en compréhension et expression orale, il leur est demandé de s’organiser

3. Les classes d'accueil, existant dans la plupart des établissements scolaires, rassemblent les élèves qui viennent d'arriver en Suisse et ne maitrisant pas ou très peu la langue.

4. La formation professionnelle en Suisse est majoritairement organisée autour des entreprises, au sein desquelles les apprentis sont formés par des maîtres d'apprentissage. En parallèle, ceux-ci suivent des cours dans des écoles professionnelles. C'est ce que l'on appelle le système dual (entreprise-école). 
une semaine de stage de découverte du monde professionnel. Alberto a beaucoup de mal à se motiver dans ses démarches de recherche d'expérience professionnelle. Il effectue finalement deux stages, un comme monteur en échafaudage et un autre comme mécanicien, grâce aux contacts de son père maçon. Ces expériences ne lui permettent pas de définir un projet qui pourrait l'intéresser. Il explique avoir choisi ces entreprises sous l'unique pression de l'école.

Lorsqu'Alberto arrive au terme de sa scolarisation en classe d'accueil, il a acquis de bonnes compétences langagières et les enseignant.e.s estiment qu'il est prêt à intégrer le système régulier. Cependant, malgré le visionnement de plusieurs vidéos d'information professionnelle, son projet n'est toujours pas défini. S'ensuivent alors de nouveaux entretiens avec le P-CO. En questionnant les projets et les motivations qu'aurait eus Alberto s'il était resté en Italie, il en ressort qu'il y a laissé une petite amie, avec laquelle il est en contact régulier par voie électronique. Il souhaiterait pouvoir la rejoindre et poursuivre ses études là-bas. Alberto étant encore mineur, une rencontre avec la mère (qui ne maîtrise pas le français) et un traducteur-interprète est proposée afin d'évaluer si ce projet est réaliste. Pour les parents, il est exclu que leurs enfants retournent en Italie (Alberto a un frère jumeau et une sœur). La famille est venue en Suisse pour que les enfants aient un avenir meilleur. Ils se formeront donc dans le pays. Alberto comprend ce projet et s'y conforme. Il choisit alors de se mettre à la recherche d'un stage d'assistant en pharmacie, apprentissage de trois ans qui lui permettrait ensuite d'intégrer le marché du travail ou de poursuivre une formation tertiaire dans le domaine de la santé. Il s'informe du contenu de cet apprentissage auprès de plusieurs de ses connaissances qui sont en train de l'effectuer. Il se rend compte qu'en comparant les programmes scolaires, cela correspond tout à fait aux possibilités d'études qu'il aurait eues en Italie. Ainsi, motivé par ce projet, il choisit de poursuivre son parcours dans une structure permettant de développer des compétences pratiques en atelier, qui le soutiendra dans ses démarches de recherche de place de formation.

L'exposé de cette situation met en évidence l'influence des attentes familiales dans le choix professionnel de jeunes migrant.e-s. Leur projet de formation est influencé par le projet de migration des parents: venir en Suisse pour favoriser un avenir économique. Les enfants doivent composer avec ce facteur dans la construction de leur projet professionnel. Il est alors essentiel, dans une démarche d'orientation, 
de prendre ces éléments en compte et de les intégrer dans le processus de définition du projet. Dans cette situation, un traditionnel test d'intérêts n'a pas été suffisant pour permettre la réflexion. Une orientation axée uniquement sur les caractéristiques de l'individu (intérêts, aptitudes, compétences, valeurs par exemple) risque donc de passer à côté d'une problématique essentielle dans le parcours de la personne: celle de la migration. Cette expérience doit donc être intégrée à la démarche d'orientation.

\subsection{LE CAS DE JAGANMAY}

Jaganmay, 23 ans, a été reçu dans un service d'orientation sur demande d'une association s'occupant de l'insertion socioprofessionnelle de jeunes réfugié.e.s ou demandeur.se.s d'asile. Jaganmay était volontaire pour effectuer cette démarche. Sa situation de vie est extrêmement complexe et fragile. Il vit avec sa famille (ses parents et un frère plus jeune) dans un foyer isolé, loin des centres urbains. L'établissement accueille beaucoup d'occupant.e.s, occasionnant du bruit à toute heure du jour ou de la nuit. La famille vit dans une seule chambre. Les conditions pour se reposer, pour apprendre le français ou tout simplement pour réfléchir à son avenir sont donc peu favorables. Le foyer est à plus d'une heure de distance en transports publics du centre urbain le plus proche, transformant tout rendez-vous en une épopée chronophage. Jaganmay est de nationalité afghane. Toutefois, ses parents ont migré en Inde avant sa naissance. Ils y vécurent durant vingt-quatre ans, sous le statut de réfugiés. Ayant de la famille en Belgique, ils souhaitaient venir en Europe pour les rejoindre. Par des circonstances peu explicitées par Jaganmay, sa famille a finalement déposé une demande d'asile en Suisse. En vertu du règlement Dublin (Règlement de l'Union européenne Nº 604/2013 du Parlement européen et du Conseil), les pays habilités à traiter une demande d'asile sont ceux dans lesquels les requérant.e.s ont déposé leur dossier en premier. Il n'est donc pas possible de tenter, successivement, de demander l'asile dans différents États membres ou en Suisse ${ }^{5}$. Pour cette raison, la famille de Jaganmay ne peut plus se rendre en Belgique.

Le jeune homme est reçu au premier entretien après avoir repoussé d'un mois la prise en charge en raison d'une surcharge de

5. Comme spécifié dans l'Accord entre la Confédération suisse et la Communauté européenne relatif aux critères et aux mécanismes permettant de déterminer l'État responsable de l'examen d'une demande d'asile introduite dans un État membre ou en Suisse, ADD, Recueil officiel, RO 2008515. 
démarches administratives. Les parents de Jaganmay ne parlant pas français, ce dernier est en effet chargé de se rendre aux différents rendez-vous de la famille. Après une anamnèse couvrant son parcours migratoire, sa scolarisation, son contexte familial et ses projets, Jaganmay fait part de son doute quant à la pertinence de continuer la prise en charge, ressentant d'importants maux de tête et douleurs à la nuque à l'évocation de sa situation de vie, des difficiles perspectives d'avenir qu'il rencontre. Il dit que cela se produit chaque fois qu'il doit raconter son histoire à de nouvelles personnes et que, finalement, personne ne peut concrètement et matériellement l'aider. Le P-CO l'invite alors à prendre quelques jours de réflexion afin de savoir s'il souhaite poursuivre le processus. Dans cet intervalle, Jaganmay contacte sa personne de référence à l'association qui l'a envoyé, qui l'encourage vivement à poursuivre la démarche. Ayant également reçu de nouvelles informations sur l'impossibilité de se rendre en Belgique, il change d'avis et décide de continuer.

Une approche narrative (Morgan, 2010; White, 2009) est alors utilisée, afin de permettre au consultant d'externaliser ses problèmes pour qu'ils n'envahissent pas l'espace de l'entretien, et de construire une histoire préférée, porteuse de solutions. En effet, durant l'anamnèse, le discours de Jaganmay tournait en boucle autour des problèmes administratifs et était très peu porteur d'espoir. Le P-CO s'assure que les sujets de discussion ne contribuent pas au mal de tête et en propose d'autres si cela devait être le cas.

Le premier entretien consiste à reprendre la demande. Le manque d'outils scientifiquement valides et utilisables avec une population migrante a amené le $\mathrm{P}-\mathrm{CO}$ à proposer un exercice à travers le photo-langage provenant d'un jeu de société, avec une méthodologie de type Q-sort (Block, 1961) afin d'aborder les questions sur une dimension différente de celle du discours rationnel. Ainsi, on propose à Jaganmay, par l'intermédiaire d'un photo-langage, de choisir quatre cartes (Figure 1): la première représentant sa situation actuelle, la deuxième une situation idéale dans laquelle il souhaiterait se trouver, la troisième les obstacles qui l'empêchent d'atteindre cette situation idéale et, finalement, la quatrième représentant les attentes qu'il a vis-à-vis de nos prestations, afin de chercher à dépasser ces obstacles. Le photo-langage utilisé provient d'un jeu appelé Dixit ${ }^{\mathrm{TM}} 6$.

6. http://www.libellud.com/jeux/dixit 


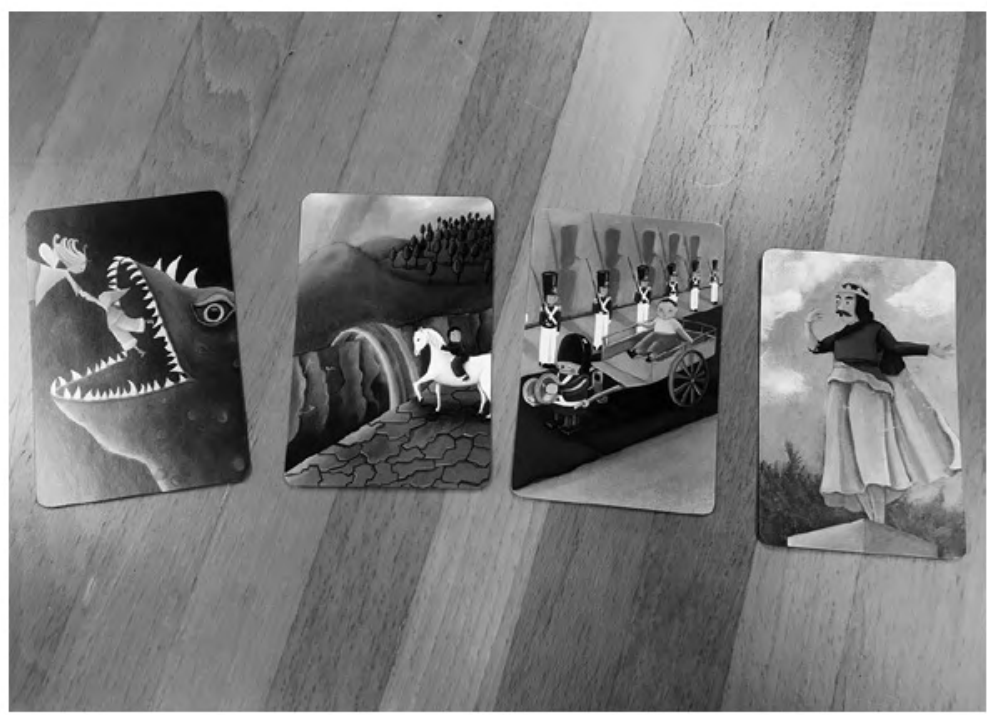

Figure 1. Cartes choisies par Jaganmay pour saisir sa demande.

La première photo à gauche de la Figure 1 illustre sa situation actuelle, vécue comme menaçante (le monstre) et risquant d'emporter notre consultant (le personnage avec un chapeau), ainsi que les personnes qui cherchent à l'aider (l'ange). La deuxième indique le pays verdoyant dans lequel il aimerait se rendre, par opposition à l'environnement aride dans lequel il se trouve actuellement. Ce nouvel environnement permettrait d'avoir des projets et d'effectuer une formation. La troisième carte représente l'administration qui le transporte, tel le personnage dans le chariot, sans qu'il puisse décider quoi que ce soit et sans que la situation change (les soldats sont constamment présents de manière menaçante). Le personnage dans le chariot n'a aucun pouvoir d'action. Cette carte révèle un important sentiment d'impuissance. La quatrième carte représente l'administration suisse, qu'il faudrait pouvoir invoquer afin de savoir quel avenir elle réserve à sa famille. Le P-CO lui explique qu'il ne dispose pas de cette marge d'action et qu'il ne peut rien faire dans ce cadre. Il lui propose de se centrer sur les consultations et de choisir une nouvelle carte, en fonction de ses attentes envers la démarche d'orientation (Figure 2). 


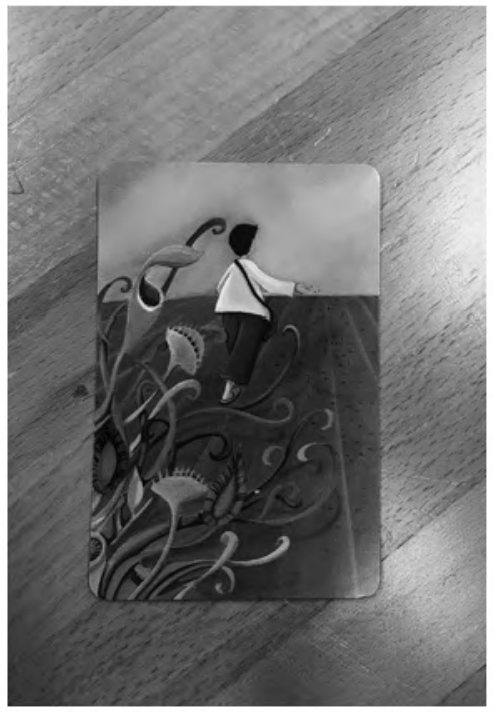

Figure 2.

Carte choisie par Jaganmay pour exprimer ses attentes envers la démarche d'orientation.

L'explication qu'il en donne n'est pas très claire. Le personnage jetant les graines représente le P-CO. Les graines que le personnage sème seraient un projet de Jaganmay, auquel le $\mathrm{P}-\mathrm{CO}$ pourrait contribuer par une aide au financement. Le jeune homme ne souhaite toutefois pas approfondir cette idée. Malgré le discours un peu confus autour de cette carte, elle permet d'entrevoir une possibilité de demande et un projet qui pourrait être porteur d'espoir: semer des graines malgré la présence des plantes menaçantes en premier plan.

Durant l'entretien suivant, le P-CO débute une activité nommée arbre de vie (Denborough, 2011). Celle-ci a pour objectif de permettre à Jaganmay de se raconter de manière différente que dans un récit saturé de problèmes, comme celui expliquant son parcours de vie en Suisse. On lui demande de dessiner un arbre et d'identifier les événements marquants dans son passé, ses ressources et ses compétences, ses projets, les personnes pouvant le soutenir et les cadeaux que lui a amenés la vie. Ces différents éléments seront représentés, respectivement, dans les racines de l'arbre, dans son tronc, dans ses branches, dans ses feuilles et dans ses fruits. Jaganmay parle de deux 
événements marquants: son expérience de travail dans la vente, en Inde, avec son père, identifiant ce dernier comme une figure essentielle dans sa vie, et la décision de sa famille de venir tenter sa chance en Europe. Il identifie deux projets pour son futur : être actif et pouvoir travailler. Plusieurs membres de sa famille sont identifiés comme des soutiens.

La prise en charge se poursuivra en terminant l'activité autour de l'arbre, afin d'aider Jaganmay à identifier ses ressources et à définir ses projets. Un meilleur ancrage dans sa situation de vie, actuellement très instable, est ainsi visé. Une réévaluation de sa demande par rapport à la prise en charge sera ensuite effectuée.

En exposant cette situation, nous pouvons constater que Jaganmay manifeste un vécu traumatique, probablement dû à son parcours migratoire et à ses conditions de vie en Suisse. Il est essentiel de prendre cette spécificité en compte dans la prise en charge, d'être attentif.ve à ce que le récit portant sur les éléments traumatisants ne le soit pas à nouveau. Une approche narrative et des outils non verbaux soutiennent le travail sur le récit de vie, ouvrant des possibilités à la personne de se raconter hors de son contexte de migrant.e, s'appuyant sur ses forces et ses ressources, et non uniquement sur ses manquements et conditions de vie difficiles. Sans occulter les éléments difficiles, cette méthode permet un travail de réflexion personnelle et d'aide à l'élaboration de projets.

\subsection{VUE D'ENSEMBLE ET ANALYSE DES CAS}

Dans les deux situations présentées, nous constatons l'importance d'inclure les éléments contextuels à la prise en charge et de les considérer comme déterminants dans le processus de prise de décision. Les attentes familiales et le projet migratoire des parents dans la situation d'Alberto et le contexte de vie difficile ainsi que l'insatisfaction à vivre en Suisse pour la famille de Jaganmay constituent des éléments incontournables dans le processus d'accompagnement proposé par le P-CO. Le bilan d'orientation doit donc permettre d'intégrer ces éléments à un récit de vie, afin que les consultants puissent se les approprier, permettant ainsi à des projets personnels et professionnels d'émerger et de s'élaborer en cohérence avec le parcours de vie global.

Une double migration rend les parcours des deux cas présentés encore plus complexes et difficiles. Bien que dans les deux situations les migrations familiales aient été volontaires et liées 
à des raisons économiques, cela n'implique pas pour autant que tous les membres de la famille vivent cette transition de la même manière. En ce qui concerne les stratégies d'acculturation, bien que la distance séparant la culture du pays d'accueil et celle du pays d'origine ne soit pas grande pour Alberto, celui-ci montre, au début de la prise en charge, une difficulté d'adaptation. Ce n'est qu'une fois qu'il a compris qu'un retour dans son pays n'est pas envisageable qu'il commence à penser à s'intégrer dans le pays d'accueil à travers un projet professionnel. Quant à Jaganmay, malgré la grande distance culturelle qui existe entre la culture de son pays d'origine et celle du pays d'accueil, l'incertitude de son séjour en Suisse et ses problèmes de santé, il est davantage dans une stratégie d'intégration (Berry, 1997) en montrant sa volonté à entrer dans une démarche d'orientation afin de définir un projet professionnel.

Concernant la démarche d'orientation et l'insertion professionnelle, dans les deux situations, le $\mathrm{P}-\mathrm{CO}$ a proposé une prise en charge adaptée et personnalisée en mettant en pratique ses compétences multiculturelles (Jacob \& Greggo, 2001). Dans le cas d'Alberto, des interventions collectives ont permis au primo-arrivant d'apprendre davantage sur le système de formation suisse et les différentes professions qui y existent. Ainsi, le P-CO a pris en compte la méconnaissance du système de formation suisse que peuvent manifester les migrants. Les interventions individuelles ont permis, quant à elles, de travailler davantage sur les caractéristiques de la personne tout en intégrant son histoire de migration et la place de la famille. Le P-CO a ainsi mobilisé ses savoirs et son savoir-être multiculturels pour une bonne compréhension et analyse de la situation. Dans le cas de Jaganmay, une prise en charge individuelle avec un bilan d'orientation classique a été mise en place dans un premier temps. Toutefois, cette prise en charge a dû être adaptée. En effet, un premier entretien avec une anamnèse classique de la situation n'ayant pas favorisé l'alliance de travail (Massoudi, Masdonati, Clot-Siegrist, Franz-Pousaz, \& Rossier, 2008), le P-CO a eu recours à une approche narrative, moins intrusive que l'anamnèse classique, afin de s'approcher au plus près de la réalité du consultant. Ainsi, il a permis à Jaganmay de s'engager et de poursuivre sa démarche d'orientation. De plus, il a mis en œuvre ses savoir-faire multiculturels en utilisant des outils d'intervention adaptés à la population migrante. 


\section{PERSPECTIVES}

\subsection{PERSPECTIVES POUR L'ACCOMPAGNEMENT}

Les deux cas cliniques présentés dans ce chapitre permettent de mettre en lumière l'hétérogénéité de la population migrante. Chaque migrant.e a un vécu propre, ses repères culturels, valeurs, expériences, compétences qui le singularisent. L'accompagnement de ce type de population exige donc de prendre en considération cette singularité, accessible au $\mathrm{P}-\mathrm{CO}$ à travers la narration. Or le stress d'immigration, l'incertitude, les vécus traumatiques pré-migratoires et d'autres facteurs peuvent provoquer chez les migrant.e.s des malaises ou troubles somatoformes (Ritsner, Ponizovsky, Kurs, \& Modai, 2000) qui pourraient sérieusement entraver leur narration. Les maux de tête et douleurs de nuque ressentis par Jaganmay à l'évocation de sa situation de vie et des difficiles perspectives d'avenir qu'il rencontre en sont une illustration. Dans de pareils cas, la maîtrise par les P-CO d'autres méthodologies qui faciliteraient l'expression des consultant.e.s se révèle capitale. Par exemple, le Q-sort permet à l'individu de retracer son propre état en classant les énoncés selon sa propre vision (Gauzente, 2005). Cet outil est également adapté aux populations n'ayant pas un niveau d'éducation élevé. Cependant, il faut veiller à ce que les cartes proposées puissent permettre aux consultant.e.s de se décrire ou d'exprimer la perception qu'elles et ils ont de leur propre situation. La formation à la maîtrise d'autres méthodologies par le $\mathrm{P}-\mathrm{CO}$ en orientation est donc nécessaire.

Pour les migrant.e.s en provenance de certains milieux culturels et se déplaçant en famille, les projets individuels font souvent partie d'un projet familial plus large (Zéroulou, 1988). De ce fait, un accompagnement centré sur les individus isolés des membres de leur famille perdra certainement en efficacité. La famille étant le premier système de soutien dont peuvent bénéficier les individus, leur inclusion dans le processus d'orientation permet aux jeunes concerné.e.s de ne pas "se sentir seul.e.s", étant donné que ce processus peut leur paraître difficile et parfois frustrant. En effet, les jeunes migrant.e.s peuvent éprouver des frustrations durant leur orientation, par exemple lorsque leurs compétences réelles ne leur permettent pas d'accéder aux formations souhaitées. Dans de pareilles situations, le fait pour ces jeunes de se 
sentir encadré.e.s et soutenu.e.s par leur famille leur permet de gérer ces moments difficiles, de développer une représentation positive de la démarche d'orientation, d'avoir confiance en soi et de développer un sentiment de compétence nécessaire à la réussite dans tout projet (Stebleton, 2007).

La validation des acquis de l'expérience (VAE) permet aux individus de valoriser leurs acquis et d'optimiser leur employabilité (Guichard \& Huteau, 2006). Elle est très développée dans certains pays, dont la France, et est récente en Suisse, où elle apparait sur le plan national en 2004 (Évéquoz, 2012). En Suisse, elle est ouverte aux adultes ayant au minimum cinq ans de pratique professionnelle ${ }^{7}$. Étant donné que les migrant.e.s ne peuvent remplir la condition d'accès à la VAE à l'arrivée, elles et ils ne sont donc pas éligibles à cette procédure. Les $\mathrm{P}-\mathrm{CO}$ en orientation peuvent parfois se sentir impuissant.e.s dans l'accompagnement des personnes migrantes lorsqu'il n'existe pas de cadre institutionnel leur permettant de prendre en compte les compétences réelles des bénéficiaires ou de les aider à les optimiser. Une difficulté fréquemment rencontrée est celle des migrant.e.s arrivant sans pièce justificative attestant d'une certification antérieure, celle-ci étant oubliée dans la fuite de la guerre, égarée ou tout simplement non délivrée, selon les cas. Certes, les compétences d'un·e migrant·e peuvent ne pas correspondre à celles attendues ou exigées dans le pays d'accueil en vue d'entrer dans une formation donnée ou d'exercer un métier en particulier, les conditions de travail pouvant différer considérablement suivant le pays. Cela n'explique pas pour autant que la reconnaissance et la validation des acquis soient avortées. Or une telle procédure pour migrant.e.s est inexistante dans la plupart des pays de l'OCDE, où le système de reconnaissance même des professions réglementées semble être complexe (OCDE, 2004). D’une part, cette procédure permettrait aux personnes migrantes de se sentir valorisées et les protègerait d'une déqualification professionnelle. D'autre part, elle leur permettrait de se rendre elles-mêmes compte de leurs insuffisances par rapport aux compétences attendues et, conséquemment, de s'investir dans la définition et la réalisation d'un projet professionnel. Ces procédures devraient intervenir après que les migrant.e.s ont atteint un certain niveau de compétences linguistiques.

7. Ordonnance sur la formation professionnelle, article 32. 


\subsection{PERSPECTIVES DE RECHERCHE}

Une adaptation au milieu scolaire, pour les plus jeunes, ainsi qu'une insertion sur le marché du travail, pour les adultes, sont capitales pour une bonne intégration dans le pays d'accueil. Cependant, les personnes migrantes rencontrent plusieurs difficultés ou barrières en essayant d'atteindre ces objectifs, nécessitant souvent un accompagnement en OSP individualisé et adapté à leur situation. Très peu de recherches ont été menées sur la pertinence des outils d'évaluation utilisés dans l'accompagnement des primo-arrivants (Leong, Bartram, Cheung, Geisinger, \& Iliescu, 2016). De même, l'adaptation des outils d'accompagnement (de type bilan de compétences) à ce type de population a été également peu explorée. Par ailleurs, beaucoup d'études ont été menées sur l'intégration des migrant.e.s en contextes anglo-saxons. La plupart de ces études se sont intéressées aux caractéristiques sociodémographiques et aux facteurs contextuels jouant un rôle important dans leur intégration. Cependant, les ressources psychologiques dont disposent les personnes migrantes et la manière dont elles les déploient dans leur adaptation sont peu explorées. Comme le relèvent Motti-Stefanidi et Masten (2017), la résilience est un exemple des ressources dont peuvent disposer les migrant.e.s et sur lesquelles les recherches devraient se centrer davantage au lieu de se focaliser uniquement sur les faiblesses que présente cette population. Travailler sur les forces dont dispose cette population revêt une grande importance, car elles représentent le moteur permettant aux individus de s'adapter au changement (Bimrose \& McNair, 2011; Savickas et al., 2009).

D'une part, la migration représente une situation éprouvante, amenant les individus à se reconstruire. Les expériences négatives vécues durant la période pré-migratoire couplées aux difficultés rencontrées dans cette quête peuvent engendrer, chez les migrant.e.s, des atteintes à la santé mentale, notamment une perte de sens (Chen, 2008). D'autre part, elle est porteuse de transitions multiples. Dans ces contextes, la construction de sens devient un moyen qui permettrait aux migrant.e.s d'être au clair sur ce qu'elles et ils sont en train de vivre et de développer des représentations réalistes de leur situation (Masdonati \& Zittoun, 2012). Selon Bernaud, Lhotellier, Sovet, Arnoux-Nicolas et Pelayo (2015), l'accompagnement psychologique à la construction du sens relève d'une "prévention tertiaire» (p. 2), étant donné qu'elle intervient ultérieurement à une transition porteuse d'un changement important dans la vie des 
migrant.e.s. De ce point de vue, les pratiques d'orientation incluant un accompagnement à la construction du sens aussi bien de leur vie que de leur travail sont d'une pertinence évidente auprès de cette population. Les outils d'accompagnement récemment développés par Bernaud et ses collègues (2015) pourraient être adaptés pour l'accompagnement de cette population. En effet, la démarche proposée par ces auteur.e.s consiste à amener les bénéficiaires à identifier elles-mêmes ou eux-mêmes leurs propres valeurs et à définir la place qu'elles occuperont dans la construction de leur nouvelle identité.

\section{RÉFÉRENCES BIBLIOGRAPHIQUES}

AbKhezr, P., McMahon, M., \& Rossouw, P. (2015). Youth with refugee backgrounds in Australia: Contextual and practical considerations for career counsellors. Australian Journal of Career Development, 24, 71-80. Doi : 10.1177/1038416215584406.

American Psychological Association (2003). Guidelines on Multicultural Education, Training, Research, Practice, and Organizational Change for Psychologists. The American Psychologist, 58, $377-$ 402. Doi : 10.1037/0003-066X.58.5.377.

American Psychological Association, Presidential Task Force on Immigration (2012). Crossroads: The psychology of immigration in the new century. En ligne: [http://www.apa.org/topics/immigration/report.aspx].

Amundson, N. E., Yeung, T., Sun, I., Chan, K., \& Cheng, J. (2011). The transition experiences of successful Chinese immigrants. Journal of Employment Counselling, 48, 129-135. Doi: 10.1002/j.2161-1920.2011.tb01119.x.

Arends-To'Th, J., \& VAn de Vijver, F. J. R. (2003). Multiculturalism and acculturation: views of Dutch and Turkish-Dutch. European Journal of Social Psychology, 33, 249-266. Doi: 10.1002/ejsp.143.

Arulmani, G. (2014). The cultural preparation process model and career development. In G. Arulmani, A. J. Bakshi, F. T. L. LeOnG, \& A. G. WATTS (éds), Handbook of career development: International perspectives (pp. 81-104). New York: Springer.

Bernaud, J. L., Lhotellier, L., Sovet, L., Arnoux-Nicolas, C., \& Pelayo, F. (2015). Psychologie de l'accompagnement: Concepts et outils pour développer le sens de la vie et du travail. Paris: Dunod. 
Berry, J. W. (1997). Immigration, acculturation, and adaptation. Applied Psychology, 46, 5-34. Doi: 10.1111/j.1464-0597.1997. tb01087.x.

Berry, J. W. (2006). Contexts of acculturation. In D. L. SAM \& J. W. BERRY (éds), Cambridge handbook of acculturation psychology (pp. 27-42). Cambridge: Cambridge University Press.

Berry, J. W., Poortinga, Y. H., Breugelmans, S. M., Chasiotis, A., \& SAM, D. L. (2011). Cross-cultural psychology: Research and applications (3e edition). Cambridge: Cambridge University Press. Doi : 10.1017/CBO9780511974274.

Berry, J. W., \& SAM, J. (1997). Acculturation and adaptation. In J.W. Berry, M. Segall, \& C. Kagitcibasi (éds), Handbook of cross-cultural psychology (pp. 291-326). Boston: Allyn \& Bacon.

Bimrose, J., \& McNair, S. (2011). Career support for migrants: Transformation or adaptation? Journal of Vocational Behavior, 78, 325-333. Doi : 10.1016/j.jvb.2011.03.012.

BLOCK, J. (1961). The Q-sort method in personality assessment and psychiatric research. Springfield (IL): Charles C. Thomas.

Blustein, D. L., McWhirter, E. H., \& Perry, J. C. (2005). An emancipatory communitarian approach to vocational development theory, research, and practice. Counseling Psychologist, 33, 141-179. Doi : $10.1177 / 0011000004272268$.

Busacca, L. A., \& Rehruss, M. C. (éds) (2016). Postmodern career counseling: A handbook of culture, context, and cases. Alexandria (VA) : American Counseling Association / Wiley.

Camilleri, C. (1990). Identité et gestion de la disparité culturelle: Essai d'une typologie. In C. Camilleri, J. Kastersztein, E. Lipianski, H. Malewska-Peyre, I. Taboada-Léonettiet, \& A. VASQUEZ (éds), Stratégies identitaires (p.85-110). Paris: PUF.

Cardu, H., \& SAnschagrin, M. (2004). Une exploration des représentations de l'identité professionnelle et de l'altérité culturelle d'intervenants de l'orientation socioprofessionnelle qui agissent auprès des femmes immigrantes. Carriérologie, 9, 595-611.

Chen, C.P. (2008). Career guidance with immigrants. In J. ATHANASOU \& R. VAN EsBROECK (éds), International handbook of career guidance (pp. 419-442). New York: Springer. 
Chen, C. P., \& Hong, J. W. L. (2016). Career Development of Chinese Canadian Professional Immigrants. The Career Development Quarterly, 64, 272-286. Doi : 10.1002/cdq.12060.

Codell, J., Hill, R., Woltz, D., \& Gore, P. (2011). Predicting meaningful employment for refugees: The influence of personal characteristics and developmental factors on employment status and hourly wages. International Journal for the Advancement of Counselling, 33, 216-224. Doi : 10.1007/s10447-011-9125-5.

Cohen, L., Arnold, J., \& O’Neill, M. (2011). Migration: Vocational perspectives on a complex and diverse transition. Journal of Vocational Behavior, 78, 321-324. Doi : 10.1016/j.jvb.2011.03.011.

Colic-Preisker, V., \& Tilbury, F. (2006). Employment niches for recent refugees: Segmented labour market in twenty-first century Australia. Journal of Refugee Studies, 19, 203-229. Doi: 10.1093/ jrs/fej016.

Constantine, M. G., Miville, M. L., Warren, A. K., Gainor, K. A., \& LEWIS-Coles, M. E. L. (2006). Religion, spirituality, and career development in African American college students: A qualitative inquiry. Career Development Quarterly, 54, 227-241. Doi : 10.1002/j.2161-0045.2006.tb00154.x.

DeEn, N. (2002). Les jeunes nouveaux immigrés dans les sociétés européennes: implications pour l'éducation et le conseil. Orientation scolaire et professionnelle, 31, 163-178. Doi : 10.4000/osp.4767.

Demes, K. A., \& Geeraert, N. (2014). Measures matter: Scales for adaptation, cultural distance, and acculturation orientation revisited. Journal of Cross-Cultural Psychology, 45, 91-109. Doi: 10.1177/0022022113487590.

Denborough, D. (2011). L'approche narrative collective. Paris: Hermann.

Duffy, R. D., Blustein, D. L., Diemer, M. A., \& Autin, K. L. (2016). The psychology of working theory. Journal of Counseling Psychology, 63, 127-148. Doi: 10.1037/cou0000140.

Durante, F., \& Favre, S. (2012). Accompagner l'insertion professionnelle de jeunes migrants: spécificités dans le travail de psychologue en orientation. In C. Durussel, E. Corbaz, E. Raimondi, \& M. Schaller (éds), Pages d'accueil (pp. 277-290). Lausanne: Antipodes. 
ÉvÉquOZ, G. (2012). La validation des acquis de l'expérience en Suisse: quel rôle pour l'orientation. L'orientation scolaire et professionnelle, 41, 1-15. Doi: 10.4000/osp.3812.

Fargues, P. (2016) Un million de migrants arrivés sans visa en Europe en 2015: Qui sont-ils? Population et Sociétés, 532, 1-3. Paris: Institut national d'études démographiques.

Flores, L. Y., Hsieh, C., \& ChiaO, H. (2011). Vocational psychology and assessment with immigrants in the United States: Future directions for training, research, and practice. Journal of Career Assessment, 19, 323-332. Doi: 10.1177/1069072710395538.

Gauzente, C. (2005). La méthodologie Q et l'étude de la subjectivité. In P. Roussel \& F. WACHEUX (éds), Management des ressources humaines: méthodes de recherche en sciences humaines et sociales, (pp. 177-206). Bruxelles: De Boeck.

Gendre, F., Capel, R., \& Rossé, R. (2006). Intérêts (LIVAP + LIMET) : manuel pratique: une analyse fonctionnelle du comportement décisionnel relatif aux préférences en matière d'intérêts professionnels mesurés par le biais de réponses à des listes de verbes (LIVAP) et de professions (LIMET). Actualités psychologiques, 2. Lausanne: Institut de psychologie/Université de Lausanne.

Guichard, J., \& Huteau, M. (2006). Psychologie de l'orientation. Paris: Dunod.

Hatoss, A., O’Neill, S., \& Eacersall, D. (2012). Career choices: Linguistic and educational socialization of Sudanese-background high-school students in Australia. Linguistics and Education, 23, 16-30. Doi : 10.1016/j.linged.2011.10.003.

Hofstede, G. (1991). Empirical models of cultural differences. In N. Bleichrodt \& P. J. D. Drenth (éds), Contemporary issues in cross-cultural psychology (pp. 4-20). Lisse: Swets \& Zeitlinger.

JACKSON, S., \& BAUDER, H. (2014). Neither temporary, nor permanent: The precarious employment experiences of refugee claimants in Canada. Journal of Refugee Studies, 27, 360-381. Doi: 10.1093/ $\mathrm{jrs} / \mathrm{fet} 048$.

JaCOB, E. J., \& GregGo, J. W. (2001). Using counselor training and collaborative programming strategies in working with international students. Journal of Multicultural Counseling and Development, 29, 73-88. Doi : 10.1002/j.2161-1912.2001.tb00504.x. 
Jong, G. F., Chamratrithirong, A., \& Tran, Q. G. (2002). For better, for worse: Life satisfaction consequences of migration. International Migration Review, 36, 838-863. Doi: 10.1111/j.17477379.2002.tb00106.x.

Kamanzi, P. C., Bastien, N., Doray, P., \& Magnan, M. O. (2016). Immigration et cheminements scolaires aux études supérieures au Canada: Qui y va et quand? Une analyse longitudinale à partir du modèle de Cox. Revue Canadienne d'Enseignement Supérieur, 46, 225-248.

Kanouté, F. (2002). Profils d'acculturation d'élèves issus de l'immigration récente à Montréal. Revue des Sciences de l'Éducation, 28, 171-190. Doi : 10.7202/007154ar.

Kennedy, T., \& Chen, C. P. (2012). Career counselling new and professional immigrants: Theories into practice. Australian Journal of Career Development, 21, 36-45. Doi: $10.1177 / 103841621202100205$.

Koert, E., Borgen, W. A., \& Amundson, N. E. (2011). Educated immigrant workers doing well with change: Helping and hindering factors. Career Development Quarterly, 59, 194-207. Doi: 10.1002/j.2161-0045.2011.tb00063.x.

Lent, R. W., Brown, S. D., \& Hackett, G. (1994). Toward a unifying social cognitive theory of career and academic interest, choice, and performance. Journal of Vocational Behavior, 45, 79-122. Doi : 10.1006/jvbe.1994.1027.

Leong, F. T. L., Bartram, D., Cheung, F. M., Geisinger, K. F., \& IliesCu, D. (2016). The ITC international handbook of testing and assessment. New York: Oxford University Press.

Leong, F. T. L., \& Flores, L. Y. (2015). Career interventions with racial and ethnic minority clients. In P. J. HARTUnG \& M. L. SAVICKAS (éds), APA Handbook of Career Intervention: Vol. 1 Foundations (pp. 225-242). Washington: American Psychological Association.

LeOng, F. T. L., \& Hartung, P. (1997). Career assessment with culturally different clients: Proposing an integrative-sequential conceptual framework for cross-cultural career counseling research and practice. Journal of Career Assessment, 5, 183-202. Doi: $10.1177 / 106907279700500205$.

Masdonati, J., \& Zittoun, T. (2012). Les transitions profession- 
nelles: processus psychosociaux et implications pour le conseil en orientation L'Orientation scolaire et professionnelle, 41, 229-253. Doi : $10.4000 /$ osp.3764.

Massoudi, K., Masdonati, J., Clot-Siegrist, E., Franz-Pousaz, S., \& Rossier, J. (2008). Évaluation des effets du counseling d'orientation: influence de l'alliance de travail et des caractéristiques individuelles. Pratiques Psychologiques, 14, 117-136. Doi: 10.1016/j. prps.2007.11.010.

Meca, A., Cobb, C., Xie, D., Schwartz, S. J., Allen, C., \& HunTER, R. (2018). Exploring adaptive acculturation approaches among undocumented Latinos: A test of Berry's model. The Counseling Psychologist, 45, 1115-1140. Doi : 10.1177/0011000017745978.

Mamgain, V., \& Collins, K. (2003). Off the boat, now off to work: Refugees in the labour market in Portland, Maine. Journal of Refugee Studies, 16, 113-146. Doi: 10.1093/jrs/16.2.113.

Morgan, A. (2010). Qu'est-ce que l'approche narrative? Paris: Hermann.

Motti-Stefanidi, F., \& Masten, A. S. (2017). A resilience perspective on immigrant youth adaptation and development. In N.J. Cabrera \& B. Leyendecker (éds), Handbook on Positive Development of Minority Children and Youth (pp. 19-34). Cham (Suisse): Springer International Publishing. Doi: 10.1007/978-3-31943645-6_2.

Muller Mirza, N., \& Alber, J.-L. (sous presse). Intégration professionnelle et transformations identitaires d'infirmières étrangères en Suisse en demande de reconnaissance: une analyse narrative. In T. Perez-Roux, M. Deltand, C. Duchesne \& J. Masdonati (éds), Parcours professionnels, transitions et transformations identitaires: le sujet au cour des évolutions dans le champ de l'éducation et de la formation. Montpellier, France: Presses universitaires de la Méditerranée.

Obschonka, M., Hahn, E., \& Bajwa, N. U. H. (2018). Personal agency in newly arrived refugees: The role of personality, entrepreneurial cognitions and intentions, and career adaptability. Journal of Vocational Behavior. Advanced online publication. Doi: 10.1016/j.jvb.2018.01.003.

Organisation de coopération et de développement économiques (OCDE) (2004). Les migrants et l'emploi, volume 3: L'intégration 
sur le marché du travail en Autriche, Norvège et Suisse. Paris: Éditions OCDE. Doi : 10.1787/9789264167919-fr.

Organisation de coopération et de développement économiques (OCDE) (2017). Perspective des migrations internationales 2017. Paris : Éditions OCDE. Doi : 10.1787/migr_outlook-2017-fr.

Organisation de coopération et de développement économiques (OCDE) (2018). Population étrangère. Paris: Éditions OCDE. En ligne: [https://data.oecd.org/fr/migration/population-etrangere.htm].

Perdrix, S., Charvoz, L., \& Rossier, J. (2009). Évaluation éthique en psychologie: une utilisation respectueuse des différents instruments psychométriques. Psychoscope, 30, 24-27.

Redfield, R., Linton, R., \& Herskovits. M. (1936). Memorandum on the study of acculturation. American Anthropologist, 38, 149-152.

ReItZ, J. G. (2007). Immigrant employment success in Canada, Part I: Individual and contextual causes. Journal of International Migration and Integration, 8, 11-36. Doi: 10.1007/s12134-007-0001-4.

Ritsner, M., Ponizovsky, A., Kurs, R., \& Modai, I. (2000). Somatization in an immigrant population in Israel: A community survey of prevalence, risk factors, and help-seeking behavior. American Journal of Psychiatry, 157, 385-392. Doi: 10.1176/appi.ajp.157.3.385.

Rossier, J., \& Duarte, M. E. (in press). Testing and assessment in an international context: Cross-and multi-cultural issues. In $\mathrm{H}$. PERERA \& J. Athanasou (éds), International handbook of career guidance ( $2^{\mathrm{e}}$ édition). New York: Springer Science.

Sarot, A., Girard, L. C., Chomentowski, M., Revah-Lévy, A., Falissard, B., \& Moro, M. R. (2017). L'insertion scolaire des élèves issus de l'immigration - enjeux et perspectives de prévention. Neuropsychiatrie de l'enfance et de l'adolescence, 65, 180-187. Doi: 10.1016/j.neurenf.2017.03.006.

Savickas, M. L., Nota L., Rossier J., Dauwalder J.-P., Duarte M. E., Guichard J., Soresi S., Van Esbroeck R., \& Van Vianen, A. E. M. (2009). Life designing: A paradigm for career construction in the 21st century. Journal of Vocational Behavior, 75, 239250. Doi : $10.1016 /$ j.jvb.2009.04.004.

Schultheiss, D., \& DAVIS, B. L. (2015). Immigrant workers: career concerns and barriers. In P. J. Hartung \& M. L. SAVICKas (éds), 
APA Handbook of Career Intervention: Vol. 1 Foundations (pp. 258271). Washington: American Psychological Association.

Secrétariat d'État à la formation, à la recherche et à l'innovation (Sefri) (2018). La formation professionnelle en Suisse. Faits et chiffres 2018. En ligne: [https://www.sbfi.admin.ch/sbfi/fr/home/services/ publications/formation-professionnelle.html].

Secrétariat d'État aux migrations (2018). Statistiques en matière d'asile. Décembre 2017. En ligne: [https:/www.sem.admin.ch/sem/ $\mathrm{fr} /$ home/publiservice/statistik /asylstatistik/archiv/ 2017/12.html].

Stebleton, M. J. (2007). Career counseling with African immigrant college students: Theoretical approaches and implications for practice. Career Development Quarterly, 55, 290-312. Doi: 10.1002/j.2161-0045.2007.tb00085.x.

Stebleton, M. J. (2012). The meaning of work for Black African immigrant adult college students. Journal of Career Development, 39, 50-75. Doi: 10.1177/0894845309358888.

Steger, M. F., Shim, Y., Rush, B. R., Brueske, L. A., Shin, J. Y., \& Merriman, L. A. (2013). The minds'eye: A photographicmethod for understandingmeaning in peoples'lives. The Journal of Positive Psychology, 8, 530-541. Doi: 10.1080/17439760.2013.830760.

Stoll, F., Jungo, D., \& Toggwiler, S. (2012). Test d'intérêts en photos (cartes de choix). Berne: SDBB / CSFO.

STUART, R. B. (2004). Twelve practical suggestions for achieving multicultural competence. Professional Psychology: Research and Practice, 35, 3-9. Doi : 10.1037/0735-7028.35.1.3.

Sultana, R. G., \& Watts, A. G. (2008). Career guidance in the Middle East and North Africa. International Journal for Educational and Vocational Guidance, 8, 19-34. Doi : 10.1007/s10775-0079133-5.

UN High Commissioner for Refugees (UNHCR) (2017). Global Trends: Forces Displacement in 2016. En ligne: [http://www. refworld.org/docid/594aa38e0.html].

Vedder, P. H., \& Motti-Stefanidi, F. (2016). Children, families, and schools. In D. L. SAM \& J. BERRY (éds), Cambridge handbook of acculturation psychology (2 édition). Cambridge: Cambridge University Press. 
WeChsler, D., \& Naglieri, J. A. (2006). Wechsler nonverbal scale of ability (WNV). San Antonio (TX): Harcourt Assessment.

Whiston, S. C., Rossier, J., \& Hernandez Barón, P. M. (2016). Working alliance in career counseling: A systematic overview. Journal of Career Assessment, 24, 591-604. Doi: 10.1177/1069072715615849.

White, M. (2009). Cartes des pratiques narratives. Bruxelles: Satas.

Yakushio, O., Backhaus, A., Watson, M., Ngaruiya, K., \& GonZALEZ, J. (2008). Career development concerns of recent immigrants and refugees. Journal of Career Development, 34, 362-396. Doi : $10.1177 / 0894845308316292$.

Yakushro, O., Watson, M., \& Thompson, S. (2008b). Stress and coping in the lives of recent immigrants and refugees: Considerations for counseling. International Journal for the Advancement of Counselling, 30, 167-178. Doi: 10.1007/s10447-008-9054-0.

Zéroulou, Z. (1988). La réussite scolaire des enfants d'immigrés: l'apport d'une approche en termes de mobilisation. Revue française de sociologie, 29, 447-470. Doi : 10.2307/3321625. 



\title{
7. INCLUSION, LIFE DESIGN, COACHING DE CARRIÈRE ET HANDICAP ${ }^{1}$
}

\author{
ILARIA DI MAGGIO, SARA SANTILLI (UNIVERSITÉ DE PADOUE), \\ MARIA CRISTINA GINEVRA (UNIVERSITÉ DE MILANO-BICOCCA), \\ GIUSEPPE MARTELLA ET LAURA NOTA (UNIVERSITÉ DE PADOUE)
}

L

e handicap «fait partie de l'histoire de l'humanité depuis toujours, mais son histoire a évolué» (Schianchi, 2012, p. 11), étant donné que les pratiques sociales, les préjugés et les interventions de réhabilitation n’ont cessé de changer au fil des siècles. Cependant, ce n'est que récemment que le handicap a commencé à faire l'objet d'études scientifiques et à devenir sujet à débats touchant à des questions légales (droit au travail, égalité des chances, etc.), mais soulevant également des enjeux en matière de développement social durable. Le handicap peut être considéré comme la manifestation de limites du fonctionnement individuel dans un contexte social donné, qui représente un inconvénient significatif pour la personne (Shogren, Luckasson, \& Schalock, 2014). Souvent, le fait de présenter un handicap se traduit par davantage d'obstacles professionnels et de défis sur le marché du travail, tels qu'un taux de chômage plus élevé et des inégalités salariales (Schartz, Schartz, \& Blanck, 2002).

Les coachs de carrière appelé.e.s à soutenir les personnes en situation de handicap désireuses de planifier une vie et une carrière futures satisfaisantes peuvent jouer un rôle essentiel pour améliorer leur inclusion ${ }^{2}$ en termes de participation à la vie sociale, civile et professionnelle, compte tenu aussi de la valeur ajoutée que ceci apporte pour la qualité de vie de tout un chacun. En fait, les questions d'inclusion et de participation devraient être mises en lien avec l'orientation professionnelle et la planification de carrière, et ce tout particulièrement lorsque l'on travaille avec des personnes en situation de handicap, plus susceptibles de subir

1. Ce chapitre a été traduit de l'anglais par A. Viscolo, K. Massoudi et J. Masdonati.

2. Le terme "inclusion" a été choisi pour signifier le terme anglais original (inclusion) utilisé par les auteur.e.s et qui représente une approche visant à l'intégration des personnes en situation de handicap, tout en reconnaissant leur droit à la singularité et à la différence. 
l'exclusion ou une marginalisation sociale ou professionnelle. À cet égard, le coaching de carrière, en tant que méthode d'accompagnement en orientation, peut être utile pour aider les personnes en situation de handicap à développer leur vie personnelle et professionnelle, à faire face aux défis professionnels, à améliorer leurs compétences et à faire des choix de carrière (Chung \& Gfroerer, 2003; Reid, 2015).

Partant du principe selon lequel l'inclusion sociale et professionnelle joue un rôle dans le processus qui consiste à concevoir et construire sa vie (Life Design), en particulier pour les personnes en situation de handicap, nous allons d'abord donner dans ce chapitre un aperçu des cadres théoriques les plus récents et les plus reconnus sur l'inclusion. Deuxièmement, autour du paradigme du Life Design, c'est-à-dire une nouvelle approche théorique de l'accompagnement en orientation fondée sur l'épistémologie du constructivisme social (Savickas et al., 2009), nous aborderons la question de l'intervention en mettant l'accent sur le rôle du coaching de carrière visant à soutenir ces personnes dans le processus de conception de leur vie future. Une revue de la littérature sur le coaching de carrière pour les personnes en situation de handicap suivra et, enfin, un cas d'intervention de coaching de carrière sera présenté.

\section{UNE VISION INCLUSIVE DE LA RÉALITÉ}

Le terme inclusion ne se réfere plus à une catégorie particulière d'individus - comme ce fut le cas par le passé et se produit encore aujourd'hui quand on parle d'intégration -, mais à l'ensemble des individus, qu'ils présentent ou non une vulnérabilité ou un handicap (Di Maggio \& Shogren, 2017). Favoriser l'inclusion signifie transformer les communautés en lieux conviviaux pour toutes et tous (Asante, 2002). La lutte pour l'inclusion exige que tou.te.s les citoyen.ne.s et que toutes les institutions jouent un rôle actif pour faciliter la participation de chacun.e dans la vie sociale et civile (Asante, 2002).

L'inclusion devient donc une problématique qui concerne tous les individus et toutes les communautés au sens large. Lorsque l'on parle d'inclusion, on ne vise pas le handicap d'une personne ou d'un groupe d'individus en particulier; on met plutôt l'accent sur les caractéristiques et variables contextuelles qui, en interaction 
avec l'unicité de chaque individu, sont susceptibles de déterminer différents niveaux de participation sociale, civile et professionnelle (Owens, 2015; Shogren, Wehmeyer, Schalock, \& Thompson, 2016). S'intéresser à l'inclusion, c'est donc s'assurer que les contextes puissent garantir à chaque personne, dans son unicité, une pleine participation à la vie sociale, civile et professionnelle. En d'autres termes, viser l'inclusion exige de bien étudier les contextes dans lesquels vivent l'ensemble des individus, car ces contextes (organisations, systèmes, politiques et pratiques sociales, par exemple) peuvent améliorer le fonctionnement et la qualité de vie de l'individu, ainsi que la co-construction de ses forces et faiblesses (Asante, 2002; Shogren et al., 2016).

Comme l'affirme Soresi (2016), ces éléments déterminants apparaissent nettement dans les approches récentes de l'inclusion, par exemple dans le «modèle social du handicap" (Social Model of Disability), l' «approche par les capabilités» (Capability Approach), l' "approche du handicap fondée sur les droits de l'homme» (Human Rights Approach to Disability) et celle du "développement positif des jeunes" (Positive Youth Development). Bien que cette dernière soit moins souvent évoquée en tant que modèle pour les questions de handicap, nous pensons qu'elle peut apporter une contribution significative.

Le «modèle social du handicap» (Owens, 2015) attribue les difficultés rencontrées dans la vie sociale et quotidienne à un écart entre les caractéristiques individuelles et les contraintes contextuelles. Le contexte joue un rôle décisif pour favoriser l'inclusion ou l'exclusion et, par conséquent, cet écart peut être réduit en modifiant les exigences contextuelles et en apportant un soutien pour faciliter la participation. L'Organisation mondiale de la santé (OMS, 2007) et l'American Association of Intellectual and Developmental Disabilities (AAIDD, Schalock et al., 2010) ont souligné qu'il convient de tenir compte du vécu de l'individu, et donc des déterminants environnementaux et contextuels de son fonctionnement. Plus particulièrement, comme le soutient également l'OMS, les actions et le parcours de vie d'un individu ne sont pas déterminés uniquement par le fonctionnement et la structure de son corps, mais influencés par des facteurs contextuels, qui peuvent être physiques, sociaux ou culturels. Selon l'OMS (2007), ces facteurs interviennent au croisement des capacités fonctionnelles de la personne et du contexte dans lequel elle vit, déterminant ainsi ses niveaux de participation 
et d'inclusion. Dans l'ensemble, la diffusion de ce modèle a favorisé les accomplissements des personnes en situation de handicap en s'opposant à la discrimination et la marginalisation, leur permettant ainsi de faire valoir leurs droits au sein de la communauté, et en associant droits civiques et activisme politique (Owens, 2015).

L'«approche centrée sur les capabilités» (Sen, 2006) a été élaborée par le Prix Nobel Amartya Sen, dont les contributions économiques et philosophiques ont favorisé une analyse axée sur l'inclusion. Selon lui, il est possible de parvenir à une répartition équitable et éthique des ressources en considérant, en valorisant et en maximisant les capabilités et le fonctionnement au cours du développement. Par "capabilités", on entend le potentiel de l'individu. Celles-ci sont donc considérées comme des occasions positives d'épanouissement. Un autre élément pris en compte par Sen, que l'on retrouve dans presque tous les modèles d'analyse de la qualité de vie, est l'agentivité, c'est-à-dire la capacité de l'individu et sa propension à déterminer sa vie en fonction du contexte. Selon Norwich (2014), l'approche par les capabilités définit le handicap comme une privation ou une limitation de capacité ou de fonctionnement. Le handicap est considéré comme le résultat de l'interaction des facteurs sociaux et individuels, allant ainsi au-delà de la traditionnelle opposition entre les modèles médical et social du handicap.

Le concept d'agentivité décrit dans l'«approche par les capabilités» est compatible avec l'"approche du handicap fondée sur les droits de l'homme». Selon cette dernière, tous les individus, qu'ils soient ou non en situation de handicap ou de vulnérabilité, sont considérés comme des personnes morales. Tout le monde, et à plus forte raison les individus vulnérables, devrait être en mesure d'exercer un contrôle accru et d'influencer son propre cadre de vie ainsi que les institutions chargées d'assurer le bien-être des citoyens (Donnelly, 2013). Cette approche considère le handicap comme un pan des droits de l'homme, les personnes concernées étant capables de faire valoir leurs droits, de prendre seules des décisions concernant leur vie et d'être des membres actifs de la société (Donnelly, 2013).

L'approche du "développement positif des jeunes" (Positive Youth Development, PYD; Gestsdottir, Lewin-Bizan, von Eye, Lerner \& Lerner, 2009), compatible avec le concept de "capabilités» élaboré par Sen, souligne la pertinence de se concentrer sur les atouts individuels afin de favoriser des trajectoires positives de développement. De plus, conformément au modèle social du handicap, 
une meilleure adéquation entre forces individuelles et ressources contextuelles favorise aussi des niveaux plus élevés de développement positif. Bien que le $P Y D$ ne soit généralement pas appliqué au handicap, Holt et al. (2017) soulignent clairement qu'il est associé à la promotion des occasions d'inclusion des individus favorisant un sentiment d'appartenance porteur de sens.

En dépit de leurs différences significatives, ces divers modèles et approches se distinguent clairement des visions médicales et particularistes du handicap, qui définissent les difficultés rencontrées dans la vie sociale et quotidienne par les personnes en situation de handicap comme des conséquences de composantes biologiques. En outre, pour favoriser le processus d'inclusion, tous ces modèles soulignent l'importance d'aider ces personnes à renforcer leur autodétermination dans leurs contextes de vie actuel et futur. Comme le soutiennent Wehmeyer et collègues (2018), cela pourrait aider tous les individus (avec ou sans handicap) à planifier une vie autonome satisfaisante sur les plans personnel et professionnel, et pourrait être particulièrement utile aux personnes en situation de handicap, qui sont plus susceptibles de souffrir d'exclusion sociale et professionnelle.

Comme nous l'avons vu, l'inclusion implique une participation à la vie sociale et professionnelle de la communauté, compte tenu également de la valeur ajoutée que cela peut apporter pour la qualité de vie des individus. Comme l'affirment Wehmeyer et collègues (2018), il pourrait s'avérer utile de soutenir les personnes en situation de handicap, qui risquent souvent l'exclusion du fait de leurs déficiences, lorsqu'elles s'engagent dans une démarche d'accompagnement en orientation visant à accroître leur niveau de participation sociale et professionnelle. Les accompagnements en orientation destinés à ces personnes devraient être fondés sur les mêmes modèles théoriques, adéquatement personnalisés, et proposer des activités et outils de développement de carrière élaborés pour et testés par des personnes non handicapées. En particulier, ces mesures devraient se concentrer sur les ressources personnelles et contextuelles utiles pour faire face aux défis quotidiens et aux obstacles à l'inclusion des personnes en situation de handicap et à la conception et la construction de leur vie (Ferrari, Sgaramella, \& Soresi, 2015). Cela évitera d'étayer la tendance généralisée qui constiste à considérer surtout les insuffisances et déficits plutôt que les atouts et les variables contextuelles (Aspinwall \& Staudinger, 2003; Ferrari et al., 2015; Keyes \& Haidt, 2003). 
Ce point de vue a récemment été soutenu et mis en évidence par l'approche du Life Design qui, comme nous le verrons ci-après et conformément au concept d'inclusion (Wehmeyer et al., 2018), considère l'individu et son écosystème comme une entité dynamique complexe, résultant d'influences mutuelles et d'une auto-organisation adaptative (Hirschi \& Dauwalder, 2015; Savickas et al., 2009). Selon le paradigme du Life Design, il est essentiel pour chacun.e que les ressources personnelles et contextuelles soient activées et réorganisées dans le but de faciliter les choix, la construction de sa vie et les changements adaptatifs (Ferrari et al., 2015). Il s'ensuit que l'objectif des interventions en orientation est d'aider la personne à construire un processus d'adaptation dynamique entre elle et son contexte, de manière à augmenter la probabilité de connaître des conditions de vie satisfaisantes et une réalisation professionnelle. De plus, cette approche vise à favoriser la construction de soi par le travail et les relations en vue d'atteindre les objectifs fondamentaux du Life Design: des activités pour façonner les intérêts, les capabilités et les aspirations; une adaptabilité pour faire face à l'évolution de soi et des situations; une narrativité permettant de livrer un récit cohérent de son histoire de vie; enfin, une intentionnalité pour donner un sens aux activités et au vécu. Concevoir et construire sa vie (Life Design) nécessite une réflexion sur soi et son environnement, ainsi qu'une réceptivité à la rétroaction et la faculté d'envisager de nouveaux récits de construction de soi (Savickas et al., 2009). C'est la raison pour laquelle l'accompagnement en orientation axé sur le Life Design se concentre sur les stratégies de survie et la dynamique d'adaptation plutôt que sur l'ajout d'informations ou de contenu (Pouyaud, 2015). À cet égard, les activités de coaching de carrière jouent un rôle crucial.

\section{LIFE DESIGN ET COACHING DE CARRIÈRE}

Selon Spence et Grant (2007), le coaching peut se définir comme une conversation collaborative axée sur les solutions et orientée vers l'action, par laquelle la ou le coach favorise une amélioration du vécu, de l'apprentissage autonome et de la performance de la personne qui la ou le consulte, ainsi que l'atteinte de ses objectifs personnels et professionnels. Il s'agit de collaborer avec les consultant.e.s en un processus de réflexion et de création qui les motive à capitaliser sur leurs atouts personnels et professionnels (International Coach Federation-ICF, 2012). 
Alors que le coaching de vie poursuit un objectif plus large, le coaching de carrière consiste en des mesures visant à promouvoir la performance et le développement personnel, généralement axées sur la planification et l'atteinte d'objectifs de carrière (Reid, 2015). Par le coaching de carrière, la ou le coach peut aider la personne à passer en revue les problèmes liés au travail et à poursuivre son développement personnel et professionnel. L'objectif global du coaching de carrière est de soutenir le développement personnel des consultant.e.s dans leur environnement et leur évolution professionnels afin de leur permettre d'identifier clairement leurs compétences, de faire de meilleurs choix de carrière et d'être des employé.e.s plus productifs et plus appréciés (Chung \& Gfroerer, 2003). Les coachs de carrière encadrent leurs consultant.e.s face à des défis professionnels et les incitent à poursuivre des objectifs de carrière réalistes (Byars-Winston, Gutierrez, Topp, \& Carnes, 2011). Elles ou ils aident la personne à planifier son développement de carrière en l'encourageant à envisager des trajectoires professionnelles alternatives et à faire face à tout problème lié au travail, par exemple par le développement de compétences de gestion, un rééquilibrage entre vie privée et vie professionnelle, l'amélioration de sa présentation ou de ses capacités de communication, etc. (Reid, 2015).

Pour bien conceptualiser le coaching de carrière, il convient de le distinguer des autres formes d'accompagnement en orientation, en particulier du conseil en orientation (career counseling). Il y a de nombreux recoupements entre les deux approches, de sorte que Duarte (2018) a conclu que la matrice épistémologique du conseil en orientation et du coaching se caractérise par plus de similitudes que de différences. Ces deux formes d'intervention portent en fait sur l'adaptation de carrière, la planification de carrière et le lien entre problèmes personnels et professionnels (Chung \& Gfroerer, 2003). De plus, ces deux formes d'accompagnement en orientation ont en commun la mise en place d'une alliance de travail, la confidentialité et la définition du nombre de séances (bien que le coaching de carrière ait tendance à durer plus longtemps). Malgré ces similitudes, le coaching de carrière est toutefois davantage axé sur l'action et la résolution de problèmes que le conseil en orientation (Duarte, 2018; Chung \& Gfroerer, 2003). Il est également moins structuré autour des contraintes contextuelles et les coachs de carrière sont susceptibles d'interagir avec les consultant.e.s sur leur lieu de travail ou à domicile, par téléphone ou par internet, voire de 
participer à leurs activités professionnelles pour observer, faire des commentaires, offrir un feed-back et renforcer et mettre en œuvre des plans de carrière. De plus, cette méthode est centrée sur les mesures visant à modifier les comportements, au moyen de stratégies et techniques axées sur les objectifs et empruntées à la thérapie cognitivo-comportementale (TCC) (Brock et al., 2016). Apporter un soutien directement dans les cadres de vie des consultant.e.s et recourir aux stratégies et techniques de la TCC (par exemple, analyse des tâches, techniques d'incitation [prompting] et d'estompage [fading], consignes verbales, rétroaction sur la performance, modélisation et jeux de rôle) peut être extrêmement utile aux personnes en situation de handicap, et ce particulièrement dans leurs processus de planification de carrière et d'insertion professionnelle (Nicholas, Attridge, Zwaigenbaum, \& Clarke, 2015).

Comme le suggèrent Van Esbroeck et Augustijnen (2015), le coaching de carrière s'inscrit dans le cadre plus large du paradigme des interventions de Life Design. Selon eux, le processus de coaching s'articule en quatre phases: (a) définition des objectifs de la consultante ou du consultant en accord avec la ou le coach et les autres personnes significatives (par exemple, l'employeur) (b) réflexion sur soi, (c) conscience de soi et (d) changements relatifs aux aspects professionnels, individuels et personnels. Au cours de ce processus, les consultant.es son considéré.es comme des auteur.e.s qui construisent leurs récits de vie et de carrière par un dialogue avec leurs contextes sociaux et professionnels, en y intégrant leurs expériences, leurs activités et leurs interprétations actuelles et passées (Van Esbroeck \& Augustijnen, 2015).

Selon l'approche du Life Design, le coaching de carrière consiste à soutenir la ou le consultant.e dans son processus de développement de carrière par une construction de soi dans tous les rôles de son parcours de vie. Simultanément, cet accompagnement joue un rôle préventif aidant la ou le bénéficiaire à identifier les évolutions futures et la manière de surmonter les obstacles (Van Esbroeck \& Augustijnen, 2015). En outre, il vise à développer son adaptabilité (faculté de s'adapter aux tâches, transitions et défis de développement de carrière), son intentionnalité (aptitude à donner un sens aux activités et au vécu), sa narrativité (capacité de raconter son histoire de vie de façon claire et cohérente) et son activité (aptitude à entreprendre des activités variées pour identifier les intérêts professionnels, les compétences et les possibles de la [re] création de soi; 
Hartung, 2015), qui sont les objectifs fondamentaux du paradigme des interventions Life Design (Savickas et al., 2009). Enfin, le coaching de carrière fondé sur le Life Design soutient la consultante ou le consultant dans le processus de développement des ressources et compétences (par exemple, courage, espoir, résilience) nécessaires pour faire face aux changements actuels et aux problèmes de transition à long terme, pour atteindre ses objectifs personnels et professionnels et faire face aux défis professionnels (Nota \& Soresi, 2018).

\section{COACHING DE CARRIÈRE}

\section{POUR PERSONNES EN SITUATION DE HANDICAP}

Bien qu'il y ait un soutien théorique au coaching de carrière, peu de recherches ont été effectuées sur ce sujet. La plupart des articles ont surtout porté sur l'impact d'interventions destinées à des adultes en emploi (Theeboom, Beersma \& van Vianen, 2014). Malheureusement, peu d'études ont été menées sur le coaching visant à soutenir les consultant.e.s en situation de handicap dans la conception de leur évolution de carrière et leurs transitions professionnelles. À cet égard, nous aimerions souligner les travaux publiés par Jordan, Gessnitzer et Kauffeld (2016) qui, moyennant un effort de transposition et d'adaptation à la problématique de l'invalidité, peuvent aussi s'avérer pertinents pour les personnes en situation de handicap. Ces auteurs ont élaboré une intervention de coaching de groupe de dix semaines pour lycéens, comprenant des activités encourageant la réflexion sur soi, des discussions avec d'autres participants et des exercices relatifs au développement de carrière. Au cours de l'intervention, les participant.e.s ont commencé par planifier des objectifs professionnels, puis identifié des stratégies et des étapes pour atteindre ces objectifs. Enfin, des activités d'évolution de carrière, telles que la participation à des cours universitaires, la réalisation d'analyses sur internet et la collecte d'informations sur les débouchés, ont été menées. Les résultats concernant l'efficacité de cette intervention de coaching suggèrent que les participant.e.s ont amélioré leur capacité de planification de carrière et leur sentiment d'efficacité personnelle par rapport à la prise de décision en comparaison avec un groupe témoin.

Le coaching de carrière peut également être utile pour aider les personnes à développer des compétences et ressources pour faire face au marché du travail actuel, gérer des transitions planifiées 
ou inattendues, et définir des objectifs personnels ou professionnels. Dans une société mondialisée et un contexte professionnel en constante mutation, l'approche du Life Design met l'accent sur des ressources telles que la capacité d'identifier les opportunités, les compétences sociales et de communication, l'autodétermination, le courage, l'espoir ou l'adaptabilité professionnelle (Nota, Ginevra, \& Santilli, 2015). Cela semble particulièrement important, en particulier pour les personnes en situation de handicap, qui subissent souvent des interventions inefficaces dans l'amélioration de telles ressources (Lindsay et al., 2014).

Signalons encore l'étude de Gilson et Carter (2016) sur une intervention de coaching de carrière réalisée auprès de trois étudiants universitaires atteints de troubles du spectre autistique (TSA) ou d'une déficience intellectuelle, afin de les aider à identifier des activités pertinentes en cours de stage et à développer leur indépendance et leurs compétences pour réussir dans le monde du travail. L'accompagnement comprenait un coaching discret permettant aux coachs de fournir en secret et en direct des retours et renforcements aux bénéficiaires par un dispositif d'oreillette, sans être physiquement présents, ce qui aurait pu être perturbant. Les coachs encourageaient les participants à demander de l'aide à leurs collègues lors de l'exécution d'une tâche et fournissaient des incitations sociales explicites lorsqu'une personne se trouvait à proximité immédiate (par exemple, «Dites bonjour à ce client» ou "À qui pouvez-vous demander ce qu'il faut faire ensuite?»). De plus, un coaching spécifique a été utilisé lorsqu'un·e consultant.e exécutait une tâche difficile ou en apprenait une nouvelle. À la fin de l'intervention, les interactions entre les personnes en situation de handicap et leurs collègues se sont améliorées et la motivation n’a pas faibli lorsque les coachs se sont éloignés pour envoyer des messages discrètement par le biais d'oreillettes. En outre, concernant la pertinence sociale de l'intervention de coaching, les consultant.e.s ont jugé que cette dernière était bénéfique et non stigmatisante.

Green, Grant et Rynsaardt (2007) ont fourni un autre exemple de promotion de l'espoir dans un programme de coaching consistant en dix séances individuelles. Plus précisément, les consultant·e.s ont été formé.e.s pour identifier leurs ressources personnelles utiles pour atteindre leurs objectifs personnels et professionnels, et pour définir leurs propres stratégies et solutions pour réaliser ces objectifs. En outre, comme le suggèrent Ginevra et Santilli (2018), un 
accompagnement pourrait aider les personnes en situation de handicap à développer l'audace nécessaire pour faire face aux difficultés et aux obstacles et pour prendre des décisions professionnelles malgré leurs craintes pour l'avenir. Une telle intervention vise le renforcement du sentiment d'efficacité en matière de gestion des risques en soulignant les avantages d'un comportement courageux et en minimisant la perception du risque.

Enfin, le coaching de carrière est particulièrement utile pour optimiser le fonctionnement professionnel des individus (par exemple, promouvoir la performance et les compétences, le bien-être, l'adaptation, les attitudes professionnelles et l'autorégulation centrée sur les objectifs) et assurer une réussite professionnelle (Theeboom et al., 2014). Il a souvent été utilisé pour promouvoir les emplois protégés, permettant aux personnes en situation de handicap d'accéder et de conserver un emploi rémunéré dans un cadre de travail régulier (Nicholas et al., 2015). Les coachs peuvent jouer plusieurs rôles dans ce processus : aider ces personnes à s'intégrer dans la culture d'entreprise, faire le lien entre elles et leurs collègues ou supérieurs, aider les employés en situation de handicap à apprendre à exécuter leurs tâches conformément aux attentes de leurs supérieurs et à surmonter les éventuelles difficultés, etc. (Nicholas et al., 2015). Comme le relèvent Bennet, Brady, Scott, Dukes et Frain (2010), dans ce processus, les coachs doivent adopter une approche peu intrusive, efficace, renforçante et systématique. De plus, elles ou ils doivent utiliser des stratégies telles que l'analyse des tâches (un ensemble de méthodes permettant de diviser en objectifs plus simples et plus accessibles une tâche initialement trop complexe), les incitations (des consignes gestuelles ou verbales favorisant la réaction souhaitée), l'estompage (la réduction progressive de l'aide apportée pendant l'activité), les rétroactions sur la performance (les retours donnés aux personnes sur leurs actions), la modélisation (une technique fondée sur l'apprentissage par observation, où l'individu observe une autre personne, le modèle, affecter le comportement), enfin, les jeux de rôle (chaque participant.e joue un rôle et agit et réagit aux situations et aux autres participant.e.s à l'exercice, de manière à stimuler l'apprentissage de comportements nouveaux ou alternatifs; Brock et al., 2016).

Mihailidis et al. (2016) ont récemment proposé un exemple d'intervention de coaching de carrière visant à suivre et aider quatre travailleurs présentant des troubles cognitifs à réaliser une tâche d'assemblage en usine, au moyen d'une technologie d'assistance 
cognitive, un système d'incitation non linéaire sensible au contexte (nonlinear contextually aware prompting system - N-CAPS). Le N-CAPS a été configuré pour réduire, par exemple, le temps nécessaire aux consultant·e.s pour exécuter une tâche d'assemblage, limiter le nombre d'erreurs dans cette tâche, etc. Les coachs ont utilisé un dispositif informatique interactif à écran tactile de 25 centimètres de large et sortie audio. L'appareil donnait des incitations automatisées sur les tâches par le biais d'illustrations, de vidéos et d'un coach animé offrant un renforcement positif lorsqu'une tâche était correctement exécutée. De plus, la ou le coach virtuel.le a aidé les consultant.e.s à surmonter les scénarios problématiques. À la fin de l'intervention, les participants ont amélioré leurs compétences dans l'exécution de la tâche, 85,7\% des étapes étant réalisées sans l'aide de la ou du coach.

Outre le soutien direct aux personnes en situation de handicap dans leur cadre de travail, les coachs doivent également promouvoir leur insertion professionnelle, réduire progressivement leur soutien et cultiver et former des soutiens naturels sur le lieu de travail (collègues, employeurs) (Villotti et al., 2017). Favoriser un soutien direct des collègues plutôt que des coachs permet de limiter les intrusions de ces derniers, d'augmenter l'aptitude des personnes en situation de handicap à jouer un rôle actif au travail et de favoriser les liens sociaux sur le lieu de travail.

Plusieurs études ont montré que le soutien naturel des collègues peut être déterminant pour la réussite professionnelle d'une personne en situation de handicap et le maintien de son poste (Corbière et al., 2014; Jenaro, Mank, Bottomley, Doose, \& Tuckerman, 2002). À cette fin, il est important de préparer les collaboratrices et collaborateurs à agir de manière inclusive dans le cadre professionnel en favorisant leur capacité à soutenir la diversité sur le lieu de travail et l'intégration des travailleurs et travailleuses en situation de handicap. Les employeuses, employeurs et collègues doivent être encouragé.e.s à mieux connaître les compétences, forces et valeurs de ces travailleurs et travailleuses, tout en évitant de mettre l'accent sur leurs déficiences (Nota et al., 2015). De plus, les employeuses, employeurs et collègues devraient être formé.e.s au type d'assistance nécessaire pour accroître leur participation et leur réussite professionnelle.

Un exemple de coaching de carrière pour le lieu de travail a été proposé par Farris et Stancliffe (2001), qui ont formé un groupe de collaborateurs et collaboratrices du secteur de la restauration au 
soutien aux employé.e.s en situation de handicap. Le coaching de deux jours comprenait des séances de brainstorming, des conférences, des vidéos, des jeux de rôle avec rétroaction, des activités de groupe, des exercices écrits avec rétroaction et la mise à disposition d'un manuel. Le premier jour, des exercices de sensibilisation aux droits, aux mythes et réalités, ainsi qu'au handicap ont permis de clarifier le rôle des collègues envers les employé.e.s en situation de handicap. La deuxième journée a été consacrée à la manière d'offrir à ces personnes une formation. À la fin de l'intervention, les participant.e.s ont reconnu que la valeur des personnes en situation de handicap sur le lieu de travail était supérieure à celle estimée avant l'intervention. De plus, lorsqu'une personne vivant un handicap était engagée dans le restaurant, ses perspectives d'emploi étaient positives, tendant ainsi à démontrer l'efficacité de cette intervention de coaching.

Dans l'ensemble, les études présentées suggèrent que le coaching de carrière peut se révéler avantageux pour soutenir les personnes en situation de handicap dans leurs projets professionnels, améliorer leurs compétences et ressources pour faire face aux conditions actuelles du marché du travail et favoriser leur accès au marché du travail. En outre, il peut également être utile pour promouvoir leur réussite et leur insertion professionnelle. Conformément à l'approche du Life Design, qui met l'accent sur les interventions et les actions individuelles pour promouvoir le développement et la planification de carrière de l'individu, une étude de cas sur le rôle d'accompagnement d'un adulte en situation de handicap dans la conception de son développement professionnel est présentée ci-dessous.

\section{4. ÉTUDE DE CAS}

Mario, un étudiant de 23 ans, souffre d'une tétraparésie spastique qui affecte la musculature du cou et du tronc et provoque une rigidité musculaire, des spasmes, des pertes d'équilibre et de coordination des mouvements, de l'ataxie et des troubles du langage et de la déglutition. À cause de cette spasticité, Mario doit utiliser un fauteuil roulant. Il est en deuxième année d'un cours de sciences humaines à l'université et a envoyé un e-mail au Centro di Ateneo per la Disabilità e Inclusione (Centre universitaire sur le handicap et l'inclusion) en vue de rencontrer un.e coach susceptible de 
l'aider à s'intégrer à l'université. Dès le début, Mario fait preuve d'une forte motivation au changement, d'espoir d'être capable de définir de nouveaux objectifs et, malgré ses difficultés, de résilience pour réaliser ses attentes.

Mario dit qu'il passe cinq ou six heures par jour devant son ordinateur pour regarder des vidéos et discuter avec ses amis sur Facebook. Il dit aussi qu'il est incapable de se concentrer sur ses études, qu'il n'a pas passé d'examens depuis plus d'un an et qu'il remet en cause le fait même de fréquenter l'université. Il indique également que la relation avec ses parents s'est récemment dégradée, avec des disputes et malentendus fréquents, car il aimerait mener une vie plus indépendante, mais se sent freiné par eux.

Lorsqu'il livre le récit de son parcours scolaire et professionnel, Mario affirme que ce sont ses parents qui ont choisi le lycée professionnel qu'il a fréquenté, alors qu'il aurait préféré aller dans une école préparatoire (lycée classique). Diplômé de son lycée professionnel, il a opté pour une majeure en sciences humaines à l'université, bien que ses parents se soient opposés à ce choix en raison de la distance entre leur domicile et l'Université de Padoue (environ 50 kilomètres), soit un trajet d'environ une heure en train et en bus.

En première année, Mario n’a pu passer que deux examens à cause d'une opération au bras qui l'a obligé à rester alité pendant environ huit mois. Ses parents semblent saisir toutes les occasions de remettre en question sa décision de fréquenter l'université en dépit de son handicap, notamment en raison des effets que ce handicap peut avoir sur ses études. En parlant de ses loisirs et passetemps, Mario montre de l'intérêt pour l'écriture et la lecture, ce qu'il ne pouvait pas faire hors de son domicile à l'époque. Interrogé sur ses amis, Mario donne les noms des volontaires qui l'aident dans ses activités quotidiennes, mais ne mentionne pas de pairs ou de collègues d'études.

Mario tient tant à s'intégrer dans les contextes sociaux que, malgré ce que disent ses parents, il décide de poursuivre ses études et tente de devenir plus autonome en fréquentant des milieux non ségrégatifs où, en plus des relations avec des personnes en situation de handicap, il peut également nouer des liens avec des personnes non handicapées.

Conformément à l'approche du Life Design, une activité de coaching de carrière de six mois a été mise en place pour faciliter la concentration sur et une redéfinition des objectifs futurs. L'activité 
a pris en compte l'agentivité de Mario, comprise comme sa faculté réelle d'agir et de poursuivre les objectifs auxquels il tient, ainsi que ses aptitudes sociales à participer à son environnement de manière inclusive. Plus précisément, selon l'approche du Life Design, les activités d'accompagnement visent à stimuler les ressources personnelles et contextuelles dans le but de faciliter la construction de sa vie et les changements pour s'adapter au contexte.

\subsection{ACTIVITÉS DE COACHING CENTRÉES SUR LA PERSONNE}

L'activité mise en place pour Mario impliquait la résolution de problèmes axée sur l'observation, la rétroaction et les renforcements, ainsi que la mise en œuvre de plans de carrière. L'accompagnement était également centré sur l'action en vue d'un changement de comportement, par le biais de techniques cognitivo-comportementales centrées sur les objectifs, telles que l'analyse des tâches, les techniques d'incitation et d'estompage, les rétroactions sur la performance, la modélisation et les jeux de rôle illustrant le comportement acceptable en société qu'il conviendrait d'adopter dans la vraie vie. Le coach a interagi avec Mario dans son propre environnement éducatif et à la maison, avec une rétroaction hebdomadaire et des mises à jour par téléphone et par SMS.

En outre, conformément à ce que relèvent Van Esbroeck et Augustijnen (2015), l'intervention de coaching de carrière a aidé Mario à concevoir le développement de sa carrière dans un cadre holistique plus large, comprenant une construction de soi dans tous les rôles de son parcours de vie. Plus précisément, le coaching a aidé Mario à prendre conscience de soi et à développer une identité professionnelle. En parallèle, cette intervention a joué un rôle préventif, permettant à Mario de bien cerner les évolutions futures et de les gérer.

L'activité de coaching a permis à Mario de prendre conscience du fait qu'il gérait les relations de façon dysfonctionnelle en adoptant un style principalement passif-agressif, selon la version italienne de l'échelle d'affirmation de soi de Rathus (Rathus Assertiveness Schedule) (Galeazzi, 1990). En analysant le mécanisme qui sous-tend l'apparition et la consolidation d'un comportement non affirmé, l'accompagnement a mis en évidence comment les convictions du consultant ont produit les conséquences qu'il craignait à cause de son manque de compétences sociales et d'affirmation de soi. En outre, le coach a cherché à améliorer les capacités de 
résolution des problèmes de relations pour surmonter certaines situations problématiques signalées par Mario en lien avec l'accès à et l'inclusion dans divers contextes universitaires (salle d'étude, activités sportives, résidence, cafétéria).

$\mathrm{Au}$ terme de six mois de coaching de carrière, Mario a pu reformuler ses objectifs en matière d'investissement dans les études. Il a décidé de recommencer à étudier et considère désormais que c'est un moyen d'augmenter ses chances de trouver un emploi qui reflète ses intérêts, qu’il a réaffirmés dans le domaine littéraire. Il a pu étudier à nouveau de manière productive et a réussi cinq examens. Comme l'indique la Figure 1, il a augmenté le nombre d'activités réalisées hors du domicile: il est devenu membre d'un groupe d'écriture et d'une chorale de jeunes; il a commencé une activité sportive. Le nombre d'interactions avec ses pairs a augmenté et, en parallèle, il passe moins de temps devant son ordinateur.

\section{Figure 1. Activités réalisées par Mario avant et après le coaching de carrière; l'axe vertical indique le nombre d'heures hebdomadaires consacrées à I'activité}

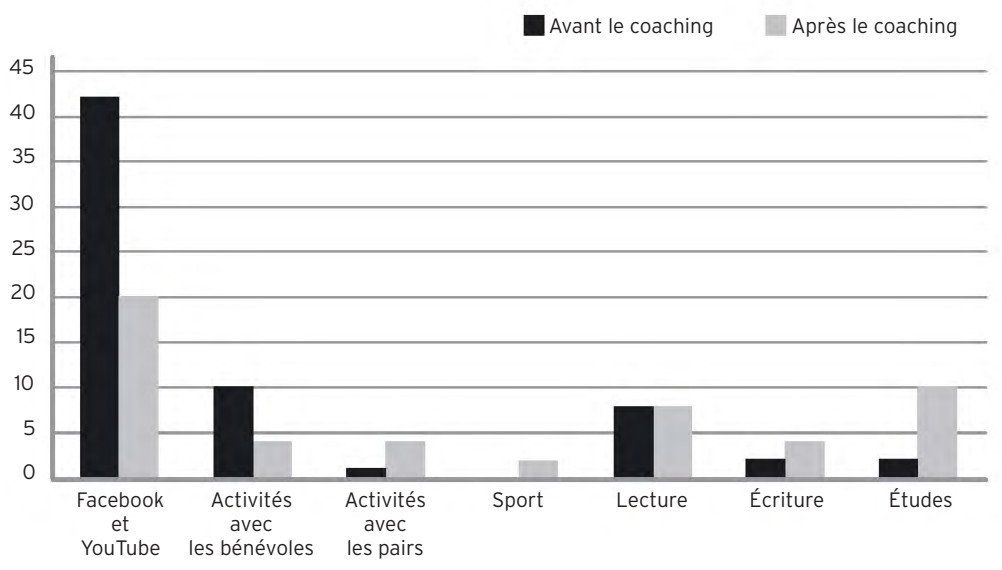

Ces résultats ressortent également des scores obtenus par Mario six mois plus tard sur l'échelle des conduites interpersonnelles (Scale for Interpersonal Behavior-SIB) (Arrindell, Nota, Sanavio, Sica, \& Soresi, 2004). La SIB est une mesure multidimensionnelle de l'affirmation de soi couvrant à la fois l'inconfort (anxiété) associé à l'affirmation de soi dans des situations données et la probabilité 
de fournir des réponses affirmées spécifiques. Elle permet d'évaluer une dimension générale d'affirmation de soi ainsi que quatre sous-dimensions: les initiatives affirmées (initiating assertiveness), comme la capacité d'exprimer ses opinions en public; l'expression de sentiments positifs (expression of positive feelings), renvoyant à la capacité de faire et de recevoir des louanges et des compliments; l'expression et la gestion des limites personnelles (expression of and dealing with personal limitations), permettant notamment d'admettre de ne pas savoir et de composer avec les critiques et la pression; et la manifestation de sentiments négatifs (display of negative feelings), conduisant par exemple à demander à autrui de changer un comportement irritant ou à défendre ses droits et intérêts. La Figure 2 indique ainsi que les niveaux de gêne de Mario ont diminué, alors que les fréquences de gestion affirmée de situations sociales ont augmenté. L'augmentation la plus marquée concernait l'expression de ses propres limites. En effet, un important travail avait été fait sur la capacité de Mario à demander et à accepter l'aide des autres et à faire connaître, si nécessaire, les limites et les difficultés liées à son handicap physique.
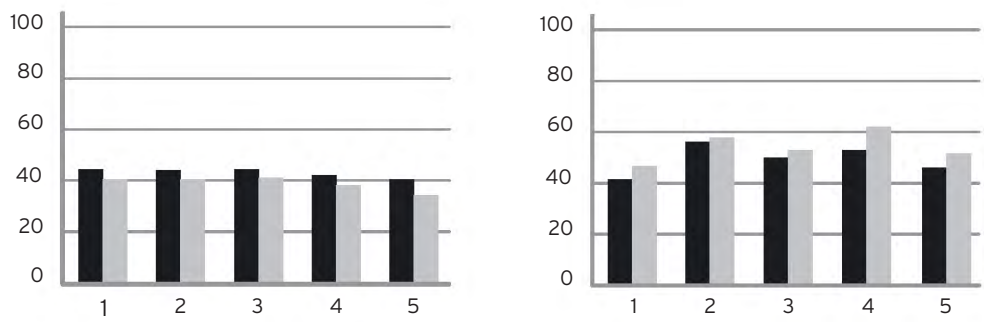

Figure 2. Profil de Mario sur l'échelle SIB en termes de gêne et de fréquence de gestion affirmée de situations sociales, avant et après le coaching; les scores ont été reportés sur une échelle de 100 points (scores T) sur laquelle les valeurs entre 40 et 60 peuvent être considérées comme «moyennes». Légende: 1 = Initiatives affirmées; 2 = Expression de sentiments positifs; 3 = Expression et gestion des limites personnelles; 4 = Manifestation de sentiments négatifs; 5 = Affirmation de soi générale .

\subsection{ACTIVITÉS DE COACHING CENTRÉES SUR LE CONTEXTE}

Au cours des séances, il s'est souvent avéré nécessaire d'aider Mario dans ses actions par rapport au contexte, en particulier par la gestion des obstacles et le soutien à la participation. Les actions de coaching consistaient principalement à accompagner Mario lorsqu'il 
devait faire face à des obstacles à sa mobilité (service de contrôle de la circulation, offices universitaires) pour renforcer son aptitude à faire respecter ses droits. En ce qui concerne la participation, le travail consistait à favoriser la présence de Mario dans des contextes universitaires tels que la cafétéria, les salles d'étude, etc., par des actions de renforcement, de modélisation et d'exposition à des situations réelles, ainsi qu'en engageant d'autres étudiants à collaborer. L'attention s'est également portée sur ses parents, afin de favoriser leur engagement envers l'autodétermination de Mario. Ils sont désormais conscients de la voie qu'a choisie leur fils et le soutiennent en le conduisant à ses activités lorsqu'il est incapable de se déplacer de manière autonome en raison de contraintes externes.

Ce cas peut être considéré comme un exemple de coaching de carrière réalisé dans un contexte de volonté politique à l'Université de Padoue (Università Inclusiva [Université inclusive] http://www. unipd.it/inclusione), qui investit dans l'aménagement d'un cadre académique inclusif en offrant des interventions diversifiées à différents niveaux. Citons par exemple des actions axées sur le conseil en orientation et le coaching de carrière, des centres et services pour la diversité et l'inclusion, un cours général sur la diversité et l'inclusion, des activités pour promouvoir une attitude positive face à l'inclusion auprès du corps enseignant, du personnel administratif et des étudiant.e.s, et enfin, des mesures de tutorat des étudiant.e.s en situation de handicap.

\section{CONCLUSION}

Le présent chapitre a mis l'accent sur les personnes en situation de handicap et sur la nécessité de mettre en œuvre des mesures de coaching de carrière telles que des interventions contribuant à promouvoir une planification professionnelle et une insertion professionnelle de qualité. Après avoir passé en revue des modèles auxquels nous avons pu faire référence à ce propos, nous avons approfondi les activités de coaching de carrière, y compris à la lumière du paradigme des interventions de Life Design qui souligne l'importance des variables contextuelles (Hirschi \& Dauwalder, 2015; Savickas et al., 2009). Comme mentionné ci-dessus, selon l'approche du Life Design, les interventions de coaching de carrière doivent viser à développer l'adaptabilité, la narrativité, l'intentionnalité et l'activité également chez les personnes en situation de 
handicap, par une auto-organisation adaptative entre chaque client.e et son écosystème. Elles doivent chercher des moyens d'augmenter la probabilité pour chacun.e de connaître des conditions de vie satisfaisantes et une réalisation professionnelle. Le coaching de carrière fondé sur le Life Design devrait aider les consultant.e.s à développer des ressources et compétences utiles pour faire face au marché du travail actuel et promouvoir une construction de leur vie et des changements pour s'adapter au contexte.

Afin de favoriser l'inclusion, le coaching de carrière selon le paradigme du Life Design devrait également mettre en place des interventions auprès des proches importants dans le contexte afin de prévenir les obstacles. Comme le relèvent Corbière et collègues (2014) ainsi que Jenaro et collègues (2002), le coaching de carrière devrait impliquer, par exemple, les collègues et les employeuses et employeurs pour les préparer à encourager et soutenir l'insertion professionnelle des personnes en situation de handicap.

Il est évident que les coachs de carrière jouent un rôle clé dans la promotion de l'inclusion professionnelle et sociale des personnes en situation de handicap. À cet égard, il est particulièrement important qu'elles et ils aient une formation adéquate et un très haut niveau de professionnalisme tant sur les questions de carrière que sur les aspects relatifs au handicap et à l'inclusion, afin d'utiliser de multiples approches pour mettre en place des activités et des interventions individuelles ou de groupe.

En conclusion, nous souhaitons souligner que les recherches portant sur des personnes en situation de handicap sont encore rares. Nous pensons qu'il faut redoubler d'efforts pour multiplier les expériences afin de tester l'efficacité des interventions de coaching de carrière visant à soutenir ces personnes dans la conception de leur vie future et à aménager des contextes respectueux des droits des personnes et soucieux de leur qualité de vie, de leur participation et de leur autodétermination. 


\section{RÉFÉRENCES BIBLIOGRAPHIQUES}

Arrindell, W. A., Nota, L., Sanavio, E., Sica, C., \& Soresi, S. (2004). SIB. Valutazione del comportamento interpersonale e assertivo [SIB. Évaluation du comportement interpersonnel et affirmé]. Trieste: Edizioni Erickson.

Asante, S. (2002). What is inclusion?'Toronto: Inclusion Press.

Aspinwall, L. G., \& Staudinger, U. M. (2003). A psychology of human strengths: Fundamental questions and future directions for a positive psychology. Washington, DC: American Psychological Association.

Bennett, K., Brady, M. P., Scott, J., Dukes, C., \& Frain, M. (2010). The effects of covert audio coaching on the job performance of supported employees. Focus on Autism and Other Developmental Disabilities, 25, 173-185. Doi: 10.1177/1088357610371636.

Brock, M. E., Cannella-Malone, H. I., Schaefer, J. M., Justin Page, E., Andzik, N. R., \& Seaman, R. L. (2016). Efficacy of training job coachs to implement evidence-based instructional strategies. Journal of Vocational Rehabilitation, 45, 351-364. Doi: 10.3233/JVR-160835.

Byars-Winston, A., Gutierrez, B., Topp, S., \& Carnes, M. (2011). Integrating theory and practice to increase scientific workforce diversity: a framework for career development in graduate research training. CBE-Life Sciences Education, 10, 357-367. Doi : $10.1187 /$ cbe.10-12-0145.

Chung, Y. B., \& Grroerer, M. (2003). Career coaching: Practice, training, professional, and ethical issues. The Career Development Quarterly, 52, 141-152. Doi: 10.1002/j.2161-0045.2003. tb00634.x.

Corbière, M., Villotti, P., Lecomte, T., Bond, G. R., Lesage, A., \& Goldner, E. M. (2014). Work accommodations and natural supports for maintaining employment. Psychiatric Rehabilitation Journal, 37, 90-98. Doi : 10.1037/prj0000033.

Di Maggio, I., \& Shogren, K. (2017). Any given context (school, work, society) will be considered inclusive only if... In L. NOTA \& S. SORESI (éds), For a Manifesto in favor of Inclusion. Concerns, ideas, intents and passwords for inclusion (pp. 29-34). Florence: Hogrefe. 
Donnelly, J. (2013). Universal human rights in theory and practice. New York: Cornell University.

Duarte, M. E. (2018). Career counseling and career coaching in working contexts: Learning from each other. In L. NOTA \& S. SORESI (éds), Counseling and Coaching in Times of Crisis and Transitions (pp. 83-102). Obingdon: Routledge

Farris, B., \& Stancliffe, R. J. (2001). The co-worker training model: Outcomes of an open employment pilot project. Journal of Intellectual and Developmental Disability, 26, 143-159. Doi: 10.1080/13668250020054459.

Ferrari, L., Sgaramella, T. M., \& Soresi, S. (2015). Bridging disability and work. Contribution and challenges of Life Design. In L. Nota \& J. Rossier (éds), Handbook of Life Design: From Practice to Theory and From Theory to Practice (pp. 219-232). Göttingen: Hogrefe.

Galeazzi, A. (1990). Uno strumento per l'assessment comportamentale: il questionario di Assertività di Rathus (RAS) [Un instrument pour l'évaluation comportementale: le questionnaire d'affirmation de Rathus (RAS)]. Bollettino di Psicologia applicata, 190, 3-12.

Gestsdottir, S., Lewin-Bizan, S., von Eye, A., Lerner, J. V., \& LERner, R. M. (2009). The structure and function of selection, optimization, and compensation in middle adolescence: Theoretical and applied implications. Journal of Applied Developmental Psychology, 30, 585-600. Doi : 10.1016/j.appdev.2009.07.001.

Gilson, C. B., \& Carter, E. W. (2016). Promoting social interactions and job independence for college students with autism or intellectual disability: A pilot study. Journal of Autism and Developmental Disorders, 46, 3583-3596. Doi: 10.1007/s10803-0162894-2.

Ginevra, M. C., \& Santilli, S. (2018). Promoting courage in children : the role of coaching. In L. NOTA \& S. SORESI (éds), Counseling and Coaching in Times of Crisis and Transitions (pp. 230-240). Obingdon: Routledge.

Green, S., Grant, A., \& Rynsaardt, J. (2007). Evidence-based life coaching for senior high school students: Building hardiness and hope. International Coaching Psychology Review, 2 (1), 24-32. 
Hartung, P. J. (2015). Life Design in childhood: Antecedents and advancement. In L. Nota \& J. Rossier (éds), Handbook of Life Design. From Practice to Theory and from Theory to Practice (pp. 89-101). Göttingen: Hogrefe.

Hirschi, A., \& Dauwalder, J. P. (2015). Dynamics in career development: Personal and organizational perspectives. In L. NotA \& J. Rossier (éds), Handbook of Life Design: From Practice to Theory and From Theory to Practice (pp. 27-39). Göttingen: Hogrefe.

Holt, N. L., Neely, K. C., Slater, L. G., Camirè, M., Côté, J., Fraser-Thomas, J., Tamminen, K. A. (2017). A grounded theory of positive youth development through sport based on results from a qualitative meta-study. International Review of Sport and Exercise Psychology, 10, 1-49. Doi: 10.1080/1750984X.2016.1180704.

International Coaching Federation (ICF) (2012). Core Coaching Competencies. En ligne: [https://coachfederation.org/core-competencies].

Jenaro, C., Mank, D., Bottomley, J., Doose, S., \& Tuckerman, P. (2002). Supported employment in the international context: An analysis of processes and outcomes. Journal of Vocational Rehabilitation, 17, 5-21.

Jordan, S., Gessnitzer, S., \& Kauffeld, S. (2016). Effects of a group coaching for the vocational orientation of secondary school pupils. Coaching: An International Journal of Theory, Research and Practice, 9, 143-157. Doi : 10.1080/17521882.2016.1210185.

Keyes, C. L. M., \& HaidT, J. (2003). Introduction: Human flourishing: The study of that which makes life worthwhile. In C. L. M. KeYES \& J. HAIDT (éds), Flourishing: Positive psychology and the life well-lived (pp. 3-12). Washington: American Psychological Association.

Lindsay, S., Adams, T., Sanford, R., McDougall., C., Kingsnorth, S., \& MENNA-DACK, D. (2014). Employers'and employment counselors'perceptions of desirable skills for entry-level positions for adolescents: how does it differ for youth with disabilities? Disability \& Society, 29, 953-967. Doi: 10.1080/09687599.2013.874330.

Mihailidis, A., Melonis, M., Keyfitz, R., Lanning, M., Van VuUren, S., \& Bodine, C. (2016). A nonlinear contextually aware prompting system (N-CAPS) to assist workers with intellectual and 
developmental disabilities to perform factory assembly tasks: system overview and pilot testing. Disability and Rehabilitation: Assistive Technology, 11, 604-612. Doi : 10.3109/17483107.2015.1063713.

Nicholas, D. B., Attridge, M., Zwaigenbaum, L., \& Clarke, M. (2015). Vocational support approaches in autism spectrum disorder: A synthesis review of the literature. Autism, 19, 235-245. Doi : $10.1177 / 1362361313516548$.

Norwich, B. (2014). How does the capability approach address current issues in special educational needs, disability and inclusive education field? Jorsen, 14, 16-21. Doi : 10.1111/1471-3802.12012.

Nota, L., Ginevra, M. C., \& Santilli, S. (2015). Life Design and prevention. In L. Nota \& J. Rossier (éds), Handbook of Life Design. From Practice to Theory and from Theory to Practice (pp. 183-199). Göttingen : Hogrefe.

Nota, L., \& Soresi, S. (2018). Counseling and coaching in times of crisis and transitions: an introduction. In L. NotA \& S. SORESI (éds), Counseling and Coaching in Times of Crisis and Transitions (pp. 1-12). Abingdon: Routledge.

Owens, J. (2015). The social model of disability and its application to dentistry. Dental Nursing, 11, 448-450. Doi: 10.12968/ denn.2015.11.8.448.

Pouyaud, J. (2015). Vocational trajectories and people's multiple identities: A life design. In L. Nota \& J. Rossier (éds), Handbook of Life Design: From Practice to Theory and From Theory to Practice (pp. 59-74). Göttingen: Hogrefe.

ReID, H. (2015). Introduction to Career Counselling \& Coaching. Londres: Sage.

Savickas, M. L., Nota, L., Rossier, J., Dauwalder, J.-P., Duarte, M. E., Guichard, J., van Vianen, A. E. M. (2009). Life designing: A paradigm for career construction in the 21st century. Journal of Vocational Behavior, 75, 239-250. Doi: 10.1016/j. jvb.2009.04.004.

Schalock, R. L., Borthwick-Duffy, S., Bradley, V., Buntix, W. H. E., Coulter, D. L., Craig, E. P. M., ... Yeager, M. H. (2010). Intellectual disability: Definition, classification, and systems of support ( $11^{\mathrm{e}}$ édition). Washington: American Association on Intellectual and Developmental Disabilities. 
Schartz, K., Schartz, H. A., \& Blanck, P. (2002). Employment of persons with disabilities in information technology jobs: Literature review for "IT works". Behavioral Science \& the Law, 20, 637-657. Doi : 10.1002/bsl.510.

Schianchi, M. (2012) (éd.). Storia della disabilità. Dal castigo degli dei alla crisi del welfare. [History of disability. From the punishment of gods to the welfare crisis]. Rome: Carocci.

SEN, A. (2006). Reason, freedom and well-being. Utilitas, 18, 80-96. Doi : 10.1017/S0953820805001846.

Shogren, K. A., Luckasson, R., \& Schalock, R. L. (2014). The definition of "context» and its application in the field of intellectual disability. Journal of Policy and practice in Intellectual Disabilities, 11, 109-116. Doi: 10.1111/jppi.12077.

Shogren, K. A., Wehmeyer, M. L., Schalock, R. L., \& ThompsON, J. R. (2016). Reframing educational supports for students with intellectual disability through strengths-based approaches. In M. L. WeHMEYER \& K. A. SHOGREN (éds), Handbook of ResearchBased Practices for Educating Students with Intellectual Disability (pp. 17-30). New York: Routledge.

Soresi, S. (2016) (éd.). Psicologia delle disabilità e dell'inclusione [Psychologie du handicap et de l'inclusion]. Bologne: Il Mulino.

Spence, G. B., \& Grant, A. M. (2007). Professional and peer life coaching and the enhancement of goal striving and well-being: An exploratory study. The Journal of Positive Psychology, 2, 185-194. Doi: 10.1080/17439760701228896.

Theeboom, T., Beersma, B., \& van Vianen, A. E. (2014). Does coaching work? A meta-analysis on the effects of coaching on individual level outcomes in an organizational context. The Journal of Positive Psychology, 9, 1-18. Doi : 10.1080/17439760.2013.837499.

Van Esbroeck, R., Augustijnen, M.-T. (2015). Coaching - A career intervention model within Life Design. In L. NoTA \& J. Rossier (éds), Handbook of Life Design: From Practice to Theory and From Theory to Practice (pp. 169-181). Göttingen: Hogrefe.

Villotti, P., Corbière, M., Dewa, C. S., Fraccaroli, F., SultanTaḮb, H., Zaniboni, S., \& Lecomte, T. (2017). A serial mediation model of workplace social support on work productivity: the role of self-stigma and job tenure self-efficacy in people with severe 
mental disorders. Disability and Rehabilitation. Advance Online Publication. Doi: 10.1080/09638288.2017.1377294.

Wehmeyer, M. L., Shogren, K., Nota, L., Sgaramella, T. M., Ferrari, L., \& Di Maggio, I. (2018). A crisis in career development: Changing paradigms and the future of transition. Career Development and Transition for Exceptional Individuals. Published online. Doi : 10.1177/2165143417750092.

World Health Organization. (2007). International classification of functioning, disability and health: Children and youth version. Genève: WHO Press. 



\title{
8. LE TRAVAIL DÉCENT: UNE AMBITION FONDAMENTALE POUR LA PSYCHOLOGIE DE L'ORIENTATION ${ }^{1}$
}

\author{
SALIHA KOZAN (BOSTON COLLEGE), \\ JONAS MASDONATI (UNIVERSITÉ DE LAUSANNE), \\ LILY KONOWITZ (BOSTON COLLEGE)
}

ET DAVID L. BLUSTEIN (BOSTON COLLEGE)

\begin{abstract}
e chapitre a pour objectifs de décrire et de définir le concept travail décent d'un point de vue psychologique et d'apporter des indications utiles pour la pratique du counseling et les politiques publiques. Les défis du monde du travail contemporain sont décrits dans un premier temps, ainsi que leurs implications psychologiques. Après une revue de la littérature sur le concept de travail décent, les interventions psychologiques et systémiques qui peuvent promouvoir le travail décent sont ensuite expliquées. Un exemple d'intervention clinique ainsi qu'une liste de recommandations pour le counseling sont également fournis. Enfin, nous discutons de la manière dont le concept de travail décent pourrait être étendu afin de bénéficier plus spécifiquement à celles et ceux qui sont privé.s de conditions et d'expériences de travail décentes.
\end{abstract}

\section{LES DÉFIS D'UN MONDE DU TRAVAIL EN MUTATION}

Les personnes à travers le monde sont confrontées à de nouvelles opportunités et de nouveaux obstacles liés au travail, tandis que ce dernier continue d'évoluer, souvent de manière imprévisible. Le développement technologique et la mondialisation ont entraîné une augmentation de la mobilité de la main-d'œuvre, la restructuration des entreprises, la sous-traitance, la délocalisation et la réduction des effectifs, couplées à l'effacement du pouvoir des syndicats (Blustein, Kenny, Di Fabio, \& Guichard, 2019). Le contrat social et psychologique entre les employé.e.s et les employeurs.ses, la qualité, la sécurité et la prévisibilité de l'emploi, la culture et les relations dans les organisations comptent parmi les principaux enjeux du travail

1. Ce chapitre a été traduit de l'anglais par M. Abessolo, E. Banet, L. Fedrigo et J. Masdonati. 
au cours des dernières décennies (Blustein, 2006; Fournier, Zimmermann, Gauthier, Masdonati, \& Lachance, 2016). En outre, la main-d'œuvre a été façonnée par les fluctuations macroéconomiques en cours, notamment par la Grande Récession de 2007-2009, qui a entraîné des tumultes financiers, politiques, sociaux et psychologiques dans le monde entier (Guichard, 2013). Alors que de nombreux pays ont encore du mal à s'en relever, ils s'efforcent d'améliorer la qualité de l'emploi et de former leurs citoyen-ne.s à répondre aux exigences d'un monde du travail en constante évolution.

Selon un récent rapport de l'Organisation internationale du travail (OIT, International Labour Organization [ILO], 2018), la croissance économique mondiale, qui a atteint son plus bas niveau en six ans en 2016, devrait rester stable ou encore s'affaiblir au cours des années à venir. Au-delà des incertitudes économiques persistantes, le nombre de chômeurs.ses dans le monde devrait augmenter de près de 1,3 million en 2019, alors que le sous-emploi des jeunes reste un problème important dans les pays développés et en voie de développement. Conjuguées à un climat économique fluctuant, les inégalités sociales et les disparités dans les opportunités d'emploi et de promotion contribuent aux migrations qui affectent les structures sociales, l'économie et la main-d'œuvre dans de nombreuses régions du monde. Les ambiguïtés concernant l'état de l'économie mondiale et ses influences durables sur le travail constituent une source de détresse, en particulier pour les personnes défavorisées (par exemple, personnes vivant dans la pauvreté ou appartenant à la classe ouvrière).

Parallèlement au problème généralisé du chômage, les changements susmentionnés dans la main-d'œuvre et le récent ralentissement économique mondial ont entraîné une intensification du travail précaire (Benach et al., 2014; Blustein, Olle, Connors-Kellgren, \& Diamonti, 2016). En Suisse, par exemple, Bonvin (2016) identifie trois problèmes principaux pour les travailleurs.ses dans un marché du travail flexible: l'accès au travail, soit les chances de trouver un emploi; sa sécurité, c'est-à-dire le maintien dans un emploi; et la dignité du travail, notamment la lutte contre son intensification, contre les risques pour la santé sur le lieu de travail et contre le déséquilibre entre vie professionnelle et vie personnelle. Par ailleurs, ces défis sont plus importants pour certaines populations, en particulier pour les femmes, les travailleurs.ses non qualifié.e.s et les migrant.e.s, qui sont surreprésenté.e-s parmi les laissés-pour-compte. 
Dans le monde du travail actuel, de nombreuses personnes, tout en étant employées, sont exposées à un travail instable, physiquement ou psychologiquement dangereux, oppressif et comportant des salaires faibles et injustes. Ceci est d'autant plus préoccupant que le travail précaire devrait augmenter dans les années à venir (Katz \& Krueger, 2016). De plus, beaucoup de personnes ont le choix entre se retrouver sans emploi ou alors avoir un travail non seulement incompatible avec leurs objectifs et leurs capacités, mais allant aussi à l'encontre de leurs besoins, c'est-à-dire demandant de travailler moins ou plus que ce qu' elles souhaiteraient (on parle alors de sous ou suremploi). Dans un contexte international de chômage persistant et de travail précaire, le travail décent est donc devenu plus difficile à atteindre (Blustein et al., 2016).

Les taux de chômage élevés et la mauvaise qualité de l'emploi sont des problèmes mondiaux difficiles à résoudre et ayant d'importants effets sociaux, psychologiques et économiques sur les individus et les communautés (Benach et al., 2014). De nombreuses recherches documentent les impacts négatifs du chômage sur la santé physique et mentale (par exemple, Paul \& Moser, 2009; Wanberg, 2012). De même, les recherches sur le travail précaire ont montré que celui-ci nuit à la santé, au bien-être et à la vie sociale, et ce, à travers des mécanismes complexes (Benach et al., 2014; Fournier, Zimmermann, Gauthier, \& Masdonati, 2014).

\section{LE TRAVAIL DÉCENT}

Étant donné le rôle central du travail dans la vie des personnes et les effets multiples des problèmes liés au travail sur les individus et les communautés, les organisations internationales ont émis des recommandations pour améliorer l'expérience de travail. L'Agenda du travail décent de l'OIT (OIT, 1999, 2008; ILO, 2012) a constitué une initiative importante visant à établir des lignes directrices ambitieuses pour les gouvernements, les organisations, les décideurs.ses et les autres interlocuteurs du monde du travail, afin de créer des politiques et des pratiques promouvant le travail décent pour tou.te-s. Cette initiative reconnaît que le travail est partie intégrante des droits humains et de l'égalité, et remonte aux principes fondamentaux de l'OIT de 1919 (Deranty \& MacMillan, 2012). Elle fait état de quatre objectifs stratégiques interdépendants visant à promouvoir «(a) [les] principes et droits fondamentaux au 
travail et [les] normes internationales du travail; (b) [les] possibilités d'emploi pour les femmes et les hommes; (c) [la] protection sociale et [la] sécurité sociale; enfin (d) [le] dialogue social et [le] tripartisme» (OIT, 2008, p. 164). Avec l'Agenda du travail décent, l'OIT a reconnu son propre rôle dans la promotion de l'objectif universel de justice sociale et de progrès, dans le développement d'une économie mondiale, dans la recherche du plein-emploi et dans la mise en place de mesures contre la pauvreté et les inégalités.

Depuis l'initiative de l'OIT, le concept de travail décent a été principalement examiné sous l'angle macroéconomique, à savoir en partant des indices du marché du travail tels que le chômage, l'emploi précaire et la pauvreté (ILO, 2018; Standing, 2008). Un certain nombre de chercheur.e.s (par exemple, Burchell, Sehnbruch, Piasna, \& Agloni, 2014; Deranty \& MacMillan, 2012; Sehnbruch, Burchell, Agloni, \& Piasna, 2015) ont critiqué cette approche du travail décent en raison de problèmes d'opérationnalisation et d'application, notamment en termes d'évaluation de ce qui constitue ou pas un travail décent. Une autre vague de critiques a porté sur le manque de considération des aspects sociaux du travail (Di Ruggiero, Cohen, Cole, \& Forman, 2015), l'OIT ayant surtout favorisé les conceptualisations guidées par les tendances du marché, en négligeant les façons dont les gens vivent et donnent un sens à leur vie professionnelle (Deranty \& MacMillan). Sehnbruch et collègues ont en outre fait valoir que l'imprécision et le recours à la littérature institutionnelle provenant de l'OIT dans l'Agenda du travail décent ont empêché l'utilisation de ce concept dans la recherche scientifique.

De plus, malgré le rôle central du travail pour la santé et le bienêtre des individus et la nécessité de promouvoir le travail décent comme un droit fondamental (Blustein, 2006, 2013; Blustein et al., 2019), les psychologues manifestent un intérêt limité envers cette question. Par ailleurs, la littérature en psychologie semble être surtout axée sur le travail salarié, plutôt que de considérer l'ensemble des formes que peut prendre le travail (Burchell et al., 2014; Sehnbruch et al., 2015). De nombreux psychologues de l'orientation ont par ailleurs évoqué le manque de conceptualisations «subjectives» du travail décent et ont appelé à approfondir la compréhension de cette notion en partant de l'expérience des travailleurs.ses (Blustein et al., 2016; Di Fabio \& Kenny, 2016; Guichard, 2013; Massoudi et al., 2018; Pouyaud, 2016; Ribeiro, Silva, \& Figueiredo, 2016). Cet appel est cohérent avec les valeurs des psychologues de 
l'orientation s'intéressant à la promotion de la santé mentale et au développement positif en s'appuyant sur des théories et des interventions qui prennent en compte le rôle des facteurs contextuels dans la vie personnelle, sociale, scolaire et professionnelle (Toporek, Gerstein, Fouad, Roysircar, \& Israel, 2006).

\section{LA PSYCHOLOGIE DE L'ACTIVITÉ DE TRAVAIL}

Comme l'a souligné Pouyaud (2016), les psychologues peuvent ainsi avoir du mal à opérationnaliser et appliquer un concept initialement défini au niveau macroéconomique. Pour tenter de combler cette lacune, Blustein et collègues (2016) ont souligné l'importance de saisir le travail décent en partant d'une perspective psychologique, afin d'explorer et de comprendre les expériences individuelles des travailleurs.ses et de se recentrer sur le souci de justice sociale inhérent au concept de travail décent. Pour relever ces défis, les auteur·e.s se sont appuyé.e.s sur le cadre théorique et sur la théorie de la psychologie de l'activité de travail (Blustein, 2001, 2006, 2008, 2013; Blustein et al., 2019; Duffy, Blustein, Diemer, \& Autin, 2016), que nous résumons ci-dessous.

\subsection{LE CADRE THÉORIQUE DE LA PSYCHOLOGIE}

\section{DE L'ACTIVITÉ DE TRAVAIL}

Le cadre théorique de la psychologie de l'activité de travail (Psychology of Working Framework, PWF, Blustein, 2001, 2006, 2013) a été développé en tant que critique des théories traditionnelles en développement de carrière, qui sont adaptées à la compréhension d'individus disposant d'une certaine marge de manœuvre dans leurs choix, mais pas de ceux ayant des choix limités et peu d'accès à des ressources et à un travail décent. Blustein a en effet fait valoir que toutes les personnes n'avaient pas les mêmes opportunités ni les mêmes ressources pour faire des choix de carrière compatibles avec leurs objectifs, leurs valeurs et leurs compétences. Considérant les interactions complexes entre de multiples facteurs individuels, identitaires, socioéconomiques, culturels et politiques, le PWF prône une approche inclusive du travail, visant à donner une place et une voix à tou.te.s celles et ceux qui travaillent ou qui veulent travailler, notamment les personnes pauvres, la classe ouvrière et les personnes marginalisées en raison de leur sexe, de leur origine ethnique ou d'autres aspects de leur identité. Ce cadre théorique conceptualise le 
travail comme un aspect fondamental de la vie et un élément crucial de la santé mentale et du bien-être. Par ailleurs, il postule que l'acte de travailler se produit conjointement aux expériences extraprofessionnelles. Enfin, le PWF considère que l'activité de travail a lieu à la fois dans le contexte du marché de l'emploi et en dehors de celui-ci, comme dans les activités du care (Blustein, 2013).

En intégrant explicitement des enjeux de justice sociale, le PWF souligne la manière dont l'expérience professionnelle et le sens du travail sont façonnés par les privilèges et les désavantages résultant du croisement de facteurs relatifs à l'identité, à la classe sociale et aux possibilités de choix. Un autre élément important du PWF est la taxonomie des besoins humains que le travail peut potentiellement remplir: le besoin de survie et pouvoir, les liens sociaux et l'autodétermination. De ce fait, le PWF met en lumière le rôle essentiel du travail pour accéder aux ressources sociales et économiques, pour promouvoir la santé et le bien-être psychologiques et pour procurer un sentiment de satisfaction et d'épanouissement dans la vie (Blustein, 2008). Au cours de la dernière décennie, le PWF a donné lieu à un riche corpus de littérature visant à explorer plusieurs concepts en lien avec l'activité de travail. Ces concepts incluent l'ethnicité, la culture, le genre et la classe sociale (Flores et al., 2011; Guerrero \& Singh, 2013); la "volition» professionnelle (work volition, cf. Duffy, Diemer, Perry, Laurenzi, \& Torrey, 2012); le chômage et le sous-emploi (Blustein, Kozan \& Connors-Kellgren, 2013; Lyons, 2011) ; le développement de carrière des jeunes (Blustein et al., 2010; Chaves et al., 2004; Ferrari et al., 2009); et les enjeux relationnels du travail (Blustein, 2011; Kenny \& Medvide, 2013).

\subsection{LA THÉORIE DE LA PSYCHOLOGIE DE LACTIVITÉ DE TRAVAIL}

Plus récemment, Duffy et collègues (2016) ont intégré les recherches et les principes du PWF dans un modèle théorique intégratif et empiriquement vérifiable: la théorie de la psychologie de l'activité de travail (Psychology of Working Theory, PWT). En positionnant le travail décent comme le résultat d'interactions entre facteurs psychologiques, contextuels et économiques, la PWT a tenté de clarifier les impacts des contraintes et des expériences sociales et économiques sur le développement de carrière. La PWT comporte l'atout de considérer les expériences de travail de tous les individus, donc autant des personnes issues de milieux plus privilégiés que de celles disposant d'une volition professionnelle limitée, manquant 
de contrôle sur leur vie professionnelle ou traversant des transitions professionnelles difficiles ou involontaires. En s'appuyant sur les définitions de l'OIT (2008; ILO, 2012), la PWT identifie cinq composantes du travail décent: (a) des conditions de travail sécuritaires des points de vue physique et interpersonnel, (b) des horaires permettant un repos adéquat et dégageant suffisamment de temps libre, (c) des valeurs organisationnelles en harmonie avec les valeurs familiales et sociales, (d) une rémunération adéquate, (e) l'accès à des soins de santé équitables et justes (Duffy et al., 2016). De plus, Duffy et collègues (2017) ont récemment conçu et validé l'échelle de travail décent (Decent Work Scale, DWS) permettant d'évaluer les composantes du travail décent d'un point de vue psychologique ${ }^{2}$. Ces auteurs ont confirmé l'existence d'une structure à cinq facteurs, chaque facteur constituant un construit unique et contribuant à un facteur général de travail décent. Ces résultats soutiennent ainsi l'utilisation de cette échelle dans les pratiques de conseil en orientation.

La Figure 1 propose une vue d'ensemble de la PWT, ainsi que les liens effectifs ou supposés entre les variables qui la composent, chaque lien soutenant des propositions empiriquement vérifiées ou vérifiables. La PWT préconise ainsi que des facteurs contextuels interdépendants, tels que les contraintes économiques et les expériences de marginalisation, sont liés à la capacité des individus d'obtenir un travail décent. Premièrement, les contraintes économiques (ressources économiques limitées, telles qu'un faible revenu) déterminent l'accès aux ressources financières et au capital social et culturel, ce qui pourrait limiter la qualité et les opportunités de formation et de travail (Duffy et al., 2016). Deuxièmement, différentes formes de marginalisation plus ou moins manifestes (par exemple, le racisme, le sexisme et l'hétérosexisme) enferment certaines personnes ou communautés dans des positions sociales désavantageuses et les excluent des ressources éducatives et des opportunités de carrière. De ce fait, selon la PWT, les personnes connaissant de plus grandes difficultés économiques et des expériences de marginalisation sont moins en mesure de faire preuve de volition et d'adaptation, ce qui, en retour, compromet leurs chances d'accéder à un travail décent. Dans ces cas, au lieu d'avoir des effets positifs sur la santé et le bien-être, le travail peut être nocif pour l'individu (Blustein, 2006).

2. L'adaptation suisse de cette échelle est actuellement en cours (cf. Masdonati, Schreiber, Marcionetti et Rossier, 2019). 


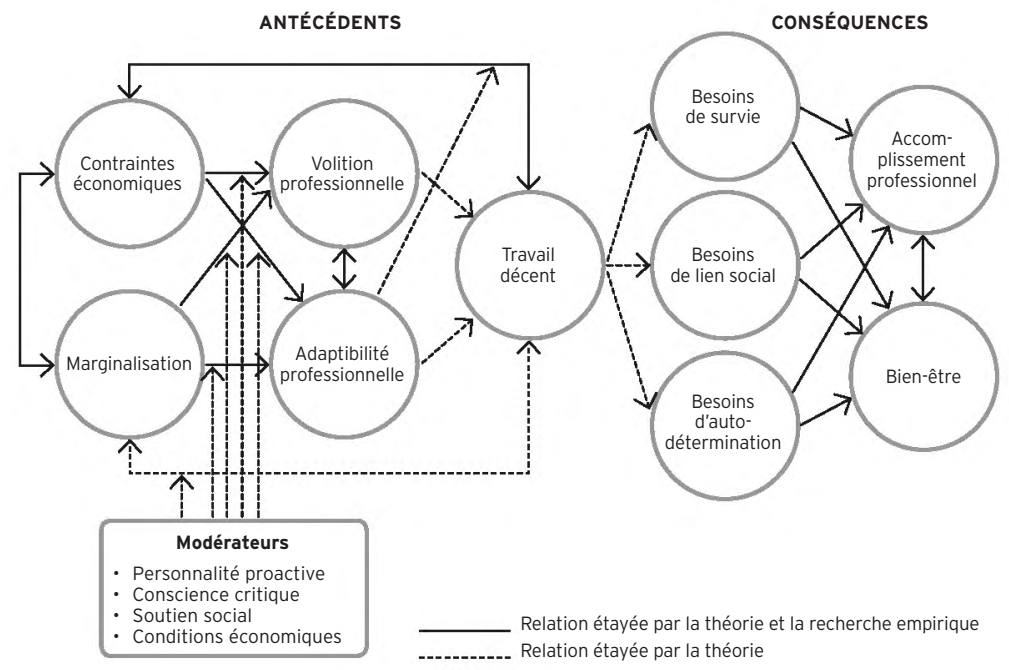

Figure 1. Modèle théorique de la psychologie de l'activité de travail (adapté et traduit de Duffy et al., 2016, p. 129).

En outre, la PWT émet l'hypothèse que la volition professionnelle (soit l'autonomie perçue de choix malgré les contraintes) et l'adaptabilité de carrière (soit la capacité d'utiliser les ressources pour gérer les tâches ou les défis liés au travail) expliquent partiellement la relation entre facteurs contextuels et travail décent. Dans cette relation, les contraintes économiques et les expériences de marginalisation sont supposées prédire négativement la volition professionnelle et l'adaptabilité de carrière, ces dernières étant interreliées. Toutefois, volition et adaptabilité constituent des concepts distincts et malléables, qui pourraient être ciblés par des interventions psychologiques visant à faciliter l'accès à un travail décent. Le PWT identifie également diverses variables susceptibles de réduire les effets négatifs des barrières contextuelles, sociales et économiques. Il s'agit d'une personnalité proactive, de la conscience critique (qui sera détaillée plus bas), du soutien social et de certaines conditions économiques. Ces facteurs peuvent ainsi jouer un rôle "tampon" sur le lien entre obstacles contextuels et accès au travail décent.

Enfin, parallèlement à la définition du travail décent de l'OIT (2008; ILO, 2012), la PWT propose que l'accès et le maintien dans un travail décent favorisent l'épanouissement et le bien-être 
global de la personne à travers la satisfaction des trois besoins de survie et pouvoir, de lien social et d'autodétermination (Blustein, 2006 ; Duffy et al., 2016). Premièrement, le besoin de survie et pouvoir dépend des besoins physiologiques (par exemple, nourriture, soins de santé et abri) et du capital qui donne accès à des opportunités professionnelles. Blustein et collègues (2016) signalent que les caractéristiques du travail, telles que la sécurité et la stabilité de l'emploi, ses conditions (couverture maladie, congés payés) et le sentiment d'autonomie et de contrôle sur le travail peuvent répondre aux besoins de survie et de pouvoir. Deuxièmement, le besoin de lien social se rapporte aux caractéristiques du travail qui permettent d'entrer en relation avec les autres et d'enrichir le sentiment d'appartenance sociale (Blustein, 2006, 2011). Le travail peut répondre à ce besoin en procurant aux personnes des relations professionnelles positives, respectueuses et valorisantes, en favorisant le sentiment d'appartenance à une communauté et en permettant de développer un lien avec le monde et la sensation de contribuer à quelque chose de plus grand que soi (Blustein, 2013). Enfin, le besoin d'autodétermination fait référence aux opportunités que le travail peut donner de participer à des activités intéressantes et significatives, motivantes et conformes à leurs systèmes de valeurs, permettant aux travailleurs.ses d'expérimenter un sentiment d'autonomie, de compétence et de lien à autrui (Blustein, 2006).

Bien que la PWT soit relativement nouvelle, une partie de ses propositions ont été étayées par des études récentes sur des employé.e.s adultes et des étudiant·e.s aux États-Unis (par exemple, Autin, Douglass, Duffy, England, \& Allan, 2017; Douglass, Velez, Conlin, Duffy, \& England, 2017). Par ailleurs, Kozan, Işık et Blustein (2019) ont récemment adopté la PWT pour comprendre la situation d'employé.e.s à faible revenu dans un cadre culturel non occidental. Cependant, d'autres recherches sont nécessaires afin de tester l'utilité et la pertinence de cette théorie dans d'autres contextes culturels.

\section{INCLURE LE TRAVAIL DÉCENT DANS LA PRATIQUE}

Jusqu'à ce jour, de nombreuses interventions de niveau "macro" ont visé à aider les pays à élaborer des cadres généraux pour promouvoir le travail décent, conçu comme un élément clé des stratégies de développement national. Par exemple, l'OIT a collaboré 
avec des organisations de travailleurs.ses, d'employeurs.ses et d'organismes gouvernementaux pour mettre au point un Diagnostic en faveur du travail décent à l'intention des États membres. Ces rapports ont par la suite été pris en compte dans les Programmes par pays de promotion du travail décent (PPTD; BIT, 2016), dans une optique de tripartisme et de dialogue social lors de la planification et de l'application du programme de soutien de l'OIT. Ainsi, les PPTD sont adaptés aux priorités et aux objectifs des pays membres, convenus avec leurs mandants tripartites.

Tout en reconnaissant que ces programmes constituent des mesures indispensables, nous préconisons également des interventions psychologiques, à même d'apporter une contribution essentielle à la promotion d'un travail décent, digne et sûr pour tou.te.s. Dans cette section, nous discutons de certaines de ces interventions. Notre objectif est de préciser la nature et la portée des interventions auxquelles les psychologues conseillers.ères peuvent avoir recours pour promouvoir le travail décent au niveau individuel. Celles-ci partent du présupposé que les problèmes liés au travail sont des aspects essentiels de la vie et qu'ils sont indissociables d'autres enjeux personnels et interpersonnels (Blustein 2006; Duffy et al., 2016).

Dans la lignée de ce qui a été préconisé par Blustein (2001, 2006), nous visons ainsi à inclure la psychologie de l'activité de travail à la fois dans le counseling personnel et dans le counseling d'orientation. Ces deux formes de counseling partagent en effet plusieurs aspects importants, tels que la création d'une alliance de travail et la facilitation du changement à travers des interprétations et des rétroactions constructives. S'appuyant sur ces fondements communs, une pratique psychologique inclusive implique le recours à une approche intégrant différents domaines de vie (domain-sensitive approach), et vise le développement du pouvoir d'agir (empowerment) et la prise de conscience critique (critical consciousness) des consultant.e.s (Blustein, 2006), augmentant par ce biais leurs chances d'accéder à un travail décent.

\subsection{UNE APPROCHE INTÉGRANT DIFFÉRENTS DOMAINES DE VIE}

Une approche intégrant différents domaines de vie (Blustein, 2006) considère l'éventail complet des expériences des consultant.e.s et valorise à égalité les domaines professionnels et non professionnels. Ici, le terme "domaine» se rapporte aux différentes préoccupations présentées par les consultant·e.s, et le terme "intégrant» 
se rapporte à une approche ouverte, empathique et authentique visant à comprendre et jongler avec leurs expériences au travail et en dehors du travail. En d'autres termes, l'approche intégrant différents domaines de vie suggère de porter attention à l'ensemble des sphères d'existence de la personne, sans négliger aucun aspect de sa vie. Cela implique de faciliter le développement et le bien-être des consultant.e.s dans leurs différents domaines de vie, de reconnaître leurs problèmes liés au travail et de les aider à les gérer tout en favorisant leur développement personnel et interpersonnel.

Cette approche a des implications tant pour le counseling d'orientation que pour le counseling personnel. Pour ce qui a trait au counseling d'orientation, les conseillers.ères ont avantage à créer un espace protégé, permettant aux consultant.e.s de partager le sens qu'elles et ils donnent au travail ou au fait de ne pas travailler (Masdonati, Fournier, \& Lahrizi, soumis). Pour intégrer les domaines de la vie professionnelle et non professionnelle, les conseillers.ères en orientation doivent également situer le rôle que joue le travail dans la vie des consultant.e.s par rapport à leurs autres priorités et engagements. En ce qui concerne le counseling personnel, le fait de poser explicitement des questions sur la vie professionnelle de la personne au début du processus montrerait que les problèmes relatifs au travail font partie intégrante du processus. L'inclusion du travail en tant que domaine dans le counseling personnel peut également donner la possibilité d'explorer le rôle du contexte sociopolitique et économique dans la vie de leurs consultant.e.s (Richardson, 1996). Ouvrir des discussions sur la qualité de la vie professionnelle des consultant.e.s peut constituer un levier pour l'amélioration de leur vie et leur bien-être général.

\subsection{DÉVELOPPER LE POUVOIR D'AGIR}

Le terme empowerment, que nous traduisons par "pouvoir d'agir", a été largement discuté dans la littérature en counseling et psychothérapie féministe (Fassinger, 2000). Richardson (2000) définit le pouvoir d'agir comme "la capacité (1) à identifier des objectifs, (2) à développer et utiliser au mieux les ressources personnelles pour atteindre ces objectifs et (3) à négocier avec l'environnement les ressources nécessaires pour les atteindre» (p. 204, traduction personnelle). Alors que la première composante de cette définition fait référence à l' "agentivité" de la personne et à des comportements dirigés vers un objectif, les deux autres éléments concernent 
le développement de la maîtrise. En responsabilisant leurs consultant.e.s, les praticien.ne.s peuvent leur donner un sentiment de contrôle sur leur vie et leur apprendre à utiliser pleinement leur potentiel afin qu'elles et ils puissent se doter des qualités actuellement valorisées sur le marché de l'emploi (Le Bossé, Gaudreau, Arteau, Deschamps, \& Vandette, 2002).

Dans le contexte d'une pratique psychologique inclusive, développer le pouvoir d'agir implique de collaborer avec les consultant.e.s pour identifier des objectifs, tant professionnels que personnels, et pour prendre des mesures pour les réaliser. En outre, cela renvoie à aider les consultant.e.s à développer des compétences cognitives (par exemple, les compétences technologiques) et interpersonnelles (comme l'affirmation de soi) et à accéder à des ressources (Blustein, 2006). En raison de la configuration contemporaine du marché du travail, les individus traversent plus fréquemment que jamais des transitions inattendues (Masdonati \& Zittoun, 2012). Par ailleurs, pour de nombreuses personnes, le travail correspond davantage à une perte de pouvoir qu'à un moyen de devenir autonome. Par conséquent, les conseillers.ères en orientation devraient être attentifs.ves à ces réalités afin d'aider leurs consultant.e.s à développer leur flexibilité, à composer avec les incertitudes et les changements constants et à gérer les émotions liées aux défis jalonnant leur parcours professionnel. Mais développer le pouvoir d'agir peut également impliquer d'apporter une aide aux consultant.e.s confronté.e.s à un traitement injuste au travail, comme de la discrimination. Les praticien-ne.s peuvent alors développer le pouvoir d'agir des consultant.e.s marginalisé.e.s en leur apprenant à se protéger et à défendre leurs droits. Si les consultant·e.s n'ont pas accès aux ressources nécessaires pour se défendre, il pourrait être utile de les mettre en lien avec des "allié.e.s» ou avec des personnes à même d'utiliser du pouvoir pour améliorer leur situation (Le Bossé et al., 2002).

\subsection{FAVORISER LA CONSCIENCE CRITIQUE}

Développée par Paulo Freire (2007), la notion de conscience critique fait référence au processus d'appréciation par lequel les personnes opprimées analysent de manière critique leur situation sociopolitique et agissent ensuite pour imposer un changement (Watts, Diemer, \& Voight, 2011). Freire a eu recours à la conscience critique pour aider des paysans brésiliens à analyser leur situation et à prendre les mesures nécessaires pour réduire les inégalités sociales (Diemer \& Li, 2011). 
Son but était d'améliorer l'alphabétisation dans la communauté tout en enseignant la conscience critique comme outil de libération.

La conscience critique a trois composantes: la réflexion critique, l'efficacité politique et l'action critique (Diemer \& Blustein, 2006; Watts, Griffith, \& Abdul-Adil, 1999). La réflexion critique consiste à examiner comment des différences sociales (liées par exemple au statut socioéconomique, à l'origine ethnique et au sexe) peuvent entraîner des disparités dans de nombreux domaines de la vie tels que la santé, le bien-être et l'éducation. L'efficacité politique est une mesure de la capacité perçue d'un individu à influencer le changement social et politique, les personnes étant généralement plus susceptibles d'agir lorsqu' elles se perçoivent comme agents possibles de changement. L'action critique fait référence à la participation des individus à des actions individuelles ou collectives visant à promouvoir le changement social et politique. Ces actions peuvent inclure des changements aux niveaux institutionnel et communautaire. Les composantes de la conscience critique sont susceptibles d'interagir les unes avec les autres, de sorte que la réflexion critique peut affecter l'action critique et vice versa (Watts et al., 2011). Autrement dit, les personnes opprimées qui acquièrent une conscience critique de leur statut sociopolitique sont plus susceptibles de prendre des mesures pour améliorer ce dernier. D'autre part, lorsque les individus s'engagent dans le changement social, ils ont davantage d'occasions d'affiner leur compréhension des injustices systémiques.

Des chercheur-e.s et des activistes américain-e.s ont adopté ce concept et un nombre croissant de recherches ont exploré le rôle de la conscience critique dans l'amélioration de la santé mentale, de l'éducation et de la vie professionnelle. Par exemple, la conscience critique aiderait les jeunes marginalisé.e.s aux États-Unis à surmonter les obstacles structurels ou à prévenir l'oppression structurelle (Watts et al., 1999). Des études ont aussi montré que, chez les jeunes marginalisés, la conscience critique est associée à l'identité professionnelle et au développement de carrière (Diemer \& Blustein, 2006), aux réalisations professionnelles à l'âge adulte (Diemer, 2009) et à l'engagement civique (Diemer \& Li, 2011).

Bien qu'encore rares, les recherches sur les fondements de la conscience critique ont souligné le rôle clé joué par le dialogue (Diemer \& Li, 2011). En utilisant la conscience critique comme outil psychopédagogique dans le counseling, les praticien.ne.s peuvent aider les consultant.e.s marginalisé.e.s à analyser et à comprendre 
comment leur contexte sociopolitique et économique peut influencer leurs expériences professionnelles, y compris l'accès à un travail décent. Sensibiliser au lien entre problèmes systémiques et vie professionnelle et donner les moyens d'agir pour se défendre aux niveaux individuel et systémique peut les aider à ne pas s'attribuer la responsabilité des difficultés à obtenir un travail digne et décent.

Les praticien.ne.s peuvent également avoir recours à des outils d'évaluation pour engager leurs consultant.e.s dans des discussions sur leur qualité de vie au travail. Par exemple, l'échelle du travail décent (DWS ; Duffy et al., 2017) peut aider à identifier les composantes du travail décent auxquelles les consultant.e.s ne parviennent pas à accéder et leur permettre ainsi de prendre conscience du rôle des facteurs sociopolitiques dans leur vie professionnelle. À l'instar de Duffy et collègues, nous pensons qu'il est primordial de compléter ce type d'évaluation par des interventions psychoéducatives amenant les consultant.e.s à considérer le travail décent comme un droit fondamental et à renforcer ainsi leur conscience critique dans la sphère du travail.

Enfin, il est nécessaire que les praticien-ne·s elles et eux-mêmes développent une certaine conscience critique envers leur propre pratique. Elles et ils peuvent par exemple se livrer à une pratique réflexive (Noble \& McIlveen, 2012; McIlveen, 2015), impliquant l'analyse critique de leurs valeurs, biais, préjugés et normes culturelles, afin d'en jauger l'impact sur leur pratique. Les conseillers.ères doivent donc être à l'écoute de leurs préjugés et de leurs présupposés concernant leurs consultant.e.s. Par exemple, un·e praticien-ne issu.e d'une culture occidentale, qui valorise l'indépendance et l'autonomie, peut être conscient.e, voire remettre en question ces valeurs. En effet, celles-ci peuvent ne pas s'appliquer aux expériences professionnelles d'un.e consultant.e issu.e d'une autre culture. En outre, les praticien-ne.s peuvent réfléchir à la manière dont leur pouvoir, leurs privilèges - ainsi que leurs éventuelles identités marginalisées - peuvent interagir avec ceux de leurs consultant.e.s et utiliser cette prise de conscience pour améliorer la relation de conseil et leurs interventions.

\subsection{ILLUSTRATION CLINIQUE: LE CAS DE LEILA}

En nous appuyant sur une psychologie inclusive de l'activité de travail, nous avons résumé la manière dont le counseling d'orientation peut favoriser l'accès au travail décent. Dans ce qui suit, nous 
exposons et discutons la situation fictive de Leila, afin d'illustrer des interventions susceptibles d'accroître la probabilité pour les individus d'accéder à un travail décent et digne. Leila, une femme musulmane de 28 ans, hétérosexuelle et qui parle plusieurs langues, a récemment migré en Suisse afin de chercher de meilleures perspectives d'emploi. Elle est née au Maroc, mais sa famille a déménagé aux Pays-Bas alors qu'elle avait 9 ans. Il y a environ un an, après avoir obtenu son doctorat au sein d'une prestigieuse filière de bio-ingénierie aux Pays-Bas, Leila a décidé de déménager en Suisse pour travailler pour une société de recherche helvétique. Elle est mariée depuis sept ans et rapporte entretenir des relations positives avec son mari, Ahmad, et leur fille de 5 ans, Sarah, qui vivent avec elle. Leila a recherché le soutien d'une conseillère en orientation en raison de ses préoccupations récentes au travail, associées à des troubles du sommeil, de la fatigue, des difficultés d'attention/de concentration, un mal du pays, un sentiment de tristesse et de solitude, et des soucis concernant sa famille.

Leila rapporte que ses parents, qu'elle perçoit comme "très proches», ont toujours été fiers de ses réalisations. Elle dit avoir reçu un fort soutien émotionnel de sa famille, en particulier de son mari et de ses deux sœurs aînées, qui vivent toujours aux Pays-Bas. Les membres de sa famille vivant à l'étranger lui manquent, et elle dit avoir très souvent le mal du pays. Elle craint que sa demande de counseling n'inquiète sa famille, à cause de leurs fausses idées sur l'aide psychologique; par conséquent, elle hésite à s'ouvrir à eux sur ce sujet. Malgré ses difficultés à gérer les tâches ménagères, elle s'en occupe, car elle ne veut pas "déranger" son mari, qu’elle décrit comme "merveilleux». Leila adore jouer avec sa fille et regarder des films avec son mari. Néanmoins, elle rapporte avoir moins d'intérêt pour ces activités ces derniers temps. En plus de sa famille, elle considère sa voisine, également une femme migrante venant d'Inde, comme une personne proche, qui l'a encouragée à demander du soutien professionnel.

Leila mentionne qu'elle n'est pas satisfaite de son emploi, bien qu'elle ait toujours rêvé de travailler dans la recherche. Elle déclare que la majorité des employé.e.s de son entreprise sont des hommes, ce qui, selon elle, contribue à sa solitude au travail. Elle révèle plus tard que deux de ses collègues masculins ont tendance à plaisanter à propos du «manque de compétences scientifiques» des femmes. Elle ajoute qu'il y a quelques semaines, ces mêmes collègues ont 
ouvertement déclaré être en faveur de l'interdiction de l'immigration des musulman.e.s en Europe, en particulier celles et ceux d'ascendance arabe. Leila nie être directement insultée par ses collègues, mais se sent déconnectée d'eux. Elle se demande également pourquoi ils ont choisi de parler de leurs opinions antimusulman.e.s/arabes dans le laboratoire, sachant qu'elle pourrait facilement les entendre.

De plus, Leila note que son employeur a des exigences exceptionnellement élevées. Pour éviter "tout problème" avec son superviseur, elle se sent obligée de mener une partie de ses recherches en dehors de ses heures régulières de travail, limitant ainsi le temps qu'il lui reste pour la famille, pour les tâches ménagères et pour elle-même. Elle ne sait pas si elle peut parler à son superviseur de ces problèmes, car cela pourrait lui faire penser qu' elle est «trop sensible» et "inefficace». Leila déclare qu'elle rentre du travail en se sentant «démoralisée» et «incompétente». Elle est de plus en plus déprimée et se sent coupable parce qu'elle pense qu'elle est incapable de s'acquitter de ses responsabilités en tant qu'épouse et mère. A cause de ces problèmes, Leila envisage la possibilité de changer de travail, mais elle pense aussi qu'elle devrait continuer à faire de son mieux pour «survivre» dans son entreprise actuelle.

Vers la fin de la première rencontre avec la conseillère, Leila évoque aussi ses défis culturels: elle se demande si elle se sent la bienvenue en Europe. Elle identifie sa foi comme une ressource importante dans sa vie. Elle pratique l'islam, mais elle n'a pas encore eu l'occasion d'aller à la mosquée locale et de rencontrer d'autres musulman.e.s de la région. Elle a également des contacts limités avec la communauté marocaine/arabe en Suisse, en raison du peu de temps dont elle dispose pour sa vie sociale. Leila déclare également souffrir du fait d'appartenir à une minorité; en raison du nombre croissant de crimes motivés par la haine dans certaines régions d'Europe, elle s'inquiète pour la sécurité de sa famille. Elle note que cela peut contribuer à sa réticence à assister aux événements sociaux ou religieux avec d'autres musulman.e.s.

\subsubsection{Conceptualisation du cas}

La situation de Leila comporte un réseau complexe d'interactions entre des facteurs psychologiques et contextuels, qui ont un effet sur sa santé mentale, ses relations sociales et ses expériences professionnelles. À première vue, les préoccupations de Leila peuvent sembler résulter principalement de problèmes personnels, tels que 
des difficultés d'adaptation à la Suisse et à son nouvel emploi, un sentiment d'isolement/de mal du pays et des difficultés de maintien de l'équilibre entre vie professionnelle et vie personnelle. Leila évite tout conflit interpersonnel et hésite à demander de l'aide à ses proches et à son superviseur, par crainte d'éventuelles conséquences négatives. Cela affecte la quantité des soutiens dont Leila peut bénéficier pour s'adapter à un nouvel environnement. En conséquence, elle se sent incompétente et en échec à plusieurs égards et a tendance à se désengager d'activités qui pourraient ajouter du plaisir et du sens à sa vie.

Néanmoins, ces problèmes personnels sont également associés à plusieurs facteurs socioculturels, contextuels et politiques. Bien qu'elle soit privilégiée en termes d'orientation sexuelle, de niveau d'éducation, de classe sociale et d'aptitudes, plusieurs de ses identités sont marginalisées: elle travaille dans un domaine scientifique où les femmes sont sous-représentées et elle appartient à une minorité ethnique et religieuse. Comme suggéré par la PWT (Blustein et al., 2019; Duffy et al., 2016), ces identités marginalisées peuvent en partie expliquer la détresse que Leila éprouve actuellement dans sa vie professionnelle. Plus précisément, le fait de travailler au sein d'une institution qui emploie majoritairement des hommes pourrait être l'un des facteurs qui exacerbent son sentiment d'isolement. Elle a également entendu ses collègues échanger des plaisanteries désobligeantes et parler de leurs préjugés, ce qui correspond à une forme plus ou moins subtile (appelée "microagression", Sue et al., 2007) de sexisme et de discrimination contre les immigré.e.s musulman.e.s. En outre, des problèmes macroéconomiques tels que la xénophobie et les crimes de haine liés à l'islamophobie en Europe semblent menacer le sentiment de sécurité et d'appartenance de Leila et faire obstacle à son lien avec sa communauté, alors même que cela pourrait constituer une source de soutien social.

Du point de vue de la PWT (Duffy et al.), les expériences de marginalisation potentielle et effective vécues par Leila entravent probablement sa capacité à utiliser ses ressources pour surmonter les défis auxquels elle est confrontée au travail (son adaptabilité de carrière), limitant ainsi son accès à un travail décent. Grâce à son travail, Leila semble satisfaire ses besoins de survie/pouvoir et d'autodétermination, ce qui lui permet de répondre à ses besoins de base, d'acquérir un capital social et de mener des activités qui ont du sens pour elle. Pourtant, elle n'est pas en mesure de combler son 
besoin de liens sociaux, ce qui l'amène à se sentir déconnectée et insatisfaite dans ses relations professionnelles. Outre des conditions de travail menaçantes sur le plan interpersonnel, le travail de Leila ne semble pas offrir suffisamment de temps de repos et de liberté, ce qui constitue un obstacle supplémentaire au travail décent.

\subsubsection{Interventions}

Leila pourrait bénéficier d'un accompagnement intégrant les domaines de vie (Blustein, 2006), couvrant à la fois ses enjeux professionnels et extraprofessionnels, et d'interventions axées sur son pouvoir d'agir et sur sa conscience critique. Comme toute intervention psychologique, cet accompagnement devrait donner la priorité à une écoute empathique, à un soutien inconditionnel et à l'acceptation des expériences et des sentiments de Leila. Avant toute intervention, il est nécessaire d'évaluer plus précisément les symptômes de Leila, qui font penser à un épisode dépressif. Par conséquent, parallèlement à une démarche d'orientation, la conseillère recevant Leila devrait envisager de la référer à un.e psychothérapeute pour un éventuel traitement des problèmes liés à son humeur.

Après cette étape, la conseillère d'orientation pourrait partager avec Leila sa compréhension de ses problèmes et planifier avec elle les étapes du processus de counseling. Il pourrait également être utile d'échanger sur la façon dont elle pourrait mieux équilibrer ses engagements professionnels, ses responsabilités familiales et du temps pour elle-même. En lien avec la notion de conscience critique (Diemer \& Blustein, 2006), intervenir avec une approche psychoéducative, par exemple en nommant les facteurs qui contribuent à la détresse de Leila, pourrait accroître sa conscience des problèmes sociopolitiques qui sous-tendent ses symptômes dépressifs. La passation de l'échelle du travail décent (Duffy et al., 2017), tout comme une réflexion sur ses cinq dimensions, peut s'avérer utile pour faciliter ce processus et engager Leila dans un dialogue sur sa vie professionnelle et les facteurs systémiques qui affectent son bien-être. Ceci l'amènerait vraisemblablement à prendre conscience de ses droits en tant qu'employée et de l'impact de son environnement sur la qualité de sa vie professionnelle, mais aussi à réduire certains reproches que Leila a tendance à se faire quant à l'origine et la responsabilité de ses problèmes.

Une autre intervention potentiellement utile serait d'aider Leila à prendre des initiatives pour améliorer sa situation. Par exemple, il pourrait être bénéfique de lui apprendre des techniques 
d'affirmation de soi, qui lui permettraient de se défendre et de faire part de ses sentiments et de ses besoins aux collègues. La conseillère de Leila pourrait également l'aider à identifier et mobiliser des allié.e.s (par exemple, des supérieur.e.s ou des syndicats) pouvant défendre ses intérêts. À cette fin, et compte tenu du sentiment de solitude éprouvé par Leila, il serait porteur de la mettre en contact avec d'autres femmes chercheuses, susceptibles de lui offrir du soutien et de l'aide pour naviguer efficacement dans ce milieu. En outre, compte tenu des réticences de Leila à s'ouvrir à son mari et à son superviseur malgré ses difficultés à gérer ses tâches à la maison et au travail, il serait bénéfique d'examiner avec elle et, au besoin, de questionner certaines de ses croyances quant à la recherche de soutien social. Il est toutefois important d'explorer les valeurs culturelles de Leila concernant l'affirmation de soi et la recherche d'aide, car certaines cultures découragent ces comportements. Par conséquent, le défi serait d'accroître le pouvoir d'agir de Leila, tout en étant conscient de ses valeurs et en les respectant. En partageant ses difficultés et ses besoins avec les personnes qui ont sa confiance, comme son mari, Leila pourrait bénéficier d'un meilleur soutien instrumental (par exemple, de l'aide dans les tâches ménagères), ce qui contribuerait à soulager sa détresse émotionnelle.

Une autre intervention envisageable serait la création d'un espace protégé pour que Leila exprime et gère ses émotions concernant les crimes haineux qui ciblent sa communauté, car cela l'aiderait à se sentir entendue et reconnue. Cet échange pourrait lui permettre de mesurer les impacts psychologiques et sociaux de ces événements sur sa vie et de prendre conscience des avantages et des inconvénients engendrés par ses propres réactions (par exemple, prendre de la distance vis-à-vis de la communauté musulmane). Aider Leila à entrer en contact avec d'autres Marocain.e.s/musulman-e.s réduirait l'isolement social dont elle souffre, ce qui, à son tour, améliorerait son humeur et son fonctionnement au travail et dans les autres domaines de sa vie. Par ailleurs, lors de ces interventions, il est essentiel que la praticienne examine ses propres biais et préjugés éventuels sur la culture de Leila, par exemple à travers un processus de pratique réflexive.

Au niveau sociopolitique, Leila pourrait développer son pouvoir d'agir en s'investissant dans des actions pour un changement sociopolitique. Par exemple, elle pourrait collaborer avec des organismes visant à créer un lieu de travail plus inclusif pour les femmes. 
Promouvoir des mesures politiques en travaillant avec des organismes communautaires qui soutiennent les musulman.e.s ou les migrant.e.s pourrait être un autre moyen de renforcer son pouvoir d'agir et de développer sa conscience critique. Par un engagement dans des actions individuelles et sociopolitiques, un sentiment d' "agentivité" et de contrôle sur sa vie pourrait émerger chez elle. En somme, des interventions qui tiennent compte des forces individuelles, sociales et politiques façonnant les expériences de Leila et qui s'attaquant en même temps à ses difficultés professionnelles et extraprofessionnelles l'aideront vraisemblablement à accroître son bien-être professionnel et de vie, facilitant ainsi ses possibilités d'accéder à un travail décent.

\section{BILAN ET PERSPECTIVES POUR LA PROMOTION DU TRAVAIL DÉCENT}

Comme l'illustre le cas de Leila, proposer une pratique qui se focalise explicitement sur le concept de travail décent - et intégrant des théories psychologiques inclusives et axées sur la justice sociale - peut faciliter l'accès à de meilleures conditions de travail et augmenter le bien-être individuel et collectif, en répondant aux besoins de survie et pouvoir, de lien social et d'autodétermination (Blustein, 2006, 2013; Blustein et al., 2019). De plus, évaluer si les conditions de travail de nos consultant.e.s répondent aux normes du travail décent et intégrer le développement de leur conscience critique et de leur pouvoir d'agir dans les interventions de counseling peut contribuer à la promotion de la justice sociale, de l'équité et des droits humains.

Comportant un niveau croissant d'incertitude et d'instabilité, le monde du travail contemporain pose des défis qui peuvent nuire à la santé et au bien-être des individus (Dejours \& Deranty, 2010). L'Agenda du travail décent de l'OIT (1999, 2008; ILO, 2012) a questionné ces défis et a suscité des dialogues utiles sur la qualité du travail, sur la justice sociale et sur les droits humains. Cependant, comme mentionné en début de chapitre, cet Agenda a également été critiqué, notamment du fait de son étroitesse (Deranty \& MacMillan, 2012) et du manque de considération des expériences psychologiques du travail. Nous partageons ainsi le point de vue de Deranty et MacMillan quant au besoin d'élargissement de l'Agenda du travail décent. Celui-ci devrait en effet inclure non seulement la promotion de l'emploi, des conditions dans lesquelles s'exerce le travail et d'une négociation équilibrée entre employé·e.s et employeurs.ses, mais également 
des contenus du travail, c'est-à-dire du type d'activités exercées au travail et de leur impact sur le fonctionnement psychologique et social des individus. De ce point de vue, un emploi répondant aux normes de la définition du travail décent de l'OIT $(1999,2008)$ n'est pas automatiquement satisfaisant pour les travailleurs.ses, lorsque l'on considère les activités professionnelles qu'elles ou ils y exercent. Indépendamment des conditions de base prônées par l'OIT, les personnes qui ne parviennent pas à exercer des activités professionnelles compatibles avec leurs aptitudes ou leurs intérêts (par exemple, qui s'engagent dans des tâches trop faciles ou ennuyeuses au travail) sont susceptibles de vivre de l'insatisfaction et de souffrir d'effets négatifs du travail sur leur bien-être et leur santé.

Une compréhension plus fine du travail décent tenant compte de ses contenus et de sa qualité pourrait donner une impulsion à l'exploration des manières complexes dont le travail permet aux personnes d'utiliser pleinement leur potentiel lorsqu'elles s'engagent dans des activités professionnelles qui ont du sens pour elles (Dejours \& Deranty, 2010). Les initiatives futures visant à améliorer la vie au travail des individus devraient prendre en compte cette perspective. Une compréhension nuancée du travail décent implique également de considérer les rapports subjectifs des personnes au travail, étant donné que la signification et le sens du travail ne sont pas les mêmes pour tou.te.s les travailleurs.ses. En conséquence, les interventions de counseling d'orientation devraient inclure une évaluation précise de ce que le travail signifie pour les personnes à un moment précis de leur vie, en particulier en termes de centralité et d'importance, de valeurs et d'objectifs professionnels (Masdonati et al., soumis). Les actions ultérieures pourraient alors être ajustées à cette évaluation et viser à aider les personnes à intégrer un travail pouvant répondre à leurs attentes professionnelles particulières (Massoudi et al., 2018).

Une autre critique adressée à l'Agenda du travail décent de l'OIT s'axe sur le passage de la philosophie de la justice sociale, qui priorise les besoins des travailleurs.ses, aux définitions du travail décent centrées sur le marché (Di Ruggiero et al., 2015; Ribeiro et al., 2016). Les efforts visant à améliorer l'accès au travail décent ne peuvent aboutir qu'en adoptant une approche qui prévoit un programme de justice sociale et de promotion des droits humains (Blustein et al., 2016). Compte tenu des impacts indéniables de la mondialisation et des récents changements macroéconomiques 
sur la nature du travail et sur la disponibilité de travail décent, il est également nécessaire de passer de lignes directrices ambitieuses à des actes juridiques favorisant des changements sociopolitiques tangibles (MacNaughton \& Frey, 2011) qui protégeraient les droits des travailleurs-ses et amélioreraient leurs expériences professionnelles. La société ne sera en effet pas en mesure de remédier au manque de travail décent sans actions systémiques et sans changements qui plaident en faveur des personnes issues de milieux marginalisés.

Bien que les psychologues conseillers.ères en orientation aient surtout l'habitude d'intervenir au niveau individuel, nous pensons qu'elles et ils devraient s'engager en faveur des personnes qui sont dans l'impossibilité d'obtenir un travail décent et digne. Une telle vision du counseling comporte une critique des approches traditionnelles de la psychologie de l'orientation, qui tendent à se concentrer sur les populations privilégiées et à minimiser l'impact des facteurs contextuels sur la vie des personnes (Blustein, 2006, 2013). Au vu de la configuration actuelle du marché mondial du travail et des nouveaux risques d'exclusion sociale et d'inégalités qu'il comporte, il est nécessaire de mettre en place une pratique du counseling socialement engagée (reformist counseling practice), qui développe le pouvoir d'agir et la conscience critique des consultant.e.s, certes, mais qui encourage également les praticien.ne.s à analyser de manière critique et à réduire les obstacles systémiques auxquels les travailleur.ses sont confronté.e.s (Pouyaud, 2016). En ce sens, les mouvements PWF/PWT constituent une invitation à mettre les théories, les pratiques et les politiques au service de toutes les personnes, y compris les populations marginalisées et les travailleurs-ses précaires (Blustein et al., 2019).

À l'instar des interventions décrites dans la section précédente, les interventions de counseling incluant des enjeux de justice sociale exigent souvent un effort supplémentaire pour s'associer aux consultant.e.s afin de lutter contre les contraintes que leur vie impose. En plus de valider les sentiments et les expériences au travail des consultant.e.s et de les connecter aux sources de soutien à disposition dans leur communauté, les conseillers.ères en orientation peuvent éclairer les pouvoirs publics en leur transmettant leurs connaissances sur les liens entre travail décent, bien-être et identités sociales (Blustein, Masdonati, \& Rossier, 2017). Bien que cela constitue une tâche difficile, ce but peut être atteint grâce à la mise en place de collaborations réunissant des psychologues conseillers.ères en orientation, des décideurs-ses politiques et des 
représentant.e.s d'organisations internationales spécialisées dans le travail (par exemple, l'OIT). L'implication d'associations d'employeurs.ses dans ces efforts conjoints d'identification de problèmes systémiques liés au travail décent peut également être fructueuse, compte tenu de leur rôle clé dans le marché du travail.

L'intérêt croissant des psychologues de l'orientation pour les enjeux liés au travail décent est prometteur. Néanmoins, il est indispensable de poursuivre les recherches sur la scène internationale afin d'explorer les diverses expériences au travail des individus et des communautés. En supposant que le travail décent soit plus difficilement atteignable dans les pays où la pauvreté, les violations des droits humains, les inégalités sociales, l'instabilité politique et l'économie informelle sont plus importantes, un examen approfondi des expériences des travailleurs.ses dans ces contextes est nécessaire. Plusieurs organisations internationales, dont le Programme des Nations unies pour le développement (United Nations Development Program, 2014), ont participé à des initiatives visant à résoudre ces problèmes et à faciliter la collaboration entre les différent.e.s acteurs.rices du monde du travail. Ces initiatives sont utiles pour identifier les préoccupations liées au travail de personnes vivant dans divers contextes et pour intégrer une perspective scientifique aux interventions au niveau macro.

Des projets futurs associant interventions individuelles et systémiques pourraient s'avérer utiles pour promouvoir le travail décent à différents niveaux. Par exemple, les établissements de formation (comme les universités), le secteur privé et les gouvernements peuvent collaborer à des projets ciblant les besoins et les problèmes liés au travail dans une ville ou une région donnée et s'atteler aux défis auxquels est confrontée la main-d'œuvre locale. Une approche sensible au contexte permettrait également de saisir les enjeux spécifiques des groupes ou des populations plus exposés au travail «indécent» ou à la marginalisation, dans un pays ou une région donnée. Dans le contexte suisse, par exemple, les personnes avec un faible niveau de formation, les travailleurs.ses jeunes et seniors, les femmes et les personnes d'origine étrangère ont une probabilité plus élevée de connaître des difficultés sur le marché de l'emploi (Bonvin, 2016; Masdonati et al., 2019; Walter et al., 2016). Dans ce contexte, les interventions centrées sur le travail décent devraient donc prioriser la compréhension et l'amélioration des expériences professionnelles de ces groupes sociaux. 
Comme ce chapitre le montre, les mouvements de justice sociale en psychologie ont ouvert la voie à des initiatives de recherche intégrative et de conseil politique permettant d'enrichir l'Agenda du travail décent. À son tour, celle-ci est de plus en plus intégrée au counseling d'orientation, ce qui a donné lieu à des propositions de pratiques innovantes, au développement de théories transformatrices (transformative theories) et à la considération des enjeux des droits humains dans les réflexions sur le travail et la carrière ${ }^{3}$. L'intégration de la psychologie aux mouvements de promotion de la justice sociale articulés par l'OIT depuis près d'un siècle a le potentiel de promouvoir et d'intensifier l'effet de réformes destinées aux populations du monde entier qui s'efforcent de trouver dans leur travail une source de soutien, de sens et de contribution sociale. Le présent chapitre se veut ainsi une contribution à cet important mouvement, dont le potentiel pour l'amélioration du bien-être des individus, des communautés et des nations est énorme.

\section{RÉFÉRENCES BIBLIOGRAPHIQUES}

Allan, B. A., Duffy, R. D., \& Blustein, D. L. (2016). Under (and over) employment: Measurement and correlates of employment discrepancy. The Counseling Psychologist, 44, 815-840. Doi : 10.1177/0011000016654766.

Autin, K. L., Douglass, R. P., Duffy, R. D., England, J. W., \& Allan, B. A. (2017). Subjective social status, work volition, and career adaptability: A longitudinal study. Journal of Vocational Behavior, 99, 1-10. Doi: 10.1016/j.jvb.2016.11.007.

Benach, J., Vives, A., Amable, M., Vanroelen, C., Tarafa, G., \& MunTANER, C. (2014). Precarious employment: Understanding an emerging social determinant of health. Annual Review of Public Health, 35, 229-253. Doi: 10.1146/annurev-publhealth-032013-182500.

Blustein, D. L. (2001). Extending the reach of vocational psychology: Toward an inclusive and integrated psychology of working. Journal of Vocational Behavior, 59, 171-182. Doi : 10.1006/ jube.2001.1823.

3. Pour plus d'informations à ce sujet, nous recommandons la lecture du livre blanc de Blustein et collègues (2017), rédigé pour l'OIT, ainsi que les travaux de Blustein et collègues (2016); Deranty et MacMillian (2012); Ribeiro et collègues (2016) et le récent ouvrage de Blustein (2019). 
BlusteIn, D. L. (2006). The psychology of working: A new perspective for career development, counseling, and public policy. New York: Routledge.

Blustein, D. L. (2008). The role of work in psychological health and well-being. The American Psychologist, 63, 228-240. Doi: 10.1037/0003-066X.63.4.228.

Blustein, D. L. (2011). A relational theory of working. Journal of Vocational Behavior, 79, 1-17. Doi: 10.1016/j.jvb.2010.10.004.

Blustein, D. L. (2013). The psychology of working: A new perspective for a new era. In D. L. BLUSTEIN (éd.), The Oxford handbook of the psychology of working (pp. 3-18). New York: Oxford University Press. Doi: 10.1093/oxfordhb/9780199758791.013.0001.

Blustein, D. L. (2019). The importance of work in an age of uncertainty: The eroding work experience in America. New York: Oxford University Press.

Blustein, D. L., Kenny, M., Di Fabio, A., \& Guichard, J. (2019). Expanding the impact of the Psychology of Working: Engaging psychology in the struggle for decent work and human rights. Journal of Career Assessment, 27, 3-28. Doi: 10.1177/1069072718774002.

Blustein, D. L., Kozan, S., \& Connors-Kellgren, A. (2013). Unemployment and underemployment: A narrative analysis about loss. Journal of Vocational Behavior, 82, 256-265. Doi : 10.1016/j. jvb.2013.02.005.

Blustein, D. L., Masdonati, J., \& Rossier, J. (2017). Psychology and the International Labor Organization: The role of psychology in the Decent Work Agenda. International Labor Organization (ILO). En ligne: [http://www.ilo.org/global/research/publications/ WCMS_561013/lang--en/index.htm].

Blustein, D. L., Murphy, K. A., Kenny, M. E., Jernigan, M., Pérez-Gualdrón, L., Castañeda, T., \& Davis, O. (2010). Exploring urban students'constructions about school, work, race and ethnicity. Journal of Counseling Psychology, 57, 248-254. Doi: 10.1037/a0018939.

Blustein, D. L., Olle, C., Connors-Kellgren, A., \& Diamonti, A. J. (2016). Decent work: A psychological perspective. Frontiers in Psychology, 7: 407. Doi: 10.3389/fpsyg.2016.00407. 
Bonvin, J.-M. (2016). Transformations des conditions de travail: quels enjeux pour les salariés? Terra Cognita, 29, 32-34.

Burchell, B., Sehnbruch, K., Piasna, A., \& Agloni, N. (2014). The quality of employment and decent work: definitions, methodologies, and ongoing debates. Cambridge Journal of Economics, 38, 459-477. Doi : 10.1093/cje/bet067.

Bureau international du travail (2016). Programme par pays de promotion du travail décent de l'OIT: Manuel pratique. En ligne: [https://www.ilo.org/wcmsp5/groups/public/---ed_mas/---program/documents/genericdocument/wcms_561027.pdf].

Chaves, A. P., Diemer, M. A., Blustein, D. L., Gallagher, L. A., DeVoy, J. E., Casares, M. T., \& Perry, J. C. (2004). Conceptions of work: The view from urban youth. Journal of Counseling Psycho$\log y$, 51, 275. Doi : 10.1037/0022-0167.51.3.275.

Dejours, C., \& Deranty, J. P. (2010). The centrality of work. Critical Horizons, 11, 167-180. Doi: 10.1558/crit.v11i2.167.

Deranty, J. P., \& MacMillan, C. (2012). The ILO's decent work initiative: Suggestions for an extension of the notion of «decent work». Journal of Social Philosophy, 43, 386-405. Doi: 10.1111/ josp. 12003.

Di Fabio, A., \& Kenny, M. E. (2016). From decent work to decent lives: positive self and relational management (PS \& RM) in the twenty-first century. Frontiers in Psychology, 7: 361. Doi: 10.3389/ fpsyg.2016.00361.

Di Ruggiero, E., Cohen, J. E., Cole, D. C., \& Forman, L. (2015). Public health agenda setting in a global context: the International Labor Organization's decent work agenda. American Journal of Public Health, 105, 58-61. Doi: 10.2105/ajph.2014.302455.

Diemer, M. A. (2009). Pathways to occupational attainment among poor youth of color the role of sociopolitical development. The Counseling Psychologist, 37, 6-35. Doi: 10.1177/0011000007309858.

Diemer, M. A., \& Blustein, D. L. (2006). Critical consciousness and career development among urban youth. Journal of Vocational Behavior, 68, 220-232. Doi: 10.1016/j.jvb.2005.07.001.

Diemer, M. A., \& Li, C. H. (2011). Critical consciousness development and political participation among marginalized youth. 
Child Development, 82, 1815-1833. Doi: 10.1111/j.14678624.2011.01650.x.

Douglass, R. P., Velez, B. L., Conlin, S. E., Duffy, R. D., \& England, J. W. (2017). Examining the psychology of working theory: Decent work among sexual minorities. Journal of Counseling Psychology, 64, 550-559. Doi: 10.1037/cou0000212.

Duffy, R. D., Allan, B. A., England, J. W., Blustein, D. L., Autin, K. L., Douglass, R. P., ... Santos, E. J. (2017). The development and initial validation of the Decent Work Scale. Journal of Counseling Psychology, 64, 206-221. Doi : 10.1037/cou0000191.

Duffy, R. D., Blustein, D. L., Diemer, M. A., \& Autin, K. L. (2016). The psychology of working theory. Journal of Counseling Psychology, 63, 127-148. Doi: 10.1037/cou0000140.

Duffy, R. D., Diemer, M. A., Perry, J. C., Laurenzi, C., \& TorREY, C. L. (2012). The construction and initial validation of the Work Volition Scale. Journal of Vocational Behavior, 80, 400-411. Doi: $10.1016 /$ j.jvb.2011.04.002.

FAssinger, R. E. (2000). Gender and sexuality in human development: Implications for prevention and advocacy in counseling psychology. In S. D. BROWN \& R. W. LENT (éds), Handbook of counseling psychology (3e édition, pp. 345-378). New York: Wiley.

Ferrari, L., Nota, L., Soresi, S., Blustein, D. L., Murphy, K. A., \& KennA, A. C. (2009). Constructions of work among adolescents in transition. Journal of Career Assessment, 17, 99-115. Doi: $10.1177 / 1069072708325829$.

Flores, L. Y., Mendoza, M. M., Ojeda, L., He, Y., Meza, R. R., Medina, V., ... Jordan, S. (2011). A qualitative inquiry of Latino immigrants'work experiences in the Midwest. Journal of Counseling Psychology, 58, 522-536. Doi : 10.1037/a0025241.

Fournier, G., Zimmermann, H., Gauthier, C., \& Masdonati, J. (2014). L'expérience du chômage chez les travailleurs seniors: de la crise identitaire à la réflexion sur l'avenir. L'Orientation scolaire et professionnelle, 43, 337-366. Doi: 10.4000/osp.4431.

Fournier, G., Zimmermann, H., Gauthier, C., Masdonati, J., \& Lachance, L. (2016). Vers l'élaboration d'un cadre d'analyse des parcours professionnels: l'éclairage de l'approche du Parcours de vie et des théories récentes en développement de carrière. 
In G. Fournier, E. POIREL \& L. LACHANCE (éds), Éducation et vie au travail: Perspectives contemporaines sur les parcours de vie professionnelle (pp. 93-143). Québec: Presses de l'Université Laval.

Freire, P. (2007). Pedagogy of the oppressed (3 édition). New York: The Continuum International.

Guerrero, L., \& Singh, S. (2013). The psychology of working: A case study of Mexican American women with low educational attainment. The Career Development Quarterly, 61(1), 27-39. Doi : 10.1002/j.2161-0045.2013.00033.x.

Guichard, J. (2013). Career guidance, education, and dialogues for a fair and sustainable human development. Paper Presented at Inaugural Conference of the Unesco Chair of Lifelong Guidance and Counselling. Wroclaw: University of Wroclaw.

International Labor Organization (2012). Decent work indicators: Guidelines for procedures and users of statistical and legal framework indicators. Genève: International Labor Office.

International Labor Organization (2018). World employment and social outlook: Trends 2018. Genève: International Labor Office.

KATZ, L., \& KRUEGER, A. (2016). The rise and nature of alternative work arrangements in the United States, 1995-2015 (Working Paper $\left.\mathrm{n}^{\circ} \mathrm{w} 22667\right)$. Cambridge (MA): National Bureau of Economic Research. En ligne: [http://www.nber.org/papers/w22667.pdf].

Kenny, M. E., \& Medvide, M. B. (2013). Relational influences on career development. In S. D. Brown \& R. Lent (éds), Career development and counseling: Putting theory and research to work ( ${ }^{\mathrm{e}}$ édition, pp. 329-356). New York: Wiley.

Kozan, S., Işı, E., \& Blustein, D. L. (2019). Decent work and well-being among low-income Turkish employees: Testing the psychology of working theory. Journal of Counseling Psychology, 66, 317-327. Doi: 10.1037/cou0000342.

Le Bossé, Y., Gaudreau, L., Arteau, M., Deschamps, K., \& VanDETTE, L. (2002). L'approche centrée sur le développement du pouvoir agir: Aperçu de ses fondements et de son application. Revue canadienne de counseling, 36 (3), 180-193.

LyONs, H. Z. (2011). Responding to hard times in the «big easy»: Meeting the vocational needs of low-income African American 
New Orleans residents. The Career Development Quarterly, 59, 290-301. Doi : 10.1002/j.2161-0045.2011.tb00070.x.

MacNaughton, G., \& Frey, D. F. (2011) Decent work for all: A holistic human rights approach. American University International Law Review, 26, pp. 441-483.

Masdonati, J., Fournier, G., \& Lahrizi, I. Z. (submitted). Work importance and purposes during the transition from vocational education and training to the labor market.

Masdonati, J., Schreiber, M., Marcionetti, J., \& Rossier, J. (2019). Decent work in Switzerland: Context, conceptualization, and assessment. Journal of Vocational Behavior, 110, 12-27. Doi: 10.1016/j.jvb.2018.11.004.

Masdonati, J., \& ZitToun, T. (2012). Les transitions professionnelles: processus psychosociaux et implications pour le conseil en orientation. L'Orientation scolaire et professionnelle, 41, 229-253. Doi: $10.4000 /$ osp.3776.

Massoudi, K., Abessolo, M., Atitsogbe, K. A., Banet, E., Bollmann, G., Dauwalder, J.-P., Handschin, P., Maggiori, C., Masdonati, J., Rochat, S. \& Rossier, J. (2018). A value-centered approach to decent work. In V. CoHEn-SCAli, J. Pouyaud, V. M. Podgórny, Drabik-Podgórna, G. Aisenson, J.-L. Bernaud, I. A. MOUMOUla \& J. Guichard (éds), Interventions in career design and education: Transformation for sustainable development and decent work (pp. 93-110). Cham, Suisse: Springer / Unesco Chair on Life Long Guidance and Counseling. Doi: 10.1007/978-3-31991968-3_6.

McIlveEn, P. (2015a). The next horizon of career counselling. In J. G. Maree \& A. di Fabio (éds), Exploring new horizons in career counselling: Turning challenges into opportunities (pp. 331-339). Rotterdam: Sense Publishers.

Noble, K., \& McIlveen, P. (2012). Being, knowing, and doing: A model for reflexivity in social constructionist practices. In P. McIlveen \& D. E. Schultheiss (éds), Social constructionism in vocational psychology and career development (pp. 105-113). Rotterdam: Sense Publishers.

Organisation internationale du travail (1999). Rapport du Directeur général: Un travail décent. In Conférence internationale du travail, 
87th session, Genève. En ligne: [https://www.ilo.org/public/french/ standards/relm/ilc/ilc87/rep-i.htm].

Organisation internationale du travail. (2008). Rapport sur le travail dans le monde 2008: Les inégalités de revenus à l'heure de la mondialisation financière. En ligne: [https:/www.ilo.org/publicl french/bureau/inst/download/world08_f.pdf].

Paul, K. I., \& Moser, K. (2009). Unemployment impairs mental health: Meta-analyses. Journal of Vocational Behavior, 74, 264-282. Doi: $10.1016 /$ j.jvb.2009.01.001.

Pouyaud, J. (2016). For a psychosocial approach to decent work. Frontiers in Psychology, 7: 422. Doi : 10.3389/fpsyg.2016.00422.

Ribeiro, M. A., Silva, F. F., \& Figueiredo, P. M. (2016). Discussing the notion of decent work: Senses of working for a group of Brazilian workers without college education. Frontiers in Psycho$\log y$, 7: 207. Doi : 10.3389/fpsyg.2016.00207.

Richardson, M. S. (1996). From career counseling to counseling/ psychotherapy and work, jobs, and career. In M. L. SAVICKAS \& W. B. WALSH (éds), Handbook of career counseling theory and practice (pp. 347-360). Palo Alto: Davies-Black.

Richardson, M. S. (2000). A new perspective for counselors: From career ideologies to empowerment through work and relationships practices. In A. Collin, \& R. A. Young (éds), The future of career (pp. 197-211). New York: Cambridge University Press.

Sehnbruch, K., Burchell, B., Agloni, N., \& Piasna, A. (2015). Human development and decent work: Why some concepts succeed and others fail to make an impact. Development and Change, 46, 197-224. Doi: 10.1111/dech.12149.

STANDING, G. (2008). The ILO: An Agency for globalization? Development and Change, 39, 355-384. Doi: 10.1111/j.14677660.2008.00484.x.

Sue, D. W., Capodilupo, C. M., Torino, G. C., Bucceri, J. M., Holder, A., Nadal, K. L., \& Esquilin, M. (2007). Racial microaggressions in everyday life: implications for clinical practice. American Psychologist, 62, 271-286. Doi: 10.1037/0003066X.62.4.271.

Toporek, R. L., Gerstein, L. H., Fouad, N. A., Roysircar, G., \& 
ISRAEL, T. (éds) (2006). Handbook of social justice in counseling psychology: Leadership, vision, and action. Thousand Oaks (CA): Sage. United Nations Development Program (2014). The role of the private sector in inclusive development: Barriers and opportunities at the base of the pyramid. Istanbul: UNDP Istanbul International Center for Private Sector in Development.

Walter, P., Bläuer Herrmann, A., Cangemi, V., Murier, T., PerrenOUd, S., Reutter, R., ... Zimmermann, M. (2016). Indicateurs du marché du travail 2016. Neuchâtel: Office fédéral de la statistique.

WANBERG, C. R. (2012). The individual experience of unemployment. Annual Review of Psychology, 63, 369-396. Doi : 10.1146/ annurev-psych-120710-100500.

Watts, R. J., Diemer, M. A., \& Voight, A. M. (2011). Critical consciousness: Current status and future directions. New Directions for Child and Adolescent Development, 134, 43-57. Doi: 10.1002/cd.310.

Watts, R. J., Griffith, D. M., \& Abdul-Adil, J. (1999). Sociopolitical development as an antidote for oppression - theory and action. American Journal of Community Psychology, 27, 255-271. Doi: 10.1023/A: 1022839818873. 

TROISIÈME PARTIE ORIENTATION ET RESSOURCES 



\title{
9. DES VULNÉRABILITÉS AUX VERTUS: UNE APPROCHE POSITIVE DU BIEN-ÊTRE AU TRAVAIL ${ }^{1}$
}

KOOROSH MASSOUDI, IEVA URBANAVICIUTE (UNIVERSITÉ DE LAUSANNE), JENNIFER HOFMANN ET FABIAN GANDER (UNIVERSITÉ DE ZURICH)

\begin{abstract}
e chapitre s'attelle à trois questions: Qu'est-ce que le bienêtre au travail? Quels en sont les déterminants? Comment peut-on le développer dans une démarche d'accompagnement en psychologie du conseil et de l'orientation? Dans un premier temps, nous tenterons de dessiner les contours du bien-être au travail et d'en proposer une définition opérationnelle afin d'aider les praticien-ne-s à évaluer le degré de bien-être actuel de leurs consultant.e.s. Puis nous chercherons à décrire les différents déterminants - en termes de ressources individuelles et environnementales - qui, de par leurs interactions, contribuent aux expériences positives au travail; il s'agira ici de proposer des pistes pour identifier les leviers et les ressources utiles et mobilisables dans une démarche d'accompagnement en psychologie du conseil et de l'orientation. Enfin, une dernière partie consistera en une proposition d'interventions; nous fondant sur une étude de cas, nous tenterons ainsi d'illustrer nos propos par des pistes d'action et d'intervention.
\end{abstract}

\section{INTRODUCTION}

Durant les trois dernières décennies, le monde du travail a connu des changements significatifs induits par des phénomènes globaux et structurels. Citons par exemple la mondialisation des marchés et ses effets sur l'intensification de la compétition et de l'insécurité de l'emploi, et l'augmentation des exigences en matière de flexibilité et d'adaptation. D'autre part, les intenses progrès technologiques

1. Cette publication a bénéficié du soutien du Pôle de recherche national LIVES-Surmonter la vulnérabilité, financé par le Fonds national suisse (n ${ }^{\circ}$ 51NF40-160590). Les auteur.e.s remercient le Fonds national suisse de la recherche scientifique de son aide financière. 
et leur impact sur la numérisation et l'automation du travail ont transformé de manière significative les modes de production, le rapport au travail et le développement des parcours professionnels, certain.e.s auteur.e.s n'hésitant pas à parler d'une "quatrième révolution industrielle» (Schwab, 2016) pour souligner l'ampleur de ces changements. Enfin, rappelons que ces changements affectent la nature même du travail dans les économies post-industrielles qui ont connu depuis les années 1990 une croissance impressionnante et continue des activités du secteur des services aux dépens des secteurs manufacturier, industriel ou agricole. Plusieurs auteur.e.s ont relevé les effets de cette "tertiarisation" des activités sur les contraintes psychosociales du travail moderne (Massoudi, 2009), à travers une recrudescence de tâches de résolution de problèmes, exigeant un haut niveau de qualification et des compétences cognitives accrues, une augmentation des procédures administratives aux finalités peu intelligibles et nuisant à la perception du sens du travail effectué, ou une complexification des relations sociales au travail et leur potentiel de conflits interpersonnels. Il s'avère que ces changements conjoncturels affectent également les modes d'organisation du travail, induisant une tension et des risques psychosociaux qui à leur tour ont une incidence sur la santé des travailleur.se.s. Il existe aujourd'hui un important corpus empirique qui signale une augmentation du stress professionnel et de ses effets, en termes de risques pour la santé des individus et de coûts pour les collectivités publiques et les entreprises. De nombreuses études révèlent ce phénomène à travers des indicateurs subjectifs, par exemple une augmentation de la perception du stress et de la pression au travail (Blaser \& Amstad, 2016; Grebner et al., 2011; Massoudi, 2009), mais également sur la base d'indicateurs objectifs comme l'absentéisme, le présentéisme, le taux de turnover dans les entreprises ou la part grandissante des coûts de la santé liée à la pression professionnelle (Ramaciotti \& Perriard, 2000 ; Paoli \& Merllié, 2001).

En préambule à sa constitution de 1946, l'Organisation mondiale de la santé définit la santé comme "un état de bien-être complet physique, mental et social, (qui) ne consiste pas seulement en une absence de maladie ou d'infirmité» (OMS, 1946, p. 100). Force est de constater, pourtant, que les chercheur.e.s et praticien.ne.s en psychologie se sont principalement intéressé.e.s à la compréhension des pathologies et au traitement des troubles mentaux (Seligman \& Csikszentmihalyi, 2000), et ce depuis la fin de 
la Seconde Guerre mondiale. Il en est de même des travaux portant sur la santé au travail, qui se sont essentiellement intéressés à l'identification des risques et à la prise en charge de la détresse psychologique sous toutes ses formes (Diener et al., 1999), parmi lesquelles le concept populaire de burnout (Maslach \& Jackson, 1986) représente vraisemblablement un archétype des «maladies professionnelles» qui caractérise les sociétés modernes (Weber \& Jaekel-Reinhard, 2000). Or il apparaît qu'une telle approche, exclusivement centrée sur les symptômes négatifs, contrevient à une définition holistique de la santé qui s'exprime également à travers des manifestations positives. Dans ce sens, Seligman rappelle dès la fin des années 1990 la nécessité de développer une psychologie positive afin de compléter et d'équilibrer les approches psychologiques existantes. Après avoir consacré sa carrière à l'étiologie de la dépression à travers la notion d'impuissance apprise (Seligman, 1972), Seligman réoriente ainsi ses travaux vers le développement du potentiel humain et la recherche du bonheur en introduisant le concept d'optimisme appris.

La psychologie positive se donne comme objectif d'œuvrer au développement du bien-être et d'accompagner les individus dans leurs efforts de développement et d'épanouissement, estimant qu'une intervention pertinente ne se résume pas à soigner ce qui est dysfonctionnel, mais devrait également viser à renforcer ce qui est fonctionnel. En d'autres termes, la psychologie positive représente "l'étude scientifique de ce qui va bien dans la vie, de la naissance à la mort et dans toutes les étapes intermédiaires" (Peterson, 2006, p. 4).

Nous estimons que la psychologie positive propose un cadre paradigmatique propice à l'élaboration d'une variété d'interventions visant au développement du potentiel des individus et à la promotion du bien-être au travail (pour une vue générale, voir Harzer \& Ruch, 2015). Dans ce sens, il nous semble utile pour les praticien-ne.s de l'orientation de s'inspirer de ce cadre et des concepts relatifs pour aider leurs consultant.e.s à identifier leurs ressources et les accompagner dans la construction de projets - de vie et de travail - porteurs de sens (Littman-Ovadia, Lazar-Butbul, \& Benjamin, 2014).

Cherchant à structurer les principaux domaines d'étude et d'intervention de la psychologie positive, Peterson (2006) suggère trois piliers essentiels de thématiques qui contribuent au bien-être, à savoir les expériences subjectives positives, les traits individuels 
positifs et les institutions positives. Nous nous proposons ainsi de transposer cette approche et ses thématiques centrales au domaine du bien-être au travail. Pour ce faire, il s'agira dans un premier temps de définir le bien-être au travail, puis d'en identifier les déterminants en termes d'expériences positives, de ressources individuelles et d'environnements professionnels salutogènes.

\section{LE BIEN-ÊTRE AU TRAVAIL: UN CONCEPT MULTIDIMENSIONNEL}

Comment définir le bien-être au travail? Vaste question, tant la conceptualisation du bien-être semble diverse et multiple, avec plus d'une vingtaine de modèles proposés à travers la littérature (Dagenais-Desmarais, 2005). Nous pouvons tout de même identifier deux consensus à travers cette nébuleuse. Tout d'abord, la majorité des auteur.e.s considèrent le bien-être au travail comme un indicateur essentiel de la santé des individus en général (Dagenais-Desmarais \& Savoie, 2012; Gilbert et al., 2011), d'où les effets collatéraux positifs des interventions visant la promotion des expériences positives au travail. Ensuite, même s'il n'existe actuellement pas de consensus quant au nombre exact et à la nature spécifique des éléments constitutifs du bien-être, la plupart des approches théoriques s'accordent sur la nécessité de considérer à la fois une composante hédonique et une composante eudémonique pour en élaborer une vision complète et équilibrée (Seligman, 2011). La composante hédonique, se fondant sur le concept de bien-être subjectif, recouvre les affects positifs qui alimentent le plaisir et la satisfaction au travail. La composante eudémonique, habituellement nommée bienêtre psychologique, relève quant à elle du sentiment d'avoir un sens au travail, de réaliser son potentiel à travers ses activités professionnelles et de construire des relations interpersonnelles satisfaisantes.

Rothmann (2008) avance que, même s'il a souvent été réduit à des mesures de satisfaction professionnelle, le bien-être au travail résulte des diverses expériences de l'individu aux prises avec son activité professionnelle et devrait donc être approché comme un construit multidimensionnel. Dans le même sens, Dagenais-Demarais et Savoie (2012) proposent de saisir les expériences positives des travailleurs «à travers cinq composantes essentielles, soit l'adéquation interpersonnelle au travail, l'épanouissement au travail, le sentiment de compétence au travail, la reconnaissance perçue au travail et la volonté d'engagement au travail» (Dagenais-Desmarais, 
Gilbert, \& Malo, 2018, p. 8.). Enfin, dans un article récent, Becker et collègues (2016) proposent une taxonomie du bien-être au travail qui décrit quatre cas de figure "prototypiques» en combinant le degré de plaisir éprouvé au travail à celui de l'énergie mobilisée par les travailleur.se.s. Il semble qu'une telle taxonomie aide à mettre en évidence le caractère à la fois hédonique et eudémonique du bien-être au travail. Au-delà des controverses et des désaccords autour de ces deux traditions, les auteur.e.s appellent aujourd'hui à une perspective unifiée et intégrative du bien-être au travail. Il nous semble donc utile, lors d'une phase préliminaire de compréhension et d'évaluation de la situation des consultant.e.s, d'explorer la dimension expérientielle et affective à travers les expériences au travail, mais également la dimension motivationnelle en questionnant les efforts, l'engagement et le sens au travail.

\section{LES EXPÉRIENCES SUBJECTIVES POSITIVES:}

\section{CULTIVER LE PLAISIR ET LA MOTIVATION AU TRAVAIL}

L'accent est porté ici sur les états psychologiques qui sont agréables et généralement désirables (Peterson, 2006). Dépassant une approche purement hédoniste et centrée sur le plaisir individuel comme une fin en soi, la théorie d'expansion et de construction (Broaden-and-build theory, BBT, Fredrickson, 2001) s'intéresse aux effets des émotions positives sur la performance. Elle postule que les émotions négatives poussent les individus à focaliser leur attention sur les menaces perçues et à puiser dans un répertoire comportemental réduit une réponse rapide à une situation d'urgence (voir par exemple l'effet du stress et de l'anxiété sur les réponses de type attaque/fuite ou approche/évitement, Zimmermann \& HaourKnipe, 1988). D’un autre côté, l'expérience d'émotions positives induit un élargissement du répertoire cognitivo-comportemental; elle permet donc d'élaborer des solutions nouvelles et créatives, de développer de nouvelles compétences et ainsi d'initier un cycle vertueux de développement personnel.

L'application de cette théorie dans le domaine de la psychologie du travail et des organisations permet d'affirmer deux éléments essentiels. Tout d'abord, un.e travailleur.se heureux.se semble être un.e travailleur-se productif.ve. Nombre d'études ont en effet mis en évidence le lien entre les états affectifs positifs et différents indicateurs de performance et de fonctionnement optimal au travail, 
notamment la coopération et les comportements prosociaux, la créativité et la résolution de problèmes ou encore la santé et l'ajustement au stress (voir par exemple Lyubomirsky, Tkach, \& DiMatteo, 2005; Roberts, Caspi, \& Moffitt, 2003).

Ensuite, les expériences positives au travail - et la motivation qui en découle - semblent étroitement associées à la reconnaissance et à la satisfaction des besoins psychologiques des travailleur.se.s (Dollard \& Bakker, 2010). La théorie de l'autodétermination (Self-Determination Theory, SDT; Deci \& Ryan, 2000) postule l'existence de trois besoins psychologiques de base universels qui motivent le comportement humain. Le besoin d'autonomie, proche du concept de volition, traduit le désir d'agir en cohérence avec soi et d'exercer son libre arbitre. Le besoin de compétence représente quant à lui le désir d'exercer une influence sur son environnement et, dès lors, de renforcer un sentiment de contrôle et de maîtrise. Enfin, le besoin d'appartenance alimente la motivation à construire des relations interpersonnelles positives et à se sentir membre d'un groupe social. Se focalisant sur les motivations sous-jacentes aux comportements humains, la SDT introduit la satisfaction de ces besoins comme une condition à une existence saine et épanouie, et ce quel que soit le domaine d'activité. Dans ce sens, la SDT a été appliquée à la sphère professionnelle et des recherches attestent de l'impact de l'environnement de travail sur la satisfaction des besoins des travailleur.se.s, et par extension sur leur bien-être. Une étude de Van den Broeck et collègues (2008) révèle par exemple que les employé.e.s actif.ve.s dans des organisations caractérisées par un soutien élevé et des ressources importantes font l'expérience d'une satisfaction de leurs besoins, s'engagent avec vigueur et enthousiasme dans leur travail et présentent un risque faible de burnout. Malgré la place accordée à l'environnement, dont les qualités et les ressources contribuent à l'épanouissement et à un fonctionnement optimal, l'individu n'est pas considéré ici comme passif et subissant les circonstances contextuelles. Certaines différences interindividuelles influencent également la tendance à percevoir des ressources dans l'environnement, à afficher des comportements autodéterminés et à persévérer dans la poursuite de motivations intrinsèques pour construire des expériences positives (Gagné \& Deci, 2005). Nous allons nous intéresser tour à tour aux caractéristiques individuelles et environnementales qui contribuent à la motivation et à l'engagement au travail. 


\section{LES TRAITS POSITIFS: IDENTIFIER ET DÉVELOPPER \\ LES RESSOURCES INDIVIDUELLES}

Les ressources personnelles, résultant des évaluations positives de l'individu quant à sa capacité à faire face à l'adversité (résilience) et à influencer son environnement professionnel de manière efficace (sentiment d'efficacité et agentivité), jouent un rôle important dans les interactions des travailleur.se.s avec leur environnement (Bakker $\&$ Demerouti, 2014). Se fondant sur le terreau fertile de la psychologie positive, de nombreux modèles théoriques visent à identifier ces ressources qui aident les individus à répondre aux demandes intenses et grandissantes d'un monde du travail turbulent, à gérer le stress, à afficher des comportements adaptatifs et, dès lors, à maintenir un état de bien-être optimal.

\subsection{LE CAPITAL PSYCHOLOGIQUE}

L'un des modèles les plus reconnus dans ce domaine est celui du capital psychologique (Luthans, Youssef, \& Avolio, 2007). Cette notion, dérivée des conceptions similaires du capital social et humain, n'en introduit pas moins une différence. Plutôt que d'investir les questions certes fondamentales que sont "Que sais-je?» (capital humain) ou "Sur qui puis-je compter?» (capital social), le capital psychologique s'attelle à des questions plus existentielles: "Qui suis-je et qui voudrais-je devenir?» Dans ce sens, le capital psychologique représente «l'état de développement psychologique positif de l'individu, caractérisé par (1) la confiance (efficacité personnelle) en ses capacités à engager et maintenir les efforts nécessaires pour venir à bout de tâches exigeantes; (2) l'attribution positive (optimisme) des causes des succès actuels et à venir ; (3) la persévérance dans la poursuite des objectifs (espoir) afin de réussir; et (4), lorsque confronté à l'adversité, la capacité à rebondir et à dépasser les difficultés (résilience) pour réussir» (Luthans et al., 2006, p. 388). Le capital psychologique se révèle à travers l'expérience d'états positifs, et ses composantes sont dans ce sens des ressources malléables et dynamiques, sujettes au changement et au développement (Luthans \& Youssef, 2007). Cette notion ouvre donc la porte à l'application d'une approche positive dans le domaine du travail. En effet, la recherche montre que ces ressources psychologiques peuvent effectivement être développées à travers des interventions (Luthans, Avey, \& Patera, 2008), que leur présence tend à diminuer les comportements contre-productifs et 
le cynisme au travail (Avey, Luthans, \& Youssef, 2010) et qu'elles représentent des ingrédients nécessaires au développement continu du bien-être au travail (Avey, Luthans, Smith, \& Palmer, 2010).

\subsection{LES FORCES DE CARACTËRE}

Les forces de caractère représentent des «traits positifs s'exprimant à travers les pensées, les sentiments et les comportements" (Park, Peterson, \& Seligman, 2004, p. 603). Il s'agit là de différences individuelles qui émanent de caractéristiques socialement valorisées de la personnalité et qui se montrent relativement stables à travers le temps et les situations. Peterson et Seligman (2004) ont proposé une classification de ces forces de caractère dans l'inventaire des valeurs en action (Values-in-Action, VIA), qui représente à leurs yeux un "Manuel de la santé mentale», véritable pendant positif du Manuel diagnostique et statistique des troubles mentaux (DSM). Pour ce faire, les auteurs ont effectué une étude des écrits philosophiques et théologiques, des chartes éthiques d'organisations et des caractéristiques de personnages fictifs, afin d'identifier une série de 24 forces de caractère, renvoyant à six vertus morales fondamentales (sagesse, courage, humanité, justice, tempérance et transcendance). Appliquées à la sphère professionnelle, ces forces se manifestent dans les comportements individuels (par exemple, bien travailler en équipe), les pensées (considérer les relations sociales harmonieuses comme un soutien important et une nécessité) et les sentiments (se sentir heureux·se d'être apprécié·e par ses collègues et éprouver de la gratitude pour leur soutien), et participent par conséquent aux expériences subjectives positives (Peterson \& Park, 2003). La recherche sur les forces de caractère a en effet permis de mettre en évidence qu'elles contribuent à une vaste palette d'indicateurs de bien-être et de fonctionnement optimal. A titre d'exemple, elles sont positivement liées à la satisfaction professionnelle indépendamment du domaine spécifique d'activité (Peterson et al., 2010), à la santé au travail (Gander et al., 2012) et aux performances professionnelles qu'elles soient auto- ou hétéro-évaluées (Harzer \& Ruch, 2014).

Malgré leur relative stabilité temporelle et transsituationnelle, les forces de caractère sont sujettes à des changements, par exemple à travers les événements critiques de vie ou par des efforts de développement ou d'intervention. L'idée principale ici est que l'identification et la mise en pratique quotidienne des forces qui caractérisent 
l'individu lui permettent de se développer et d'atteindre un niveau optimal de fonctionnement (développement des compétences et du sentiment d'efficacité), d'augmenter les expériences subjectives positives (émotions positives, motivation et engagement au travail) et de renforcer sa capacité d'interagir de manière efficace avec l'environnement (faire face aux exigences et résister à la pression professionnelle).

\section{LES INSTITUTIONS POSITIVES:}

\section{CULTIVER DES ENVIRONNEMENTS PROFESSIONNELS SALUTOGÈNES}

Le modèle des exigences et ressources au travail (Job demands-ressources model, JDR, Demerouti \& Bakker, 2011) représente une approche récente qui se fonde sur l'interaction entre l'environnement organisationnel, les ressources individuelles et les stratégies comportementales pour expliquer la performance et le bien-être au travail (Bakker \& Demerouti, 2014; 2017). Tout système d'organisation du travail présente deux catégories distinctes de caractéristiques en termes d'exigences et de ressources. Les exigences découlent des charges - aussi nommées stresseurs - d'ordre physique, psychologique, social et organisationnel qui requièrent un effort important de la part du ou de la travailleur-se et supposent donc un coût physiologique ou psychologique chez elles et eux (Demerouti et al., 2001). Les ressources, quant à elles, renvoient aux caractéristiques de l'organisation du travail qui facilitent le travail des employé.e.s. Elles visent par exemple à modérer la charge de travail (soutien effectif à travers une aide technique ou instrumentale, ou soutien affectif à travers la reconnaissance et des relations interpersonnelles positives) ou à stimuler le développement personnel et les apprentissages (par exemple, les procédures de promotion et programmes de formation continue) (Bakker, 2011).

Il est intéressant de rappeler que le JDR met en avant un effet d'interaction entre les exigences et les ressources au travail, ce qui présente un bénéfice pragmatique évident pour les praticien.ne.s et autres responsables de la santé au travail. En effet, alors qu'il est souvent difficile de réduire la charge de travail dans des environnements hautement compétitifs sans nuire aux normes de productivité et de rendement des entreprises, il semble au contraire plus approprié de mener une action sur l'identification et le développement des ressources pertinentes qui permettent de protéger les employéee.s des 
effets délétères des stresseurs et ainsi, de promouvoir et maintenir leur bien-être (Xanthopoulou et al., 2007). Une telle approche, centrée sur les solutions et les ressources, rappelle l'importance d'identifier les facteurs contextuels qui facilitent le fonctionnement individuel optimal, afin de cultiver des environnements positifs dans lesquels les individus peuvent exercer et développer leurs compétences et de vivre des expériences gratifiantes (Peterson \& Park, 2003).

\subsection{IDENTIFIER LES RESSOURCES ENVIRONNEMENTALES}

Nombre de modèles théoriques ont tenté d'identifier et de décrire les caractéristiques centrales du travail qui influencent le bien-être des travailleur-se.s. Parmi les précurseurs de ces approches, citons Hackman et Oldham (1980), dont le modèle des caractéristiques du travail décrit cinq dimensions fondamentales qui contribuent au bien-être: (1) l'autonomie, qui représente le degré de liberté dont jouissent les travailleur-se.s pour organiser et effectuer leurs tâches, renforce le sentiment de contrôle et l'agentivité individuelle; (2) le feed-back, soit l'information à disposition pour évaluer l'efficacité et la qualité de son travail, qui renforce le sentiment d'efficacité; (3) la variété des activités qui, via l'utilisation de compétences et aptitudes différentes, contribue au sentiment de développement de soi; (4) l'identité des tâches qui, de par l'intelligibilité des activités et des objectifs qu'elles visent, contribue au sentiment d'utilité; (5) le sens (ou la signifiance) des tâches, qui traduit le degré perçu de contribution sociale de son travail.

D’une manière similaire, mais cette fois focalisée sur les caractéristiques d'un travail pourvoyeur de sens, Morin et Forest (2007) identifient cinq composantes principales que sont l'utilité sociale, la rectitude morale, les occasions d'apprentissage et de développement, l'autonomie et la qualité des relations. Il semble donc que, quelle que soit l'approche théorique ou la conceptualisation adoptée, il s'avère que les ressources répondent de manière générale aux besoins psychologiques de base, soit ceux d'agir de façon autonome, efficace et socialement significative.

Même si, pour des raisons de simplification et de structuration de nos propos, nous avons présenté les ressources individuelles et environnementales sous des points séparés, nous aimerions à ce stade rappeler la centralité de l'interaction entre l'individu et son environnement social comme clé de compréhension des processus psychologiques qui portent la motivation (Baard, Deci, \& Ryan, 2004) et alimentent le bien-être au travail (Bakker \& Demerouti, 2017). 
Les recherches montrent en effet une relation bidirectionnelle entre les ressources personnelles et celles de l'environnement de travail. Ainsi, les ressources environnementales (comme le feed-back formatif) contribuent au renforcement des ressources personnelles (par exemple, le sentiment d'efficacité et la perception de contrôle), ce qui à son tour renforce l'individu dans ses efforts à chercher activement et à mieux exploiter les ressources disponibles pour venir à bout de ses tâches (Xanthopoulou et al., 2009). Ceci a une importance cruciale pour les interventions des praticien-ne.s de l'orientation, qui à travers une démarche d'identification et de renforcement des ressources de leurs consultant.e.s peuvent les aider à mieux interagir avec leur environnement professionnel et développer leur bien-être au travail. C'est une telle logique d'intervention que nous aimerions illustrer à l'aide de l'étude de cas qui suit.

\section{ILLUSTRATION D'UNE INTERVENTION : LE CAS D'ISABELLA}

Isabella, âgée de 29 ans, fait appel au service de consultations de l'Université de Lausanne en raison d'une intense insatisfaction quant à sa situation professionnelle. Isabella travaille actuellement en tant qu'assistante administrative dans une filiale d'une multinationale du domaine textile. À propos de son travail, elle nous dit que, mis à part des relations agréables avec ses collègues et de rares tâches, elle n'éprouve aucun plaisir particulier.

Lors de notre première rencontre, nous revenons sur son parcours de formation afin de mieux comprendre son évolution et ses questionnements actuels. Isabella a effectué une première formation professionnelle dans le domaine de la création de vêtements, directement poursuivie par une formation supérieure de technicienne de l'industrie de la mode. Concernant les raisons de son premier choix de formation, elle dit être intéressée par l'aspect créatif de ce métier, mais moins par l'activité manuelle de couture en soi. Dès lors, sa deuxième formation a permis de poursuivre sa curiosité et ses intérêts pour des applications techniques et industrielles dans le domaine de la mode. En effet, ce qui l'intéresse au-delà de la création et de l'expression d'une idée est le processus de résolution de problèmes et la réflexion liée à la production et à la diffusion de cette idée à une échelle industrielle. Toutefois, considérant la faible implantation de l'industrie de la mode en Suisse et les risques et incertitudes liés à la poursuite d'une carrière "créative», Isabella a 
opté pour une reconversion « raisonnable» directement après ses formations. Elle a d'abord occupé un poste dans le secrétariat/administration dans une entreprise du domaine textile, puis a effectué une formation d'employée de commerce en cours d'emploi. Elle reconnaît avoir opté pour un métier "sûr et répandu» en Suisse. Lorsque nous évoquons ses journées au travail, elle relate une intense insatisfaction: elle effectue des tâches répétitives et dénuées de sens que "même un enfant de 12 ans pourrait effectuer"; non seulement elle n'a pas l'opportunité d'exploiter ses compétences créatives et techniques dans son travail, mais elle dit ne même pas pouvoir utiliser ses facultés de jugement et de réflexion. Elle aimerait donc identifier un nouveau projet professionnel afin de se réorienter.

\subsection{ANALYSE DE LA DEMANDE:}

\section{UNE APPROCHE CENTRÉE SUR LES SOLUTIONS}

De manière générale, nous avons adopté, à travers nos entretiens, une posture préconisée par l'approche orientée sur les solutions (Solution-focused career counseling, Miller, 2017). Dans cette approche, les conseillers.ères appliquent des interventions communes à tout type d'entretien, mais avec une visée centrale légèrement différente. Alors que, habituellement, la demande des consultant.e.s est conceptualisée en tant que "problème» en analysant l'inconfort de la situation actuelle et en identifiant les obstacles à dépasser pour résoudre l'insatisfaction ressentie, l'accent est ici porté clairement sur la définition d'une situation souhaitée et les ressources à disposition pour l'atteindre. Il s'agit d'observer une écoute sélective, centrée sur les réponses du ou de la consultant·e qui expriment l'optimisme, l'autodétermination et le sentiment d'auto-efficacité. Les interventions verbales du ou de la conseiller.ère visent essentiellement au renforcement des ressources qui contribuent à la réussite du projet du ou de la consultant.e. Enfin, les clarifications et les questions visent à développer la construction de sens et la recherche de solutions.

Afin de clarifier la demande de notre consultante, nous l'interrogeons sur les raisons qui la poussent à se réorienter. Pour ce faire, nous nous fondons sur les travaux de Masdonati, Fournier et Lahrizi (2017, voir également le chapitre 3 de cet ouvrage), qui proposent une distinction parmi les raisons réactives et proactives qui motivent le désir de reconversion. Il est apparu que le désir de reconversion d'Isabella vise en partie un changement réactif afin de 
contrer une insatisfaction de type intrinsèque (le travail qu' elle fait ne lui plaît pas). Néanmoins, la plupart des raisons qu'elle évoque décrivent un désir de changement proactif: elle vise à développer un sentiment d'utilité à travers un travail qui a du sens et qui apporte une contribution sociale, à se développer personnellement par de nouveaux apprentissages et, finalement, à poursuivre sa vocation en revenant vers ses premiers penchants pour la créativité. Nous identifions dès cette phase initiale les signes d'une ressource importante en matière de stratégies proactives (Gawke, Gorgiewski, \& Bakker, 2017) et n'hésitons pas à relever et à renforcer cet atout: le recours à notre service pour engager une démarche de réflexion et de changement peut être compris comme un comportement autonome et proactif pour atteindre un plus grand bien-être au travail. Cette tentative de clarification nous semble importante dans une approche positive, puisque nombre de consultant.e.s ayant recours à un service psychologique font l'expérience d'un "aveu de faiblesse", ne s'estimant pas capable de résoudre seuls leurs "problèmes». Dans ce sens, Isabella nous dit ne pas avoir parlé de cette démarche à son entourage (surtout pas à sa famille), craignant leur jugement quant à une "énième tergiversation" et à son incapacité à se satisfaire de son travail et à opter enfin pour une certaine stabilité. Malgré tout, elle affirme tout de même son droit à l'épanouissement professionnel et reconnaît se sentir soulagée d'avoir enfin décidé de passer à l'action et de mener explicitement une réflexion qui, jusqu'alors, était inhibée et s'exprimait à travers une insatisfaction diffuse et quotidienne.

\subsection{ANALYSE DE L'ENVIRONNEMENT PROFESSIONNEL}

Notre deuxième séance vise à l'analyse et à la compréhension du contexte professionnel actuel. Isabella nous indique que, malgré son insatisfaction actuelle, le changement envisagé suppose également des coûts, puisque sa situation financière risque d'en être bouleversée. Faisant référence au concept de job crafting (Tims, Bakker, \& Derks, 2013), nous interrogeons avec elle la possibilité de mobiliser sa proactivité et son énergie afin de redéfinir ou "façonner» son poste actuel pour créer un environnement plus favorable à son épanouissement, sans pour autant perdre sa stabilité actuelle. Nous analysons ainsi son poste en termes de charges et de ressources. L'interrogeant sur les sources de pression et les charges, Isabella nous dit très vite qu'elle se sent plutôt sous-sollicitée: ses 
tâches sont simples (peu de charge mentale) et peu nombreuses (peu de charge quantitative). Elle reporte néanmoins une grande charge émotionnelle: s'acquittant rapidement de ses tâches de la journée, elle se trouve face à un vide à combler et culpabilise de "toucher un salaire sans vraiment fournir d'efforts». Pire, lorsque ses responsables se rendent compte de cette "oisiveté», ils lui attribuent des tâches supplémentaires qu'elle perçoit comme une activité de façade, totalement dénuée de sens. Il s'agit le plus souvent de classer et d'archiver des documents qui, elle en est certaine, ne seront jamais consultés par personne. Elle avoue que,s parfois, pour contrer la nature rébarbative et inutile de ces tâches, il lui arrive de consulter les documents à archiver, cherchant ainsi à satisfaire un minimum sa curiosité et à échapper quelques instants à l'ennui et à l'absurdité immédiate. On pourrait voir là les prémices d'un job crafting implicite, puisqu'Isabella cherche à dépasser les limites des tâches prescrites. Toutefois, cette initiative n'est pas soutenue par l'organisation du travail et ne vise pas des objectifs utiles à l'entreprise. Isabella se sent alors déprimée et coupable: elle perçoit non seulement son travail comme inutile, mais, de plus, elle juge son comportement comme moralement répréhensible. Quand on s'intéresse aux ressources de l'environnement, il s'avère que le poste et le niveau de responsabilité d'Isabella ne lui laissent que très peu d'autonomie: outre des procédures administratives très strictes et des interactions formelles et limitées avec les client.e.s, elle ne se sent pour ainsi dire libre que dans ses échanges informels avec ses collègues. Poussant plus loin l'analyse de ses relations avec ses collègues, Isabella nous dit que, même si elle a des rapports cordiaux et agréables avec ceux-ci, elle ne partage pas de points d'intérêt avec eux et se sent finalement assez différente, ce qui ne lui permet pas de renforcer un sentiment d'identification à un groupe professionnel valorisé. Enfin, son poste ne présente aucune possibilité de mobilité ou de promotion. Il apparaît dès lors que le poste d'Isabella, avec d'une part une absence totale de charges positives ou de défis, et d'autre part très peu d'autonomie et d'opportunités de développement, correspond à un environnement de travail "passif», par ailleurs popularisé par Graeber (2018) sous le terme de bullshit jobs. Ce sont des postes, souvent dans les activités tertiaires ou le domaine de l'administration, qui comprennent des tâches qui sont perçues par celles et ceux qui les réalisent comme totalement dénuées d'utilité sociale et ne contribuant en rien à leur développement personnel (Guichard, 2016). 


\subsection{ANALYSE DES AUTRES SPHËRES DE VIE}

Afin de dépasser cette impression d'impasse et le manque d'opportunités d'expression de soi dans son poste actuel, nous avons dirigé la discussion vers les activités extraprofessionnelles. Nous inspirant du concept de leisure crafting (Petrou, Bakker, \& van den Heuvel, 2017), nous cherchons à identifier d'autres sphères de vie comme sources de plaisir et de sens et à renforcer des comportements proactifs en dehors du travail. Isabella éprouve du plaisir, à ses heures perdues, à revenir vers ses premiers centres d'intérêt et à exprimer sa créativité: elle élabore des idées, fait des croquis et développe des patrons de couture, sans pour autant les réaliser. Elle estime que son taux d'emploi actuel ne lui laisse que peu de temps libre et d'énergie disponible; elle estime devoir diminuer son temps de travail pour s'investir plus dans des activités de loisirs et pousser jusqu'à la réalisation concrète de ses idées. Isabella exprime clairement ses doutes quant à la viabilité d'une telle solution: elle estime qu'à ce stade de sa vie, le travail reste le moyen central pour construire une identité sociale positive, réaliser son potentiel et satisfaire ses besoins expressifs. Nous reconnaissons là la centralité du travail pour notre consultante et le caractère saillant du rôle professionnel comme une ressource pour l'expression de soi et le développement du bien-être (Amatea, Cross, Clark, \& Bobby, 1986). Ensemble, nous estimons donc que notre démarche devrait se recentrer sur le remaniement et la redéfinition du rôle professionnel.

\subsection{RENFORCEMENT DE LA MOTIVATION AU CHANGEMENT}

Nous fondant sur le modèle JDR, nous avons identifié la situation professionnelle actuelle comme source d'une double menace pour le bien-être d'Isabella, via les processus d'atteinte à la santé (fatigue persistante, sentiment d'inutilité et angoisse de retourner au travail) et d'atteinte à la motivation (engagement réduit et perte de sentiment de compétence, d'autonomie et d'appartenance). À la suite de cette analyse, Isabella estime que, même si elle hésitait jusque-là à renoncer à sa stabilité d'emploi, elle ne peut plus rien espérer de son poste actuel et que, dès lors, elle ressent le besoin de s'engager clairement dans une reconversion. À ce stade, nous avons identifié une réelle motivation intrinsèque qui, portée par le désir de progresser et de se réaliser, l'emportait sur sa satisfaction partielle quant aux conditions extrinsèques (stabilité de l'emploi). Isabella se 
dit prête à dépasser son ambivalence et à décider de manière autonome à s'engager dans un projet de reconversion professionnelle.

Nous inspirant de l'approche centrée sur la solution (Miller, 2017), nous demandons à Isabella d'identifier son degré d'assurance et de discernement quant à son projet professionnel futur sur une échelle graduée de 1 (très confus) à 10 (très clair). Nous lui demandons d'indiquer son degré de certitude: a) avant la démarche; b) actuellement; et c) le degré visé. Isabella estime que, alors qu'avant d'engager cette démarche elle se sentait extrêmement confuse et ambivalente (à 2), elle éprouve aujourd'hui une certaine confiance quant à la nécessité de sa décision et un espoir quant à un changement possible (à 6), mais désirerait tout de même avoir une idée plus précise d'une piste et des actions à mettre en œuvre (8). Le recours à un tel exercice de "graduation" permet en effet à notre consultante de relever d'une part, le progrès déjà effectué jusqu'ici et d'identifier, d'autre part, la manière dont ses forces et ressources personnelles contribuent à ce progrès. Isabella reconnaît et verbalise sa tendance à rester optimiste et à garder espoir malgré les incertitudes et l'adversité. Nous identifions là des éléments constitutifs du capital psychologique (Luthans, Youssef, \& Avolio, 2007) et saisissons l'opportunité d'une transition pour recentrer notre travail sur l'analyse des ressources personnelles.

\subsection{IDENTIFICATION DES RESSOURCES PERSONNELLES}

Avant de terminer la deuxième séance, nous proposons à Isabella un exercice à domicile en prévision de notre prochaine rencontre. Il s'agit pour elle, en se fondant sur une liste de 24 forces de caractère (VIA-IS), de déterminer trois à cinq forces qui la caractérisent, puis de les "contextualiser» en décrivant les situations dans lesquelles elle peut les exploiter, et le ressenti face à cela. Lorsque nous la retrouvons, Isabella dit avoir reconnu parmi la liste quatre traits qui la caractérisent: la curiosité, la faculté de jugement et ouverture, l'intelligence sociale et le sens du beau et de l'excellence. Elle nous dit par exemple que sa curiosité et son envie d'apprendre représentent à ses yeux une réelle force et une source de motivation intrinsèque qui lui permettent d'éprouver du plaisir et de la satisfaction, même à travers des périodes particulièrement chargées. Ainsi en est-il du moment où elle a entrepris sa formation en gestion et administration en cours d'emploi : malgré la double charge de cette formation en emploi et le peu d'intérêt pour le domaine spécifique étudié, Isabella nous a dit éprouver du plaisir à se plonger dans une matière 
nouvelle, d'y chercher du sens et de la maîtriser. Concernant sa faculté de jugement, elle dit prendre plaisir à s'atteler à une tâche complexe, à conduire une réflexion rigoureuse et approfondie et à prendre des décisions pour résoudre les problèmes rencontrés. Pour illustrer cette ressource, elle se réfere à sa formation supérieure, à travers laquelle elle a appris à développer et à appliquer des solutions techniques et pragmatiques pour la production industrielle à grande échelle d'une création de mode. Quant à l'intelligence sociale, elle décrit sa tendance naturelle à se mettre parfois en retrait dans un groupe afin d'entendre et comprendre les positions différentes. Elle estime que cette capacité d'écoute lui permet de se former une vision complète des enjeux d'une problématique rencontrée, de s'adapter aux besoins de chacun pour cultiver une atmosphère de travail harmonieuse et d'intégrer les avis différents afin de développer des solutions collectives et efficaces à un problème. Enfin, le sens $d u$ beau renvoie à sa capacité à se laisser toucher par la beauté et l'harmonie; elle dit ainsi pouvoir se ressourcer et éprouver du plaisir en se promenant dans la nature ou en visitant des monuments architecturaux, se laissant facilement émerveiller par la perfection des formes naturelles et la beauté des créations humaines.

Isabella a pu, à travers cet exercice, reconnaître des ressources qui renforcent sa résilience, son sentiment de compétence (curiosité) et son sentiment d'autonomie (faculté de jugement), qui l'aident à créer et consolider des liens sociaux (intelligence sociale) et, enfin, qui lui ouvrent l'accès à des états de plaisir et de plénitude (sens du beau). Elle se déclare ainsi décidée à identifier une formation menant vers un domaine professionnel dans lequel elle pourrait mobiliser ses différentes ressources et satisfaire ses besoins.

Avant de terminer notre séance, nous proposons à Isabella d'initier une phase prospective: il s'agit pour elle d'imaginer le prototype d'un travail qui a du sens. Pour ce faire, nous lui proposons la lecture d'un bref article (Morin \& Forest, 2007) et lui demandons de s'en inspirer pour identifier les principaux ingrédients du sens au travail pour elle, puis de formuler un ou plusieurs domaines professionnels qui pourraient contribuer à son épanouissement.

\subsection{FORMULATION D'OBJECTIFS: À LA RECHERCHE DU SENS PERDU}

Au début de cette quatrième séance, Isabella dit éprouver des difficultés à nommer clairement un "métier", puis formule à demi-mot vouloir occuper une position de «cheffe de projet». Pour illustrer sa 
vision, Isabella se réfere spontanément à son réseau social. Elle nous dit avoir beaucoup d'amis architectes, et qu'elle ressent beaucoup d'admiration, une certaine excitation et un plaisir "par procuration" à les entendre parler de leurs activités et de leurs responsabilités de gestion d'un chantier regroupant différents corps de métier autour d'un résultat commun. Par ailleurs, elle voit dans cette profession une opportunité de concilier une approche technique et pragmatique avec sa sensibilité à l'esthétique et son besoin de créativité. Elle doute néanmoins de sa capacité à venir à bout d'une formation aussi longue et exigeante.

Se référant à sa lecture, Isabella dit avoir été fortement interpellée par la dimension de "rectitude morale», qui caractérise un travail qui est «fait d'une façon responsable, non seulement dans son exécution, mais aussi dans les produits et les conséquences qu'il engendre» (Morin \& Forest, 2007, p. 33). Isabella dit avoir pris conscience d'une dimension centrale qui, même si elle ne l'avait jamais explicitement verbalisée, contribuait par son absence à un sentiment d'insatisfaction diffuse dans ses activités jusqu'ici. Elle explique ainsi qu'elle fait face à un dilemme moral: celui de participer à une industrie qui, à son sens, exploite une main-d'œuvre vulnérable dans des conditions de travail discutables par de fortes injonctions à la réduction des coûts de production, et qui, en visant la consommation de masse, engendre une mauvaise gestion des ressources naturelles et des conséquences néfastes pour l'environnement. Elle dit ne plus vouloir y participer, ni de manière active (en revenant vers sa formation de technicienne de l'industrie de mode), ni de manière passive (en travaillant dans l'administration dans ce même domaine).

Il nous semble que cette réflexion autour du sens au travail a initié une sorte de "déclic». Déjà consciente de ses besoins personnels de stimulation et de maîtrise pour éprouver du plaisir au travail (dimension hédonique), Isabella a pu affirmer ses valeurs écologiques et éthiques. Elle dit ainsi chercher le sens de son travail (dimension eudémonique) à travers sa contribution à la réduction de l'impact environnemental et de l'injustice sociale. Nous invitons Isabella à appliquer ce critère de déontologie à ses recherches de formation.

Lors de notre dernière rencontre, Isabella dit contempler avec plus d'assurance deux voies de formation: l'architecture du paysage et l'ingénierie en gestion de la nature. Elle considère que les deux voies correspondent non seulement à ses attentes subjectives du travail, mais répondent également à des critères objectifs et 
pragmatiques (en termes de durée et de conditions d'admission). Enfin, malgré l'identification assez claire de ses objectifs de formation (les deux options sont offertes par une même institution et ont les mêmes exigences en matière de prérequis), Isabella rapporte ses doutes quant à sa capacité à les atteindre. Nous investiguons avec elle son réseau social afin d'identifier une personne pouvant lui servir de soutien et de modèle positif. L'idée ici est de recourir à l'apprentissage vicariant afin de développer le sentiment d'efficacité (Luthans, Avecy, Avolio, \& Peterson, 2010). Très spontanément, Isabella nomme sa cousine, avec qui elle partage beaucoup en ce moment. Également dans une situation de transition et désirant entreprendre une formation artistique, sa cousine lui semble avoir non seulement beaucoup de talents, mais également l'assurance nécessaire pour avancer dans son projet. Elle se dit décidée à cultiver cette ressource sociale essentielle et de continuer à échanger des stratégies avec sa cousine et, lorsque les doutes l'assaillent, de se laisser inspirer par sa détermination.

Isabella désire à ce stade suspendre les consultations afin d'investiguer plus précisément chaque institution de formation, de s'atteler à des prévisions budgétaires et ainsi d'élaborer un plan d'action. Lors d'un entretien de suivi, sollicité par Isabella après deux mois, elle nous relate avoir décidé de donner sa démission, se considérant prête à "couper les ponts» et à se lancer dans son nouveau projet. Elle profite ainsi de ses derniers mois de travail pour réaliser des économies et mobiliser son réseau pour préparer une expérience pratique d'une année dans le domaine de la gestion de la nature. Cette expérience lui permettrait non seulement de mettre ses attentes et intérêts à l'épreuve de la réalité et ainsi de départager les deux pistes de formation qu' elle contemple encore, mais elle correspond de plus aux exigences d'admission, quelle que soit la formation finalement choisie. Nous saluons cet élan et sa détermination renouvelée et restons à sa disposition pour toute question ultérieure.

\section{BILAN ET PERSPECTIVES}

Deux arguments appellent à une meilleure intégration des concepts et des méthodes de la psychologie positive dans le domaine du conseil et de l'orientation. D'abord, si l'on considère le rôle significatif du travail dans la vie des gens et la contribution importante de la satisfaction professionnelle au bien-être général, le processus 
de conseil en orientation représente un terreau particulièrement propice pour la construction de sens et le développement du bien-être (Walsh \& Eggerth, 2005). De plus, Savickas (2003) rappelle que la psychologie positive ne représente pas une tendance nouvelle ou secondaire, mais bien un paradigme central et constitutif de l'identité des chercheur.se.s et des praticien.ne.s de ce champ. En effet, la visée principale de la psychologie du conseil et de l'orientation a été, depuis ses origines, de soutenir les individus dans leurs efforts d'identifier leurs ressources et de développer des projets professionnels - et de vie - satisfaisants (Jones, 1994). Toutefois, la littérature révèle peu de tentatives d'adaptation conceptuelle et de mise à l'épreuve empirique dans le champ spécifique du conseil en orientation. Nous aimerions ici définir deux chantiers importants pour une meilleure intégration.

\subsection{ADAPTATION SPÉCIFIQUE}

Des auteur.e.s appellent à un développement des approches inspirées de la psychologie positive, estimant que l'approche classique de l'orientation, avec un fort accent sur l'évaluation psychométrique - en termes d'aptitudes, de compétences ou de personnalité - est susceptible d'avoir des effets délétères à différents niveaux. D'abord, l'évaluation peut activer une appréhension qui peut en biaiser les résultats, ou encore mettre l'accent sur des lacunes qui peuvent être vécues comme un échec par certain·ess consultant.e.s déjà mis.es à mal dans leurs sentiments d'efficacité (Littman-Ovadia, Lazar-Butbul, \& Benjamin, 2014). D'autre part, il est vraisemblable que l'évaluation puisse induire une dépendance vis-à-vis de l'avis de «l'expert.e» et, dès lors, nuire au développement de l'implication autonome du ou de la consultant.e dans la démarche (McIlveen \& Patton, 2006). Afin de mettre à l'épreuve ces arguments, des études de comparaison entre ces deux types d'approche - l'une "classique» et l'autre ouvertement "positive» - et leurs effets semblent utiles. Aussi, afin de dépasser une dimension idéologique et ainsi neutraliser l'approche intuitive ou le biais de la conviction des praticien.ne.s, il semble utile d'élaborer des protocoles standardisés des interventions avant d'en étudier les effets. Il s'agit par exemple de développer un manuel précis et des techniques spécifiques d'intervention, puis de former les praticien.ne·s à leur application standardisée. Citons dans ce sens Rochat et Rossier (2016), qui, à travers l'adaptation de 
l'entretien motivationnel au champ de l'orientation et l'examen de ses effets spécifiques, montrent l'exemple d'une tentative rigoureuse de transfert d'une méthode jusque-là essentiellement appliquée dans le domaine de la thérapie et du traitement des addictions.

\subsection{MISE À L'ÉPREUVE SCIENTIFIQUE}

Nous avons jusqu'ici tenté de donner une définition du bien-être au travail, d'en décrire les sources et de proposer des leviers d'action pour son développement. Voici venu le moment de nous poser une question essentielle: peut-on vraiment modifier le bien-être - et ses dénominations alternatives communes en termes de bonheur ou de succès? Si l'on en croit le nombre impressionnant de livres et de manuels qui jonchent les rayons de développement personnel et l'intense intérêt populaire pour ce sujet, la réponse est oui. Mais qu'en est-il des interventions évaluées et validées selon une méthodologie scientifique robuste? Même si les premières études scientifiques sur ce sujet ont été publiées il y a longtemps déjà (Fordyce, 1977), ce n'est qu'avec l'avènement de la psychologie positive que l'on constate une augmentation des interventions développées et évaluées selon une méthodologie fiable. Ces interventions, généralement appelées PPI (Positive Psychology Interventions), correspondent à "des méthodes de traitement ou des activités intentionnelles qui visent à cultiver les sentiments, comportements et cognitions positifs» (Sin \& Lyubomirsky, 2009, p. 468) et doivent dans ce sens être distinguées des interventions dont le but principal est le rétablissement des troubles et la diminution des symptômes. Les PPI consistent en des exercices qui couvrent une vaste palette de traits et de ressources positives, comme la promotion de la pleine conscience (Zautra et al., 2008), de l'espoir et de l'optimisme (Cheavens, Feldman, Gum, Michael, \& Snyder, 2006) ou encore de l'humour (Ruch \& Hofmann, 2017). Deux méta-analyses portant sur ce type d'interventions montrent un effet positif significatif sur l'augmentation du bien-être subjectif et la réduction des symptômes de détresse psychologique (Bolier et al., 2013; Sin \& Lyubomirsky, 2009). Pourtant, il s'avère que la majorité des études considérées dans ces méta-analyses ont porté sur des interventions cliniques ou des démarches de développement personnel. Il est donc difficile de spéculer sur l'effet des PPI sur des indicateurs spécifiques au domaine de l'orientation, comme l'indécision vocationnelle, l'employabilité perçue ou encore des indicateurs 
objectifs tels que la réussite effective d'une formation ou l'insertion professionnelle stable. Il nous semble utile de développer cet axe de recherches spécifiques pour souligner et défendre la véritable plus-value d'une «orientation positive».

\subsection{LIMITES}

Avant de terminer, il nous semble important de préciser que, même si l'accent porté sur les ressources et les initiatives personnelles présente d'importants avantages pour développer l'autonomie et l'agentivité de nos consultant.e.s, nous devrions nous garder d'une approche exclusivement «individuelle» et excessivement "positive» du bien-être. En effet, au-delà des responsabilités et des ressources individuelles, le bien-être au travail reste également tributaire de facteurs contextuels et de pratiques organisationnelles (conditions de travail, style de management, culture d'entreprise, soutiens à l'articulation des sphères de vie, etc.) sur lesquels les individus n'ont que peu de prise. D'autre part, pour se garder d'une vision trop "angélique» des choses, rappelons que certains groupes de la population active se retrouvent en position de vulnérabilité à différents moments de leur parcours professionnel en raison de leurs caractéristiques sociodémographiques (âge, sexe ou origine) ou d'obstacles relevant de pratiques discriminatoires. Ainsi en est-il des jeunes adultes sans qualification, qui connaissent de grandes difficultés d'insertion professionnelle et courent le risque d'une exclusion à long terme. Citons également certains groupes de travailleur.se.s «seniors" qui, fortement sollicités par les exigences de formation continue et de flexibilité, et menacés par une compétition intense, courent le risque d'une perte d'emploi et d'un chômage de longue durée. Dans ce sens, il nous semble qu'une pratique responsable du conseil et de l'orientation devrait également viser, au-delà de l'accompagnement individuel, à dénoncer les contextes délétères et à combattre les inégalités sociales. 


\section{RÉFÉRENCES BIBLIOGRAPHIQUES}

Amatea, E. S., Cross, E. G., Clark, J. E., \& Bobby, C. L. (1986). Assessing the work and family role expectations of career-oriented men and women: The life role salience scales. Journal of Marriage and the Family, 48, 831-838. Doi: 10.2307/352576.

Avey, J. B., Luthans, F., Smith, R. M., \& Palmer, N. F. (2010). Impact of positive psychological capital on employee well-being over time. Journal of Occupational Health Psychology, 15, 17-28.

Avey, J. B., Luthans, F., \& Youssef, C. M. (2010). The additive value of positive psychological capital in predicting work attitudes and behaviors. Journal of Management, 36, 430-452.

BaARD, P. P., Deci, E. L., \& Ryan, R. M. (2004). Intrinsic need satisfaction: A motivational basis of performance and weil-being in two work settings. Journal of Applied Social Psychology, 34, $2045-$ 2068.

BAKKer, A. B. (2011). An evidence-based model of work engagement. Current Directions in Psychological Science, 20, 265-269.

Bakker, A. B., \& Demerouti, E. (2017). Job Demands-Resources theory: Taking stock and looking forward. Journal of Occupational Health Psychology, 22, 273-285.

BAKKer, A. B., \& Demerouti, E. (2014). Job demands-resources theory. In P. Y. Chen \& C. L. Cooper (éds), Work and Wellbeing: Wellbeing: A complete reference guide (vol. III ; pp. 37-64). Chichester: Wiley-Blackwell.

Becker, J., R., de Bruin, G. P., Györkös, C., Rossier, J., \& MasSOUDI, K. (2016). Extending the nomological network of wellness at work. Management Dynamics, 25 (4), 2-18.

Blaser, M., \& Amstad, F. T. (éds) (2016). La santé psychique au cours de la vie. Rapport de base (Rapport 6). Berne/Lausanne: Promotion Santé Suisse.

Bolier, L., Haverman, M., Westerhof, G. J., Riper, H., Smit, F., \& Bohlmeijer, E. (2013). Positive psychology interventions: a meta-analysis of randomized controlled studies. BMC Public Health, 13 (1), 119. Doi: 10.1186/1471-2458-13-119.

Cheavens, J. S., Feldman, D. B., Gum, A., Michael, S. T., \& SnyDER, C. R. (2006). Hope therapy in a community sample: A pilot 
investigation. Social Indicators Research, 77, 61-78. Doi: 10.1007/ s11205-005-5553-0.

Dagenais-Desmarais, V., \& Savoie, A. (2012). What is psychological well-being, really? A grassroots approach from organizational sciences. Journal of Happiness Studies, 13, 659-668. Doi: 10.1007/ s10902-011-9285-3.

Dagenais-Desmarais, V., Gilbert, M.-H., \& Malo, M. (2018). La thèse du travailleur heureux-productif revisitée: une analyse par profils. Psychologie du travail et des organisations, 24, 6-20. Doi: 10.1016/j.pto.2017.09.002.

Deci, E. L., \& Ryan, R. M. (2000). The "what" and "why» of goal pursuits: Human needs and the self-determination of behavior. Psychological Inquiry, 11, 227-268.

Demerouti E., Bakker, A. B., Nachreiner, F., \& Schaufeli, W. B. (2001). The Job Demands - Resources model of burnout. Journal of Applied Psychology, 86, 499-512.

Diener, E., Suh, E. M., Lucas, R. E., \& Smith, H. L. (1999). Subjective well-being: Three decades of progress. Psychological Bulletin, 125, 276-302.

Dollard, M., \& Bakker, A. B. (2010). Psychosocial safety climate as a precursor of conducive work environments, psychological health problems employee engagement. Journal of Occupational \& Organizational Psychology, 83 (3), 579-599. Doi: 10.1348/096317909X470690.

Fordyce, M. W. (1977). Development of a program to increase personal happiness. Journal of Counseling Psychology, 24, 511-521. Doi : 10.1037/0022-0167.24.6.511.

GaGné, M., \& Deci, E. L. (2005). Self-determination theory and work motivation. Journal of Organizational Behavior, 26, 331-362.

Gander, F., Proyer, R. T., Ruch, W., \& Wyss, T. (2012). The good character at work: an initial study on the contribution of character strengths in identifying healthy and unhealthy work-related behavior and experience patterns. International Archives of Occupational and Environmental Health, 85 (8), 895-904. Doi: 10.1007/ s00420-012-0736-x.

Gawke, J. C. L., Gorgievski, M. J., \& Bakker, A. B. (2017). 
Employee intrapreneurship and work engagement: A latent change score approach. Journal of Vocational Behavior, 100, 88-100.

Gilbert, M.H., Dagenais-Desmarais, V., \& Savoie, A. (2011). Validation d'une mesure de santé psychologique au travail. Revue européenne de psychologie appliquée, 61 (4), 195-203. Doi: 10.1016/j.erap.2011.09.001.

Graeber, D. (2018). Bullshit Jobs: a theory. Londres: Allen Lane

Grebner, S., Berlowitz, I., Alvarado, V., \& Cassina, M. (2011). Le stress chez les personnes actives occupées en Suisse. Liens entre conditions de travail, caractéristiques personnelles, bien-être et santé. Berne: SECO/Conditions de travail.

Guichard, J. (2016). Life-and working-design interventions for constructing a sustainable human(e) world. Studia Parodoznawczel Journal of Counsellogy, 5, 179-190.

Hackman, J. R., \& Oldham, G. R. (1980). Work redesign. Reading (MA) : Addison-Wesley.

Harzer, C., \& Ruch, W. (2014). The role of character strengths for task performance, job dedication, interpersonal facilitation, and organizational support. Human Performance, 27, 183-205. Doi : 10.1080/08959285.2014.913592.

Harzer, C., \& Ruch, W. (2015). The relationships of character strengths with coping, work-related stress, and job satisfaction. Frontiers in Psychology, 6. Doi: 10.3389/fpsyg.2015.00165.

JonEs, L. K. (1994). Frank Parsons'contribution to career counseling. Journal of Career Development, 20, 287-294.

Littman-Ovadia, H., \& Steger, M. (2010). Character strengths and well-being among volunteers and employees: toward an integrative model. The Journal of Positive Psychology, 5 (6), 419-430. Doi : $10.1080 / 17439760.2010 .516765$.

Littman-Ovadia, H., Lazar-Butbul, V., \& Benjamin, B. A. (2014). Strenghts-Based Career Counseling: Overview and Initial Evaluation. Journal of Career Assessment, 22 (3), 403-419.

Luthans, F., Avey, J. B., \& Patera, J. L. (2008). Experimental analysis of a web-based training intervention to develop positive psychological capital. Academy of Management Learning \& Education, 7, 209-221. 
Luthans, F., Avey, J. B., Avolio, B. J., Norman, S. M., \& Combs, G. M. (2006). Psychological capital development: toward a microintervention. Journal of Organizational Behavior, 27, 387-393.

Luthans, F., \& Youssef, C. M. (2007). Emerging positive organizational behavior. Journal of Management, 33, 321-349.

Luthans, F., Youssef, C. M., \& Avolio, B. J. (2007). Psychological capital: Developing the human competitive edge. Oxford: Oxford University Press.

Luthans, F., Avey, J. B., Avolio, B. J., \& Peterson, S. J. (2010). The development and resulting performance impact of positive psychological capital. Human Resource Development Quarterly, 21, 41-67. Doi: $10.1002 /$ hrdq.20034.

Lyubomirsky, S., TKach, C., \& DiMatteo, M. R. (2005). What are the differences between happiness and self-esteem. Social Indicators Research, 78 (3), 363-404. Doi : 10.1007/s11205-005-0213-y.

Maslach, C., \& Jackson, S. E. (1986). The Maslach Burnout Inventory: Research Edition. Palo Alto: Consulting Psychologist.

Masdonati, J., Fournier, G., \& Lahrizi, I. Z. (2017). The Reasons Behind a Career Change Through Vocational Education and Training. International Journal for Research in Vocational Education and Training, 4 (3), 1-35.

MAssoudi, K. (2009). Le stress professionnel: Une analyse des vulnérabilités individuelles et des facteurs de risque environnementaux. Berne: Peter Lang.

McIlveen, P., \& PAtTon, W. (2006). A critical reflection on career development. International Journal for Educational and Vocational Guidance, 6, 15-27.

Miller, J. (2017). Solution-focused career counseling. In M. McMahon (éd.), Career counseling: Constructivist approaches (2e édition, pp. 127-138). Londres / New York: Routledge.

Morin, E. M., \& Forest, J. (2007). Promouvoir la santé mentale au travail: donner un sens au travail. Gestion, 32, 31-36. Doi : 10.3917/ riges.322.0031.

OMS (1946). Préambule à la Constitution de l'Organisation mondiale de la santé. Actes officiels de l'Organisation mondiale de la santé, $\mathrm{N}^{\circ} 2$. 
Paoli, P., \& Merllié, D. (2001). Troisième enquête européenne sur les conditions de travail. Luxembourg: Office des publications officielles des Communautés européennes.

Park, N., Peterson, C., \& Seligman, M. E. P. (2004). Strengths of character and well-being. Journal of Social and Clinical Psychology, 23 (5), 603-619. Doi : 10.1521/jscp.23.5.603.50748.

Peterson, C. (2006). A Primer in Positive Psychology. New York: Oxford University Press.

Peterson, C., \& PARK, N. (2003). Positive psychology as the evenhanded positive psychologist views it. Psychological Inquiry, 14 (2), 143-147. Doi : 10.1207/S15327965PLI1402_03.

Peterson, C., \& Seligman, M. E. P. (2004). Character strengths and virtues: a handbook and classification. New York: Oxford University Press.

Petrou, P., Bakker, A.B., \& van den Heuvel, M. (2017). Weekly job crafting and leisure crafting: Implications for meaning-making and work engagement. Journal of Occupational and Organizational Psychology, 90, 129-152.

Ramaciotti, D., \& Perriard, J. (2000). Les coûts du stress en Suisse. Zurich: SECO / Ressort Arbeit und Gesundheit.

Roberts, B. W., Caspi, A., \& Moffitt, T. E. (2003). Work experiences and personality development in young adulthood. Journal of Personality and Social Psychology, 84 (3), 582-593.

Rochat, S., \& Rossier, J. (2016). Integrating motivational interviewing in career counseling: A case study. Journal of Vocational Behavior, 93, 150-162.

Rothmann, S. (2008). Job satisfaction, occupational stress, burnout and work engagement as components of work-related wellbeing. South African Journal of Industrial Psychology, 34 (3), 11-16.

Ruch, W., \& Hofmann, J. (2017). Fostering humor. In C. Proctor (éd.), Positive psychology interventions in practice (pp. 65-80). New York: Springer. Doi : 10.1007/978-3-319-51787-2_5.

SAVICKAS, M. L. (2003). Toward a taxonomy of human strengths: Career counseling's contribution to positive psychology. In W. B. WaLSH (éd.), Counseling psychology and optimal human function (pp. 229-249). Mahwah (NJ): Lawrence Erlbaum. 
SCHWAB, K. (2016). The fourth industrial revolution. New York: Crown Business.

Seligman, M. E. P., \& Csikszentmihalyi, M. (2000). Positive psychology. An introduction. The American Psychologist, 55 (1), 5-14. Doi : 10.1037/0003-066X.55.1.5.

Seligman, M. E. P. (1972). Learned helplessness. Annual Review of Medicine, 23, 407-412.

Seligman, M. E. P. (2011). Flourish: A Visionary New Understanding of Happiness and Well-being. New York: Free Press.

Sin, N. L., \& LyUbOMirsky, S. (2009). Enhancing well-being and alleviating depressive symptoms with positive psychology interventions: a practice-friendly meta-analysis. Journal of Clinical Psycho$\log y, 65$ (5), 467-487. Doi : 10.1002/jclp.20593.

Tims, M., Bakker, A.B., \& Derks, D. (2013). The impact of job crafting on job demands, job resources, and well-being. Journal of Occupational Health Psychology, 18, 230-240.

Van den Broeck, A., Vansteenkiste, M., De Witte, H., \& Lens, W. (2008). Explaining the relationships between job characteristics, burnout, and engagement: The role of basic psychological need satisfaction. Work \& Stress, 22, 277-294.

Walsh, W. B., \& EgGerth, D. E. (2005). Vocational psychology and personality: The relationship of the five-factor model to job performance and job satisfaction. In W. B. WALSH \& M. L. SAVICKAS (éds), Handbook of vocational psychology (3 édition, pp. 267-295). Mahwah (NJ) : Lawrence Erlbaum.

Weber, A., \& Jaekel-Reinhard, A. (2000). Burnout syndrome: a disease of modern societies? Occupational Medicine, 50 (7), 512-517.

Xanthopoulou, D., Bakker, A.B., Demerouti, E., \& Schaufeli, W.B. (2009). Reciprocal relationships between job resources, personal resources, and work engagement. Journal of Vocational Behavior, 74, 235-244.

Xanthopoulou, D., Bakker, A. B., Dollard, M. F., Demerouti, E., Schaufeli, W. B., Taris, T. W., \& Schreurs, P. J. G. (2007). When do job demands particularly predict burnout? The moderating role of job resources. Journal of Managerial Psychology, 22, 766-786. 
Zautra, A. J., Davis, M. C., Reich, J. W., Nicassario, P., Tennen, H., Finan, P., ... IrWin, M. R. (2008). Comparison of cognitive behavioral and mindfulness meditation interventions on adaptation to rheumatoid arthritis for patients with and without history of recurrent depression. Journal of Consulting and Clinical Psychology, 76 (3), 408-421. Doi : 10.1037/0022-006X.76.3.408

Zimmermann, E., \& Haour-Knipe, M. (1988). Stress psychosocial, adaptation et santé. Médecine \& Hygiène, 46, 2640-2651. 



\section{DU BON USAGE DE L'INCERTITUDE:}

UN EXEMPLE D'APPLICATION

DE L'APPROCHE CENTRÉE SUR LE

DÉVELOPPEMENT DU POUVOIR D'AGIR

DES PERSONNES

ET DES COLLECTIVITÉS (DPA-PC)

AU DOMAINE DE L'ORIENTATION

YANN LE BOSSÉ, BRUNO BOURASSA, MANON CHAMBERLAND,

GENEVIÈVE FOURNIER, SIMON-PIERRE TREMBLAY

(UNIVERSITÉ LAVAL, QUÉBEC)

ET BERNARD VALLERIE

(UNIVERSITÉ PIERRE MENDÈS FRANCE, GRENOBLE)

L

es défis auxquels font actuellement face les praticien·ne.s dans le domaine de la psychologie du conseil et de l'orientation s'inscrivent dans le contexte plus global de l'évolution récente des pratiques sociales dans les pays développés (Castel, 2001, 2009; Espin-Andersen, 2007; Thomas, 1997). Au cours des dernières décennies, la multiplication des accords de libre-échange a placé la question de la compétitivité de la main-d'œuvre au cœur des stratégies de développement économique sur les plans local, régional et national (OCDE, 2005). Cette compétition à l'échelle internationale a notamment eu pour conséquence d'entraîner une profonde mutation du marché de l'emploi, qui a eu des impacts directs sur les pratiques sociales en général et celles du domaine de l'orientation en particulier (Duffy, Blustein, Diemer, \& Autin, 2016). On peut regrouper ces conséquences autour de trois éléments centraux: une augmentation de la demande d'accompagnement, une réduction des ressources disponibles et une remise en question des pratiques traditionnelles.

\section{UNE AUGMENTATION DE LA DEMANDE D'ACCOMPAGNEMENT}

La mondialisation des échanges commerciaux exerce une pression directe sur les populations par l'intermédiaire des exigences de 
spécialisation de la main-d'œuvre, d'accroissement de la productivité, de mobilité géographique et de flexibilité des conditions de travail (Castel, 2009; Di Fabio \& Bernaud, 2010). Longtemps cantonnée dans le seul domaine de l'orientation en milieu scolaire, la profession de conseiller-ère en orientation connaît un essor notable dans tous les secteurs qui touchent de près ou de loin aux relations entre la vie personnelle et la vie professionnelle (Hansen, 2010 ; Southern, Gomez, Smith, \& Devlin, 2010). Cette augmentation de la demande se manifeste à la fois auprès de la population générale et des publics spécifiques (chômeurs de longue durée, personnes immigrantes, etc.).

\section{LA RÉDUCTION DES RESSOURCES DISPONIBLES}

L'impératif d'attractivité des économies nationales impose une politique de réduction des déficits budgétaires de l'État qui conduit la majorité des pays occidentaux à réduire l'enveloppe destinée aux dépenses sociales (Espin-Andersen, 2007). La gestion managériale des interventions bouleverse les conditions de travail des praticien.ne.s du social. Elle remet en question la finalité de leur mission, de leurs méthodes et des fondements de leur identité professionnelle (Chauvière, 2007). C'est particulièrement le cas des conseiller.ère-s en orientation (c.o.) qui, devant la complexification croissante des parcours professionnels, doivent composer avec l'élargissement manifeste de leur mission (Bain, 2012; Hansen, 2010).

\section{UNE REMISE EN QUESTION DES PRATIQUES TRADITIONNELLES}

Les pratiques issues de l'État providence, notamment en sciences de l'orientation, reposent sur une conception techniciste de l'intervention inspirée du modèle médical (Collin \& Suissa, 2007; Lee-Rife, 2010). L'expertise professionnelle y est généralement considérée comme suffisante pour établir un diagnostic et une stratégie d'intervention. La personne accompagnée est cantonnée dans un rôle passif qui consiste pour l'essentiel à appliquer les recommandations de l'intervenant (McLaughlin, 2010b). Or l'évolution des connaissances et des pratiques milite clairement en faveur d'une contribution beaucoup plus active des personnes accompagnées (Savickas, 2012).

La convergence contextuelle de ces trois caractéristiques crée une situation nouvelle pour laquelle il est nécessaire d'opérer un 
changement de paradigme (Di Fabio \& Bernaud, 2010). En effet, les praticien.ne.s du social sont concrètement placé.e.s dans une position très délicate, dans laquelle il leur est demandé de faire plus pour répondre à la demande avec moins de ressources et en prenant appui sur leur seule expertise (Chauvière, 2007). La perspective constructiviste, aujourd'hui largement partagée par les chercheur.e.s et formateur-trice-s en sciences sociales, conduit à repenser jusqu'à la finalité même des pratiques sociales. Il ne s'agit plus de combler de supposées carences qui altèreraient les capacités d'adaptation des personnes accompagnées, mais plutôt de les soutenir dans leur capacité à conduire les changements qu'elles ont elles-mêmes déterminés à partir d'une compréhension négociée de leur situation (Hansen, 2010). En ce qui concerne l'accompagnement des personnes et des groupes dans le champ des sciences de l'orientation, la fonction de la ou du c.o. consiste essentiellement à créer les conditions pour que les protagonistes puissent élaborer les modalités de leur transition socioprofessionnelle (Guichard, 2008).

Dans cette perspective, les transitions sont appréhendées comme des configurations ponctuelles d'une existence constamment en mouvement. Ce sont des occasions où les personnes accompagnées sont invitées à renégocier le sens de leur expérience passée en vue de mieux construire celui de leur future direction. Savickas et ses collègues (2010) parlent de leur capacité à utiliser un "pouvoir d'agir biographique».

Cette notion de "pouvoir d'agir" joue un rôle important dans la formulation de la conception de la finalité des pratiques en sciences de l'orientation proposée par ce regroupement de chercheur.e.s. Elle capte de manière imagée l'imbrication très dense des facteurs personnels et structurels dans l'évolution des parcours socioprofessionnels tout au long de la vie. Elle est présentée comme un résultat à rechercher dans cette nouvelle conception de l'accompagnement en orientation (Savickas et al., 2010). On y souligne explicitement la nécessité de «favoriser l'augmentation du pouvoir d'agir de la personne» (p. 11).

À notre connaissance, la finalité du développement du pouvoir d'agir (DPA), régulièrement évoquée dans les écrits récents en sciences de l'orientation, se traduit encore peu en termes de pratiques spécifiques. Le plus souvent, elle est utilisée pour incarner une vague intention (Van Hal, Meershoek, Nijhuis, \& Horstman, 2012). Bref, si beaucoup considèrent que leur pratique contribue 
à soutenir le DPA des personnes accompagnées, très peu sont en mesure de donner des indications claires sur la manière dont elles ou ils s'y sont pris et les résultats tangibles qu'elles ou ils ont obtenus (Parazelli \& Bourbonnais, 2017). Or, si on souhaite que les praticien-ne.s centrent leur intervention sur le soutien au DPA, il est nécessaire qu'elles ou ils disposent d'indications suffisamment précises et structurées pour guider leur action (Tengland, 2007). Toutefois, un tel objectif constitue un défi conséquent. En effet, l'impérative nécessité d'intégrer les dimensions contextuelles dans la mise au point de chaque intervention destinée à soutenir le DPA personnel ou collectif (DPA-PC) exclut le recours à une procédure standardisée. D'un autre côté, si les contenus et les outils d'intervention peuvent grandement varier d'un contexte à l'autre, on doit pouvoir retrouver une cohérence dans la logique d'action à la fois entre les interventions d'un·e même praticien-ne et entre celles des professionnel-le.s qui visent le soutien au DPA. Il faut donc élaborer un outil de référence commun qui puisse permettre de systématiser la démarche d'accompagnement tout en étant entièrement ajustable à chaque contexte d'intervention. Une solution consiste à développer un cadre conceptuel transversal, captant les principaux éléments qui caractérisent une démarche de soutien au DPA selon l'état des connaissances sur le sujet. C'est la tâche à laquelle nous avons dédié nos efforts au cours des dernières années. Le produit de ce travail est présenté dans les pages ci-dessous en trois volets distincts. Le premier est consacré à la présentation de la démarche conceptuelle que nous avons suivie, ainsi qu'à l'outil d'analyse des pratiques qui en découle. Le second présente un exemple d'application de cette approche à partir d'un cas fictif, mais qui demeure très proche de la réalité. Le dernier rend compte du processus d'émergence d'une nouvelle communauté de pratique à la suite d'une démarche de diffusion sous la forme de projets pilotes.

\section{UNE DÉMARCHE DE MODÉLISATION:}

\section{À LA RECHERCHE D'UNE SYNTHÈSE FÉCONDE}

$\mathrm{Au}$ cours des dernières années, de nombreuses études ont été conduites pour explorer la pertinence potentielle de la notion d'empowerment, que nous avons traduite par "Développement du pouvoir d'agir (DPA)»(Le Bossé, 2003), afin d'appréhender 
les problématiques propres au champ des pratiques sociales. Trois questions fondamentales peuvent être utilisées pour caractériser le développement de ce champ d'études.

\section{LE PHÉNOMÈNE DU DPA EST-IL UN SUJET D'INTÉRÊT POUR LE DOMAINE DES PRATIQUES SOCIALES?}

Cette question s'est principalement posée dans le cadre de la réflexion sur le nécessaire renouvellement des pratiques (Cattaneo \& Goodman, 2015; Hur, 2006; Pantazidou, 2012; Parazelli \& Bourbonnais, 2017; Partridge, 2008; Tengland, 2007; Vallerie, 2012). Pour une large part, la réponse fait l'objet d'un consensus. Pour des raisons diverses et parfois contradictoires, acteurs.trices politiques, responsables d'organismes prestataires de services, praticien-ne.s et personnes accompagnées s'accordent sur la pertinence de soutenir le DPA personnel ou collectif. Ceci dans des champs d'études aussi variés que la promotion de la santé (Bloch et al., 2014; Guareschi \& Jovchelovitch, 2004; McLaughlin, 2010a), l'évaluation de programme (Craig, 2002; Fetterman \& Wandersman, 2007), les études féministes (Lee-Rife, 2010), la toxicomanie (Shearer \& King, 2001), l'éducation (Mohajer \& Earnest, 2009), l'économie sociale (Ninacs, 2008) ou encore la relation d'aide (Evans et al., 2011; Lemay, 2007; Prilleltensky \& Prilleltensky, 2003; Vallerie, 2012).

\section{QUE SAIT-ON DE SON PROCESSUS ET DE SES IMPACTS?}

Une grande diversité de recherches attestent de la présence de liens empiriques entre certains éléments de pratique et plusieurs variables telles que le sentiment de compétence (Hur, 2006), la capacité à gérer ses émotions (Haswell et al., 2010), l'inclusion sociale (McCubbin et al., 2010), le processus de prise de décision (Clarke et al., 2015), le changement personnel (Song, 2015), l'autodétermination (Boudrias, Gaudreau, \& Laschinger, 2004), l'engagement politique, la capacité de leadership (Christens, Speer, \& Peterson, 2011), etc. Cependant, ces résultats sont fragiles en raison de la grande hétérogénéité de leurs fondements théoriques et de leur étendue. Pour une large part, la notion d'empowerment est beaucoup plus utilisée pour son potentiel évocateur que pour désigner une réalité précise (Adamson, 2010; Cattaneo \& Goodman, 2015; Handy 
\& Kassam, 2006; Tengland, 2007). Le même problème se pose en ce qui concerne les modélisations du processus (Hur, 2006).

\section{PEUT-ON CONTRIBUER À SA MANIFESTATION?}

Cette question constitue le véritable talon d'Achille des travaux actuels sur le développement du pouvoir d'agir personnel ou collectif. Si l'idée générale de soutenir le DPA est globalement acquise, seule une poignée d'études propose une manière concrète de passer de cette intention à sa mise en œuvre (Enn, 2012; Everett, Homstead \& Drisko, 2007; Franco et al., 2008; Miley \& DuBois, 2007; Windsor, Jemal, \& Benoit, 2014). Pourtant, il s'agit d'une question cruciale si l'on veut éviter que cette alternative attrayante ne devienne un leurre ou même, dans certains cas, une mystification (Handy \& Kassam, 2006; Lupton, Fonnebo, Sogaard, \& Fylkesnes, 2005; Van Hal et al., 2012). Pour éviter ces écueils, il est essentiel que toute intention affichée de contribuer à soutenir le DPA s'appuie sur un cadre de référence explicite.

C'est avec l'objectif de créer un tel cadre que nous avons entrepris, il y a quelques années déjà, la mise au point d'un outil d'analyse $^{1}$ des pratiques susceptible de guider des intervenant.e.s désireux-euses de centrer leur propre pratique sur le soutien au DPA des personnes ou des groupes qu'ils accompagnent. Pour élaborer cet outil, nous avons, dans un premier temps, réalisé une revue complète de la littérature scientifique et professionnelle sur la question générale de l'empowerment (Le Bossé, 2003). Cet exercice a tout d'abord permis de statuer que cette expression renvoyait au passage d'un sentiment d'impuissance à l'égard d'une réalité donnée à une possibilité réelle d'influencer le cours des événements importants pour une personne, ses proches ou la collectivité à laquelle elle s’identifie (Rappaport, 1981). Il a ensuite conduit à l'identification de quatre grands axes autour desquels se répartissait la quasi-totalité des publications scientifiques et professionnelles sur le sujet.

1. L'expression "outil d'analyse des pratiques» renvoie à une manière concrète d'appréhender les situations dans lesquelles nous sommes appelés à intervenir. Dans notre cas de figure, il s'agit plus précisément de questionner systématiquement quatre aspects spécifiques de la réalité à laquelle on est confronté; soit les quatre axes dont il est ici question. S’il est identique d'une situation à l'autre, ce questionnement peut conduire à utiliser une grande variété de stratégies d'intervention en fonction des réponses obtenues, du profil de l'intervenant.e et des conditions de l'intervention. 


\section{$1^{\text {ER }}$ AXE: L'ADOPTION D'UNE UNITÉ D'ANALYSE "ACTEUR·TRICE EN CONTEXTE »}

D'une manière ou d'une autre, l'ensemble de travaux consultés portait sur des variables impliquant la prise en compte simultanée de dimensions individuelles et structurelles. Il ressortait également que ces dimensions pouvaient avantageusement être appréhendées sous la forme de transactions ponctuelles (Altman \& Rogoff, 1987), mettant ainsi systématiquement l'accent sur les caractères à la fois idiosyncratiques et génériques des processus de DPA, qu'ils soient anticipés (modélisation), déduits (études empiriques) ou directement observés (rapports d'intervention). Face à une littérature particulièrement éclatée, l'adoption d'une unité d'analyse "acteur-trice en contexte" permettait d'identifier un premier trait d'union entre les études.

C'est plus précisément sous l'angle interactionniste que nous avons abordé cette unité d'analyse. Il s'agit d'un point de vue qui privilégie l'étude de ce qui se passe entre les humains d'une part, et entre les humains et les systèmes sociaux d'autre part (Barus-Michel, Enriquez, \& Lévis, 2003). Ces relations sont appréhendées sous un angle qui aborde les phénomènes sociaux en tant qu'espaces d'interactions stratégiquement organisées dans le but de poursuivre des finalités constamment en définition. Conséquemment, cet «interactionnisme stratégique» (I.S.) envisage la finalité des interactions comme un produit ponctuel et non comme un processus organisateur (Le Bossé, 2011; Pépin, 2018). Pour cette raison, elle accorde une place particulièrement importante aux enjeux (ce que chacun veut obtenir et éviter) des différents acteurs-trices et à la manière dont ceux-ci sont négociés. Du point de vue I.S., seule une définition de la réalité à l'étude qui intègre, au moins en partie, les principaux enjeux en présence peut produire une démarche de changement viable.

Cette lecture psychosociologique très spécifique nous conduit à analyser les acteurs.trices à partir de la mise au jour de leurs enjeux et des stratégies qu'elles ou ils utilisent pour les rencontrer. Les contextes y sont appréhendés comme des espaces d'interactions ponctuelles dont la configuration particulière détermine les marges de manœuvre disponibles pour la conduite des changements personnels et structurels. Sur le plan philosophique, l'approche DPA présentée dans ces pages s'inscrit dans une conception réformatrice des pratiques sociales. Elle considère que la finalité de ces pratiques consiste à contribuer ouvertement à l'avènement d'une société 
plus juste. Pour cela, elle considère qu'il est incontournable d'appréhender simultanément les aspects personnels et structurels qui contribuent au maintien des problèmes sociaux. Dans la lignée des travaux d'Amartya Sen (2009) et de Martha Nussbaum (2011), elle subordonne la réalisation de cette justice sociale à la réunion de l'ensemble des conditions nécessaires à l'exercice effectif des droits fondamentaux (capabilités).

\section{E AXE: LA NÉGOCIATION DE LA DÉFINITION DU PROBLÈME ET DES SOLUTIONS AVEC LES PERSONNES CONCERNÉES}

A priori, la notion de "participation» constituait un autre fil d'Ariane entre les différents types d'études. Mais, à y regarder de près, cette notion s'avérait être beaucoup trop vague pour refléter la manière dont les participant.e.s aux recherches ou les personnes accompagnées étaient appréhendés. De façon constante, on retrouvait des références aux "rapports égalitaires", "accents mis sur les compétences", "co-constructions" ou encore "prises de parole» de la population à l'étude. Toutes ces formulations reposaient sur une prémisse selon laquelle le point de vue des personnes participantes ou accompagnées devait être systématiquement pris en compte. Plus précisément, ces personnes disposaient d'une véritable expertise expérientielle en matière de pertinence et de viabilité du changement. Ainsi, pour être compatibles avec la logique de DPA et éviter le risque d'une instrumentalisation, les différentes formes de participation devaient inclure la contribution des personnes dans la définition de la problématique et des solutions envisageables. Cette position relative à la participation dans une perspective centrée sur le DPA s'est progressivement approfondie à partir des travaux de Ricœur (1990) sur son anthropologie de l'homme capable et de Hannah Arendt (2003) sur l'action collective. Ces développements ultérieurs ont notamment permis d'asseoir la notion de DPA sur des fondements conceptuels qui ont en commun d'accorder une importance centrale aux savoirs tirés du passage de l'action.

\section{$3^{E}$ AXE: LA PRISE EN COMPTE DES CONTEXTES D'APPLICATION}

Un des éléments les plus communs à l'ensemble des études recensées était que les modélisations tout comme les données de recherche et les rapports d'intervention insistaient explicitement 
sur le fait que les impacts du DPA prennent des formes différentes selon les contextes.

Lorsqu'on réfléchit les choses en termes de recherche, le défi de la prise en compte des contextes réside dans notre capacité à dégager des enseignements transposables à partir de l'étude de cas particuliers. Sur le plan plus général de la conduite du changement, c'est essentiellement la capacité des acteurs-trices à appréhender la situation dans sa globalité tout en tenant compte de son caractère dynamique (Morin, 1990) qui permet d'affiner les stratégies d'intervention. Philosophiquement ancrés dans une perspective pragmatique (Rorty, 2008), selon laquelle la recherche de la viabilité d'une solution est en pratique plus efficiente que la recherche d'une forme de vérité absolue, nous appréhendons les contextes comme des réalités ponctuelles ne nécessitant pas d'être analysées selon une perspective temporelle linéaire. L'adoption de ce troisième axe place l'intervenant.e devant la nécessité d'enraciner sa démarche sur la situation telle qu'elle se présente ici et maintenant plutôt qu'en s'appuyant sur d'hypothétiques liens de causalité avec des expériences passées ou la formulation de pronostics pour le futur.

\section{E AXE: L'INTRODUCTION D'UNE DÉMARCHE D'ACTION CONSCIENTISANTE}

À l'époque, tout un pan de la documentation scientifique et professionnelle sur le DPA prenait appui sur la notion de conscientisation pour décrire le processus et une partie de ses impacts. De manière évidente, il y avait une ligne de fracture entre les promoteur.trice.s d'un DPA collectif, pour lesquels l'application de cette notion sur le plan individuel constituait une forme de récupération, voire même de détournement, et ceux et celles qui s'intéressaient à ce DPA dans une logique d'amélioration des capacités individuelles d'adaptation. Mais, au-delà de ces divergences, l'ensemble des auteur.e.s faisaient plus ou moins directement référence à une nécessaire démarche de "prise de conscience» de la part des personnes engagées dans un processus de DPA.

À partir de notre compréhension des travaux de Francisco Varela sur la relation action/cognition (Varela, Thompson, \& Rosch, 1993), nous avons proposé de considérer la question de la prise de conscience dans le processus de DPA sous la forme de "l'introduction d'une démarche d'action conscientisante» (IDAC), qui appréhende 
le rapport pensées/action comme une relation circulaire dont les composantes ont, tour à tour, le statut de cause et d'effet.

Une fois cette synthèse réalisée, nous sommes partis de l'hypothèse que les quatre axes décrits ci-dessus constituaient des points de repère susceptibles de permettre aux praticien.ne.s d'appréhender n'importe quelle situation sous l'angle du DPA-PC. Plus précisément, cette grille d'analyse des pratiques devait pouvoir faire émerger les possibilités concrètes de soutien effectif au DPA-PC des personnes accompagnées dans chaque situation. À des fins d'illustration, nous avons construit un exemple type d'application du cadre d'analyse fondée sur les quatre axes décrits plus haut à partir d'un cas fictif inspiré de descriptions réelles de pratiques par les nombreux intervenant.e.s que nous avons été amenés à accompagner avec cette approche.

\section{DE LA THÉORIE À LA PRATIQUE: ILLUSTRATION DE L'APPLICATION DE L'APPROCHE DPA-PC À LA QUESTION DE L'INDÉCISION VOCATIONNELLE} L'étude de cas présentée ci-dessous illustre le type de pratique que peut produire l'utilisation du cadre d'analyse de l'approche DPA-PC. Chaque axe conduit à un questionnement spécifique qui permet d'appréhender la complexité de la situation d'une manière simple et pragmatique tout en prenant en considération son caractère dynamique.

\section{AXE 1: ADOPTION D'UNE UNITÉ D'ANALYSE "ACTEUR·TRICE EN CONTEXTE ॥: QUEL EST LE CONTEXTE?}

Marina a 17 ans, elle termine son secondaire $5^{2}$ et n'a toujours pas fait de choix d'orientation pour le CEGEP ${ }^{3}$. Elle explique sa difficulté par le fait qu'elle se sent incapable de choisir entre trois options. Ce n'est pas la première fois qu'elle est plongée dans cette situation. Au cours de sa jeune existence, elle a souvent été confrontée à des situations critiques, qu'elle attribue à son ambivalence «chronique». Les demandes d'admission doivent être

2. L'équivalent de la $11^{\mathrm{e}}$ année de scolarité.

3. Collège d'enseignement général et professionnel (CEGEP). C'est une étape intermédiaire dans le cheminement scolaire entre le secondaire et l'université. 
expédiées avant le 1er mars. Devant l'absence de disponibilité de la part du c.o. de l'établissement de Marina, ses parents, inquiets, ont décidé de lui offrir les services d'une ou un conseiller •ère en orientation en pratique privée. Comme nous sommes à la fin du mois de janvier, la praticienne qui l'accueille dispose d'un maximum de trois rencontres d'une heure pour l'aider à faire un choix définitif.

Dans cet exemple, il est possible de repérer immédiatement un élément contextuel qui colore fortement l'ensemble de la situation. Il s'agit bien évidemment du fait que l'étudiante est «mise en demeure» de faire un choix d'ici à trois semaines. Un deuxième élément de contexte concerne la préoccupation des parents à l'égard du choix professionnel de leur fille. Enfin, il apparaît clairement que Marina attribue une bonne part de ses difficultés à ses caractéristiques personnelles (indécision supposément "chronique», anxiété, etc.).

\section{QUI SONT LES ACTEURS·TRICES?}

Dans l'exemple de Marina, il y a potentiellement deux acteurs.trices concerné.e.s ${ }^{4}$ : Marina et ses parents. Les acteurs.trices impliqué.e.s sont ceux et celles qui contribuent, au niveau macro et micro-social, à définir le cadre dans lequel Marina est amenée à réfléchir à son orientation.

Avant tout, Marina veut être sûre de faire le «bon» choix. Tout d'abord pour elle-même. Elle est particulièrement inquiète du vide qu'elle ressent lorsqu'elle tente de se projeter dans l'avenir. Dans un deuxième temps, son désir de ne pas se tromper est également fondé sur son inquiétude à l'égard de ses parents. En effet, cette situation de choix professionnel a pris une importance démesurée dans la maison depuis que le père s'est retrouvé licencié pour des raisons économiques.

4. Dans cette approche, nous faisons une distinction opérationnelle entre les personnes "concernées", qui sont celles qui auront à composer avec les conséquences concrètes des interventions, et les personnes "impliquées", qui interviennent, à un titre ou à un autre, dans la définition du changement visé sans que le résultat ait d'impact significatif sur leur réalité quotidienne. 


\section{QUELS SONT LES ENJEUX DES DIFFÉRENT·E·S ACTEURS·TRICES (CE QU'ELLES ET ILS CHERCHENT À OBTENIR OU À ÉVITER)?}

Au niveau macro et micro-social, on peut avancer qu'a priori, tous les acteurs-trices cherchent à obtenir que Marina puisse dépasser les obstacles (sociologiques, économiques, politiques, psychologiques ou psychosociologiques) à sa démarche de choix professionnel. Dès lors se pose la question suivante: "Qu'est-ce qui, dans la situation actuelle et compte tenu des enjeux en présence, fait obstacle à la démarche de choix de Marina?"

Sur le plan macro-social, le caractère rigide de la date butoir des inscriptions aux études collégiales constitue une difficulté certaine. Par ailleurs, on constate une contradiction importante entre l'enjeu global de soutenir cette démarche de choix et l'absence de ressources suffisantes au sein de l'établissement scolaire de Marina qui auraient pu lui permettre de bénéficier d'un accompagnement plus précoce et donc moins anxiogène.

Sur le plan micro social, les parents de Marina cherchent apparemment à obtenir une forme d'apaisement de leur inquiétude en ce qui concerne l'avenir de leur fille. En corollaire, ils cherchent à éviter que Marina ne "se ferme des portes». En même temps, leur décision d'offrir à Marina une série de rencontres avec un.e conseiller-ère en orientation illustre leur désir de permettre à Marina de trouver une forme de sérénité à l'égard de sa démarche de choix professionnel.

En ce qui concerne Marina, son désir ardent de faire «le bon choix » illustre l'omniprésence d'une peur "de se tromper». Marina cherche à obtenir un sentiment de certitude et à éviter de ressentir de la peur à l'égard de son orientation professionnelle.

\section{AXE 2: LA NÉGOCIATION DE LA DÉFINITION DU PROBLÈME ET DES SOLU- TIONS AVEC LES PERSONNES CONCERNÉES}

Chaque situation est singulière et doit être définie à partir de deux sources d'expertises complémentaires. D'un côté, il y a l'expertise professionnelle, qui peut contribuer à restaurer les conditions pour permettre à Marina de conduire sa démarche de choix professionnel de manière autonome. De l'autre côté, il y a l'expertise expérientielle, indispensable à toute démarche de changement viable (seul.e.s Marina et ses proches sont en mesure de déterminer ce qui est viable pour elles ou eux). Le défi consiste donc à négocier 
une définition opérationnelle du problème qui soit suffisamment représentative des enjeux en présence.

Le milieu socio-éducatif dans lequel Marina évolue contribue à faire en sorte qu'elle est implicitement amenée à concevoir sa difficulté comme "un trouble de la décision" ou un trait personnel stable (elle serait "de nature indécise»). Plus Marina s'accroche à l'idée qu'elle est de nature indécise, plus elle se place en situation d'impuissance face à l'échéance qui se rapproche quant à son choix professionnel. Il y a donc là un premier obstacle lié à la manière dont cette jeune personne se représente «son problème». La ou le conseiller.ère en orientation, qui adopte cette approche centrée sur le DPA, cherchera à obtenir une définition plus concrète et plus personnelle des difficultés que rencontre Marina. On assistera donc à un dialogue qui ressemblera à ceci (disons que Marina rencontre une conseillère en orientation qui s'appelle Dominique).

Dominique: «Alors, dit-elle en s'installant, comment ça va?»

Marina: «Ben, j'ai de la misère à faire mon choix pour le CEGEP... »

Dominique: «OK et puis...?»

Marina: «Puis ça m'inquiète beaucoup.»

Dominique: «Ah oui? Pourquoi ça?»

Marina: «Parce que j'ai peur de ne pas être capable de choisir...»

Dominique: «Tu ne sais pas écrire?»

Marina: «Bien sûr que si, pourquoi?»

Dominique: «Tu as de la difficulté à comprendre ce qui est inscrit sur le formulaire?»

Marina: «Bien évidemment non.»

Dominique: «Est-ce que tu comprends entre quoi et quoi tu dois choisir?»

Marina (de plus en plus impatiente): «Je ne suis pas idiote quand même!»

Dominique: «C'est bien ce qui me semble. Alors d'où vient cette peur de ne pas être capable?»

Marina: «C'est que je suis une personne indécise...»

Dominique: «Que veux-tu dire?»

Marina: «C'est difficile pour moi de faire un choix.»

Dominique: «Tout le temps, dans toutes les circonstances? »

Marina: «Bien non! Seulement quand c'est important pour moi!»

Dominique: «Quel est ton plat favori?» 
Marina (écarquillant les yeux): «La lasagne, pourquoi?»

Dominique: «Si l'on te propose une lasagne ou un spaghetti pour le souper, auras-tu du mal à choisir?»

Marina (souriant spontanément): «Pas du tout, ce serait même très facile!»

Dominique: «ll y a donc ce qui est important pour toi pour lequel il est facile de choisir et ce qui est important pour toi pour lequel il est plus difficile de choisir.»

Marina: «C'est ça!»

Dominique: «Je suis désolée de te dire que c'est le sort de tout le monde et que je ne vois pas en quoi ça te rend plus indécise que les autres.»

Dans ce court dialogue, on voit comment la conseillère en orientation travaille à ébranler les fondements du problème que Marina "pense» avoir. Voyons maintenant comment elle s'y prend pour identifier concrètement le problème tel qu'il se manifeste au moment de la rencontre.

Marina (dubitative): «Je croyais que c'était moi qui avais un problème... Je hais ça, être obligée de choisir.»

Dominique: «Es-tu vraiment obligée?»

Marina: «Comment ça?»

Dominique: «Rien ne t'oblige à choisir toi-même. Tu peux y aller au hasard, demander à tes parents, suivre les avis d'un conseiller en orientation ou de tes amis, etc.»

Marina: «Ah, mais non! J'veux pas me retrouver dans n'importe quoi! Même si j'haïs choisir, faut que ce soit moi qui décide.»

Dominique: «Tu voudrais donc à la fois choisir par toi-même et être sûre de ne pas te tromper?»

Marina: «Bien... j'crois que c'est ça... en tout cas... c'est pour ça que je suis là...»

Dominique: «Pour avoir la garantie de ne pas te tromper?»

Marina: «Bien... j'aimerais bien être certaine de mon choix... pis, comme ça, mes parents aussi arrêteraient de se stresser avec ça...»

Dominique: «Donc, si je comprends bien, tu voudrais faire un choix 
de carrière rapide, sûr et définitif pour rassurer tes parents et toimême par la même occasion?»

Marina: «C'est ça, mais je veux aussi être sûre de faire le bon choix.»

Dominique: «Comment vas-tu faire ça? Comment sauras-tu que c'est le bon choix...? ?

Marina: «Bien... Je pensais que vous aviez des tests ou des trucs pour me dire dans quoi je serais bonne...»

Dominique: «Aucun test n'est capable de te dire une fois pour toutes et sans risque d'erreur quel serait le bon choix pour toi... On peut uniquement les utiliser comme une source d'informations complémentaires. Donc je reviens à ma question: comment peux-tu être sûre de faire le bon choix? Ou, plus simplement, qu'est-ce que serait le bon choix pour toi?»

Marina: «Je ne sais pas... un choix qui me ferait me sentir bien avec ma décision?»

Dominique: «Qu'est-ce qui t'empêche maintenant de te sentir bien?»

Marina: «Bien, c'est toujours pareil, la peur de me tromper. J'y pense tout le temps et plus le 1er $^{\text {er }}$ mars approche, plus j'ai peur... »

Dominique: «Est-ce que l'on peut dire que c'est surtout la peur de te tromper qui te pose un problème en ce moment?»

Marina: «Je ne sais pas...»

Dominique: «Imaginons que le système soit fait de manière différente et que chaque étudiant ait la possibilité d'explorer, pendant un an, différents types de formation qui l'intéressent, serais-tu encore aux prises avec cette peur de te tromper?»

Marina: «Bien non! Parce que je pourrais toujours changer d'idée en cours de route... »

Dominique: «Aurais-tu du mal à choisir les formations que tu voudrais essayer?»

Marina: «Pas vraiment, parce que j'hésite entre deux ou trois possibilités.»

Dominique: «Alors, qu'est-ce qui fait la différence avec la situation actuelle?»

Marina: «Bien, c'est qu'il faut que je prenne une décision, alors que je ne suis pas sûre de mon choix...»

Dominique: «Est-ce que l'on pourrait reformuler le problème ainsi: 
«Comment remplir le formulaire d'inscription dans un établissement collégial, d'ici au $1^{\text {er }}$ mars, en étant sûre de mon choix?»

Marina: «C'est sûr que j'aimerais bien trouver une réponse à ça!» Dominique: "C'est un objectif que I'on peut atteindre en trois rencontres. Si l'on s'entend là-dessus, on peut commencer tout de suite.»

Marina: «OK, ça me va.»

Durant cette partie de l'entrevue, Dominique pousse un peu Marina dans ses retranchements. Elle l'amène progressivement à préciser sa demande. Il apparaît que la peur de se tromper domine les préoccupations de l'étudiante. Nous voilà confrontés à un problème concret: 1) une peur omniprésente qui «brouille les cartes" et qui constitue un obstacle à la poursuite de la conduite autonome d'une démarche de choix professionnel et 2) la nécessité de compléter un formulaire de choix d'ici à trois semaines. Quelles vont être les solutions viables pour Marina d'ici là?

\section{DEUXIÈME PARTIE DE L'AXE 2: LA NÉGOCIATION DES SOLUTIONS ENVI- SAGEABLES AVEC LES PERSONNES CONCERNÉES}

Dans ce contexte précis, il peut être pertinent de tenter une forme de recadrage cognitif, c'est-à-dire de présenter à Marina une interprétation alternative de son problème qui soit susceptible de l'aider à appréhender la situation de manière moins anxiogène. Voyons comment Dominique procède.

Dominique: «Pour commencer, voyons comment tu pourrais t'y prendre pour être sûre de ton choix...»

Marina: «Justement, c'est ça que je ne sais pas!»

Dominique: «Tu disais, tout à I'heure, que tu souhaitais te sentir «bien avec ta décision ». Concrètement, qu'est-ce qui ferait que tu te sentes bien?»

Marina: «Bien, j'arrêterais de me stresser avec ça.»

Dominique: «Est-ce que c'est possible? Est-ce que l'on peut faire un choix pour notre avenir sans se stresser avec ça?» 
Marina (qui commence à fatiguer avec toutes ces questions): «Bien oui, si l'on est sûr de notre choix!»

Dominique: «Comment peut-on être sûr d'aimer ce que l'on n'a pas encore vécu? Est-ce que ce n'est pas toujours un peu incertain?» Marina: «Bien, à l'école, il y en a qui savent depuis qu'ils sont petits ce qu'ils veulent faire dans la vie... Mais c'est vrai que la plupart ont du mal à se démêler, pis changent souvent...»

Dominique: «En fait, on ne peut choisir qu'à partir de ce que l'on connaît. Même quand on imagine ce que ce serait d'avoir telle ou telle profession, c'est toujours à partir de notre expérience et de nos connaissances actuelles (ce que l'on a vu à la télé, les livres que I'on a lus, etc.). Choisir son orientation, c'est forcément prendre une chance.»

Marina: «C'est ça qui m'énerve! Parce qu'en fait on peut jamais être sûr à $100 \% . »$

Dominique: «Donc, comment faire un choix d'études en étant sûr à $100 \%$ de ne pas se tromper?»

Marina: «Bien... finalement, c'est impossible, on ne peut pas y arriver.»

Dominique: «Si ça c'est impossible, qu'est-ce qui est possible?»

Marina: «Je ne sais plus, je suis toute mêlée.»

Dominique: «C'est normal, ça fait beaucoup de choses à penser en même temps. En fait, on peut résumer ça de la manière suivante: d'ici à trois semaines, tu dois faire un choix pour t'inscrire au collégial, tu as peur de te tromper et, en même temps, on vient de voir qu'il est impossible d'être certain à $100 \%$ de son choix. Qu'est-ce que tu peux faire concrètement?»

Marina: "Je peux choisir quand même, mais je vais toujours avoir peur de me tromper.»

Dominique: «C'est inévitable, on ne peut pas être sûr à 100\% de son choix, mais ce n'est pas forcément un problème.»

Marina: «Comment ça?»

Dominique: «As-tu choisi ta robe de bal?»

Marina (à la fois étonnée et réjouie de la digression): «Oui, j'ai hésité entre deux pendant un bout de temps, je me suis finalement décidée pour la plus claire, car je ne voulais pas avoir l'air d'une madame.»

Dominique: «Et pendant que tu hésitais, avais-tu peur de te tromper?» 
Marina: «Oui, mais ça ne m'empêchait pas de dormir...»

Dominique: «Tu constates donc par toi-même que l'on fait un choix en fonction d'un futur hypothétique (ici: de quoi j'aurai l'air le soir du bal des finissants); la possibilité de se tromper est toujours là, mais elle est plus ou moins stressante en fonction de l'importance que l'on accorde au choix que l'on va faire.»

Marina: «Justement, c'est pour ça que ça me stresse de faire un choix d'ici au $1^{\text {er }}$ mars, parce que c'est mon avenir professionnel qui se joue!»

Dominique: «Tu le penses vraiment?»

Ce processus de dialogue relève d'une démarche circulaire. Toute négociation des solutions envisageables revient à préciser encore plus le problème. Nous pourrions donc, à ce stade-ci de l'échange entre Dominique et Marina, considérer que le problème est en train de prendre la forme suivante: étant donné que l'incertitude est inhérente à toute démarche de choix tournée vers l'avenir, qu'est-ce qui, dans les circonstances actuelles, peut contribuer à diminuer de manière efficiente le stress de Marina afin de lui permettre de compléter plus sereinement son formulaire d'ici à trois semaines?

Jusqu'ici, les deux premiers axes du modèle d'analyse ont permis de disposer d'une définition très opérationnelle du problème et des solutions envisageables. Nous allons maintenant nous intéresser aux conditions concrètes du passage à l'action.

\section{AXE 3: LA PRISE EN COMPTE DES CONTEXTES D'APPLICATION}

Nous avons vu qu'en matière de changement social, la prise en compte des contextes constitue une nécessité incontournable. L'adoption de ce troisième axe de pratique constitue une application concrète de cette nécessité et elle a un effet direct sur la manière dont la ou le conseiller ère en orientation conduira son intervention. Reprenons l'exemple de Marina pour illustrer les conséquences de ce que nous avons appelé «la prise en compte des contextes d'application". 
Après trente-cinq minutes d'échanges et de recadrages successifs de la part de Dominique, Marina est parvenue à une compréhension beaucoup plus contextualisée de son problème, qui est par ailleurs formulé maintenant en termes opérationnels. La conversation s'oriente dès lors sur les critères de choix que Marina pourrait bien adopter. En une quinzaine de minutes, Marina parvient à dégager un ensemble de critères personnels assez précis. Auparavant, sa peur de se tromper dominait tellement ses réflexions qu'elle était incapable d'adopter des critères stables.

À première vue, l'intervention est un succès, Marina est «sur la bonne voie» et Dominique peut raisonnablement s'attendre à ce que la prochaine rencontre conduise à la formulation d'un choix précis dans un climat de sérénité. Pourtant, il ne faut pas perdre de vue qu’à ce jour les éventuels changements de posture qu’a pu réaliser Marina au cours de cet entretien n’ont pas encore conduit à compléter le formulaire. Ce point est très important, parce qu'il montre que le changement ne peut se résumer à une seule opération de recadrage cognitif. Il est nécessaire qu'au moment opportun l'ensemble des ressources (matérielles, d'informations, personnelles, structurelles, etc.) puissent être disponibles et accessibles.

C'est la raison pour laquelle Dominique prend soin, à la fin de son entrevue, de vérifier auprès de Marina si elle dispose des ressources requises (ici: connaissance des sources d'information, disponibilité et motivation) pour pouvoir réaliser les tâches qu'elle se donne d'ici à la prochaine rencontre. Par ailleurs, en matière de pratiques sociales comme dans bien d'autres domaines, la réalité n'est pas statique, mais dynamique. En gros, «tout bouge tout le temps». Dans le cas de Marina, la position de Dominique vis-à-vis de la nature de la réalité (statique ou dynamique) peut complètement changer la manière dont elle va réagir à la situation suivante.

Marina se présente la semaine suivante dans un état très agité. Les derniers jours ont été pleins de rebondissements. Son petit ami lui a proposé de venir s'installer en appartement avec lui et d'étudier dans un CEGEP de la province spécialisé dans les formations techniques. Cette institution offre aussi une formation collégiale géné- 
rale, mais avec peu d'options étant donné sa vocation première. II est très amoureux de Marina, qui lui est également très attachée. Ses parents refusent de financer ses études si elle prend sa décision sur la base de sa relation avec son copain. Pour la convaincre de donner la priorité à ses études, ils lui ont appris qu'au fil des ans ils avaient suffisamment épargné pour financer ses études jusqu'au troisième cycle! Elle qui, il y a encore une semaine, hésitait entre trois programmes bien précis, se retrouve aujourd'hui divisée entre l'attrait pour une vie plus indépendante et affectivement épanouissante, mais matériellement plus difficile, et la possibilité d'avoir accès à un appui financier auquel elle n'aurait même pas osé rêver. Évidemment, avec tous ces événements, elle n'a pas eu le temps ni l'énergie d'approfondir ses choix de programme.

Face à cette nouvelle situation, Dominique va aligner sa pratique sur le troisième axe du modèle, qui propose de toujours tenir compte des contextes d'application. En effet, puisque «tout bouge tout le temps» et que chaque instant est unique, alors le seul espace du temps dans lequel on peut agir est le présent et, encore plus concrètement, la situation telle qu'elle se manifeste ici et maintenant. Voici une illustration du type d'échange auquel l'adoption d'une telle posture peut conduire

Dominique (après avoir écouté le récit un peu décousu de Marina): «Eh bien dis donc... Les choses ont bougé en une semaine! Qu'est-ce qui te préoccupe le plus aujourd'hui dans toute cette histoire?»

Marina: «Je ne veux pas avoir à choisir!»

Dominique: «Entre quoi et quoi?»

Marina: «Entre mon chum [amoureux] et mes parents.»

Dominique: «D'accord! Comment peux-tu ne pas choisir?»

Marina: « J'en sais rien! Si au moins je savais ce que je veux...»

Dominique: «Tu sais que tu ne le sais pas, c'est déjà ça...»

Marina: «Pour ce que ça m'avance!»

Dominique: «Au contraire, c'est un bon point de départ. Tu es dans une situation où plusieurs personnes veulent que tu prennes une décision et toi tu sais que tu ne sais pas ce que tu veux. À partir de là, que peux-tu faire?» 
Marina: «Je n'ai rien compris!»

Dominique: «Si tu ne te sens pas prête à prendre une décision, que peux-tu faire?»

Marina: «Bien, je ne vois pas... À moins que je dise non aux deux? » Dominique: «Qu'est-ce qui se passerait alors?»

Marina: «Je perdrais mon chum et je serais en chicane avec mes parents!»

Dominique: «En effet, ce n'est pas réjouissant... Comment tu pourrais faire pour éviter cela tout en évitant de décider tout de suite?»

Marina: «Bien, c'est pas possible à cause de cette histoire du 1er mars...»

Dominique: «Que veux-tu faire pour le $1^{\text {er }}$ mars exactement ?»

Marina: «Je dois faire ma demande d'inscription si je veux avoir une place quelque part.»

Dominique: «Qu'est-ce qui t'empêche de faire ça?»

Marina: «Bien là, je ne sais toujours pas dans quoi je veux m'en aller et en plus je dois savoir si je vais dans le CEGEP de mon chum ou non.»

Dominique: «N'as-tu pas la possibilité de faire plusieurs choix par ordre de priorité?»

Marina: «Oui... Je pourrais toujours faire les trois choix autorisés pour garder toutes les possibilités ouvertes...»

Dominique: «Ça serait quoi ces trois choix?»

Marina: «Les deux dont on a parlé (sciences de la nature et littérature) dans la région et puis l'un ou l'autre de ces programmes dans le CEGEP de mon chum.»

Dominique: «Il ne reste plus qu'à choisir un ordre de priorité... Comment peux-tu faire pour que ce choix donne le maximum de marge de manœuvre?»

Paradoxalement, en tenant compte de ce qui se passe ici et maintenant dans la vie de Marina, la stratégie de Dominique a consisté à l'aider à ne pas choisir! Pourquoi? Parce que la demande qui domine dans la situation présente est celle de sortir de la double contrainte (Nardone \& Watzlawick, 2002) dans laquelle Marina se trouve. En fait, dans cette situation, il s'avère urgent d'attendre que les choses se clarifient. Le type de soutien offert par la conseillère en orientation a délibérément placé Marina dans une posture qui lui 
permettait de faire concrètement l'expérience d'une gestion lucide et stratégique de l'incertitude. C'est en effet parce qu'elle ne sait pas ce qu'elle veut et qu' elle l'assume que Marina est maintenant en mesure de percevoir (et éventuellement de revendiquer) son incertitude comme un motif pour ne pas prendre de décision précipitée et non plus comme une carence plus ou moins pathologique. Mais cette compréhension intégrée de ce qu'a permis l'accompagnement se fait rarement spontanément. C'est la raison pour laquelle le modèle d'analyse proposé ici comprend un quatrième axe, intitulé «introduction d'une démarche d'action conscientisante». Ce dernier a aussi pour fonction de stimuler des initiatives de changements structurels.

\section{AXE 4: INTRODUCTION D'UNE DÉMARCHE D'ACTION CONSCIENTISANTE}

Qu'est-ce qu'une prise de conscience? En gros, c'est le fameux "déclic" qui nous amène à dire: "J'ai pris conscience que..." Dans l'approche centrée sur le DPA, la prise de conscience est alimentée par la confrontation constante de l'ensemble de l'appareil perceptif (tête, cœur, corps) avec l'expérience issue du passage à l'action. Elle génère un ensemble d'impacts sur les plans cognitif (elle affecte la manière dont je comprends ce que je vis), affectif (elle modifie ma manière de ressentir mon expérience quotidienne) et comportemental (elle transforme ma façon d'agir à l'égard des difficultés que je rencontre). Voyons comment tout ceci peut être illustré à l'aide de la situation de Marina.

Marina et Dominique en sont à leur quatrième rencontre. Au cours de la semaine, Marina a finalement complété son formulaire d'inscription aux études collégiales en plaçant trois choix, par ordre de priorité, selon des critères précisément identifiés avec Dominique au cours de la troisième rencontre. À cette occasion, Marina avait soudainement pris conscience qu'elle ne pouvait pas se fonder uniquement sur des critères abstraits. Elle a donc retenu des critères qui tenaient compte à la fois de ses aspirations générales en matière professionnelle et de ses préoccupations à court terme. Ainsi, elle avait choisi de reporter sa réponse à l'offre de son chum tout en choisissant son futur CEGEP en troisième position. Avant de prendre une décision définitive à ce sujet, elle souhaitait tester la solidité 
de sa relation. En ce qui concerne ses parents, elle avait décidé de ne rien leur promettre, tout en s'inscrivant, dans un CEGEP local, dans un programme de sciences de la nature, comme deuxième choix. Enfin, puisqu'elle savait qu'elle possédait potentiellement un petit pécule pour faire ses études, elle avait choisi de s'inscrire, en premier choix, dans un CEGEP plus éloigné de chez elle, mais dans lequel il y avait un programme qui l'intéressait. Si elle était admise (ce qui, lui avait-on dit, était fort probable), elle devrait vivre en résidence, et se trouverait à distance comparable entre le domicile de ses parents et la résidence du CEGEP de son chum. Bien que toujours préoccupée par son avenir professionnel, Marina apparaît beaucoup plus détendue lorsqu'elle entre dans le bureau de Dominique pour cette quatrième rencontre.

D'une certaine manière, la question de l'orientation professionnelle de Marina reste entière. L'intervention centrée sur le DPA, telle qu'elle a été conduite par Dominique, a d'abord et avant tout permis à la jeune étudiante de composer avec l'incertitude de manière beaucoup plus optimale. Il s'agit là d'un changement majeur. Le fait d'avoir expérimenté une gestion stratégique de l'incertitude va permettre à Marina de se positionner différemment dans de nombreux domaines de sa vie quotidienne. En plus de constituer une base solide pour stimuler la conduite autonome de ses futures démarches de choix professionnel, Marina pourra éventuellement utiliser ses nouveaux acquis dans sa manière de se positionner à l'égard de ses parents, dans la gestion de ses relations affectives, etc. Dominique va donc consacrer cette quatrième entrevue à l'intégration du cheminement réalisé par Marina au cours des trois dernières semaines.

Dominique: «Alors, il n'y a plus de problème?»

Marina: «Bien, je dirais pas ça... mais ça va mieux en tout cas.»

Dominique: «Qu'est-ce qui va mieux?»

Marina: «Je suis beaucoup moins stressée, puis surtout j'ai réussi à faire mon inscription!»

Dominique: «Y a-t-il d'autres gains au passage?»

Marina: "C'est sûr, je me sens moins coincée entre mon chum et mes parents.» 
Dominique: «Comment ça se fait?»

Marina: «Ben, je crois que j'ai compris des affaires...»

Dominique (sur un ton qui encourage Marina à préciser sa pensée): «Des affaires?»

Marina: «Par exemple, ce n'est pas parce que quelqu'un demande de choisir qu'on est obligé de le faire. Tu sais, on a le droit d'être mêlée un peu. Pis c'est pas parce qu'on aime quelqu'un qu'on doit toujours être d'accord avec.»

Dominique: «OK. Et puis es-tu contente de tes choix d'inscription?» Marina: «Ah oui! Je vais avoir la paix pour quelques mois et ça va me donner le temps de me brancher un peu plus...»

Dominique: «Effectivement, ça n'a pas l'air de t'énerver plus que ça!»

Marina: «Bien non, je me dis qu'après tout ce n'est pas la fin du monde de ne pas savoir où on s'en va, qu'on a du temps pour y penser. Puis je me suis rendu compte que ça sert à rien d'essayer de savoir à l'avance. On verra bien quand on y sera.»

Dominique: «Parfait... J'ai l'impression que tu es à ton affaire. $Y$ a-t-il d'autres choses que je peux faire pour toi?»

Si Dominique a terminé, pour le moment, son accompagnement avec Marina, elle n'a pas fini pour autant son intervention. En effet, ce n'est pas la première fois qu'elle reçoit les finissant.e.s qui vivent des «pics» d'anxiété au moment de choisir dans quel établissement elles ou ils vont s'inscrire pour faire leurs études collégiales. Dominique a donc décidé de contacter son ordre professionnel pour voir ce qui pourrait être fait pour interpeller les autorités responsables de ce dossier. Elle est personnellement prête à donner quelques heures par mois pour siéger dans un comité qui pourrait étudier le problème plus à fond et faire des propositions de changement à la procédure d'inscription au CEGEP. Par ailleurs, comme elle connaît bien la conseillère en orientation de l'école de Marina, elle a décidé de la contacter pour voir ce qui pourrait être fait avec elle au sein de l'établissement.

Le dialogue et le récit présentés ici illustrent la manière dont un accompagnement centré sur le DPA peut conduire concrètement à renforcer la conduite autonome d'une démarche de choix professionnel. Que l'on compare la situation initiale de Marina avec celle qui 
prévaut lors de la quatrième rencontre, ou que l'on s'intéresse à l'évolution de sa posture vis-à-vis du problème du choix professionnel à la suite de l'accompagnement reçu, il apparaît manifeste qu'elle a bougé à la fois sur le plan du processus et sur celui des résultats. Ces changements sont ponctuels et contextuels et il est très probable que la question de l'orientation professionnelle de Marina se posera à nouveau à court ou moyen terme. Mais ils peuvent aussi avoir des impacts plus fondamentaux. Ainsi en est-il de son changement de posture à l'égard de sa démarche de choix et de ses relations avec ses proches. En effet, même si le problème se représente, Marina est maintenant beaucoup plus en mesure d'y faire face par elle-même et, si toutefois une forme de soutien s'avérait nécessaire, il serait probablement plus court et plus circonscrit.

\section{D'UNE APPLICATION LOGIQUE À L'ÉMERGENCE CONCRÈTE D'UNE NOUVELLE COMMUNAUTÉ DE PRATIQUE}

Si cette étude de cas fictive permet d'illustrer concrètement le potentiel de cette approche, cela reste un cas d'école. Pour tester la viabilité de cette manière d'intervenir, il était indispensable de la soumettre à l'épreuve des faits tout en veillant à ne pas exposer des personnes vulnérables à d'éventuels effets iatrogènes.

Pour ce faire, nous avons initié une série d'études pilotes auprès de praticien.ne.s chevronné.e.s au cours desquelles les participant.e.s étaient placé.e.s en situation d'intégrer l'approche en l'appliquant à leur propre pratique. À ce stade du processus de recherche et développement, le but principal de ces études était de soumettre l'outil d'analyse et le cadre de formation à un test de réalité. Mais le temps du terrain n'est pas le temps de la recherche. Très rapidement, on a assisté à un mouvement de diffusion plus ou moins formalisé. À ce jour, plus de 10500 personnes ont été sensibilisées ou formées dans quatre pays et cinq contextes culturels différents (Québec, France, Belgique, Suisse, île de la Réunion). Des séminaires d'approfondissement sont régulièrement organisés par les personnes formées, qui ont également tenu plusieurs congrès internationaux. Ces "personnes ressources" sont également à l'origine de la publication de trois ouvrages collectifs (Jouffray, 2014; Portal, Desomer, \& Dutrieux, 2017; Vallerie, 2012) et d'au moins une vingtaine d'articles dans des revues professionnelles (Cometti, 2017; Étienne, 2017; Jouffray \& Vallerie, 2009; Petit, 2016a, 2016b; Portal, 2016). Le fait que les 
praticien.ne.s se soient emparée.s de cette approche et contribuent à son enrichissement suggère qu'elle répond à la nécessité d'enraciner la notion de DPA dans une pratique concrète. Avec quelques années de recul et dans un contexte où la notion de pouvoir d'agir devient de plus en plus centrale dans le champ des pratiques sociales francophones, il semble bien que nous disposions d'un outil permettant aux intervenant.e.s de soutenir et de traduire dans les faits leur volonté de soutenir le DPA des personnes qu'elles ou ils accompagnent individuellement ou collectivement. Si cela s'avérait être le cas, il s'agirait d'une avancée certaine dans notre effort collectif pour faire en sorte que cette notion de développement du pouvoir d'agir dépasse le stade des bonnes intentions.

\section{PISTES DE RECHERCHE}

Toutefois, la multiplication des initiatives de mise en pratique ne constitue pas directement une démonstration de leur efficience réelle. Parmi les nombreuses perspectives de recherche que suscite l'émergence de cette approche, la question de sa validité empirique constitue la plus centrale. L'approche proposée parvient-elle à soutenir concrètement le pouvoir d'agir des personnes et des collectivités? Pour répondre à cette question, il faudra tout d'abord développer des indicateurs qui soient à la fois constants dans leur nature et différents dans leurs manifestations afin de tenir compte de la spécificité de chaque contexte d'intervention. Beau défi! Dans un autre ordre d'idées, même si cette mise en pratique s'avérait plutôt efficiente, il restera à mettre à jour les mécanismes fondamentaux qui contribueraient à ce résultat.

\section{LIMITES DE CETTE APPROCHE}

Au cours des dernières années, nous avons eu l'occasion de constater un certain nombre de contraintes associées à cette approche. Premièrement, sa transmission passe par une forme ou une autre d'expérimentation personnelle. En effet, l'expérience montre que le caractère expérientiel de la formation joue un rôle central dans l'intégration de l'approche. Il apparaît que le fait de vivre personnellement l'expérience d'un processus de DPA sur sa propre réalité contribue activement à un ajustement de posture professionnelle qui semble être un passage incontournable lorsqu'on centre sa pratique sur le DPA 
des personnes accompagnées. Ce nécessaire repositionnement semble bien constituer un élément central du processus d'appropriation de l'approche. On peut donc avancer que la formation à cette approche est personnellement exigeante pour les professionnel.le.s qui s'y engagent. Par ailleurs, la nécessité d'intégrer, au moins en partie, les enjeux des personnes concernées dans la définition du problème et des solutions envisageables (axe 2) limite logiquement sa portée potentielle dans les situations où l'intervention se déroule dans un contexte d'autorité (protection de la jeunesse; mandat de suivi de probation, etc.).

\section{CONCLUSION}

Le présent chapitre a permis d'illustrer de manière très concrète la manière dont l'approche centrée sur le DPA-PC s'applique aux pratiques en orientation. Bien que l'illustration proposée porte sur une intervention en contexte individuel, elle est également mobilisable dans les accompagnements de collectifs. Même s'il faudra attendre une confirmation empirique en bonne et due forme, il semble bien que cette approche constitue un moyen concret de contribuer au soutien du pouvoir d'agir que Savickas et ses collègues (2010) appellent de leurs voux. À tout le moins s'agit-il d'un pas dans la bonne direction.

\section{RÉFÉRENCES BIBLIOGRAPHIQUES}

AdAMSON, D. (2010). Community empowerment: Identifying the barriers to "purposeful» citizen participation. International Journal of Sociology and Social Policy, 30 (3/4), 114-126.

Altman, I., \& Rogoff, B. (1987). World view in psychology: Trait, interactional, organismic, and transactional perspectives. In D. Stokols \& I. Altman (dir.), Handbook of Environmental Psycho$\log y, 1$. New York: Wiley \& Sons.

Arendt, H. (2003). Condition de l'homme moderne. Paris: Calmann-Lévy (coll. Agora).

BAIN, S. F. (2012). School counselors: A review of contemporary issues. Research in Higher Education Journal, 18, 1-7. 
Barus-Michel, J., Enriquez, E., \& Lévis, A. (2003). Vocabulaire de psychosociologie: références et positions. Paris: Érès.

Bloch, P., Toft, U., Reinbach, H. C., Clausen, L. T., Mikkelsen, B. E., Poulsen, K., \& Jensen, B. B. (2014). Revitalizing the setting approach - supersettings for sustainable impact in community health promotion. The International Journal of Behavioral Nutrition and Physical Activity, 11 (118), 1-15.

Boudrias, J., Gaudreau, P., \& Laschinger, H. K. (2004). Testing the structure of psychological empowerment: Does gender make a difference? Educational and Psychological Measurement, 64 (5), 861-877.

Castel, R. (2001). Les métamorphoses de la question sociale: une chronique du salariat. Paris: Gallimard.

Castel, R. (2009). La montée des incertitudes. Paris: Seuil.

Cattaneo, L. B., \& Goodman, L. A. (2015). What is empowerment anyway? A model for domestic violence practice, research, and evaluation. Psychology of Violence, 5, 84-94.

Chauvière, M. (2007). Trop de gestion tue le social: enquête sur une discrète chalandisation. Paris: La Découverte.

Christens, B. D., Speer, P. W., \& Peterson, N. A. (2011). Social class as a moderator of the relationship between (dis) empowering processes and psychological empowerment. Journal of Community Psychology, 39 (2), 170-182.

Clarke, E., Puschner, B., Jordan, H., Williams, P., Konrad, J., KaWohl, W., Bär, A., Rössler, W., Del Vecchio, V., Sampogna, G., Nagy, M., Süveges, A., Krogsgaard Bording, M., \& Slade, M. (2015). Empowerment and satisfaction in a multinational study of routine clinical practice. Acta Psychiatrica Scandinavica, 131(5), 369-378.

Collin, J., \& Suissa, A.J. (2007). Les multiples facettes de la médicalisation du social. Nouvelles pratiques sociales, 19 (2), 25-33.

Cometti, E. (2017). Participation de tous: passer des intentions aux actes. Vie sociale, 3 (19), 131-152.

Craig, G. (2002). Towards the measurement of empowerment: The evaluation of community development. Community Development, 33 (1), 124-146. 
Di Fabio, A. D., \& Bernaud, J. L. (2010). Un nouveau paradigme pour la construction de la carrière au $21^{\mathrm{e}}$ siècle: bienvenue! L'Orientation scolaire et professionnelle, 39 (1), 111-118.

Duffy, R. D., Blustein, D. L., Diemer, M. A., \& Autin, K. L. (2016). The Psychology of Working Theory. Journal of Counseling Psychology, 63 (2), 127-148.

ENN, R. (2012). Indigenous empowerment through collective learning. Multicultural Education \& Technology Journal, 6 (3), 149-161.

EsPING-ANDERsEn, G. (2007). Les trois mondes de l'État-providence: essai sur le capitalisme moderne. Paris: PUF.

ÉTIENNE, C. (2017). L'approche centrée sur le développement du pouvoir d'agir dans le management d'équipe. Vie sociale, 19 (3), 165-179.

Evans, S. D., Prilleltensky, O., McKenzie, A., Prilleltensky, I., Nogueras, D., Huggins, C., \& Mescia, N. (2011). Promoting strengths, prevention, empowerment, and community change through organizational development: Lessons for research, theory, and practice. Journal Of Prevention \& Intervention In The Community, 39 (1), 50-64.

Everett, J. E., Homstead, K., \& Drisko, J. (2007). Frontline worker perceptions of the empowerment process in community-based agencies. Social Work, 52 (2), 161-170.

Fetterman, D., \& Wandersman, A. (2007). Empowerment Evaluation: Yesterday, Today, and Tomorrow. American Journal of Evaluation, 28 (2), 179-198.

Franco, L. M., McKay, M., Miranda, A., Chambers, N., PauLino, A., \& LaWrence, R. (2008). Voices from the community: Key ingredients for community collaboration. Social Work in Mental Health, 5 (3/4), 313-331.

Guareschi, P. A., \& Jovchelovitch, S. (2004). Participation, Health and the Development of Community Resources in Southern Brazil. Journal of Health Psychology, 9 (2), 311-322.

Guichard, J. (2008). Proposition d'un schéma d'entretien constructiviste de conseil en orientation (Life Design counseling) pour des adolescents ou de jeunes adultes. L'Orientation scolaire et professionnelle, 37 (3), 413-440. 
Handy, F., \& Kassam, M. (2006). Practice what you preach? The role of rural NGOs in women's empowerment. Journal of Community Practice, 14 (3), 69-91.

Hansen, J. T. (2010). Consequences of the postmodernist vision: Diversity as the guiding value for the counseling profession. Journal of Counseling \& Development, 88 (1), 101-107.

Haswell, M., Kavanagh, D., Tsey, K., Reilly, L., Cadet-James, Y., Laliberte, A., Wilson, A., \& Doran, C. (2010). Psychometric validation of the growth and empowerment measure (GEM) applied with Indigenous Australians. The Australian and New Zealand Journal of Psychiatry, 44 (9), 791-799.

Hur, M. H. (2006). Empowerment in Terms of Theoretical Perspectives Exploring a Typology of the Process and Components across Disciplines. Journal of Community Psychology, 34 (5), 523-540.

Jouffray, C. (2014). Développement du pouvoir d'agir, une nouvelle approche de l'intervention sociale. Rennes, France: Presse de l'École des hautes études en santé publique.

Jouffray, C., \& Vallerie, B. (2009). Le développement du pouvoir d'agir des personnes et des collectivités: une autre approche des pratiques sociales. La Revue française de service social, 232 (1), 46-59.

Le Bossé, Y. (2003). Vous avez dit «empowerment»? De «l'habilitation" au "pouvoir d'agir»: vers une définition plus circonscrite de la notion d'empowerment. Nouvelles pratiques sociales, 16 (2), 30-51.

Le Bossé, Y. (2011). Psychosociologie des sciences de l'orientation: Un point de vue interactionniste et stratégique. Québec: Éditions Ardis.

LeE-Rife, S. M. (2010). Women's empowerment and reproductive experiences over the lifecourse. Social Science and Medecine, 71 (3), 634-642.

LEMAY, L. (2007). L'intervention en soutien à l'empowerment: du discours à la réalité. La question occultée du pouvoir entre acteurs au sein des pratiques d'aide. Nouvelles pratiques sociales, 20 (1), 165180.

Lupton, B. S., Fonnebo, V., Sogand, A. J., \& Fylkesnes, K. (2005). The Finnmark Intervention Study: Do community-based intervention programmes threaten self-rated health and well-being? 
Experiences from Batsfjord, a fishing village in North Norway. European Journal of Public Health, 15 (1), 91-96.

McCubbin, M., Dallaire, B., Lagrange, V., Wallot, H., Bergeron-Leclerc, C., Cormier, C., \& Nelson, G. (2010). Reconstruction et rééquilibrage du lien social: Une étude exploratoire sur les rôles de l'inclusion sociale, de l'appropriation du pouvoir d'agir et de l'espoir dans le rétablissement [Rebuilding and rebalancing of the social link: An exploratory study on the roles of social inclusion, empowerment, and hope in rehabilitation]. Canadian Journal of Community Mental Health, 29 (1), 1-18.

McLaughlin, K. (2010a). Control and Social Work: A reflection on some twenty-first century developments. Practice, 22 (3), 143154.

McLaughlin, K. (2010b). Psychologisation and the Construction of the Political Subject as Vulnerable Object. Annual Review of Critical Psychology, 8, 63-79.

Miley, K., \& DuBois, B. (2007). Ethical Preferences for the Clinical Practice of Empowerment Social Work. Social Work in Health Care, 44 (1/2), 29-44.

Mohajer, N., \& EARnest, J. (2009). Youth empowerment for the most vulnerable: a model base on the pedagogy of Freire and experiences in the Field. Health education, 109 (5), 424-438.

Morin, E. (1990). Introduction à la pensée complexe. Paris: ESF Éditeur.

Nardone, G., \& WatzlaWick, P. (2002). L'art du changement: thérapie stratégique et hypnothérapie sans transe. Bordeaux: L'Esprit du temps (coll. Psychologie).

Ninacs, W. A. (2008). Empowerment et intervention: développement de la capacité d'agir et de la solidarité. Québec: Presses de l'Université Laval.

Nussbaum, M. (2011). Creating Capabilities: The Human Development Approach. Cambridge (MA): The Belknap Press of Harvard University Press.

Organisation mondiale de la santé (2005). Commissions des déterminants sociaux de la santé. En ligne: [http://www.who.int/social_ determinants/strategy/QandAs/fr/index.html]. 
Pantazidou, M. (2012). What Next For Power Review Of Recent Experience With The Powercube And Related Frameworks. IDS Working Paper, (400), 1-46.

Parazelli, M., \& Bourbonnais, M. (2017). L'empowerment en travail social: perspectives, enseignements et limites. Sciences et actions sociales, (6).

Partridge, W. L. (2008). Praxis and Power. Journal of Community Psychology, 36 (2) 161-172.

PÉPIN, Y. (2018). Intervention psychosociale: perspective interactionniste stratégique. Coll. Pratiques d'accompagnement professionnel. Québec: Presses de l'Université Laval.

Petit, C. (2016a). La contribution de «l'empowerment» ou approche centrée sur le développement du pouvoir d'agir aux pratiques d'ergothérapie pour accompagner le changement et mobiliser les capacités. In M. H. Izard (dir.). Expériences en ergothérapie. $29^{e}$ série (pp. 356365). Montpellier: Sauramps Medical.

PetrT, C. (2016b). Le développement du pouvoir d'agir (empowerment) en ergothérapie; une pratique innovante avec les personnes vulnérables en santé. Ergothérapies, (63), 5-12.

PorTal, B. (2016). De l'empowerment anglo-saxon au développement du pouvoir d'agir européen. Le Sociographe, 55 (3), 83-97.

Portal, B., Desomer, V., \&Dutrieux, B. (2017). Changer le monde au quotidien. L'approche DPA-PC: récits d'expériences, analyses et regards critiques. Namur: Éditions UVCW/Fédération des CPAS.

Prilleltensky, I., \& Prilleltensky, O. (2003). Synergies for wellness and liberation in counseling psychology. The Counseling Psychologist, 31 (3), 273-281.

RAPPAPORT, J. (1981). In praise of paradox: A social policy of empowerment over prevention. American Journal of Community Psychology, 9 (1), 1-25.

Riceur, P. (1990). Soi-même comme un autre. Paris: Seuil.

Rorty, R. (2008). Objectivity, relativism and truth. Philosophical papers (6 édition), vol 1. Cambridge: Cambridge University Press.

Savickas, M. L., Nota, L., Rossier, J., Dauwalder, J. P., Duarte, M. E., Guichard, J., Soresi, S., Van Esbroeck, R., Van Vianen, A., \& 
Bigeon, C. (2010). Construire sa vie (life designing): Un paradigme pour l'orientation au $21^{\mathrm{e}}$ siècle. L'Orientation scolaire et professionnelle, 39 (1), 5-39.

SAVICKAS, M. L. (2012). Life design: A paradigm for career intervention in the 21st century. Journal of Counseling \& Development, 90 (1), 13-19.

SEN, A. (2009). Lidée de justice. Paris: Flammarion.

SheArer, R. A., \& King, P. A. (2001). The Powerless-Empowerment Scale: Measurement of Substance Abuse Counseling Orientation. Journal of addiction and offenders counseling, 21 (2), 68-75.

SonG, L. (2015). The Association between the Utilisation of Empowerment Strategies and Clients'Changes of Self in the Field of Intimate Partner Abuse: From the Perspective of Social Workers. British Journal of Social Work, 45 (2), 527-548.

Southern, S., Gomez, J., Smith, R. L., \& Devlin, J. M. (2010). The transformation of community counseling for 2015 and beyond. En ligne: [http://counselingoutfitters.com/vistas/vistas10/Article75.pdf].

Tengland, P.-A. (2007). Empowerment: A Goal or a Mean. The Health Promotion? Medecine Health Care and Philosophy, 10 (2). 197-207.

Thomas, H. (1997). La production des exclus. Politiques sociales et processus de désocialisation sociopolitique. Paris: PUF.

Van Hal, L. B. E., Meershoek, A., Nijhuis, F., \& Horstman, K. (2012). The "empowered client" in vocational rehabilitation: The excluding impact of inclusive strategies. Health Care Analysis, 20 (3), 213-230.

VALlerie, B. (2012). Interventions sociales et empowerment (Développement du pouvoir d'agir). Coll. Savoirs et formation. Paris: L'Harmattan.

Varela, F., Thompson, E., \& Rosch, E. (1993). Linscription corporelle de l'esprit. Paris: Seuil.

Windsor, L. C., Jemal, A., \& Benoît, E. (2014). Community Wise: Paving the way for empowerment in community reentry. International Journal of Law and Psychiatry, 37 (5), 501-511. 



\title{
11. CHOIX PROFESSIONNEL, INDÉCISION ET ÉMOTIONS
}

\author{
SHÉKINA ROCHAT (OFFICE CANTONAL D'ORIENTATION SCOLAIRE \\ ET PROFESSIONNELLE, LAUSANNE) \\ ET ÉLOÏSE BANET (UNIVERSITÉ DE LAUSANNE)
}

\section{uelle profession souhaitez-vous exercer?» L'explora- $\ll Q$ tion des différentes options possibles nécessaire pour répondre à cette question va très certainement susciter} en vous des réactions émotionnelles très variées, telles que la joie, l'excitation, l'ennui, la crainte ou le dégoût. C'est du moins ce que suggèrent les travaux empiriques qui se sont penchés sur le type d'émotions suscitées par l'exploration des options professionnelles (Puffer, 2015; Young, Pasluikho, \& Valach, 1997). Néanmoins, pendant de longues années, les théoricien-ne.s de l'orientation ont principalement proposé des modèles «rationnels» du choix professionnel, faisant l'impasse sur le rôle des émotions dans ce processus (par exemple, Gati et al., 1996; Sampson, Lenz, Reardon, \& Peterson, 1999). Un manque particulièrement préjudiciable au vu du nombre croissant de travaux qui tendent à souligner le caractère «irrationnel» des décideurs (par exemple, Kahneman \& Tversky, 1979), ainsi que le rôle central joué par les émotions dans le processus de choix (par exemple, Damasio, 1994). Ces dernières années ont ainsi conduit à des appels répétés à prendre en compte les émotions dans les modèles du choix professionnel et dans les interventions visant à favoriser le développement de carrière (par exemple, Barak, 2001; Di Fabio, Palazzeschi, Asulin-Peretz, \& Gati, 2013; Emmerling \& Cherniss, 2003; Kidd, 1998; Murtagh, Lopes, \& Lyons, 2012; Puffer, 2011, 2015; Saka \& Gati, 2007; Saka, Gati, \& Kelly, 2008; Young, Paseluikho, \& Valach, 1997).

En effet, dans un climat ambiant de plus en plus complexe, instable et imprévisible, être à l'écoute de ses émotions va devenir une compétence essentielle pour la gestion efficace des trajectoires professionnelles (Emmerling \& Cherniss, 2003). Ainsi, les psychologues conseillers.ères en orientation vont de plus en plus devoir 
être en mesure d'aider leurs consultant.e.s à prendre conscience de leur vécu émotionnel et à y donner un sens au vu des alternatives considérées (Puffer, 2015). Développer ce savoir-faire professionnel nécessite toutefois d'accroître au préalable notre compréhension quant au rôle et au fonctionnement des émotions dans le choix professionnel (Kidd, 2004). À cette fin, il nous semble essentiel de partir d'une définition claire des émotions, qui permettra de faire le point sur l'état actuel de la recherche en orientation à ce sujet, ainsi que les pistes d'intervention possibles. Toutefois, cette tâche n'est pas aisée, car les questions quant à la nature, au fonctionnement et au développement des émotions fait débat de longue date parmi les scientifiques (par exemple, D. H. Ford, 1987) et le nombre de modèles théoriques proposés est intimidant.

Dans le cadre de sa théorie du living system framework (LSF), qui rend compte de l'ensemble des processus et fonctions qui régissent les comportements (en interaction) et le développement de l'être humain, D. H. Ford (1987) propose une définition des émotions qui synthétise les travaux de ses prédécesseurs. Récemment, Vondracek, Ford et Porfeli (2015) ont adapté cette théorie dans leur tentative plus vaste de rendre compte des comportements vocationnels et des trajectoires professionnelles. Ce chapitre adopte cette définition des émotions de manière à clarifier les implications potentielles de celles-ci sur le processus de choix professionnel. Cela ouvrira à des propositions d'intervention de conseil en orientation permettant de travailler sur et avec les émotions pour faciliter les choix professionnels.

\section{VERS UNE DÉFINITION DES ÉMOTIONS}

Intuitivement, le terme "émotions " peut évoquer chez tout un chacun l'idée de réactions instinctives associées à des contenus identifiables (joie, tristesse, colère, peur, dégoût...) pouvant survenir dans des situations variées et à différents niveaux (physiologique, subjectif, expressif, cognitif et comportemental) (par exemple, Nugier, 2009). Les émotions font d'ailleurs partie intégrante de la vie des êtres humains. Selon une étude récente, les individus tendent à ressentir des émotions $90 \%$ du temps de leur vie éveillée, et les émotions positives seraient 2,5 fois plus fréquentes que les émotions négatives (Trampe, Quoidbach, \& Taquet, 2015). Face à la difficulté de définir les émotions, D. H. Ford (1987) tente 
de proposer une synthèse cohérente de l'ensemble des nombreux travaux des chercheurs qui se sont intéressés à cette notion (en particulier Ekman, 1984; Izard, 1977; Plutchik, 1980). Selon lui, il y a deux types d'affects: (1) ceux qui donnent des informations sur les perceptions sensorielles, et (2) ceux qui reflètent différents états émotionnels. L'individu naîtrait ainsi avec un set d'affects directement lié à ses perceptions sensorielles et qui va servir de point de départ pour la construction progressive d'un répertoire émotionnel, sur la base des situations rencontrées, des mécanismes d'apprentissage de l'individu et de la combinaison d'émotions entre elles (par exemple, la présence simultanée de joie et de tristesse peut être identifiée comme de la mélancolie).

Ce répertoire émotionnel va alors servir à réguler l'ensemble des comportements de l'individu, et ce, de trois manières: (1) en offrant à l'individu des informations évaluatives immédiates sur des situations actuelles ou anticipées; (2) en ajustant le fonctionnement biologique de l'individu pour le préparer à exécuter les actions requises; et (3) en produisant des comportements d'action (par exemple, approche ou évitement) et de communication (par exemple, les expressions faciales) appropriés pour gérer la situation en question. Ce faisant, D. H. Ford (1987) propose de définir les émotions comme des réponses complexes de préparation à l'action et de régulation des comportements qui vont faciliter l'adaptation efficace de l'individu à son environnement. En particulier, cet auteur avance que certaines émotions (telles que la curiosité ou la peur) vont servir à réguler l'ensemble des transactions de l'individu avec son contexte alors que d'autres émotions (telles que l'affection ou la jalousie) ont évolué pour réguler spécifiquement les interactions sociales. Dans les premiers temps de la vie, ce sont les cinq sens qui vont constituer la principale source d'affects, alors que, chez l'adulte, les émotions vont principalement être suscitées par les pensées. À leur tour, en raison de leurs implications dans le processus de traitement et de l'encodage subséquent de l'information, les émotions vont également avoir une influence sur le contenu des pensées.

En précisant la nature, le contenu et les fonctions des émotions dans le comportement et le développement humain, le cadre théorique de D. H. Ford permet d'identifier trois grands axes selon lesquels les émotions sont susceptibles d'influencer les comportements et le développement vocationnel: (1) le traitement et 
l'encodage des informations; (2) la préparation du corps à l'action, notamment via l'ajustement du champ attentionnel; et (3) la production d'une réponse comportementale. En effet, ainsi que l'ont exposé Lerner, Li, Valdesolo et Kassam (2015), non seulement les émotions influencent les processus de décision, mais elles sont omniprésentes dans ce processus et y interviennent de multiples manières. Ces trois grands axes vont alors à présent nous permettre de synthétiser le corpus de la littérature existant quant à l'impact des émotions sur le processus de choix professionnel.

\section{RÔLE DES ÉMOTIONS DANS LE PROCESSUS DE CHOIX PROFESSIONNEL}

\subsection{TRAITEMENT ET ENCODAGE DES INFORMATIONS}

Dans l'approche du living system framework, D. H. Ford (1987) propose de considérer les trajectoires développementales comme des successions de séquences de comportements au sein desquelles la fonction première des émotions est de produire une évaluation de la situation actuelle (ou anticipée). Ce faisant, les émotions vont renseigner l'individu sur les conséquences désirables ou indésirables des comportements adoptés, et ainsi contribuer au processus d'apprentissage en connotant l'expérience vécue. Les émotions joueraient alors un rôle clé dans la motivation à s'engager dans des objectifs, en indiquant à l'individu quels sont les buts qui valent la peine d'être poursuivis (par exemple, M. E. Ford, 1992). Ces considérations sont cohérentes avec les propositions de la théorie des marqueurs somatiques (Bechara, Damasio, Damasio, \& Lee, 1999; Bechara, Tranel, \& Damasio, 2000; Damasio, 1994) qui postulent que les émotions positives et négatives associées à nos expériences passées sont encodées dans la mémoire et sont réactivées lors des processus de choix pour évaluer de manière rapide et efficace la désirabilité des options considérées. De la sorte, les émotions seraient directement impliquées dans le processus de raisonnement pour que celui-ci ne s'applique qu'aux options potentiellement prometteuses.

Emmerling et Cherniss (2003) suggèrent alors qu'il est probable que les individus fassent appel à des souvenirs émotionnels pour évaluer leurs intérêts et valeurs professionnelles, par exemple lorsqu'ils répondent à des questionnaires ou aux questions du ou de 
la psychologue conseiller.ère en orientation. Cette proposition est appuyée par les résultats de l'étude de Murtagh, Lopes et Lyons (2012), qui tendent à indiquer que les individus se servent de leurs émotions positives comme d'une "boussole» pour guider leur choix au fur et à mesure que de nouvelles options se présentent à eux. Par ailleurs, dans le domaine de l'orientation, plusieurs auteurs (par exemple, Barak, 2001; Krumboltz, 1996; Lent, Brown, \& Hackett, 1994; Vondracek et al., 2015) ont proposé de considérer les trajectoires professionnelles comme des successions de multiples "expériences d'apprentissage» au sujet de contenus professionnels. Cependant, jusqu'alors, le rôle des émotions dans ces expériences d'apprentissage a été relativement négligé ou sous-estimé par ces théories (Meijers, 2002). Le modèle cognitif des intérêts de Barak (2001) fait toutefois exception. Contrairement à ce que le nom du modèle pourrait laisser présager, ce dernier propose en effet de considérer les intérêts professionnels comme des émotions qui refléteraient le degré d'attraction ou d'aversion qu'éprouve un individu à l'égard de différentes activités professionnelles. En cela, il est intéressant de constater que la proposition de Barak (2001) s'accorde avec la taxonomie de D. H. Ford (1987), qui présente l' «intérêt» comme une émotion de même nature que la curiosité ou l'excitation, ayant pour fonction de faciliter les comportements d'approche et d'encourager la récolte d'informations sur soi, sur l'environnement et sur le lien qui existe entre les deux.

Selon Barak, les émotions survenant aux cours d'expériences d'apprentissage liées à des contenus professionnels seraient suscitées par les évaluations que l'individu effectuerait au sujet de (1) ses aptitudes, (2) ses chances de succès et (3) la satisfaction escomptée à la suite de l'engagement dans l'activité. Cependant, Barak souligne que de telles informations sont souvent biaisées, y compris par les émotions elles-mêmes. En effet, ainsi que le relève D. H. Ford (1992), dans un processus d'interaction réciproque, les émotions ont également la capacité d'altérer les cognitions. Il apparaît cependant que, dans le cadre des comportements et du développement vocationnels, l'influence de ces émotions differe selon que leur valence est positive ou négative.

Impact des émotions négatives. L'interférence entre émotions et pensées dans le cadre du développement et des comportements vocationnels peut notamment être illustrée par l'impact bien documenté de l'anxiété sur l'indécision (Emmerling \& Cherniss, 
2003). En effet, de nombreuses études ont démontré l'existence d'un lien étroit entre l'anxiété (trait et état) et l'indécision vocationnelle (Braunstein-Bercovitz, Benjamin, Asor, \& Lev, 2012; Campagna \& Curtis, 2007; Fuqua, Seaworth, \& Newman, 1988; Fuqua, Seaworth, \& Newman, 1987; O’Hare \& Tamburri, 1986; Vignoli, 2015). L'anxiété est ainsi souvent considérée comme l'une des sources clés de l'indécision vocationnelle (Brown et al., 2012; Brown \& Rector, 2008; Dickinson \& Tokar, 2004; Germeijs, Verschueren, \& Soenens, 2006; Hacker, Carr, Abrams, \& Brown, 2013), voire l'une des raisons principales des difficultés chroniques à prendre des décisions (par exemple, Saka \& Gati, 2007; Saka et al., 2008) et à former une identité vocationnelle (Santos, 2001). Néanmoins, il est intéressant de constater que les individus ne sont pas nécessairement conscients de l'impact de l'anxiété sur leurs capacités à prendre des décisions d'ordre vocationnel (Campagna \& Curtis, 2007). De plus, il existe vraisemblablement une influence réciproque entre l'indécision et l'anxiété (Newman, Fuqua, \& Seaworth, 1989).

Certaines recherches ont également mis en évidence le lien positif entre les difficultés au choix et d'autres émotions négatives, telles que les symptômes dépressifs (Saunders, Peterson, Sampson, \& Reardon, 2000; Sweeney \& Schill, 1998; Walker \& Peterson, 2012), le stress perçu (Meldahl \& Munchinsky, 1997), la peur de l'engagement (Leong \& Chervinko, 1996) et, plus récemment, la "détresse de carrière» (Creed \& Hennessy, 2016; Creed, Hood, Praskova, \& Makransky, 2016), qui représente une gamme de différents sentiments négatifs tels que «l'impuissance, la dépression, le stress, le manque de but, l'anxiété, le blâme et le désespoir» (Creed et al., 2016, p. 733, traduction des auteures). De manière similaire, certains auteurs (Betz, Hammond, \& Multon, 2005; Multon, Heppner, \& Lapan, 1995) rapportent une association négative entre les affects négatifs (se sentir agité.e, apeuré·e, bouleversé·e, coupable, effrayé.e, honteux·se, hostile, irritable, nerveux·se ou perturbé.e) ressentis au cours de la semaine précédente et mesurés par la Positive and Negative Affect Schedule (PANAS; Watson, Clark, \& Tellegen, 1988) et le sentiment d'efficacité à effectuer des choix professionnels. L'ensemble de ces travaux tendant donc à indiquer qu'éprouver des émotions négatives est susceptible de perturber les processus cognitifs permettant de prendre une décision quant à son avenir professionnel. 
Impact des émotions positives. À l'inverse, il semblerait que les émotions positives aient un effet diamétralement opposé sur le processus de choix, en le favorisant au lieu de l'entraver. En effet, les rares travaux qui ont étudié les corrélats entre les affects positifs mesurés par la PANAS (se sentir actif·ve, attentif.ve, déterminé.e, enthousiaste, excité.e, fier.ère, fort·e, inspiré·e, intéressé·e ou vigilant·e) et l'indécision (Meldahl \& Munchinsky, 1997; Multon, Heppner, \& Lapan, 1995) rapportent, en général, une association négative entre ces construits. De manière similaire, il convient de noter que la "curiosité» est une composante à part entière de l'adaptabilité de carrière (Savickas \& Porfeli, 2012). À ce titre, cette émotion a été associée positivement et de manière récurrente à une attitude affirmée quant à son avenir professionnel (par exemple, Ginevra, Pallini, Vecchio, Nota, \& Soresi, 2016; Hirschi, Hermann, \& Keller, 2015; Urbaniviciute, Kairys, Pociute, \& Liniauskaite, 2014) ou encore au sentiment d'efficacité au choix professionnel (Duffy et al., 2015; Douglass \& Duffy, 2015), et associée de manière négative aux difficultés à faire un choix professionnel (par exemple, Neureiter \& Traut-Mattaush, 2017; Hirschi \& Valero, 2015; Hirschi, Hermann, \& Keller, 2015). De façon plus générale, il existe une relation positive entre les affects positifs et le sentiment d'efficacité au choix (Betz, Hammond, \& Multon, 2005; Larson \& Majors, 1998). Multon et al. (1995) rapportent également en lien positif entre les affects positifs évalués par la PANAS et l'agentivité manifestée dans le contexte du choix professionnel.

Ces différents résultats tendent à mettre en relief le rôle central que les émotions positives sont susceptibles de jouer pour faciliter le processus de choix professionnel. Les travaux d'Isen (2001) soulignent d'ailleurs l'impact bénéfique des émotions positives sur la résolution de problème et la prise de décision. Les émotions sont donc capables d'altérer, de biaiser ou de faciliter la prise de décision vocationnelle.

\subsection{AJUSTEMENT DU CHAMP ATTENTIONNEL}

Dans le modèle de D. H. Ford (1987), une fois qu'une réaction émotionnelle est produite en réponse à l'évaluation de la situation par le système nerveux central, les émotions vont ajuster le fonctionnement du corps (via les informations transmises au corps par le système nerveux autonome et le système endocrinien) pour produire les comportements d'approche ou d'évitement susceptibles 
de produire des conséquences désirées. Parmi ces modifications physiologiques, l'une consiste notamment en une modification du champ de l'attention (par exemple, Damasio, 1994). Ici encore, les émotions négatives et positives vont avoir un impact différent sur ce processus. Ainsi, il apparaît que le ressenti d'émotions négatives aura pour effet de rétrécir le champ de l'attention, de manière à se fixer sur l'élément qui a induit l'état en question (par exemple, Beck, 1979). À l'inverse, les émotions positives vont élargir le champ de l'attention de l'individu, ce qui va lui permettre de prendre en compte un set plus grand d'alternatives dans une situation de choix (Fredrickson, 2001; Fredrickson \& Branigan, 2005). Ces réactions physiologiques pourraient alors influencer le nombre et le type d'options considérées par un individu au moment du choix professionnel (Emmerling \& Cherniss, 2003; Vignoli, 2015).

Ainsi, le vécu d'émotions négatives pourrait conduire l'individu soit à se focaliser sur les informations réellement pertinentes pour son choix professionnel, soit à se concentrer de manière excessive sur les informations menaçantes (Vignoli, 2015). Au vu de ce qui précède, nous pourrions émettre l'hypothèse qu'un tel rétrécissement de l'attention pourrait également conduire l'individu à prendre en considération un set plus limité d'alternatives professionnelles. À l'inverse, il nous semble probable que, en élargissant le champ d'attention de l'individu, le vécu d'émotions positives pourrait accroître le répertoire des options considérées. Ceci pourrait permettre d'effectuer un choix dans une gamme d'options plus large, mais pourrait également contribuer à complexifier le processus de prise de décision en raison du plus grand nombre de possibilités considérées.

\subsection{PRODUCTION D'UNE RÉPONSE COMPORTEMENTALE}

L'aboutissement des processus d'évaluation de la situation et de préparation du corps à l'action est la production d'une réponse comportementale susceptible d'engendrer des conséquences désirées par l'individu. Ici encore, le ressenti d'émotions positives ou négatives est susceptible d'avoir un impact différent sur ces comportements, les premières stimuleraient l'exploration et la recherche d'informations tandis que les secondes auraient pour effet de mettre fin aux activités entreprises (par exemple, D. H. Ford, 1987). Selon les auteurs des théories traditionnelles de l'apprentissage dans le domaine de l'orientation (par exemple, 
Krumboltz, 1996; Lent, Brown, \& Hackett, 1994), le vécu d'émotions négatives va conduire l'individu à mettre en place des stratégies d'évitement des situations susceptibles d'activer à nouveau des émotions négatives, par exemple en remettant à plus tard le moment de faire un choix. Cependant, les recherches empiriques menées sur le lien entre l'anxiété et l'exploration professionnelle suggèrent que les réactions comportementales suscitées par les émotions négatives sont complexes. Ainsi, certaines études montrent effectivement que les individus anxieux sont moins susceptibles de s'engager dans des activités d'exploration de carrière que ceux qui ne présentent pas d'anxiété (Blustein \& Philips, 1988; Park, 2017; Vignoli, Croity-Belz, Chapeland, de Fillipis, \& Garcia, 2005), tandis que d'autres études attestent qu'être anxieux peut susciter des conduites d'exploration vocationnelle (Vignoli, 2015; Park et al., 2017) qui pourraient viser à alléger l'anxiété suscitée par la perspective du choix. Selon Park et al. (2017), si l'anxiété est initialement associée à une faible exploration vocationnelle, son rôle est susceptible de varier au fil du temps et de favoriser par la suite l'exploration de carrière et, au final, la prise de décision.

En ce qui concerne le rôle des émotions positives sur l'exploration vocationnelle, force est de constater que, jusqu'à présent, aucune recherche n'a été entreprise sur ce sujet. Toutefois, les travaux de Fredrickson (1998) et d'Isen (2001) suggèrent que les émotions positives stimuleraient l'engagement dans des conduites d'exploration. Néanmoins, il semblerait que les individus éprouvant des émotions positives soient moins prompts à prendre des risques que ceux vivant des affects neutres ou négatifs (Isen \& Geva, 1987; Isen, Nygren, \& Ashby, 1988; Isen \& Patrick, 1983). Sur cette base, il nous semble possible d'émettre l'hypothèse que le vécu d'émotions positives pendant le processus de choix serait susceptible de conduire à davantage de comportements d'exploration de carrière, mais à des choix plus traditionnels que les individus présentant des affects négatifs ou neutres.

\section{PERSPECTIVES PRATIQUES ET OUTILS NOVATEURS}

Emotions provide the fulcrum for revising the self during counseling. Before meaning may be reorganised and action engaged, feelings must change.

(Savickas, 2011, p. 53) 


\subsection{SUSCITER LES ÉMOTIONS POSITIVES}

Dans l'ensemble, le travail de synthèse effectué ci-dessus tend à mettre en évidence la centralité des émotions dans le processus de choix professionnel, et donc l'importance de les prendre en compte dans les démarches d'orientation. En particulier, il convient de relever le rôle considérable des émotions positives pour faciliter le choix professionnel. En ce qui concerne la pratique du conseil en orientation, ces données encouragent à stimuler le vécu d'affects positifs lors du processus de choix vocationnel et des transitions susceptibles de jalonner les parcours professionnels. En effet, les trajectoires professionnelles contemporaines non linéaires sont susceptibles de susciter de nombreuses émotions, et notamment des émotions négatives (Meijers, 2002). Cependant, il apparaît compliqué, voire contre-productif, de tenter de supprimer ou de réduire ces dernières (Wenzlaff \& Wegner, 2000), qui peuvent par ailleurs se révéler une source d'apprentissage précieuse pour les individus (Meijers, 2002). Ainsi, plutôt que de tenter de diminuer l'occurrence des émotions négatives, il serait plus judicieux de chercher à augmenter le nombre d'émotions positives vécues quotidiennement (par exemple, Fredrickson, 2001). En effet, hormis leur impact sur le processus de choix, le fait d'augmenter le nombre d'émotions positives éprouvées quotidiennement contribuerait au bien-être des individus à long terme (par exemple, Fredrickson, 2001). À cette fin, les différents travaux réalisés dans le domaine émergeant de la psychologie positive sont susceptibles d'ouvrir des pistes d'intervention intéressantes.

\subsection{UTILISER DES APPROCHES INNOVANTES}

Rochat et Bellier-Teichman (2017) proposent ainsi que, lors des séances, les psychologues conseillers.ères en orientation encouragent leurs consultant.e.s à identifier les ressources personnelles, sociales et de loisirs qui sont susceptibles de leur faire du bien dans les moments de transitions professionnelles, par exemple au moyen d'outils ludiques tels que le jeu de cartes AERES (Bellier-Teichman \& Pomini, 2015). En effet, de nombreux travaux réalisés dans le domaine de la psychologie positive mettent en évidence l'impact positif de l'identification et de l'utilisation des ressources sur le bienêtre des individus (par exemple, Wood, Linley, Maltby, Kashdan, \& Hurling, 2011). De plus, une étude récente de Dambrun et Dubuy (2013) montre que le fait de cultiver des ressources personnelles et sociales via des exercices issus du courant de la psychologie positive 
(telles que l'utilisation de ses forces, la réalisation d'actions altruistes et le fait de noter quotidiennement ses sujets de gratitude) chez des individus en recherche d'emploi contribuait significativement à diminuer leurs symptômes dépressifs et anxieux, ainsi qu'à augmenter leur bien-être psychologique et leur estime d'eux-mêmes. L'identification et l'utilisation des ressources sont ainsi susceptibles de conduire à amplifier le vécu d'émotions positives et à diminuer le vécu d'émotions négatives, et ce notamment lors des transitions professionnelles.

Il a également été démontré que l'humour constitue un moyen efficace pour induire des humeurs et des émotions positives chez les individus (par exemple, Isen \& Gorgoglione, 1983; Danzer, Dale, \& Klions, 1990; Martin \& Labott, 1991; pour une revue voir Deckers, 1998). En ce sens, l'humour pourrait constituer une approche prometteuse pour augmenter le vécu d'émotions positives au quotidien et au sein même de la relation de conseil. Selon Apter (2014), il pourrait constituer un cadre protecteur - à l'image du ring pour la boxe - qui permettrait de donner une tonalité plaisante aux activations émotionnelles dépassant une certaine intensité, même lorsque ces dernières auraient pu être vécues comme déplaisantes dans un autre cadre (voir aussi Svebak \& Apter, 1987). Par exemple, l'anxiété ressentie par un individu présente les mêmes caractéristiques physiologiques que l'excitation positive; elle peut donc être recadrée de manière plus plaisante comme le fait de se réjouir (par exemple, Brooks, 2014). Ceci pourrait avoir des implications intéressantes dans le domaine du conseil en orientation, notamment pour accompagner les choix vocationnels sous l'angle des émotions positives. De plus, l'humour permet de réduire ou de réguler les humeurs et les émotions négatives, notamment parce qu'il agit à court terme comme un distracteur cognitif (Strick, Holland, van Baaren, \& van Knippenberg, 2009). En ce sens, il peut être utilisé comme stratégie consciente de régulations de l'anxiété (Thayer, Newman, \& McClain, 1994). En modulant la perception (plus ou moins menaçante) d'un événement stressant (Kuiper, Martin, \& Olinger, 1993) et en permettant de l'aborder sous différents points de vue (Kuiper, Mackenzie, \& Belanger, 1995), l'humour favoriserait ainsi le recadrage des situations et l'adaptation du comportement de l'individu.

En outre, étant donné qu'il contribue à une meilleure estime de soi (Martin, Kuiper, Olinger, \& Dance, 1993), l'humour pourrait constituer une ressource pour la personne en situation de 
transition ou de questionnement vocationnel, susceptible de générer un stress important (Meijers, 2002). Nevo (1986) avait d'ailleurs déjà proposé d'y avoir recours pour remettre en question les croyances dysfonctionnelles des consultant.e.s à l'égard du choix professionnel. Dans une étude récente, Banet et Masdonati (2017) ont du reste montré que les interventions humoristiques spontanées étaient présentes à toutes les étapes du processus d'orientation et que les psychologues conseillers ères en orientation qui y recouraient avaient tendance à le faire pour soutenir le déroulement de ce processus. L'humour pourrait donc être bénéfique pour la relation de conseil et la construction du projet vocationnel. D'ailleurs, comme l'humour est lié aux compétences sociales (Yip \& Martin, 2006), à la manière de gérer les conflits (Bippus, 2003), et qu'il permet d'augmenter l'attention et la sympathie pour une source (par exemple, Megdell, 1984; Weinberger \& Gulas, 1992), il serait particulièrement indiqué en tant qu'approche favorisant la qualité relationnelle. C'est peut-être pourquoi les conseillers.ères en orientation semblent y avoir particulièrement recours pour établir et maintenir un lien positif avec leurs consultant.e.s (Banet \& Masdonati, 2017).

Enfin, les effets bénéfiques de l'humour sont susceptibles de dépasser le cadre de la relation de conseil et du processus de choix professionnel. Il a pu être associé négativement au burnout (Van den Broeck, Vander Elst, Dikkers, De Lange, \& De Witte, 2012) et positivement à la performance au travail (Lehmann-Willenbrock \& Allen, 2014), ainsi qu'au fonctionnement professionnel de manière générale (voir Mesmer-Magnus, Glew, \& Viswesvaran, 2012, pour une méta-analyse à ce sujet). L'humour a aussi été mis en lien avec certains apprentissages informels dans le cadre du travail (Tews, Michel, \& Noe, 2017) et, plus généralement, avec le bien-être (par exemple, Kuiper, Martin, \& Dance, 1992; Lefcourt \& Thomas, 1998). Au vu de ce qui précède, il nous semble donc prometteur de réfléchir à la conception d'outils d'intervention utilisant l'humour dans le domaine de l'orientation.

\subsection{ENCOURAGER L'ÉQUILIBRE ÉMOTIONNEL}

Bien qu'il apparaisse essentiel de chercher à favoriser les émotions positives dans le processus de conseil en orientation et au cours des transitions professionnelles, il est nécessaire de rester attentif à ne pas nier pour autant le vécu émotionnel négatif des consultant·e.s. 
En effet, si les interventions présentées précédemment visaient essentiellement à stimuler le vécu d'émotions positives chez les individus, il s'agit toutefois de souligner, à l'instar de Meijers (2002), qu'il n'est pas toujours possible, ni même souhaitable, d'éviter ou de pallier l'apparition d'émotions négatives. Selon cet auteur, les affects négatifs peuvent constituer des opportunités essentielles d'apprentissage, et non pas uniquement des obstacles au processus de choix. Ainsi, selon lui, lorsqu'un individu est sous l'emprise d'un vécu émotionnel négatif, il est important que les psychologues conseillers.ères en orientation y prêtent attention et aident les personnes à lui donner du sens. Ceci implique, pour les psychologues de l'orientation, de faire preuve d'une certaine sensibilité dans le choix de l'approche employée (susciter des émotions positives versus accueillir les émotions négatives) en fonction de ce qui est exprimé par le ou la consultant.e ainsi que de la qualité de l'alliance établie. Si certains auteurs ont mis en évidence l'impact des émotions positives sur le bien-être (Fredrickson, 2001), d'autres se sont plus récemment intéressés à la richesse émotionnelle. Ainsi, à travers son conceptual act model, Barret (2009) souligne la variabilité interindividuelle et interculturelle des émotions, ainsi que leurs liens avec des phénomènes psychologiques distincts comme la cognition. Quoidbach, Gruber, Mikolajzak, Kogan, Kotsou et Norton (2014) montrent, quant à eux, les bienfaits d'éprouver une grande diversité émotionnelle pour la santé physique et mentale. Ces auteurs abordent les émotions humaines comme un écosystème dans lequel il est fondamental de trouver de la variété et un certain équilibre entre les différentes émotions expérimentées. Cette "émodiversité» (Quoidbach et al., 2014, p. 2057) pourrait refléter une plus grande attention envers ses propres ressentis et favoriserait l'adaptation à des contextes variés. Ceci peut être rapproché de l'idée de "psychodiversité» (Apter, 2013, p. 4) reflétant la richesse des états motivationnels d'un individu - richesse qui permettrait de s'adapter plus aisément à un environnement en constante évolution. En effet, le changement constant étant une caractéristique du monde du travail actuel (par exemple, Bauman, 2000), l'adaptation (par exemple, Savickas, 1997) et la mobilité (par exemple Arthur \& Rousseau, 1996) constituent des préoccupations centrales, tant pour les individus s'attachant à construire leur trajectoire vocationnelle que pour les professionnel.le.s qui les accompagnent. Enfin, pour Quoidbach et al. (2014), l'équilibre 
des émotions préviendrait le risque de domination de l'écosystème par une seule émotion, potentiellement destructrice. Ces idées viennent soutenir l'importance de travailler avec les émotions pour accompagner au mieux le processus de choix professionnel, en particulier en cherchant à développer l'intelligence émotionnelle.

\subsection{FAVORISER LE DÉVELOPPEMENT}

\section{DE L'INTELLIGENCE ÉMOTIONNELLE}

Pour plusieurs auteurs (Di Fabio \& Kenny, 2010; Di Fabio, Palazzeschi, Asulin-Peretz, \& Gati, 2013; Emmerling \& Cherniss, 2003 ; Poon, 2004; Puffer, 2011, 2015; Udayar, Fiori, Thalmayer, \& Rossier, en préparation) l' "intelligence émotionnelle» - en tant que capacité à (1) percevoir, (2) intégrer, (3) comprendre, et (4) gérer les émotions (Mayer \& Salovey, 1997) - constitue ainsi une compétence clé pour la réussite des trajectoires professionnelles et du processus de choix. Ainsi, les individus capables d'identifier et de gérer efficacement leurs émotions seraient mieux préparés à affronter les nombreux défis inhérents au choix de carrière dans le monde incertain et imprévisible du XXI ${ }^{\mathrm{e}}$ siècle (Emmerling \& Cherniss, 2003). En effet, plusieurs travaux (Di Fabio et al., 2013; Puffer, 2011) contribuent à indiquer qu'une faible intelligence émotionnelle est un prédicteur important de l'indécision vocationnelle, au-delà d'autres antécédents classiques tels que la personnalité, l'anxiété et le sentiment d'efficacité au choix (Di Fabio et al., 2013). Selon Puffer (2015), l'intelligence émotionnelle serait ainsi susceptible de jouer un rôle central dans le traitement de l'information lors des choix professionnels, ainsi qu'en témoignent les travaux qui rapportent qu'une intelligence émotionnelle élevée est associée à peu de pensées dysfonctionnelles ou d'anxiété au choix, ainsi qu'à des niveaux plus élevés d'identité vocationnelle, de promptitude au choix, de sentiment d'efficacité au choix et de disposition à explorer les options vocationnelles. Récemment, Udayar et collègues (en préparation) ont ainsi également mis en évidence l'importance de l'intelligence émotionnelle et des processus de régulation émotionnelle dans les processus de choix professionnel et d'adaptabilité de carrière, ainsi que dans la perception de son employabilité.

Ces résultats intéressants ont des implications importantes pour la pratique du conseil en orientation; ils soulignent la nécessité pour les psychologues conseillers.ères d'aider leurs consultant.e.s à être davantage capables d'identifier, d'intégrer, de comprendre et 
de gérer leur vécu émotionnel. À ce sujet, les travaux de Di Fabio et Kenny (2010) offrent des résultats encourageants, car ils tendent à indiquer que les compétences inhérentes à l'intelligence émotionnelle peuvent être accrues significativement au moyen d'un entrâ̂nement ciblé.

\section{DISCUSSION}

La définition des émotions proposées par D. H. Ford (1987) a permis, dans ce chapitre, de proposer une organisation cohérente des résultats de la recherche sur l'implication des émotions dans le processus de choix professionnel, ainsi que des pistes d'intervention possibles pour aborder le vécu émotionnel dans les démarches de conseil en orientation. Ce faisant, ce travail souligne le rôle crucial des émotions dans le processus de choix et ouvre de nouvelles perspectives, notamment en termes d'interventions concrètes. En dépit de ces avancées, il convient de préciser que l'approche de D. H. Ford (1987), choisie ici comme point d'ancrage, ne correspond qu’à une définition des émotions parmi de nombreuses autres. Partir d'une autre théorie aurait pu conduire à l'obtention de résultats sensiblement différents.

\subsection{S'AFFRANCHIR DES IDÉES REÇUES}

Si les recherches sur les émotions positives offrent des résultats prometteurs, l'étude de ces dernières n'en est toutefois qu'à ses prémices, ce qui peut parfois contribuer à donner l'impression d'une simplification outrancière de la réalité. De plus, des efforts supplémentaires sont nécessaires pour mieux cerner leurs effets spécifiques, notamment dans le champ du conseil en orientation. Comme nous l'avons montré au fil de ces lignes, les émotions constituent assurément des phénomènes complexes susceptibles d'influencer le processus de décision vocationnelle à plusieurs niveaux : traitement de l'information, champ attentionnel et réponse comportementale (D.H. Ford, 1987). Évoquer cette complexité laisse aussi entrevoir les problèmes qui peuvent survenir lorsque le vécu émotionnel est atténué, par exemple dans les cas de troubles psychiques tels que l'alexithymie ou de consommation de certaines substances psychoactives comme le cannabis. Par ailleurs, l'attention grandissante portée aux émotions positives pourrait être assimilée à un optimisme inconsidéré, faisant l'impasse sur la souffrance et la 
complexité de la réalité. Ce type de reproches a, par exemple, déjà été adressé au champ de la psychologie positive (voir Shankland, 2014, pour une revue à ce sujet). Cependant, l'accent mis sur les émotions positives ne vise pas à désavouer les difficultés, mais incarne plutôt une volonté de redonner place aux ressources et au fonctionnement adéquat de la personne. Il compense ainsi l'importance traditionnellement accordée en psychothérapie au symptôme et au dysfonctionnement (Shankland \& André, 2014).

En ce qui concerne plus spécifiquement l'humour, une certaine prudence est de mise vis-à-vis de l'idée (non fondée scientifiquement) que toute intervention humoristique s'avérerait bénéfique. Lynch (2002) souligne que l'humour est avant tout un processus de communication; par conséquent, il ne serait ni positif ni négatif en lui-même. Plusieurs auteurs (De Koning \& Weiss, 2002; Martin, Puhlik-Doris, Larsen, Gray, \& Weir, 2003) relèvent ainsi que certains styles d'humour seraient positifs pour la relation, alors que d'autres seraient potentiellement néfastes. Au-delà des considérations sur les styles d'humour en tant que tels, certaines études en psychologie du travail mettent en lumière l'importance de la qualité relationnelle préalable pour que l'humour soit perçu comme bienveillant par l'interlocuteur et qu'il engendre des émotions positives (Robert, Dunne, \& Lun, 2016; voir aussi Wijewardena, Härtel, \& Samaratunge, 2017). En effet, comme le montre Bippus (2003), lorsqu'une personne recourt à l'humour, son interlocuteur infere les motifs pour lesquels elle le fait et ces inférences influent sur l'évaluation (positive ou négative) de l'intervention. Ainsi, il convient de différencier l'impact spécifique de chaque style d'humour et de tenir compte du contexte dans lequel il s'insère. Le modèle proposé par Martin et collègues (2003) fournit un bon appui pour explorer plus profondément, par des études empiriques, les phénomènes humoristiques dans le contexte du travail, ainsi qu'en orientation scolaire et professionnelle.

\subsection{INTENSIFIER ET MODERNISER LES RECHERCHES}

Dans le contexte du choix professionnel, il apparaît que les recherches sur l'impact des émotions sur le processus de traitement de l'information se sont principalement concentrées sur le rôle des émotions négatives, et en particulier sur le rôle de l'anxiété. Ce faisant, les travaux ultérieurs gagneraient à s'intéresser au rôle potentiel des émotions positives (par exemple, intérêt, plaisir et surprise) 
sur l'évaluation des alternatives possibles et l'indécision, ainsi qu'à une plus grande variété d'émotions négatives (par exemple, désintérêt, découragement, colère et dégoût). En ce qui concerne l'impact des émotions sur les processus physiologiques, la synthèse effectuée montre qu'il est nécessaire de rechercher à étudier le rôle des émotions positives et négatives sur l'ampleur des options considérées. Enfin, ce travail permet de souligner qu'il conviendrait également d'étudier le rôle joué par les émotions positives et par d'autres émotions de valence négative dans les comportements d'exploration de carrière. Néanmoins, il s'agit de souligner que les propositions de D. H. Ford (1987) gagneraient à être confrontées avec d'autres travaux plus récents sur les émotions, et que l'intégration cohérente de ce modèle des émotions au sein des modèles théoriques actuels de l'orientation nécessiterait davantage d'efforts.

Il apparaît également que l'ensemble des recherches présentées dans cette revue de la littérature portent sur des émotions autoreportées. Or les travaux récents menés dans d'autres champs de la psychologie ont conduit au développement d'outils de mesure permettant d'appréhender de manière plus expérientielle les réactions émotionnelles à certains types de stimuli, notamment au travers de marqueurs physiologiques, comme la conductance épidermique et les taux hormonaux contenus dans la salive. Afin d'appréhender de manière plus rigoureuse le rôle des émotions dans le choix professionnel, il conviendrait donc que les chercheurs en orientation s'ouvrent à l'utilisation de tels procédés pour leurs recherches, par exemple en étudiant les réactions émotionnelles suscitées par des photos représentant des activités spécifiques à des métiers. Bien que ces approches soient encore limitées dans le type d'émotions qu'elles permettent de distinguer, ce style de mesures permettrait d'aller au-delà des émotions reportées par les participant.e.s et d'ouvrir la porte à d'autres moyens d'investiguer plus finement les processus en œuvre lors du choix ou des séances de conseil en orientation. Par ailleurs, il convient de souligner que la définition des émotions présentée en début de chapitre relève que ces dernières surviennent toujours dans un contexte particulier. Ce faisant, il s'agirait, pour les recherches futures, de ne pas négliger les caractéristiques environnementales susceptibles de contribuer à la réaction émotionnelle de l'individu en situation de choix ou de transition.

De même, afin de mieux comprendre le vécu de l'humour spontané et son influence sur la relation entre les psychologues 
conseillers.ères en orientation et leurs consultant.e.s, il serait tout spécialement instructif d'en analyser, avec les personnes concernées, les aspects émotionnels, cognitifs et physiologiques. En effet, cerner plus précisément les enjeux et processus qui entourent les différents styles d'humour fournirait des éclaircissements précieux quant à leur impact sur la relation de conseil (notamment en termes d'alliance de travail), ainsi que sur l'engagement de la ou du consultant.e et sa progression dans la démarche d'orientation. Une telle étude pourrait permettre aux chercheurs.euses et aux praticien-ne.s de concevoir des outils novateurs visant par exemple à favoriser l'établissement et le maintien d'une relation positive avec les consultant.e.s, à susciter des émotions positives chez ces derniers.ères, à réguler des émotions négatives ou encore à favoriser l'équilibre entre les deux.

\subsection{INTÉGRER LES ÉMOTIONS DANS LA PRATIQUE}

En dépit des limitations évoquées ci-dessus et des efforts qui doivent encore être entrepris, le travail de synthèse effectué dans ce chapitre permet de mettre en évidence l'importance centrale que le vécu émotionnel est susceptible de jouer dans le processus de choix professionnel. Ce faisant, il apparaît crucial que les psychologues conseillers.ères en orientation reconnaissent, prennent en compte et travaillent avec les émotions. Ceci implique d'être sensibilisé à cette thématique dans le cadre de son cursus de formation initiale et continue. L'objectif serait alors de permettre aux futur.e. praticien-ne.s de mieux appréhender théoriquement les enjeux du ressenti émotionnel sur le choix professionnel, ainsi que de disposer d'outils pour moduler ce vécu. En effet, étant donné que la vaste majorité des théories actuelles de l'orientation font l'impasse sur ces aspects ou ne le traitent que de manière superficielle (Vondracek et al., 2015), les psychologues conseillers.ères en orientation se trouvent relativement démuni.e.s pour aborder ces questions. Introduire le thème des émotions dans la formation initiale et continue de ces professionnel.le.s s'avère donc un enjeu essentiel pour le futur de l'orientation scolaire et professionnelle. Toutefois, il convient de souligner qu'une attention accrue portée aux émotions dans les démarches d'orientation pourrait conduire à craindre un glissement vers une pratique d'ordre psychothérapeutique plutôt que de conseil à proprement parler. Ce faisant, les limites entre ces deux types de suivi gagneraient à être clairement spécifiées. 


\section{CONCLUSION}

Au travers de la proposition d'une définition concrète des émotions, ce chapitre visait à réaliser une synthèse de la littérature sur le rôle des émotions dans le processus de choix professionnel et à relever les avancées dans ce domaine. Ce travail a également permis de souligner pourquoi et comment une meilleure prise en compte des émotions (du point de vue de leur variété, de leur nature et de leur intensité) pourrait favoriser la prise de décision et le processus vocationnel. Par ailleurs, il met en lumière les aspects qui seraient encore à développer pour parvenir à une meilleure intégration de cette thématique tant dans les théories de l'orientation que dans la pratique du conseil, ouvrant ainsi quelques perspectives d'avenir.

\section{RÉFÉRENCES BIBLIOGRAPHIQUES}

APTER, M. J. (2013). Developing reversal theory: Some suggestions for future research. Journal of Motivation, Emotion, and Personality, 1, 1-8. Doi: 10.12689/jmep.2013.101.

Apter, M. J. (2014). Towards a theory of things: Reversal theory and design. Journal of Motivation, Emotion, and Personality, 2, 3-11. Doi : 10.12689/jmep.2014.302.

Arthur, M. B., \& Rousseau, D. M. (1996). Introduction: The boundaryless career as a new employment principle. In M. B. Arthur \& D. M. Rousseau (éds), The boundaryless career: A new employment principle for a new organizational era, (pp. 3-20). New York: Oxford University Press.

Banet, E., \& Masdonati, J. (2017). The spontaneous use of humour by career counsellors. The Canadian Journal of Career Development/Revue canadienne de développement de carrière, 16 (2), 32-37.

BARAK, A. (2001). A cognitive view of the nature of vocational interests: Implications for career assessment, counseling, and research. In T. L. LEONG \& A. BARAK (éds), Contemporary models in vocational psychology: A volume in honor of Samuel H. Osipow. Mahwah (NJ) : Lawrence Erlbaum Associates.

Barrett, L. F. (2009). Variety is the spice of life: A psychological construction approach to understanding variability 
in emotion. Cognition and Emotion, 23, 1284-1306. Doi: 10.1080/02699930902985894.

Bauman, Z. (2000). Liquid modernity. Cambridge: Polity Press.

Bechara, A., Damasio, H., Damasio, A. R., \& Lee, G. P. (1999). Different contributions of the human amygdala and ventromedial prefrontal cortex to decision-making. Journal of Neuroscience, 19 (13), 5473-5481.

Bechara, A., Tranel, D., \& Damasio, A. R. (2000). Poor judgement in spite of high intellect. In R. BAR-On, J. V. Chan \& J. D. A. PARKER (éds), Handbook of emotional intelligence (pp. 192-214). San Francisco: Jossey-Bass.

Beck, A. T., Rush, A. J., Shaw, B., \& Emery, G. (1979). Cognitive therapy of depression. New York: Guilford Press.

Bellier-Teichmann, T., Fusi, M., \& Pomini, V. (2017). Évaluer les ressources des patients: une approche centrée sur le rétablissement. Pratiques Psychologiques, 23, 41-59. Doi: 10.1016/j. prps.2016.03.004.

Betz, N. E., Hammond, M. S., \& Multon, K. D. (2005). Reliability and validity of five-level response continua for the Career Decision Self-Efficacy Scale. Journal of Career Assessment, 13, 131149. Doi : 10.1177/1069072704273123.

Bippus, A. M. (2003). Humor motives, qualities, and reactions in recalled conflict episodes. Western Journal of Communication, 67, 413-426. Doi : 10.1080/10570310309374781.

Blustein, D. L., \& Phillips, S. D. (1988). Individual and contextual factors in career exploration. Journal of Vocational Behavior, 33, 203-216. Doi : 10.1016/0001-8791 (88) 90056-5.

Braunstein-Bercovitz, H., Benjamin, B. A., Asor, S., \& Lev, M. (2012). Insecure attachment and career indecision: Mediating effects of anxiety and pessimism. Journal of Vocational Behavior, 81, 236-244. Doi : 10.1016/J.Jvb.2012.07.009.

Brooks, A.W. (2014). Get excited: Reappraising pre-performance anxiety as excitement. Journal of Experimental Psychology: General, 143, 1144-1158. Doi: 10.1037/a0035325.

Brown, S. D., \& Rector, C. C. (2008). Conceptualizing and diagnosing problems in vocational decision making. In S. D. Brown 
\& R. W. LENT (éds), Handbook of counseling psychology (4e édition, pp. 392-407). Hoboken (NJ): Wiley.

Brown, S. D., Hacker, J., Abrams, M., Carr, A., Rector, C., Lamp, K., ... SienA, A. (2012). Validation of a four-factor model of career indecision. Journal of Career Assessment, 20, 3-21. Doi: $10.1177 / 1069072711417154$.

Campagna, C. G., \& Curtis, G. J. (2007). So worried I don't know what to be: Anxiety is associated with increased career indecision and reduced career certainty. Australian Journal of Guidance and Counselling, 17, 91-96. Doi: 10.1375/Ajgc.17.1.91.

Creed, P. A., \& Hennessy, D. A. (2016). Evaluation of a goal orientation model of vocational identity. Career Development Quarterly, 64, 345-359. Doi: 10.1002/cdq.12070.

Creed, P. A., Hood, M., Praskova, A., \& Makransky, G. (2016). The career distress scale. Journal of Career Assessment, 24, 732-746. Doi : $10.1177 / 1069072715616126$.

Dambrun, M. \& Dubuy, A.-L. (2013). A positive psychology intervention among long-term unemployed people and its effect on psychological distress and well-being. Journal of Employment Counseling, 51, 75-88. Doi: 10.1002/j.2161-1920.2014.00043.x.

Damasio, A. R. (1994). Decartes'error: Emotion, reason, and the buman brain. New York: Avon Books.

Danzer, A., Dale, J. A., \& Klions, A. H. L. (1990). Effect of exposure to humorous stimuli on induced depression. Psychological Reports, 66, 1027-1036. Doi: 10.2466/pr0.1990.66.3.1027.

De Koning, E., \& WeIss, R. L. (2002). The relational humor inventory: Functions of humor in close relationships. American Journal of Family Therapy, 30, 1-18. Doi : 10.1080/019261802753455615.

DeCKers, L. (1998). Influence of mood on humor. In W. RuCH (éd.), The sense of humour - Explorations of a Personality Characteristic (pp. 309-328). New York: Mouton de Gruyter.

Di Fabio, A., \& Kenny, M. E. (2010). Promoting emotional intelligence and career decision making among Italian high school students. Journal of Career Assessment, 19, 21-34. Doi: $10.1177 / 1069072710382530$.

Di Fabio, A., Palazzeschi, L., Asulin-Peretz, L., \& Gati, I. 
(2013). Career indecision versus indecisiveness: Associations with personality traits and emotional intelligence. Journal of Career Assessment, 21, 42-56. Doi : 10.1177/1069072712454698.

Dickinson, J., \& ToKar, D. M. (2004). Structural and discriminant validity of the career factors inventory. Journal of Vocational Behavior, 65, 239-254. Doi: 10.1016/J.Jvb.2003.07.002.

Douglass, R. P., \& Duffy, R. D. (2015). Calling and career adaptability among undergraduate students. Journal of Vocational Behavior, 86, 58-65. Doi : 10.1016/j.jvb.2014.11.003.

Duffy, R. D., Douglass, R. P., \& Autin, K. L. (2015). Career adaptability and academic satisfaction: Examining work volition and self efficacy as mediators. Journal of Vocational Behavior, 90, 46-50. Doi: 10.1016/j.jvb.2015.07.007.

EKMAN, P. (1984). Expression and the nature of emotions. In K. Scherer \& P. EkMAn (éds), Approaches to emotions. Hillsdale (NJ): Lawrence Earlbaum Associates.

Emmerling, R. J., \& Cherniss, C. (2003). Emotional intelligence and the career choice process. Journal of Career Assessment, 11, 153167. Doi : 10.1177/1069072702250425.

Emmons, R. A., \& McCullough, M. E. (2003). Counting blessings versus burdens: An experimental investigation of gratitude and subjective well-being in daily life. Journal of Personality and Social Psychology, 84, 377-389. Doi : 10.1037/0022-3514.84.2.377.

FORD, D. H. (1987). Humans as self-constructing living systems: A developmental perspective on behavior and personality. Hillsdale (NJ) : Erlbaum.

Ford, M. E. (1992). Motivating humans: Goals, emotions and personal agency beliefs. Londres: Sage.

Fredrickson, B. L. (1998). What good are positive emotions? Review of General Psychology, 2, 300-319. Doi: 10.1037/10892680.2.3.300.

Fredrickson, B. L. (2001). The role of positive emotions in positive psychology: The broaden-and-build theory of positive emotions. American Psychologist, 56, 218-226. Doi: 10.1037//0003066x.56.3.218.

Fredrickson, B. L., \& Branigan, C. (2005). Positive emotions broa- 
den the scope of attention and thought-action repertoires. Cognition and Emotion, 19, 313-332. Doi: 10.1080/02699930441000238.

Fuqua, D. R., Seaworth, T. B., \& Newman, J. L. (1987). The relationship of career indecision and anxiety: A multivariate examination. Journal of Vocational Behavior, 30, 175-186. Doi: 10.1016/0001-8791 (87) 90017-0.

Fuqua, D. R., Seaworth, T. B., \& Newman, J. L. (1988). Relation of state and trait anxiety to different components of career indecision. Journal of Counseling Psychology, 35, 154-158. Doi: 10.1037/0022-0167.35.2.154.

Gati, I., Krausz, M., \& Osipow, S. H. (1996). A taxonomy of difficulties in career decision making. Journal of Counseling Psychology, 43, 510-526. Doi : 10.1037//0022-0167.43.4.510.

Germeijs, V., Verschueren, K., \& Soenens, B. (2006). Indecisiveness and high school students'career decision-making process: Longitudinal associations and the mediational role of anxiety. Journal of Counseling Psychology, 53, 397-410. Doi: 10.1037/00220167.53.4.397.

Ginevra, M. C., Pallini, S., Vecchio, G. M., Nota, L., \& Soresi, S. (2016). Future orientation and attitudes mediate career adaptability and decidedness. Journal of Vocational Behavior, 95-96, 102110. Doi: 10.1016/j.jvb.2016.08.003.

Hacker, J., Carr, A., Abrams, M., \& Brown, S. D. (2013). Development of the Career Indecision Profile: Factor structure, reliability, and validity. Journal of Career Assessment, 21, 32-41. Doi: $10.1177 / 1069072712453832$.

Hirschi, A., \& VAlero, D. (2015). Career adaptability profiles and their relationship to adaptivity and adapting. Journal of Vocational Behavior, 88, 220-229. Doi: 10.1016/j.jvb.2015.03.010.

Hirschi, A., Herrmann, A., \& Keller, A. (2015). Career adaptivity, adaptability, and adapting: A conceptual and empirical investigation. Journal of Vocational Behavior, 87, 1-10. Doi : 10.1016/j. jvb.2014.11.008.

IsEN, A. M. (2001). An influence of positive affects on decision-making in complex situations: Theoretical issues with practical implications. Journal of Consumer Psychology, 11, 75-85. Doi : 10.1207/ S15327663JCP1102_01. 
IsEN, A. M., \& GEVA, N. (1987). The influence of positive affect on acceptable level of risk: The person with a large canoe has a large worry. Organizational Behavior and Human Decision Processes, 39, 145-154. Doi : 10.1016/0749-5978 (87) 90034-3.

Isen, A. M., \& Gorgoglione, J. M. (1983). Some specific effects of four affect-induction procedures. Personality and Social Psychology Bulletin, 9, 136-143. Doi: 10.1177/0146167283091019.

Isen, A. M., \& PATrick, R. (1983). The effect of positive feelings on risk-taking: When the chips are down. Organizational Behavior and Human Decision Processes, 31, 194-202. Doi : 10.1016/00305073 (83) 90120-4.

Isen, A. M., Nygren, T. E., \& Ashby, F. G. (1988). Influence of positive affect on the subjective utility of gains and losses: It is just not worth the risk. Journal of Personality and Social Psychology, 55, 710-717. Doi : 10.1037//0022-3514.55.5.710.

Izard, C. E. (1977). Human emotions. New York: Plenum Press.

Kahneman, A., \& Tversky, A. (1979). Prospect theory: An analysis of decision under risk. Econometrica, 47, 263-292. Doi: $10.2307 / 1914185$.

KIDD, J. M. (1998). Emotion: An absent presence in career theory. Journal of Vocational Behavior, 52, 275-288. Doi: 10.1006/ Jvbe.1997.1629.

KIDD, J. M. (2004). Emotions in career context: Challenges for theory and research. Journal of Vocational Behavior, 64, 441-454, Doi : $10.1016 /$ j.jvb.2003.12.009.

Krumboltz, J. D. (1996). A learning theory of career counseling. In M. L. SAVICKAS \& W. B. WALSH (éds), Handbook of career counseling theory and practice (pp. 55-80). Palo Alto: Davies-Black Publishing.

Kuiper, N. A., Martin, R. A., \& Dance, K. A. (1992). Sense of humour and enhanced quality of life. Personality and Individual Differences, 13, 1273-1283. Doi: 10.1016/0191-8869 (92) 90169-P.

Kuiper, N. A., Martin, R. A., \& Olinger, L. J. (1993). Coping humour, stress, and cognitive appraisals. Canadian Journal of Behavioural Science/Revue canadienne des sciences du comportement, 25, 81-96. Doi: 10.1037/h0078791. 
Kuiper, N. A., McKenzie, S. D., \& Belanger, K. A. (1995). Cognitive appraisals and individual differences in sense of humor: Motivational and affective implications. Personality and Individual Differences, 19, 359-372. Doi : 10.1016/0191-8869 (95) 00072-E. LARSON, L. M., \& MajORS, M. S. (1998). Application of the coping with career indecision instrument with adolescents. Journal of Career Assessment, 6, 163-179. Doi : 10.1177/106907279800600204.

Lefcourt, H. M., \& Thomas, S. (1998). Humor and stress revisited. In W. Ruch (éd.), The sense of humour: Explorations of a personality characteristic (pp. 179-202). New York: Mouton de Gruyter. Lehmann-Willenbrock, N., \& Allen, J. A. (2014). How fun are your meetings? Investigating the relationship between humor patterns in team interactions and team performance. Journal of Applied Psychology, 99, 1278-1287. Doi : 10.1037/a0038083.

Lent, R. W., Brown, S. D., \& Hackett, G. (1994). Toward a unifying social cognitive theory of career and academic interest, choice, and performance. Journal of Vocational Behavior, 45, 79-122. Doi : 10.1006/Jvbe.1994.1027.

Leong, F. T. L., \& Chervinko, S. (1996). Construct validity of career indecision: Negative personality traits as predictors of career indecision. Journal of Career Assessment, 4, 315-329. Doi: $10.1177 / 106907279600400306$.

Lerner, J. S., Li, Y., Valdesolo, P., \& Kassam, K. S. (2015). Emotion and decision making. Annual Review of Psychology, 66, 799823. Doi : 10.1146/annurev-psych-010213-115043.

LYNCH, O. H. (2002). Humorous communication: Finding a place for humor in communication research. Communication Theory, 12, 423-445. Doi : 10.1111/j.1468-2885.2002.tb00277.x.

Martin, R. A., Kuiper, N. A., Olinger, L. J., \& Dance, K. A. (1993). Humor, coping with stress, self-concept, and psychological well-being. Humor-International Journal of Humor Research, 6, 89-104. Doi: 10.1515/humr.1993.6.1.89.

Martin, R. A., Puhlik-Doris, P., Larsen, G., Gray, J., \& Weir, K. (2003). Individual differences in uses of humor and their relation to psychological well-being: Developpment of the Humor Styles Questionnaire. Journal of Research in Personality, 37, 48-75. Doi : 10.1016/S0092-6566 (02) 00534-2 
Martin, R. B., \& Labott, S. M. (1991). Mood following emotional crying: Effects of the situation. Journal of Research in Personality, 25, 218-244. Doi : 10.1016/0092-6566 (91) 90017-K.

Mayer, J. D., \& Salovey, P. (1997). What is emotional intelligence? In P. Salovey \& D. Sluyter (éds), Emotional development and emotional intelligence: Implications for educators (pp. 3-34). New York: Basic Books.

Megdell, J. I. (1984). Relationship between counselor-initiated humor and client's self-perceived attraction in the counseling interview. Psychotherapy: Theory, Research, Practice, Training, 21, 517523. Doi: 10.1037/h0085997.

MejJers, F. (2002). Career learning in a changing world: The role of emotions. International Journal for the Advancement of Counselling, 24, 149-167. Doi: 10.1023/A: 1022970404517.

Meldahl, J. M., \& Munchinsky, P. M. (1997). The neurotic dimension of vocational indecision: Gender comparability? Journal of Career Assessment, 5, 317-331. Doi: 10.1177/106907279700500305.

Mesmer-Magnus, J., Glew, D. J., \& Viswesvaran, C. (2012). A meta-analysis of positive humor in the workplace. Journal of Managerial Psychology, 27, 155-190. Doi : 10.1108/02683941211199554.

Multon, K. D., Heppner, M. J., \& Lapan, R. T. (1995). An empirical derivation of career decision subtypes in high school sample. Journal of Vocational Behavior, 47. Doi : 10.1006/jvbe.1995.1030.

Murtagh, N., Lopes, P. N., \& Lyons, E. (2012). Decision making in voluntary career change: An other-than-rational perspective. The Career Development Quarterly, 59, 249-263. Doi: 10.1002/ j.2161-0045.2011.tb00067.x.

Neureiter, M., \& Traut-Mattausch, E. (2017). Two sides of the career resources coin: Career adaptability resources and the impostor phenomenon. Journal of Vocational Behavior, 98, 56-69. Doi : 10.1016/j.jvb.2016.10.002.

Nevo, O. (1986). Uses of humor in career counseling. The Career Development Quarterly, 34, 188-196. Doi: 10.1002/j.2164585X.1986.tb01122.x.

Newman, J. L., Fuqua, D. R., \& Seaworth, T. B. (1989). The 
role of anxiety in career indecision: Implications for diagnosis and treatment. Career Development Quarterly, 37, 221-231. Doi : 10.1002/j.2161-0045.1989.tb00826.x.

Nugier, A. (2009). Histoire et grands courants de recherche sur les émotions. Revue électronique de psychologie sociale, 4 (4), 8-14.

O'Hare, M. M., \& Tamburri, E. (1986). Coping as a moderator of the relation between anxiety and career decision-making. Journal of Counseling Psychology, 33, 255-264. Doi: 10.1037//00220167.33.3.255.

Park, K., Woo, S., Park, K., Kyea, J., \& Yand, E. (2017). The mediation effects of career exploration on the relationship between trait anxiety and career indecision. Journal of Career Development, 44, 440-452. Doi: 10.1177/0894845316662346.

Sampson, J. P., Lenz, J. G., Reardon, R. C., Peterson, G. W. (1999). A cognitive information processing approach to employment problem solving and decision making. Career Development Quarterly, 48, 3-18. Doi: 10.1002/j.2161-0045.1999.tb00271.x.

Plutchik, R. (1980). Emotion: A psychoevolutionary synthesis. New York: Harper \& Row.

Poon, J. M. L. (2004). Career commitment and career success: Moderating role of emotion perception. Career Development International, 9, 374-390. Doi: 10.1108/13620430410544337.

Puffer, K. A. (2011). Emotional intelligence as a salient predictor for collegians'career decision making. Journal of Career Assessment, 19, 130-150. Doi : 10.1177/1069072710385545.

Puffer, K. A. (2015). Facilitating emotional awareness in a career counseling context. Journal of Career Assessment, 23, 265-280. Doi : $10.1177 / 1069072714535027$.

Quoidbach, J., Gruber, J., Mikolajczak, M., Kogan, A., KotsOu, I., \& NORTON, M. I. (2014). Emodiversity and the emotional ecosystem. Journal of experimental psychology: General, 143, $2057-$ 2066. Doi: $10.1037 / \mathrm{a} 0038025$.

Robert, C., Dunne, T. C., \& Lun, J. (2016). The impact of leader humor on subordinate job satisfaction: The crucial role of leader - subordinate relationship quality. Group \& Organization Management, 41, 375-406. Doi : 10.1177/1059601115598719. 
Rochat, S., \& Bellier-Teichmann, T. (2017). Psychologie positive et conseil en orientation: guide pratique et pistes d'interventions. Le Journal des psychologues, 4, 37-41. Doi : 10.3917/jdp.346.0037. SAKA, N., \& GATI, I. (2007). Emotional and personality-related aspects of persistent career decision-making difficulties. Journal of Vocational Behavior, 71, 340-358. Doi : 10.1016/J.Jvb.2007.08.003.

SAKa, N., Gati, I., \& Kelly, K. R. (2008). Emotional and personality-related aspects of career-decision-making difficulties. Journal of CareerAssessment, 16,403-424. Doi : 10.1177/1069072708318900.

Santos, P. J. (2001). Predictors of generalized indecision among Portuguese secondary school students. Journal of Career Assessment, 9, 381-396. Doi : 10.1177/106907270100900405

Saunders, D. E., Peterson, G. W., Sampson, J. P., \& Reardon, R. C. (2000). Relation of depression and dysfunctional career thinking to career indecision. Journal of Vocational Behavior, 56, 288-298. Doi : 10.1006/jvbe.1999.1715.

SaviCKaS, M. L. (1997). Career Adaptability: An integrative construct for life-span, life-space theory. The Career Development Quarterly, 45, 247-259. Doi: 10.1002/j.2161-0045.1997. tb00469.x.

SAVICKAS, M. L. (2011). Career counseling. Washington, DC: American Psychological Association.

Savickas, M. L., \& Porfeli, E. J. (2012). Career Adapt-Abilities Scale: Construction, reliability, and measurement equivalence across 13 countries. Journal of Vocational Behavior, 80, 661-673. Doi: $10.1016 /$ j.jvb.2012.01.011.

Shankland, R. (2014). La psychologie positive (2 édition). Paris: Dunod.

Shankland, R., \& André, C. (2014). Pleine conscience et psychologie positive: incompatibilité ou complémentarité. Revue québécoise de psychologie, 35 (2), 157-178.

Strick, M., Holland, R. W., van BaAren, R. B., \& van KnipPenberg, A. D. (2009). Finding comfort in a joke: Consolatory effects of humor through cognitive distraction. Emotion, 9, 574578. Doi: 10.1037/a0015951.

Svebak, S., \& Apter, M.-J. (1987). Laughter: an empirical test of 
some reversal theory hypothesis. Scandinavian Journal of Psychology, 28, 189-198. Doi : 10.1111/j.1467-9450.1987.tb00755.x.

Sweeney, M. L., \& Schill, T. R. (1998). The association between self-defeating personality characteristics, career indecision, and vocational identity. Journal of Career Assessment, 6, 69-81. Doi: $10.1177 / 106907279800600105$.

Tews, M. J., Michel, J. W., \& Noe, R. A. (2017). Does fun promote learning? The relationship between fun in the workplace and informal learning. Journal of Vocational Behavior, 98, 46-55. Doi : 10.1016/j.jvb.2016.09.006.

Thayer, R. E., Newman, R. J., \& McClain, T. M. (1994). Selfregulation of mood: Strategies for changing a bad mood, raising energy, and reducing tension. Journal of Personality and Social Psychology, 67, 910-925. Doi : 10.1037/0022-3514.67.5.910.

Trampe, D., Quoidbach, J., \& Taquet, M. (2015). Emotions in everyday life. PloS ONE, 10, e0145450. Doi: 10.1371/journal. pone. 0145450 .

Udayar, S., Fiori, M., Thalmayer, A., \& Rossier, J. (en préparation). The role of cognitive and emotional regulatory processes in explaining the impact of dispositions on career prospects.

Urbanaviciute, I., Kairys, A., Pociute, B., \& Liniauskaite, A. (2014). Career adaptability in Lithuania: A test of psychometric properties and a theoretical model. Journal of Vocational Behavior, 85, 433-442. Doi: 10.1016/j.jvb.2014.09.005.

Van den Broeck, A., Vander Elst, T., Dikkers, J., De Lange, A., \& De Witte, H. (2012). This is funny: On the beneficial role of self-enhancing and affiliative humour in job design. Psicothema, 24 (1), 87-93.

Vignoli, E. (2015). Career indecision and career exploration among older French adolescents: The specific role of general trait anxiety and future school and career anxiety. Journal of Vocational Behavior, 89, 182-191. Doi: 10.1016/j.jvb.2015.06.005.

Vignoli, E., Croity-Belz, S., Chapeland, V., De Fillipis, A., \& GarCiA, M. (2005). Career exploration in adolescents: The role of anxiety, attachment, and parenting style. Journal of Vocational Behavior, 67, 153-168. Doi: 10.1016/j.jvb.2004.08.006. 
VondraceK, F. W., Ford, D. H., \& Porfeli, E. (2015). A living systems theory of vocational behavior and development. Rotterdam: Sense Publishers.

Walker, J. V., \& Peterson, G. W. (2012). Career thoughts, indecision, and depression. Journal of Career Assessment, 20, 497-506. Doi : $10.1177 / 1069072712450010$.

Watson, D., Clark, L. A., \& Tellegen, A. (1988). Development and validation of brief measure of positive and negative affect: The PANAS Scales. Journal of Personality and Social Psychology, 54, 1063-1070. Doi: 10.1037/0022-3514.54.6.1063.

Weinberger, M. G., \& Gulas, C. S. (1992). The impact of humor in advertising: A review. Journal of Advertising, 21, 35-59. Doi: 10.1080/00913367.1992.10673384.

Wenzlaff, R. M., \& Wegner, D. M. (2000). Thought suppression. Annual Review of Psychology, 51, 59-91. Doi: 10.1146/ annurev.psych.51.1.59.

Wijewardena, N., Härtel, C. E., \& Samaratunge, R. (2017). Using humor and boosting emotions: An affect-based study of managerial humor, employees'emotions and psychological capital. Human Relations, 70, 1316-1341. Doi: 10.1177/0018726717691809.

Wood, A. M., Linley, P. A., Maltby, J., Kashdan, T. B., \& HurLING, R. (2011). Using personal and psychological strengths lead to increase in well-being over time: A longitudinal study and the development of the Strengths Use Questionnaire. Personality and Individual Differences, 50, 15-19. Doi: 10.1016/j.paid.2010.08.004.

Yip, J. A., \& Martin, R. A. (2006). Sense of humor, emotional intelligence, and social competence. Journal of Research in Personality, 40, 1202-1208. Doi : 10.1016/j.jrp.2005.08.005.

Young, R. A., Paseluikho, M. A., \& Valach, L. (1997). The role of emotions in the construction of career in parent-adolescent conversation. Journal of Counseling and Development, 76, 46-44. Doi : 10.1002/j.1556-6676.1997.tb02374.x. 


\title{
12. LA CONVERSATION, MÉTAPHORE DE L'APPROCHE NARRATIVE DU COUNSELING D'ORIENTATION
}

\author{
PETER MCILVEEN (UNIVERSITY OF SOUTHERN QUEENSLAND, AUSTRALIA) \\ ET ALLISON CREED (UNIVERSITY OF MELBOURNE, AUSTRALIA)
}

es métaphores sont omniprésentes dans la vie quotidienne. Dans leur ouvrage Metaphors We Live By, Lakoff et Johnson (1980) font valoir que la cognition humaine est constituée par le langage et regorge de métaphores. La pensée, la parole, la gestuelle sont en effet structurées par des concepts métaphoriques, si bien que, sans métaphores, la communication est presque impossible. Les métaphores abondent dans la terminologie relative à la carrière (Inkson, 2004). Les conseillers.ères comme les client.e.s évoquent des passerelles, des échelles, des cycles, des stades, des schémas, des voyages et des histoires pour donner un sens partagé au concept de carrière. En counseling, impossible de créer du sens commun sans utiliser les métaphores pour comprendre, déconstruire et reconstruire les idées sur la carrière - en d'autres termes, sans être sur la même longueur d'onde. Si l'on opte pour une acception radicale du constructionnisme social, qui considère l'identité personnelle comme le produit du discours (Gergen, 1991; McAdams, 1993; Polkinghorne, 1988; Sarbin, 1986) et une théorie dialogique du soi (Hermans, 2006; Hermans \& Gieser, 2012) et de sa carrière (McIlveen \& Patton, 2007), alors le dialogue entre conseiller.ère et client.e représente à la fois le processus de construction de sens et le sens lui-même (McIlveen, 2012, 2017). Nous articulons ainsi le conseil en orientation dans le cadre métaphorique du dialogue et le conceptualisons en tant que conversation entre conseiller $\cdot$ ère et client.e.

Nous passerons premièrement en revue la théorie et la pratique originelle de l'approche narrative du counseling d'orientation et son extension au modèle de la métaphore de la conversation. Dans un second temps, nous décrirons la centralité de l'alliance de travail dans le conseil en orientation, car c'est dans la relation entre client.e et conseiller.ère qu'abondent le dialogue et les métaphores. 
Troisièmement, nous introduirons une théorie de la métaphore qui clarifie le rôle de la conversation dans le cadre du counseling. Quatrièmement, nous présenterons une méthode narrative de conseil en orientation pour illustrer ces principes théoriques. Enfin, nous mettrons au défi la méthode narrative proposée en appelant à une explicitation de sa philosophie sous-jacente et à une évaluation empirique de son efficacité.

\section{L'APPROCHE NARRATIVE DU COUNSELING D'ORIENTATION}

L'approche narrative du counseling d'orientation considère le soi en tant qu'identité narrative (McAdams \& McLean, 2013). À travers des termes comme "counseling de carrière narratif» et "identité narrative», le jargon professionnel reflète une conceptualisation du counseling de carrière et de l'identité en termes de récit (narrative). L'une des principales métaphores de la carrière, l'histoire (Hartung, 2015), est illustrée par des méthodes narratives de counseling (Cochran, 1997; Savickas, 2011), qui impliquent un façonnage de l'histoire (shaping the story) (Maree, 2011) lors du travail avec des client.e.s qui sont des narrateurs.trices (storytellers) (McMahon, 2017) et scénaristes (storywriters) (Lengelle \& Meijers, 2014; Meijers \& Lengelle, 2012). Le counseling de carrière narratif est une manifestation pragmatique des principes du constructionnisme social radical: la création de sens est un processus constitué dans le discours; il en est de même pour l'identité personnelle; et tant la création de sens que l'identité sont délimitées et encadrées par leur discours. Ainsi, l'histoire, qui est intrinsèquement discursive, est une métaphore idéale pour le counseling de carrière narratif, car les client.e.s et les praticien.ne.s parlent souvent de la carrière en ces termes. Ce faisant, ces personnes conçoivent la carrière comme une histoire de vie professionnelle, dans laquelle elles campent le personnage principal et, ce faisant, agissent en accord avec leur propre conception de la carrière. Par conséquent, la métaphore LA CARRIÈRE EST UNE HISTOIRE peut aider client.e.s comme praticien-ne.s, car elle facilite la communication et la co-construction d'une réalité partagée.

Les modèles de counseling de carrière narratif considèrent les client.e.s comme des narrateurs.trices (McMahon, 2017) et scénaristes (Lengelle \& Meijers, 2014; Meijers \& Lengelle, 2012). Ces modèles reposent sur des processus semi-dirigés permettant aux client.e.s de raconter et d'écrire leur histoire. Le modèle du nar- 
rateur d'histoire de McMahon (McMahon \& Watson, 2012), par exemple, utilise des questions pour orienter le récit (story crafting questions) à trois niveaux:

- $1^{\text {er }}$ niveau: histoires d'informations compartimentées sur le contenu et l'expérience, informations;

$-2^{\mathrm{e}}$ niveau: processus relatifs à la connexion (connectedness) et à l'interaction récursive entre l'individu et ses influences et expériences subjectives;

- $3^{\mathrm{e}}$ niveau: "Qu'est-ce que cela dit de vous en tant que personne?"; thèmes et modèles pouvant servir d' "ingrédients" à la future histoire (p. 221).

Ces amorces servent à enrichir et à étoffer l'histoire existante des client.e.s pour en faire un vecteur de compréhension de soi, à utiliser pour l'exploration de leur carrière, la prise de décisions et d'éventuelles mesures en vue d'un changement.

Tous les modèles de narration ne sont pas identiques, mais il existe des principes susceptibles d'en guider les applications dans le but de créer du sens. McAdams (2008) articule six principes des récits de vie:

1. Le soi est une mise en récit;

2. Les histoires intègrent des vies;

3. Les histoires se racontent au travers des relations sociales;

4. Les histoires évoluent au fil du temps;

5. Les histoires sont des textes culturels;

6. Certaines histoires sont meilleures que d'autres.

Ces principes se retrouvent dans les travaux sur les récits de vie et dans les méthodes de recherche visant à identifier les aspects saillants de ces histoires (McAdams \& McLean, 2013). Les histoires peuvent faire ressortir le sentiment d'agentivité d'une personne, exprimé comme une volonté de maîtriser les circonstances de la vie. L'amour, les relations, les liens avec autrui s'expriment sous forme de communion. Les événements perçus comme négatifs ou douloureux peuvent se transformer en occasions positives ou de renaissance par une thématique de rédemption. Les histoires peuvent exprimer la contamination de choses positives qui deviennent négatives, voire insurmontables. Apprendre des événements de la vie équivaut à une construction de sens. L'exploration de soi, le développement de la compréhension et de la connaissance de soi sont exprimés sous forme de 
traitement narratif exploratoire. Une résolution positive cohérente favorisera un sentiment d'apaisement, d'achèvement ou de finalisation.

Ces caractéristiques distinctives des récits (McAdams \& McLean, 2013) peuvent être observées dans le cadre de l'approche narrative du counseling d'orientation. L' "agentivité», par exemple, représente la quintessence du conseil par laquelle les individus engendrent un sentiment d'efficacité personnelle et la détermination à prendre des mesures pour faire progresser leur carrière. De même, la construction de sens rend compte d'une histoire d'apprentissages par le biais de l'éducation et des expériences de vie, susceptible de mener à prendre une décision importante, comme le projet d'obtenir un diplôme.

Alors que l' "agentivité» et la construction de sens apparaissent clairement dans les pratiques de counseling d'orientation, l'émotion est curieusement absente de la littérature en la matière (Hartung, 2011; Kidd, 2008), cette dernière se concentrant majoritairement sur les processus cognitifs et comportementaux (par exemple, la prise de décision). Il en est de même dans la littérature sur l'approche narrative du counseling d'orientation, à l'exception des travaux de Hartung (2011), qui intègrent l'émotion et la narration de la manière suivante:

Les récits chargés d'émotion incarnent les histoires qu'une personne se doit de raconter à un moment donné pour se rappeler la principale passion dans sa vie. Exploiter les émotions liées aux souvenirs autobiographiques favorise une précieuse réflexion sur soi et une perspicacité qui peuvent avoir des effets positifs similaires à ceux qui seraient produits par une expression écrite centrée sur les émotions. (p. 301, traduction des auteur.e.s)

L'approche narrative du counseling d'orientation invite à se concentrer sur le dialogue entre conseillers.ères et client.e.s. Ce lien relationnel crée un espace interpersonnel pour améliorer le traitement affectif du vécu et des futurs scénarios, en mettant éventuellement l'accent sur la communion et les aspects relationnels du travail et de la carrière.

\section{LE LIEN RELATIONNEL}

La relation entre conseiller ère et client.e se définit comme une alliance de travail (Horvath \& Greenberg, 1989; Tracey \& Kokotovic, 1989) et constitue le fondement des processus et de l'efficacité 
du counseling (Xu \& Tracey, 2015). Pour le counseling d'orientation, la qualité de cette alliance de travail prédit l'efficacité de l'intervention (Masdonati, Perdrix, Massoudi, \& Rossier, 2014; Whiston, Rossier, \& Barón, 2015). Elle peut aider dans l'exploration du parcours professionnel (Elad-Strenger \& Littman-Ovadia, 2012) ainsi que des perspectives de carrière (Alchin, McIlveen, \& Perera, 2018). Une alliance de travail se développe sur la base d'un dialogue intime, le conseil ne se limitant pas à poser des questions et à y répondre. Il ne suffit pas de fournir des informations aux client.e.s et de reformuler leurs propos pour établir une alliance de travail (Multon, Ellis-Kalton, Heppner, \& Gysbers, 2003). Le counseling se fonde sur une relation intime par laquelle les histoires d'un·e client.e sont racontées, entendues, révisées et, en fin de compte, revécues.

La création et le récit d'histoires en counseling d'orientation sont l'œuvre d'un·e auteur·trice et d'un.e narrateur.trice (Mcllveen $\&$ Patton, 2007). La mise en récit est une forme de communication active et générative par laquelle la personne peut examiner un élément sous une lumière nouvelle et, ce faisant, affranchir "ses propres réflexions des contraintes des pratiques existantes, en discutant avant d'agir, ce qui constitue une caractéristique essentielle de la communication humaine» (Krippendorff, 1990, p. 2, traduction des auteur·e.s). La mise en récit peut être assimilée à une construction de l'identité au sens de la théorie du soi dialogique (Dialogical Self Theory, DST; Hermans, 2006; Hermans \& Gieser, 2012). Selon cette théorie, la personne peut être conceptualisée métaphoriquement comme un théâtre à plusieurs voix (Hermans, 2006). Imaginez une pièce de théâtre, une scène de marché animée, un plateau rempli d'acteurs-trices qui jouent leurs rôles en discutant, que ce soit de manière bruyante ou plus discrète et réfléchie. Cette cacophonie représente les conversations qu'ont les individus avec d'autres personnes dans leur vie quotidienne. Certaines conversations sont vocalisées avec d'autres personnes dans des contextes réels, tandis que d'autres sont imaginées et sous-vocalisées, sous forme de souvenirs de conversations passées ou d'anticipations de conversations futures. Selon la TSD, les acteurs.trices sur scène représentent les nombreuses voix qui constituent une personne, chaque voix étant unique par rapport aux autres; ainsi, chaque voix évoque une position $d u$ Je. En conséquence, l'auteur-trice et le narrateur.trice renvoient à différentes positions $d u$ Je constitutives 
de la même personne. L'auteur-trice et le narrateur-trice incarnent tou.te.s deux la ou le client.e, mais chacun.e est doté.e de voix et de perspectives distinctes. Et il existe une troisième voix: celle de l'éditeur-trice. Ensemble, l'auteur·trice, le narrateur-trice et l'éditeur-trice livrent des histoires sur une vie composée, racontée, révisée et racontée à nouveau dans un processus itératif et continu.

Le but ultime du counseling n'est pas simplement de raconter une histoire, mais bien de la réifier - l'appliquer à la vie quotidienne pour la vivre. L'espace psychologique confiné de l'entretien de conseil est différent du monde dans lequel une histoire, doit être vécue et sa véracité mise à l'épreuve. Le rôle de l'éditeur.trice est donc d'assurer la véracité idiographique d'une histoire qui doit être réifiée dans son contexte discursif. Une histoire "bidon» sera bien vite en butte à des critiques littéraires dans la vraie vie, et il est peu probable que celles-ci soient assez indulgentes pour permettre à un.e client.e d'habiter un mensonge. Par conséquent, les conversations entre auteur-trice, narrateur-trice et éditeur-trice doivent être franches et sans crainte.

Une autre personne participe aux conversations: la ou le conseiller.ère - à la fois interlocuteur·trice, auditeur-trice, public et ami-e critique. Les conseillers.ères fournissent aux client.e.s l'espace psychologique dans lequel se déroulent les histoires. Elles et ils s'engagent activement dans le processus de narration en posant des questions pour orienter le récit (McMahon \& Watson, 2012) et en demandant aux client.e.s d'approfondir les thèmes essentiels de l' «agentivité», de la communion, de la rédemption, de la contamination, de la construction de sens, du traitement narratif exploratoire ou d'une résolution positive cohérente (McAdams \& McLean, 2013). Récolter du contenu narratif en posant des questions qui visent le façonnage du récit et son orientation vers des thèmes saillants ne relève pas de l'interrogatoire; il s'agit d'une conversation entre deux personnes. Au sens habituel du terme conversation, le dialogue peut fluctuer au sein du récit, au gré d'émotions légères ou intenses, et il peut y avoir des digressions, des ruptures et des dénouements. Une conversation n'est pas forcément ordonnée et son intensité et sa sincérité dépendent de la relation entre les participant.e.s. En conséquence, les qualités psychologiques de la conversation doivent non seulement être comprises, mais également mises à profit pour assurer un milieu propice à l'espace psychologique créatif nécessaire à la mise en récit. 


\section{LA CONVERSATION EN TANT QUE MÉTAPHORE DÉLIBÉRÉE POUR LE COUNSELING}

La communication humaine est ancrée dans le discours et le langage peut être considéré comme un outil générateur de changement. Le phénomène de la communication a été décrit par des métaphores comme contenant, porteur de message, partage, dispute, canal ou maîtrise. Chacun de ces cadres figuratifs a des implications pour les théories du constructionnisme social, notamment lorsqu'elles s'appliquent à la vie professionnelle et au counseling d'orientation. Étant donné que le dialogue au sein du récit est au cœur des approches narratives de la carrière, la métaphore LA COMMUNICATION EST PARTAGE constitue le fondement de la discussion.

À des fins de clarification, notre compréhension de la métaphore se situe dans la théorie de la métaphore conceptuelle (Lakoff \& Johnson, 1980) et repose sur des perspectives linguistiques cognitives (Croft \& Cruse, 2004). À cette fin, la métaphore relève d'une cartographie qui relie différents domaines, puisant ses sources dans un domaine de connaissances pour éclairer un autre domaine cible (par exemple, LA COMMUNICATION EST PARTAGE, LA CARRIÈRE EST UNE HISTOIRE); elle est structurée de façon systématique (ce qui donne des expressions comme LE TEMPS, C'EST DE L'ARGENT, par exemple, "vous me faites perdre mon temps»; "cela en vaut-il la peine?»; le temps lui est compté»); elle est située physiquement et culturellement (le HAUT indique une idée de quantité ou de valeur supérieure, le BAS renvoie à ce qui est moindre ou moins bon et l'avenir est situé DEVANT). Le phénomène de la métaphore implique une mise en action coordonnée où «le discours et les actions d'une personne ne sont pas une simple manifestation extérieure d'une métaphore conceptuelle intérieure précédemment encodée", mais plutôt la conceptualisation et l'expression d'une métaphore par la personne "au moment même de son mouvement et de son discours" (Gibbs, 2017 , p. 252, traduction des auteur.e.s).

Selon la théorie de la métaphore conceptuelle (Conceptual Metaphor Theory, CMT) de Lakoff et Johnson (1980), la métaphore est bien plus qu'une figure de style ou un dispositif rhétorique. Le langage métaphorique se manifeste quotidiennement dans le discours, la pensée et la communication. En substance, «une métaphore permet de comprendre et de faire l'expérience d'une chose donnée du point de vue de quelque chose d'autre» (Lakoff \& Johnson, 1980, p. 5, traduction personnelle). Loin de se confiner au langage, la 
métaphore est incarnée dans la cognition. Par conséquent, le langage métaphorique est directement accessible aux sens (Forceville, 2006). On peut par exemple observer le voyage que représente une carrière. Plus abstrait ou non physique, le domaine CIBLE de la CARRIÈRE peut être éclairé dans le contexte de la conversation en s'appuyant sur des éléments du domaine sOURCE du VOYAGE. Ce faisant, une expérience plus concrète, plus physique de la CARRIÈRE est évoquée à travers la métaphore conceptuelle UNE CARRIÈRE EST UN VOYAGE, qui procède de la métaphore primaire SOURCE-CHEMIN-BUT. Au lieu d'évoquer le domaine cible de la CARRIÈrE en tant qu'état ou événement, la conceptualisation de la carrière s'anime avec des implications qui suggèrent un point de départ, une progression linéaire, des arrêts possibles ou des détours éventuels vers le point final qui pourrait être un nouvel emploi ou la retraite. Plus subtilement, la métaphore du VOYAGE peut receler (ou révéler) un manque de sentiment d' "agentivité» (le voyage dans lequel m'a embarqué ma carrière) où MA CARRIÈRE EST UNE CAUSALITÉ, indiquant une force externe par opposition à MA CARRIÈRE EST UN MOUVEMENT AUTONOME qui fait partie de moi ou dont je décide.

De plus, la littérature récente affirme que le langage métaphorique peut impliquer une pensée consciente ou inconsciente (Baumeister \& Masicampo, 2010; Cameron, 2003; Steen, 2009, 2017). Par exemple, Inkson (2004) soutient que la métaphore de l'histoire se situe dans le passé d'une personne et sert à comprendre et à expliquer sa vie actuelle: "Nous scrutons nos propres carrières avec avidité; nous les racontons, les reconstituons et les analysons en nous-mêmes; et nous faisons de notre mieux pour donner sens à notre passé, prendre des décisions au présent et faire des projets pour le futur ${ }^{1}$ " (p. 69, traduction des auteur·e.s). Par conséquent, la métaphore de l'histoire est omniprésente dans les choix linguistiques quotidiens que font les gens pour parler de leur carrière et de leur vie professionnelle. Cependant, ces choix linguistiques peuvent ne pas être remarqués par la ou le locuteur.trice ou son public en raison de la banalité de leur usage courant. De telles métaphores sont définies comme conventionnelles ou non délibérées, car elles ne sont pas choisies consciemment. En revanche, le langage métaphorique peut être délibéré, le choix de la métaphore étant alors conscient et stratégique (Steen, 2009, 2017). Les métaphores

1. "We watch our own careers avidly; narrate, compose, and analyze them in our minds; and try as best we can to make sense of the past, decisions for the present, and plans for the future." (p. 96) 
délibérées peuvent faciliter les interactions sociales et culturelles, car elles sont reconnues et leur emploi est choisi délibérément pour le contexte discursif situé. Par conséquent, une même métaphore (c'est-à-dire LA CARRIÈRE EST UNE HISTOIRE) peut être délibérée ou non en fonction de son utilisation.

L'utilisation intentionnelle de la métaphore est évidente dans les travaux actuels de Musolff $(2004,2017)$ sur le discours politique. Musolff souligne le rôle dialogique joué par la métaphore corporelle dans le discours politique pour décrire l'État ou la société. Les études de cas de Musolff (2004) démontrent que la métaphore du cœur géopolitique a été utilisée par les médias britanniques dans les années 1990 pour parler de l'intégration économique et politique avec l'Union européenne (UE) (le CCEUR DE L'EUROPE). pour marquer son scepticisme et son opposition à la poursuite de l'intégration, la presse a usé de scénarios métaphoriques comme le CEUR DE LA MALADIE ou encore l'ARRÊT DU CEUR. Et avant même qu'on ne parle du Brexit, Musolff (2000) rapportait que les symboles évoquant la maison ou la construction étaient fréquemment utilisés pour décrire les problématiques de politique européenne (par exemple UNE MAISON SANS ISSUE). Musolff a contesté les hypothèses linguistiques cognitives en affirmant qu'on ne pouvait pas affirmer qu' "une interprétation spécifique de l'image de la maison domine le débat sur l'UE» (p. 216, traduction personnelle). De même, des études à grande échelle sur des patient.e.s, des travailleurs.ses de la santé et des soignant.e.s suggèrent que la métaphore a une influence puissante sur le sens et le vécu de la maladie et de la fin de vie (Demmen et al., 2015; Semino, Demjén, \& Demmen, 2016; Semino et al., 2017). Aucune métaphore particulière n'est entièrement bonne ou mauvaise, mais différentes métaphores contribuent à différentes réactions auprès des patient.e.s, des soignant.e.s et des travailleurs.ses de la santé.

Pour en revenir à la notion de communication, la conceptualisation et l'énaction (Varela, Thompson, \& Rosch, 2017) de la communication en tant que partage a des implications sur les cadres théoriques de la psychologie vocationnelle et sur les méthodes de conseil en orientation (par exemple sur les interventions standardisées) en ce qu'elle affecte l'autonomie cognitive de la personne. Les méthodes de conseil en orientation, telles que la narration et la rédaction d'histoires, dépendent des conversations entre client.e.s et conseillers.ères. Ces conversations ne sont pas de simples inter- 
rogations, elles sont de nature relationnelle. La notion de partage suggère une symétrie relationnelle et une volonté commune d'effectuer une action impliquant "l'autre" (interlocuteur-trice ou correspondant.e). La communication avec l'autre offre la possibilité d'une meilleure compréhension ou d'une autre interprétation du sens (ou d'une vérité). Ceci est important car, comme le soulignent Gibbs et Franks (2002), dans les récits personnels l'utilisation de la métaphore peut être multiple, voire contradictoire, lorsque le narrateur cherche à créer et à transmettre un sens. Du point de vue de la TSD et du soi en tant que théâtre, imaginez la confusion qu'engendrent des conversations multiples déployant différentes métaphores.

La notion de partage est une éthique socialement reconnue et mise en évidence par des pratiques comportementales qui impliquent l'utilisation d'un objet en même temps qu'un autre, de donner ou de posséder quelque chose ou, dans la communication, de raconter quelque chose. Par conséquent, il semble raisonnable de postuler que la métaphore LA COMMUNICATION EST UN PARTAGE implique que les messages soient conçus comme des entités délimitées (vectrices de sens) et transportables (susceptibles d'être déplacées d'un endroit à un autre). Partager une histoire nécessite ainsi une autre personne qui porte l'entité délimitée à une autre encore qui la reçoit (pour pouvoir en partager les significations). La compréhension du sens reçu est implicite, mais ne devrait pas être présumée. L'acte de partage implique une part ou un élément d'un tout dont la division est considérée comme raisonnable ou même égale. Pour développer un lien relationnel, le partage implique la mise en commun de parties d'un tout, c'est-à-dire d'éléments partagés. Par exemple, les membres d'une communauté peuvent avoir des valeurs et des croyances similaires ou, en d'autres termes, partager une façon de penser. Par conséquent, certains éléments sont inclus, alors que d'autres sont exclus.

Dans le contexte du conseil en orientation, la métaphore LA COMMUNICATION EST UN PARTAGE suggère que les significations peuvent être partagées à travers un récit (UNE HISTOIRE) en déduisant que le sens partagé (c'est-à-dire le message envoyé, reçu et décrypté) peut être interprété ou compris (c'est-à-dire qu'il a une signification donnée). Comme le souligne Krippendorff (1990), "par la métaphore de la communication en tant que partage, le statut objectif du contenu de la communication ne fait aucun doute, sauf si les 
communicateurs le sortent de son contenant» (p. 22, traduction personnelle). Selon ce courant de pensée, la communication est asymétrique et favorise la capacité à comprendre (c'est-à-dire à partager le sens correct) pour en assurer la réussite. L'alternative est une incapacité ou un manque de talent de communication dans le contexte situé de la conversation. Ce paradoxe remet en question la fiabilité du processus de communication, ou postule que le communicateur est peu compétent, au risque que sa signification soit rejetée, ou du moins remplacée en partie par ce que l'autre comprend comme étant correct ou véridique. En outre, la fixation des critères pour juger le sens repose sur l'hypothèse qu'il existe un sens ou une vérité (par exemple, vous ne comprenez pas ce que je veux dire) et que la réussite de la communication dépend de ce fait d'une ligne de raisonnement et de la position ou du rôle du locuteur (LA COMMUNICATION, C'EST LA MAÎTRISE).

Une métaphore de partage suppose de la confiance, des confidences, de l'amitié et des pratiques professionnelles. À l'inverse, le partage implique également des aspects moins souhaitables tels que le pouvoir, la domination, l'inégalité et la dépendance. Pour en revenir à la métaphore LA COMMUNICATION EST UN PARTAGE, ce partage lui-même implique des éléments partagés et non partagés. Ces deux éléments doivent être reconnus dans le processus de création de sens, étant donné que l'approche narrative du conseil en orientation se fonde sur la métaphore LA CARRIÈRE EST UNE HISTOIRE, elle-même encadrée par une métaphore selon laquelle LA COMMUNICATION EST UN PARTAGE, qui est intégrée dans une procédure normalisée, routinière ou institutionnalisée. À cette fin, nous présentons ci-après "Mon chapitre de carrière" (My Career Chapter) en tant qu'approche pratique du conseil de carrière narratif qui exprime métaphoriquement la carrière comme une conversation.

\section{MY CAREER CHAPTER, UNE CONVERSATION SEMI-DIRECTIVE}

My Career Chapter (MCC; McIlveen, 2006) est un outil pour l'évaluation et l'accompagnement en orientation qui permet à la ou au client.e d'écrire une brève autobiographie en lien avec sa carrière. Une caractéristique centrale du MCC est son format semi-dirigé. Les client.e.s sont invitéee.s à compéter des phrases (Loevinger, 1985) à partir d'une racine (par exemple, «mes principaux intérêts sont...»). Les phrases sont des sujets choisis, compris comme des 
influences professionnelles dans le cadre de la théorie systémique de la carrière (Patton \& McMahon, 2014). Ces sujets comprennent des influences intrapersonnelles (par exemple, intérêts, objectifs, santé, sexualité) et interpersonnelles proches des client.e.s (par exemple, pairs, famille) en plus des influences distales se situant au niveau social et environnemental (par exemple, conjonctures politique et économique). Les phrases s'expriment au passé, au présent et au futur, et permettent également de décrire la signification affective d'une influence professionnelle et son impact sur la ou le client.e.

Le manuscrit de l'autobiographie est au cœur de la conversation entre client.e et conseiller.ère. En outre, le MCC exige que les client.e.s entament une conversation avec elles ou eux-mêmes lors de la rédaction du manuscrit. Ce processus est emprunté à la théorie du soi dialogique (Hermans, 2006; Hermans \& Gieser, 2012; McIlveen \& Patton, 2007), au sens ou la ou le client.e raconte à haute voix le manuscrit autobiographique à un autre soi imaginaire - habituellement à soi-même cinq ans plus jeune. Ainsi, les client.e.s entendent leurs propres paroles à mesure qu'elles sont lues à haute voix. Les évaluations du MCC en matière de conseil ont révélé que le fait que les clients entendent leurs propres mots constitue une expérience significative (McIlveen, Patton, \& Hoare, 2008). Il y a donc deux phases: d'abord la ou le client.e du présent lit l'histoire à la ou au client.e du passé, puis la ou le client.e du passé parle à la ou au client.e du présent pour lui faire part de ses réactions à l'écoute de l'histoire de son propre avenir.

Le processus d'interprétation entre client.e.s et conseillers.ères nécessite que les conseillers.ères lisent le manuscrit à haute voix aux client.e.s. Cette lecture est une variante de la lecture que les client.e.s se font à elles ou eux-mêmes. Elles ou ils entendent ainsi leur histoire de l'extérieur et d'une autre voix, comme s'il s'agissait d'un livre. Imaginez comment vous ressentiriez le fait que quelqu'un d'autre vous lise votre autobiographie. Certain.e.s client.e.s peuvent être émus à l'écoute de leurs propres paroles lues à haute voix par quelqu'un d'autre. Les conseillers.ères et les client.e.s entament alors une discussion sur tout contenu ou moment particulier qui semble important pour l'un.e ou l'autre.

Le MCC n'est pas une simple autobiographie écrite comme un artefact de la vie d'une personne. Son processus assure la poursuite de conversations client.e-client.e et client.e-conseiller-ère. Une particularité importante du MCC est qu'il exige que la ou le 
client.e décrive par écrit (et en parle) un large éventail d'influences proximales et distales tirées du cadre de la théorie systémique de la carrière. Cela évite que client.e.s et conseillers.ères ne limitent leur discussion exploratoire à quelques facteurs, ce qui produit une histoire et une expérience plus étoffées ${ }^{2}$.

En résumé, le MCC offre aux conseillers.ères et aux client.e.s un outil pour générer un récit à la fois écrit et oral. Les processus d'écriture, de narration, d'écoute et de réflexion, mis en acte à partir de points de vue différents (en tant qu'auteur.e et éditeur-trice), mettent en pratique les principes théoriques du soi dialogique et de la théorie de la métaphore conceptuelle.

\section{LE CAS DE MARYANNE, INFIRMIÈRE}

Nous présentons ici le cas de Maryanne, afin de mettre en évidence certaines des caractéristiques du MCC. Le conseil en orientation de Maryanne a débuté par un entretien sur les systèmes de carrière (Career Systems Interview; McIlveen, 2003, 2015a), qui s'apparente à un entretien d'embauche normalisé, mais avec des caractéristiques empruntées au cadre de la théorie systémique de la carrière (Patton \& McMahon, 2014).

\subsection{ANALYSE DE LA DEMANDE}

Maryanne fait une demande pour un accompagnement en orientation, car elle remet en question son intérêt pour une formation en sciences infirmières. Maryanne occupe un poste d'accompagnatrice de personnes handicapées. Elle apprécie beaucoup ce travail et retire une grande satisfaction personnelle du fait de venir en aide à ses client.e.s et répondre à leurs besoins. N'ayant suivi qu'une formation de six mois, et ce, il y a quelques années déjà, elle ressent aujourd'hui le besoin d'un travail plus complexe et mieux rémunéré. Elle s'inquiète toutefois au sujet du coût de la formation pour devenir infirmière - non seulement en termes de frais d'écolage, mais également par le manque à gagner pendant les trois ans du bachelor en sciences infirmières. Bien que Maryanne puisse compenser l'investissement immédiat dans les études par de meilleures conditions de travail et de rémunération futures, elle se sent déconcertée et parfois en colère en raison de son indécision.

2. Les ressources pour l'administration du MCC sont disponibles gratuitement en ligne, en anglais (McIlveen, 2006, 2015a, 2015c; Mcllveen \& du Preez, 2012). 


\subsection{EXAMEN DE L'ÉTAT DE SANTÉ PSYCHOLOGIQUE}

Maryanne paraît plus âgée que ses 35 ans. Habillée avec soin, elle se montre courtoise et coopérative tout au long de l'entretien. Elle s'exprime normalement, avec l'accent de quelqu'un qui a grandi dans une zone rurale de l'Australie. D'humeur maussade et irritable, elle déclare qu'elle "veut juste prendre une décision et poursuivre sa vie». Ses affects semblent de portée et d'intensité normales, évoluant en fonction des sujets de conversation et de son humeur. Il n'y a aucune indication de troubles au niveau des pensées et des processus cognitifs ou perceptuels. De toute évidence, Maryanne est une personne lucide et prête à l'introspection, qui souhaite mieux comprendre ses difficultés actuelles et ses tendances à la procrastination.

\subsection{ANAMNÈSE}

Le premier entretien révèle d'importants éléments biographiques et un certain nombre de thèmes centraux. Concernant les premiers stades de son développement, Maryanne semble avoir grandi dans un contexte harmonieux. Elle a toutefois perdu son frère - décédé à 16 ans dans un accident tragique - alors qu'elle avait 12 ans, et a vécu son adolescence comme enfant unique, dans une petite communauté rurale où elle a terminé ses études secondaires, à 17 ans.

Maryanne a ensuite pris une année sabbatique pour parcourir l'Europe. Elle a trouvé du travail dans le secteur de la restauration, comme serveuse et barmaid. Son année sabbatique s'est prolongée sur trois ans, car Maryanne adorait le style de vie européen et ne voulait pas rentrer en Australie. Ses parents ont cependant insisté pour qu'elle rentre afin d'obtenir un diplôme et un emploi convenable. Â son retour, elle a entamé un cursus d'études commerciales, estimant que son travail dans les restaurants et les bars en Europe justifiait de poursuivre une carrière dans le secteur de l'hôtellerie et de la restauration. Elle fut pourtant loin d'être motivée par ses études, estimant que les cours théoriques ne ressemblaient en rien à la réalité du service et du contact avec la clientèle. Ses notes ont constamment baissé jusqu'à ce qu'elle finisse par abandonner le cursus en deuxième année. Ses parents, qui avaient financé ses études, étaient furieux et ont alors exigé qu'elle trouve un travail.

Après deux ans de petits boulots dans des bars, Maryanne a décidé de tout tenter pour sortir de la voie de garage dans laquelle elle s'était engagée. Le gouvernement recrutait à ce moment des 
accompagnateurs-trices de personnes en situation de handicap et offrait une formation entièrement subventionnée. Maryanne a posé sa candidature et a commencé à travailler dans une institution pour personnes âgées et invalides. Les premières années, Maryanne appréciait le travail, en particulier dans sa dimension d'aide et de soin aux personnes. Le salaire était toutefois modeste et le travail est devenu progressivement routinier et ennuyeux.

\subsection{ADMINISTRATION DU MCC}

Après avoir livré son histoire et exprimé son irritation et sa frustration face à son indécision, Maryanne a convenu avec la conseillère d'utiliser le MCC pour l'aider à élaborer une réflexion à partir de son récit et parvenir ainsi à de nouvelles perspectives quant à son passé, son présent et son avenir. De retour à la séance suivante avec son autobiographie MCC dûment rédigée, Maryanne a dit avoir trouvé le processus plutôt ardu, car elle rechignait à écrire les pensées qui lui venaient à l'esprit en réponse aux sujets proposés et aux phrases à compléter. Ce n'est qu'après de longues délibérations avec elle-même que Maryanne a pu écrire ses pensées et ses sentiments, terminant progressivement le récit de ce chapitre de sa vie.

\subsection{INTERPRÉTATION DU MCC}

Lors de la séance, la conseillère a lu l'autobiographie de Maryanne à haute voix. Au cours de la lecture, la conseillère a relevé des évocations systématiques de culpabilité associée à l'échec, de fuite face à ses peurs et du sentiment de ne jamais être «à la hauteur». C'est le sujet de la famille qui a suscité les préoccupations de Maryanne. La conseillère a lu à haute voix ce qu'elle avait écrit :

Il fut un temps où ma famille ne voulait pas de moi. Mon frère était le préféré de mes parents. Ma famille dit que je devrais grandir et commencer à me comporter comme une adulte. Je pense que ma famille ne sera jamais satisfaite de ce que je suis parce que j'ai toujours été une source de déception. J'ai des sentiments particulièrement négatifs par rapport à ma famille parce que je crois qu'ils n'ont jamais surmonté la mort de mon frère et que je les ai toujours déçus. La famille a un impact très négatif sur ma carrière, car je doute qu'ils s'intéressent réellement à mes aspirations ${ }^{3}$.

3. Le texte en italique représente les ébauches de phrases à compléter dans le MCC. 
En entendant ses propres mots, Maryanne éclate en sanglots et s'écrie: "Je porte cette honte et cette culpabilité en moi depuis des années et je ne sais pas qu'en faire", et "Je sais que je veux être infirmière, mais j'ai peur d'échouer, d'être la ratée que mes parents disent que j'ai toujours été».

Le thème de la honte et de la culpabilité, émergeant en réaction au processus d'écriture de son autobiographie, est devenu l'objectif central de la démarche d'orientation. Le travail de réflexion et d'accompagnement a porté sur la façon dont Maryanne a formé ces idées sur elle-même et ses relations avec ses parents. Les interventions visant à soulager sa souffrance psychologique et son indécision ont porté à une nouvelle lecture et interprétation de son passé à l'aide de la thérapie d'acceptation et d'engagement (ACT; Hoare, McIlveen, \& Hamilton, 2012), mais également à une reprise de contact avec ses parents afin de leur communiquer ses ambitions pour l'avenir.

Pour Maryanne, le but de l'administration du MCC n'était pas de découvrir ce qu'elle voulait faire de sa carrière. Il s'agissait plutôt de dévoiler ce qu'elle avait été jusque-là incapable d'exprimer explicitement, et de l'intégrer dans une histoire d'elle-même qui ait un effet de rédemption pour le passé et le présent, et qui soit source d'ouverture et de possibilités pour l'avenir. Ainsi, le MCC facilite les conversations avec soi-même et autrui, qui constituent le processus de mise en récit d'une histoire.

\section{PERSPECTIVES FUTURES DE RECHERCHE}

\section{ET DE DÉVELOPPEMENT : IMPASSE OU INVITATION?}

Sous le prisme de la métaphore, le conseil en orientation en tant que conversation peut être compris à travers des expressions comme LA COMMUNICATION EST PARTAGE Et LA COMMUNICATION EST CONTRÔLE. Ce paradoxe entre partage et contrôle questionne la vocation émancipatrice du conseil de carrière narratif, qui vise à donner la parole aux client.e.s. Comment cette louable intention peut-elle être mise en œuvre en présence du paradoxe partage-contrôle qui pourrait altérer la transparence du dialogue? Y a-t-il une solution? Est-ce une impasse ou une invitation à approfondir l'analyse philosophique du conseil en orientation?

Jusqu'ici, l'approche narrative du conseil en orientation n'a pas fait l'objet d'une grande analyse critique ou de suffisamment de recherches empiriques démontrant son efficacité, ce qui est compréhensible au vu 
de son apparition relativement récente. Il est donc temps d'éclaircir l'axiologie de l'approche narrative du conseil en orientation: le récit et la conversation en représentent le substrat, et ces phénomènes sont soumis aux intentions de partage et aux tentatives de contrôle. Les tenants du conseil de carrière narratif ne peuvent nier que le dialogue intra et intersubjectif est en constante évolution à travers les entretiens, parfois de façon fortuite. Comment les praticien-ne.s réussissent-elles ou ils à rester centré.e.s sur les client.e.s, tout en sachant que leurs pratiques dialogiques ne sont ni objectives, ni transparentes et qu'elles sont, de plus, soumises à un examen minutieux? La réponse à cette question nécessite une analyse philosophique de l'approche narrative du conseil en orientation en termes d'axiologie et d'éthique.

McIlveen (2015b) soutient que le conseil en orientation est actuellement à un carrefour où les principes éthiques devraient être au centre des préoccupations de la théorie et de la pratique. Même s'il est a priori louable que les conseillers.ères adoptent des valeurs qui glorifient l'histoire de leurs client.e.s, il semble tout aussi important que le caractère éthique de ces valeurs fasse l'objet d'une analyse critique. En dépit de l'attrait conceptuel et pragmatique de l'approche narrative du conseil en orientation et de la métaphore de la conversation, il faut mener davantage de recherches sur les processus et les résultats du conseil de carrière narratif, et ce non seulement pour assurer qu'il reste fidèle à la théorie, mais aussi pour établir son efficacité. L'alliance de travail est pertinente pour l'efficacité du conseil et un corpus de recherches empiriques en matière de conseil en orientation est en train de se constituer (Elad-Strenger \& Littman-Ovadia, 2012; Masdonati, Massoudi, \& Rossier, 2009; Masdonati et al., 2014; Whiston et al., 2015). Étant donné le manque de travaux sur la prise en compte des émotions en orientation professionnelle (Hartung, 2011; Kidd, 2008), alors même que l'émotion est intrinsèque à la narration, nous recommandons de poursuivre les recherches sur l'alliance de travail en mettant davantage l'accent sur les émotions suscitées lors de l'écriture, la narration et l'écoute d'une histoire et dans les réflexions entourant ce récit.

\section{CONCLUSION}

Nous avons retracé les fondements de l'approche narrative du conseil en orientation dans l'histoire, le récit et la conversation. Le constructionnisme social est le paradigme idéal pour comprendre 
ces phénomènes, car ce sont essentiellement des actes sociaux, accomplis en présence d'autrui, qui font de l'identité professionnelle une construction sociale susceptible de se manifester dans les conversations de tous les jours. Le dispositif MCC met en pratique la métaphore de la conversation dans le conseil en orientation. Il permet ainsi la tenue de conversations entre différents acteurs trices dédié.e.s au développement de la carrière des client.e.s, l'autobiographie étant le point de mire autour duquel s'articulent ces conversations.

\section{RÉFÉRENCES BIBLIOGRAPHIQUES}

Alchin, C., Mcllveen, P., \& Perera, H. N. (2018). Negative career outlook and the working alliance in career counseling. Career Development Quarterly, 66 (3), 279-284. Doi : 10.1002/cdq.12149 Baumeister, R. F., \& Masicampo, E. J. (2010). Conscious thought is for facilitating social and cultural interactions: How mental simulations serve the animal - culture interface. Psychological Review, 117 (3), 945-971. Doi: 10.1037/a0019393.

Cameron, L. (2003). Metaphor in educational discourse. Londres: Continuum Press.

Cochran, L. (1997). Career counseling: A narrative approach. Thousand Oaks (CA): Sage Publications.

Croft, W., \& Cruse, D. A. (2004). Cognitive linguistics. Cambridge: Cambridge University Press.

Demmen, J., Semino, E., Demjén, Z., Koller, V., Hardie, A., RaYSON, P., \& PAYNe, S. (2015). A computer-assisted study of the use of Violence metaphors for cancer and end of life by patients, family carers and health professionals. International Journal of Corpus Linguistics, 20 (2), 205-231. Doi: Doi : 10.1075/ijcl.20.2.03dem.

Elad-Strenger, J., \& Littman-Ovadia, H. (2012). The contribution of the counselor - client working alliance to career exploration. Journal of Career Assessment, 20 (2), 140-153. Doi: 10.1177/1069072711420850.

Gergen, K. J. (1991). The saturated self: Dilemmas of identity in contemporary life. New York: Basic Books. 
Gibis, R. W. (2017). Metaphor wars: conceptual metaphors in human life. Cambridge: Cambridge University Press.

GibBs, R. W., \& Franks, H. (2002). Embodied metaphor in women's narratives about their experiences with cancer. Health Communication, 14 (2), 139-165. Doi: 10.1207/S15327027HC1402_1.

Hartung, P. J. (2011). Barrier or benefit? Emotion in lifecareer design. Journal of Career Assessment, 19 (3), 296-305. Doi: $10.1177 / 1069072710395536$.

Hartung, P. J. (2015). Career as story: Making the narrative turn. In B. W. Walsh, M. L. Savickas, \& P. J. Hartung (éds), Handbook of vocational psychology: Theory, research, and practice ( $4^{\mathrm{e}}$ édition, pp. 33-52). New York: Routledge.

Hermans, H. J. M. (2006). The self as a theater of voices: Disorganization and reorganization of a position repertoire. Journal of Constructivist Psychology, 19(2), 147-169. Doi: 10.1080/10720530500508779. Hermans, H. J. M., \& Gieser, T. (éds). (2012). Handbook of dialogical self theory. Cambridge: Cambridge University Press.

Hoare, P. N., McIlveen, P., \& Hamilton, N. (2012). Acceptance and commitment therapy (ACT) as a career counselling strategy. International Journal for Educational and Vocational Guidance, 1-17. Doi : $10.1007 / \mathrm{s} 10775-012-9224-9$.

Horvath, A. O., \& Greenberg, L. S. (1989). Development and validation of the Working Alliance Inventory. Journal of Counseling Psychology, 36, 223-233. Doi : 10.1037/0022-0167.36.2.223.

InKSON, K. (2004). Images of career: Nine key metaphors. Journal of Vocational Behavior, 65 (1), 96-111. Doi: 10.1016/S0001-8791 (03) 00053-8.

KIDD, J. M. (2008). Exploring the components of career wellbeing and the emotions associated with significant career experiences. Journal of Career Development, 35 (2), 166-186. Doi: $10.1177 / 0894845308325647$.

KrippendorfF, K. (1990). Models and metaphors of communication. Media and communication, construction of realities. En ligne: [http://repository.upenn.edu/asc_papers/276].

Lakoff, G., \& Johnson, M. (1980). Metaphors we live by. Chicago: University of Chicago Press. 
Lengelle, R., \& Mejers, F. (2014). Narrative identity: Writing the self in career learning. British Journal of Guidance \& Counselling, 42 (1), 52-72. Doi: 10.1080/03069885.2013.816837.

LOEVINGER, J. (1985). Revision of the sentence completion test for ego development. Journal of personality and social psychology, 48, 420-427. Doi: 10.1037/0022-3514.48.2.420.

Maree, K. (éd.). (2011). Shaping the story: A guide to facilitating narrative counselling. Rotterdam: Sense Publishers.

Masdonati, J., Massoudi, K., \& Rossier, J. (2009). Effectiveness of career counseling and the impact of the working alliance. Journal of Career Development, 36 (2), 183-203. Doi: $10.1177 / 0894845309340798$.

Masdonati, J., Perdrix, S., Massoudi, K., \& Rossier, J. (2014). Working alliance as a moderator and a mediator of career counseling effectiveness. Journal of Career Assessment, 22 (1), 3-17. Doi : $10.1177 / 1069072713487489$.

McAdams, D. P. (1993). The stories we live by. Personal myths and the making of the self. New York: William Morrow \& Company.

McAdams, D. P. (2008). Personal narratives and the life story. In J. Oliver, R. Robins, \& L. Pervin (éds), Handbook of personality: Theory and research ( $3^{\mathrm{e}}$ édition). New York: The Guilford Press.

McAdams, D. P., \& McLean, K. C. (2013). Narrative identity. Current Directions in Psychological Science, 22 (3), 233-238. Doi: 10.1177/0963721413475622.

McIlveen, P. (2003). Career Systems Interview (non publié). University of Southern Queensland, Toowoomba, Australie.

McIlveen, P. (2006). My Career Chapter: A dialogical autobiography. En ligne: [http://eprints.usq.edu.au/id/eprint/23797].

McIlveen, P. (2012). Extending the metaphor of narrative to dialogical narrator. In P. McIlveen \& D. E. SchultheIss (éds), Social constructionism in vocational psychology and career development (pp. 59-75). Rotterdam: Sense Publishers.

McIlveen, P. (2015a). My Career Chapter and the Career Systems Interview. In M. McMaHOn \& M. WATSON (éds), Career assessment: Qualitative approaches (pp. 123-128). Rotterdam: Sense Publishers. 
MCIlveEn, P. (2015b). The next horizon of career counselling. In J. G. Maree \& A. DI FABIO (éds), Exploring new horizons in career counselling: Turning challenges into opportunities (pp. 331-339). Rotterdam: Sense Publishers.

MCIlveen, P. (2015c). Using My Career Chapter in career counselling. In M. McMahon \& W. Patton (éds), Ideas for career practitioners (2 édition, pp. 188-190). Samford Valley: Australian Academic Press.

McIlveen, P. (2017). Dialogical self: Co-investigator in career self-research. In M. McMaHON (éd.), Career counselling: Constructivist approaches (pp. 153-163). Londres: Routledge.

McIlveen, P., \& DU Preez, J. (2012). A model for the co-authored interpretation of My Career Chapter. Cypriot Journal of Educational Sciences, 7 (4), 276-286.

McIlveen, P., \& Patton, W. (2007). Dialogical self: Author and narrator of career life themes. International Journal for Educational and Vocational Guidance, 7 (2), 67-80. Doi: 10.1007/s10775-007-9116-6.

McIlveen, P., Patton, W., \& Hoare, P. N. (2008). An interpretative phenomenological analysis of clients'reactions to My Career Chapter. Australian Journal of Career Development, 17 (3), 51-62. Doi: $10.1177 / 103841620801700308$.

McMahon, M. (2017). Working with storytellers: A metaphor for career counselling. In M. McMahon \& W. PatTon (éds), Career counselling: Constructivist approaches (pp. 17-28). Londres: Routledge.

McMahon, M., \& Watson, M. (2012). Story crafting: strategies for facilitating narrative career counselling. International Journal for Educational and Vocational Guidance, 12 (3), 211-224. Doi: 10.1007/s10775-012-9228-5.

Meijers, F., \& Lengelle, R. (2012). Narratives at work: The development of career identity. British Journal of Guidance \& Counselling, 40 (2), 157-176. Doi : 10.1080/03069885.2012.665159.

Multon, K. D., Ellis-Kalton, C. A., Heppner, M. J., \& Gysbers, N. C. (2003). The relationship between counselor verbal response modes and the working alliance in career counseling. The Career Development Quarterly, 51 (3), 259-273. Doi: 10.1002/j.21610045.2003.tb00606.x.

Musolff, A. (2000). Political Imagery of Europe: A House Without 
Exit Doors? Journal of Multilingual and Multicultural Development, 21 (3), 216-229. Doi : 10.1080/01434630008666402.

Musolff, A. (2004). The Heart of the European Body Politic. British and German Perspectives on Europe's Central Organ. Journal of Multilingual and Multicultural Development, 25 (5-6), 437-452. Doi: 10.1080/01434630408668917.

Musolff, A. (2017). Truths, lies and figurative scenarios. Journal of Language and Politics, 16 (5), 641-657. Doi: Doi: 10.1075/ jlp.16033.mus.

Patton, W., \& McMahon, M. (2014). Career development and systems theory: Connecting theory and practice. ( $3^{\mathrm{e}}$ édition). Rotterdam: Sense Publishers.

Polkinghorne, D. E. (1988). Narrative knowing and the human sciences. Albany: State University of New York Press.

SARBIN, T. R. (éd.) (1986). Narrative psychology: The storied nature of human conduct. New York: Praeger.

SAVICKAS, M. L. (2011). Career counseling. Washington, DC: American Psychological Association.

Semino, E., Demjén, Z., \& Demmen, J. (2016). An integrated approach to metaphor and framing in cognition, discourse, and practice, with an application to metaphors for cancer. Applied Linguistics. Doi : 10.1093/applin/amw028.

Semino, E., Demjen, Z., Demmen, J., Koller, V., Payne, S., HarDIE, A., \& RaYson, P. (2017). The online use of violence and journey metaphors by patients with cancer, as compared with health professionals: a mixed methods study. BMJ Supportive and Palliative Care. Doi : 10.1136/bmjspcare-2014-000785.

STEEN, G. (2009). Deliberate metaphor affords conscious metaphorical cognition. Cognitive Semiotics, 5 (1-2), 179-197).

SteEn, G. (2017). Deliberate Metaphor Theory: Basic assumptions, main tenets, urgent issues. Intercultural Pragmatics, 14 (1), 1-24. Doi : 10.1515/ip-2017-0001.

Tracey, T. J., \& Kokotovic, A. M. (1989). Factor structure of the Working Alliance Inventory. Psychological Assessment: A Journal of Consulting and Clinical Psychology, 1 (3), 207-210. Doi: 10.1037/1040-3590.1.3.207. 
Varela, F. J., Thompson, E., \& Rosch, E. (2017). The embodied mind: Cognitive science and human experience. Cambridge: MIT press. Whiston, S. C., Rossier, J., \& Barón, P. M. H. (2015). The working alliance in career counseling: A systematic overview. Journal of Career Assessment, 24 (4), 591-604. Doi: 10.1177/1069072715615849.

Xu, H., \& Tracey, T. J. G. (2015). Reciprocal influence model of working alliance and therapeutic outcome over individual therapy course. Journal of Counseling Psychology, 62 (3), 351-359. Doi: $10.1037 /$ cou0000089. 



\section{CONCLUSION}

JÉRÔME ROSSIER ET JEAN-PIERRE DAUWALDER

(UNIVERSITÉ DE LAUSANNE\}

es psychologues conseillers.ères en orientation ont depuis toujours la tâche importante, mais compliquée de faire le lien entre les aspirations et envies individuelles et les besoins et exigences de la société, en particulier de ses acteurs économiques et politiques. Ainsi, l'orientation consiste à la fois à accompagner des transitions vocationnelles et professionnelles et à aider les individus à se positionner sur le marché du travail. L'orientation répond donc à une double ou triple exigence dont les finalités sont différentes, centrées sur le développement de l'individu en soutenant l'insertion sociale et professionnelle. Les professionnel.le.s de la psychologie de l'orientation doivent ainsi savoir gérer l'ensemble de ces attentes et exigences en maintenant un équilibre parfois fragile, ce qui nécessite une sensibilité particulière à ces enjeux multiples et un niveau de compétences élevé (Schiersmann, Ertelt, Katsarov, Mulvey, Reid, \& Weber, 2012).

Les cinq dernières décennies ont donné lieu à une accélération de l'évolution de nos sociétés, qui se trouvent ainsi confrontées à de nouveaux défis qui transforment aussi notre profession. Ces évolutions sont à la fois technologiques, avec la digitalisation et la robotisation, économiques, avec une internationalisation des échanges, des parcours et des savoirs, une réorganisation des outils de production ainsi qu'une désindustrialisation des sociétés occidentales, et sociales, avec une augmentation des flux migratoires et une perte de confiance dans les classes dirigeantes. Ces évolutions ont une incidence importante sur la structure du marché du travail (par exemple, le secteur des médias imprimés a perdu la moitié des places de travail ces dix dernières années) et sur la nature même du travail, la plupart des emplois ayant été impactés par le tournant numérique (Armez-Droz, 2015). De manière générale, les tâches 
manuelles routinières et administratives semblent être en diminution, alors que les emplois du secteur des services, ainsi que les emplois intermédiaires et intellectuels connaissent, quant à eux, une augmentation (Aepli, Angst, Iten, Kaiser, Lüthi, \& Schweri, 2017). L'incidence à long terme de ces changements sur le nombre d'emplois reste encore débattue (voir, par exemple, Arntz, Gregory, $\&$ Zierahn, 2016). Cependant, il est certain que la nature du travail va continuer à évoluer rapidement. Il semblerait que ces évolutions induisent une augmentation des emplois précaires, qui tendent à être occupés plutôt par des personnes qui sont en situation de vulnérabilité. On peut donc parler de dynamiques de précarisation et de dualisation (Hipp, Bernhardt, \& Allmendingen, 2015). Ces évolutions rapides entraînent également une obsolescence rapide des savoirs acquis et la nécessité pour la plupart des individus de s'engager dans un processus d'apprentissage permanent. Nos sociétés sont également confrontées à d'autres défis importants, tant démographiques que climatiques, qui remettent en cause nos modes d'existence et qui pourraient contribuer à une instabilité sociale à venir (Werrel \& Femia, 2018).

Dans cet environnement dynamique, les parcours de vie et professionnels tendent à se diversifier. On observe une dé-standardisation des parcours et l'apparition de carrières dites protéiformes (Abessolo, Hirschi, \& Rossier, 2017). Le contexte exigeant que l'individu soit l'acteur de son parcours, c'est à cet individu que revient la tâche de lui trouver du sens, en lien notamment avec ses valeurs et ses objectifs, le champ social offrant un cadre de valeurs partagées plus diversifié et moins normatif (Massoudi et al., 2018). Cet environnement implique aussi que les individus sont confrontés à davantage de transitions professionnelles, chacune pouvant potentiellement représenter une situation de vulnérabilité. Si la question de la gestion des transitions devient cruciale dans un monde globalisé, il en est de même de celle des conditions de travail. Bénéficier d'un travail décent est essentiel au bien-être et à la dignité des individus (Masdonati, Schreiber, Marcionetti, \& Rossier, 2019), comme souligné par l'Organisation des Nations unies (ONU) dans sa Déclaration universelle des droits de l'homme de 1948: "Toute personne a droit au travail [...]. Quiconque travaille a droit à une rémunération équitable et satisfaisante lui assurant [...] une existence conforme à la dignité humaine» (art. 23, al. 1 et 3, p. 75). Il est également spécifié que «toute personne a droit à 
un niveau de vie suffisant pour assumer sa santé, son bien-être et ceux de sa famille [...]; elle a droit à la sécurité [...]» (art. 25, al. 1, p. 76). Si l'on veut garantir l'accès à un travail décent et à une carrière durable pour tou.te.s comme le préconise l'Agenda 2030 des Nations unies (2015), il faut notamment garantir l'accès à des prestations d'accompagnement à l'orientation et à l'apprentissage tout au long de la vie. Une carrière durable implique de pouvoir collectivement garantir une sécurité lors de ces transitions professionnelles (De Vos \& Van Der Heijden, 2015). Ces évolutions ont également une incidence sur le rôle des psychologues conseillers.ères en orientation, qui sont amené·e.s à gérer une grande diversité de situations qui surviennent à différents moments de la vie et à travailler en collaboration avec d'autres professionnel.le.s, notamment dans le cadre de prestations d'aide à l'insertion sociale et professionnelle, mais de plus en plus aussi au-delà, au sein des organisations politiques, économiques et sociales multiples. Pour ces raisons, notre société semble avoir toujours davantage besoin de professionnel.le.s hautement qualifié.e.s dans notre domaine.

Différentes approches théoriques nouvelles dans le domaine de l'orientation, comme celle du Life Design (Savickas et al., 2009) ou de la théorie de la psychologie de l'activité de travail (Blustein, 2013), mettent l'accent sur l'importance de considérer les individus dans leurs contextes et de proposer des interventions qui leur permettent de développer leurs subjectivités, leurs réflexivités, leurs intentionnalités, leurs ressources et leurs capacités à gérer leurs parcours de vie (Nota \& Rossier, 2015). Ces approches entendent favoriser l'émancipation, l'agentivité et le bien-être individuel en encourageant notamment le développement de ressources comme la volition professionnelle, l'adaptabilité de carrière, la capacité à se projeter dans l'avenir ou encore l'espoir. Un des avantages d'une approche centrée sur les ressources individuelles est qu'il est possible de les activer et de les développer (Rossier, 2015). Les prestations dans le domaine de l'orientation ont pour vocation de soutenir l'insertion professionnelle, notamment celle des populations les plus vulnérables, et leur efficacité a été largement démontrée (Whiston, Rossier, \& Hernandez Barón, 2017). Il serait cependant intéressant de poursuivre l'étude des dynamiques du changement lors d'interventions d'orientation et de développer davantage la recherche sur les interventions préventives systématiques. Un autre défi consistera à continuer à garantir l'accès à nos prestations, notamment aux 
personnes qui en ont le plus besoin, sans toujours la solliciter. Sans avoir la prétention de traiter de l'ensemble des thématiques importantes qui mettent à l'épreuve notre pratique, cet ouvrage s'est proposé de stimuler la réflexion des professionnel-le.s de l'orientation sur un certain nombre d'enjeux contemporains. Les questions de l'insertion, des inégalités et de l'inclusion ont été illustrées à travers les cas spécifiques des jeunes adultes, des migrants ou des personnes en situation de handicap. De toute évidence, la promotion d'une société inclusive et socialement juste implique de tenir compte d'un plus grand nombre d'aspects ou réalités. Il ne faut pas perdre de vue que la question de la justice sociale est centrale si l'on souhaite promouvoir une société et des carrières durables (IAEVG, 2013).

Pour que nos pratiques puissent continuer à répondre aux attentes des individus et de la société, il est important que nos modèles de développement de carrière et les interventions qui en découlent (1) soient adaptés aux problèmes émergents du monde du travail, tels que la précarisation des emplois, la multiplication des transitions ou la promotion du sens au travail ; (2) tiennent compte tout particulièrement des populations qui ne parviennent pas à accéder à un travail décent ou à une insertion socioprofessionnelle satisfaisante en réduisant l'impact des barrières et obstacles qu'elles peuvent rencontrer; et (3) considèrent que le travail sous ses différentes formes et dans ses différents contextes culturels. S’il est crucial de comprendre l'individu dans son parcours et sa singularité, comme en témoigne la première partie de cet ouvrage, nos interventions devraient également tenir compte des contraintes et vulnérabilités des individus, tout en visant au développement de leurs ressources, ainsi que relevé dans les deux autres parties de notre ouvrage. Pour terminer, il ne faut jamais oublier que nos actions s'inscrivent dans un contexte social, politique et économique. Elles devraient ainsi être menées sur un plan tant individuel que collectif, si l'on veut relever le défi d'une insertion sociale et professionnelle durable et digne pour tou.te.s. Nos interventions, tout comme les actions des pouvoirs publics, devraient donc avoir pour vocation de favoriser le développement du capital humain, social et psychologique. 


\section{RÉFÉRENCES BIBLIOGRAPHIQUES}

Abessolo, M., Hirschi, A., \& Rossier, J. (2017). Work values underlying protean and boundaryless career orientations. Career Development International, 22, 241-259. Doi : 10.1108/CDI-102016-0167.

Aepli, M., Angst, V., Iten, R., Kaiser, H., LÜthi, I., Schweri, J. (2017). Die Entwicklung der Kompetenzanforderungen auf dem Arbeitsmarkt im Zuge der Digitalisierung. Zollikofen: OBS IFFP/ INFRAS.

Armez-Droz, Ph. (2015). Médias suisses à l'ère numérique. Lausanne: Presses polytechniques et universitaires romandes.

Arntz, M., Gregory, T., \& Zierahn, U. (2016). The risk of automation for jobs in OECD countries: A comparative analysis. OECD Social, Employment and Migration Working Papers, $\mathrm{n}^{\circ} 189$. Doi : $10.1787 / 5$ jlz9h56dvq7-en.

Blustein, D. L. (éd.). (2013). The Oxford handbook of the psychology of working. New York: Oxford University Press. Doi: 10.1093/ oxfordhb/9780199758791.001.0001.

De Vos, A., \& Van Der Heijden, B. I. J. M. (2015). Handbook of research on sustainable careers. Cheltenham: Edward Elgar Publishing.

Hipp, L., Bernhardt, J., \& Allmendinger, J. (2015). Institutions and the prevalence of nonstandard employment. Socio-Economic Review, 13, 351-377. Doi: 10.1093/ser/mwv002.

IAEVG (2013, septembre). Communiqué on social justice in educational and career guidance and counselling. Montpellier. En ligne: [https://iaevg.com/Resources\#Communiques].

Masdonati, J., Schreiber, M., Marcionetti, J., \& Rossier, J. (2019). Decent work in Switzerland: Context, conceptualization, and assessment. Journal of Vocational Behavior, 110, 12-27. Doi: 10.1016/j.jvb.2018.11.004.

Massoudi, K., Abessolo, M., Atitsogbe, K. A., Banet, E., Bollmann, G., DauWalder, J.-P., ... Rossier, J. (2018). A value-centered approach to decent work. In V. Cohen-Scali, J. Pouyaud, V. M. Podgorny, V. Drabik-Podgorna, G. Aisenson, J.-L. Bernaud, I. A. Moumoula, \& J. Guichard (éds), Interventions in 
career design and education: Transformation for sustainable development and decent work (pp. 93-110). Cham: Springer.

Nota, L., \& Rossier, J. (éds) (2015). Handbook of the Life Design paradigm: From practice to theory and from theory to practice. Göttingen: Hogrefe.

Organisation des Nations unies, Assemblée générale (10 décembre 1948). Déclaration universelle des droits de l'homme. A/RES/217 (III) A. En ligne: [http://www.un.org/en/ga/search/view_doc. asp?symbol=A/RES/217 (III)]

Organisation des Nations unies, Assemblée générale (21 octobre 2015). Transformer notre monde: le Programme de développement durable à l'horizon 2030. A/RES/70/1. En ligne: [http://www. un.org/fr/documents/view_doc.asp?symbol=A/RES/70/1].

Rossier, J. (2015). Personality assessment and career interventions. In P. J. Hartung, M. L. Savickas, \& W. B. Walsh (éds), APA handbook of career intervention: Foundations (vol. 1, pp. $327-$ 350). Washington, DC: American Psychological Association. Doi : 10.1037/1438-018.

Savickas, M. L., Nota, L., Rossier, J., Dauwalder, J.-P., Duarte, E., Guichard, J., Soresi, S., Van Esbroeck, R., \& van Vianen, A. E. M. (2009). Life designing: A paradigm for career construction in the 21st century. Journal of Vocational Behavior, 75, 239-250. Doi: $10.1016 / j . j v b .2009 .04 .004$.

Schiersmann, C., Ertelt. B. J., Katsarov, J., Mulvey, R., Reid, H., \& WeBER, P. (éds) (2012): NICE Handbook for the Academic Training of Career Gidance and Counseling Professionals. Heidelberg: IBW der Universität Heidelberg. En ligne: [www.nice-network.eu].

Werrel, C.E. \& Femia, F. (2018) : Avec le changement climatique, la menace de nouveaux conflits. Courrier de l'Unesco, Grand Angle. 2018-2. e-ISSN : 2220-2227.

Whiston, S. C., Rossier, J., \& Hernandez Barón, P. M. (2017). Evidence-based practice in career and workforce development interventions. In V. S. H. SOlbERG \& S. R. Ali (éds), The handbook of career and workforce development: Research, practice, and policy (pp. 39-56). New York: Routledge/Taylor \& Francis Group. 


\section{LES AUTEUR·E·S}

Marc Abessolo, docteur en psychologie, est psychologue du travail et des organisations, ainsi que psychologue conseiller en orientation professionnelle, universitaire et de carrière. Ses travaux et intérêts de recherche portent sur l'évaluation des valeurs professionnelles, des orientations contemporaines de carrière et du succès de carrière. Il a notamment publié une nouvelle échelle de mesure des valeurs de carrière (le Career Values Questionnaire) dans le Journal of Career Development.

Kokou A. Atitsogbe est chercheur FNS junior à l'Institut de psychologie de l'Université de Lausanne, ainsi que doctorant et membre du Centre de recherche en psychologie du conseil et de l'orientation (CePCO). Ses recherches portent sur les intérêts professionnels, la transition formation-emploi, la personnalité, la psychométrie et la psychologie interculturelle. Il a récemment publié "Vocational interests and career indecision in Switzerland and Burkina Faso" dans la revue Journal of Vocational Behavior.

Éloise Banet est psychologue conseillère en orientation au Service de l'orientation professionnelle et de la formation des adultes du Canton de Fribourg et titulaire d'une licence en droit de l'Université de La Réunion. Elle intervient plus spécifiquement auprès des jeunes adultes et des adultes. Ses travaux de recherche de doctorat en psychologie du conseil et de l'orientation à l'Université de Lausanne portent sur l'utilisation de l'humour dans le cadre du processus d'orientation.

Jean-Luc Bernaud est professeur en psychologie au Conservatoire national des arts et métiers (CNAM) à Paris et membre du laboratoire CRTD (Centre de recherches sur le travail et le développement). 
Ses recherches portent sur le conseil en carrière et le sens du travail et de la vie dans une perspective existentielle. Auteur de plus de 130 publications, il a récemment fait paraître Introduction à la psychologie existentielle (Éditions Dunod).

David L. Blustein est professeur au Département de psychologie du counseling, du développement et de l'éducation du Boston College (États-Unis). Il est l'auteur des ouvrages The Psychology of Working: A New Perspective for Career Development, Counseling, and Public Policy et The Importance of Work in an Age of Uncertainty: The Eroding Experience of Work in America. Sa page web institutionnelle est la suivante: https://www.bc.edu/bc-web/schools/ lsoe/faculty-research/faculty-directory/david-blustein.html.

Grégoire Bollmann, $\mathrm{PhD}$, est enseignant-chercheur à l'Université de Zurich. Au croisement de la psychologie du conseil en orientation et du management, ses travaux portent sur les dynamiques entre les ressources d'adaptabilité, la carrière et le bien-être à la suite d'événements de vie majeurs, et sur les contextes d'émergence des comportements contre-productifs et du sentiment de justice au travail. Ils ont paru entre autres dans le Journal of Management Studies et le Journal of Vocational Behavior.

Bruno Bourassa est professeur au Département des fondements et pratiques en éducation et chercheur régulier du Centre de recherche et d'intervention sur l'éducation et la vie au travail (CRIEVAT) de l'Université Laval (Québec). Ses travaux portent plus spécifiquement sur l'analyse et la transformation des pratiques d'intervention socioéducative et d'orientation en contexte de formation et de recherche collaborative. L'étude des processus d'apprentissage expérientiel et d'accompagnement éducatif et clinique y occupe une place privilégiée.

Manon Chamberland est professeure au Département des fondements et pratiques en éducation à l'Université Laval (Québec) et membre du Centre de recherche et d'intervention sur l'éducation et la vie au travail (CRIEVAT). Ses enseignements et recherches portent notamment sur l'éducation des adultes, la supervision et l'accompagnement, les pratiques éducatives émancipatrices, le développement du pouvoir d'agir des personnes et des collectivités, ainsi que l'interculturalité. 
Eva Clot-Siegrist est maître d'enseignement et de recherche à l'Institut de psychologie et membre du Centre de recherche en psychologie du conseil et de l'orientation (CePCO) de l'Université de Lausanne. Elle supervise et forme les étudiant.e.s de master lors de leur initiation à la pratique professionnelle en tant que psychologues, conseiller-ère.s en orientation au Service de consultations en conseil et orientation.

Valérie Cohen-Scali est professeure en psychologie de l'orientation et de l'insertion à l'Institut national d'études du travail et de l'orientation professionnelle (Inetop) du Conservatoire national des arts et métiers (CNAM, Paris). Elle dirige l'équipe de recherche Inetop-Psychologie de l'orientation du Centre de recherche sur le travail et le développement (CRTD) et le master de psychologie "Travail, santé et parcours professionnels». Ses travaux concernent les transitions scolaires et professionnelles, l'évolution du rapport au travail et les interventions de conseil basées sur les dialogues.

Allison Creed, $\mathrm{PhD}$, est conceptrice de programmes à l'Université de Melbourne, avec une expertise en éducation et en linguistique appliquée. Elle est membre du Researching and Applying Metaphor Executive Committee et des équipes de recherche du University of Amsterdam Metaphor Lab, du Inland Norway University of Applied Sciences Faculty of Education et de la University of Southern Queensland ACCELL. Ses recherches s'appuient sur une analyse de la métaphore pour cerner des questions relatives à l'enseignement supérieur, à l'employabilité et à la commercialisation du vin.

Jean-Pierre Dauwalder est professeur émérite, ancien titulaire de la Chaire de psychologie du conseil et de l'orientation de l'Université de Lausanne. Ses recherches portent sur le counseling, le Life Design, les thérapies cognitivo-comportementales, la réhabilitation et l'orientation professionnelle. Il préside la Commission fédérale des professions de la psychologie (PSYKO), est ancien président de l'European Society for Vocational Designing and Career Counseling (ESVDC) et membre de l'International Life Design Research Group.

Ilaria Di Maggio, PhD, est chargée de recherche au Département de philosophie, sociologie, éducation et psychologie appliquée de l'Université de Padoue (Italie). Elle collabore avec le Laboratoire 
de recherche et d'intervention en orientation professionnelle (La.R.I.O.S.) et le Centre universitaire sur le handicap et l'inclusion. Elle est membre de la Société italienne pour l'orientation (SIO) et de la Société italienne pour l'analyse et la modification du comportement et la thérapie cognitivo-comportementale (AIAMC). Ses domaines de recherche sont le counseling d'orientation et la psychologie du handicap et de l'inclusion.

Federico Durante est psychologue conseiller en orientation à l'Office pour l'orientation, la formation professionnelle et continue à Genève. Depuis 1998, il exerce essentiellement auprès de jeunes en transition professionnelle, allophones et/ou primo-arrivants. En parallèle, maître d'enseignement et de recherche à l'Université de Lausanne, il supervise des étudiant.e.s en master de psychologie du conseil et de l'orientation lors de leur pratique de terrain au Service de consultations de l'Institut de psychologie.

Sylvie Franz est maître d'enseignement et de recherche à l'Institut de psychologie et membre du Centre de recherche en psychologie du conseil et de l'orientation (CePCO) de l'Université de Lausanne. Ses enseignements portent sur la supervision des étudiant.e.s dans leur formation pratique et l'orientation scolaire. Elle accompagne également des adultes en transition au sein d'une association privée. Ses domaines d'intérêt sont notamment l'analyse réflexive des pratiques professionnelles, les interventions en groupe et les enjeux carriérologiques des adultes.

Ariane Froidevaux est professeure assistante au College of Business de l'Université du Texas à Arlington. Ses recherches et enseignements portent sur les transitions de carrière, le vieillissement au travail, l'identité et la gestion des ressources humaines. Après l'obtention de son doctorat au sein du Centre de recherche en psychologie du conseil et de l'orientation (CePCO) de l'Université de Lausanne, elle a travaillé comme chercheuse postdoctorale à l'Université de Floride avec le soutien du Fonds national suisse.

Geneviève Fournier est professeure en sciences de l'orientation et chercheure régulière au Centre de recherche et d'intervention sur l'éducation et la vie au travail (CRIEVAT) de l'Université Laval (Québec). Ses recherches et ses enseignements portent 
principalement sur les parcours professionnels, les transitions professionnelles et de vie, le rapport au travail ainsi que sur les modèles d'intervention en counseling individuel.

Fabian Gander est docteur en psychologie, assistant d'enseignement et de recherche senior et coordinateur de la formation postgrade en psychologie positive à l'Université de Zurich. Ses recherches portent sur l'évaluation des forces de caractère, sur les interventions visant à améliorer le bien-être, ainsi que sur le rôle des différences individuelles, telles que les forces de caractère, le rôle dans une équipe ou l'orientation vers le bonheur, sur le bienêtre au travail et les trajectoires professionnelles.

Maria Cristina Ginevra est professeure assistante au Département de philosophie, sociologie, éducation et psychologie appliquée de l'Université de Padoue (Italie). Elle collabore avec le La.R.I.O.S. (Laboratoire de recherche et d'intervention en orientation professionnelle) et le Centre universitaire sur le handicap et l'inclusion de l'Université de Padoue. Ses activités de recherche portent sur l'orientation et le coaching de carrière et sur la psychologie du handicap et de l'inclusion.

Jean Guichard est professeur émérite de psychologie au Conservatoire national des arts et métiers (CNAM, Paris). Ancien directeur de l'Institut national d'études du travail et d'orientation professionnelle (Inetop), il est président d'honneur du réseau UNITWIN et de la Chaire Unesco "pour l'accompagnement à l'orientation des personnes vers le travail décent et le développement durable». Ses recherches actuelles portent sur le rôle des dialogues de Life Design dans la construction de soi et sur l'accompagnement à l'orientation vers un développement humain, équitable et durable.

Philippe Handschin est membre du Centre de recherche en psychologie du conseil et de l'orientation (CePCO) et doctorant à l'Institut de psychologie de l'Université de Lausanne. Il y réalise une thèse sur les difficultés d'insertion professionnelle des jeunes adultes et participe à la validation de tests psychologiques. Il a enseigné les statistiques et les méthodes de recherche en psychologie. Ses intérêts portent notamment sur la psychologie de la personnalité et la sociologie des sciences. 
Levke Henningsen est post-doctorante en psychologie sociale et économique à l'Université de Zurich. Ses intérêts d'enseignement et de recherche comprennent la diversité de genre, le leadership des organisations, le rôle de l'autosélection et de la discrimination dans l'avancement professionnel des femmes et des hommes, et l'incivilité au travail. Sa thèse examine l'impact des facteurs individuels et contextuels dans la sous-représentation des femmes au sein des directions d'universités.

Jennifer Hofmann, PhD, est senior teaching et research fellow au Département de psychologie, section personnalité et évaluation, de l'Université de Zurich. Ses intérêts de recherche actuels couvrent la personnalité, l'évaluation et la psychologie positive, l'humour, les émotions positives, les interventions sur l'humour en milieu de travail et le comportement non verbal (application du Facial Action Coding System). Elle est responsable d'enseignements universitaires sur la personnalité, la théorie des tests, l'humour et les émotions positives, et propose des ateliers sur l'humour dans divers contextes.

Lily Konowitz est doctorante à la Lynch School of Education du Boston College (États-Unis). Étudiante en psychologie du conseil, elle est titulaire d'une maîtrise en théories du counseling. Elle a obtenu son bachelor à l'Université Bucknell en Pennsylvanie. Ses recherches portent notamment sur la manière dont les différentes opportunités de carrière permettent à une personne de trouver une finalité et donner un sens à sa vie.

Saliha Kozan a obtenu son PhD en psychologie du counseling au Boston College et sa maîtrise en counseling à la Michigan State University (États-Unis). En plus d'enseigner et de superviser des stagiaires psychologues depuis de nombreuses années, elle a dirigé des équipes de recherche dans le cadre de divers projets au Boston College. Elle a coécrit des articles scientifiques et des chapitres de livre sur la santé mentale, la psychologie du travail, la culture et la justice sociale. Elle est actuellement psychologue du counseling au Fawzia Sultan Healthcare Network (Koweït).

Yann Le Bossé est professeur titulaire à la Faculté d'éducation de l'Université Laval à Québec. Ses travaux se concentrent sur l'analyse des pratiques sociales et plus spécifiquement sur la contribution 
potentielle de l'approche centrée sur le pouvoir d'agir des personnes et des collectivités (DPA-PC). Il est l'auteur d'un grand nombre de publications scientifiques et de plusieurs ouvrages sur ce sujet, dont notamment un petit livret d'introduction destiné au grand public intitulé Soutenir sans prescrire, qui présente les fondements de cette conception de l'intervention.

Christian Maggiori a obtenu un $\mathrm{PhD}$ en psychologie à l'Université de Fribourg et a ensuite travaillé auprès de l'Office fédéral de la statistique, de l'Université Lausanne, ainsi que du Pôle de recherche national LIVES. Depuis 2014, il est professeur à la Haute École de travail social de Fribourg (HES-SO-HETS-FR). Ses intérêts de recherche portent notamment sur les trajectoires professionnelles, le bien-être au travail, la transition à la retraite, ainsi que la vieillesse et l'âgisme.

Giuseppe Martella est psychologue et psychothérapeute cognitivo-comportemental en formation. Ses intérêts de recherche et ses activités cliniques portent sur la mise en ouvre d'activités psychoéducatives et de réhabilitation pour les enfants et les jeunes en situation de handicap, en vue de leur inclusion scolaire et professionnelle.

Jonas Masdonati est professeur à l'Institut de psychologie et membre du Centre de recherche en psychologie du conseil et de l'orientation (CePCO) de l'Université de Lausanne, où il est également responsable du Service de consultations en conseil et orientation. Ses enseignements et recherches portent notamment sur la psychologie du counseling, les transitions et les reconversions professionnelles, l'insertion et la formation professionnelle, la construction identitaire et le rapport au travail.

Koorosh Massoudi, PhD, est maittre d'enseignement et de recherche à l'Institut de psychologie, membre du Centre de recherche en psychologie du conseil et de l'orientation (CePCO) de l'Université de Lausanne et chercheur avancé au Pôle de recherche national LIVES. Outre ses travaux de recherche qui visent l'identification des risques et des ressources au travail, il enseigne les méthodes d'évaluation et d'accompagnement psychologiques. Enfin, il intervient régulièrement en tant que consultant dans des institutions publiques et des entreprises privées aux prises avec les enjeux de la promotion de la santé au travail. 
Peter McIlveen est professeur et directeur de recherche du Australian Collaboratory for Career, Employability, \& Learning for Living (ACCELL), à la University of Southern Queensland. Il est membre de l'Australian Psychological Society, de la Career Development Association of Australia et de l'International Institute for Career Education and Counselling. Il siège aux comités éditoriaux du Journal of Vocational Behavior, du Journal of Career Assessment, du Career Development Quarterly et de l'International Journal of Educational and Vocational Guidance.

Laura Nota est professeure ordinaire au Département de philosophie, sociologie, éducation et psychologie appliquée de l'Université de Padoue (Italie). Elle est déléguée du recteur pour les questions liées au handicap, ainsi que présidente de la European Society for Vocational Designing and Career Counseling (ESVDC) et de la Société italienne pour l'orientation (SIO). Ses activités de recherche portent sur l'orientation et le coaching de carrière et sur la psychologie du handicap et de l'inclusion.

Jacques Pouyaud, PhD, est maître de conférences en psychologie à l'Université de Bordeaux (EA 4132). Il est aussi membre associé au Centre de recherche sur le travail et le développement (CRTDCNAM). Ses travaux portent sur l'étude des processus psychosociaux de construction des personnes en contextes scolaires et professionnels de transition, sur les processus diaboliques et relationnels de conseil, et sur l'efficacité des dispositifs d'orientation dans une perspective de Life Design.

Shékina Rochat, $\mathrm{PhD}$, est responsable des prestations pour la formation postobligatoire à l'Office cantonal d'orientation scolaire et professionnelle (canton de Vaud). Elle poursuit également des activités de recherche et d'enseignement dans le domaine de l'orientation sur la thématique des ressources psychologiques et de la résilience dans les trajectoires professionnelles. Elle s'apprête à publier un livre proposant une "carte» des interventions permettant de répondre aux différentes problématiques d'orientation.

Jérôme Rossier est professeur ordinaire en psychologie du conseil et de l'orientation à l'Université de Lausanne. Il est actuellement éditeur de la revue International Journal for Educational and Vocational 
Guidance. Ses thèmes d'enseignement et de recherche concernent la psychologie vocationnelle, de l'orientation, de la personnalité et interculturelle, ainsi que l'évaluation psychologique. Il a publié près de 200 articles scientifiques et chapitres d'ouvrage. Il est le coéditeur du Handbook of the life design paradigm: From practice to theory, from theory to practice (Hogrefe).

Sara Santilli, PhD, est chargée de recherche au Centre universitaire sur le handicap et l'inclusion de l'Université de Padoue (Italie). Elle est psychologue, thérapeute CBT et au bénéfice d'un diplôme postgrade en orientation de l'Université de Padoue, où elle collabore également avec La.R.I.O.S. Ses domaines de recherche couvrent l'orientation, la construction de carrière et l'inclusion professionnelle des personnes en situation de handicap ou pas. Elle est vice-présidente de la Société italienne pour l'orientation (SIO) et membre de la European Society for Vocational Designing and Career Counseling (ESVDC).

Marco Schiavetta est psychologue, psychothérapeute et docteur en sciences sociales. Il est actif dans l'éducation, la formation et l'orientation tout au long de la vie, collabore avec l'Université de Gênes (Disfor) et est enseignant de lycée. Il a publié Lavorando sapendo ascoltare: competenze di counseling per il sociale (2017), Transumanze generazionali: complessità e complessificazioni sociali (2018) et NEET study: Beliefs, fears and perceptions of the young stigmatized generations (2019), chez Aracne.

Simon-Pierre Tremblay est conseiller en orientation et doctorant en sciences de l'orientation à l'Université Laval (Québec). Il est également assistant de recherche au Laboratoire de recherche sur le développement du pouvoir d'agir des personnes et des collectivités (LADPA). Ses intérêts de recherche portent essentiellement sur la santé mentale au travail, la formation pratique et le développement du pouvoir d'agir des personnes et des collectivités.

Shagini Udayar est doctorante à l'Institut de psychologie et membre du Centre de recherche en psychologie du conseil et de l'orientation (CePCO) de l'Université de Lausanne. Ses recherches, menées dans le cadre du pôle de recherche national LIVES, portent principalement sur l'impact des vulnérabilités et ressources sur les 
trajectoires professionnelles des individus en Suisse. L'orientation avec une population multiculturelle et/ou migrante fait également partie de ses thèmes de recherche.

Ieva Urbanaviciute, $\mathrm{PhD}$, est chargée de recherche au sein du pôle de recherche national LIVES, à l'Université de Lausanne, où elle mène des analyses sur les effets à court et à long terme des vulnérabilités dans le monde du travail et des ressources individuelles sur le bien-être personnel et professionnel. Ses intérêts de recherche portent sur l'insécurité au travail, les conditions psychosociales de travail ainsi que sur les ressources de développement de carrière et le bien-être des employé.e.s.

Bernard Vallerie a exercé pendant vingt-cinq ans en tant qu'éducateur spécialisé. Puis, de 2004 à 2018, il a été maître de conférences à l'Université Grenoble Alpes. Chercheur statutaire dans l'équipe "Éducation familiale et interventions sociales auprès des familles" de l'Université Paris Nanterre et chercheur associé au Centre de recherche et d'intervention sur l'éducation et la vie au travail (CRIEVAT) de l'Université Laval de Québec, il a consacré ses travaux à l'accompagnement, par des intervenants sociaux, des personnes rencontrant des obstacles dans leurs parcours de vie. 


\section{LISTE DES ACRONYMES}

ACT

ARV

BBT

BIT

C.O.

CCI

CCId (système)

CCIdS (système) CDD

CEGEP

CMT

DCLD

DPA

DPA-PC

DSM

DWS

ECC

ESDE

FIS

FISA

FORJAD

FSE

HCR

I.S.

IDAC

ISA

JDR

LSF

MCC
Thérapie d'acceptation et d'engagement

Analyse des rôles de vie

Théorie d'expansion et de construction

(Broaden-and-Build Theory)

Bureau international du travail

Conseillers.ères en orientation

Career Construction Interview

Système de cadres cognitifs identitaires

Système des cadres cognitifs identitaires subjectifs

Contrat à durée déterminée

Collège d'enseignement général et professionnel

Théorie de la métaphore conceptuelle

(Conceptual Metaphor Theory)

Dialogues de conseil en Life Design

Développement du pouvoir d'agir

DPA personnel ou collectif

Manuel diagnostique et statistique

des troubles mentaux

Échelle de travail décent (Decent Work Scale)

Entretien de construction de carrière

Évolution de l'emploi

et de la situation sociale en Europe

Formes identitaires subjectives

Forme identitaire subjective anticipée

Formation pour les jeunes adultes en difficulté

Fonds social européen

Haut Commissariat des Nations unies

pour les réfugiés

Interactionnisme stratégique

Introduction d'une démarche

d'action conscientisante

Inventaire du système des activités

Modèle des exigences et ressources au travail

(Job Demand-Rressources Model)

Living System Framework

My Career Chapter 
MIS

N-CAPS

NEET

OCDE
OFAS
OFFT
OIM
OIT (ILO)

OMS
OSP
P-CO
PANAS
PPIs
PPTD
PRISMA
PWF

PWT

PYD

RH

SDT

SIB

TCC

TSA

TSD (DST)

VAE

VIA
Mesure d'insertion sociale

Système d'incitation non linéaire sensible au contexte

(nonlinear contextually aware prompting system) Not in Employment, Education or Training (ni en emploi, ni en formation, ni en stage)

Organisation de coopération

et de développement économiques

Office fédéral des assurances sociales

Office fédéral de la formation professionnelle et de la technologie

Organisation internationale pour les migrations

Organisation internationale du travail

(International Labour Organization)

Organisation mondiale de la santé

Orientation scolaire et professionnelle

Psychologues conseillers.ères en orientation

Positive and Negative Affect Schedule

Positive Psychology Interventions

Programmes par pays de promotion

du travail décent

Fondation d'investissement à but non lucratif

Cadre théorique de la psychologie de l'activité

de travail (Psychology of Working Framework)

Théorie de la psychologie de l'activité de travail

(Psychology of Working Theory)

Développement positif des jeunes

(Positive Youth Development)

Ressources humaines

Théorie de l'autodétermination

(Self-Determination Theory)

Échelle des conduites interpersonnelles

(Scale for Interpersonal Behavior)

Thérapie cognitivo-comportementale

Troubles du spectre autistique

Théorie du soi dialogique

(Dialogical SelfTheory)

Validation des acquis de l'expérience

Inventaire des valeurs en action

(Values-in-Action) 


\section{LEXIQUE}

Acculturation (Chapitre 6). Issu du domaine de l'anthropologie sociale et culturelle, ce concept est employé en psychologie pour désigner et étudier les changements chez l'individu en termes d'identité et pratique culturelles suite au contact direct et continu avec une culture différente, et la manière dont ces changements affectent son bien-être psychologique et son fonctionnement social.

Adaptabilité de carrière (Chapitre 2). Ressources mobilisées par l'individu à travers son parcours professionnel pour s'acquitter des tâches de développement actuelles et à venir, gérer les transitions en matière d'emploi et faire face aux traumas professionnels. Ces ressources se subdivisent en quatre dimensions principales, que sont (1) la capacité à être conscient de son futur vocationnel et à le planifier (concern), (2) le sentiment de contrôle et la prise de responsabilité quant à son futur vocationnel (control), (3) la tendance à activement explorer différents "soi» et scénarios possibles (curiosity) et (4) la confiance quant à ses capacités à faire face aux défis et exigences du parcours professionnel (confidence).

Adaptation à la retraite (Chapitre 4). Processus au travers duquel les individus s'habituent aux changements intervenus dans leur vie quotidienne pour atteindre un certain niveau de bien-être psychologique.

Adulte émergent.e (Chapitre 1). Période de la vie entre 18 et 25 ans (s'étendant parfois jusqu'à la fin de la vingtaine) durant laquelle la personne explore des activités variées, en bénéficiant du fait d'être moins soumise à des contraintes normatives et à des 
attentes sociales qu'à d'autres moments de sa vie. Cela offre également davantage de latitude dans la construction de soi. Durant cette période, l'individu apprend progressivement à faire des choix concernant son avenir et à gérer sa vie professionnelle.

Alliance de travail (Chapitre 12). L'alliance de travail caractérise le lien affectif entre conseiller-ère et client·e, ainsi que leur degré d'accord quant aux objectifs et aux moyens de les réaliser. Elle se crée par un dialogue intime dans lequel les client.e.s racontent leur histoire, ce qui facilite l'exploration du parcours professionnel et des perspectives de carrière. Processus fondamental du counseling, la qualité de l'alliance de travail prédit l'efficacité d'une intervention.

Analyse des rôles de vie (ARV) (Chapitre 2). Inspirée des approches constructivistes et narratives de l'orientation, cette méthode d'intervention vise à aider les personnes à adopter une posture distanciée et à développer une réflexion au-delà du domaine professionnel, invitant ainsi à l'exploration et à l'intégration des autres domaines de vie. L'analyse de l'évolution passée ou projetée des différents rôles de vie, ainsi que de leurs interrelations, y occupe une place importante.

Approche inclusive du counseling (Chapitre 7). Approche du counseling qui s'adresse à tous les individus, incluant les personnes en situation de vulnérabilité ou de handicap. L'objectif des interventions s'appuyant sur cette approche est de proposer des lieux conviviaux pour tou.te.s et de favoriser la participation de chacun.e à la vie sociale et civile, en misant sur l'action de l'ensemble des citoyen-enne.s et des institutions.

Approche intégrant différents domaines de vie (domain sensitive approach) (Chapitre 8). Approche de l'intervention de counseling qui invite à considérer l'ensemble des sphères d'existence de l'individu. Cela implique de favoriser son bien-être dans les différents domaines de vie et de l'aider à gérer ses problèmes professionnels tout en favorisant son développement personnel et interpersonnel.

Approche narrative du counseling (Chapitre 12). Approche qui considère le soi en tant qu'identité narrative et qui reflète une conceptualisation du counseling de carrière et de l'identité en 
termes de récit. Les client.e.s sont ainsi considéré.e.s comme des narrateurs.rices dont le discours permet de créer du sens. Le dialogue entre conseillers.ères et client.e.s permet de créer un espace interpersonnel favorisant la mise en récit de soi.

Bien-être au travail (Chapitre 9). Construit multidimensionnel qui regroupe aussi bien une composante subjective ou hédonique recouvrant les affects positifs et la satisfaction au travail qu'une composante psychologique ou eudémonique, qui relève quant à elle du sentiment d'avoir un sens au travail, de réaliser son potentiel à travers ses activités professionnelles et de construire des relations interpersonnelles satisfaisantes.

Bilan de compétences (Chapitre 3). Démarche d'accompagnement d'un.e adulte en transition professionnelle pouvant se diviser en trois phases: rétrospective (analyse du déroulement de la vie de la personne); présente (exploration des valeurs, intérêts, image de soi, compétences, etc.); prospective (projection dans l'avenir et formulation d'un projet).

Capital psychologique (Chapitre 9). «État de développement psychologique positif de l'individu, caractérisé par (1) la confiance en ses capacités à engager et maintenir les efforts nécessaires pour venir à bout de tâches exigeantes (efficacité personnelle); (2) l'attribution positive des causes des succès actuels et à venir (optimisme); (3) la persévérance dans la poursuite des objectifs afin de réussir (espoir); et (4), lorsque confronté à l'adversité, la capacité à rebondir et à dépasser les difficultés pour réussir (résilience)» (Luthans et al., 2006, p. 388, traduction personnelle).

Carrières tardives (late careers) (Chapitre 4). Nouvelles formes de carrière concernant les individus dès 55 ans et jusqu'au-delà de 65 ans.

Coaching de carrière (Chapitre 7). Forme d'intervention visant à favoriser la performance et le développement personnel par la planification et la réalisation d'objectifs de carrière. Plus spécifiquement, selon l'approche Life Design, le coaching de carrière fait référence à un accompagnement de l'individu dans son développement personnel et professionnel qui met l'accent sur l'action et la 
résolution de problèmes. Il permet à la personne d'identifier et de développer les ressources et les compétences nécessaires à l'atteinte de ses objectifs.

Conciliation des domaines de vie (Chapitre 2). Stratégies mobilisées par la personne pour faire face à ses différents rôles et responsabilités et pour favoriser les interactions et gérer les interdépendances entre ses différents domaines de vie, notamment le travail, la famille, la vie sociale et associative, les études ou les loisirs.

Conscience critique (Chapitre 8). Processus par lequel des personnes opprimées analysent de manière critique leur situation sociopolitique et agissent pour stimuler un changement. La conscience critique se divise en trois composantes: la réflexion critique, l'efficacité politique et l'action critique.

Démarche d'action conscientisante (Chapitre 10). Paradigme d'accompagnement qui, pour susciter un changement plus global et durable, vise à permettre à la personne accompagnée de tirer des enseignements de l'analyse de ses actions pour prendre conscience de la complexité des éléments qui contribuent à ses difficultés et de la nécessité d'agir à différents niveaux.

Développement du pouvoir d'agir (DPA, empowerment) (Chapitres 8 et 10). "La capacité (1) à identifier des objectifs, (2) à développer et utiliser au mieux les ressources personnelles pour atteindre ces objectifs et (3) à négocier avec l'environnement les ressources nécessaires pour les atteindre" (Richardson, 2000, p. 204, traduction personnelle). Selon la définition donnée par le Laboratoire de recherche sur le développement d'agir des personnes et des collectivités de l'Université Laval, il s'agit d'un processus par lequel des personnes accèdent ensemble ou séparément à une plus grande possibilité d'agir sur ce qui est important pour elles-mêmes, leurs proches ou la collectivité à laquelle elles s'identifient.

Empathie culturelle (Chapitre 6). Capacité à identifier et à saisir les valeurs et les attentes caractéristiques de la culture d'origine de son interlocuteur et à comprendre ses raisonnements, émotions ou attitudes en référence à celles-ci. 
Emploi-pont (Chapitre 4). Nouvel emploi se situant entre le départ de l'emploi exercé dans la "carrière principale» d'un individu avant son passage à la retraite et son désengagement complet du monde du travail.

Entretien de construction de carrière (ECC) (Chapitre 1). Entretiens de conseil qui ont pour objectif d'aider la ou le demandeur.se à s'appuyer sur certaines de ses caractéristiques personnelles (son caractère, sa personnalité, ses intérêts) pour faire face au problème d'orientation auquel elle ou il est confronté.e.

Formes identitaires subjectives (FIS) (Chapitres 1 et 4). Une FIS correspond à l'activation-actualisation d'un cadre cognitif identitaire subjectif (CCIdS) dans une circonstance donnée. Les FIS d'un individu correspondent à l'ensemble de ses représentations de soi dans différents contextes d'interaction. Elles renvoient donc à la manière dont une personne se voit, se pense et parle d'ellemême dans un rôle social donné, en relation avec son passé et son futur.

Indécision vocationnelle (Chapitre 11). Difficultés - pouvant parfois mener à l'incapacité - à effectuer des choix de profession ou de formation. Ces difficultés peuvent avoir différentes sources et raisons, comme le manque de motivation à s'engager dans un choix ou des croyances dysfonctionnelles et des craintes excessives quant aux conséquences du choix, un manque de connaissance de soi ou des options disponibles, ou encore la difficulté à dépasser une ambivalence personnelle ou à concilier des attentes externes contradictoires.

Insertion professionnelle (Chapitre 5). Désigne à la fois (1) un processus comprenant un ensemble de démarches réalisées par l'individu afin d'obtenir un rôle et une place stables dans le marché du travail, et (2) sa capacité à exercer une activité professionnelle et à fonctionner dans le monde du travail. De par sa contribution en matière d'intégration et de socialisation, l'insertion professionnelle fait souvent partie des objectifs visés par les politiques sociales.

Intégration socioprofessionnelle (Chapitre 6). Processus qui mène l'individu, à travers l'acquisition des compétences nouvelles et 
nécessaires, à s'adapter à un nouveau contexte social et à accéder de manière durable au marché du travail.

Intelligence émotionnelle (Chapitre 11). Capacité à percevoir, à intégrer, à comprendre et à gérer ses émotions.

Interactionnisme stratégique (Chapitre 10). Approche qui privilégie l'étude des interactions entre les acteurs.rices impliqué.e.s dans une situation donnée, en accordant une place particulièrement importante à leurs enjeux spécifiques (ce que chacun·e veut obtenir et éviter) et à la manière dont ceux-ci sont négociés.

Inventaire du système des activités (ISA) (Chapitre 2). Outil d'exploration de quatre domaines de vie fondamentaux - professionnel, familial, social et personnel - dont le but est d'initier une réflexion chez la personne sur les interrelations entre ces domaines et sur la manière dont elle désire les prioriser et s'y investir.

Life Design (Chapitres 1, 2 et 7). Approche qui propose, depuis 2009, une réflexion sur les modalités d'accompagnement à l'orientation permettant de faire face aux défis de la construction de soi dans les sociétés de la modernité tardive. Une de ces modalités introduit les dialogues de conseil en Life Design, dont l'objectif est d'aider les personnes à déterminer des perspectives d'avenir qui donnent du sens à leur vie et à s'engager dans leur réalisation.

Ligne de vie (Chapitre 3). Outil qualitatif qui s'inscrit dans les approches constructivistes et narratives de l'orientation et qui vise à retracer l'histoire de la personne et à y ancrer ses projets d'avenir. Elle permet de repérer, de revisiter et de donner un sens aux périodes et aux événements marquants de la vie.

Métaphore (Chapitre 12). Plus qu'une figure de style, la métaphore se retrouve constamment dans le discours, la pensée et la communication. De ce fait, elle peut être utilisée dans l'accompagnement d'orientation afin de comprendre, déconstruire et reconstruire les idées sur la carrière.

Migrant.e.s (Chapitre 6). Personnes qui s'expatrient dans l'objectif d'améliorer leurs conditions. 
Modèle des exigences et ressources au travail (Job DemandsRessources model) (Chapitre 9). Modèle théorique qui se base sur l'interaction entre les exigences émanant de l'environnement organisationnel et les ressources à disposition des employé.e.s pour expliquer leur bien-être au travail, aussi bien en termes d'indicateurs positifs, comme la motivation et l'engagement, qu'en termes d'indicateurs négatifs, comme l'épuisement et le burnout.

Modèle social du handicap (Chapitre 7). Modèle qui considère que les difficultés rencontrées par une personne dans la vie sociale et quotidienne sont déterminées par un écart entre ses caractéristiques et les contraintes contextuelles. Cet écart peut être réduit en modifiant les exigences du contexte et en apportant du soutien à la personne.

Mon chapitre de carrière (My Career Chapter) (Chapitre 12). Intervention semi-dirigée pour l'évaluation et l'accompagnement en orientation, où il est demandé aux client.e.s de rédiger une brève autobiographie en lien avec leur carrière en complétant des phrases telles que "mes principaux intérêts sont...». Cet exercice donne lieu à un récit écrit et oral de la carrière qui sert de support au dialogue entre client.e et conseiller.ère.

NEET (Not in education, employment or training) (Chapitre 5). Acronyme d'origine britannique désignant la catégorie des jeunes adultes, généralement de 15 à 29 ans, qui ne sont ni en éducation, ni en emploi, ni en stage. Même si cette situation soulève un problème sociétal en augmentation - celui de la vulnérabilité croissante des jeunes adultes face au marché de l'emploi -, elle est le plus souvent temporaire et concerne des individus aux parcours et conditions de vie très diverses.

Normes/rôles de genre (Chapitre 2). Croyances et attentes quant aux rôles sociaux différents attribués aux hommes et aux femmes. Selon la théorie des rôles sociaux (social role theory), la distribution inégale des femmes et des hommes dans diverses professions ainsi que dans le travail non rémunéré renforcent ces normes et les stéréotypes de genre.

Psychologie positive (Chapitre 9). Courant de la psychologie qui se donne comme objectif l'étude scientifique des conditions et des 
processus qui contribuent au développement, à l'épanouissement et au fonctionnement optimal des individus, des communautés et des institutions.

Psychologisation (Chapitre 5). Au sens large, il s'agit de la réduction d'un phénomène ou d'un individu à des dimensions psychologiques, notamment en privilégiant les causes intrinsèques et individuelles des comportements au détriment des influences sociales, contextuelles et culturelles. Le terme est aussi employé pour dénoncer les dérives des politiques sociales visant essentiellement à surresponsabiliser les personnes en situation de précarité.

Reconversion professionnelle (Chapitre 3). Transition vers un nouvel emploi qui éloigne l'individu d'une progression de carrière typique et attendue. Elle comporte un changement d'employeur.se et la modification des activités et rôles au travail. Les compétences déployées dans l'ancien emploi deviennent en partie caduques, et la personne est donc obligée à entreprendre de nouveaux apprentissages, formels ou informels.

Transition à la retraite (Chapitre 4). Sortie du monde du travail, accompagnée par une diminution de l'engagement psychologique de l'individu envers le travail et par un retrait comportemental.

Travail décent (Chapitre 8). Selon la théorie de la psychologie de l'activité de travail (Psychology of Working Theory), le travail décent est un emploi offrant des conditions de travail sécuritaires, des horaires permettant du repos et laissant suffisamment de temps libre, des valeurs en harmonie avec les valeurs familiales et communautaires, une rémunération adéquate, ainsi que l'accès à des soins de santé. Un travail décent est censé permettre de satisfaire les besoins de base de survie, de lien social et d'autodétermination.

Équilibre vie privée-vie professionnelle (work-life balance) (Chapitre 2). Concept couvrant l'ensemble des domaines de vie d'une personne et défini comme «l'évaluation globale du degré de cohérence entre l'efficacité et la satisfaction d'une personne dans ses rôles professionnels et familiaux et ses valeurs personnelles à un moment donné» (Greenhaus \& Allen, 2011, p. 174, traduction personnelle). 


\section{TABLE DES MATIÈRES}

Jean-Luc Bernaud

Préface ................................... 7

Koorosh Massoudi \& Jonas Masdonati

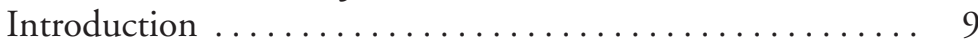

\section{PREMIÈRE PARTIE. ORIENTATION ET PARCOURS}

Valérie Cohen-Scali, Jacques Pouyaud et Jean Guichard

1. Les méthodes de Life Design pour la construction de soi et l'orientation professionnelle des adultes émergent.e.s . . . . . 19

Grégoire Bollmann, Eva Clot-Siegrist et Levke Henningsen

2. Conciliation des domaines de vie:

le rôle du contexte social et des ressources d'adaptabilité . . . . 49

Jonas Masdonati, Sylvie Franz et Marc Abessolo

3. Comprendre et accompagner

les reconversions professionnelles . . . . . . . . . . . . . . . . . 79

Ariane Froidevaux et Christian Maggiori

4. Transitions à la retraite ........................ 111

DEUXIÈME PARTIE. ORIENTATION ET VULNÉRABILITÉS

Philippe Handschin et Marco Schiavetta

5. Linsertion professionnelle des jeunes adultes ni en emploi, ni en formation, ni en stage (NEET) . ................ 145 
Kokou A. Atitsogbe, Shagini Udayar et Federico Durante 6. Orientation et insertion en contexte migratoire . . . . . . 173

Ilaria Di Maggio, Sara Santilli, Maria Cristina Ginevra, Giuseppe Martella et Laura Nota

7. Inclusion, Life Design, coaching de carrière et handicap. . . 207

Saliha Kozan, Jonas Masdonati, Lily Konowitz et David L. Blustein

8. Le travail décent: une ambition fondamentale pour la psychologie de l'orientation

\section{TROISIÈME PARTIE. ORIENTATION ET RESSOURCES}

Koorosh Massoudi, Ieva Urbanaviciute, Jennifer Hoffman et Fabian Gander

9. Des vulnérabilités aux vertus: une approche positive du bien-être au travail .

Yann Le Bossé, Bruno Bourassa, Manon Chamberland, Geneviève Fournier, Simon-Pierre Tremblay et Bernard Vallerie 10. Du bon usage de l'incertitude: un exemple d'application de l'approche centrée sur le développement du pouvoir d'agir des personnes et des collectivités (DPA-PC) au domaine de l'orientation . . . . . . . . . . . . . . . . . . 297

Shékina Rochat et Éloïse Banet

11. Choix professionnel, indécision et émotions . . . . . . . 331

Peter McIlveen et Allison Creed

12. La conversation, métaphore de l'approche narrative

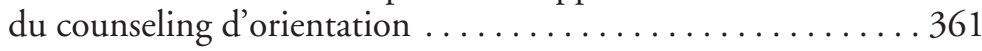

Jérôme Rossier et Jean-Pierre Dauwalder Conclusion.............................. 385

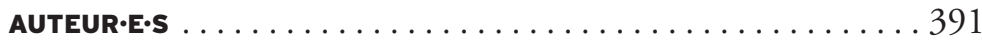

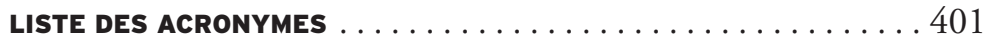

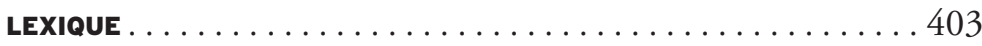




\section{AUX ÉDITIONS ANTIPODES \\ CATALOGUE COMPLET SUR WWW.ANTIPODES.CH}

\section{ACTUALITÉS PSYCHOLOGIQUES}

Samuel Berthoud, 50 ans de

l'Institut de psychologie (1968-2018), 2018.

Christiane Moro, Nathalie Müller

Mirza et Pascal Roman (dir.),

L'intersubjectivité en questions.

Agrégat ou nouveau concept

fédérateur pour la psychologie?,

2014.

Valentino Pomini, Yves de Roten,

Fabrice Brodard et Vincent

Quartier (dir.), L'étude de cas.

Dialogue entre recherche et pratique

en psychologie clinique

et en psychothérapie, 2016.

Christian Staerklé et Fabrizio

Butera, Conflits constructifs, conflits destructifs. Regards psychosociaux, 2017.

\section{BoÎTE À OUTILS}

Pascal Roman, L'adolescence, un passage. Guide à destination des parents et des professionnel.le.s, 2018.

\section{REGARDS ANTHROPOLOGIQUES}

Giada de Coulon, Lillégalité régulière. Ethnographie du régime de l'aide d'urgence en Suisse, 2019.

Laurence Ossipow, Marc-Antoine Berthod, Gaëlle Aeby, Les miroirs de l'adolescence. Anthropologie du placement juvénile, 2014.

\section{LE LIVRE POLITIQUE - CRAPUL}

Olivier Fillieule, Vanessa Monney et Hervé Rayner, Le métier et la vocation de syndicaliste.

L'enquête suisse, 2019.

David Pichonnaz, Devenirs policiers. Une socialisation professionnelle en contrastes, 2017.

\section{HISTOIRE}

Jacqueline Heinen... et 110 autres, 1968... des années d'espoirs, 2018.

Geneviève Heller, Ceci n'est pas une prison. La maison d'éducation de Vennes. Histoire d'une institution pour garçons délinquants en Suisse romande (1805-1846-1987), 2012. 
André Holenstein, trad. Marianne Enckell et Diane Gilliard, Au cœur de l'Europe. Une histoire de la Suisse entre ouverture et repli, 2018.

Sarah Kiani, De la révolution féministe à la Constitution. Mouvement des femmes et égalité des sexes en Suisse (1975-1995), 2019.

Anne-Françoise Praz, De l'enfant utile à l'enfant précieux.

Filles et garçons dans les cantons de Vaud et Fribourg (1860-1930), 2005.

Martine Ruchat, Edouard Claparède (1873-1940). À quoi sert l'éducation?, 2015.

Caroline Rusterholz, Deux enfants, c'est déjà pas mal. Famille et fécondité en Suisse (1955-1970), 2017.

Carole Villiger, Le choix de la violence en politique. Témoignages, 2019.

Carole Villiger, Usages de la violence en politique (1950-2000), 2017.

\section{HISTOIRE MODERNE}

Daniel Tröhler, Pestalozzi et le "tournant pédagogique», 2016.

\section{HISTOIRE.CH}

Alain Clavien, La presse romande, 2017.

Damir Skenderovic et Christina Späti, Les années 68, 2012.

\section{MÉDIAS ET HISTOIRE}

Izabella Pluta (dir.), Salle d'attente de Krystian Lupa. Création et transmission, 2019.

Dominique Vinck, Les métiers de l'ombre de la Fête des vignerons, 2019.

\section{UNIVERS VISUELS}

Gianni Haver, Jean-François Fayet, Valérie Gorin et Emilia Koustova, Le spectacle de la Révolution.

La culture visuelle des commémorations d'Octobre, 2017.

\section{HISTOIRE}

\section{ET SOCIÉTÉ CONTEMPORAINES}

Hadrien Buclin, Les intellectuels de gauche. Critique et consensus dans la Suisse d'après-guerre (1945-1968), 2019.

Malik Mazbouri et François Vallotton, Scandale et histoire, 2016.

\section{REVUE HISTORIQUE VAUDOISE}

Éducation et société, No 117, 2009.

\section{CONTRE-PIED}

Lorenzo Tomasin, L'empreinte digitale. Culture humaniste et technologie, 2018. 


\section{EXISTENCES ET SOCIÉTÉ}

Roland J. Campiche

et Yves Dunant (dir.), À la retraite, les cahiers au feu? Apprendre tout au long de la vie. Enjeux et défis, 2018.

Roland J. Campiche et Afi Sika Kuzeawu, Adultes aînés: les oubliés de la formation, 2014.

Alexandre Dubuis, Grands brûlés de la face. Epreuves et luttes pour la reconnaissance, 2014.

Chris Durussel, Étienne Corbaz, Émilie Raimondi et Marisa Schaller (dir.), Pages d'accueil. Vingt-ans de classes post-obligatoires dans le canton de Vaud, 2012.

Arnaud Frauenfelder, Christophe Delay et Laure Scalambrin, "Joindre l'utile à l'agréable». Jardin familial et modes de vie populaires, 2015.

Françoise Gavillet-Mentha, Un métier désenchanté. Parcours d'enseignants secondaires 1970-2010, 2011.

Nadia Lamamra et Jonas Masdonati, Arrêter une formation professionnelle. Mots et maux d'apprenti.e.s, 2009.

Marylène Lieber, Ellen Hertz et Janine Dahinden (dir.), "Cachez ce travail que je ne saurais voir." Ethnographies du travail du sexe, 2010.
Lorraine Odier, Métamorphoses de la figure parentale. Analyse des discours de l'école des parents de Genève (1950-2010), 2018.

Marion Repetti, Les bonnes figures de la vieillesse. Regard rétrospectif sur la politique de la vieillesse en Suisse, 2018.

Marc Perrenoud et Pierre Bataille, Vivre de la musique?

Enquête sur les musicien.ne.s et leurs carrières en Suisse romande (2012-2016), 2019.

Valérie Rolle et Olivier Moeschler, De l'école à la scène. Entrer dans le métier de comédien.ne, 2014.

Magdalena Rosende et Natalie Benelli (dir.), Laboratoires du travail, 2008.

\section{ANTILOGIES \\ ÉCRITS PHILOSOPHIQUES}

Jean-François Aenishanslin, Les pensées parallèles.

Husserl et Freud, 2019.

\section{NOUVELLES QUESTIONS FÉMINISTES \\ Vol. 38/2, 2019, Le physique de l'emploi.}

\section{A CONTRARIO}

Martine Ruchat, Le "Roman de Solon". Enfant placé Voleur de métier. 1840-1896, 2008. 
Impression

La Vallée - Aoste

Février 2020 